\title{
Impulse Mitigation of Structures Impacted by Granular Media
}

\author{
A Dissertation \\ Presented to \\ the faculty of the School of Engineering and Applied Science \\ University of Virginia

\begin{abstract}
in partial fulfillment
of the requirements for the degree

Doctor of Philosophy
\end{abstract} \\ by \\ Anne Fay Kyner \\ August 2017
}




\section{APPROVAL SHEET}

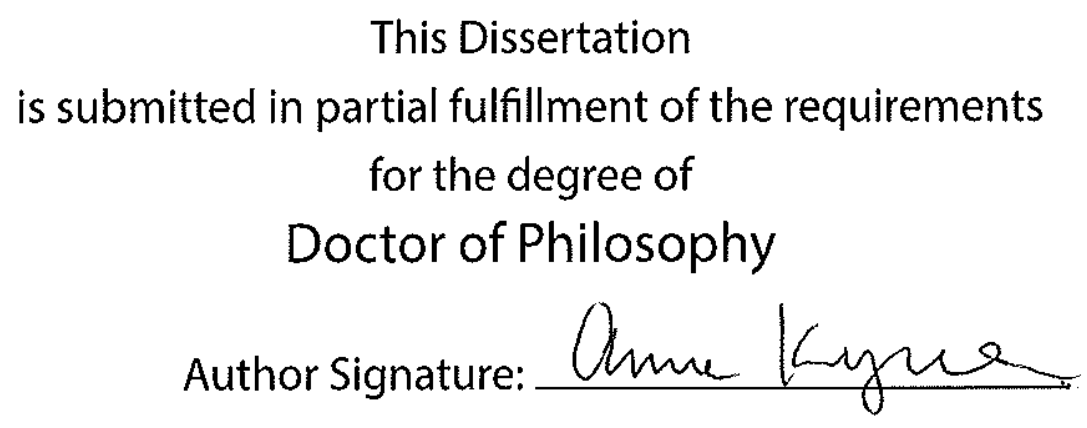

This Dissertation has been read and approved by the examining committee:

Advisor: Haydn Wadley

Committee Member: James Fitz-Gerald

Committee Member: Vikram Deshpande

Committee Member: Robert Hirosky

Committee Member: Matthew Panzer

Committee Member:

Accepted for the School of Engineering and Applied Science:

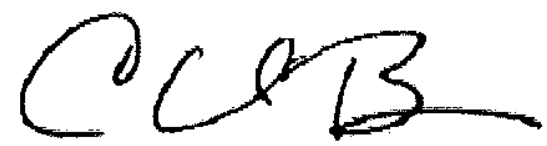

Craig H. Benson, School of Engineering and Applied Science

August 2017 


\section{Executive Summary}

The momentum and pressure applied to stationary structures by the impact of a high velocity granular medium such as soil that has been accelerated by a buried explosion, is a subject of considerable scientific and technical interest. During the detonation of an explosive material, the (solid or liquid) energetic material is converted to gaseous detonation products across a detonation front that propagates at high velocity $(4-10,000 \mathrm{~m} / \mathrm{s})$ across the energetic material. This front separates the condensed energetic material at ambient temperature and pressure from its gaseous detonation products at high temperature and pressure. The momentum of the expanding gas is transferred to particles of the surrounding granular media causing granular compaction across an outward propagating shock front. Upon reflection of the shock at the air/granular medium interface, granular particles are accelerated away from the soil surface and can impact a nearby structure, transferring all or a part of their momentum to the structure. If strong particle reflection occurs, conservation of momentum shows that it is possible to transfer more than the incident particles momentum to the impacted structure. This granular impact process results in the development of a pressure load distribution on the structure that can be sufficient to initiate elastic, and in some cases, high rate plastic deformation which is sometimes sufficient to initiate dynamic fracture.

Efforts to design impulse resistant structures has led to interest in understanding the mechanisms by which momentum is transferred to a structure following the detonation of a buried explosive. Recently discrete particle contact approaches have begun to be investigated for developing numerical methods since they have the potential to accurately implement the physics of granular media acceleration and momentum transfer. A particle based method implemented in the IMPETUS Afea Solver code allows fully coupled calculations with discrete particles and finite element models in which particle/structure interactions can be directly analyzed. This dissertation has investigated the validity of this approach by performing a simulation of an experiment in which a well-defined explosive/granular particle test charge system is used to load an edge clamped 
square, ductile 304 stainless steel plate whose dynamic properties are well characterized. The test charge system consisted of a $3 \mathrm{~kg}$ mass sphere of high explosive encased by a concentric sphere of water-saturated $\sim 200 \mu \mathrm{m}$ diameter glass microspheres (synthetic wet sand) with a mass of 23.8 $\mathrm{kg}$ (18.8 kg of dry sand). This charge was suspended above the $2.54 \mathrm{~cm}$ thick, $1.32 \mathrm{~m} \mathrm{x} 1.32 \mathrm{~m}$, edge gripped 304 stainless steel test plate, and the time dependent motion of the surface of the granular medium was measured using high-speed video imaging techniques. The pressure applied by impact of the granular medium was directly measured using a Kolsky bar technique and found to be well approximated by the hydrodynamic pressure $\rho v^{2}$ (where $\rho$ is the granular media density and $v$ its incident velocity) created by stagnation of the granular medium against a stationary target. Both sets of measurements were well predicted by the simulation approach. The final deflected shape of the test plate was also predicted and compared very well with the measured distribution. The structure used to grip the test plate was discovered to have suffered significant strain (resulting in partial fracture during later testing). The simulation approach was used to investigate the mechanisms of failure to successfully design an improved testing facility.

A series of five experimental tests were then performed with suspended water-saturated sand charges of systematically increased explosive and granular media mass using the same target plate system. For some of the tests, the glass microspheres were replaced with higher density zirconia particles to enable higher granular mass charges to be used. The total charge mass varied from $\sim 25$ to $150 \mathrm{~kg}$. The motion of each spherical test charge following detonation was again tracked by high-speed video imaging. The expansion velocity of the granular media varied from approximately $500-1200 \mathrm{~m} / \mathrm{s}$ and was dependent upon the ratio of the granular particle to high explosive mass. The test charges with a lower mass ratio had the highest sand front expansion velocities and exhibited a sand fingering instability phenomena at the leading edge of their hypersonic fronts. As the mass ratio increased, the expansion velocity of the granular front decreased, and the sand fingering instability gradually disappeared. For the highest mass ratio zirconia (higher density granular media) charges, this instability was almost absent at the leading edge of these more slowly expanding sand fronts (velocities 500-600 m/s).

The impulsive loading response for each of the five test charges was characterized by the Kolsky bar technique and four regions of loading by granular impacts were identified. These corresponded directly to impacts by various regions of the granular media. As the spherical sand 
shell expanded (stretched) and suffered momentum transfer with surrounding air particles, density and velocity gradients developed within the expanding volume. Examination of the simulations helped to resolve these regions of loading and corresponding impacts by the different particle types (air, sand, and high explosive particles).

Recent studies of the impulsive loading of edge clamped panels has shown that some sandwich panel constructions have a higher deflection resistance than solid equivalent (same mass per unit area and material type) plates. Since reduction of the dynamic deflection is an important goal of many blast resistant target designs, this potential mitigation strategy was investigated. Square honeycomb sandwich panels with identical mass per unit area to the $2.54 \mathrm{~cm}$ thick solid plates were fabricated from 304 stainless steel by machining square pockets from a thick plate and using an electron beam welding method to attach a rear face sheet. These experiments confirmed that permanent plastic deflections could be reduced by the use of sandwich panel constructions. The strong core of the square honeycomb sandwich panel resulted in very little core compression during loading. The main structural response benefit was attributed to the increased bending and stretching resistance of the sandwich panel design. The IMPETUS Afea code was then used to simulate all the target structural responses and investigate the phenomena governing the target dynamic response.

Motivated by recent laboratory scale studies at the University of Cambridge (UK), the effect of target inclination (obliquity of impact) upon momentum transfer was investigated using a vertical impulse test rig and shallow buried planar explosive charges. These experimental studies and subsequent simulations revealed that substantial momentum transfer reductions could be achieved when the granular medium was not stagnated against a test structure. However, the experiments at Cambridge University showed that more momentum was transferred to the test structure than predicted by a simple resolution of momentum onto the inclined surface. The extra transferred momentum resulted from frictional interactions of the sand particles with the target surface. In the resolved frictionless limit, the resolved vertical impulse transferred to the target depended on the angle of inclination, and the ratio of transferred and incident impulse is $I_{T} / I_{O}=$ $\sin ^{2} \alpha$.

A novel impulse reduction concept was then investigated both experimentally and with validated simulations. This concept used a lubricated (low dynamic friction) sliding plate attached 
to the inclined $\mathrm{V}$-shaped target (whose impacted surface were inclined at $53^{\circ}$ angle to the soil impact direction) and was found to offer significant reductions in impulse transfer beyond that of surface inclination. An impulse reduction of approximately 17\% was achieved when impact surfaces were inclined from a $90^{\circ}$ (normal) to $53^{\circ}$ angle from the incident granular media. An additional $11 \%$ reduction was then achieved when sliding plates were attached to these inclined $\left(53^{\circ}\right)$ surface. This dissertation explores the response of this new target design and uses the simulation solver to explore the efficiency of the sliding plates through a parametric study. This study explored the effects of the sliding plate mass, explosive charge mass, and the number of attached (stacked) sliding plates on the impulse reduction. These parametric studies revealed that reductions in the transferred impulse could be achieved by increasing the sliding plate mass and that increasing the number of stacked plates resulted in approximately the same benefit (impulse reduction) achieved by increasing the plate mass. The impulse reduction achieved by the sliding plates was also observed for sand accelerated by higher explosive charge masses, indicating the efficiency of the sliding plates as the applied impulse loading is increased. 


\section{Acknowledgements}

I would like to thank my advisor, Haydn Wadley, for all his mentoring and encouragement the last few years. He has taught me so much about the research process and exploring all the limitations of the simulation approach. I could not have done this without his advice and guidance.

This research was funded by the Defense Advanced Research Projects Agency (DARPA) under grant number W91CRB-11-1-0005 (Program manager, Dr. J. Goldwasser). I am very grateful for the opportunity to work on this project, which has inspired me to continue working in the field of energetic materials. I am also extremely grateful for all the opportunities I had for expanding my knowledge base with various trainings for the IMPETUS Afea Solver and Hyperworks (Altair).

I would like to acknowledge my committee members, James Fitz-Gerald, Vikram Deshpande, Robert Hirosky, and Matt Panzer, for their advice and review of my work.

I would also like to acknowledge Kumar Dharmasena for all his help with target design and fabrication and mentoring me as a new graduate student. There were many discussions about experimental design and expectations that really aided and guided me and my research.

This project was made possible with the help of Keith Williams and NEWTEC Services. Thank you for the use of their blasting site and all their help and advice for experimental setup and testing. They offered valuable insight and guidance for the experimental work in this dissertation.

I am very grateful for the opportunity to travel to Norway and work with Arve Hanssen and Harald Vistnes in Flekkefjord. Thank you for hosting me, treating me like family, and teaching me more about IMPETUS Afea Solver as well as encouraging me to see more of your beautiful country. I learned so much about using the solver and modeling simulations. It was truly an unforgettable experience that I will cherish forever. Further, thank you for the continued communication and support for my work with IMPETUS and understanding different aspects of the solver. 
Thank you to the IPM group. To my lab mates Chris, Andrew, Jeroen, and Prabha thank you for all the encouragement and support as well as not judging me for my endless coffee consumption. Thank you to Sherri Sullivan for everything she does and keeping us all in line. Special thanks to Ryan Thompson for helping with all my computer issues, particularly the last few months. Finally, Tommy Eanes for all his help and technical support. I know the trips to South Carolina for our experimental testing were not always the most enjoyable (either in the freezing cold or in the middle of summer) but we could not have done it without him.

Thank you to my family and their encouragement for my continued education.

Special thanks to my friends and roommates Megan and Leslie for being my sounding board for life and work issues. Thanks for all their support and fun times in Charlottesville. Your friendship was truly invaluable.

Lastly to Julia and Mike, you two have shown incredible patience and love throughout the last few years. I could not have down this without their support and continued encouragement. Thank you for never letting me give up and listening to all my talks about research and presentations even though science is not your preferred area. 


\section{Table of Contents}

Executive Summary .......................................................................................................

Acknowledgements ...................................................................................................................................... vii

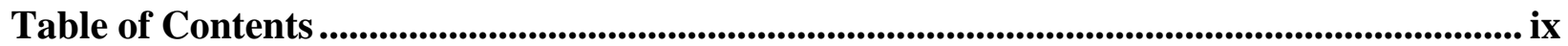

List of Figures......................................................................................................................................................... xiv

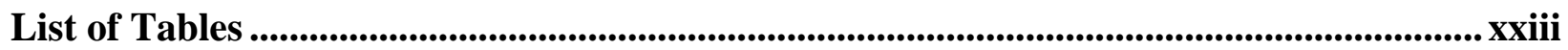

Chapter 1. Introduction ............................................................................................................................ 1

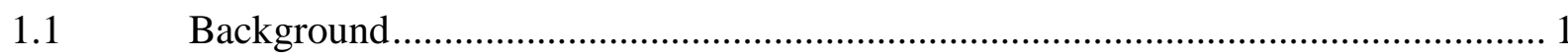

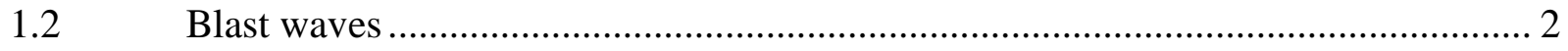

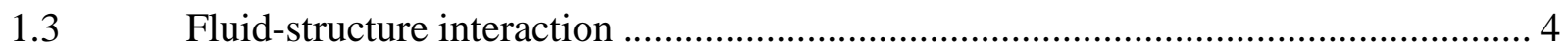

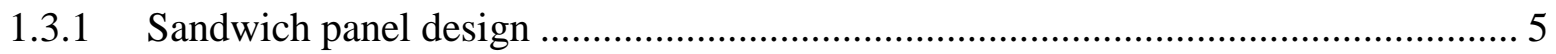

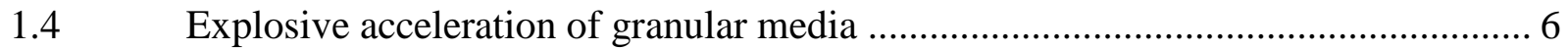

$1.5 \quad$ Numerical simulation approach ………………................................................... 7

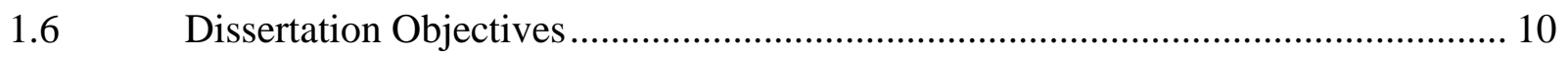

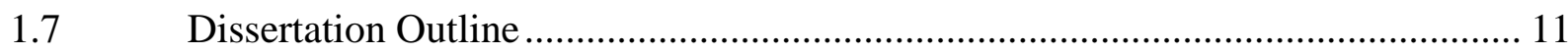

Chapter 2. Granular media impacts ……................................................................................................. 13

2.1 Loading by impact of granular media.................................................................. 14

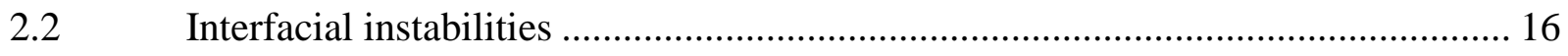

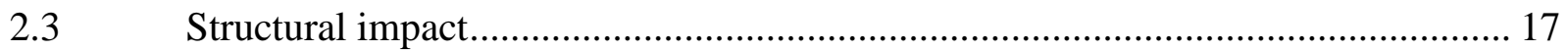

2.3.1 Sandwich panel benefits .............................................................................. 19

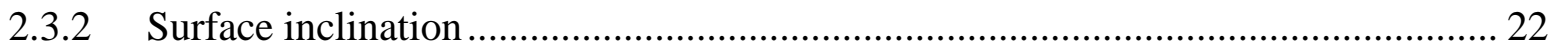

Chapter 3. High intensity impulsive loading by explosively accelerated granular matter 25

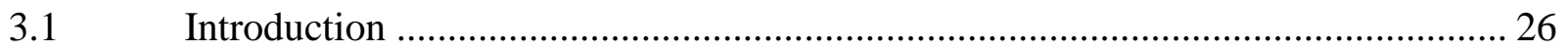




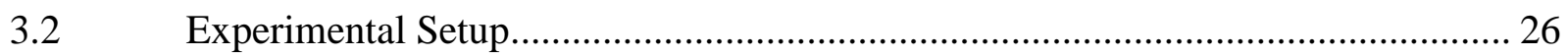

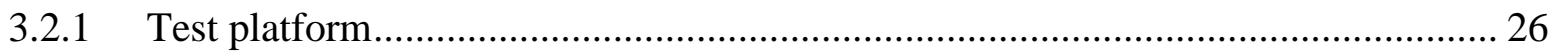

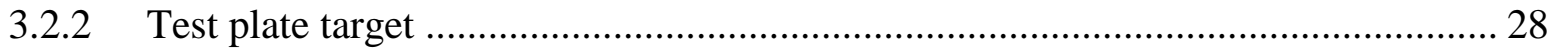

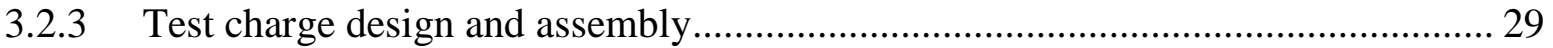

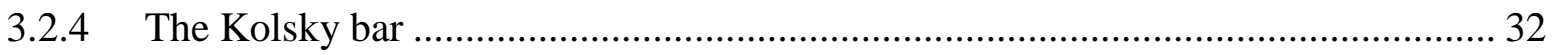

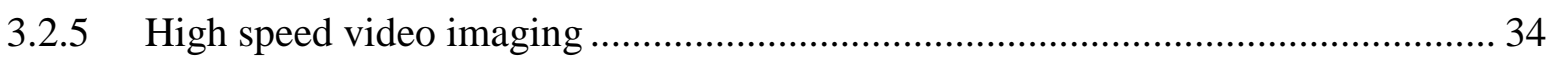

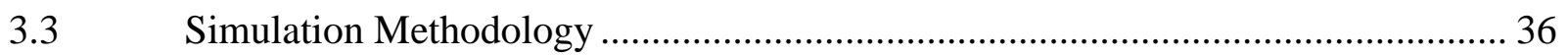

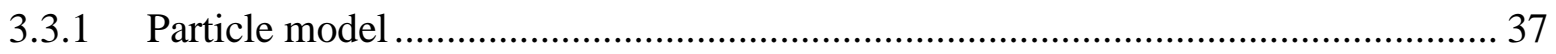

3.3.2 Finite Element model...................................................................................... 39

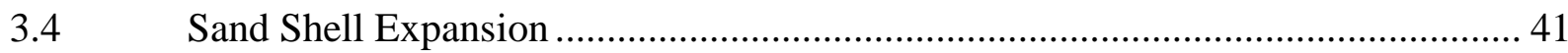

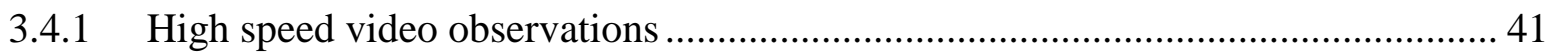

3.4.2 Simulated sand front ................................................................................. 47

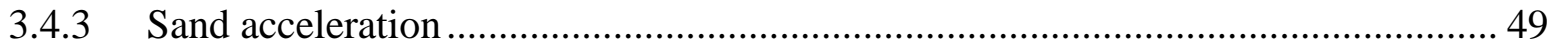

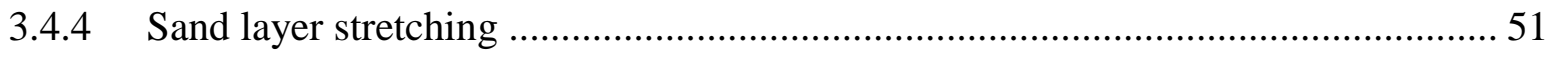

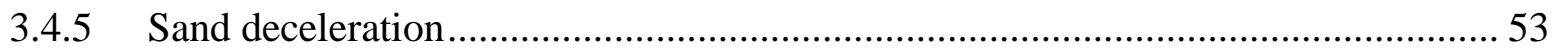

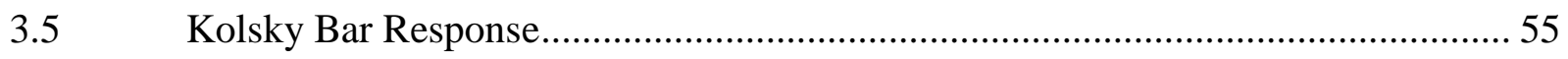

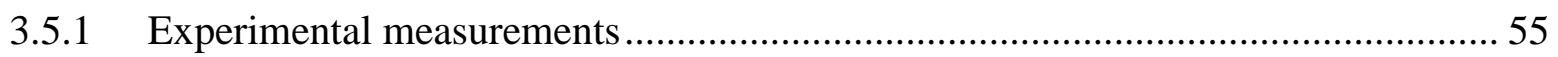

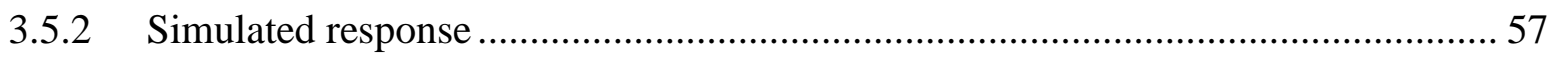

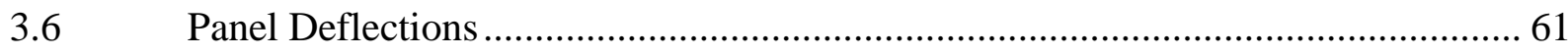

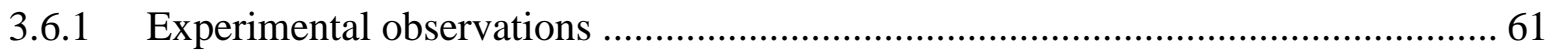

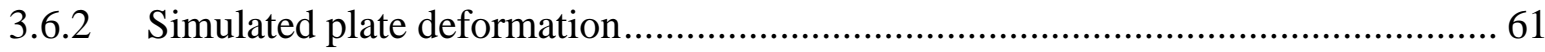

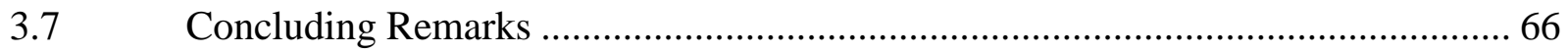

Chapter 4. High intensity impact of granular matter with edge clamped ductile plates... 68

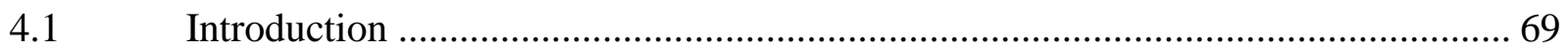

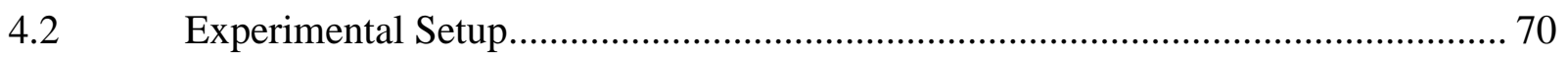




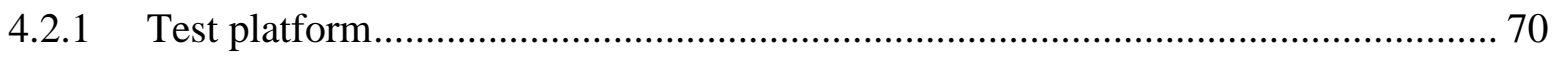

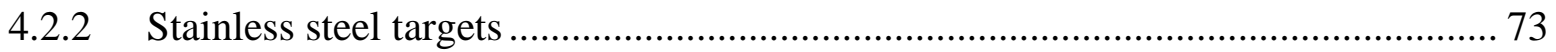

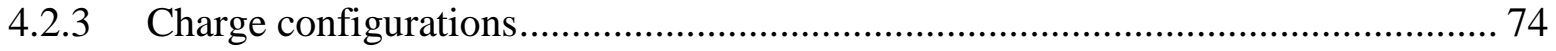

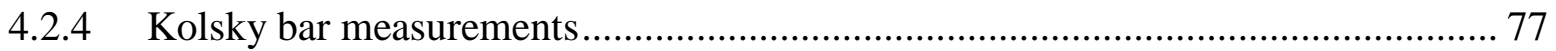

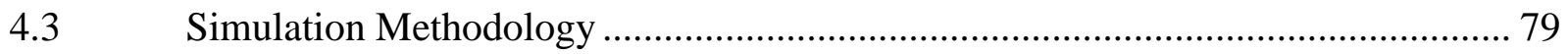

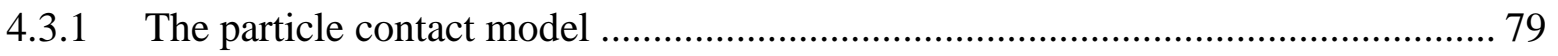

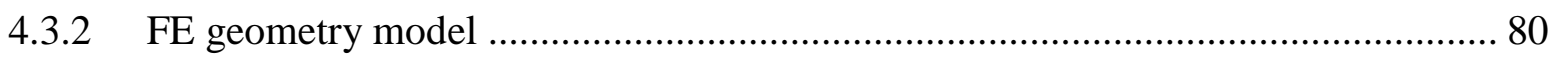

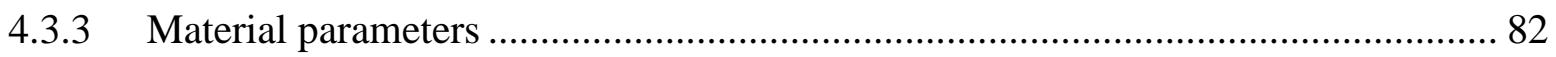

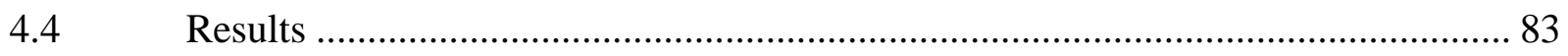

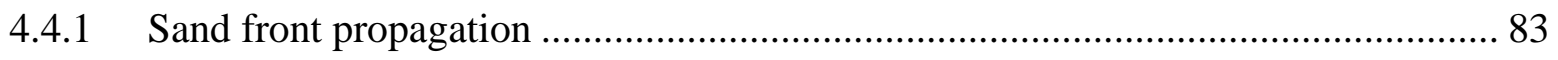

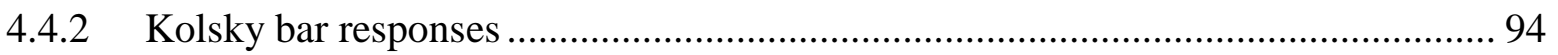

4.4.3 Panel deformations .................................................................................. 100

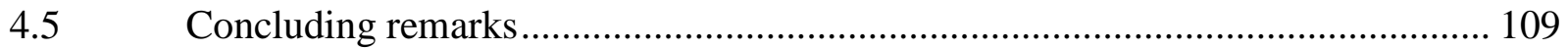

Chapter 5. Impact of granular matter with edge clamped square honeycomb sandwich panels ................................................................................................................................................. 111

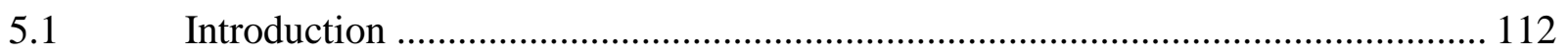

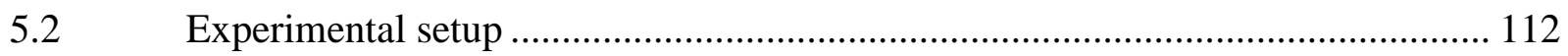

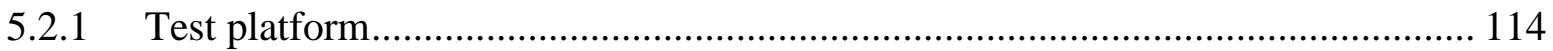

5.2.2 Honeycomb panel design and fabrication ............................................................ 114

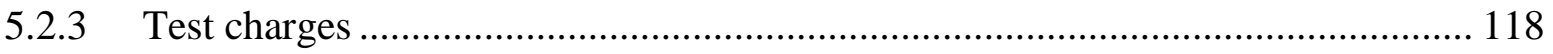

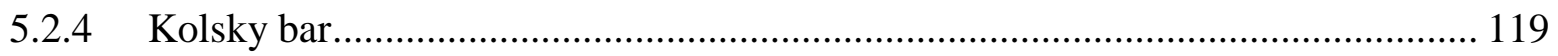

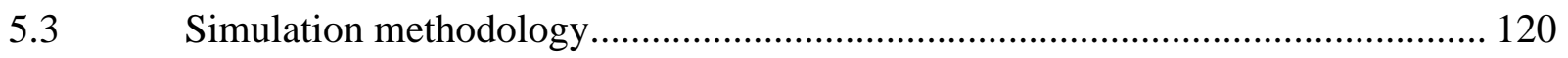

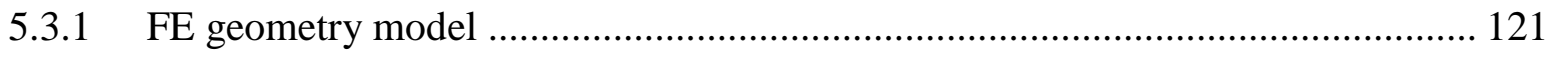

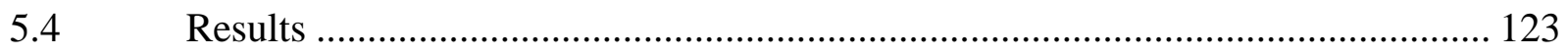

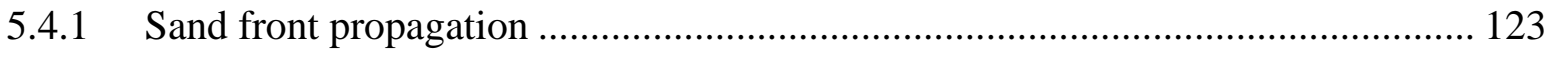




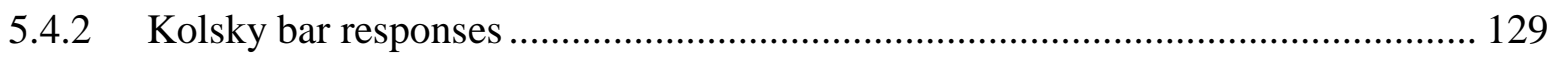

5.4.3 Panel deflection ........................................................................................ 130

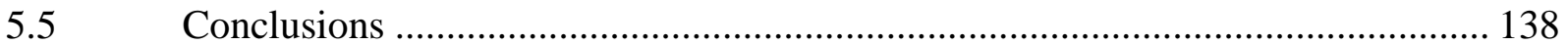

Chapter 6. Momentum transfer during the impact of granular matter with inclined sliding

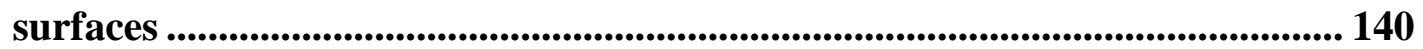

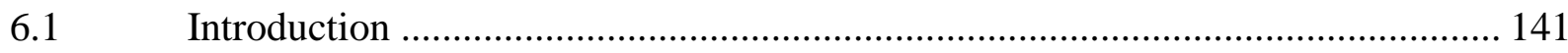

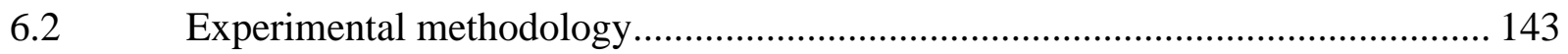

6.2.1 Sample designs and fabrication ................................................................... 143

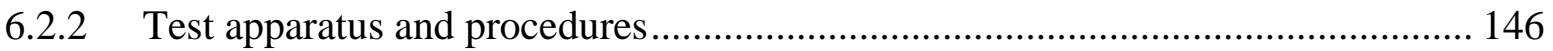

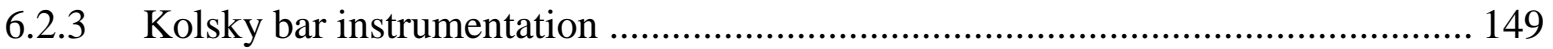

6.3 Numerical discrete particle simulations ……………………........................... 150

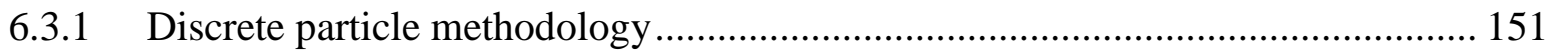

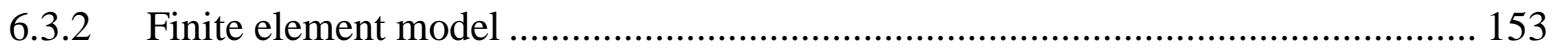

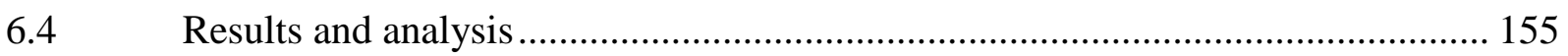

6.4.1 Flat bottom target...................................................................................... 155

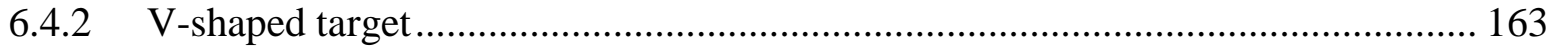

6.4.3 V-shaped target with sliding plates ........................................................................ 167

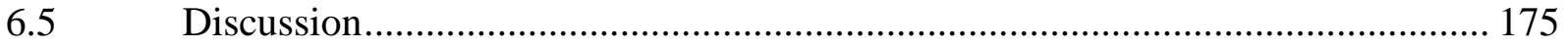

6.6 Concluding Remarks ………………………................................................... 182

Chapter 7. Parametric study of impulse mitigation of granular impacts to inclined sliding

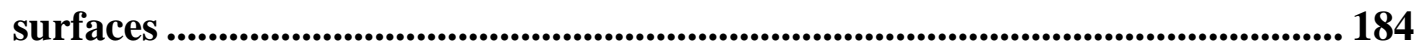

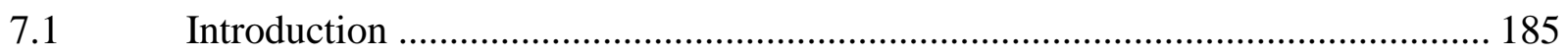

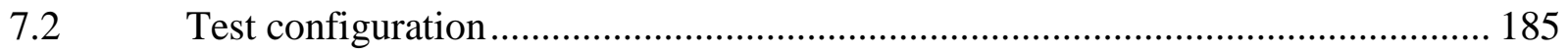

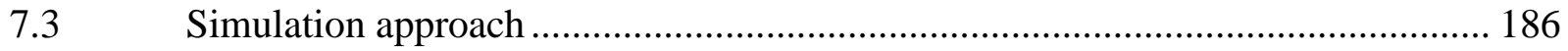

7.4 Steel sliding plate results ............................................................................. 187

7.4.1 Sand particle impacts .................................................................................... 187 


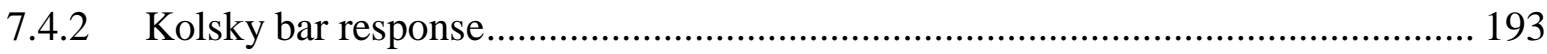

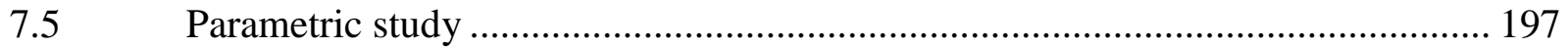

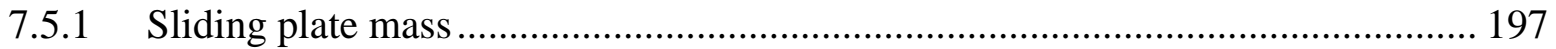

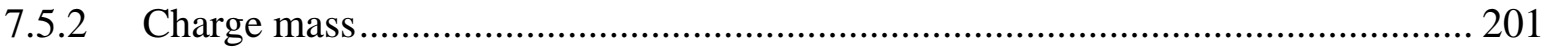

7.5.3 Number of sliding plates..................................................................... 202

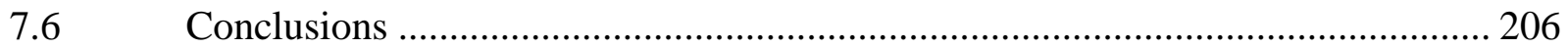

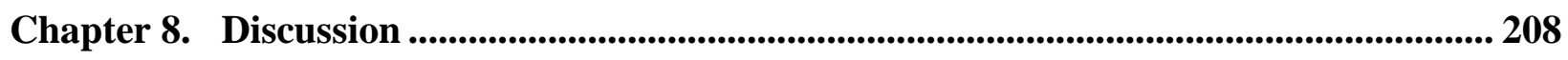

8.1 Explosively accelerated granular media propagation .................................... 209

8.2 High Impact Loading and Structural Response of Solid Plates.......................... 213

8.3 Structural Response of Sandwich Panels.................................................... 215

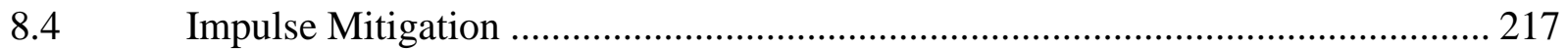

Chapter 9. Conclusions......................................................................................................................... 224

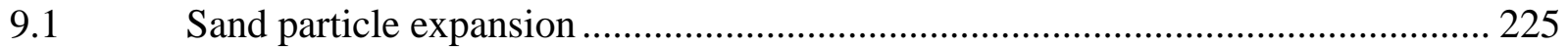

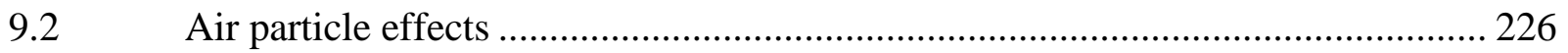

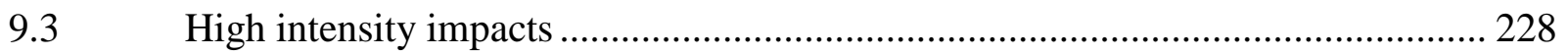

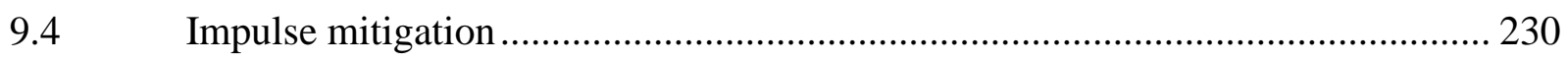

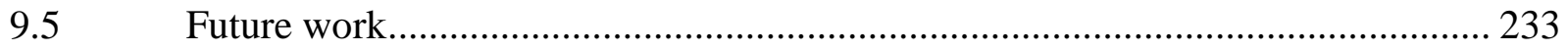

Appendix A. Effect of air particles in simulation .................................................................. 235

Appendix B. Rotation of I-beam support frame ................................................................... 238

Appendix C. Shock front propagation of five test shots....................................................... 241

Appendix D. Kolsky bar measurements for the sandwich panel tests .............................. 244

References 247 


\section{List of Figures}

Figure 1.1. Pressure profile of shock blast wave. Adapted from Ngo et al. [21]......................... 3

Figure 1.2. Schematic of soft-particle model for soil interaction. ............................................... 8

Figure 2.1. Schematic of buried explosive beneath a zero obliquity vehicle structure design... 18

Figure 2.2. (a) Edge clamped monolithic plate and (b) sandwich panel normally impacted by sand slugs. (c) Discrete particle predictions of the dimensionless maximum (elastic plus plastic) dynamic deflection of an edge clamped monolithic plate or sandwich panel versus dimensionless impulse for collimated sand slug impacts at a $90^{\circ}$ angle of incidence. (Adapted from Liu et al. [32])

Figure 2.3. (a) A schematic illustration of the impact of a sand column of length $h$ and momentum $\mathrm{mv}_{\mathrm{o}}$ with an inclined coated surface. The transmitted impulse to the structure, $\mathrm{I}_{\mathrm{T}}$, is balanced by a reaction momentum for a rigidly clamped (or massive) target. (b) The measured effect of target surface inclination and roughness upon the fraction of the incident momentum transferred to the target. The fraction of the incident momentum resolved onto the frictionless $\alpha=45^{\circ}$ plane is also shown as the resolved limit. (Modified from reference [79]) ...................................................... 24

Figure 3.1. Schematic illustration of the test geometry. A spherical test charge consisting of an annular shell of wet sand encasing an explosive sphere was suspended a distance $\mathrm{H}$ over the center of an edge clamped test structure with a Kolsky bar positioned a similar distance from the center of the charge. To ensure that similar ejecta from the charge impacted the center of the plate and the face of the Kolsky bar, the detonator was placed at a position that bisected the directions from the charge center to the test plate center and to the Kolsky bar axis.

Figure 3.2. Schematic illustration of edge constrained test panel geometry showing the location of the Kolsky bar (including its strain gauge location), the test charge geometry and the standoff distances to the Kolsky bar $\left(\mathrm{H}_{\mathrm{k}}\right)$ and test plate $\left(\mathrm{H}_{\mathrm{p}}\right)$ at the instant of detonation... 27

Figure 3.3. Illustration of the construction of the test plate with the welded frame used for attachment to the test stand. Steel dowel pins were press fitted to strengthen the welded connection between the test plate and the four rectangular bars.

Figure 3.4. The geometry and procedure used to assemble the spherical test charge with its concentric shell of water-saturated synthetic sand. 31

Figure 3.5. (a) The strain gauge sensor arrangement used to instrument the Kolsky bar. (b) Shows the signal conditioning and digital data recording arrangement. The trigger signal used to begin digital data recording was initiated by rupture of a break wire attached to the outer shell of the test charge. This trigger event was initiated $40 \mu$ s after the start of the detonation process. 33

Figure 3.6. Plan view of the X-Y plane showing camera locations. An expanded view of the test charge and plate shows the plate center, the charge center at detonation, and the point of maxiumum plate deflection $(\mathrm{X}=71.3 \mathrm{~cm}$ and $\mathrm{Y}=66.0 \mathrm{~cm})$ after the event. The location of the section cut used to obtain a plate deflection profile is also shown. The origin of the coordinate system used for the study was at the front (lower) left corner of the test plate. 35 
Figure 3.7. A cross-section through the mid-plane of the FE model geometry used for the IMPETUS Afea discrete particle based simulation. The coordinate axis origin was located at the front left corner of the top of the test plate (out of the plane of this figure) as shown in Figure 3.6.

Figure 3.8. Sand front propagation observed with the wide-angle video camera; (a) $t=0 \mathrm{~s}$ (instant of detonation), (b) $t=72 \mu \mathrm{s}$ (c) $\mathrm{t}=215 \mu \mathrm{s}$, (d) $\mathrm{t}=358 \mu \mathrm{s}$, (e) $\mathrm{t}=643 \mu \mathrm{s}$, and (f) $=1.5 \mathrm{~ms}$. The distance from the charge center to the test plate was $\mathrm{H}_{\mathrm{p}}=43.2 \mathrm{~cm}$ and to the Kolsky bar end $\mathrm{H}_{\mathrm{k}}=$ $48.5 \mathrm{~cm}$. (e) A magnified region of the outermost sand front shows the presence of sand spikes (fingers). The white box in (f) indicates the region observed in Figure 3.10. ..... 42

Figure 3.9. Images of the sand using the higher spatial resolution high speed camera at times (a) $t$ $=0 \mathrm{~s}$ (instant of detonation), (b) $\mathrm{t}=72 \mu \mathrm{s}$, (c) $\mathrm{t}=120 \mu \mathrm{s}$, (d) $\mathrm{t}=168 \mu \mathrm{s}$, (e) $\mathrm{t}=310 \mu \mathrm{s}$, and (f) $=$ $453 \mu \mathrm{s}$. The sand progression towards the Kolsky bar, the main sand front expansion, and the sand

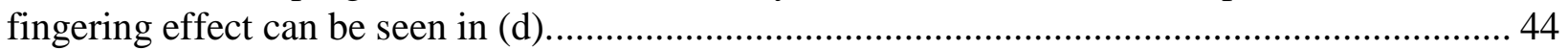

Figure 3.10. Sand front expansion for the white box region in Figure 3.8(f). The sand front consisted of many circumferentially expanding conical regions. As propagation of the front advanced in the radial direction, the cones evolved into arrays of smaller diameter cones. 45

Figure 3.11. (a) Experimental and IMPETUS Afea predicted sand front positions measured in the Kolsky bar direction (horizontal X direction) and (b) sand front velocity vs time after detonation. The velocity of the fastest experimentally observable sand spike (finger) is also indicated on (b). The estimated time for the sand to reach both the Kolsky bar and test plate are also shown...... 46

Figure 3.12. IMPETUS Afea predicted (tan colored) sand front positions. (a) Corresponds to the instant of detonation $(\mathrm{t}=0 \mathrm{~s})$. The position of the (red) HE particles is also shown, but air particles are hidden to improve visualization of sand and HE particle locations. Sand particle probability density contours are identified in (e) showing that the low volume fraction of high speed sand advanced well ahead of the main sand front. 48

Figure 3.13. A simulation sequence following detonation of the test charge. The propagation of a detonation front through the HE and the locations of compressive shock front within the annular wet sand shell are also shown. (f) Shows the distance the sand and HE particles were displaced from their initial locations at $\mathrm{t}=45 \mu \mathrm{s}$ after initiation of detonation. 50

Figure 3.14. Sand particle shell with color contours corresponding to the magnitude of the particle displacement at $\mathrm{t}=50 \mu \mathrm{s}$ after detonation. The spikes in the green-blue contours are consistent with development of an instability at the interface between HE particles and the sand. 51

Figure 3.15. (a) The variation in sand particle density and (b) the sand particle probability density as a function of distance from the charge center (measured in the Kolsky bar direction) at simulation times $\mathrm{t}=102,182$, and $208 \mu \mathrm{s}$. 52

Figure 3.16. Evolution of the air, HE, and wet sand particle regions during the first $500 \mu \mathrm{s}$ following detonation. The presence of an air shock (darker blue) in front of the expanding sand shell can be seen. 54

Figure 3.17. (a) The measured and (b) simulated Kolsky bar pressure versus time after detonation. (c) Shows the measured and simulated impulse versus time response and the four regions of impulsive loading. 56 
Figure 3.18. The simulated specific impulse contributions from the sand, air, and HE particles versus time at the strain gauge location. 58

Figure 3.19. The position of sand and high explosive (HE) particles that impacted the Kolsky bar at selected times after detonation $($ at $\mathrm{t}=0 \mathrm{~s})$.

Figure 3.20. (a) Cross sectional view of the test plate sectioned through the point of maximum deflection (along $X=71.3 \mathrm{~cm}$ ). The grey square marks on the underside of the test structure indicate the locations of the picture frame support while the grey dashed lines show the location of the I-beam flanges of the support structure. (b) The measured and simulated deflection profile of the underside of the edge clamped plate. The vertical dashed line corresponds to the center of the plate in the $\mathrm{Y}$-direction (at $\mathrm{Y}=66 \mathrm{~cm}$ ) which was also the position of maximum deflection. 62

Figure 3.21. A comparison of the IMPETUS predicted transient deflection of the plate at the region of maximum deflection $(X=71.3 \mathrm{~cm} ; \mathrm{Y}=66 \mathrm{~cm})$, and the experimentally measured final maximum deflection. 63

Figure 3.22. Specific impulse distribution at the plate surface after the first $1 \mathrm{~ms}$ of loading. ... 63

Figure 3.23. Effective plastic strain contours of the test plate. (a) On the front and (b) the back face of the test plate after it had ceased reverberation $(t=20 \mathrm{~ms})$. 65

Figure 4.1. (a) The experimental setup showing test plate and suspended charge location together with the strain gage instrumented Kolsky bar. (b) A side view of the test arrangement showing the position of the Kolsky bar and test plate at similar distances and orientations to the test charge. 72

Figure 4.2. Schematic illustration showing explosive charge, detonator, and Kolsky bar placement relative to the test plate. Each test plate had a $5.1 \mathrm{~cm}$ wide perimeter lip to provide edge restraint. 73

Figure 4.3. Optical image of the (a) fused silica and (b) zirconia particles used for experimental testing.

Figure 4.4. Simulation model of test setup with suspended test charge above an edge clamped test plate with a Kolsky bar positioned to measure the applied pressure and impulse loading. 81

Figure 4.5. High-speed video images of glass microsphere tests. (a) Shot 1 (R1 = $80 \mathrm{~mm}$; R2 = $152 \mathrm{~mm} ; 3 \mathrm{~kg}$ charge) (b) Shot $2(\mathrm{R} 1=80 \mathrm{~mm}$; R2 = $203 \mathrm{~mm} ; 3 \mathrm{~kg}$ charge) and (c) Shot 3 (R1 = $90 \mathrm{~mm}$; R2 = $203 \mathrm{~mm} ; 4.5 \mathrm{~kg}$ charge). The insets in (c), (f) and (i) show "sand fingers" emanating from the main sand fronts. 84

Figure 4.6. High-speed video images from the zirconia particle tests. (a) R1 = $90 \mathrm{~mm}$; R2 $=203$ $\mathrm{mm} ; 4.5 \mathrm{~kg}$ charge (b) R1 $=90 \mathrm{~mm}$; $2=229 \mathrm{~mm} ; 4.5 \mathrm{~kg}$ charge. The insets in (c) and (f) indicate little particle finger formation emanating from the main particle front. 86

Figure 4.7. (a) Main particle front position and (b) velocity versus time for the glass microsphere test configurations (shots 1-3). In (a) the filled in markers represent the experimental results and the empty markers are from the simulations.

Figure 4.8. (a) Main sand front particle position and (b) velocity versus time for the zirconia test configurations (Shots 4 and 5). In (a) the filled in markers represent the experimental results and the empty markers those from the simulation..... 88 
Figure 4.9. Simulation of particle positions for the three glass microsphere tests at times $t=0 \mathrm{~s}, \mathrm{t}$ $=250 \mu \mathrm{s}$, and $650 \mu \mathrm{s}$. The model is section cut through the center of each test plate.

Figure 4.10. Simulation of particle positions for the zirconia particle tests. (a) Shot 4 and (b) Shot 5 at times $\mathrm{t}=0 \mathrm{~s}, \mathrm{t}=250 \mu \mathrm{s}$, and $650 \mu \mathrm{s}$. The model is section cut along the center of each test plate. 92

Figure 4.11. High-speed video images showing the air shock that developed in front of the expanding sand shell for Shot 2 (a) $t=333 \mu$ s and (b) $t=555 \mu$ s after detonation. The insets use a dashed white line to indicate the position of expanding air shock ahead of the particles. Simulation images are shown at approximately equivalent times in (c) at $t=330 \mu$ s and in (d) at $t$ $=550 \mu \mathrm{s}$ after detonation. The blue dots are air particles, and the region of concentration ahead of the expanding particle front corresponds to the shock. The black line on the Kolsky bar in (c) and (d) indicates the $10 \mathrm{~cm}$ length painted region of the Kolsky bar in the high-speed video image in (a).

Figure 4.12. Kolsky bar data for the three glass microsphere tests. The waveforms in (a), (b) and (c) show the pressure measured at the strain gage location for Shots 1, 2 and 3. Figures (d), (e) and (f) show the impulse for test Shots 1, 2 and 3 95

Figure 4.13. Kolsky bar data for the two zirconia tests (Shots 4 and 5). The waveforms in (a) and (b) show the pressure versus time response measured at the stain gage location for Shots 4 and 5 . The corresponding impulses are given in (c) and (d). 96

Figure 4.14. Test plate out of plane displacement contour plots for the glass particle tests (Shots 1, 2 and 3). Experimental profilometry results are shown in (a), (b) and (c). The simulated responses at $\mathrm{t}=20 \mathrm{~ms}$ after plate oscillations had decayed to an approximate steady state displacement are shown in (d), (e) and (f). The white dashed lines indicate the location of the underlying support structure. Within this region, the panels were not back supported. 101

Figure 4.15. Out of plane displacement contour plots for the zirconia tests (Shots 4 and 5). Experimental results are shown in (a) and (b) while the simulated responses after the plate oscillations had decayed to an approximate steady state $(\mathrm{t}=20 \mathrm{~ms})$ are shown in (c) and (d). The white dashed lines indicate the outline of the underlying support structure. 102

Figure 4.16. Mid-section out of plane deflection profiles for the five test panels. (a) Measured and (b) simulated results. 103

Figure 4.17. Simulated transient out of plane deflection at the center of the test plates impacted by each test charge. 104

Figure 4.18. The simulated specific impulse distribution applied to the test plates for the five test charge configurations. The maximum deflection $(\delta)$ is also shown for each test and the $81.3 \mathrm{~cm}$ wide region where no back support of the panels was provided. 105

Figure 4.19. (a) The simulated test plate total strain in the Y-direction for Shot 3 at $20 \mathrm{~ms}$ after detonation. The samples were edge restrained by the picture frame support (with a span of 122 $\mathrm{cm})$. The inner, $81.3 \mathrm{~cm}$ wide center of the panels was not back supported allowing unconstrained out of plane deflection as well as inplane stretching. The outer $20.35 \mathrm{~cm}$ wide picture frame provided rigid back support of the panels but did not restrain in-plane stretching. Three node locations are indicated where the nodal strain and displacement magnitude were recorded for each 
test shot. (b) The effective stress for Shot 3 at $1 \mathrm{~ms}$ after detonation showing regions of the plate that were stressed beyond the yield strength of the plate material $(310 \mathrm{MPa})$. 106

Figure 4.20. The simulated permanent plate deflection scaled by the half span, L versus dimensionless impulse in which $\mathrm{I}_{0}$ corresponded to the maximum simulated specific impulse applied near the test plate centers. Results are shown for the $81.3 \mathrm{~cm}$ wide span where no back support existed and the $122 \mathrm{~cm}$ span over which unconstrained inplane panel stretching was allowed. The experimentally measured deflections normalized by the two span lengths are also shown (solid circle and square data). The $\mathrm{x}$ data points correspond to simulations in which the background air particles were omitted. 108

Figure 5.1. Test setup with strain gage instrumented Kolsky bar for impact load measurements. 113

Figure 5.2. Schematic diagram showing the test arrangement for edge clamped panels, solid plate (left) and equivalent mass honeycomb sandwich panel (right). The standoff distance to the back face of both the solid plate and sandwich panel, $\mathrm{H}_{\mathrm{b}}$, is consistent for all tests...... 114

Figure 5.3. Machined, square honeycomb core sandwich panel. The pockets are machined such that the impact side face sheet is integrated to the core structure. (a) Top and section view of the cells. (b) Machined front face sheet/core integrated panel before back face sheet was welded on.

Figure 5.4. Electron beam welding of linear welds attaching the back face sheet to the square honeycomb core in alignment with the honeycomb web walls. 117

Figure 5.5. Electron beam weld bonding the back face and the integral sandwich panel core structure. 117

Figure 5.6. Model geometry used for simulations for solid plate and honeycomb sandwich panel showing standoff distance of charge to the back face of the panels. 122

Figure 5.7. Silica glass microsphere sand shots with honeycomb sandwich panel target showing approximately equivalent time snapshots for Shot $1(\mathrm{R} 1=80 \mathrm{~mm} ; \mathrm{R} 2=152 \mathrm{~mm} ; 3 \mathrm{~kg}$ charge) Shot $2(\mathrm{R} 1=80 \mathrm{~mm} ; \mathrm{R} 2=203 \mathrm{~mm} ; 3 \mathrm{~kg}$ charge $)$ and Shot $3(\mathrm{R} 1=90 \mathrm{~mm} ; \mathrm{R} 2=203 \mathrm{~mm} ; 4.5 \mathrm{~kg}$ charge)...... 124

Figure 5.8. Zirconia sand shots with honeycomb sandwich panel target showing approximately equivalent time snapshots for Shot $4(\mathrm{R} 1=90 \mathrm{~mm}$; R2 = $203 \mathrm{~mm} ; 4.5 \mathrm{~kg}$ charge) and Shot 5 (R1 $=90 \mathrm{~mm} ; \mathrm{R} 2=229 \mathrm{~mm} ; 4.5 \mathrm{~kg}$ charge $)$.

Figure 5.9. Simulated sand front propagation of the three silica test shots (Shots 1-3) for $t=0 \mathrm{~s}$ the moment of detonation, $\mathrm{t}=250 \mu \mathrm{s}, \mathrm{t}=650 \mu \mathrm{s}$, and $\mathrm{t}=2 \mathrm{~ms}$. The sand particles are tan and the inner red particles are the HE. 127

Figure 5.10. Simulated sand front propagation of the two zirconia test shots (Shots 4 and 5) for $t$ $=0 \mathrm{~s}$ the moment of detonation, $\mathrm{t}=250 \mu \mathrm{s}, \mathrm{t}=650 \mu \mathrm{s}$, and $\mathrm{t}=2 \mathrm{~ms}$. The sand particles $\operatorname{are} \tan$ and the inner HE particles are red. 128

Figure 5.11. Test panel permanent plastic displacement contour plot in the $Z$ direction for the silica test shots (Shots 1-3). Experimental profilometry results are shown in (a), (b) and (c). The simulated responses at $\mathrm{t}=20 \mathrm{~ms}$ are shown in (d), (e), and (f). The larger black box indicates the 
underlying support base and region of honeycomb cells. The inner black box indicates the refined mesh region in the FE simulated model. 131

Figure 5.12. Test panel permanent plastic displacement contour plot in the $\mathrm{Z}$ direction for the zirconia test shots (Shots 4 and 5). Experimental profilometry results are shown in (a) and (b). The simulated responses at $\mathrm{t}=20 \mathrm{~ms}$ are shown in (c) and (d). The larger black box indicates the underlying support base and region of honeycomb cells. The inner black box indicates the refined mesh region of the FE model. 132

Figure 5.13. The cross section of the sandwich panels shows the specific impulse distribution applied to the surface by the five test shots. The $81.3 \mathrm{~cm}$ wide region of the honeycomb cells is indicated along with the refined mesh center region of $40.6 \mathrm{~cm}$. The permanent deflection $(\delta)$ is also shown for each test.

Figure 5.14. Strain in the $X$ and $Y$ directions ( $\varepsilon X X$ and $\varepsilon Y Y$ ) on the top face of the sandwich panel for Shot 3 at $\mathrm{t}=20 \mathrm{~ms}$. 135

Figure 5.15. Normalized plot of center permanent deflection versus the impulse comparing the solid plates and the honeycomb sandwich panels (measured solid circle and square and simulated open circle and square). The deflection is normalized by the panel span $2 \mathrm{~L}=81.3 \mathrm{~cm}$......... 136

Figure 5.16. Simulated panel displacement for different cases of back face sheet bonding to the sandwich panel. Case 1: A merge failure allowed debonding of the back face sheet during impact. Case 2: A merged sandwich panel where the back face sheet remained bonded ("welded") to the sandwich panel for all high intensity tested impacts. Case 3: The back face sheet was never connected (non bonded) to the sandwich panel.

Figure 6.1. Reaction momenta following impact of a granular matter slab of thickness $h$ with (a) a V-block target and (b) a V-shaped target with attached slider plates. 143

Figure 6.2. The three Al 6061-T6 targets used for the impulse transfer study: (a) The flat bottom target, (b) the $\mathrm{V}$-shaped target with surfaces inclined at $53^{\circ}$ to the incident sand and (c) the Vshaped target with a pair of sliding plates attached. 145

Figure 6.3. The sliding plate target geometry showing the use of four edge clamps that act as guides for the sliding plates during sand impact. 146

Figure 6.4. (a) Schematic illustration of the vertical impulse test rig with a flat-bottomed solid aluminum target attached. (b) An expanded view of the interior of the steel box, the test charge design, the sample location, and the strain gauge location on the Kolsky bars. 147

Figure 6.5. Schematic illustration of the test charge. 149

Figure 6.6. (a) The FE model of the vertical impulse test rig. Each color-coded part used a different model and material combination. (b) The simulation geometry within the sandbox. The V-block target with sliding plates is shown for a standoff distance of $29 \mathrm{~cm}$. The model geometries of the flat block and V-block targets are also shown. 154

Figure 6.7. (a) A photograph of the sand front at $0.47 \mathrm{~ms}$ after detonation showing the main front position and the presence of sand fingers for a test with the flat bottom sample at a standoff distance of $29 \mathrm{~cm}$. (b) The measured and simulated main sand front position versus time and measured fingers position versus time. (c) The measured and simulated main sand front velocity versus time with the impact times for the flat bottom sample for each of the standoffs. 157 
Figure 6.8. Simulation of sand particle progression towards the baseline test target for a standoff distance of $29 \mathrm{~cm}$. The particle velocity is color coded. The position of the sensors used to monitor the particle position and velocity are indicated in (a).

158

Figure 6.9. Comparison of the strain gauge measured (black line) and simulated (red dashed line) pressure-time waveforms for the flat-bottomed target at standoff distances of (a) $19 \mathrm{~cm}$, (c) $24 \mathrm{~cm}$ and (e) $29 \mathrm{~cm}$. The impulse-time waveforms are also shown for each standoff distance and the arrival times of the $1^{\text {st }}$ and $2^{\text {nd }}$ distal reflection at the strain gauges are indicated on (b), (d), and (f). The time $\mathrm{t}=0 \mathrm{~s}$ corresponds to initiation of detonation of the Detasheet. 160

Figure 6.10. (a) Impulse at the first distal reflection (up to $558 \mu$ s after the signal first arrives at the strain gauge) for each of the three standoff distances for the flat (baseline) target, the solid Vblock target, and the V-block target with the aluminum slider plates. (b) Impulse at the second reflection (748 $\mu$ s after the first reflection) for each of the targets. 162

Figure 6.11. High-speed video image sequence of sand impact with the V-shaped target at a standoff distance of $29 \mathrm{~cm}$. The target geometry is indicated by white lines in (d)-(f). 164

Figure 6.12. Simulation of sand impact with the V-shaped target for a standoff distance of $29 \mathrm{~cm}$. 165

Figure 6.13. Comparison of the strain gauge measured (black line) and simulated (red dashed line) pressure-time waveforms for the $\mathrm{V}$-shaped target at standoff distances of (a) $19 \mathrm{~cm}$, (c) $24 \mathrm{~cm}$ and (e) $29 \mathrm{~cm}$. Figures (b), (d), and (f) show the impulse-time waveforms for each standoff distance together with the arrival times of the $1^{\text {st }}$ and $2^{\text {nd }}$ distal reflections at the strain gauges. Time $t=0$ $\mathrm{s}$ again corresponds to the instant of detonation. 166

Figure 6.14. High-speed video image sequence for the V-shaped target with aluminum slider plates impacted by sand for a $29 \mathrm{~cm}$ standoff distance. The target geometry is indicated by white lines in (d)-(f). 168

Figure 6.15. Simulation time sequence of sand impact with the V-shaped target with attached slider plates for $29 \mathrm{~cm}$ standoff distance. 169

Figure 6.16. Multiple exposure image of a sliding plate target test at a $29 \mathrm{~cm}$ standoff distance. The sliding plate (left) location at $\mathrm{t}=370 \mathrm{~ms}$ after detonation is shown and its trajectory over time is indicated by the dotted line. The time for each slider plate position is shown (in milliseconds) at approximately $100 \mathrm{~ms}$ intervals. 170

Figure 6.17. High-speed video images of V-block target with aluminum slider plates at $19 \mathrm{~cm}$ standoff distance. (a) The test rig at $\mathrm{t}=0 \mathrm{~s}$ (the moment of detonation), (b) $\mathrm{t}=0.3 \mathrm{~ms}$, (c) $\mathrm{t}=1.2$ $\mathrm{ms},(\mathrm{d}) \mathrm{t}=5.5 \mathrm{~ms}$, (e) $\mathrm{t}=35.6 \mathrm{~ms}$, and (f) $\mathrm{t}=48.2 \mathrm{~ms}$. The two aluminum slider plates are visible as they were ejected from the target in (e) and (f). The white dotted lines show the approximate trajectory. 171

Figure 6.18. Sand particle propagation simulations for a V-block target with sliding plates showing the sliding plate movement after impact. The standoff distance was $19 \mathrm{~cm}$. The dark red particles represent high explosive particles and the brown particles the wet sand. 172

Figure 6.19. Comparison of the strain gauge measured (black line) and simulated (red dashed line) pressure-time waveforms for the $\mathrm{V}$-shaped target with aluminum slider plates at standoff distances of (a) $19 \mathrm{~cm}$, (c) $24 \mathrm{~cm}$ and (e) $29 \mathrm{~cm}$. The impulse-time waveforms for each standoff distance 
are shown in (b), (d), and (f) together with the arrival times of the $1^{\text {st }}$ and $2^{\text {nd }}$ distal reflections. 174

Figure 6.20. Impulse distributions on the bottom of the flat block, V-block target, and the V-block with slider plates accumulated during the first $2 \mathrm{~ms}$ after detonation for each standoff distance. $\xi$ is the distance the sliding plates have traveled along the inclined underlying surface. The simulations used a coefficient of friction $\mu_{\mathrm{s}}=0.5$ between the sand and roughened target surface, and a friction value of $\mu_{p}=0.05$ for the lubricated interface between the slider and target....... 176

Figure 6.21. (a) Comparison of simulated impulse transfer to the V-block target (solid lines) and the V-block target with aluminum sliding plates (dashed lines) for the three standoff distances. (b) The normalized plate sliding distance versus time during sand impact for the three standoff distances. The distance and time of sliding for plate escape from the V-block are also shown for each standoff distance.

Figure 6.22. (a) The total simulated transferred impulse in the Z-direction after $4 \mathrm{~ms}$ calculated at the strain gauge location. (b) The impulse contributions transferred to the test targets and to the periphery of their attachment plate. 179

Figure 6.23. The simulated transferred impulse ratio in the Z-direction versus average sand particle velocity. The impulse of the (flat-bottomed) target with $\alpha=90^{\circ}$ dropped from 1.02 to 0.85 when $\alpha=53^{\circ}$ and a particle-target friction coefficient $\mu_{\mathrm{s}}=0.5$ was used. The effect of the frictional interaction between sand particles and the inclined plate can be seen by comparing the $\mu_{\mathrm{s}}=0.5$ and 0.0 results. This resulted in a decrease in impulse ratio from 0.85 to 0.72 . The use of a sliding plate reduced the impulse ratio from 0.85 to 0.76 . A fraction of the impulse was transferred to the targets by air and detonation product particles with no frictional interaction. When their contribution was subtracted from the results, the impulse transferred by frictionless sand particles approached the $\alpha$ $=53^{\circ}$ resolved frictionless limit. 181

Figure 7.1 High-speed video images of particle impacts on V-shaped target with attached steel plates at $29 \mathrm{~cm}$ standoff distance. These times represent the time after detonation $(\mathrm{t}=0$ is moment of detonation). 188

Figure 7.2. Simulation images of sand particle impacts on the V-target with attached sliding plates, transferring momentum to the plate movement for $29 \mathrm{~cm}$ standoff distance. 189

Figure 7.3. Impulse distributions on the impact surfaces of the V-block target, and the V-block targets with aluminum and steel slider plates $2 \mathrm{~ms}$ after detonation for each standoff distance. $\xi$ is the distance the slider plate has traveled along the inclined surface.

Figure 7.4. Impulse distributions on the impact surfaces of the V-block targets with (a)-(c) aluminum and (d)-(f) steel slider plates at $19 \mathrm{~cm}$ standoff distance, showing snapshots of the slider plate movement 1,2 , and $3 \mathrm{~ms}$ after detonation. 192

Figure 7.5. Comparison of the strain gauge measured (black line) and simulated (red dashed line) pressure-time waveforms for the V-block target with attached steel slider plates at standoff distances of (a) $19 \mathrm{~cm}$, (b) $24 \mathrm{~cm}$ and (c) $29 \mathrm{~cm}$. The impulse-time waveforms are also shown for each standoff distance and the arrival times of the $1^{\text {st }}$ and $2^{\text {nd }}$ distal reflection at the strain gauges are indicated on (d), (e), and (f). The time $t=0 \mathrm{~s}$ corresponds to initiation of detonation of the Detasheet. 
Figure 7.6. Impulse experimentally measured by the Kolsky bars at the arrival times of the (a) $1^{\text {st }}$ and (b) $2^{\text {nd }}$ distal reflections at each standoff distance for each of the targets; flat bottomed, Vblock, and V-block with slider plates ( $\mathrm{Al}$ and steel). 195

Figure 7.7. (a) Transferred impulse in the vertical Z-direction to all targets measured in the simulation after $4 \mathrm{~ms}$. (b) Calculated impulse ratio for each target. 196

Figure 7.8. The sliding plate escape velocity for the five different sliding plate masses. (a) The escape velocity at the three standoff distances. (b) The plates' normalized escape velocity (normalized by the incident sand velocity) versus the normalized mass of the slider plates, $\mathrm{m}_{\mathrm{p}} / \mathrm{m}_{\mathrm{s}}$ $\left(\mathrm{m}_{\mathrm{s}}=5.36 \mathrm{~kg}\right.$ is the initial mass of the sand $)$. 199

Figure 7.9. Impulse transferred to the $\mathrm{V}$-shaped target with varying slider plate mass. (a) The transferred impulse versus the normalized slider plate mass, $\mathrm{m}_{\mathrm{p}} / \mathrm{m}_{\mathrm{s}}$, where the initial mass of the sand is $\mathrm{m}_{\mathrm{s}}=5.6 \mathrm{~kg}$. (b) Change in impulse, $\Delta \mathrm{I}$, between the V-block target without sliding plates and the targets with different mass sliding plates as a function of the normalized plate mass... 200

Figure 7.10. Impulse transferred to test targets as the charge mass increased at a fixed $19 \mathrm{~cm}$ standoff distance. (a) Transmitted impulse to the flat block, V-block, and V-block target with aluminum and steel slider plates. (b) Difference in transferred impulse between the V-block target and V-block target with slider plates $(\Delta \mathrm{I}=$ IVblock - Isliders) for the aluminum (filled triangles) and steel (empty triangles) sliding plates. 202

Figure 7.11. The targets with varied number of sliding plates, (a) one, $6.35 \mathrm{~mm}$ thick plate, (b) two, $3.18 \mathrm{~mm}$ thick plates, (c) three, $2.12 \mathrm{~mm}$ thick plates. 203

Figure 7.12. Effect of changing the number of sliding plates attached to the V-block target. Impulse transferred to the V-block target without slider plates ( 0 sliders) compared to the impulse transferred to the target with 1-3 attached slider plates (Al and steel) at standoff distance (a) $19 \mathrm{~cm}$ and (b) $29 \mathrm{~cm}$. The difference in transferred impulse between the V-block target without slider plates and the target with attached plates (both Al and steel) at standoff distances (c) $19 \mathrm{~cm}$ and (d) $29 \mathrm{~cm}$. 204 


\section{List of Tables}

Table 2.1. Select materials with relevant material properties for the normalized impulse

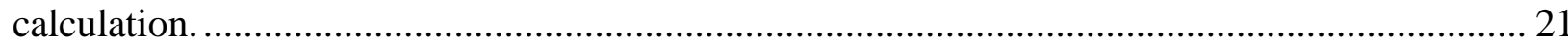

Table 3.1. Material constants for 304SS, A-36 steel, C-350 maraging steel, and AISI 1040...... 41

Table 4.1. Charge configurations for the five test shots............................................................. 74

Table 4.2. Standoff distances from the test charge center to the top of the test plate and impacted end of the Kolsky bar, measured from the high-speed videos prior to detonation. ....................... 76

Table 4.3. Time for detonation and shock front propagation through the explosive and granular

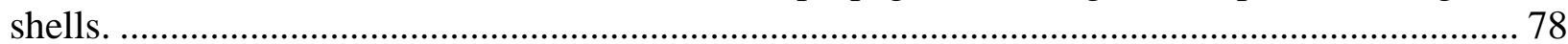

Table 4.4. Distribution of air, HE, and soil particles in the simulations for each test shot.......... 79

Table 4.5. The impact times of the main particle front with the Kolsky bar deduced from experimental main particle front position and the peak velocity for each test charge. The calculated air shock pressures and speeds are also listed.

Table 4.6. The experimentally measured and simulated impulse applied to the Kolsky bar and the permanent plate displacement of each test plate. The simulated (maximum) impulse applied to the test plate and maximum transient displacement are also listed.

Table 4.7. The measured and simulated start times (in microseconds) for each region of the sand loading on the Kolsky bar at the signal arrival time at the strain gauge location (corresponding to impacts at the front of the bar $125 \mu$ s earlier).

Table 4.8. The maximum out of plane dynamic $\left(\delta_{\max }\right)$ and permanent $(\delta)$ displacements and the $\mathrm{Y}$ direction tensile strains at nodes 1,2 , and 3 . The permanent $\mathrm{Y}$ displacement $\left(\delta_{\mathrm{Y}}\right)$ for node 3 determined at $20 \mathrm{~ms}$ after detonation is also listed.

Table 5.1. The design parameters defined for the square honeycomb panels with a relative core density of $\sim 30 \%$. 115

Table 5.2. Charge configurations for the five sandwich panel test shots 119

Table 5.3. The standoff distance from the center of the test charge to the front face of the sandwich panel and to the impact end of the Kolsky bar. The delay time for the Kolsky bar signal initiation and the impact time of the main sand front on the Kolsky bar are also listed. 119

Table 5.4. Distribution of air, HE, and soil particles in the simulations for each test shot 121

Table 5.5. Experimental permanent Z-displacement for the sandwich panels from each test shot for both the integral core/front face sheet structure and the back face, as well as the permanent and maximum simulation Z-displacement of the sandwich panels. 130

Table 5.6. The maximum out of plane dynamic $\left(\delta_{\max }\right)$ and permanent $(\delta)$ displacements along with the $\mathrm{Y}$ direction tensile strains at nodes 1,2 , and 3 . The permanent $\mathrm{Y}$ displacement $\left(\delta_{\mathrm{Y}}\right)$ for node 3 determined at $20 \mathrm{~ms}$ after detonation is also listed. 135 
Table 5.7. The measured and simulated (after $20 \mathrm{~ms}$ ) core compression (change in core height) and the compression strain for each test shot.

Table 6.1. Soil parameters for predefined options in IMPETUS [19] and calibrated wet sand by Holloman et al. [13]. 152

Table 6.2. Johnson-Cook material constants for AA6061-T6 aluminum and A514-grade B steel. 155

Table 6.3. Incident main sand front speed at the time of impact with the flat bottom target and experimentally measured initial pressure peaks and impulse rates for the three targets for each standoff distance. 161

Table 6.4. Experimentally measured transmitted impulses at the arrival time of the first and second distal reflections for the three targets. 163

Table 6.5. Simulation predicted slider plate escape time for a V shaped target, together with the plates $\mathrm{Y}$ and $\mathrm{Z}$ component velocities and plate speed at the time of escape. The $\mathrm{P}_{\mathrm{Z}}$ momentum and $\mathrm{I}_{\mathrm{Z}}$ impulse component of the each plate is also given. 173

Table 6.6. Simulated impulse and normalized impulse applied to the three target designs for each standoff distance. 180

Table 6.7. Impulse transferred to the V-block target by each particle type (sand, air, and HE). The sand particles were modeled with a friction coefficient $\mu \mathrm{s}=0.0$. 182

Table 7.1. Material properties and Johnson Cook parameters for aluminum [37], titanium [106], steel [104], and tungsten alloy $(0.07 \mathrm{Ni}, 0.03 \mathrm{Fe})$ [107] used for the sliding plates.

Table 7.2. The simulated velocity and momentum at escape times for both the aluminum and steel sliding plates for the three standoff distances. 192

Table 7.3. Impulse measured at the arrival times of the $1^{\text {st }}$ and $2^{\text {nd }}$ distal reflections for each standoff distance for the V-shaped targets with aluminum and steel slider plates attached....... 195

Table 7.4. Incident velocity and impulse of incoming sand particles and the Z-direction impulse transferred to the rigid flat block and V-block target (without sliding plates).

Table 7.5. The material density and mass of the sliding plates, along with their mass normalized by the $5 \mathrm{~cm}$ thick wet sand mass, $\mathrm{m}_{\mathrm{s}}=5.36 \mathrm{~kg}$. The Z-direction impulse transferred to the targets for each different material slider plate is also listed for each standoff distance. 198

Table 7.6. Impulse transferred to each target when the charge mass was varied from $100 \mathrm{~g}$ to 1.2 $\mathrm{kg}$. 201

Table 7.7. Transferred impulse to the targets at 19 and $29 \mathrm{~cm}$ standoff distance with varying number of stacked aluminum or steel sliding plates, $\mathrm{n}=0,1,2$, or 3 . 204

Table 7.8. Impulse transferred to the sliding plate targets at a standoff distance of $19 \mathrm{~cm}$ with two plates and three plates with varying material properties for the series of stacked plates. 205 


\section{Chapter 1. Introduction}

\subsection{Background}

Understanding the mechanisms by which an explosion transfers momentum and applies pressure to nearby structures via the arrival of blast shock fronts is important if improved concepts are to be developed to mitigate the damage caused by deliberate or accidental explosions. The detonation of an explosive material results in a detonation wave front that propagates away from the detonation location through the explosive material. Ahead of the front, the explosive material exists as a solid or liquid (condensed phase) containing a significant stored chemical energy per unit mass. Behind the front, the condensed phase has been converted to a gaseous state under high pressure and at a high temperature [1]. When the detonation wave front reaches the interface between the explosive and surrounding medium (air, water, or soil), it accelerates the medium to a speed in excess of its sound wave speed. This results in the formation of a compressive shock in the surrounding medium that propagates outward, away from the explosion [2, 3]. Numerous studies have explored the near-field structural interactions of targets impacted by shock waves generated by a free-field blast in air and [4-7] water [7-10] and also by shallow buried explosions [11-15].

The need to understand structural loading by shallow buried explosives has grown in importance. Since World War II, there has been an increased use of landmines and other buried explosive devices due to the simplicity of their production and deployment, and their effectiveness for disabling vehicles. Thus, the momentum and resulting pressure applied to stationary structures by the impact of a high velocity granular medium such as soil is a subject of considerable scientific $[12,15-17]$ as well as technical interest [18-20]. The impact of the granular media on a structure

applies a net force for the duration of the loading. This change in momentum, $\Delta p=F \Delta t$, results in a total impulse, $I$, applied to the structure with a specific impulse, $I_{o}$, that represents the impulse per unit area on the impacted surface. The need for robust structural designs has led to the 
exploration of novel methods for impulse mitigation. The need to understand their dynamic structural response during and after loading by granular media impacts has resulted in increased interest in numerical methods that more accurately implement the physics of a buried explosive event $[3,19,20]$ in order to predict the local pressures, displacements, and the global acceleration of the structure [1].

\subsection{Blast waves}

An explosion is characterized by a sudden, rapid energy release of significant magnitude [21, 22]. The detonation of an explosive material generates high temperature and high pressure gases by converting the (solid or liquid) energetic material to (mostly) gaseous detonation products. This leads to the formation and propagation (away from the detonation location) of a detonation wave front across the energetic material at high velocities between $4-10 \mathrm{~km} / \mathrm{s}$ [23]. The gases behind the detonation front have been reported to reach pressures of the order of 9 to $21 \mathrm{GPa}$ and temperatures from $2000^{\circ} \mathrm{C}$ and $6000^{\circ} \mathrm{C}$ [1]. In a free field explosion, a compressed layer of air (i.e. blast wave) forms in front of the expanding volume of hot explosive gases [21] with a shock front traveling at a supersonic speed relative to the undisturbed air into which it is traveling [22]. The general pressure profile, as a function of time at a fixed location, is approximated by the Friedlander equation:

$$
P(t)=P_{O P} \mathrm{e}^{-\frac{t}{t^{*}}}\left(1-\frac{t}{t^{*}}\right)
$$

where $t^{*}$ represents the decay constant [24]. The modified Friedlander equation [25] for the pressure waveform shown in Figure 1.1 has the form

$$
P(t)=P_{O}+P_{O P} \mathrm{e}^{-b \frac{t}{t^{*}}}\left(1-\frac{t}{t^{*}}\right)
$$

The arrival of the pressure waveform at time, $t_{a}$, results in a peak overpressure, $P_{O P}$, which decays over time with the decay constant, $b$. This blast wave pressure profile is defined by a positive phase (above ambient air pressure, $P_{o}$ ) and a negative phase (below ambient air pressure). For this modified Friedlander equation, the positive phase duration is defined by the parameter $t^{*}=t_{d}{ }^{+}$. During the positive phase, the blast wave instantaneously peaks to an overpressure value greater 


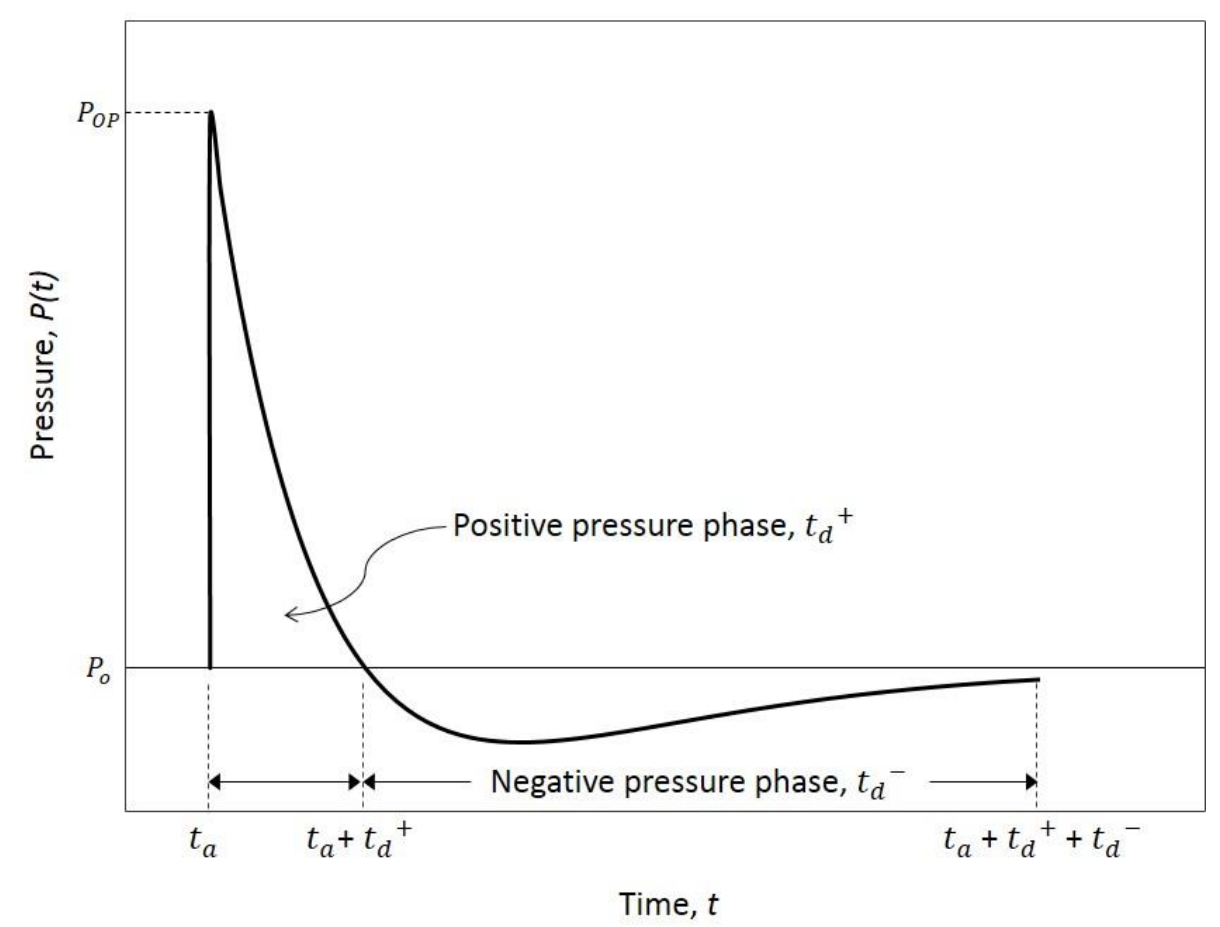

Figure 1.1. Pressure profile of shock blast wave. Adapted from Ngo et al. [21].

than the ambient atmospheric pressure. The pressure then decays from this peak overpressure over time as the shock propagates outward away from the detonation location. After some time, $t_{d}{ }^{+}$ (positive time duration), the pressure may even drop below the ambient pressure forming a negative phase that lasts for some time, $t_{d}{ }^{-}$(negative time duration), in which a partial vacuum is created into which air is sucked inward [21].

When only considering the positive pressure phase, the pressure waveform at a fixed location can be approximated as

$$
P(t)=P_{O P} \mathrm{e}^{-\frac{t}{t^{*}}}
$$

The pressure waveform is also dependent on the distance the blast has traveled. The positive phase of the incident pressure, $P_{i}$, on a normally impacted structure located at a distance x (measured perpendicular to the plate surface) is approximately

$$
P_{i}(t, x)=P_{O P} \mathrm{e}^{-\frac{t-x / c}{t^{*}}}
$$


where $\mathrm{c}$ is the speed of sound in air [26].

\subsection{Fluid-structure interaction}

If the blast wave encounters an object or structure in its path, it is reflected and the peak overpressure applied to the structure is amplified by a shock reflection factor which is greatest for normal incident impacts (perpendicular to the blast wave). For normal incident impacts of weak shock waves with a flat rigid, fully supported structure (that does not move during interaction), the pressure is fully reflected and the impulse applied is

$$
I=2 \int_{0}^{\infty} P_{O P} \mathrm{e}^{-t / t^{*}} d t=2 P_{O P} t^{*}
$$

where the shock reflection factor in the acoustic limit is approximately 2 (twice the incident pressure). For increasingly stronger shock waves, this reflection factor can increase up to a maximum factor of 8 [27]. The rise in the shock reflection factor causes the pressure and impulse applied to the structure to increase.

Taylor [26] developed a linear theory for the fluid-structure interaction (FSI) on unsupported plates for impulsive loading in water and showed that the pressure applied by shock reflection was also approximately 2 for heavy plates. This study also revealed that the acceleration of an unsupported plate away from the shock during impact could reduce the reflected pressure and thus transferred impulse, particularly for low mass plates that accelerate faster [26]. Taylor's approach has been extended by Kambouchev et al. [27] and Hutchinson [28] to investigate the pressure applied to a structure by air blasts. Their results show that nonlinear compressibility during fluidstructure interaction (FSI) mitigates the impulse transferred to a structure during loading caused by an explosion. As Taylor presented [26], this is a consequence of the structural (plate) movement and acceleration of unsupported plates upon impact which relieves the applied pressure (results in less reflected pressure) and leads to reductions in the transmitted impulse. This is intensified for lighter plates where the plates are accelerated faster, leading to a greater decrease in the reflected

pressure and thus reduction in the transmitted impulse [26, 27]. For very heavy plates, the impulsive loading is similar to that experienced by a rigid boundary where the reflected pressure and transmitted impulse are twice the incident values. The FSI effect suggested that structural 
compressibility could enhance impulse mitigation which supported the idea of a sandwich panel design as a mitigation strategy [27].

\subsubsection{Sandwich panel design}

Sandwich panels have been extensively studied as a blast resistant structure for sustaining smaller deflections compared to an equivalent mass (and material) solid monolithic plate when subjected to large impulses. This is a consequence of the acceleration of the light impacted face of the panel upon impact (resulting in a reduced reflected pressure and transferred impulse) and the higher bending resistance of a well-designed sandwich panel [7, 29]. For the latter, the sandwich panel benefit is restricted to the bending dominated region of loading, when the deflections are small relative to the width of the panel. In this regime, the high bending stiffness of the panel reduces panel deflections at constant applied pressure. During larger deflections, the structural response becomes stretching dominated, and the sandwich panel benefit over the monolithic plate diminishes [29]. The core topology of the panel is an important factor in the panel performance. Ideal core designs are those that are optimized to provide both the core crushing needed to enable pressure reduction by the FSI effect (in which the impacted face is accelerated away from the shock) while providing sufficient face sheet separation to retain bending resistance $[7,29]$.

The enhanced performance of a sandwich panel structure has been well established for underwater explosive events [7-10, 29-31]. The benefit in this case arose from two effects (i) an enhanced (higher) panel bending resistance and (ii) the FSI effect of reduced momentum transferred from the water to the structure. Shocks that impact a rigid steel surface at zero obliquity underwater are almost perfectly reflected, resulting in a doubling of momentum transferred to the plate. The FSI benefit of a sandwich panel occurs because the face sheet impacted by a water propagated shock was able to move (away from the shock) during the shock interaction, thereby reducing the reflected shock intensity and the reaction momentum applied to the structure.

The sandwich panel also retains a benefit over a monolithic plate for air blasts and shallow buried explosions. However, assessments these explosive shocks (in air and soil) impacting sandwich panels revealed that the FSI effect was much weaker in air $[4,5,7,27]$ and for granular media impacts $[14,17,32]$. As such, the primary benefit of the sandwich panel for impacts by both an impulsive air shock and granular media was the panel's greater bending resistance and 
core strength $[7,14,17,32]$. An ideal design has a strong core that offers high strength in the normal and longitudinal directions. This makes a square honeycomb sandwich panel design a potentially ideal candidate since it has a very high core compressive strength and also retains stretching resistance [7].

\subsection{Explosive acceleration of granular media}

As previously described, the detonation of an energetic material results in conversion of a solid or liquid energetic material to its gaseous detonation products across a high-pressure detonation front that travels through the explosive at the energetic materials characteristic detonation velocity [33]. For a buried event, this detonation front eventually reaches the interface between the explosive and the surrounding granular media [2], compressing it across a shock front that propagates away from the detonation at the granular media's shock velocity $[1-3,16]$. This compressive shock eventually reaches the granular media/air interface. The high shock impedance difference at this interface results in a strongly reflected (sign converted) tensile (release) shock returning towards the detonation center [2]. To conserve momentum during this reflection, granular media is accelerated (spalled) from the reflecting surface with a velocity that can approach twice that of the incident shock. The spalled ejecta travels from the surface, and eventually impacts a target leading to its impulse loading [1]. For some combinations of depth of burial and soil properties, the majority of the impulse that loads a nearby structure results from soil particle impact rather than the momentum transferred by the air shock or detonation products [2]. Since the focus of this dissertation is on the high intensity impulse loading of explosively accelerated granular media, Chapter 2 discusses granular media impact loading in greater detail.

The impulse created during buried explosive tests at outdoor test ranges can be sensitive to the composition, moisture content, temperature, and degree of compaction of the foundation beneath the test charge [34, 35]. Many of these factors are difficult to control, and the resulting irreproducibility of such tests greatly complicates experimental assessment of mitigation strategies. Dharmasena et al. [17] recently developed an experimental setup to controllably load test structures with explosively accelerated sand. In this approach, a suspended spherical explosive charge was encased by an annular shell of silica glass particles (synthetic sand) of known mass, particle size and shape, and water content. Detonation of explosive charges with a mass of 0.1 $0.3 \mathrm{~kg}$ encased in $5 \mathrm{~cm}$ thick sand shells resulted in sand front velocities of 300-600 m/s [17, 36, 
37]. While the deformation of structures tested this way are readily measured after testing, the dynamic interaction of the sand with the structure was much more difficult to monitor because of obscuration by incident and reflected sand, and by escaping detonation products. However, simulations do not suffer from such problems.

\subsection{Numerical simulation approach}

Several numerical techniques have been proposed to simulate the blast loading of structures [18, 19, 38-42]. The widely used LS-DYNA code [43] allows several different approaches to be used for blast modeling [18] including pure Lagrangian approaches, sequential Eulerian followed by Lagrangian simulations, and fully coupled Eulerian-Lagrangian simulations. A fully coupled simulation allows the blast loading and structural response to be simulated during the dynamic deformation of the structure where fluid-structure interactions can be significant [18]. In this approach, the response of the soil to impulsive loading by the detonation event and the loads applied by the soil to the test structure are calculated using a soil constitutive model. Many empirical continuum soil models have been proposed for this purpose [44-46], but all require careful calibration $[34,45,47]$, and causality between soil structure/composition and impulse loads is obscured.

Deshpande et al. [3] proposed a soil constitutive model based upon a particle-particle contact mechanics analysis that defined interactions between soil particles. This granular model examined two regimes of particle interaction corresponding to the dispersed and high-density particle packing limits. For dispersed particles, typical of conditions during soil propagation through air, the model defined particle contact law was analogous to that for gas molecule collisions in the kinetic theory of gases. The high packing density limit corresponded to a regime of semipermanent contacts dominated by particle deformation and friction, which is representative of conditions during impact of the particles with a structure.

Pingle et al. [48] and Liu et al. [32] proposed a different approach based upon the use of a discrete particle contact model for representing the soil and a finite element model to analyze the response of a structure to particle impacts. This approach treated a soil column as an aggregate of sand particles with an inter-particle contact law governing the aggregate behavior. Figure 1.2 shows a schematic of the soft-particle contact model that has been used in this approach. This contact model was comprised of a linear spring $\mathrm{k}$, in parallel with a linear dashpot with a damping 


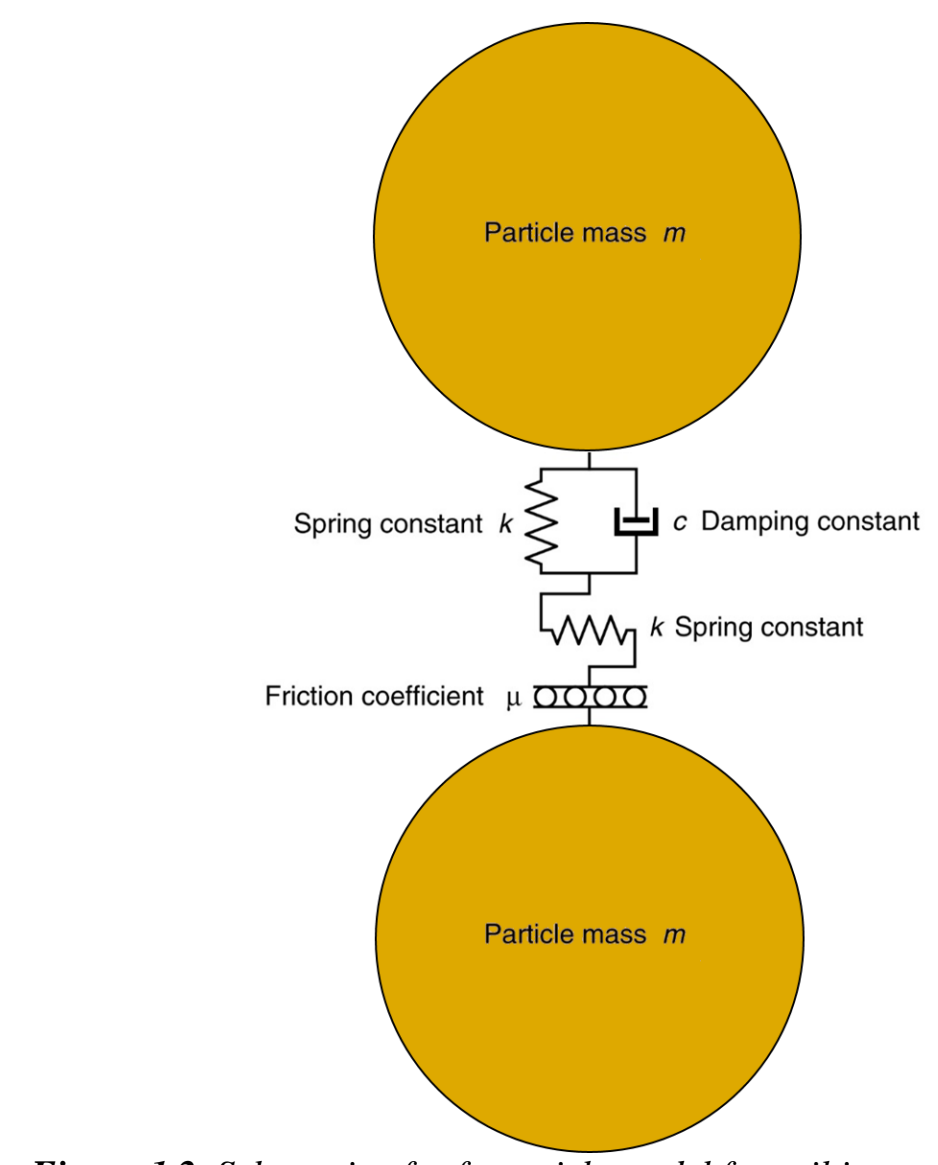

Figure 1.2. Schematic of soft-particle model for soil interaction.

constant $\mathrm{c}$, which are in series with a Coulomb friction component characterized by a coefficient of friction, $\mu$. The values selected for the normal spring constant and the damping parameter govern the normal motion while a horizontal spring constant and the friction coefficient govern the tangential motions during inter-particle contact. These simulations, when coupled with reasonable estimates for the spring constants, damping and friction coefficients, successfully predicted experimental results by Park et al. [49], confirming that the transferred momentum during impact of sand with a zero obliquity rigid (stationary) beam was approximately that of the incident sand column impulse (i.e. the impact was inertial in nature with negligible reflection of the sand on impact).

Related corpuscular simulations have begun to be used for explosive loading simulations [19, 20]. They use the discrete particle approach to model the momentum transfer via contact forces between particles and between particles and a structure. This approach uses different rigid, 
spherically symmetric particles to represent high explosive detonation products, the surrounding air, and the soil. The contact laws, governing interactions between the various particles and with a structure, result in contact forces that are used in a finite element analysis to predict dynamic structural response.

A study by Borvik et al. [19] investigated the validation of this model by experimental testing [17]. Small (150 g) spherical explosive test charges encased in concentric spheres of dry or watersaturated spherical silica particles were suspended at various standoff distances above an edge clamped stainless steel plate. The deflection profile of the test plate was measured, and the velocity of the sand fronts was determined with a high-speed video camera. Good agreement was seen between this experimental data and the numerical simulations using reasonable calibration parameters that predicted the sand velocity and panel deformations. These studies $[19,20]$ have shown the particle-based (corpuscular) method-based codes were capable of reducing the CPU time compared to the Eulerian and coupled Lagrangian-Eulerian approaches. Further, this method avoided problems associated with averaging errors and issues associated with particle/structure contact [19]. These successful experiments then led to the commercial release of the IMPETUS Afea Solver.

The IMPETUS Afea Solver [50] is a non-linear finite element code, which implements the constitutive soil interaction model [3] via a discrete particle (corpuscular) method. Parameters for high explosive (HE), air, and soil particles were calibrated by Borvik et al. [19]. This commercially available code was developed to be computationally robust and efficient and allow users to easily specify design input parameters including material properties, 3D structural designs, boundary conditions, and any other relevant factors. Based on a Lagrangian formulation for finite element structures, the code is a robust analysis method that allows for the investigation of the particle/structure impact and structural deformation and failure. Several studies using this solver have begun to investigate the accuracy of predicted structural responses [13, 14, 19, 20, 37]. In these studies, the explosive charges were small (less than $0.5 \mathrm{~kg}$ ), sand front velocities were usually comparable to the speed of sound in air, and the effects of momentum transfer from the soil to the surrounding air did not appear to be significant. In this regime, once the soil particle contact model parameters were established, the simulated responses were in good agreement with the experimental observations. 


\subsection{Dissertation Objectives}

The motivation for this dissertation extends from the ongoing desire to mitigate the impulse applied to structures during high impact loading by granular media and subsequently reduce the structural deformation response. The impulse transferred to a structure from a shallow buried explosion depends upon the granular media (moisture content, density, etc.) but also upon the angle of impact and the frictional force on the impacted surface. This dissertation employs both experimental and simulation approaches to investigate granular media impacts on several structural designs and the subsequent applied pressure and impulse. First, it investigates the mechanisms by which pressure and impulse are applied to edge clamped plates by the high intensity impact of granular media and the subsequent structural response of the plates. It then investigates the structural response of square honeycomb sandwich panels, and their bending resistance that results in reduced panel deformation compared to its equivalent mass solid plate. Lastly, it investigates a novel target design that has been developed for reducing the impulse transferred to an inclined plate surface. This concept exploits the FSI effect of granular impacts by the application of sliding plates attached to a lubricated (low friction) inclined surface of a structure. The hypothesis is that when the sliding plate's exterior surface is impacted by granular media, it is accelerated and acquires a momentum with resolved components perpendicular and parallel to the plate surface. The latter induce plate sliding across a very low friction coefficient interface, reducing the momentum transferred to the underlying structure. The goals of this dissertation are the following:

(i) To validate discrete particle simulations and their use in quantitatively analyzing high velocity granular media propagation and impulsive impacts on structures

(ii) To investigate granular media expansion and characterize the high intensity impacts on edge clamped plates

(iii) To explore the assumption that at these high impact impulsive loads a sandwich panel design maintains a benefit for reducing structural deformation compared to its equivalent mass monolithic plate counterpart

(iv) To examine the impulse reduction of granular impacts when a normally oriented surface is inclined and the supposition that a novel target design, in which momentum 
from the granular media is transferred to sliding plates, can be implemented to reduce structural impulse loading.

\subsection{Dissertation Outline}

This chapter has presented an introduction of shock waves generated by explosives and the subsequent structural interaction (and FSI effect). Further, the numerical discrete particle simulation approach used in this dissertation to model particle-particle and particle-structural interactions has been introduced. This dissertation focuses on impulsive loading applied by explosively accelerated granular media. As such, Chapter 2 provides a more detailed discussion of granular media impact loading. Chapter 3 presents a validation of the discrete particle simulation solver for high velocity impacts on an edge clamped plate. Simulations are compared to experimental testing of a spherically suspended test charge that was explosively accelerated towards a solid, square, edge clamped test plate. The solver was then used to create a test series of five test charge configurations in which the impulse loading conditions were systematically increased by adjusting either the explosive charge or granular media mass. This series of five test charges were experimentally tested against solid test plates and their equivalent mass (and material) square honeycomb sandwich panel counterparts. Chapter 4 analyzes the radial expansion of these spherical charges, as well as their applied impulse and the resulting structural deformation of the five solid test plates. The sand front expansion and impact loading on a strain gauge instrumented Kolsky bar are analyzed in detail to investigate the effects of the granular mass to explosive mass ratio for the various charge configurations. Chapter 5 analyzes the applied impulse and structural deformation of square honeycomb sandwich panels subjected to the same high impact loading conditions as in Chapter 4. The benefit of the sandwich panel design is investigated, comparing the final permanent deflection of the solid plates and the square honeycomb sandwich panels.

Chapter 6 presents a novel target design implementing sliding plates on a low friction (lubricated) inclined target. The impulse mitigation that arises from surface inclination is first investigated, and it is then shown that a further impulse reduction is achieved with the addition of sliding plates attached to inclined surface. Simulations are shown to be in good agreement with the experimental results. They are used to further examine the particle structure interactions with the test targets and investigate the impulse reduction resulting from the momentum transfer from 
the incident granular particles to the sliding plates. The simulation approach was then used to perform a parametric study on several variables and their influence on the sliding plate effectiveness. This parametric study is presented in Chapter 7, examining the effect of the sliding plate mass, explosive charge mass, and the number of the attached (stacked) sliding plates on the impulse transferred to the test target. Chapter 8 presents a discussion and Chapter 9 provides concluding remarks on the work presented in this dissertation along with suggestions for future research. 


\section{Chapter 2. Granular media impacts}

Soil can be accelerated to very high velocities by the detonation of a shallow buried explosive [51]. While air drag can eventually slow the particles [13, 52-54], the impact of high momentum particle flows with nearby structures can result in the application of significant pressures and momentum transfer, causing structural deformation or failure $[1,15,21,37,55,56]$. The mechanisms by which a rapidly expanding gas transfers momentum to granular media, the processes that slow or disperse the particles during propagation through the air, and those by which the structure subsequently responds to granular impact are therefore areas of considerable research interest $[11,12,15,17]$.

The experimental assessment of potential blast mitigation strategies can be a difficult, timeconsuming challenge, especially as the severity of the impulsive load on a structure increases. The problem is further complicated because the transient deformations responsible for failure are very difficult to directly observe due to obscuration by the ejecta and detonation products [35]. In addition, the placement of sensitive cameras near large detonations is hazardous to the equipment. Numerical simulation provides a complementary means for investigating potential mitigation concepts, provided the physics of soil acceleration [3, 44, 47], its impact loading of the structure $[12,15,16,49]$, and the structures dynamic mechanical response are properly captured. Bergeron et al. [2] utilized a pulse x-ray method to characterize the evolution of a sand plume (ejecta) after detonation of a small explosive charge. This method offered significant insight into the physical phenomena activated by an explosive charge in the early stages of soil deformation and provided relevant time variation of variables (ejecta density and velocity) required to represent an explosion for computer codes.

There are numerous variables that influence the loading of a structure by granular media including the type of soil (sand, clay, pebble content, etc.), its water content, and the standoff distance from the soil surface to a nearby structure. Hlady [35] investigated the effect of soil 
parameters on the applied loading to a nearby structure and reported that a granular medium with high moisture content (i.e. higher density) transferred greater momentum than dry particles (of the same type). Further studies have confirmed this result with saturated granular media having greater impulse [57] and resulting in larger panel deflections [17]. The effect of standoff distance from the charge to the structure has been investigated in numerous studies $[13,15,17,36,58]$. These have shown (while keeping other variables fixed), that as the standoff distance increases, the impulse applied to structures decreases. This appears consistent with spreading of the sand over the expanding diameter ejecta front which reduces the local density of the sand. Park et al. [49] examined sand slugs launched from a gas gun which do not suffer from lateral spreading and found that the sand particles also spread out in the direction of travel. This results in a stretching of the sand that increases with increasing travel time (and therefore standoff distance) to the target $[13,49,106]$. As a result, the ejecta density (and so pressure applied upon arrest on a target) can fall rapidly with standoff distance. Additionally, reductions in applied pressure and impulse are expected to arise if the ejecta is significantly decelerated by air drag. The applied impulse and structural response of the impacted structure obviously must depend on the soil and target to soil (standoff) distance as well as the structural design of the target.

\subsection{Loading by impact of granular media}

In order to understand the dynamic structural response and develop model-based design methods, it is essential to understand the physics of impulsive loading of structures by high velocity granular media. Suppose a finite volume of a granular material impacts a target and applies a constant force of magnitude $\mathrm{F}$ for a period of time $\Delta \mathrm{t}$, the momentum, $\Delta \mathrm{p}(\mathrm{N} \cdot \mathrm{s})$, transferred to the target by the granular material is simply

$$
\Delta p=F \Delta t
$$

The incident momentum of the granular material is determined by its mass and incident velocity, $v$. If the granular material is brought to rest against the target, all of its momentum is transferred to the target, and so its change in momentum is

$$
\Delta p=m \Delta v
$$


The change in momentum of the target (Eqn. (2.2)) is the incident impulse, $I$, applied to the total structure,

$$
I=\Delta p
$$

The specific incident impulse, $I_{0}(\mathrm{~Pa} \cdot \mathrm{s})$, is the incident impulse divided by the impacted area, A $\left(\mathrm{m}^{2}\right)$ :

$$
I_{0}=\frac{I}{A}
$$

Using sand column (cylinder) slugs launched from a gas gun, a laboratory scale study by Park et al. [10] measured the pressure exerted on a Kolsky bar by incoming sand slugs and found that the change in pressure, $\Delta P$, during impact was approximately given by,

$$
\Delta P \approx \rho_{s} v^{2}
$$

where $\rho_{s}$ is the sand particle density and $v$ the incident particle velocity. High-speed video observations showed that the sand particles stagnated against the bar and then flowed laterally off the face of the Kolsky bar, suffering negligible particle rebound (reflection) during impact. Holloman et al. [13] also reported calculated values of the hydrodynamic pressure as $P=\rho_{s} v^{2}$ applied to a normally impacted rigid structure by sand particles. This was equal to the incident sand pressure and indicated the absence of the FSI effect observed in underwater impulse loading $[7-9,30]$.

If particle rebound (reflection) were observed upon impact, this would cause an increase of the momentum (and therefore pressure) applied to the structure. The total change in pressure would then become

$$
\Delta P=\rho v^{2}+p_{r}
$$

where $p_{r}$ is the additional pressure due to particle reflection.

The specific impulse, $I_{t}$, transferred to a structure by the impact of a granular slug is the time integral of the applied pressure: 


$$
I_{t}(t)=\int_{t_{i}}^{t_{f}} \Delta P(t) d t
$$

The ratio of the transmitted to incident impulses, $I_{t} / I_{0}$, is then a measure of the fluid-structure interaction (FSI) for a granular fluid impact. If no rebound of the granular media occurs upon impact, this ratio equals 1 , but if the ratio is greater than unity 1 , this corresponds to some fraction of the particles bouncing back off of the target with a nonzero velocity component normal to the target surface indicating a form of the FSI effect. In cases where the ratio is less than unity, the granular medium will have retained some of its original momentum (for example during interaction with an inclined surface).

\subsection{Interfacial instabilities}

A jetting phenomena or instability occurs along the expanding front of some explosively accelerated granular media $[53,59,60]$. This results in the formation of jets or "fingers" forming at the leading edge of the particles which have a higher initial velocity than the main bulk of the granular media. This was originally attributed to a Rayleigh Taylor instability (RTI) which occurs along an interface where a dense fluid is decelerated by a lighter fluid (i.e. heavy granular particles flowing into light air particles). Milne et al. [61] concluded that while this jetting showed characteristic signs of RTI behavior, RTI occurs at a much later time scale than observed for the jetting phenomena and as such did not accurately predict the observed behavior. Taylor et al. [62] postulated that this jetting phenomena (irregular "fingers") results from the unstable interface between the granular media/air which resembles the Richtmyer-Meshkov instability (RMI) [63, 64]. RMI occurs when an impulsive shock accelerates an interface between two fluids of different densities and amplifies initial perturbations that exist at the interface. This instability develops features of heavier fluid spikes (jets) penetrating a lighter fluid. Holmes et al. [65] and Grove et al. [66] showed excellent agreement with experimentally measured growth rate of granular jets when modeling the interfacial perturbations as RMI.

The jetting phenomena is observed to be driven by the detonation shock, while the main bulk of the sand is driven by the expanding gas bubble of the high explosive products [62]. Any initial perturbations that were initially present on the surface of the granular media interface are amplified by the shock refraction when the compressive shock reaches the granular media/air interface. 
Initially, the jets accelerate very quickly but then decelerate due to air drag, and eventually if enough time elapses, the main bulk of the sand catches up with the jets [53, 62]. This implies that the jetting phenomena is important to understanding near-field effects as these higher velocity jet formations effect the pressure and impulse loading on a target.

Rodriguez et al. [67-69] performed a series of quasi two dimensional experiments within a Hele-Shaw cell where a shock wave was generated in the center of a ring of granular media to impulsively accelerate it. These studies examined the formation of particle jets, characterizing the number of jets formed and their dependence on the particle material properties as well as the ratio between the particle mass and explosive mass. The number of jets was shown to be dependent on the particle layer acceleration and mass, increasing with increasing initial shock pressure and with decreasing material density [67, 69]. Recently, Kandan et al. [70] investigated the development of these instabilities at the front face of a granular sand slug. Results revealed that these instabilities developed from the impingement of air and were dependent on the material properties of the granular particles as well as the magnitude of the applied shock. It was determined that the front face instability was only triggered once a critical velocity was reached, below which no instabilities were observed. Further instabilities were observed at the trailing end of the sand slug where the granular particles were exposed to the high-pressure explosive gases. Their study confirmed that these instabilities are reminiscent of an RMI instability developing at the interface of two fluids as a result of a shock wave propagating through the interface.

\subsection{Structural impact}

Figure 2.1 shows a schematic illustration of a practical problem where the underside of a vehicle is impacted by ejecta from a shallow buried explosion. In this case, the vehicle floor plate is parallel to the ground surface, and so the ejecta impacts the underside of the horizontal flat floor surface at approximately zero obliquity (normal incident impact). The transfer of specific impulse (momentum per unit area) by granular media to structures has been both experimentally and numerically investigated for normal incident impacts in a series of sand slug investigations [32, 48,49 ]. For a slug brought to rest (stagnated with no rebound) against a flat rigid structure oriented perpendicular to the slug propagation direction (at an inclination angle $\alpha=90^{\circ}$ ), all the momentum would be transferred to the structure causing light structures to suffer significant accelerations. As mentioned earlier, several experimental $[13,49]$ and simulation [48] studies have shown that the 
hydrodynamic pressure applied by the soil to the structure is $\sim \rho_{s} v^{2}$, where $\rho_{s}$ is the density of the sand slug and $v$ its incident impact velocity normal to the structure surface. Typically, the soil at the outer surface of the slug is more strongly accelerated, and the sand slug acquires a distribution of velocities, causing the sand column to stretch (and its density to decrease) as it travels towards a target $[49,54]$. If the soil impacting the structure is not strongly reflected, the pressure applied by arrest of the soil particles against a zero obliquity rigid plate is approximately equal to the stagnation pressure of the impacting soil particles (a granular flow) [13,49]. The average pressure exerted by impacts with a structure can be sufficient to cause substantial deformation and rupture of lightweight structures [37]. In practical situations, the application of a high intensity dynamic load to a horizontal floor plate causes the floor plate to bend, resulting in upward deflections. In severe cases, the plastic strains associated with the deflection are sufficient to rupture the floor and allow ejecta and detonation products to enter the crew compartment. Even in less severe loadings, the dynamic deflection of the floor plate can cause rapid accelerations to objects that are in contact with the floor. The impulse applied to the vehicle also results in a vertical acceleration of the entire vehicle. The majority of injuries to vehicle occupants due to blast loading arise from some combination of these effects [1].

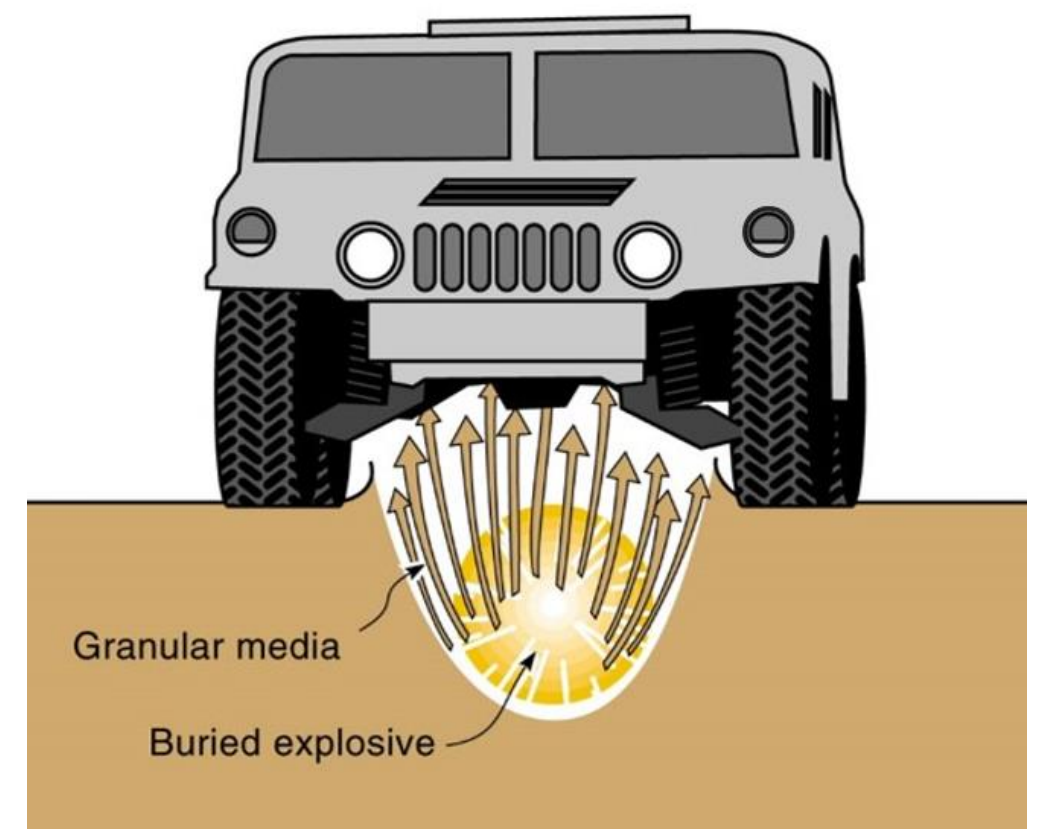

Figure 2.1. Schematic of buried explosive beneath a zero obliquity vehicle structure design. 
Numerous concepts have been proposed for mitigating the effects of granular media impacts with the underside of vehicles $[15,17,36,37,58]$. These include reinforcing the floor plate to lessen its deflections and reduce the risk of rupture (entry of the blast into a vehicle interior), increasing the height of the floor above the ground to reduce the applied pressure, and changing the geometry of the floor plate to reduce the momentum transferred to it [1]. Increasing panel bending resistance can be achieved by replacing a solid plate with a well-designed sandwich panel of identical mass. Raising the height of a vehicle above the surface increases the standoff distance and is beneficial since the sand density is reduced by axial and lateral expansion and by additional time for air drag to decelerate the soil particles [54]. The use of a V-shaped hull that reduces the fraction of momentum that is transferred to the impacted surface and increases the time over which the momentum transfer occurs has also been investigated in numerous studies [12,71-75]. When these approaches are implemented in conjunction with the use of damage resistant materials and structures, the susceptibility of light vehicles to damage by shallow buried landmines can be significantly reduced [76].

\subsubsection{Sandwich panel benefits}

Lui et al. [32] used a coupled particle/finite element modeling approach to investigate the dynamic displacement of edge clamped monolithic (solid) plates and sandwich panels, Figure 2.2(a) and (b), impacted normal to the surface by high velocity granular media (sand slugs) like that ejected from a buried explosion. The sand slugs impacted at various velocities (i.e. incident impulses). Figure 2.2(c) shows the numerically computed dimensionless out of plane maximum transient displacement, $\delta_{\max }$, of an edge clamped plate of span length 2L (Figure 2.2(a)), following the impact of a sand slug with incident specific impulse, $I_{O}(\mathrm{kPa} \cdot \mathrm{s})$, at an incidence angle of $90^{\circ}$ [32]. In this scenario, the sand particles were of uniform incident velocity. They did not appreciably bounce from the surface and instead flowed, like a laminar wall jet laterally across the surface [32,77]. The transferred specific impulse was therefore approximately equal to the incident momentum per unit area of the sand slug. The dimensionless impulse,

$$
\bar{I}_{0}=\frac{I_{0}}{m_{b} \sqrt{\sigma_{Y} / \rho_{m}}}
$$


shown in Figure 2.2(c), is the impulse divided by the mass per unit area (areal mass) of the plate $\left(\mathrm{kg} / \mathrm{m}^{2}\right)$ and the plastic wave speed of the plate, $\left(\sigma_{\mathrm{y}} / \rho_{\mathrm{m}}\right)^{1 / 2}$, where $\sigma_{\mathrm{y}}$ is the plate's yield strength (Pa) and $\rho_{\mathrm{m}}$ its density $\left(\mathrm{kg} / \mathrm{m}^{3}\right)$. The areal mass of a solid monolithic plate is

$$
m_{b}=\rho_{m} h
$$

in which $h$ is the plate thickness shown in Figure 2.2(a). This analysis indicates that the out of plane displacement is a strong function of specific impulse for impacts by particles at normal incidence, but can be ameliorated by use of high strength plate materials of low density [32].

Results for impacts on an equivalent mass sandwich panel of span $2 \mathrm{~L}$ are also shown in Figure 2.2(c). The areal mass of a sandwich panel (Figure 2.2(b)) is dependent on its material density and thickness $\left(t_{f}\right.$ and $\left.t_{b}\right)$ of the front and back face sheets as well as the core density $\rho_{c}$ and height $H_{c}$,

$$
m_{b}=\rho_{m} t_{f}+\rho_{m} t_{b}+\rho_{c} H_{c}
$$

Here the front and back face are assumed to have the same material density, $\rho_{m}$ (equivalent to the solid plate density). A summary of these results is also shown with the normalized maximum panel transient displacement, $\delta_{\max } / \mathrm{L}$ as a function of the normalized (dimensionless) impulse.

Little evidence of a beneficial FSI effect was observed for granular media impacts with the sandwich panel as was seen in water [4-7]. However, the investigation revealed that the sandwich panel back face deflection was less than that of the monolithic plate for the range of incident impulses studied. Greater benefits were observed for sandwich panels with thicker cores and shorter spans between the edge grip locations. Figure 2.2(c) shows the substantial reduction that can be achieved with a sandwich panel construction for two normalized core strength values,

$$
\overline{\sigma_{c}}=\frac{\sigma_{c}}{\overline{\rho_{c}} \sigma_{Y}}
$$

where $\sigma_{c}$ is the sandwich panel core strength and $\overline{\rho_{c}}$ is the relative density of the core. Results showed that increasing the sandwich panel core strength further reduces panel deflection significantly [32]. 
(a) Monolithic plate

sand column

$\mathrm{H}$
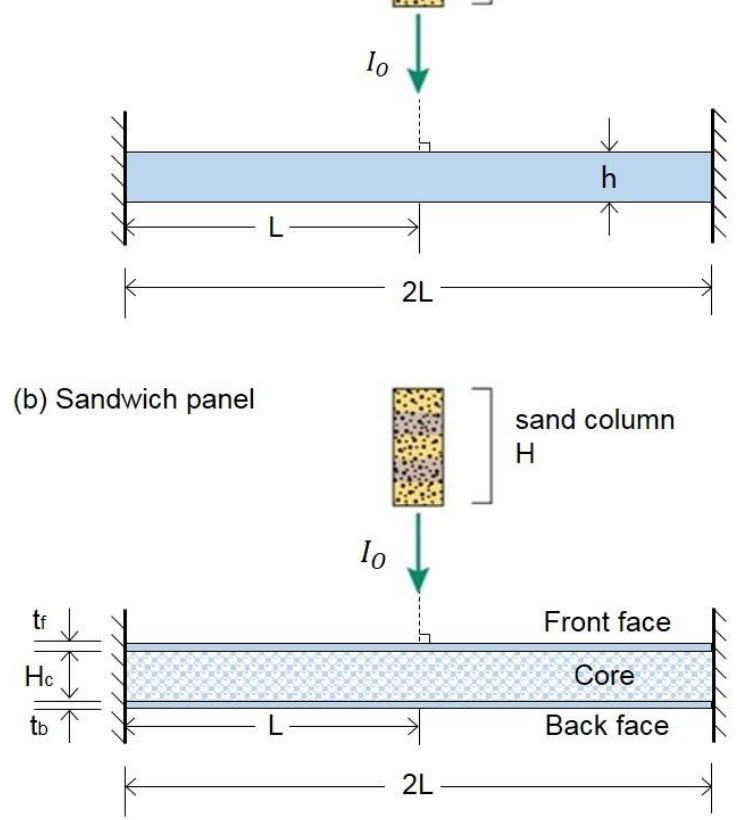

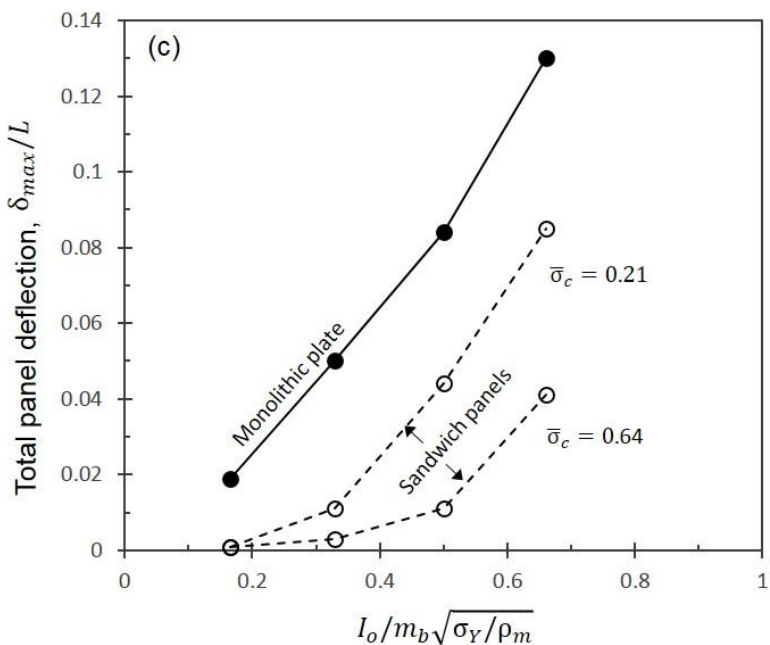

Figure 2.2. (a) Edge clamped monolithic plate and (b) sandwich panel normally impacted by sand slugs. (c) Discrete particle predictions of the dimensionless maximum (elastic plus plastic) dynamic deflection of an edge clamped monolithic plate or sandwich panel versus dimensionless impulse for collimated sand slug impacts at a $90^{\circ}$ angle of incidence. (Adapted from Liu et al. [32])

Table 2.1. Select materials with relevant material properties for the normalized impulse calculation.

\begin{tabular}{cccccc}
\hline Candidate material & Yield strength & Density & Areal mass & $\sqrt{\sigma_{Y} / \rho_{m}}$ & $m_{b} \sqrt{\sigma_{Y} / \rho_{m}}$ \\
& $\sigma_{Y}(\mathrm{MPa})$ & $\rho_{m}\left(\mathrm{~kg} / \mathrm{m}^{3}\right)$ & $m_{b}\left(\mathrm{~kg} / \mathrm{m}^{2}\right)$ & & \\
\hline 304 stainless steel & 275 & 8030 & 200.75 & 0.19 & 38.14 \\
60601-T6 aluminum & 275 & 2700 & 67.5 & 0.32 & 21.60 \\
17-4 PH stainless steel & 1170 & 7810 & 195.25 & 0.40 & 78.10 \\
Ti-6-Al-4V & 1000 & 4420 & 110.5 & 0.48 & 53.04 \\
\hline
\end{tabular}


The dimensionless analysis above (Eqn. (2.8)), indicated that two material properties, yield strength and density, as well as the thickness (i.e. $m_{b}$ ) of the plate dictate the deflection distance for a fixed half-span of the plate/sandwich panel width for a fixed transferred impulse. Table 2.1 lists four selected materials with yield strength, density, and the calculated values of $m_{b,} \sqrt{\sigma_{Y} / \rho_{m}}$, and the resultant denominator of Eqn. (2.8) for a $25 \mathrm{~mm}$ thick plate.

While the wave speed of the plate, $\sqrt{\sigma_{Y} / \rho_{m}}$, increases from 0.19 to 0.48 for the selected candidate materials of 304 stainless steel to a Ti-6-Al-4V alloy (row 1 to row 4), for plates of equal thickness, 17-4 PH SS has the largest denominator (78.10) and thus benefit for mitigating the deflection of monolithic plates. However, such steels are very costly and difficult to fabricate. In this case, a Ti-6-Al-4V plate would be less costly (and more easily fabricated) alternatively followed by 304 stainless steel. For plates of identical $m_{b}$ (varied thicknesses), the plate deflection varies only with the value of the wave speed, $\sqrt{\sigma_{Y} / \rho_{m}}$. Thus for an equivalent mass plate, a titanium plate will outperform a 304 or 17-4 PH stainless steel plate when subjected to the same incident momentum.

\subsubsection{Surface inclination}

To gain a deeper understanding of these granular media/structure interactions, Uth et al. [78] and Goel et al. [79] recently conducted laboratory studies in which wet sand slugs were launched from a gas gun with velocities of 70-150 m/s and impacted against rigid targets supported on piezoelectric sensors that measured the three reaction force components. This enabled determination of the reaction momentum components for rigidly clamped surfaces inclined at either $\alpha=90^{\circ}$ or $45^{\circ}$ to the slug propagation direction, Figure 2.3(a). The use of striped sand allowed the axial stretching of the sand slug to be visualized while the rigid targets reaction momentum components in the $\mathrm{Z}$ and $\mathrm{Y}$ directions $\left(I_{T}^{Z}\right.$ and $\left.I_{T}^{Y}\right)$, resulting from impact of a slug with free field momentum $\left(I_{O}\right)$ travelling in the $-\mathrm{Z}$ direction, were determined from the force sensor data. These reaction momenta are exerted by the supports on the rigid structure and are subsequently referred to as the transmitted momentum. The transmitted impulse $I_{T}$ was determined from that in the two directions using

$$
I_{T}=\sqrt{\left(I_{T}^{Z}\right)^{2}+\left(I_{T}^{Y}\right)^{2}}
$$


The momentum components were shown to depend upon combinations of the normal, $f_{n}$, and tangential, $f_{t}$, reaction forces created during impact of the sand with the surface. Since surface friction significantly affected the tangential force, the transferred momentum depended upon both the orientation of the impact surface and the frictional interaction between the particles and this surface.

For a perpendicularly oriented $\left(\alpha=90^{\circ}\right)$ surface, conservation of momentum gives $I_{T}^{Y}=0$ and $I_{T}=I_{T}^{Z}$. The measured transmitted impulse was approximately 1.03 times that of the incident slug $\left(I_{O}\right)$, consistent with a weak rebound (reflection) of sand from the target surface (in the $\mathrm{Z}$ direction), Figure 2.3(b) [79]. Prediction of the momentum transferred to an inclined surface requires knowledge of the tangential force, $f_{t}$. However, when the interaction of the sand with the target is frictionless, Goel et al. [79] showed that the impulse transferred in the $\mathrm{Z}$ and $\mathrm{Y}$ directions is

$$
I_{T}^{Z}=I_{O} \sin ^{2} \alpha
$$

and

$$
I_{T}^{y}=I_{O} \sin \alpha \cos \alpha
$$

For the case of $\alpha=45^{\circ}, \cos \alpha$ and $\sin \alpha$ are identical, so $I_{T}^{Z} / I_{O}=I_{T}^{y} / I_{O}=0.5$, and therefore from Eqn. (2.12), $I_{T} / I_{O}=0.707$ which is shown on Figure 2.3(b) as the resolved (frictionless) limit.

The experimental study confirmed that the momentum transferred to an inclined target was significantly reduced, but exceeded the (frictionless) limit by an amount that depended upon the coefficient of friction between the impact surface and sand slug. Smooth, very hard surfaces with the lowest friction coefficient were found to acquire the least momentum, while targets with very rough surfaces (or that became very rough during granular impact) acquired substantially more of the sand slugs momentum, Figure 2.3(b). Furthermore, the changes in momentum transfer arising from variations of the friction coefficient were of a similar magnitude to those achieved by inclining the target. These findings suggest that the use of hard polished coatings applied to the surface of a V-shaped structure might result in substantially reduced momentum transfer to the underside of a vehicle. However, the very high contact pressure of soil particles whose impact velocity can exceed $1000 \mathrm{~m} / \mathrm{s}$, is likely to cause significant local plastic indentation, erosion and 
(a)

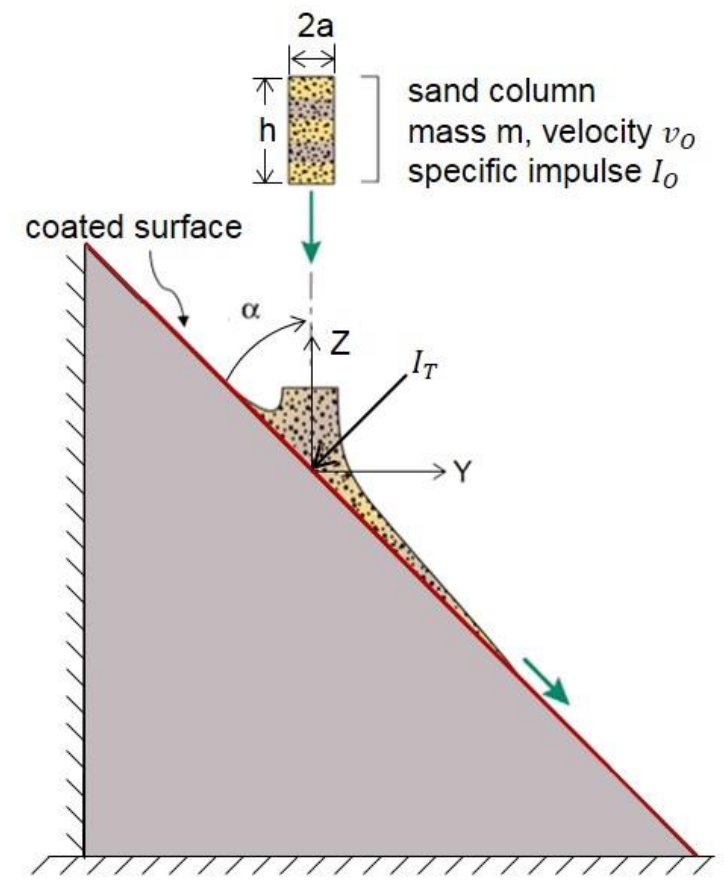

(b)

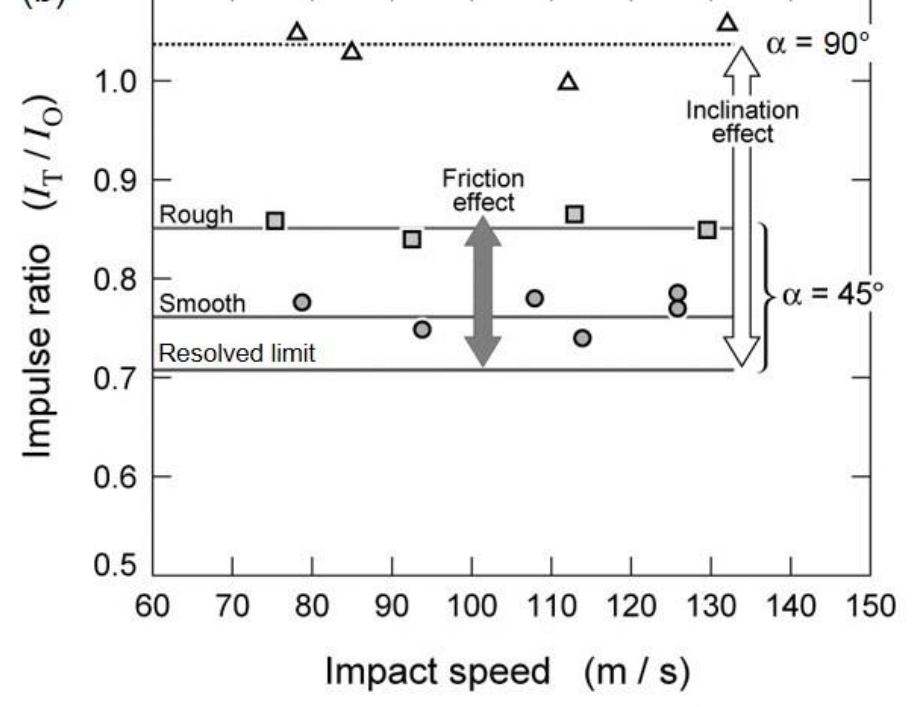

Figure 2.3. (a) A schematic illustration of the impact of a sand column of length $h$ and momentum mv $v_{o}$ with an inclined coated surface. The transmitted impulse to the structure, $I_{T}$, is balanced by a reaction momentum for a rigidly clamped (or massive) target. (b) The measured effect of target surface inclination and roughness upon the fraction of the incident momentum transferred to the target. The fraction of the incident momentum resolved onto the frictionless $\alpha=45^{\circ}$ plane is also shown as the resolved limit. (Modified from reference [79])

surface roughening during the impact event. Such a dynamic increase of the friction coefficient is therefore likely to limit the benefits of such an approach. 


\section{Chapter 3. \\ High intensity impulsive loading by explosively accelerated granular matter}

This chapter is based on a paper published in the International Journal of Impact Engineering ${ }^{1}$. It investigates the acceleration of sand particles created by a model explosive event consisting of a spherical, water-saturated (synthetic) sand encased high explosive test charge that launched a dispersed sand front with velocities of more than $1200 \mathrm{~m} / \mathrm{s}$. The study investigates the deformation response of a $2.54 \mathrm{~cm}$ thick, high ductility, stainless steel edge clamped plate when impacted by the ejecta from the model charge whose center was suspended above the center of the plate. It also explores the validity of particle-based simulations of the test. The temporal evolution of pressure applied by the sand particles was measured by simultaneously stagnating the sand against the end of an instrumented Kolsky bar. A pair of high-speed video cameras were also used to make a full observation of soil front fragmentation and propagation towards the structure. The IMPETUS Afea code was then used to analyze the impulse loading of the Kolsky bar and the plate, and to examine the dynamic response of the test structure to which the plate was attached. The combination of sand impulse measurement, observations of the sand front evolution, and the measured plate deflection then enabled identification of the important physical phenomena associated with high intensity impulsive loading by granular media, and an assessment to be made of the extent to which a particle-based code is able to analyze them.

\footnotetext{
${ }^{1}$ A. Kyner, K. Dharmasena, K. Williams, V. Deshpande, H. Wadley, High intensity impulsive loading by explosively accelerated granular matter, International journal of impact engineering, (2017).
} 


\subsection{Introduction}

The detonation of a shallow buried explosive can result in granular media being accelerated to very high velocities [51]. These high velocity granular particles impact nearby structures, and they can apply large impulsive loads that result in inelastic structural deformations and even rupture of the structure $[1,21]$. It is difficult and time-consuming to assess potential mitigation strategies by experimental means, especially as the impulsive load increases. This is further complicated by obscuration of the loading event by the ejecta [35]. Numerical simulations that accurately model the physics of soil acceleration [3, 44, 47], its impact and loading of a test structure $[12,15,16,49]$, and the structures dynamic response provides a complementary means for investigating potential mitigation concepts. The study presented here uses a combination of an instrumented experiment and particle-based simulations to investigate the dynamic loading and response of a model test structure following detonation of a spherically symmetric granular material encased test charge. The validity of the discrete particle simulation approach is investigated for high intensity impulsive loading conditions where the granular media is explosively accelerated to a high (supersonic) velocity and impacted against a ductile edge clamped test plate.

\subsection{Experimental Setup}

A high intensity soil impact loading experiment was conducted at an outdoor testing facility operated by the NEWTEC Services Group, Inc. (Edgefield, SC). A schematic illustration of the test setup is shown in Figure 3.1, with additional details given in Figure 3.2. The test utilized a rigid platform to support a square edge clamped test plate. The detonation of a suspended high explosive sphere encased within a spherical annulus of water-saturated synthetic sand provided the impulse loading. A $3.81 \mathrm{~m}$ long, strain gauge instrumented, maraging steel Kolsky bar system was used for measurement of the sand impact pressure and impulse applied at a location symmetrically equivalent to the center of the test plate.

\subsubsection{Test platform}

A test platform was designed to enable the testing of edge clamped square test plates, Figure 3.1. A square support base with an overall footprint of $1.22 \mathrm{~m} \times 1.22 \mathrm{~m}$ was constructed using a 


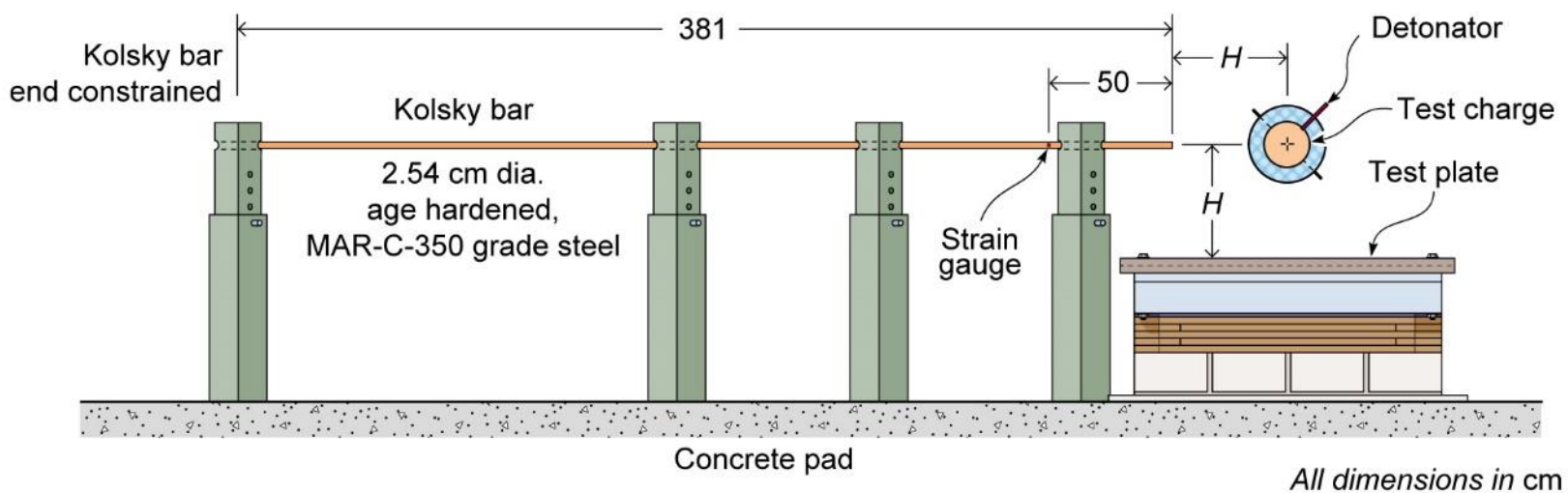

Figure 3.1. Schematic illustration of the test geometry. A spherical test charge consisting of an annular shell of wet sand encasing an explosive sphere was suspended a distance $H$ over the center of an edge clamped test structure with a Kolsky bar positioned a similar distance from the center of the charge. To ensure that similar ejecta from the charge impacted the center of the plate and the face of the Kolsky bar, the detonator was placed at a position that bisected the directions from the charge center to the test plate center and to the Kolsky bar axis.
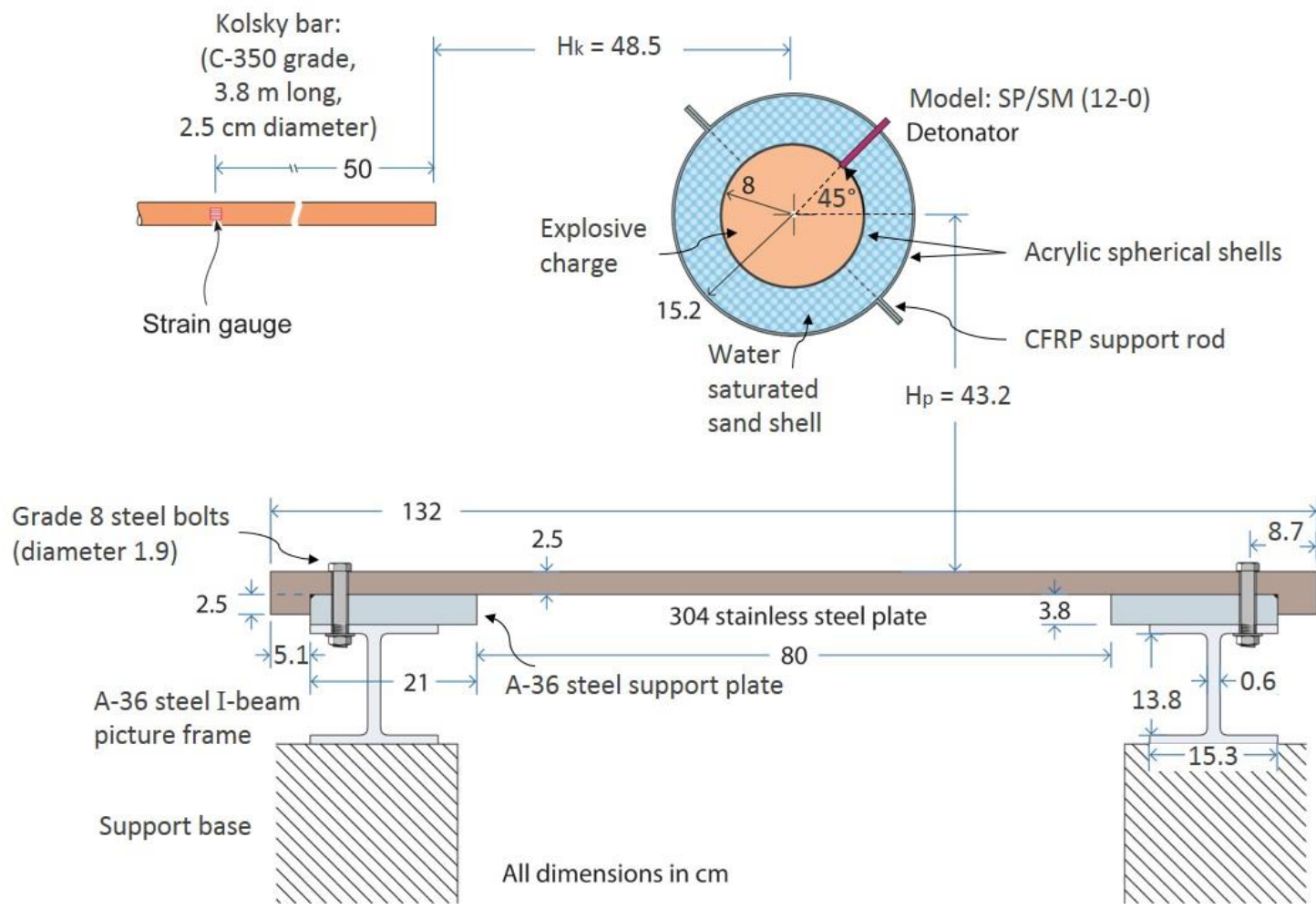

Figure 3.2. Schematic illustration of edge constrained test panel geometry showing the location of the Kolsky bar (including its strain gauge location), the test charge geometry and the standoff distances to the Kolsky bar $\left(H_{k}\right)$ and test plate $\left(H_{p}\right)$ at the instant of detonation. 
single layer of cinder block laid down on a concrete pad. Several wooden beam layers were placed on the cinder block to raise the platform to a height that enabled unobstructed observation of the test. A $0.95 \mathrm{~cm}$ thick rubber mat was placed on the wooden supports to cushion impacts, and reduce damage to the support structure. The rest of the platform was then assembled by positioning a square picture frame, constructed from welded, $15.3 \mathrm{~cm}$ deep, A-36 steel I-beams, on the support structure. To avoid plate shear-off near the test plate edge clamping, a $3.8 \mathrm{~cm}$ thick, $1.22 \mathrm{~m} \mathrm{x} 1.22$ m, A-36 steel support plate with an $80 \mathrm{~cm}$ x $80 \mathrm{~cm}$ square, center cutout was attached to the steel I-beam picture frame assembly, Figure 3.2. The test plate was then attached to the periphery of this support plate where its center, $80 \mathrm{~cm} \times 80 \mathrm{~cm}$ opening, defined the span during test plate testing, Figure 3.2. This enabled the total length of the edge clamped region to be large enough that the pull-in stresses at the edge attachment location were maintained below the yield strength of the test plate, thereby avoiding otherwise difficult to analyze edge effects during the test [17, 36].

\subsubsection{Test plate target}

The test plate target consisted of a $2.54 \mathrm{~cm}$ thick, $1.32 \mathrm{~m} \mathrm{x} 1.32 \mathrm{~m}, 304 \mathrm{~L}$ grade stainless steel plate, Figure 3.3. The plate was supplied by Rolled Alloys, Inc. (Temperance, MI) and was tested in the (as received) hot rolled, annealed and pickled (HRAP) condition. For testing, very large forces must be supported at the gripped edges of the test sample. Furthermore, studies with square plates that utilized edge grips that extended above the top surface of the plate were found to create very significant local impulse amplification due to the reaction momentum created during upward redirection of soil [17]. To prevent plate pull-in at its gripped edges during center impact loading, while also avoiding out-of-plane sand reflection at the periphery of the structure, $5.1 \mathrm{~cm}$ wide, 2.54 $\mathrm{cm}$ thick, rectangular cross section 304 grade stainless steel bars were welded in a picture frame pattern along the four edges of the eventual underside of the test plate, Figure 3.3.

To strengthen the edge restraint system, a series of holes were drilled through the edges of the test plate and the picture frame to enable insertion of $\sim 19 \mathrm{~mm}$ diameter, press-fitted tool steel dowel pins. This combination of welded and pinned bars robustly connected the $2.54 \mathrm{~cm}$ thick test plate to the picture frame support, Figure 3.3. This edge reinforced test plate was secured to the test platform with four $19 \mathrm{~mm}$ diameter, Grade 8 steel bolts located near the corners of the test plate, Figure 3.2. 


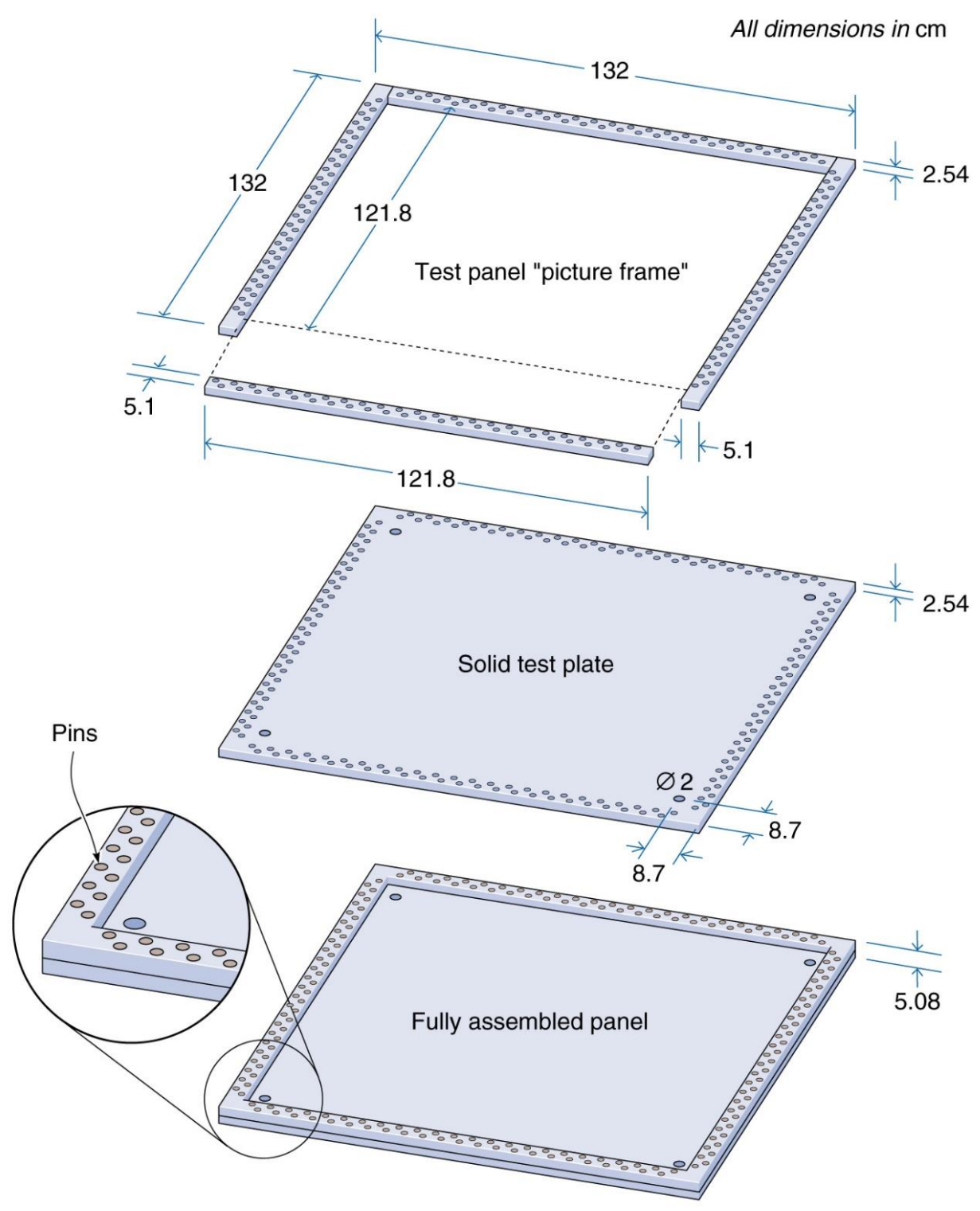

Figure 3.3. Illustration of the construction of the test plate with the welded frame used for attachment to the test stand. Steel dowel pins were press fitted to strengthen the welded connection between the test plate and the four rectangular bars.

\subsubsection{Test charge design and assembly}

A high explosive charge was suspended directly above the center of the test plate. The charge was constructed using two thin wall, acrylic plastic, concentric sphere assemblies with thicknesses ranging from $4.8 \mathrm{~mm}$ at the equator to $1.6 \mathrm{~mm}$ at the poles of each hemisphere. The concentric 
test charge was assembled by filling a $160 \mathrm{~mm}$ diameter, inner sphere with $3 \mathrm{~kg}$ of C-4 explosive [80]. A thin walled cylindrical plastic pipe with an internal diameter of $10 \mathrm{~mm}$ penetrated a pole of one of the hemispheres for subsequent placement of a detonator in contact with the explosive surface, Figure 3.4(a). The explosive sphere was then centered inside a $304 \mathrm{~mm}$ diameter, outer sphere with a carbon fiber reinforced polymer (CFRP) suspension rod passing through the center of both spheres for eventual positioning of the charge above the test plate. The outer sphere was again constructed from two hemispheres, each with a $3.8 \mathrm{~cm}$ flange around the equator which aided in the test charge assembly but resulted in a ledge around the sphere. The approximately 72 mm wide annular space between the two spheres was filled with $18.8 \mathrm{~kg}$ of glass microspheres with a diameter of 150-200 $\mu \mathrm{m}$, Figure 3.4(b). These Grade GL-0191 soda-lime glass spheres were obtained from Mo-Sci Corporation (Rolla, Missouri), and were identical to those used in previous studies by Hollomon et al. [13] and by Borvik et al. [19] for sand model calibration. Finally, $5.0 \mathrm{~kg}$ of water was added to fill the void spaces between the packed silica microspheres forming a water-saturated model test "soil", Figure 3.4(c). An instantaneous (0 ms delay) model SP/SM (12-0) detonator with a 3.5 m copper lead wire manufactured by Dyno Nodel Inc. (Salt Lake City, Utah) was inserted into the explosive through the plastic pipe just prior to testing.

To ensure that the center of the Kolsky bar and center of the test plate were subjected to similar impulsive loads, the orientation of the sphere was adjusted such that the detonator axis was at an angle of 45 degrees to both the test plate normal and the axis of the Kolsky bar. The center of the spherical charge was initially suspended $45 \mathrm{~cm}$ above the center of the top surface of the test plate and at the same standoff distance from the Kolsky bar end face. However, after the sphere was suspended, sufficient time elapsed during placement of the detonator for a small displacement of the charge to occur. The actual standoff distances (shown in Figure 3.2) to the plate and Kolsky bar could be measured from the high-speed camera images captured immediately before detonation. These measurements indicated the test charge was displaced $1.8 \mathrm{~cm}$ downward towards the plate and $3.5 \mathrm{~cm}$ further away from the Kolsky bar prior to the detonation. The actual standoff distance from the charge center to the end of the Kolsky bar was $\mathrm{H}_{\mathrm{k}}=48.5 \mathrm{~cm}$. If the top surface of the plate is taken to lie in the $\mathrm{X}-\mathrm{Y}$ coordinate plane with the $\mathrm{X}$-axis parallel to the Kolsky bar and the origin at the plate corner, the coordinates for the center of the test plate are located at $\mathrm{X}=66 \mathrm{~cm}$ and $\mathrm{Y}=66 \mathrm{~cm}$. The actual location of the charge center above the plate was $\mathrm{X}=69.5$ 
(a) Concentric explosive / sand charge preparation

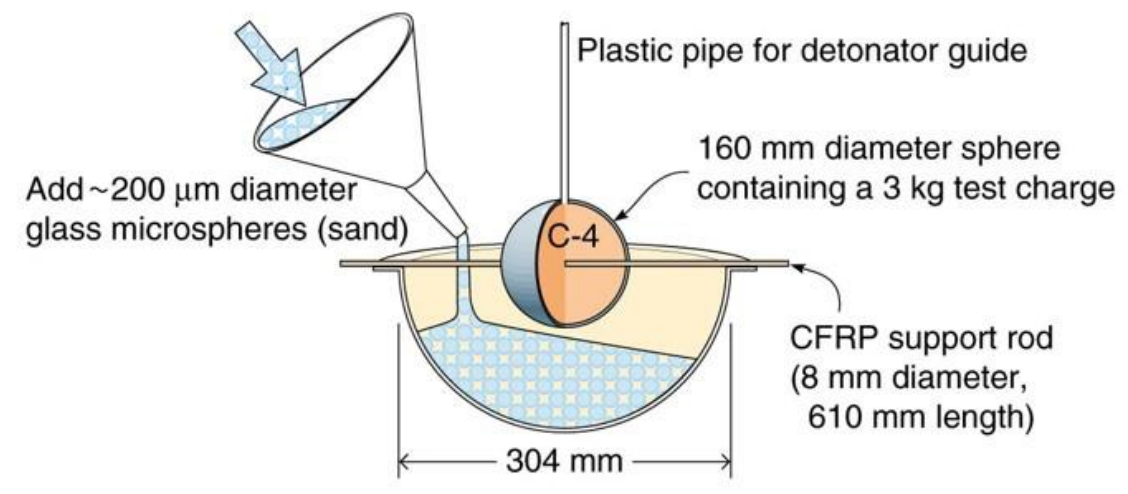

(b) Partially filled dry sand charge

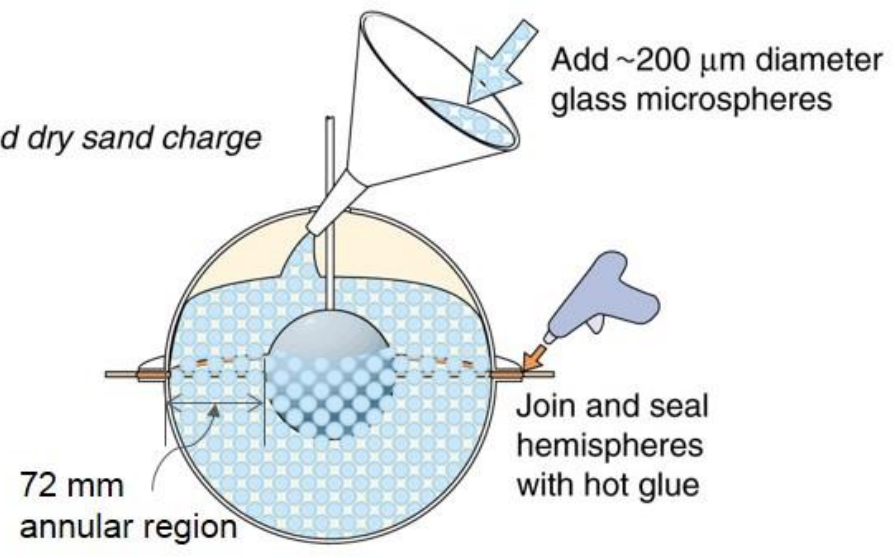

(c) Water saturated sand charge

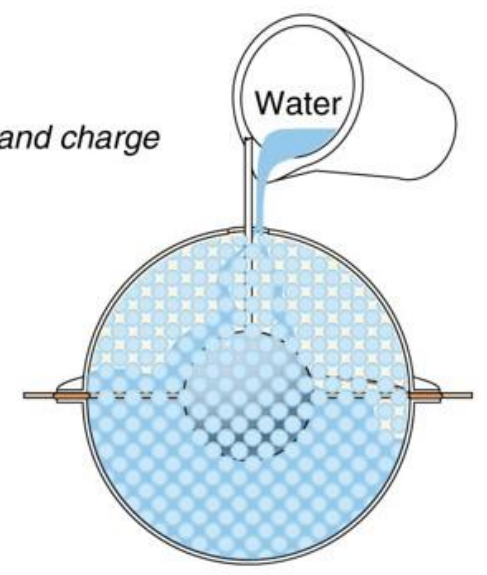

Figure 3.4. The geometry and procedure used to assemble the spherical test charge with its concentric shell of water-saturated synthetic sand. 
$\mathrm{cm}$ and $\mathrm{Y}=66 \mathrm{~cm}$, and the standoff distance from the charge center to the plate surface was $\mathrm{H}_{\mathrm{p}}=$ $43.2 \mathrm{~cm}$.

\subsubsection{The Kolsky bar}

The pressure applied by impact of the water-saturated sand was measured using a $2.54 \mathrm{~cm}$ diameter, $3.81 \mathrm{~m}$ long, age hardened C-350 grade, maraging steel Kolsky bar (Figure 3.1). Plastic bushings were placed around the Kolsky bar where it was attached to a series of pedestals to minimize energy leakage of the elastic modes of the Kolsky bar [81]. The end of the Kolsky bar was positioned so that a spherically expanding wet sand front reached the test target and the Kolsky bar almost simultaneously. Even though the charge had shifted slightly and was not equidistant to the test plate and end of the Kolsky bar, the Kolsky bar measurement still provided a quantitative measure of the pressure and impulse loading experienced near the center of the test plate. The simulation of its response provided a critical test of the validity of the numerical simulation approach. To minimize plastic deformation during impulsive loading, the maraging steel bar was aged before use at a temperature between 480 and $510^{\circ} \mathrm{C}$ for a period of 6 hours to achieve a Rockwell "C" scale hardness value of $\sim 58$ (equivalent to a tensile strength of $~ 2.07 \mathrm{GPa}$ ). Strain gauges were mounted on the Kolsky bar to enable the impact pressure to be measured, Figure 3.5. Two, T-rosette type strain gauges (Vishay Precision group, CEA-06-125UT-350), each with a pair of axial and transverse oriented strain gauge grid patterns, were adhesively bonded to the Kolsky bar, $0.5 \mathrm{~m}$ from the impact end, using Vishay AE-10 epoxy adhesive. The gauges were diametrically opposite to each other and were wired in a full-bridge Wheatstone bridge voltage measurement mode, Figure 3.5(a). A protective coating recommended for outdoor gauge installations (Vishay Gage Kote \#5), was applied over the strain gauges and the fine lead wires.

Figure 3.5(b) shows the signal conditioning and digital signal acquisition arrangement used to record the strain gauge voltages. The output of the strain gauge circuit was fed through a $7 \mathrm{~m}$ long 8723 Multi-Conductor, Shielded Twisted Pair cable to a $1 \mathrm{MHz}$ bandwidth amplifier (A.A. Lab Systems Ltd., G-3020) with an amplifier gain of 100. The output signal from the amplifier was then recorded with a digital oscilloscope (Tektronix, TPS2014) using a sampling rate of $1 \mathrm{MHz}$ at 8-bit resolution. In all, 2500 digitized samples were recorded (a $2.5 \mathrm{~ms}$ record length). The recording of the signal was initiated by a trigger event created by the breaking of a wire attached to the outer surface of the polymer shell containing the synthetic sand. This resulted in a time 
(a) Strain gauges

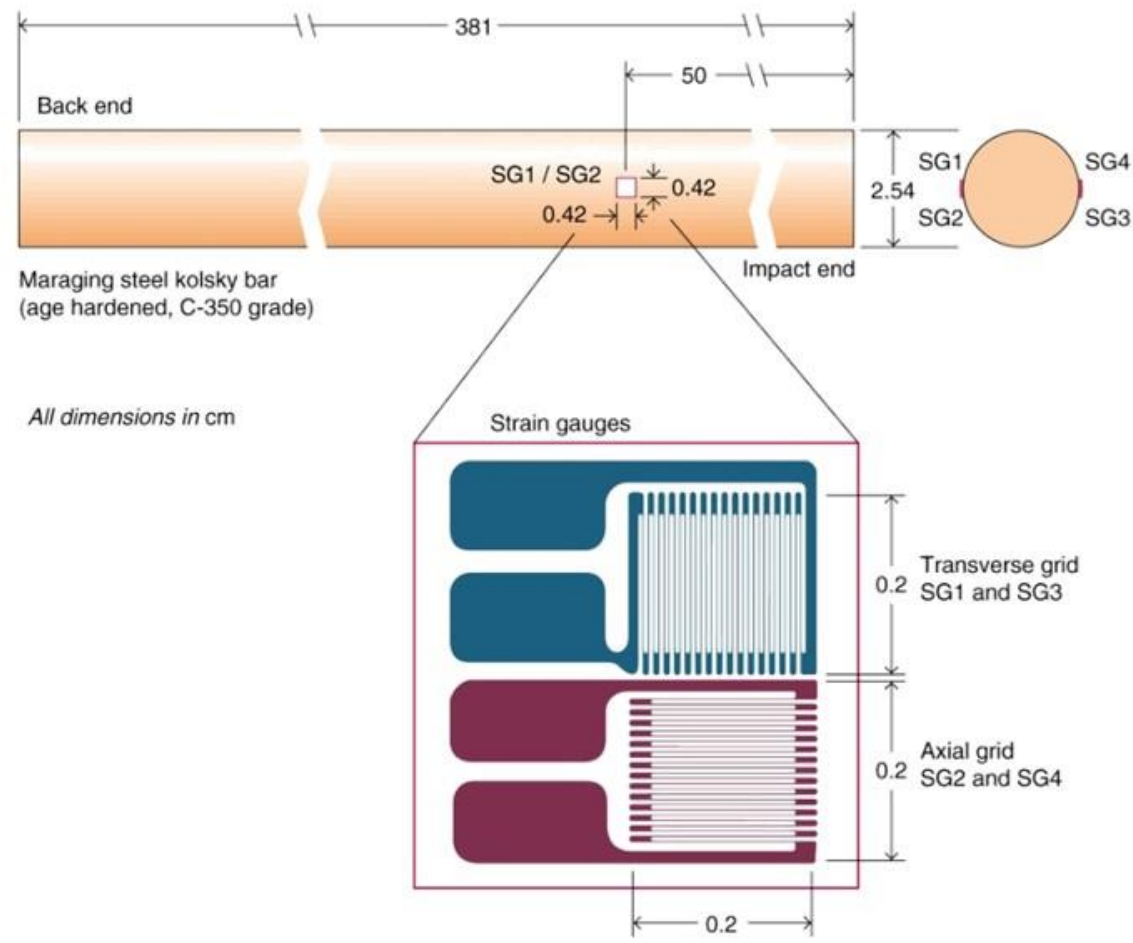

(b) Signal processing and recording

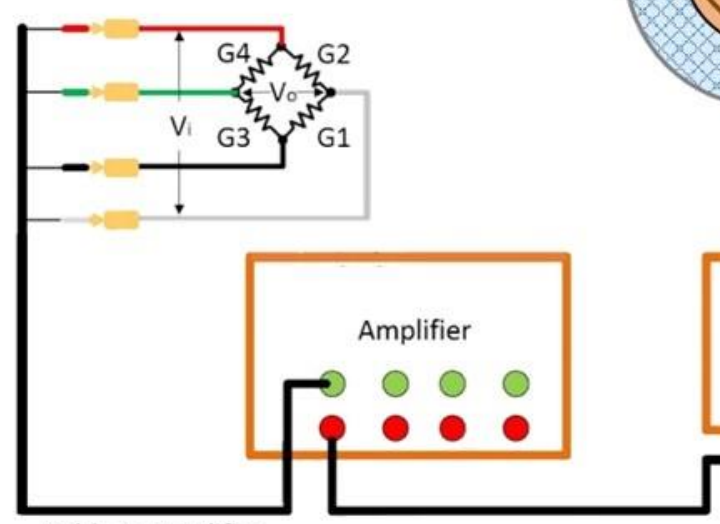

Cable to amplifier 6.4 -7.6 m length

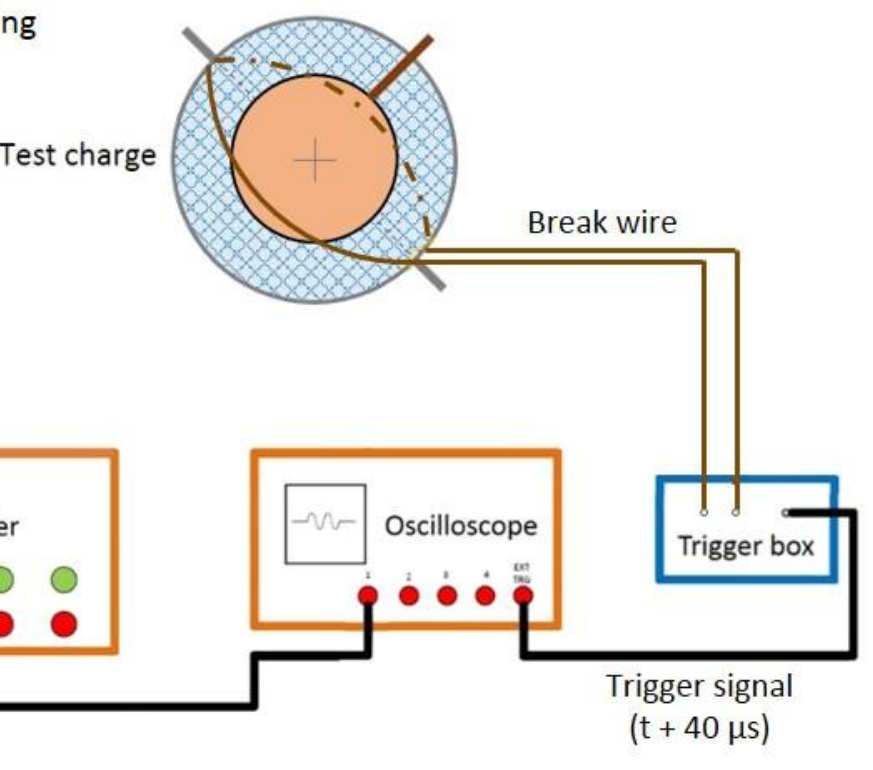

Figure 3.5. (a) The strain gauge sensor arrangement used to instrument the Kolsky bar. (b) Shows the signal conditioning and digital data recording arrangement. The trigger signal used to begin digital data recording was initiated by rupture of a break wire attached to the outer shell of the test charge. This trigger event was initiated $40 \mu$ s after the start of the detonation process. 
delay of $40 \mu$ s between the beginning of detonation and the time at which the trigger wire was broken. To avoid confusion, the initial time $\mathrm{t}=0 \mathrm{~s}$ is defined as the moment of detonation, and 40 $\mu$ s has been added to the experimental Kolsky bar data in all the results to follow. For protection from the blast, the instrumentation was placed to the side of the Kolsky bar in a robust metal box and covered with sand bags.

The input-output voltage relationship for the full-bridge wired measurement circuit used here is given by,

$$
\frac{V_{o}}{V_{i}}=\frac{G F * \varepsilon(1+v)}{2+G F * \varepsilon(1-v)}
$$

where GF $=2.15$ is the gauge factor for the Vishay strain gauge, $\varepsilon$ the axial strain in the Kolsky bar, $v=0.28$ the Poisson ratio of the bar material, $V_{i}=10 \mathrm{~V}$ the input excitation voltage, and $V_{o}$ the measured output voltage. The axial strain deduced from the voltage ratio, $V_{o} / V_{i}$, and equation (3.1) was then used with Hooke's Law and Young's modulus of the bar (E = 180.7 GPa), to determine the axial compressive stress (pressure) within the Kolsky bar. The transmitted specific impulse (units of $\mathrm{kPa} \cdot \mathrm{s}$ ) was found by integration of the pressure waveform over time from $\mathrm{t}=0$ s defined as the initiation of detonation.

\subsubsection{High speed video imaging}

Two Vision Research Inc., Phantom V7.3 high-speed cameras were positioned side by side in front of the test stand at $\sim 20 \mathrm{~m}$ from the charge to enable the wet sand front to be observed after detonation of the charge. A plan view of the X-Y plane, Figure 3.6, shows the experimental setup with the locations of the cameras (placed side by side). An expanded view of the test plate in the $\mathrm{X}-\mathrm{Y}$ plane indicates the distances to the plate center and the charge center location from the edge of the test plate (measured with the cameras). To provide a reference for distance and velocity calculations, a $20 \mathrm{~cm}$ long section of the front end of the Kolsky bar, and the full width of the 132 $\mathrm{cm}$ wide test plate front side were spray painted in white prior to the experiment. One camera provided a wide view of the entire event with a $640 \times 480$ pixel resolution and a $30 \mu$ s exposure time at $142 \mu$ s intervals. The second camera used a 512 × 256 resolution and $20 \mu$ s exposure time at $47 \mu$ s intervals to provide a magnified image of the test charge. These images were used to measure the sand front position. The cameras spatial resolution was reduced to allow the capture 


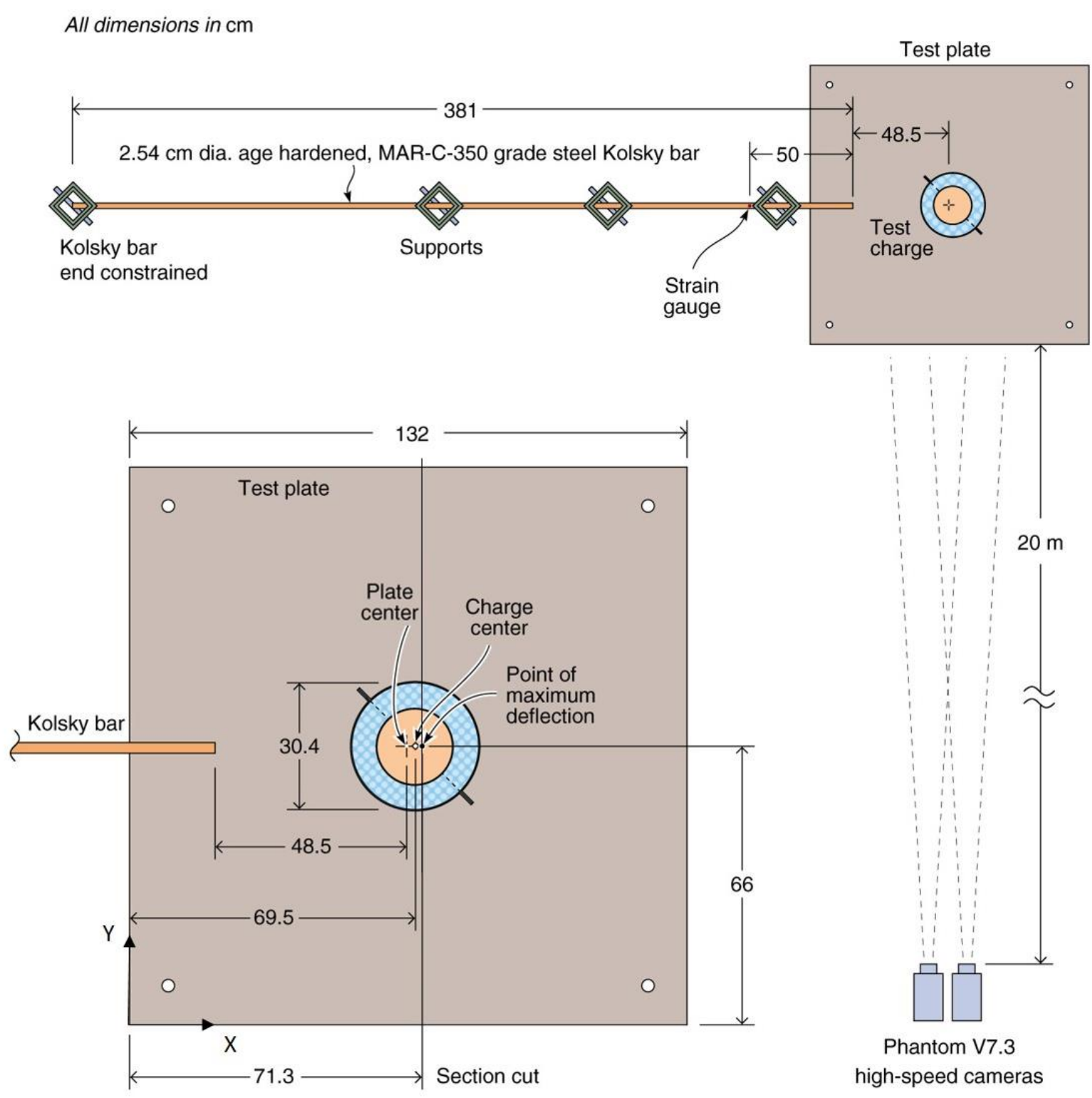

Figure 3.6. Plan view of the X-Y plane showing camera locations. An expanded view of the test charge and plate shows the plate center, the charge center at detonation, and the point of maxiumum plate deflection $(X=71.3 \mathrm{~cm}$ and $Y=66.0 \mathrm{~cm})$ after the event. The location of the section cut used to obtain a plate deflection profile is also shown. The origin of the coordinate system used for the study was at the front (lower) left corner of the test plate.

of more frames per second and therefore increase the temporal resolution. The higher magnification camera captured images that were $1.14 \times 0.57 \mathrm{~m}^{2}$ in area, with each pixel corresponding to $2.23 \times 2.23 \mathrm{~mm}^{2}$ area in the observation plane. 


\subsection{Simulation Methodology}

The suspended charge test geometry was simulated using the IMPETUS Afea Solver [19]. This code employs a discrete particle based method using air, high explosive (HE), and soil particles. A particle contact interaction model was used to determine the interaction between these discrete particles while contact forces created by particle impact with the test structure were coupled to a finite element (FE) model of the test plate and support structure to determine dynamic deformations. The particle model approach used here considers only the translational degrees of freedom of the system. Particle rotation is not addressed, which is equivalent to ascribing an infinite angular moment of inertia to the particles. The discrete particle method used here was first described by Borvik et al. [18] and Olovsson et al. [20], and has been validated as an analysis tool for low soil velocity loading by several studies [13, 14, 17, 19]. Particle contact parameter calibration was performed by Borvik et al. [19] with data from an experimental study [17] using spherical, sand encapsulated, $150 \mathrm{~g}$ high explosive charges. The sand in those tests was identical to that used here.

The simulation model used the geometry of the experimental setup with a charge suspended over a $2.54 \mathrm{~cm}$ thick, edge gripped solid plate that was bolted at its four corners to a steel support plate placed on an I-beam picture frame base structure, Figure 3.7. A $3.81 \mathrm{~m}$ long Kolsky bar was placed $45 \mathrm{~cm}$ above the test plate to determine the predicted pressure and impulse loading. The spherical charge was constructed with an inner sphere of high explosive material surrounded by an outer annular region of wet sand particles contained within a $3 \mathrm{~mm}$ thick acrylic plastic shell of $152 \mathrm{~mm}$ radius. The ledge around the outer shell was not modeled as it appeared to have no effect on the loading of the Kolsky bar or test plate as a result of its inclination.

The inner sphere was also confined in a $3 \mathrm{~mm}$ thick acrylic plastic shell with a radius of 80 mm. The inner sphere was filled with sufficient HE particles to represent a $3 \mathrm{~kg}$ charge with the properties of C-4. This concentric sphere charge was suspended in air at a vertical standoff distance of $43.2 \mathrm{~cm}$ from the center of the charge to the top of the solid plate. The horizontal standoff distance from the center of the charge to the front of the Kolsky bar was $48.5 \mathrm{~cm}$. Both distances were consistent with those measured from the experimental high-speed video images just prior to detonation. This data set the center of the charge at $X=69.5 \mathrm{~cm}, Y=66 \mathrm{~cm}$, and Z $=$ $43.2 \mathrm{~cm}$. The detonation point was defined at a 45 -degree angle from the top center of the spherical 


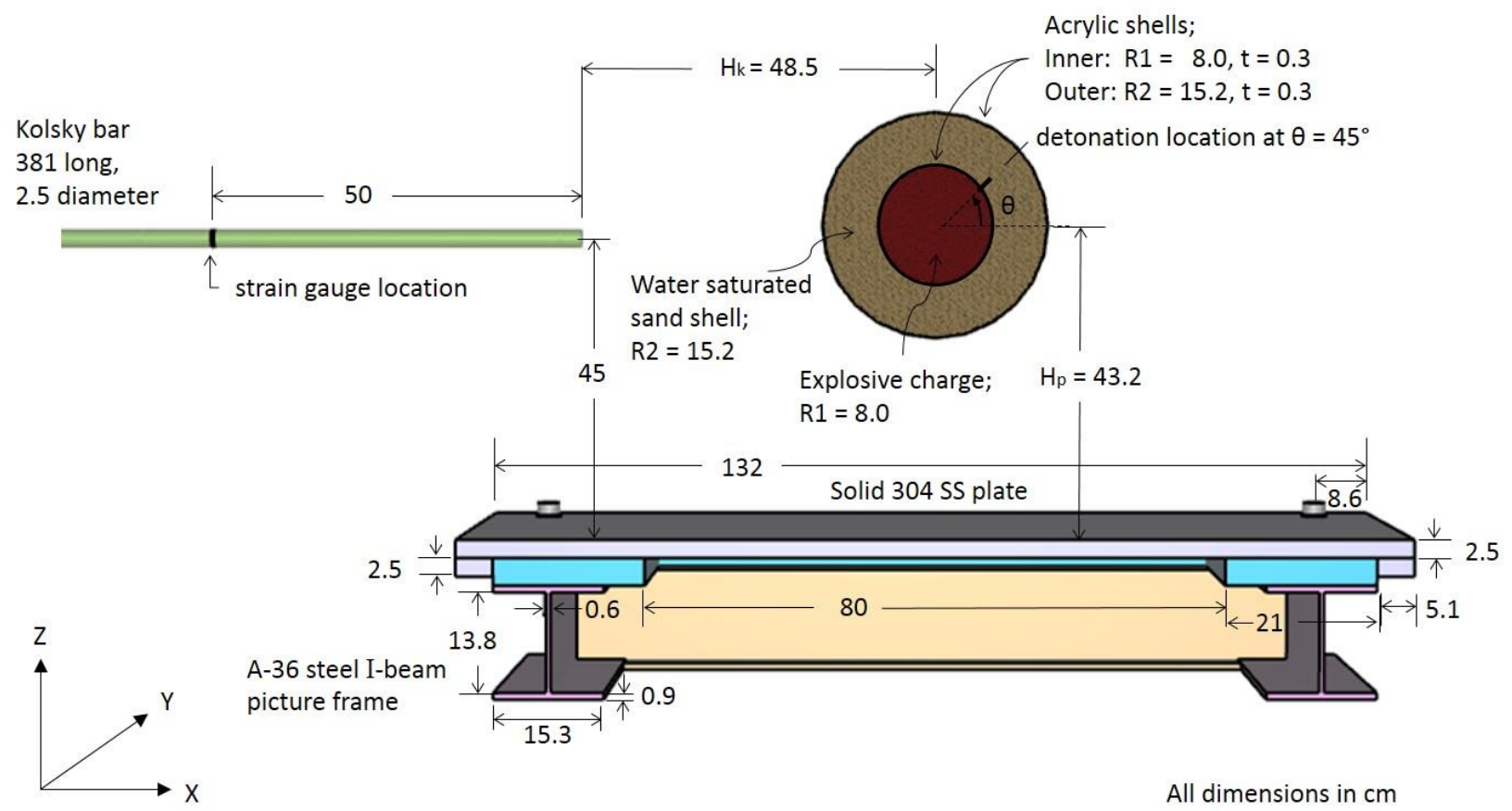

Figure 3.7. A cross-section through the mid-plane of the FE model geometry used for the IMPETUS Afea discrete particle based simulation. The coordinate axis origin was located at the front left corner of the top of the test plate (out of the plane of this figure) as shown in Figure 3.6.

charge and at the edge of the C-4, located on the opposite side of the Kolsky bar. One additional simulation test was performed with a second Kolsky bar positioned at the center of the test plate location $(X=66 \mathrm{~cm}, Y=66 \mathrm{~cm}$, and $\mathrm{Z}=0 \mathrm{~cm}$ with the front of the Kolsky bar in the $\mathrm{X}-\mathrm{Y}$ plane $45 \mathrm{~cm}$ below the center of the test charge). It confirmed that the impulse applied to both Kolsky bars (when each was placed at a $45 \mathrm{~cm}$ standoff distance from the test charge center) were the same, validating the assumption that radial expansion and loading are equivalent in these two directions, and confirming that the horizontal Kolsky bar could be used as a witness of the blast load near the center of the test plate.

\subsubsection{Particle model}

The air and HE particles used by the solver were modeled as rigid particles each representing many actual particles that transfer momentum via collisions. Following Olovsson et al. [20], particle interactions between air and HE particles were taken to be elastic, consistent with Maxwell's kinetic theory of gases [82]. In this approach, IMPETUS models the air particles as an ideal gas with density $\rho=1.3 \mathrm{~kg} / \mathrm{m}^{3}$, initial internal energy $\mathrm{E}_{0}=253 \mathrm{~kJ} / \mathrm{m}^{3}$, and a ratio of specific 
heats $\gamma=1.4$ with initial directions and velocities distributed at the start of the simulation according to the Maxwell-Boltzmann distribution [13]. The IMPETUS code has several predefined HE particle options that have been calibrated by Borvik et al. [19] by iterative simulation of a standard explosive filled pipe test. For the simulations reported here, the C-4 high explosive option was selected with predefined parameters of initial density $\rho=1601 \mathrm{~kg} / \mathrm{m}^{3}$, initial internal energy $\mathrm{E}_{0}=$ $8.7 \mathrm{GJ} / \mathrm{m}^{3}$, ratio of heat capacities $\gamma=1.4$, particle initial solid-fill fraction $\mathrm{b}=0.35$, and a detonation speed $\mathrm{D}=8190 \mathrm{~m} / \mathrm{s}$. The explosion was initiated at the detonator location by releasing $\mathrm{HE}$ into motion at a release time R/D where $\mathrm{R}$ was the radial distance from the point of detonation and $\mathrm{D}$ the detonation velocity [19].

The water-saturated (wet) sand was modeled with a penalty based (soft-particle) inter-particle contact law governing the behavior of the soil particles [19]. This contact model comprised a linear spring $\mathrm{k}$, in parallel with a linear dashpot with a damping constant $\mathrm{c}$, which were in series with a Coulomb friction component characterized by a coefficient of friction, $\mu$. The values selected for the normal spring constant and the damping parameter govern the normal motion while a horizontal spring constant and the friction coefficient govern the tangential motions during interparticle contact. The sand input parameter model was calibrated for 150-200 $\mu \mathrm{m}$ diameter, silica glass microspheres (density of $2700 \mathrm{~kg} / \mathrm{m}^{3}$ ) for both dry and water-saturated (wet) sand. The dry sand fill fraction was $60 \%$ for the glass spheres with initial density $\rho=1620 \mathrm{~kg} / \mathrm{m}^{3}$. The solver, rather than directly modeling the water in the wet sand, accounts for water effects by modifying the sand particle contact friction, contact stiffness, and damping as proposed by Borvik et al. [19]. The wet sand was given an initial density $\rho=2020 \mathrm{~kg} / \mathrm{m}^{3}$. The calibrated wet sand parameters include the soil-soil particle contact stiffness $\mathrm{k}_{0}=4.0 \mathrm{GN} / \mathrm{m}$, the soil-soil particle contact coefficient of friction $\mu=0.0$, and soil-soil particle damping coefficient $\xi=0.005$.

To analyze the sand particle distribution, the sand particle density was calculated at specified time steps after detonation. The simulated volume was segmented into approximately $1 \mathrm{~cm}^{3}$ volume rectangular cells in a radial direction of interest. The number of simulated sand particles in each cell was determined to calculate the simulation particle density,

$$
\left(\frac{N}{V}\right)_{i}
$$


(number of particles $/ \mathrm{cm}^{3}$ ) where $N$ is the number of sand particles in the segmented cell $i$ with volume $V$ (approximately $1 \mathrm{~cm}^{3}$ ). The sum of the particles in all cells was also calculated,

$$
\sum_{j}\left(\frac{N}{V}\right)_{j}
$$

The particle density was then divided by the total number of simulated sand particles to calculate a particle probability density,

$$
\frac{\left(\frac{N}{V}\right)_{i}}{\sum_{j}\left(\frac{N}{V}\right)_{j}}
$$

A convergence study indicated the simulation converged at 2 million total particles divided (by the solver) into 803,849 air, 439,911 sand, and 756,240 HE particles. For the 2 million particle simulation results shown here, one simulated sand particle was equivalent to approximately 5,200 actual sand particles.

\subsubsection{Finite Element model}

A cross sectional view of the model and the suspended spherical charge with the model coordinate system defined is shown in Figure 3.7. The coordinate axis origin was located at the top front left corner of the test plate, shown in Figure 3.6. The $3.81 \mathrm{~m}$ long Kolsky bar was modeled using the IMPETUS Afea Solver finite element package as a series of four cylindrical parts each of diameter $2.54 \mathrm{~cm}$ merged together, using the merger option in the solver. A $2.54 \mathrm{~cm}$ length cylindrical section located at the front of the bar was used to measure the impact force on the front of the Kolsky bar. The second cylindrical part was $47.86 \mathrm{~cm}$ in length and ended at the location of the strain gauges where the pressure was experimentally measured. The third part consisted of a $4 \mathrm{~mm}$ cylinder representing the region where strain gauges were located and was used to measure the bar pressure. The fourth cylindrical section covered the remaining length of the bar. The full bar model was constructed from 39,000 linear hexahedra elements with 43,248 nodes.

The $2.54 \mathrm{~cm}$ thick solid plate with in plane dimensions of $1.32 \mathrm{~m} \mathrm{x} 1.32 \mathrm{~m}$, was supported on a test platform consisting of a $3.8 \mathrm{~cm}$ thick support plate, with an $80 \mathrm{~cm}$ x $80 \mathrm{~cm}$ center opening on top of an I-beam picture frame structure. To clamp the edges of the plate securely to the test 
platform, an outer $(5.1 \mathrm{~cm}$ wide and $2.54 \mathrm{~cm}$ thick) picture frame was merged to the solid $2.54 \mathrm{~cm}$ thick plate to represent the four rectangular bars that were welded and pin reinforced to the test plate. The bottom plane of the model was constrained in the $\mathrm{X}, \mathrm{Y}$, and $\mathrm{Z}$ directions using a fixed boundary condition. A mesh sensitivity analysis was performed to determine the number of elements needed for solution convergence. The final full model geometry, including the inner and outer spherical acrylic shells, test plate, test platform, attachment bolts, and the Kolsky bar, was meshed with a total of 44,284 elements; 288 linear pentahedra elements, 42,320 cubic elements, and 1,676 linear hexahedra elements with 129,432 nodes. The test plate used a more refined mesh than the support structure and the Kolsky bar to better observe the plate deflection during impulse loading. The full test plate with rectangular bars forming the picture frame edge consisted of 1,676 cubic hexahedra elements with 62,964 nodes.

\subsubsection{Material models}

The test plate, support frame, bolts, and Kolsky bar were all modeled as isotropic, JohnsonCook materials. The IMPETUS Afea Solver uses the Johnson-Cook (J-C) constitutive model to calculate the von Mises flow stress defined by [13],

$$
\sigma_{y}=\left(A+B\left(\left(\varepsilon_{e f f}\right)^{n}\right)\right) \cdot\left(1+C \ln \frac{\dot{\varepsilon}_{e f f}}{\dot{\varepsilon}_{0}}\right) \cdot\left(1-\left(\frac{T-T_{0}}{T_{m}-T_{0}}\right)^{m}\right)
$$

where $\mathrm{A}, \mathrm{B}, \mathrm{n}, \mathrm{C}$, and $\mathrm{m}$ are material constants and $\varepsilon_{\text {eff }}$ is the effective plastic strain of the material. The strain hardening constant parameters are the initial yield strength $\mathrm{A}$, the two hardening parameters $\mathrm{B}$ and $\mathrm{n}$, the strain rate parameter $\dot{\varepsilon}_{0}$, and the strain rate hardening parameter $\mathrm{C}$. The $\mathrm{J}-\mathrm{C}$ model also included the ambient temperature $\mathrm{T}_{0}$, melting temperature $\mathrm{T}_{\mathrm{m}}$, and the thermal softening parameter $\mathrm{m}$, for the material. The J-C model parameters for 304SS were reported by Dean et al. [83] and by Mori et al. [84] for annealed 304SS. For the A-36 steel constitutive model, the model parameters for ASTM-A36 were assumed [85]. The J-C parameters for 350 grade, maraging steel were taken from parameters for a similar VascoMax 300 alloy [86], assuming approximately equivalent material properties with an adjusted yield strength parameter for 350 grade steel. The reported value for the yield strength of 350-grade commercial maraging steel is 2.195 GPa with an ultimate tensile strength of $2.227 \mathrm{GPa}$ [87]. The bolts were modeled as AISI 1040 carbon steel with J-C model parameters reported by Cohen et al. [88]. The J-C parameters 
Table 3.1. Material constants for 304SS, A-36 steel, C-350 maraging steel, and AISI 1040.

\begin{tabular}{|c|c|c|c|c|c|c|c|c|c|c|c|}
\hline \multirow[t]{2}{*}{ Material } & \multicolumn{3}{|c|}{$\begin{array}{l}\text { Density and } \\
\text { elastic constants }\end{array}$} & \multicolumn{3}{|c|}{$\begin{array}{l}\text { Yield stress and } \\
\text { strain hardening }\end{array}$} & \multicolumn{2}{|c|}{$\begin{array}{l}\text { Strain rate } \\
\text { hardening }\end{array}$} & \multicolumn{3}{|c|}{$\begin{array}{l}\text { Temperature } \\
\text { softening } \\
\text { and adiabatic } \\
\text { heating }\end{array}$} \\
\hline & $\begin{array}{c}\rho \\
\left(\mathrm{kg} / \mathrm{m}^{3}\right)\end{array}$ & $\begin{array}{c}\mathrm{E} \\
(\mathrm{GPa})\end{array}$ & $v$ & $\begin{array}{c}\mathrm{A} \\
(\mathrm{MPa})\end{array}$ & $\begin{array}{c}\mathrm{B} \\
(\mathrm{MPa})\end{array}$ & $\mathrm{n}$ & $\mathrm{C}$ & $\begin{array}{c}\varepsilon_{0} \\
\left(\mathrm{~s}^{-1}\right)\end{array}$ & $\begin{array}{l}\mathrm{T}_{0} \\
(\mathrm{~K})\end{array}$ & $\begin{array}{l}\mathrm{T}_{\mathrm{m}} \\
(\mathrm{K})\end{array}$ & $\mathrm{m}$ \\
\hline $304 \mathrm{SS}$ & 7900 & 200 & 0.3 & 310 & 1000 & 0.65 & 0.07 & 1.0 & 293 & 1673 & 1.0 \\
\hline A-36 & 7850 & 199 & 0.3 & 244 & 484 & 0.235 & 0.0165 & $2 \cdot 10^{-4}$ & 300 & 1790 & 1.03 \\
\hline C-350 & 7900 & 180.7 & 0.28 & 2195 & 9400 & 1.175 & 0.0046 & 1.0 & 300 & 1685 & 0.78 \\
\hline $\begin{array}{l}\text { AISI } \\
1040\end{array}$ & 7845 & 200 & 0.28 & 533 & 601 & 0.234 & 0.0134 & 1.0 & 293 & 1521 & 1.0 \\
\hline
\end{tabular}

used with Equation (3.5) are summarized in Table 3.1 for the three materials. There was no fracture of the test plate so a failure model for 304SS was not included in the simulation model.

The acrylic polymer spherical shells used for both the inner shell (containing the HE) and the outer shell (containing the wet sand), were modeled using a linear elastic constitutive model with acrylic plastic values of density $\rho=1180 \mathrm{~kg} / \mathrm{m}^{3}$, Young's modulus $\mathrm{E}=2.80 \mathrm{GPa}$, and Poisson's ratio $v=0.37$. The failure criteria for the plastic model was set at an effective geometric strain of $10 \%$. When elements in the geometric shell, reached the failure strain $\varepsilon_{\text {fail }}=0.1$, they were eroded.

\subsection{Sand Shell Expansion}

\subsubsection{High speed video observations}

Figure 3.8 shows a sequence of images of the test charge recorded with the wide field of view camera following detonation. Both the white painted side of the test plate and $20 \mathrm{~cm}$ length end of the Kolsky bar can be seen. The $t=0 \mathrm{~s}$ image in Figure 3.8(a) corresponds to the moment of detonation. This image was used to confirm that the test charge was displaced to the right of the plate center by $3.5 \pm 0.3 \mathrm{~cm}$ and displaced downwards from the Kolsky bar center axis by $1.8 \pm$ $0.3 \mathrm{~cm}$. This resulted in actual standoff distances (measured from the test charge center) to the plate $\mathrm{H}_{\mathrm{p}}=43.2 \pm 0.3 \mathrm{~cm}$ and to the Kolsky bar end $\mathrm{H}_{\mathrm{k}}=48.5 \pm 0.3 \mathrm{~cm}$.

The white sand front expanded almost isotropically with only a slight asymmetry in sand front shape. It can be seen to have impacted the test plate between the images shown in Figure 3.8(c) 

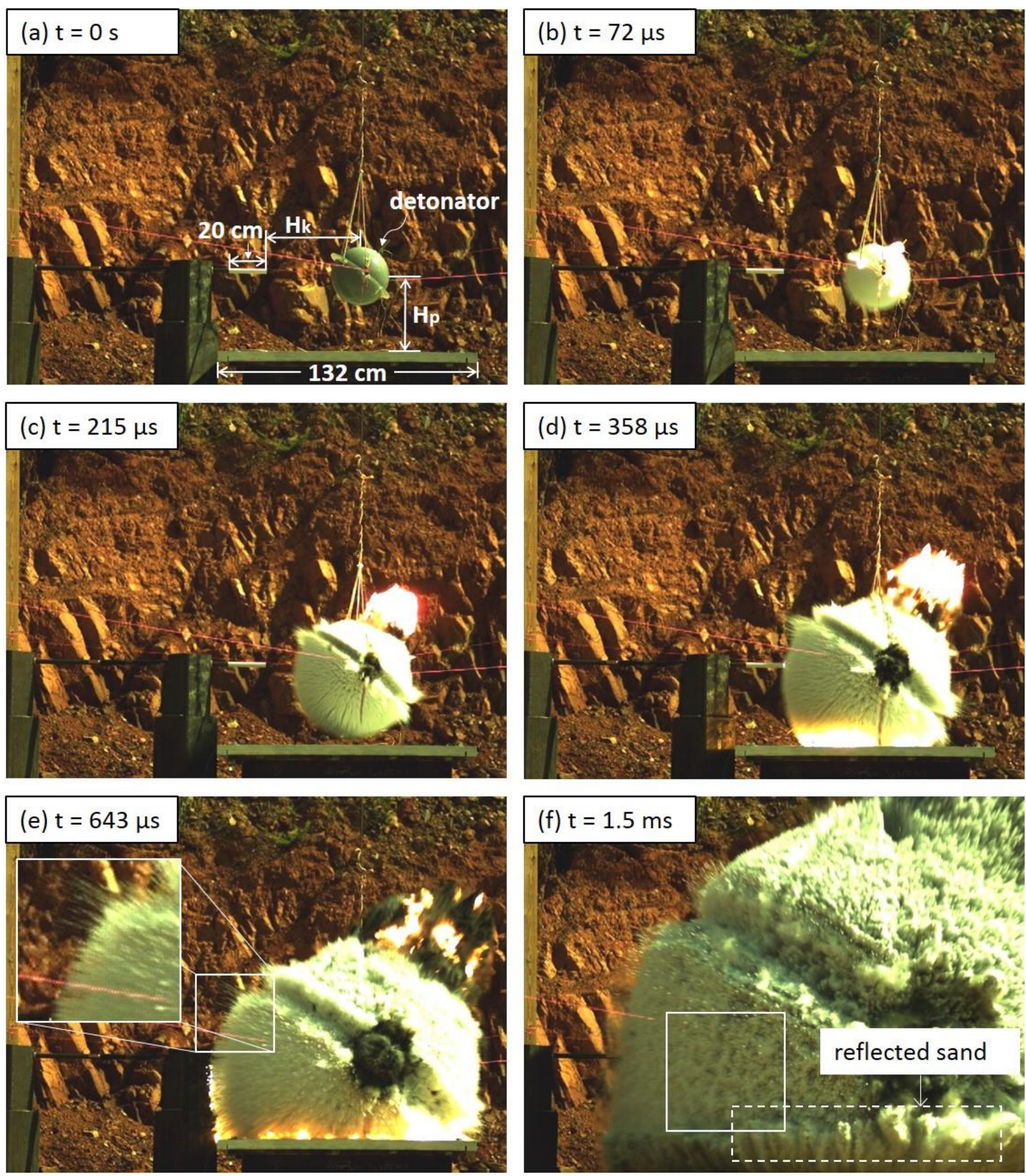

Figure 3.8. Sand front propagation observed with the wide-angle video camera; (a) $t=0 \mathrm{~s}$ (instant of detonation), (b) $t=72 \mu \mathrm{s}$ (c) $t=215 \mu \mathrm{s}$, (d) $t=358 \mu \mathrm{s}$, (e) $t=643 \mu \mathrm{s}$, and $(f)=1.5 \mathrm{~ms}$. The distance from the charge center to the test plate was $H_{p}=43.2 \mathrm{~cm}$ and to the Kolsky bar end $H_{k}=48.5 \mathrm{~cm}$. (e) A magnified region of the outermost sand front shows the presence of sand spikes (fingers). The white box in $(f)$ indicates the region observed in Figure 3.10. 
and (d) at approximately $\mathrm{t}=290 \mu \mathrm{s}$, slightly before impact with the Kolsky bar; consistent with the reduced standoff distance to the plate surface. The lower part of Figure 3.8(f) shows that after impact with the test plate the sand front had flowed laterally over the plate surface and off the edges of the test plate. This sand had a small upward component of motion consistent with sand refection at an increasingly acute angle of impact near the plate periphery. Careful examination of the sand front images in Figure 3.8 indicates the presence of locally faster sand spikes (sometimes referred to as "fingering") similar to those observed by Holloman et al. [29] for buried explosive events. Figure 3.8(e) shows a magnified region where the sand spikes/fingers with small radius of curvature leading edges are more clearly observed. The images in Figure 3.8(d) and (e) indicate significant (white) optical luminescence associated with the first sand finger impacts with various parts of the test plate and the Kolsky bar support structure. The significant additional mass of the acrylic polymer ledges around the equator of the test charge can be seen to have locally slowed the sand front. However, due to the inclination of the charge, this had no effect upon sand front expansion towards the test plate or end of the Kolsky bar.

Figure 3.9 shows a similar sequence of images obtained with the higher magnification camera using the same definition of time as Figure 3.8. Figure 3.8(b) and Figure 3.9(b) indicate that, at $t$ $=72 \mu \mathrm{s}$, the sand sphere had already begun to radially expand. By backward extrapolation, it is estimated that the sand front began its movement at $\mathrm{t}=40 \pm 20 \mu \mathrm{s}$, consistent with the propagation of an explosive detonation front with handbook velocity of $8040 \mathrm{~m} / \mathrm{s}$ [80] across the $160 \mathrm{~mm}$ diameter charge (in $\mathrm{t}=20 \mu \mathrm{s}$ ) followed by the propagation of a shock through the $72 \mathrm{~mm}$ thick annular sand shell at a shock velocity of $3600 \mathrm{~m} / \mathrm{s}[89,90]$.

The main sand front position is indicated with a black dotted line in Figure 3.9(d), and shows the fast moving sand spikes well ahead of this main front. The image sequence in Figure 3.9 shows the length of the sand front fingers increased with time consistent with their higher velocity. The images in Figure 3.9(f) indicate the observable tips of the sand fingers had advanced between 2 and $5 \mathrm{~cm}$ ahead of the main sand front at $453 \mu \mathrm{s}$ after detonation. Impact of the stretching sand with both the end of the Kolsky bar and the center of the test plate was therefore distributed over time. Examination of the high speed video also showed that the sand front developed a fractal like topology consisting of outwardly propagating sand cones that themselves became composed of smaller diameter cones as the sand radially expanded. Figure 3.10 shows the square region 

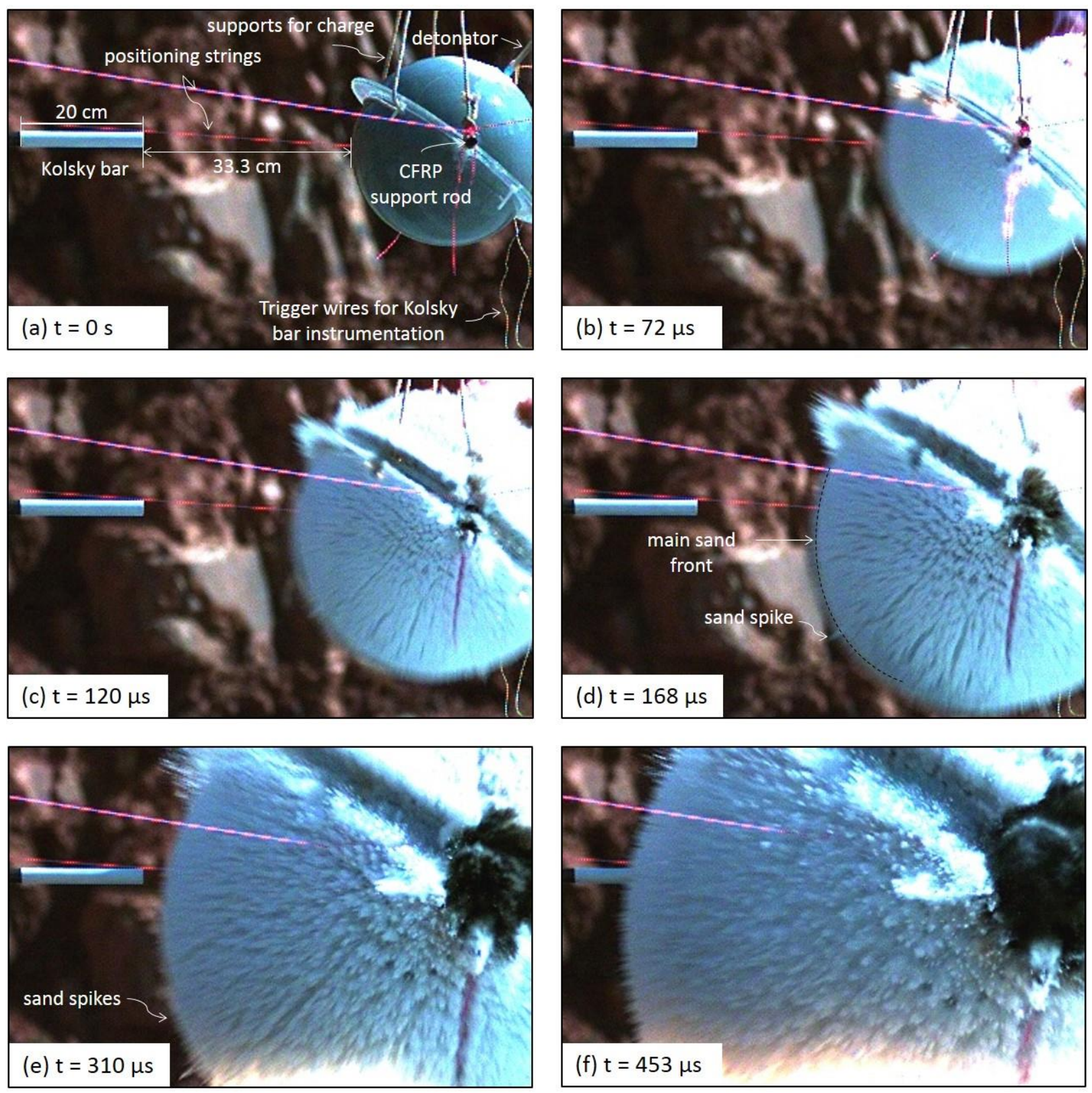

Figure 3.9. Images of the sand using the higher spatial resolution high speed camera at times $(a) t=0 \mathrm{~s}$ (instant of detonation), (b) $t=72 \mu \mathrm{s}$, (c) $t=120 \mu \mathrm{s}$, (d) $t=168 \mu \mathrm{s},(e) t=310 \mu \mathrm{s}$, and $(f)=453 \mu \mathrm{s}$. The sand progression towards the Kolsky bar, the main sand front expansion, and the sand fingering effect can be seen in $(d)$.

indicated in Figure 3.8(f) where conical features in the sand front decompose into smaller diameter cones over time. 
(a) $t=1.64 \mathrm{~ms}$

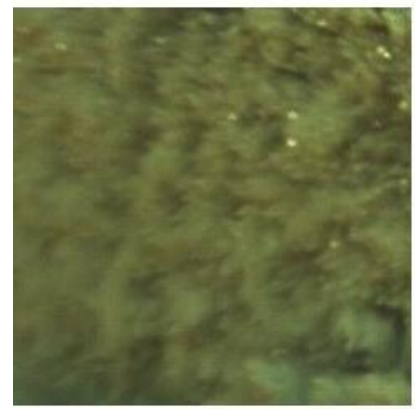

(b) $\mathrm{t}=1.79 \mathrm{~ms}$

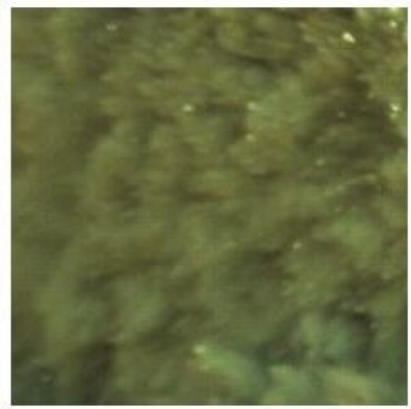

(c) $\mathrm{t}=1.93 \mathrm{~ms}$

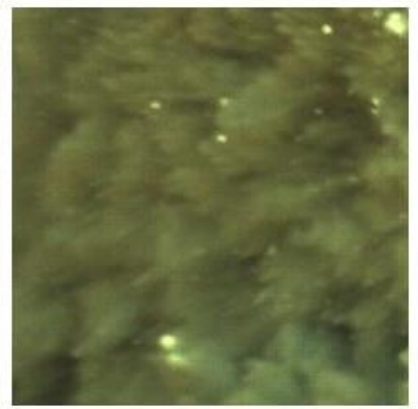

(d) $\mathrm{t}=2.07 \mathrm{~ms}$

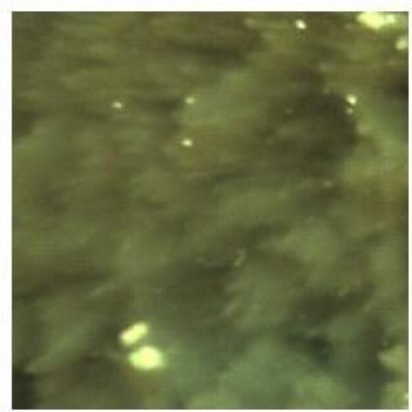

Figure 3.10. Sand front expansion for the white box region in Figure 3.8(f). The sand front consisted of many circumferentially expanding conical regions. As propagation of the front advanced in the radial direction, the cones evolved into arrays of smaller diameter cones.

A full analysis of the main sand front position was performed in the Kolsky bar horizontal direction by assuming the observed radial expansion was in the plane formed by the charge diameter and the axis of the Kolsky bar. Since it was difficult to identify a consistent front for the sand fingers, particularly early in the expansion process, only the main spherical sand front expansion (indicated by the black dotted line in Figure 3.9(d)) was measured. This experimentally deduced sand front location is plotted as solid black circles in Figure 3.11(a). Numerical differentiation of the main sand front leading edge position data gave a sand front velocity that is also shown as solid black circles in Figure 3.11(b). Error propagation analysis was used to estimate the uncertainty in sand front velocity [91], and resulted in a radial velocity error that decreased with time from $\pm 96 \mathrm{~m} / \mathrm{s}$ error for the earliest velocity estimate to $\pm 15 \mathrm{~m} / \mathrm{s}$ for the last data point. It is evident from Figure 3.11(b) that the main sand front was rapidly accelerated to a maximum velocity of $\sim 1200 \mathrm{~m} / \mathrm{s}$ at $110 \mu$ s after detonation, and was followed thereafter by a slower, but prolonged period of deceleration. Given a measured standoff distance of $48.5 \mathrm{~cm}$ from the charge center to the edge of Kolsky bar, and an initial outer sand sphere radius (including polymer shell thickness) of $15.2 \mathrm{~cm}$, the distance from the front of the initially stationary annular sand shell to the front of the Kolsky bar was $33.3 \mathrm{~cm}$. The distance of sand front propagation to the front surface of the target plate was $28 \mathrm{~cm}$, since the standoff distance to the plate was $43.2 \mathrm{~cm}$. Projection of these propagation distances on the position vs time plot, Figure 3.11(a), shows that the main sand front required a time of $\sim 330 \mu$ s to reach the Kolsky bar and $\sim 290 \mu$ s to reach the target plate. Figure 3.11(b) indicates that at these instances, the main sand front's instantaneous velocity was beginning to decrease from its maximum value, and at impact with the Kolsky bar, the velocity 

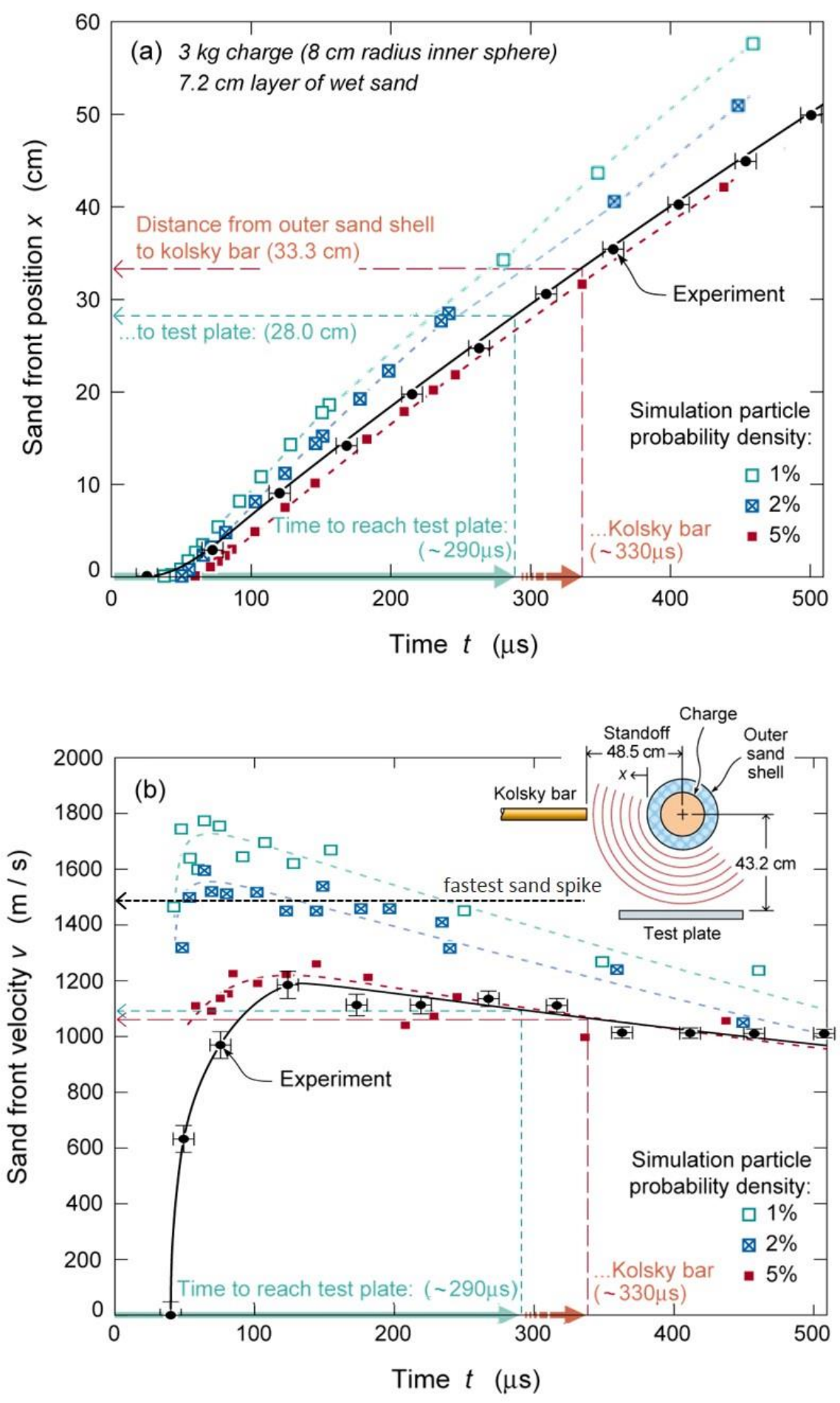

Figure 3.11. (a) Experimental and IMPETUS Afea predicted sand front positions measured in the Kolsky bar direction (horizontal $X$ direction) and (b) sand front velocity vs time after detonation. The velocity of the fastest experimentally observable sand spike (finger) is also indicated on (b). The estimated time for the sand to reach both the Kolsky bar and test plate are also shown. 
was $1050 \pm 44 \mathrm{~m} / \mathrm{s}$, and $1100 \pm 50 \mathrm{~m} / \mathrm{s}$ at impact with the plate. The maximum estimated velocity of the fastest sand spike, $v=1470 \mathrm{~m} / \mathrm{s}$, shown in Figure 3.9(d), is indicated by the horizontal dotted black line in Figure 3.11(b). These sand spikes appear to be a manifestation of an interfacial instability that exists at high velocity granular matter/air interfaces $[62,68,70]$.

\subsubsection{Simulated sand front}

The IMPETUS predicted HE and sand particle distributions for a sectional plane through the center of the plate and charge containing the axis of the Kolsky bar are shown in Figure 3.12. This view is analogous to that of the experimental time sequence images shown in Figure 3.9. Particle positions at the moment of detonation $(t=0 \mathrm{~s})$ are shown in Figure 3.12(a). The subsequent simulated images show that the sand shell velocity was slightly asymmetric with the fastest sand propagating in the (southwest) direction; normal to the expanding detonation front. Significant sand velocity dispersion was present in all directions with some sand particles having traveled significantly further (at substantially higher velocity) than others. To help visualize this effect, Figure 3.12(e) shows the approximate location of the 1,2, and 5\% sand particle probability density contours at $310 \mu$ s after detonation (calculated using the procedure detailed in Section 3.3.1).

Figure 3.12(e) and (f) also show the initial interaction of the sand particles with the test plate surface. They indicate that sand impact with the test structure occurred slightly earlier than that with the end of the Kolsky bar, consistent with experimental observations. The sand particles that made impact near the center of the test plate at zero obliquity suffered weak reflection, and accumulated in this region. This was consistent with the results of previous calculations of the impact of unconstrained sand slugs with rigid beams where the incident momentum of the sand slug was transferred to the test structure with little amplification by reflection [32, 48]. However, most particles did not behave in this manner. Instead, they made oblique impact with the plate, and were reflected along the plate surface with only a fraction of their incident momentum transferred to the plate. These sand particles eventually (not shown) left the surface as a thin sheet travelling radially outwards from the center of impact, consistent with the reflected sand identified in Figure 3.8(f). 

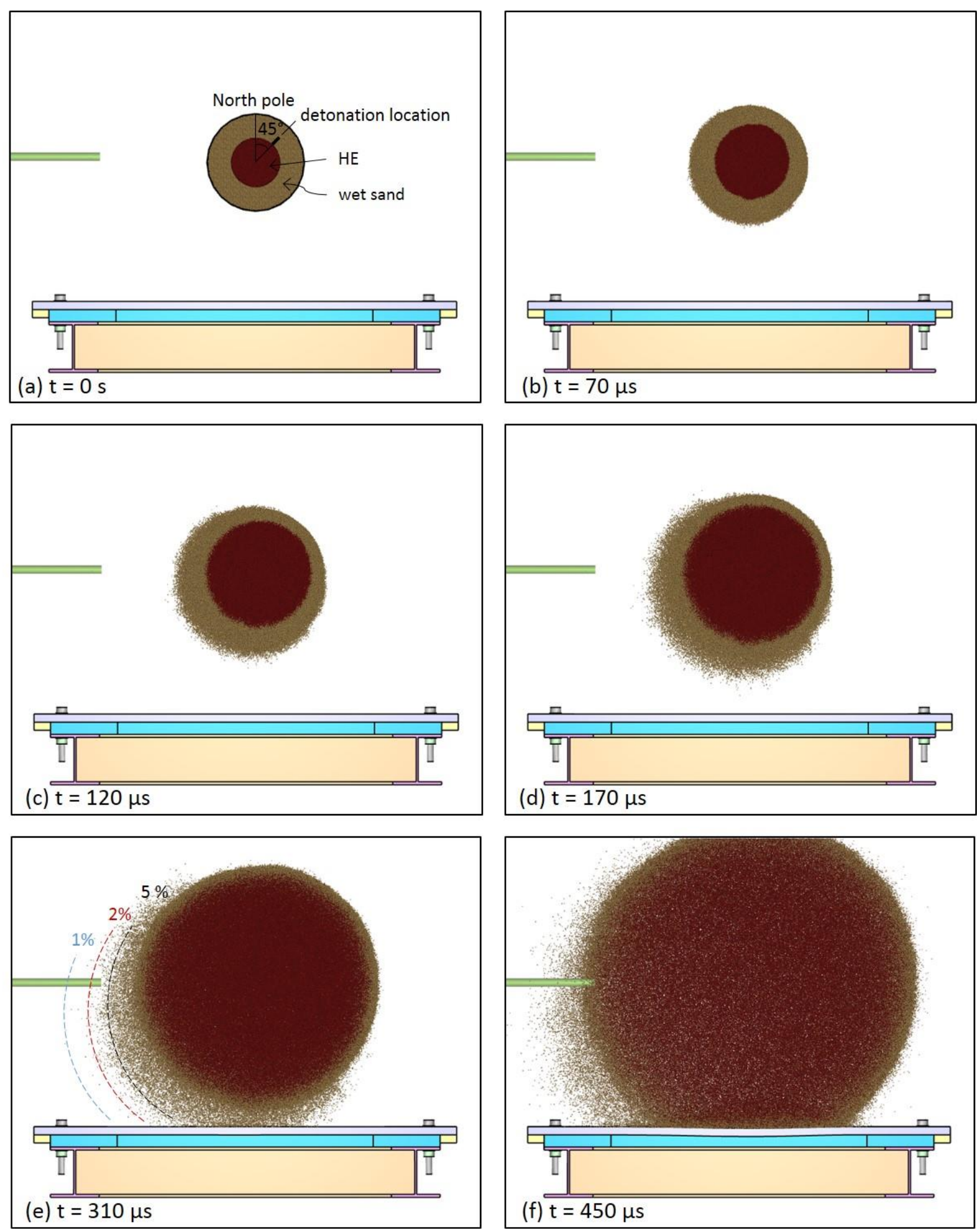

Figure 3.12. IMPETUS Afea predicted (tan colored) sand front positions. (a) Corresponds to the instant of detonation $(t=0 \mathrm{~s}$ ). The position of the (red) $\mathrm{HE}$ particles is also shown, but air particles are hidden to improve visualization of sand and HE particle locations. Sand particle probability density contours are identified in (e) showing that the low volume fraction of high speed sand advanced well ahead of the main sand front. 


\subsubsection{Sand acceleration}

To better understand the sand shell acceleration process, Figure 3.13 shows sand and HE particle positions for the first $45 \mu$ following detonator activation. The dotted white line in Figure 3.13(a) indicates the position of the detonation front at $10 \mu$ s after the initiation of detonation. The detonation shock front required $19.5 \pm 0.5 \mu$ s to propagate the $160 \mathrm{~mm}$ diameter of the inner $\mathrm{HE}$ sphere and reach the southwestern edge of sand/HE interface (diametrically opposite the detonation location). This gives a detonation velocity of $8205 \pm 210 \mathrm{~m} / \mathrm{s}$; consistent with the detonation velocity parameter, $\mathrm{D}=8190 \mathrm{~m} / \mathrm{s}$, given as input to the solver, and is close to the handbook value for the detonation velocity of the C-4 explosive $(8040 \mathrm{~m} / \mathrm{s})$ [80]. A compressive shock wave was then launched into the $72 \mathrm{~mm}$ thick annular sand region, and can be seen as the line separating the darker (compressed) sand from the (original contrast) stationary sand. The approximate sand shock location is indicated by the black dashed line in Figure 3.13(b)-(e). The sand shock front reached the outer surface of the sand shell at $\mathrm{t}=38.5 \pm 0.5 \mu \mathrm{s}$, corresponding to a wet sand shock velocity of $3790 \pm 100 \mathrm{~m} / \mathrm{s}$ which is consistent with reported shock velocities in water-saturated granular media (sands) [90]. The acrylic shell (not shown in Figure 3.13) failed as the sand shock front reached the polymer shell inner boundary at $\mathrm{t}=38.5 \mu \mathrm{s}$. At $45 \mu \mathrm{s}$ after initiation of detonation, Figure 3.13(f), the sand shock had undergone reflection/sign conversion at the sand/acrylic interface and had begun to propagate back into the sand shell with concomitant spalling of the sand behind this release wave. The initial HE and sand front locations are indicated by dashed lines in Figure 3.13(f) to show how far the sand particles had been displaced from their initial locations at this time.

Detailed examination of the simulations showed that the radial distance of propagation of the sand particles within the sand shell was not uniform in the circumferential direction. To illustrate this, Figure 3.14 shows the displacement magnitude of the sand particles at $t=50 \mu$ s after detonation. Spikes at the green to blue interface can be seen within the inner region of the sand shell. They are consistent with the development of a shock induced interfacial instability whose conditions for initiation were recently analyzed by Kandan et al. [70] and shown to be a manifestation of a Richtmyer-Meskov type instability. 

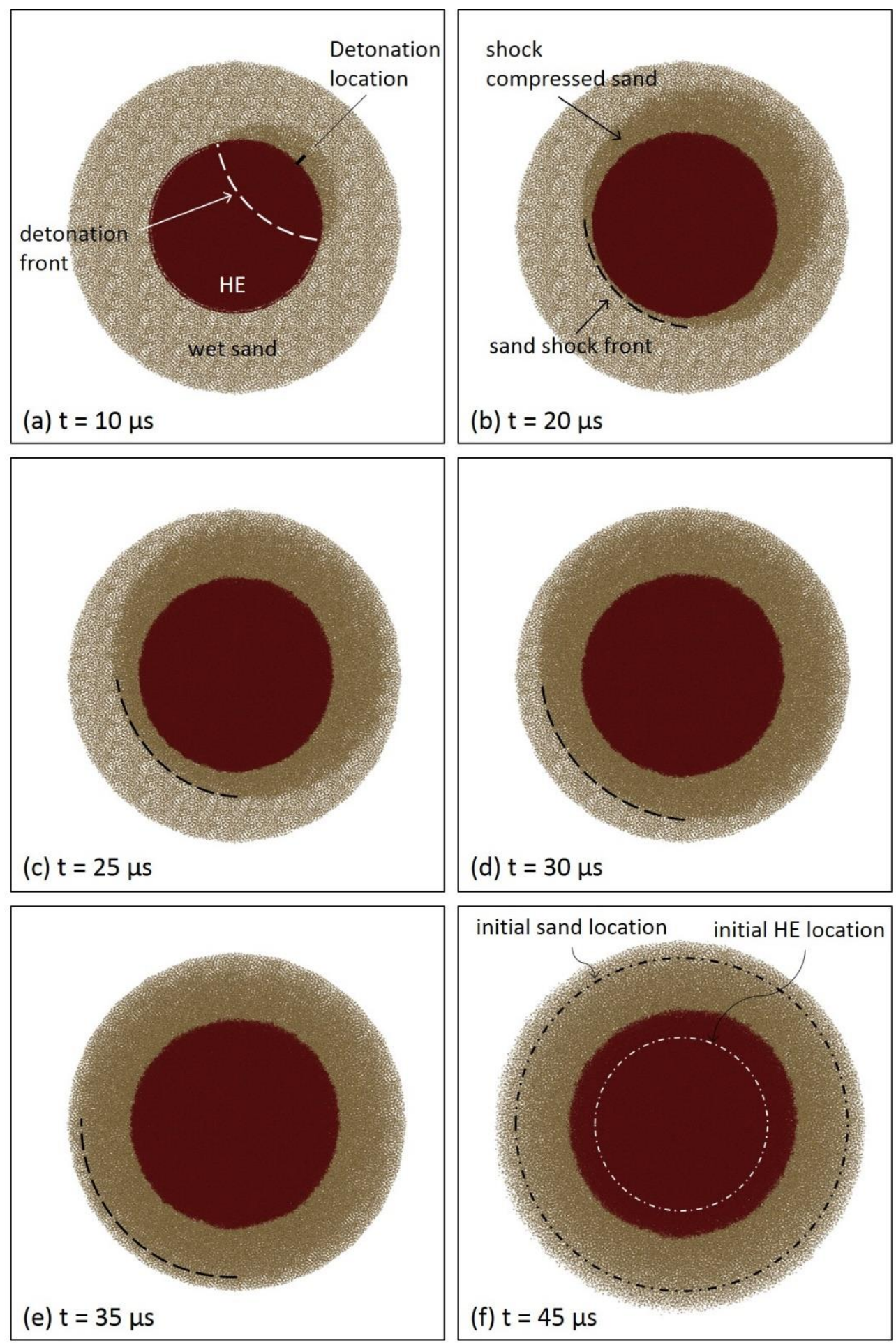

Figure 3.13. A simulation sequence following detonation of the test charge. The propagation of a detonation front through the HE and the locations of compressive shock front within the annular wet sand shell are also shown. (f) Shows the distance the sand and HE particles were displaced from their initial locations at $t=45 \mu \mathrm{s}$ after initiation of detonation. 


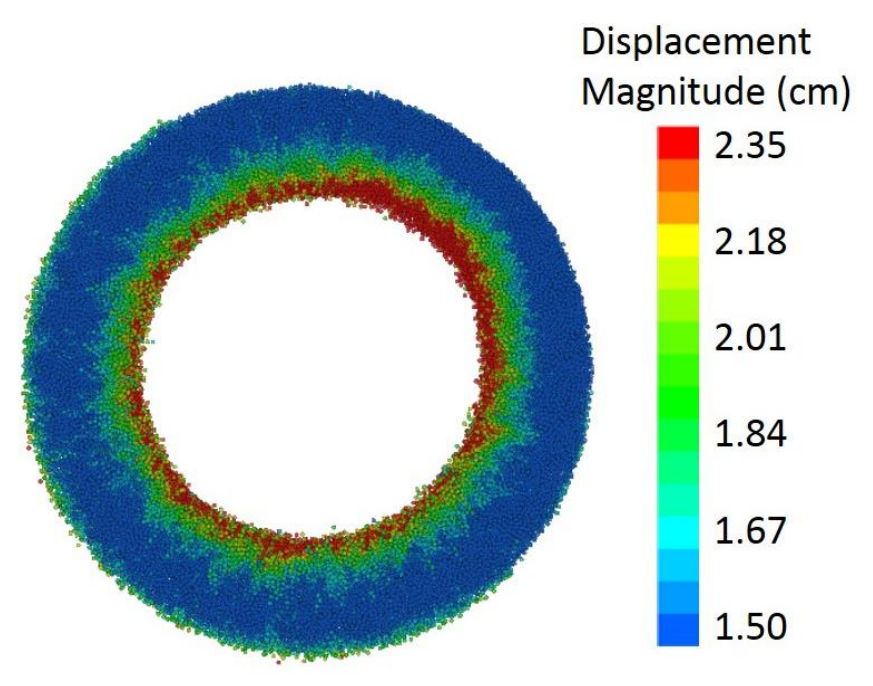

Figure 3.14. Sand particle shell with color contours corresponding to the magnitude of the particle displacement at $t=50 \mu \mathrm{s}$ after detonation. The spikes in the green-blue contours are consistent with development of an instability at the interface between HE particles and the sand.

\subsubsection{Sand layer stretching}

Figure 3.12(c)-(f) showed the presence of a significant variation in the sand particle displacement, resulting in the development of a substantial sand particle density gradient. A significant fraction of sand was accelerated well ahead of the main sand front, while a trailing, much denser sand region existed directly in front of the expanding detonation products. To quantify the sand particle distribution, the simulated sand particle density was calculated (as detailed in Section 3.3.1, equation (3.2)-(3.4), and is plotted as a function of distance from the test charge center in the Kolsky bar (X-axis) direction in Figure 3.15(a). It is evident from Figure 3.15(a) that at $102 \mu$ s after detonation, the density of sand particles was essentially zero beyond approximately $29 \mathrm{~cm}$ from the charge center. The sand particle density then increased behind the fast sand to reach a peak value (of about 5 simulated particles per $\mathrm{cm}^{3}$ ) at a radial distance of $\sim 15$ $\mathrm{cm}$ from the charge center before falling very rapidly. During this period, the sand front expanded from an initial width of $7.2 \mathrm{~cm}$ to $17 \mathrm{~cm}$ at $102 \mu \mathrm{s}$ after detonation (and $24 \mathrm{~cm}$ at $182 \mu \mathrm{s}$ and 26 $\mathrm{cm}$ at $208 \mu \mathrm{s}$ after detonation). This was accompanied by a decrease of the sand particle density due to a combination of inverse square law spreading arising from growth of surface area of the sand shell, and radial stretching of the sand shell. 

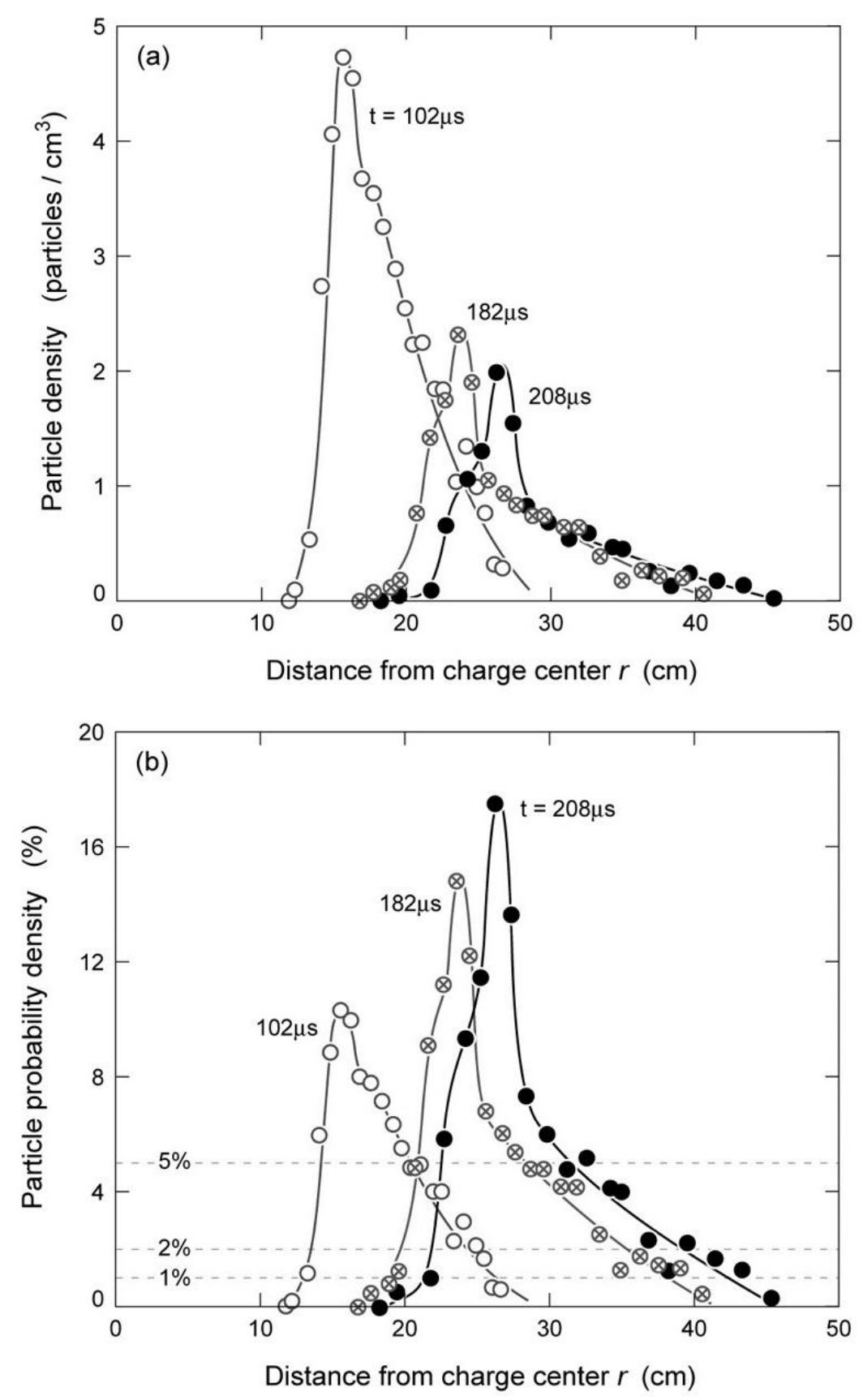

Figure 3.15. (a) The variation in sand particle density and (b) the sand particle probability density as a function of distance from the charge center (measured in the Kolsky bar direction) at simulation times $t=$ 102,182 , and $208 \mu \mathrm{s}$.

The sand probability density is shown for each time step in Figure 3.15(b). The horizontal lines shown on the figure correspond to particle probability densities of 1,2, and 5\%. Their intersection with the probability distributions in Figure 3.15(b) show the radial location of each 
probability density. These particle probability values were transposed as probability contours on the image shown in Figure 3.12(e). The fastest sand (with a sand probability density of 1 to $2 \%$ ) was consistent with the leading tips of the sand fingers (spikes) observed experimentally in Figure 3.9, and had advanced well ahead of the main front with a 5\% particle probability density. The positions of the 1, 2, and 5\% sand probability density contours are also overlaid on the experimental sand front location data obtained with the high-speed camera in Figure 3.11(a) and their corresponding velocity in Figure 3.11(b).

\subsubsection{Sand deceleration}

There was a well-defined decay in sand front velocity after the peak velocity was attained in both the experimental and the simulation data, Figure 3.11(b). Examination of simulation results in which air particles are shown, Figure 3.16, indicates the development of an air shock front (a dense air layer) in front of the sand. A red dashed line in Figure 3.16(a) and (c) indicate the approximate location of this shock. The darker shade of blue indicates the presence of significantly higher air pressure behind the air shock front. This air shock had an estimated radial speed of approximately $1300 \mathrm{~m} / \mathrm{s}$.

Examination of simulations conducted without air particles (Appendix A) showed no sand front velocity decay, indicating that the sand deceleration was a result of momentum-transferring collisions between sand and air particles associated with the formation of the air shock. Its presence in the experiment may also have contributed to the formation of sand fingers ahead of the main sand front; a hypothesis that is investigated by more appropriate shock instability analysis methods in a recent paper [70].

Kandan et al. [70] analyzed the propagation of a sand front moving with a velocity $V_{s}$ into air at atmospheric pressure, $p_{O}$ and ambient temperature. The front generates an air shock wave as it pushed through air. The shock pressure $p_{f}$ at the front of the slug was shown to be related to $V_{s}$ by

$$
\frac{V_{s}}{a_{0}}=\frac{1}{\gamma}\left(\bar{p}_{f}-1\right)\left[\frac{2 \gamma}{\bar{p}_{f}(\gamma+1)+\gamma-1}\right]^{1 / 2}
$$



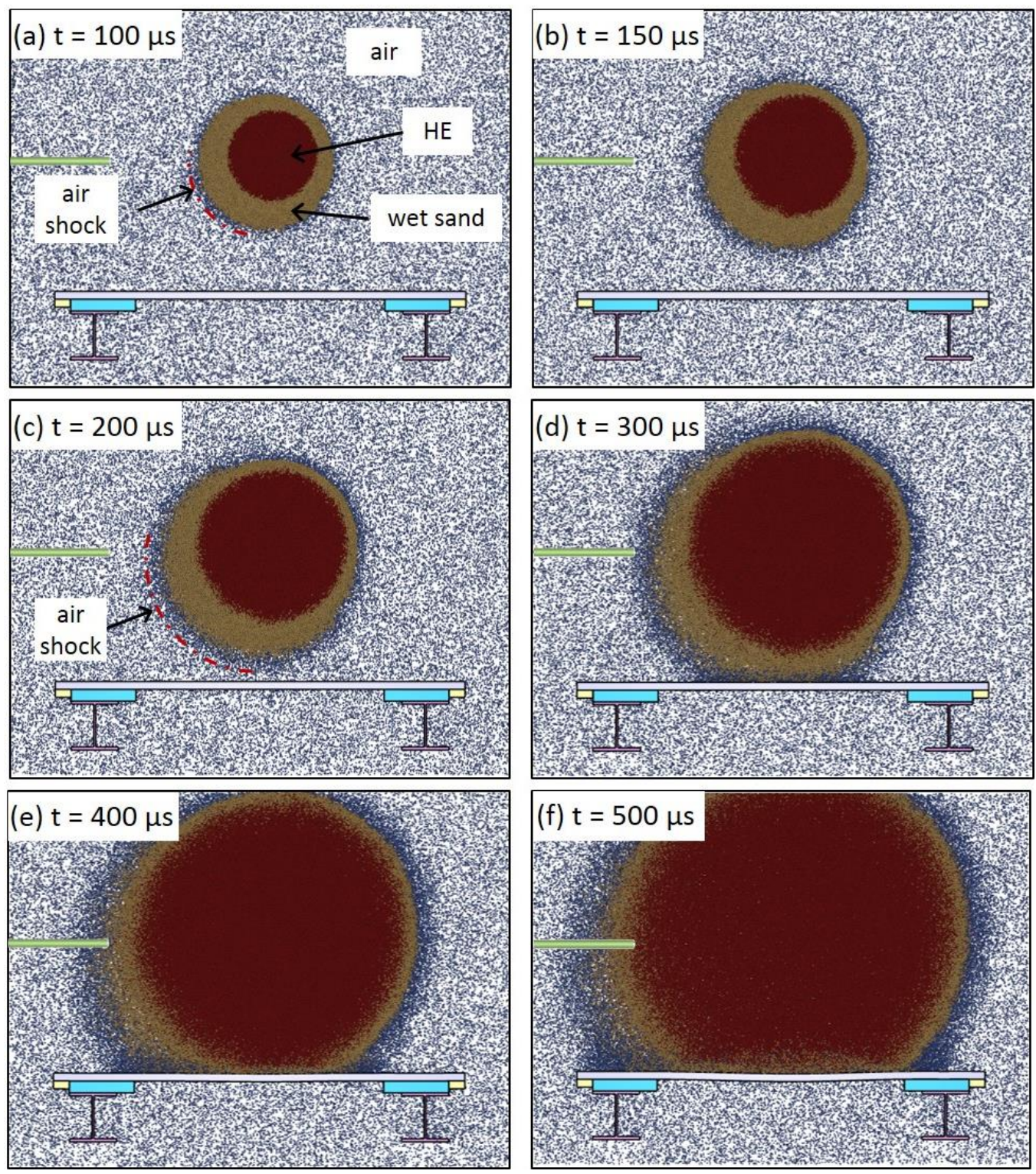

Figure 3.16. Evolution of the air, HE, and wet sand particle regions during the first $500 \mu$ s following detonation. The presence of an air shock (darker blue) in front of the expanding sand shell can be seen.

where $\bar{p}_{f} \equiv p_{f} / p_{0}$ is the normalized shock pressure and $\gamma$ is the ratio of specific heat and $a_{o}$ the speed of sound for air. The air shock speed, $c_{f}$ can be obtained from the normalized air shock pressure

$$
\frac{c_{f}}{a_{0}}=\left[\frac{\gamma-1}{2 \gamma}+\bar{p}_{f} \frac{\gamma+1}{2 \gamma}\right]^{1 / 2} .
$$


The main sand front approached the test plate with an incident velocity, $V_{s}$ of $\sim 1200 \mathrm{~m} / \mathrm{s}$, resulting in a (calculated) shock pressure $p_{f} \sim 2.3 \mathrm{MPa}$ (23 times atmospheric pressure) and a shock speed of $\sim 1520 \mathrm{~m} / \mathrm{s}$. The high air pressure loading on this hypersonic sand front (which scales with the sand front speed) results in an instability that causes breakdown of the front into an array of conical shaped protrusions [70]. We note that by increasing the standoff distance, momentum would be progressively transferred to the air shock from the sand, and the nature of test plate loading would increasingly be governed by the fluid structure interaction between the plate and an air shock [4, 5, 27].

\subsection{Kolsky Bar Response}

\subsubsection{Experimental measurements}

The time corrected (with $\mathrm{t}=0 \mathrm{~s}$ corresponding to the moment of detonation) axial stress (pressure) within the Kolsky bar pressure-time response is shown in Figure 3.17(a). Observations at the strain gauge location were delayed by the time for a longitudinal elastic wave (with a speed of $4800 \mathrm{~m} / \mathrm{s}$ for the C-350 maraging steel bar) to propagate $0.5 \mathrm{~m}$ along the bar (a time of $104 \mu \mathrm{s}$ ). The first (sign reversed) distal reflection from the end of the $3.81 \mathrm{~m}$ long Kolsky bar arrived 1.38 ms after the initial direct signal. The data from the Kolsky bar is therefore only plotted for the first $1 \mathrm{~ms}$ of recorded data and is not complicated by distal reflections. However, complex Pochhammer-Chree modes for the large diameter bar used here (to avoid buckling instabilities) resulted in a complex wavetrain that distorted the pressure response [81, 92, 93]. Figure 3.17(a) shows that the first compressive stress whose amplitude significantly exceeded the background noise was detected by the Kolsky bar strain gauges at $\sim 420 \mu$ s after detonation. However, it was preceded by a sequence of very weak arrivals whose amplitude was no more than two times the background.

The compressive stress associated with the first significant signal (at $420 \mu \mathrm{s}$ ) had an amplitude of less than $50 \mathrm{MPa}$, and was followed by similarly weak signals until a large compressive pulse with a peak pressure of $\sim 750 \mathrm{MPa}$ arrived at 560 to $575 \mu \mathrm{s}$ after detonation. Integration of the pressure-time curve gave the specific impulse transmitted to the bar, Figure 3.17(c). The impulse signal first began to rise very slowly between 350 and $400 \mu$ s, and was followed by small impulse 
(a) Measured pressure waveform

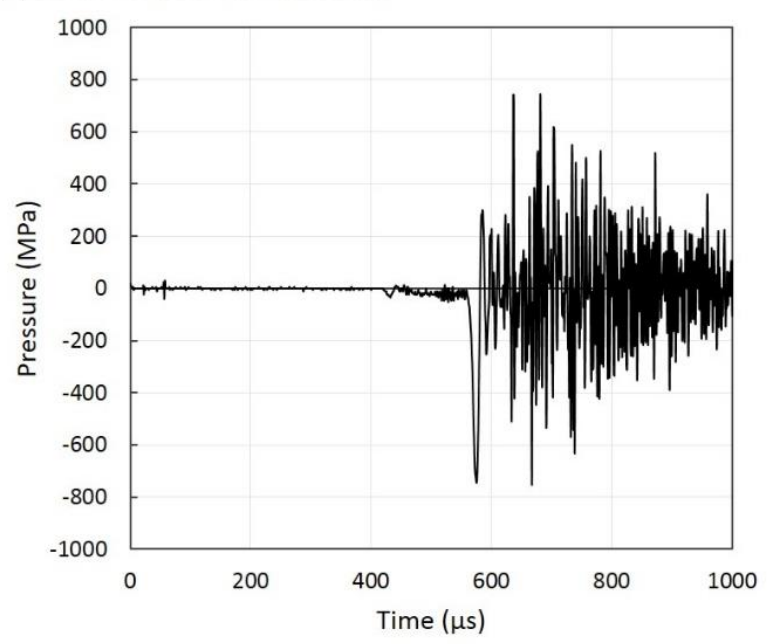

(b) Simulated pressure waveform

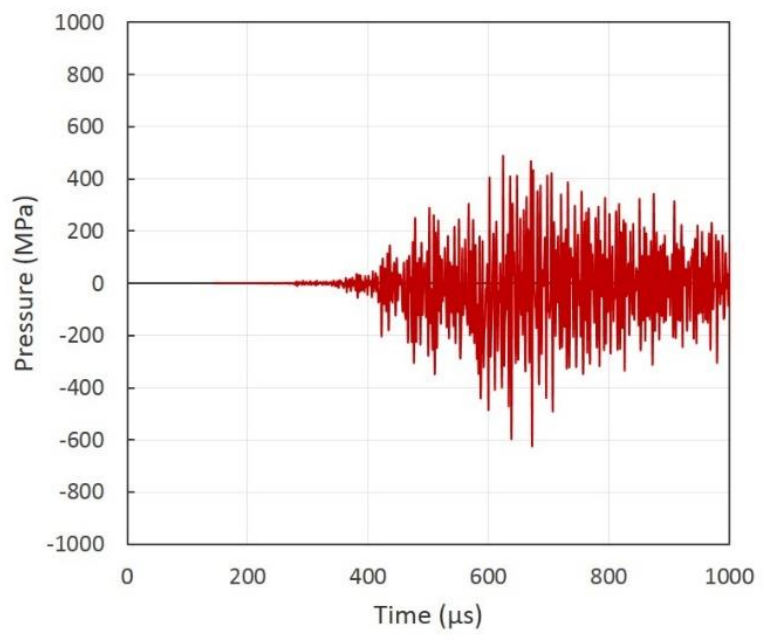

(c) Specific Impulse

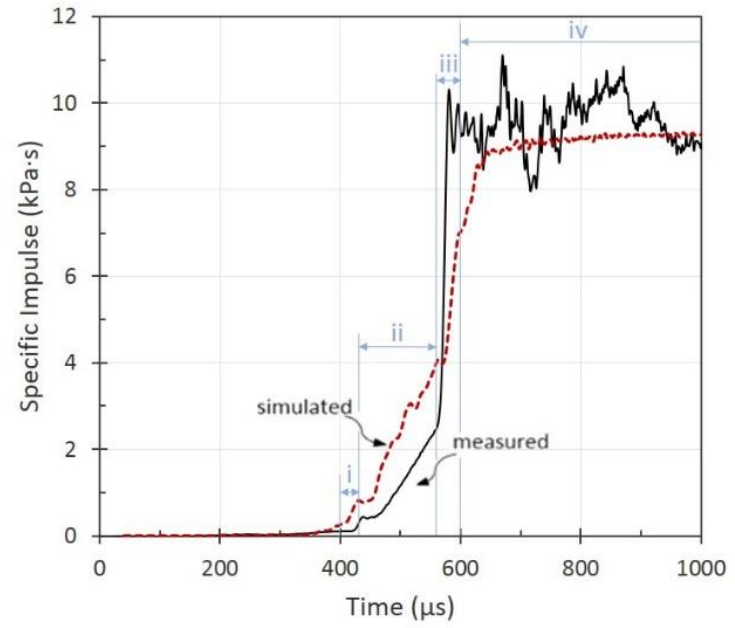

Figure 3.17. (a) The measured and (b) simulated Kolsky bar pressure versus time after detonation. (c) Shows the measured and simulated impulse versus time response and the four regions of impulsive loading. 
jump of $\sim 0.4 \mathrm{kPa} \cdot \mathrm{s}$ between 410 and $445 \mu \mathrm{s}$. This was followed by a $\sim 120 \mu$ s period of increasing impulse with an approximately constant impulse rate. The arrival of the large pressure pulse at approximately $575 \mu$ s after detonation caused a rapid rise in impulse to a final value of $\sim 9.5 \pm 1.5$ $\mathrm{kPa} \cdot \mathrm{s}$ that persisted to the end of observation $(1 \mathrm{~ms})$. The plateau specific impulse is consistent with simple estimates of the impulse per unit area of $8.5 \mathrm{kPa} \cdot \mathrm{s}$ calculated by distributing the momentum for $23.8 \mathrm{~kg}$ of water-saturated sand accelerated to an average velocity of $1050 \mathrm{~m} / \mathrm{s}$ evenly over a sphere of $48.5 \mathrm{~cm}$ radius (the standoff distance to the end of the Kolsky bar).

\subsubsection{Simulated response}

The axial stress due to the simulated sand impact with the end of the Kolsky bar was calculated from the simulated force-time signal at the strain gauge location and the cross sectional surface area of the Kolsky bar $\left(5.07 \mathrm{~cm}^{2}\right)$, Figure $3.17(\mathrm{~b})$. The impulse was then calculated as the time integral of the pressure-time response, and compares well with the experimental data in Figure 3.17(c). Four response regimes can be identified and are indicated in Figure 3.17(c); (i) an initial step in impulse arriving at the strain guage position at approximately $400 \mu$ s after detonation and persisting for 20-30 $\mu$ s, (ii) a region of slowly rising impulse between approximately 430 to 560 $\mu \mathrm{s}$, (iii) a rapidly rising impulse regime that began at $560 \mu \mathrm{s}$, and persisted for $\sim 40 \mu \mathrm{s}$ in the experiment and about twice this time in the simulation and (iv) a plateau region of no further increase in impulse that persisted from about $600 \mu$ s to beyond $1 \mathrm{~ms}$. Again, careful examination of the response prior to region (i) reveals that the step in impulse was preceded by a weak, slowly rising impulse beginning approximately $360 \mu$ s after detonation.

The simulation can be used to calculate the impulse applied to the impacted end of the Kolsky bar by each particle type (air, HE, and sand). The impulse contribution to the signal at the strain guage location for each of the different particle types is shown in Figure 3.18. These sum to the specific impulse calculated at the strain guage location in Figure 3.17(c). The air shock initially impacted the end of the Kolsky bar at approximately $250 \mu$ s after detonation, resulting in a signal reaching the strain gauges at $\sim 355 \mu \mathrm{s}$. This appears to be responsible for a very weak, slowly rising impulse prior to the onset of region (i) step response. Figure 3.18 shows that the impulse from the air particle impacts leveled out at $\sim 0.3 \mathrm{kPa} \cdot \mathrm{s}$ at $\sim 400 \mu$ s after detonation. 


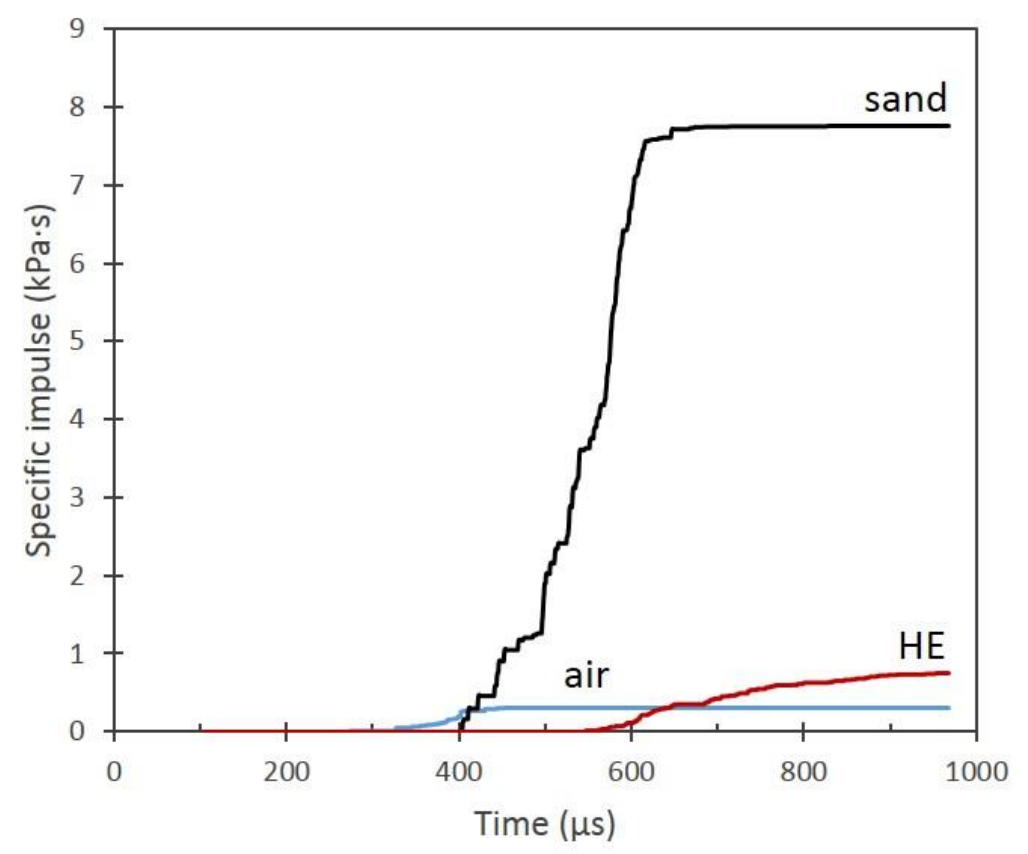

Figure 3.18. The simulated specific impulse contributions from the sand, air, and HE particles versus time at the strain gauge location.

The first (fastest) sand particles impacted the front of the Kolsky bar at around $300 \mu$ s after detonation, Figure 3.11, and their impulse signal began to be observed at the strain gauge at about $400 \mu \mathrm{s}$. This initial sand impact, when combined with the impulse from the air particles, led to the first jump in region (i) impulse. The region (ii) response in Figure 3.17(c) corresponded with a period of impact by low density, but high velocity sand while region (iii) corresponded to impact by the densest sand front directly in front of the HE particles and ended at $600 \mu \mathrm{s}$ after detonation. The HE particles began to impact the end of Kolsky bar at $470 \mu$ s with their associated impulse arriving at the strain gauge location at $\sim 575 \mu$ s after detonation (as the last of the sand impulse was recorded). The HE particle impacts contributed very little additional impulse to the total response, consistent with a leveling out of impulse beyond $600 \mu$ s (the region (iv) response), Figure 3.17(c).

Further insight can be gained by examining the position of only the sand and HE particles that eventually impacted the end of the Kolsky bar, Figure 3.19. Radial stretching of the sand front with time (distance of propagation) can be clearly seen. The simulation sequence is analogous to that observed in a laboratory scale study of the impact of sand columns on a Kolsky bar [49]. The laboratory scale study also observed the sand column lengthening over time as it traveled through 

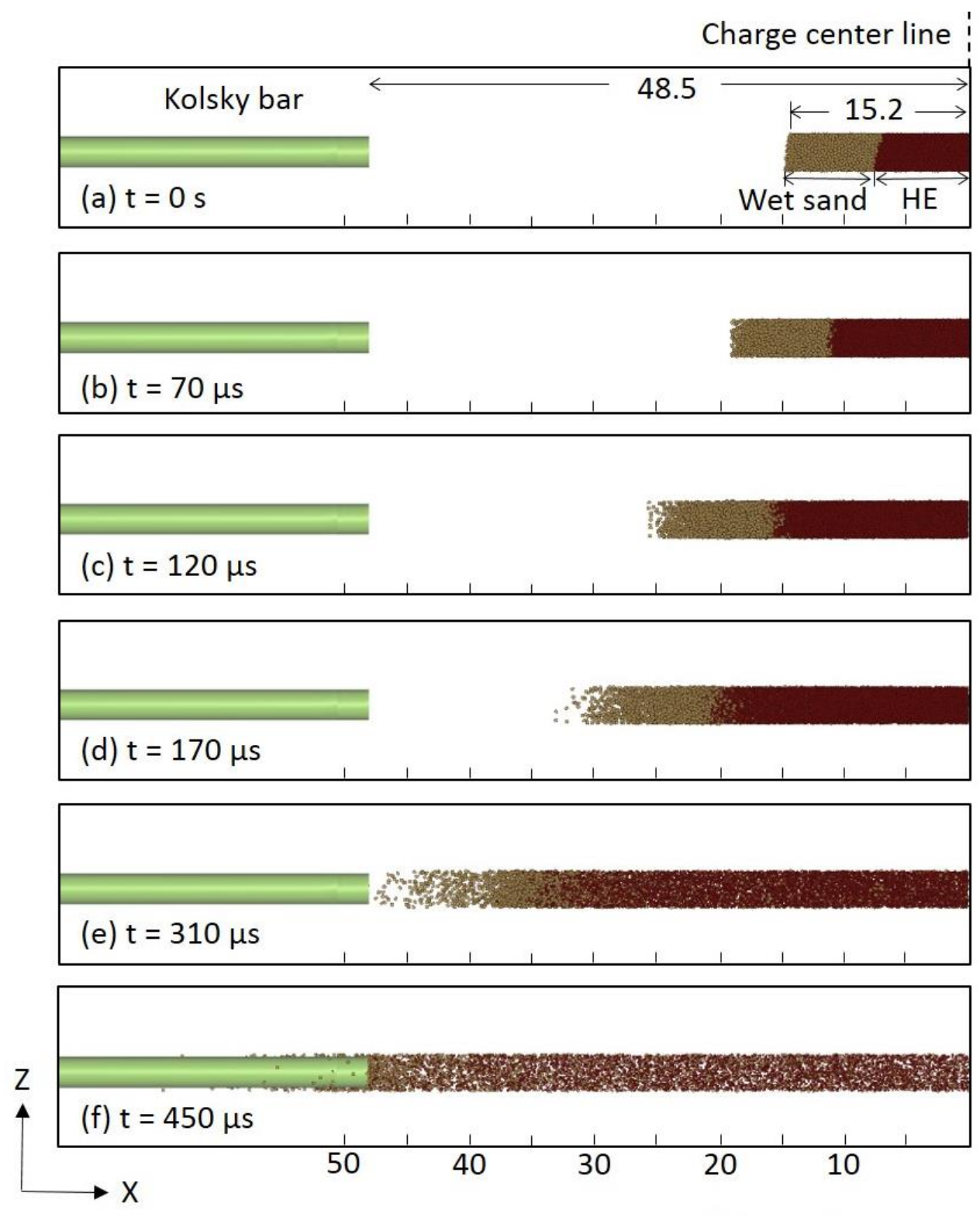

All dimensions in $\mathrm{cm}$

Figure 3.19. The position of sand and high explosive $(H E)$ particles that impacted the Kolsky bar at selected times after detonation (at $t=0 \mathrm{~s}$ ).

air. In both cases, this arose from progressive spallation of sand at the sand/air interface during reflection of a sand shock launched here by the detonation wave. Examination of Figure 3.19(b)(d) clearly shows that the highest velocity sand particles had advanced well ahead of the main front while lower velocity sand accumulated directly ahead of the detonation products. Careful analysis of the simulations indicated that the fastest (1\% contour) sand, shown in Figure 3.12(e), reached 
the end of the Kolsky bar at approximately $280 \mu$ s after detonation, and was detected at the strain gauges at approximately $394 \mu$ s after detonation consistent with the simulation plot, Figure 3.17(c). However, Figure 3.18 showed the initial loading experienced on the bar was from the air shock particles at this time rather than the fastest moving sand. The $2 \%$ probability density contour arrived at the end of the Kolsky bar at $\sim 300 \mu \mathrm{s}$. This is consistent with the first sand impact shown in Figure 3.18 and, coupled with the loading from the air particles, the existence of a region (i) response $\sim 400 \mu$ s after detonation (Figure 3.17(c)).

The region (ii) response corresponds to the sand arrival with a probability density of $\sim 5 \%$ or higher. The impulse loading from this sand reached the strain gauge location around $440 \mu \mathrm{s}$, consistent with a time during which the impulse was rising at a constant rate. This constant rate of loading persisted to $560 \mu$ s and was followed by a sharp jump in impulse to a plateau impulse of $9.2 \mathrm{kPa} \cdot \mathrm{s}$. This region (iii) response corresponds to the time at which the sand directly in front of the HE particles impacted the bar. This region of lower velocity but higher density sand remained just in front of the detonation products, Figure 3.19(f), and impacted the front of the Kolsky bar at about $450 \mu$ s after detonation and would therefore have begun to be detected at the strain gauges at $\sim 555 \mu \mathrm{s}$ after detonation. It is interesting to note that the arrival of the slower moving HE particles after the sand did not cause significant additional impulse to be transferred during region (iv); a consequence of efficient momentum transfer from lower mass HE particles to the denser sand.

There was good agreement between the measured $(\sim 9.5 \pm 1.5 \mathrm{kPa} \cdot \mathrm{s})$ and simulated plateau impulse $(9.2 \mathrm{kPa} \cdot \mathrm{s})$ results and the timing of the main features observed in the impulse waveform, Figure 3.17(c). It is also noted that while the simulations showed significant interactions between the air and sand particles, when simulations were performed without air particles, only a small difference in the impulse transferred to the Kolsky bar was observed. Figure 3.18 indicates that less than $10 \%$ of the impulse was transferred by air particles, and it is therefore concluded that the majority of the impulse loading occurred by the impact of sand. 


\subsection{Panel Deflections}

\subsubsection{Experimental observations}

The deformed $2.54 \mathrm{~cm}$ thick, 304 stainless steel test plate was sectioned through the point of maximum deflection at $\mathrm{X}=71.3 \mathrm{~cm}$ and $\mathrm{Y}=66.0 \mathrm{~cm}$ as shown in Figure 3.6. The deflection as a function of distance $\mathrm{Y}$ along the plate (with $\mathrm{Y}=0 \mathrm{~cm}$ corresponding to the white painted near edge of the plate) is shown in Figure 3.20(b) for the full $132 \mathrm{~cm}$ length of the test plate. A permanent center deflection, $\mathrm{U}_{\max }=3.56 \mathrm{~cm}$ was measured near the midpoint of the section cut. This deflection profile was aligned with the cross-section image of the test plate, Figure 3.20(a), and lines corresponding to the locations of the steel picture frame and I-beam supports are shown on the plot. The center of the test charge was located vertically above the $\mathrm{Y}=66 \mathrm{~cm}$ line in Figure 3.20 (b) at $\mathrm{X}=71.3 \mathrm{~cm}$ which was also the location of maximum permanent deflection. There was no evidence of fracture observed in the plate after impact or of permanent rotational deformations at the edge grips. However, the I-beam support structure did suffer plastic deformation (and in a subsequent test, the flange underwent partial rupture).

\subsubsection{Simulated plate deformation}

The calculated displacement response of the solid plate is shown in Figure 3.21. It shows the transient damped oscillatory displacement as the plate asymptotes to a steady state permanent displacement of $3.51 \mathrm{~cm}$; a $1.4 \%$ difference to the experimentally measured maximum experimental displacement at $X=71.3 \mathrm{~cm}$ and $\mathrm{Y}=66 \mathrm{~cm}$. The simulated plate was then virtually sectioned along the $\mathrm{Y}$ axis at $\mathrm{X}=71.3 \mathrm{~cm}$ (the same as the section in the experiment). This simulated permanent deflection profile is compared with the measured profile in Figure 3.20(b) and can be seen to be in excellent agreement with the measurement. The location of maximum simulated and measured deflection was slightly off the center of the plate due to the small drift in charge position prior to detonation. 
(a) Cross section

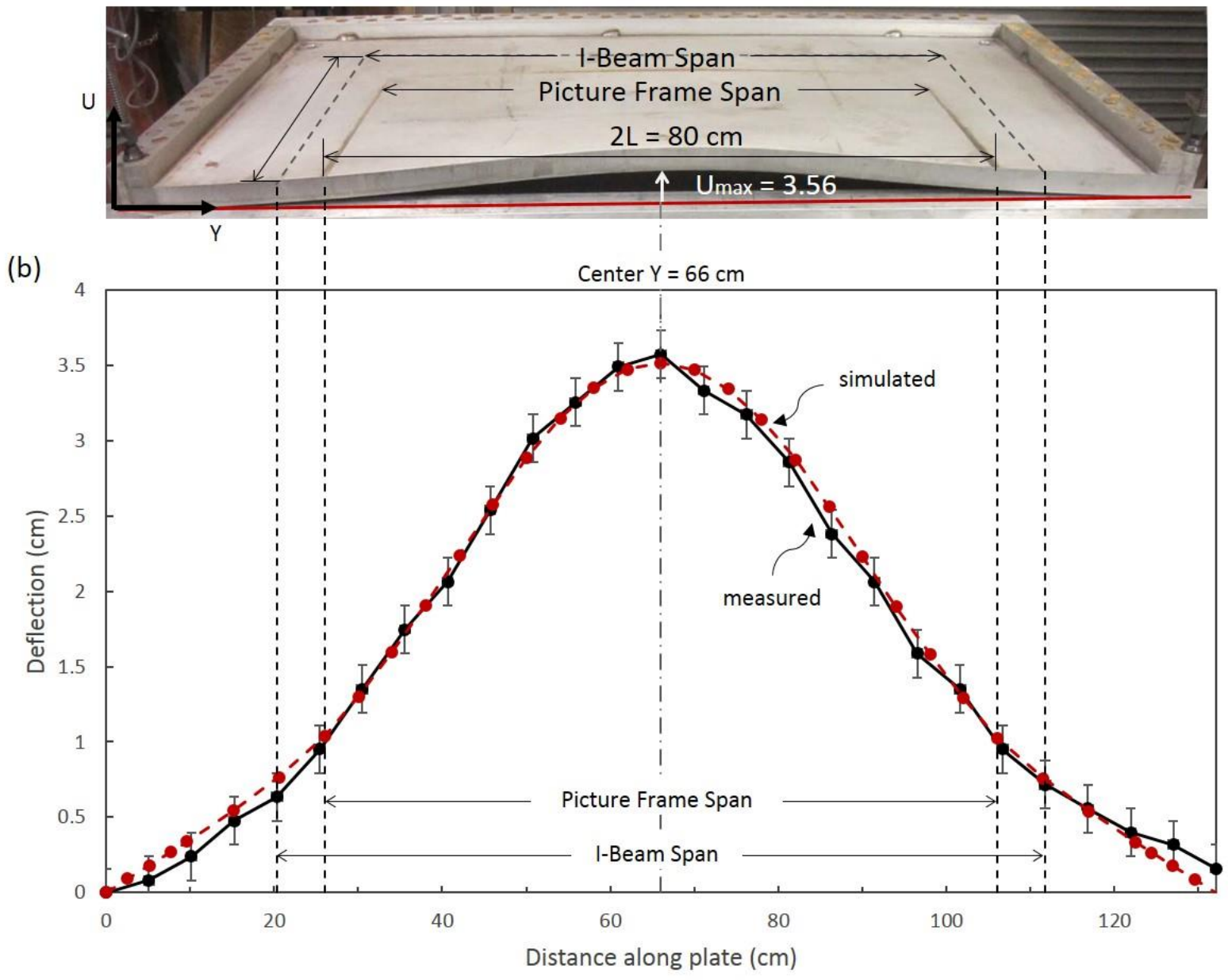

Figure 3.20. (a) Cross sectional view of the test plate sectioned through the point of maximum deflection (along $X=71.3 \mathrm{~cm}$ ). The grey square marks on the underside of the test structure indicate the locations of the picture frame support while the grey dashed lines show the location of the I-beam flanges of the support structure. (b) The measured and simulated deflection profile of the underside of the edge clamped plate. The vertical dashed line corresponds to the center of the plate in the $Y$-direction (at $Y=66 \mathrm{~cm})$ which was also the position of maximum deflection. 


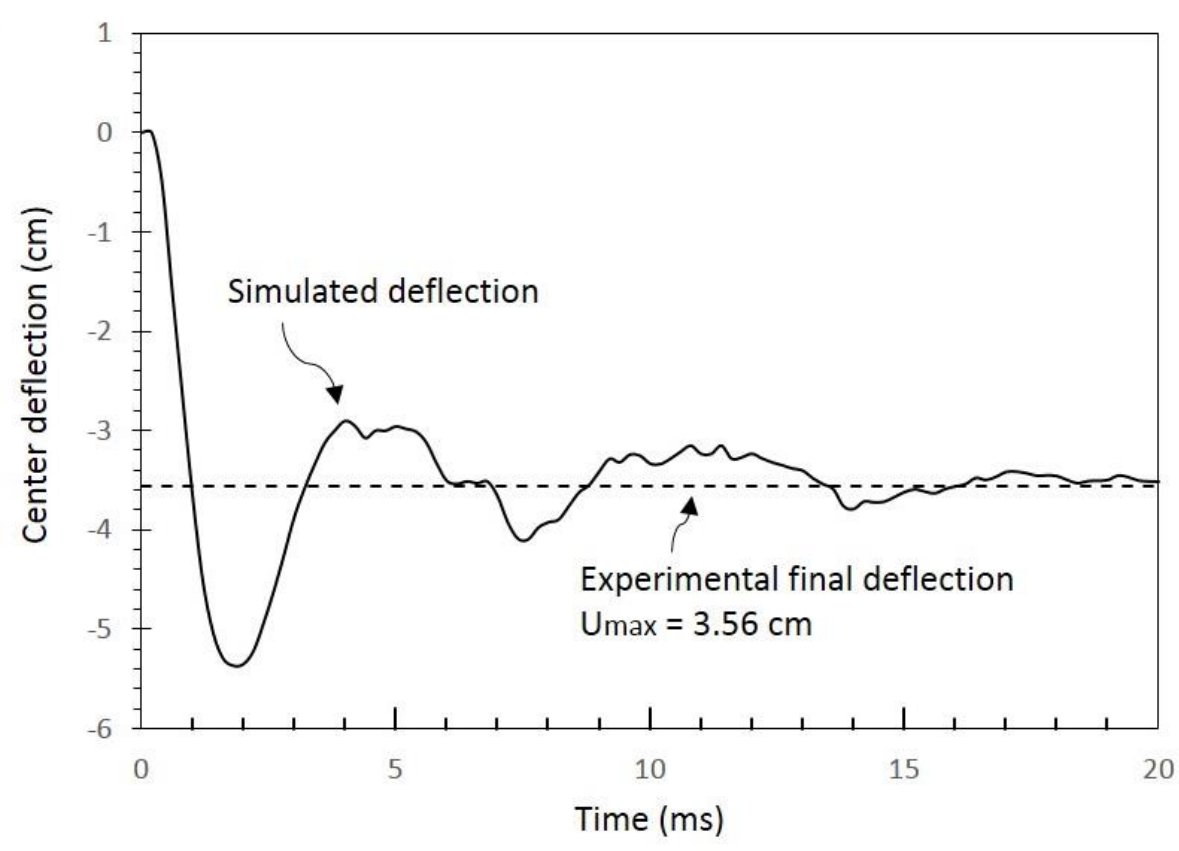

Figure 3.21. A comparison of the IMPETUS predicted transient deflection of the plate at the region of maximum deflection $(X=71.3 \mathrm{~cm} ; Y=66 \mathrm{~cm})$, and the experimentally measured final maximum deflection.

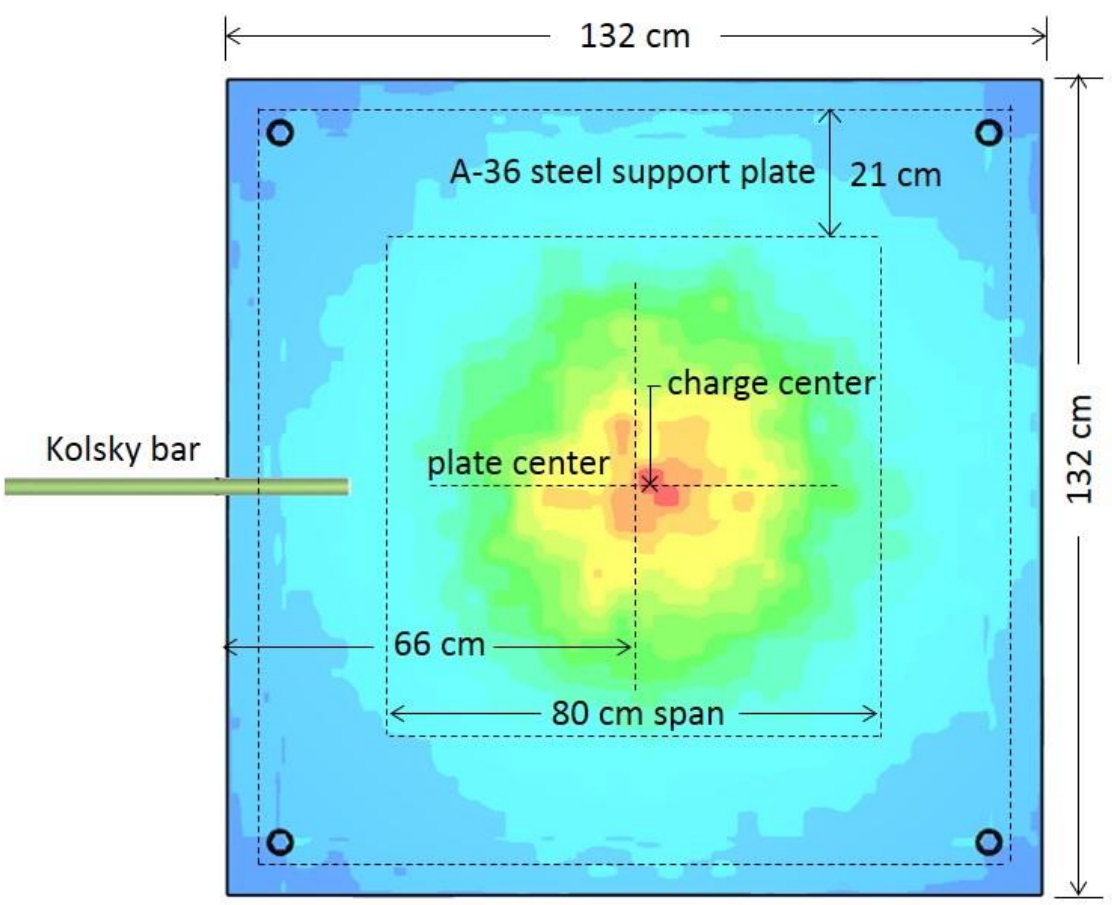

Specific Impulse (Pa.s)

$1.350 \mathrm{e}+04$

$1.260 \mathrm{e}+04$

$1.170 \mathrm{e}+04$

$1.080 e+04$

$9.900 \mathrm{e}+03$

$9.000 e+03$

$8.100 \mathrm{e}+03$

$7.200 \mathrm{e}+03$

$6.300 e+03$

$5.400 \mathrm{e}+03$

$4.500 \mathrm{e}+03$

$3.600 \mathrm{e}+03$

$2.700 \mathrm{e}+03$

$1.800 \mathrm{e}+03$

$9.000 e+02$

$0.000 e+00$

Figure 3.22. Specific impulse distribution at the plate surface after the first $1 \mathrm{~ms}$ of loading. 
Figure 3.22 shows the specific impulse distribution applied to the plate during the first $1 \mathrm{~ms}$ of loading. The maximum specific impulse was registered along the $\mathrm{Y}=66 \mathrm{~cm}$ (center) axis of the test plate at a value of $X=71 \mathrm{~cm}$; directly beneath the center of the test charge. This maximum impulse has a value, $\mathrm{I}_{\mathrm{t}}=12.8 \mathrm{kPa} \cdot \mathrm{s}$; slightly higher than that incident upon the Kolsky bar because of the $\sim 5 \mathrm{~cm}$ shorter standoff distance to the plate surface. The impulse then rapidly decayed with radial distance from this location falling to less than $1 \mathrm{kPa} \cdot \mathrm{s}$ at the corners of the plate as a result of the longer standoff distance (inverse square law spreading of the sand and its deceleration by interaction with the air) and the oblique angle of incidence which reduces the fraction of particle momentum transferred to the plate surface.

A simulation was performed without the test plate present to determine the hydrodynamic pressure, $P=\rho v^{2}$ (where $\rho$ is the sand density and $v$ its velocity), and the incident impulse at the surface of the plate directly below the charge center. A sand particle detecting "sensor" with a cross sectional area of $0.04 \times 0.04 \mathrm{~m}^{2}$ was placed at the location of maximum impulse in Figure 3.22 and detected the passage of sand particles. The maximum calculated pressure was $560 \mathrm{MPa}$, similar to that observed experimentally. The incident impulse was calculated as $I_{o}=12.4 \mathrm{kPa} \cdot \mathrm{s}$ resulting in a transmitted to incident impulse ratio $\mathrm{I}_{\mathrm{t}} / \mathrm{I}_{\mathrm{o}}=1.03$, consistent with weak sand particle reflection [48].

Figure 3.23(a) shows the effective plastic strain on the top surface of the target plate, where the consequence of stretching of the plate during its impulsive loading can be seen along the diagonal lines connecting pairs of bolts. Figure 3.23(b) shows the effective plastic strain on the back face of the test plate where the highest strain was found to be located directly beneath the charge center and at stress concentrations near the bolt hole locations. Black dotted lines show the location of the beam span defining the support plate. Even though the loading to the plate was circularly symmetric in Figure 3.22, the strain distribution was strongly influenced by the square symmetry of the test plate and edge grip system.

The simulation methodology also enables detailed analysis of the response of the test frame during sand loading. Appendix B shows that significant elastic rotation of the support frame accompanied later stages of the test. This resulted in a softened edge restraint. It also resulted in large inelastic deformations of some parts of the support structure indicating a need for a sturdier support structure for tests at this scale. 


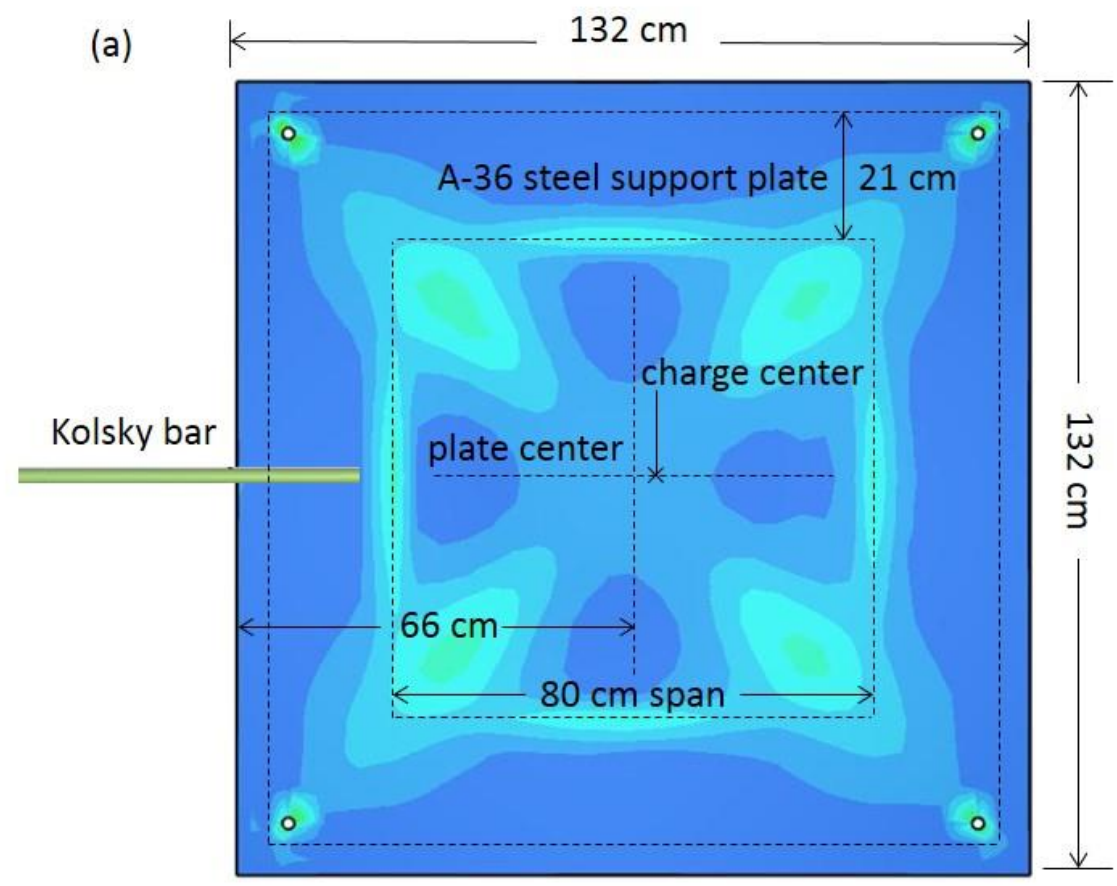

\section{Effective plastic strain}

$4.000 \mathrm{e}-02$

$3.733 \mathrm{e}-02$

$3.467 \mathrm{e}-02$

3.200e-02

$2.933 \mathrm{e}-02$

$2.667 \mathrm{e}-02$

2.400e-02

2.133e-02

$1.867 \mathrm{e}-02$

$1.600 \mathrm{e}-02$

$1.333 \mathrm{e}-02$

$1.067 \mathrm{e}-02$

$8.000 \mathrm{e}-03$

$5.333 \mathrm{e}-03$

$2.667 \mathrm{e}-03$

$0.000 e+00$

$\mathrm{t}=20 \mathrm{~ms}$

Figure 3.23. Effective plastic strain contours of the test plate. (a) On the front and (b) the back face of the test plate after it had ceased reverberation $(t=20 \mathrm{~ms})$. 


\subsection{Concluding Remarks}

A suspended spherical test charge consisting of a $3 \mathrm{~kg}$ high explosive sphere surrounded by a $23.8 \mathrm{~kg}$ water-saturated annular sand shell has been detonated above a $2.54 \mathrm{~cm}$ thick edge clamped stainless steel plate to explore the impulse transfer mechanisms and dynamic response of a ductile plate. A combination of high-speed video and instrumented Kolsky bar measurements during the test were used in conjunction with particle-based simulations to analyze the impulse loading response of the 304 stainless steel plate by high velocity sand. The fidelity of the predicted pressure and impulse distributions for scenarios in which particle velocities exceeded $1200 \mathrm{~m} / \mathrm{s}$ have been confirmed, and therefore enabled sand front propagation and spreading to be investigated at a fundamental level. It has also allowed the contributions to the total impulse from the air shock, sand, and HE particles to be evaluated.

The study has shown that for the test geometry used here, $90 \%$ of the impulse was delivered to the test plate by the impact of sand particles. During propagation of the sand through background air, a strong air shock developed immediately in front of the sand. This air shock and the eventual impact of the detonation product impacts was responsible for the remaining impulse.

The highest velocity sand particles acquired their momentum by spallation from the outer surface of the sand shell during reflection of a compressive sand shock front initiated by a detonation front that had crossed the HE charge. The experimental study showed that this fast sand was distributed in sand spikes (fingers) whose tip speeds significantly exceeded that of the main sand front. However, the majority of the impulse was carried by a denser sand front that was driven from the rear by repeated collisions with expanding HE particles. The main sand front was accelerated to a velocity of $\sim 1200 \mathrm{~m} / \mathrm{s}$ during the first $130 \mu \mathrm{s}$ of the event, but then began to decelerate. Simulations conducted with and without the presence of background air particles showed that the deceleration resulted from momentum transfer from sand to air particles. The impact of the sand particles with the plate resulted in little outward reflection, and therefore transferred little more than the incident momentum for zero obliquity impacts directly beneath the charge.

The IMPETUS solver was also used to simulate the structural response of the I-beam frame support structure used for the test. The simulation identified a region on the I-beam flange where very large localized plastic strains were developed during rotation of the gripping system. This 
corresponded with the location of the support structure rupture during a subsequent test. The solver was therefore found to be well suited for the engineering level design of impulsive resistant test structures. While the simulations reproduced the impulse transfer from the detonation particles to the test plate well, they failed to provide insight into the origin of the shock induced instability at the sand/air interface, to capture the fractal-like evolution of the sand front topology, and predict the shape of sand spikes. The study of these phenomena will require the emergence of computational methods that can treat the coupling between the shape of the array of highly deformable sand front instabilities and the aerodynamic forces upon them in the hypersonic regime.

We note that the test conducted here was an example of a more general problem. During detonation, the release of the chemical (stored) energy of an energetic material creates an expanding shell of detonation products. While in any direction the detonation product momentum is high, the principle of conservation of momentum requires its integral about the charge center to be zero. Over time, the detonation product momentum in any direction is fully transferred to the sand shell, causing its rapid acceleration to a maximum value (dictated by detonation product momentum divided by the sand mass). For the test conducted here, most of the impulse was transferred to the structure by sand whose speed was near this maximum value. However, over time (or distance of propagation), the sand eventually transfers all its momentum to the surrounding air, and at large standoff distances, loading of a structure then occurs by air shock reflection. In this case, the impulse is sensitive to the air shock pressure created during the sand front expansion. Because of inverse square law spreading, the momentum transferred to a small area structure in this limit is likely to be low. 


\section{Chapter 4. \\ High intensity impact of granular matter with edge clamped ductile plates}

This chapter is based on a paper submitted to the International Journal of Impact Engineering ${ }^{2}$. It investigates the deflection of edge clamped solid plates subjected to a series of increasing intensity impacts by explosively accelerated granular matter. Numerical simulations were used to determine configurations of different test charges to achieve these increases in impulse. These charge configurations were then used to evaluate the deflection of 304 stainless steel edge clamped plates using a reinforced (but otherwise) similar test setup to that presented in Chapter 3 . This chapter presents a detailed analysis of the five charge configurations and their impact with the five solid test plates. Three of the tests used glass microspheres as a simulated sand whose mass was varied. Two others replaced the glass microspheres by much denser zirconia particles, enabling application of a higher impulse, but with a substantially lower velocity impact. The series of tests also varies the ratio of granular material to explosive charge mass to investigate its effect upon the applied pressure and impulse transferred to the test samples. High-speed video imaging was used to observe the sand front shape during propagation, and to measure the granular particle front velocities. An instrumented Kolsky bar was again used to measure the applied pressure and impulse loading by the accelerated granular media. Simulations implemented via the IMPETUS Afea Solver were compared with the Kolsky bar measurements and plate deformation mapping to ascertain the validity of the simulations. They were then used to analyze the sand front movement and mechanisms of particle interaction with the test plate for tests that applied specific impulses up to $25 \mathrm{kPa} \cdot \mathrm{s}$.

\footnotetext{
${ }^{2}$ A. Kyner, K. Dharmasena, K. Williams, V. Deshpande, H. Wadley, High intensity impact of granular matter with ductile edge clamped plates, (Submitted 2017).
} 


\subsection{Introduction}

As discussed in Section 2.3, a series of sand slug investigations has investigated the momentum transfer by granular media to nearby structures [32, 48, 49]. Lui et al. [32] numerically computed the dimensionless out of plane maximum transient displacement, $\delta_{\max }$, of an edge clamped plate of span length $2 \mathrm{~L}$, following the normal incident impact of a sand slug with incident specific impulse, $I_{o}$, Figure 2.2. The dimensionless impulse, $\bar{I}_{o}=I_{o} / m_{b} \sqrt{\sigma_{Y} / \rho_{m}}$, is the specific impulse divided by the mass per unit area of the plate, $m_{b}=\rho_{m} h$, while $\left(\sigma_{\mathrm{y}} / \rho_{\mathrm{m}}\right)^{1 / 2}$ (where $\sigma_{\mathrm{y}}$ is the plate's yield strength and $\rho_{\mathrm{m}}$ its density) is the plastic wave speed of the plate. The dimensionless analysis shown in Figure 2.2 indicates that the out of plane displacement is an approximately linear function of specific impulse for normal incidence impacts by granular particles, but can be reduced by use of high strength plate materials of low density [32].

In the problems of interest here, the granular particles suffer differential accelerations and are subject to different velocity dependent air drag forces during propagation [13, 52-54]. They therefore possess a distribution of impact velocities and angles of incidence upon impact with a planar structure. Since the momentum transferred by each particle impact is a function of the particle mass, speed, and its angle of incidence, and depends upon the mass and local velocity of the surface being impacted, prediction of the impulse can be complicated. For example, late arriving granular impacts with an already deforming plate surface no longer encounter a flat or stationary surface, and the impulse transferred to the plate can differ from that during early stage impacts. These granular particle-structure interactions [14, 17] result in effects akin to Taylor's fluid structure interaction (FSI) [8, 26], and the time-dependent loads that cause plate deformation can be difficult to estimate. The manner in which a test structure is supported (especially the end gripping conditions) further complicates the displacement response of the structure. During high intensity impulsive loading, edge clamped panels are susceptible to pull-in, and to plate failure at the grips by shear localization or necking $[56,94,95]$. As previously mentioned, the edge effects can be severe, especially when the grips extend above the impacted surface of the plate since this causes outward reflection of the granular wall jet with an associated (inward) reaction momentum that promotes shear failure $[17,94]$.

In the previous chapter, a granular impact test method was developed based upon a suspended spherical charge that radially accelerated a thick shell of water-saturated silica (glass) particles 
toward the center of an edge clamped test plate. The plate was edge gripped in a manner that avoided grip induced outward reflection of the particles that flowed across the plate surface, thereby avoiding shear-off. The study showed that a particle-based numerical simulation method implemented in the IMPETUS Afea Solver [3, 19, 20] successfully modeled the detonation of a high explosive and the transfer of momentum from the detonation products to a granular medium. It also successfully modeled their impact with a Kolsky bar and predicted the applied pressure and impulse, as well as that applied to a stainless steel plate, and the plate's subsequent dynamic deformation.

While the particle-based simulation approach has produced similarly encouraging results for lower intensity loading scenarios [13, 14, 19, 37], its scalability to higher intensity loading situations has not been investigated. Its utility for modeling other granular materials with a different density, elastic stiffness, and shape from spherical glass particles (silica) has also not yet been established. The study reported in this chapter extends the study in Chapter 3 to investigate the effects of increasing the impulsive loads applied by water-saturated granular media.

\subsection{Experimental Setup}

To investigate the deformation of structures under very high intensity granular media loading conditions, a five shot experimental test series was conducted at the same outdoor blast testing facility (NEWTEC Services Group, Inc. in Edgefield, SC) used for the study reported in Chapter 3. It also used the same edge clamped 304 stainless steel plates. Three of the tests were designed to enable an exploration of the effects of changing the mass of water-saturated fused silica (glass) particles to that of the explosive driver. The two additional tests used a higher mass of watersaturated zirconia particles, and enabled the consequences of changing the density (and mass) of the particles to be assessed.

\subsubsection{Test platform}

A schematic illustration of the test arrangement is shown in Figure 4.1. It is similar to that described in the previous chapter, but used a more heavily reinforced support base structure to avoid rupture of the supports during repeated testing. The test system consisted of; (i) a solid picture frame test platform to support the test plates, (ii) a suspended, spherical explosive charge encased by an annular shell of water-saturated fused silica or zirconia particles, and (iii) a steel 
Kolsky bar instrumented with strain gauges to measure the applied pressure and impulse loading at a symmetrically equivalent location to the center of the test plate, Figure 4.1(b). The test plates were mounted on two A-36 steel, square picture frame support plates with an $81.3 \mathrm{~cm} \times 81.3 \mathrm{~cm}$ square, center cutout, Figure 4.2. The upper most picture frame plate was $5.1 \mathrm{~cm}$ thick and was placed on a second $15 \mathrm{~cm}$ thick plate. This thicker support plate replaced the less robust I-beam used in the Chapter 3, which underwent permanent deformation and eventual fracture under the high intensity loading conditions (Appendix B) like those used here. Steel stock shim pieces were used as necessary to fill any small gaps between the test plates and the picture frame support plate to which the test plates were secured, Figure 4.2. The plate span, $2 \mathrm{~L}$, between the edge clamps (where inplane plate stretching occurred) was $1.22 \mathrm{~m}$ while that where out-of-plane panel bending was permitted was $81.3 \mathrm{~cm}$ (defined by the center cutout of the picture frame steel supports), Figure 4.2 .

A 3D view of the test arrangement with the reinforced support plate is shown in Figure 4.1(a). Both picture frame plates had four corner bolt-holes that aligned with holes in the test plate corners to secure the test plate to the support base using $1.9 \mathrm{~cm}$ diameter, Grade 8 bolts. Similar to the test setup in the previous chapter, the steel picture frame support structure was mounted on a wooden frame placed above cinder blocks that raised the platform to an optimal height for visual observation of the tests. To reduce structural damage to the test structure, a $0.95 \mathrm{~cm}$ thick rubber mat was placed between the steel support plates and the wooden structural frame.

A pair of Vision Research Inc., Phantom V7.3 high-speed cameras were used for observation of the test shots after detonation. These cameras were positioned side by side approximately $20 \mathrm{~m}$ in front of the test platform. One camera provided a wide view of the test event while the other provided a more magnified image of the test charge, test plate, and impact end of the Kolsky bar. The magnified image used a reduced number of pixels to allow the recording of more frames per second. The images from the high-speed video cameras were used to visualize and track the granular material front position during its propagation towards the Kolsky bar and test plate. The $1.32 \mathrm{~m}$ length front edge of the test plates was spray painted prior to each test shot to provide a length scale reference for the high-speed video calculations of sand front position and velocity. 
(a)

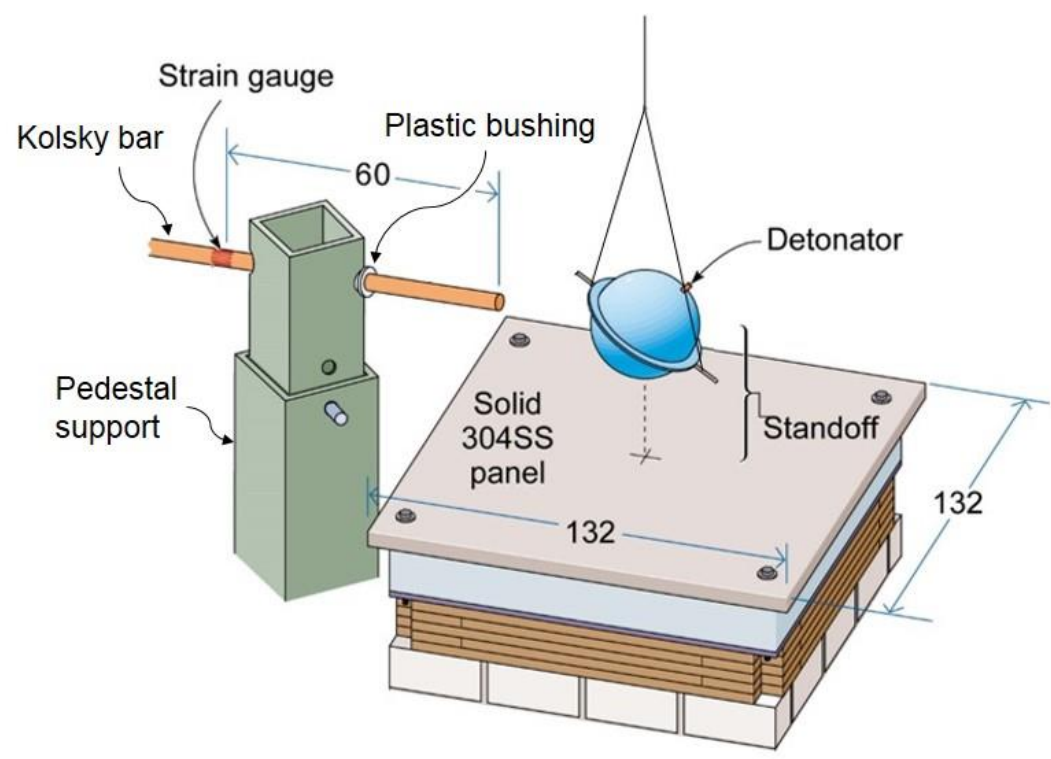

(b)

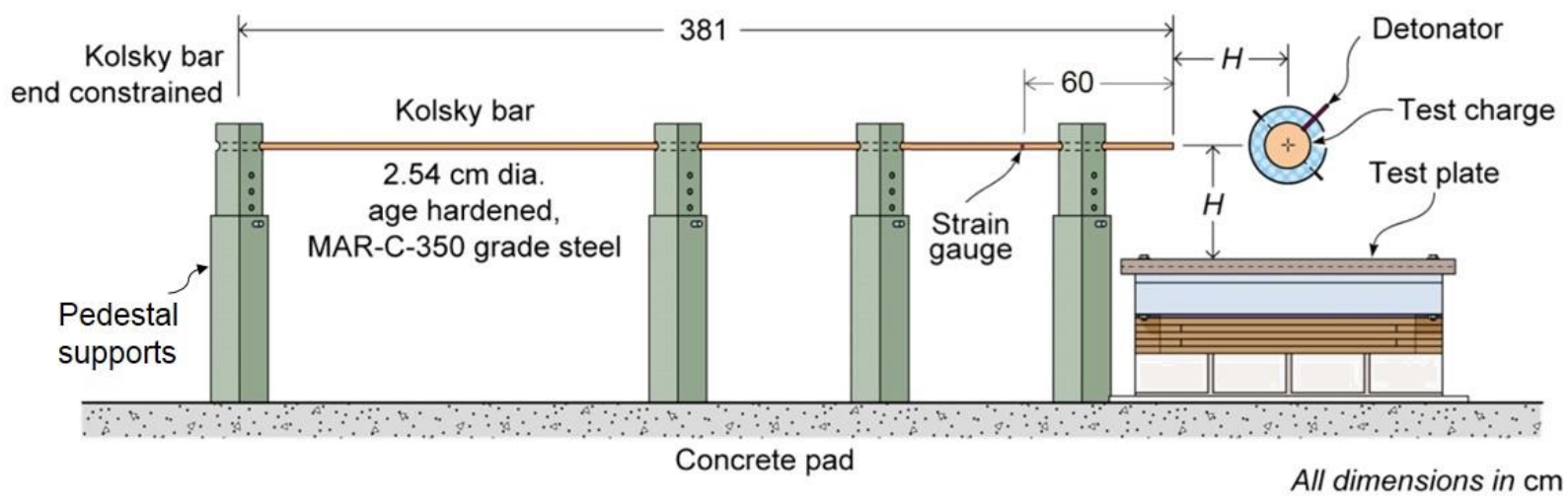

Figure 4.1. (a) The experimental setup showing test plate and suspended charge location together with the strain gage instrumented Kolsky bar. (b) A side view of the test arrangement showing the position of the Kolsky bar and test plate at similar distances and orientations to the test charge. 


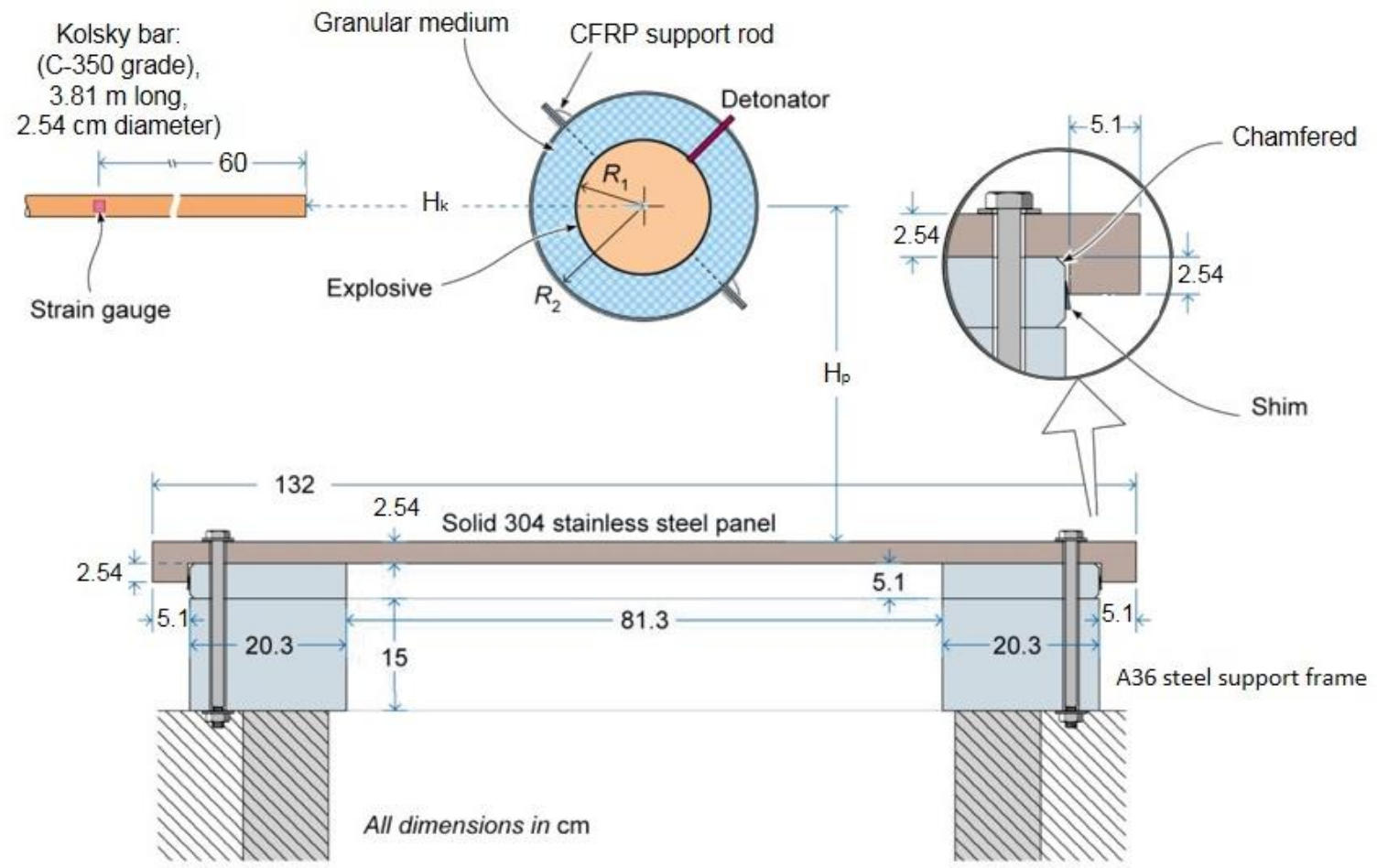

Figure 4.2. Schematic illustration showing explosive charge, detonator, and Kolsky bar placement relative to the test plate. Each test plate had a $5.1 \mathrm{~cm}$ wide perimeter lip to provide edge restraint.

The front end of the Kolsky bar was also painted to provide a reference scale. The painted length was $10 \mathrm{~cm}$ for test Shots $1-3$ and $15 \mathrm{~cm}$ for Shots 4 and 5. The optimum sampling rate of the highspeed videos varied from shot to shot due to variations in lighting. The data shown later used inter-frame times of 100, 111, 87, 48, and $29 \mu$ s for Shot 1 through Shot 5 respectively.

\subsubsection{Stainless steel targets}

Five, $2.54 \mathrm{~cm}$ thick, $1.32 \mathrm{~m}$ x $1.32 \mathrm{~m} 304$ stainless steel plates were used for the study. The test plates were first annealed at $538^{\circ} \mathrm{C}$ for eight hours (Rex Heat Treat, Lansdale, PA) to remove internal stress and then machined to their square shape at KVK Precision Specialties Inc. (Shenandoah, VA). As in the test plate fabrication presented in Chapter 3, to resist the large pullin stresses at the periphery of the edge clamped test plate, four, $1.32 \mathrm{~m}$ length, $5.1 \mathrm{~cm}$ wide by 2.54 $\mathrm{cm}$ thick rectangular 304 stainless steel bars were welded along the four edges of the underside of 
the test plates forming a frame on the (eventual) underside of the plates. These edge grips were then fitted over the A-36 steel support frame to provide effective edge restraint during impact loading while preventing local impulse amplification observed in studies where the edge grips extended above the top surface of the test plate [17,94]. The edge grip welded attachment was reinforced using high strength steel dowel pins that were press-fitted through a series of predrilled holes that penetrated the test plate and edge grips.

\subsubsection{Charge configurations}

A spherical Composition-4 (C-4) explosive charge surrounded by a concentric granular media shell was suspended above the center of each test plate to provide impulse loading to the square 304 stainless steel test plates, Figure 4.2. To optimize investigation of the effect of impulse upon the test plate deflection, a series of simulations were first performed using the IMPETUS Afea particle based simulation code to identify appropriate explosive mass and granular shell thickness/density combinations. The five charge configurations developed using this approach are summarized in Table 4.1.

Each charge was constructed with the aid of two thin-walled acrylic polymer, concentric spheres. Each spherical shell consisted of two hemispheres that were adhesively connected at the equator. The plastic hemispheres varied in thickness from $4.8 \mathrm{~mm}$ at the equator to $1.6 \mathrm{~mm}$ at the top of each hemisphere. Figure 4.2 shows the charge consisted of an internal sphere of radius, $\mathrm{R}_{1}$, containing the explosive surrounded by an outer sphere of radius $\mathrm{R}_{2}$, defining the outer diameter of the sphere containing the granular material and test charge. The radius of both the inner and outer sphere was defined by the outside of the acrylic shell. The annular region between the two spheres was approximated by $\mathrm{R}_{2}-\mathrm{R}_{1}$, less the thickness of the outer acrylic shell (which varied between 1.6-4.8 $\mathrm{mm}$ ) and was filled with the water-saturated granular material.

Table 4.1. Charge configurations for the five test shots.

\begin{tabular}{lcccccccc}
\hline $\begin{array}{c}\text { Test } \\
\text { shot }\end{array}$ & $\begin{array}{c}\text { Inner } \\
\text { radius, } \\
\mathrm{R}_{1}(\mathrm{~mm})\end{array}$ & $\begin{array}{c}\text { Explosive } \\
\text { mass } \\
(\mathrm{kg})\end{array}$ & $\begin{array}{c}\text { Outer } \\
\text { radius, } \\
\mathrm{R}_{2}(\mathrm{~mm})\end{array}$ & $\begin{array}{c}\text { Annular } \\
\text { shell width } \\
(\mathrm{mm})\end{array}$ & $\begin{array}{c}\text { Particle } \\
\text { type }\end{array}$ & $\begin{array}{c}\text { Particle } \\
\text { mass } \\
(\mathrm{kg})\end{array}$ & $\begin{array}{c}\text { Water } \\
\text { mass } \\
(\mathrm{kg})\end{array}$ & $\begin{array}{c}\text { Annular } \\
\text { shell mass } \\
(\mathrm{kg})\end{array}$ \\
\hline 1 & 80 & 3.0 & 152 & 72 & Glass & 19.87 & 4.18 & 24.05 \\
2 & 80 & 3.0 & 203 & 123 & Glass & 51.76 & 12.95 & 64.71 \\
3 & 90 & 4.5 & 203 & 113 & Glass & 50.26 & 12.66 & 62.92 \\
4 & 90 & 4.5 & 203 & 113 & $\mathrm{ZrO}_{2}$ & 86.41 & 15.27 & 101.68 \\
5 & 90 & 4.5 & 229 & 139 & $\mathrm{ZrO}_{2}$ & 125.63 & 23.15 & 148.78 \\
\hline
\end{tabular}



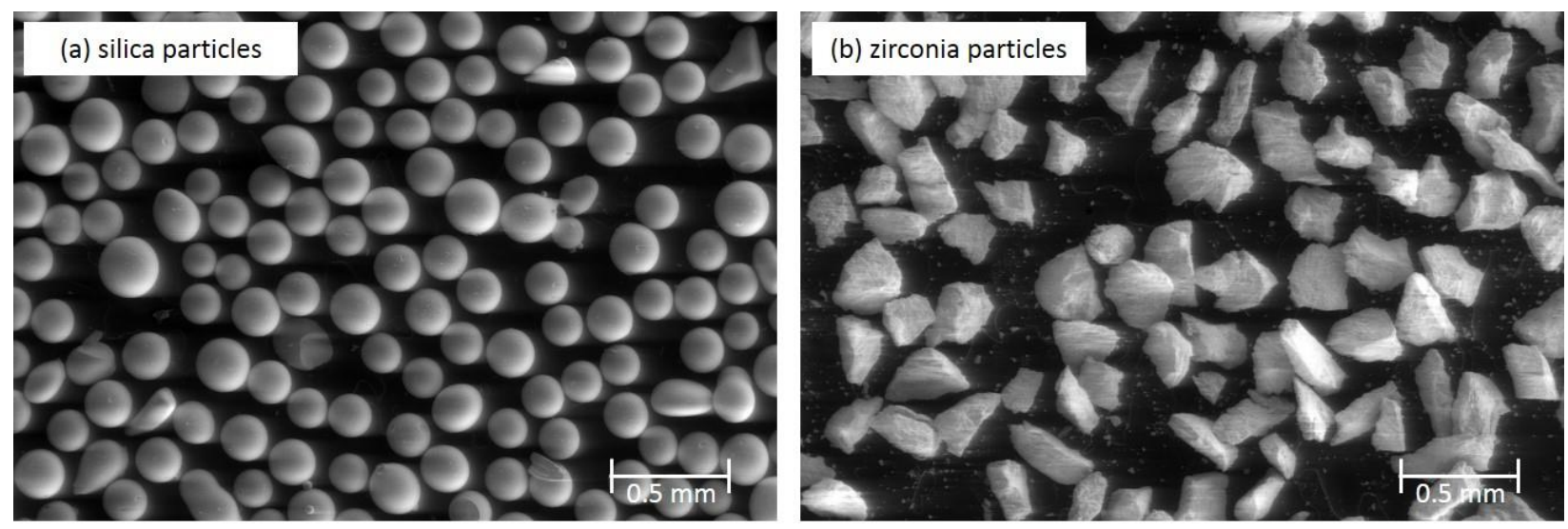

Figure 4.3. Optical image of the (a) fused silica and (b) zirconia particles used for experimental testing.

The test charge assembly procedure (similar to that presented in Chapter 3, Figure 3.4) began by filling the inner polymer sphere (with radius $\mathrm{R}_{1}$ ) with either $3 \mathrm{~kg}$ or $4.5 \mathrm{~kg}$ of $\mathrm{C}-4$ explosive. This sphere was then centered within the larger polymer sphere with radius $R_{2}$. A carbon fiber reinforced polymer (CFRP) suspension rod was inserted through the center of both spheres to enable the charge to be later suspended above the test plate, Figure 4.1(a). The outer sphere was composed of two hemispheres, with a $3.8 \mathrm{~cm}$ circumferential flange that enabled them to be joined together with a high strength epoxy adhesive. A $10 \mathrm{~mm}$ diameter, cylindrical, thin-walled plastic pipe was inserted through the top of the outer and inner concentric spheres to provide access for subsequent placement of a detonator in contact with the C-4 charge. The annular space between the two spheres was filled with the granular material and then sufficient water was added to fill all voids between the particles. Three test charges (Shots 1-3) used fused silica (glass) particles with a diameter of 150-200 $\mu \mathrm{m}$, Figure 4.3(a). These grade GL-0191 soda-lime glass particles from Mo-Sci Corporation (Rolla, Missouri) had a density of $2700 \mathrm{~kg} / \mathrm{m}^{3}$, and were identical to those used in Chapter 3 with this experimental setup [54] and in several other lower impulse level tests $[13,14,17]$. Two of the charges (Shots 4 and 5) used zirconia particles obtained from Saint-Gobain (Huntsville, AL; product 9826 70/100), Figure 4.3(b). These particles had a similar effective diameter but were more angular in shape and had a much higher density of $5930 \mathrm{~kg} / \mathrm{m}^{3}$. Sufficient water was again added to fill the void space between the $\mathrm{ZrO}_{2}$ particles. The very high mass of these charges, Table $4.1(\sim 102$ and $149 \mathrm{~kg})$, required the use of a coarse mesh net for their suspension over the test plates. 
The charges were suspended above the center of the test plates as shown in Figure 4.1. Their standoff distance was defined from the charge center to the top of the test plate, $\mathrm{H}_{\mathrm{p}}$. The distance

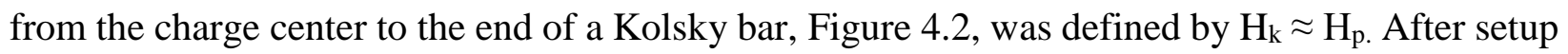
of the test arrangement, an instantaneous (zero millisecond delay) detonator manufactured by Dyno Nodel Inc. (Salt Lake City, Utah; model SP/SM (12-0)) was inserted into the explosive charge through the cylindrical, plastic pipe just before the detonation event. To ensure equivalent loading of both the test plate and end of the Kolsky bar, the charge orientation was adjusted as shown in Figure 4.1 and Figure 4.2, so that the detonator was situated $45^{\circ}$ to the East of the charges' North Pole. The significant mass of test Shot 5 made it difficult to suspend the charge at the desired rotation angle, and so this test used a detonator inclination angle $\theta=70^{\circ}$ from the $\mathrm{X}$-axis as defined in Figure 4.4.

The initial placement of the center of the test charge was $45 \mathrm{~cm}$ above the center of the test plate and $45 \mathrm{~cm}$ from the impact end of the Kolsky bar. However for each of the five test shots, enough time elapsed between test setup and charge detonation that the charge location suffered some drift, resulting in small changes to the standoff distance between the charge center and end of the Kolsky bar and surface of the plate. The actual standoff distances, to the test plate, $\mathrm{H}_{\mathrm{p}}$, and to the Kolsky bar, $\mathrm{H}_{\mathrm{k}}$, were measured from the high-speed video images just prior to detonation and are summarized in Table 4.2.

Table 4.2. Standoff distances from the test charge center to the top of the test plate and impacted end of the Kolsky bar, measured from the high-speed videos prior to detonation.

\begin{tabular}{ccc}
\hline Test shot & $\begin{array}{c}\text { Standoff to test plate } \\
\mathrm{H}_{\mathrm{p}}(\mathrm{cm})\end{array}$ & $\begin{array}{c}\text { Standoff to Kolsky bar } \\
\mathrm{H}_{\mathrm{k}}(\mathrm{cm})\end{array}$ \\
\hline 1 & 41.7 & 41.6 \\
2 & 48.6 & 44.5 \\
3 & 44.6 & 44.6 \\
4 & 44.4 & 44.9 \\
5 & 45.6 & 45.3 \\
\hline
\end{tabular}




\subsubsection{Kolsky bar measurements}

In order to measure the impulse applied by the different test charges, a strain gage instrumented, $2.54 \mathrm{~cm}$ diameter, $3.81 \mathrm{~m}$ long (age hardened) C-350 grade maraging steel Kolsky bar was positioned $45 \mathrm{~cm}$ above the top of the test plate. To estimate the impulse applied to the center of the test plate, the end of the bar was placed at approximately the same distance from the center of the test charge as that to the top of the test plate center, Figure 4.2 and Table 4.2. Despite some experimental variability in these distances, the pressure applied to the Kolsky bar provided an experimental estimate of the loading experienced by the center of the test plates. It was also used to test the validity of a subsequent simulation of the test. After validation, this simulation was then used to determine the impulse distribution applied to the plate. The Kolsky bar was aligned such that its cylindrical axis intersected the center of the explosive charge using four adjustable height pedestal supports, Figure 4.1. The end of the bar experienced a considerable force during the tests, and was therefore clamped to each of the pedestals which in turn were bolted to a thick reinforced concrete foundation. The square cross section pedestals were rotated so that they presented $45^{\circ}$ symmetrically inclined faces to the ejecta to reduce the applied load. To reduce leakage of the Kolsky bar's elastic wave propagation modes [81], plastic bushings were placed on the Kolsky bar where it was secured to the pedestals, Figure 4.1(a).

Two, T-rosette type strain gauges (Vishay Precision group, CEA-06-125UT-350) were mounted $0.6 \mathrm{~m}$ from the impact end of the Kolsky bar to measure the axial stress (pressure) and transmitted impulse resulting from impact by the water-saturated granular material. The strain gauges were bonded to the bar diametrically opposite each other with Vishay AE-10 epoxy adhesive. A protective coating (Vishay Gage Kote \#5) was applied to the strain gauges and the connecting wires for protection from the test events. The wires led to a digital signal recording system located in a metal box to the side of the test arrangement and covered with sand bags for protection from the blast events. For the full Wheatstone bridge circuit, the relationship between the input, $V_{i}$ and output $V_{o}$ voltages is described by in Chapter 3 by Eqn. (3.1). For these test shots, test Shots 1 and 2 used an input excitation voltage $V_{i}=10 \mathrm{~V}$ while test Shots 3-5 used $V_{i}=5 \mathrm{~V}$ to keep the signal amplitude from overloading the analogue to digital converter. A low-pass filter applied an upper cutoff frequency of $1 \mathrm{MHz}$ to the recorded signal. The axial strain deduced from the data using Eqn. (3.1) was used with Hooke's law to determine the axial stress (applied pressure) on the Kolsky bar, and by its time integration, the impulse transmitted to the bar. 
As for the previous model test shot in Chapter 3, the signal recording was initiated by a break wire that was attached to the external surface of each test charge. For consistency, $t=0 \mathrm{~s}$ was defined as the time of detonation of the event. Since the trigger wire was attached to the outer shell of the test charge, this meant there was a delay from the time of detonation until the trigger signal was initiated. To adjust for this delay, the data for each test charge was shifted by the time for a detonation wave to propagate through the explosive and for a shock wave to penetrate the shell of granular material, break its surface and trigger the recoding system. Appendix $\mathrm{C}$ presents discrete particle simulations of the charge detonation and shock compression of the granular materials surrounding each charge configuration. These simulations enabled the detonation and granular shock transit times to be estimated which are summarized in Table 4.3.

The Kolsky bar data has been time-shifted (to maintain a consistent definition of time) by adding the time delay between initiation of detonation and breakage of the trigger wire, whereupon signal recording was started, Table 4.3. The strain gauge sensors from which the pressure was deduced were positioned $0.6 \mathrm{~m}$ from the impacted end of the Kolsky bar. The signal at the strain gauge location was therefore delayed by the time for the longitudinal elastic wave to propagate along this $0.6 \mathrm{~m}$ distance along the bar. Since the longitudinal elastic wave speed in C-350 maraging steel bar is $4800 \mathrm{~m} / \mathrm{s}$, it required $125 \mu \mathrm{s}$ for a signal, caused by impact with the end of the bar, to reach the sensor location. The first reflection from the distal end of the Kolsky bar (length $3.81 \mathrm{~m}$ ) arrived $1.34 \mathrm{~ms}$ after the initial signal. To avoid complexities associated with this reflected signal, only data prior to the arrival of the distal reflection was used.

Table 4.3. Time for detonation and shock front propagation through the explosive and granular shells.

\begin{tabular}{cccccc}
\hline $\begin{array}{c}\text { Test } \\
\text { shot }\end{array}$ & $\begin{array}{c}\text { Explosive } \\
\text { radius, } \\
\mathrm{R}_{1}(\mathrm{~mm})\end{array}$ & $\begin{array}{c}\text { Outer } \\
\text { radius, } \\
\mathrm{R}_{2}(\mathrm{~mm})\end{array}$ & $\begin{array}{c}\text { Detonation wave } \\
\text { transit time } \\
(\mu \mathrm{s})\end{array}$ & $\begin{array}{c}\text { Granular material } \\
\text { shock transit time } \\
(\mu \mathrm{s})\end{array}$ & $\begin{array}{c}\text { Total delay } \\
\text { time } \\
(\mu \mathrm{s})\end{array}$ \\
\hline 1 & 80 & 152 & 20 & 20 & 40 \\
2 & 80 & 203 & 20 & 34 & 54 \\
3 & 90 & 203 & 22 & 31 & 53 \\
4 & 90 & 203 & 22 & 36 & 58 \\
5 & 90 & 229 & 22 & 45 & 67 \\
\hline
\end{tabular}




\subsection{Simulation Methodology}

The five test shots were simulated using the IMPETUS Afea Solver [19], previously described in Section 1.5 and Section 3.3. The solver uses a discrete particle blast code that tracks collisions between air, soil, and high explosive (HE) particles using a particle contact model. Its validation for the water-saturated soda lime glass particles (synthetic sand) used here has been presented in the previous chapter and by Borvik et al. [19] using a similar experimental test [17], but at a much lower impulse level. Chapter 3 and several prior studies have shown this solver to be a reasonably accurate tool for the analysis of high intensity granular loading problems $[13,14,17,19,54]$. Briefly, the solver is based on a Lagrangian formulation in which the discrete particles are fully coupled and allowed to interact with a finite element (FE) model of a test structure. The particles momentum transferring collisions are governed by a contact interaction model. To reduce computational time, the particles are allowed three translational degrees of freedom, and so rotation of the particles is not treated by the solver. It is presumed that prior calibration of the contact model parameters compensates for this simplifying assumption.

\subsubsection{The particle contact model}

Air and HE particles were modeled as rigid, spherical particles with elastic particle interactions consistent with Maxwell's kinetic theory of gases [20]. The air is treated as an ideal gas with a density $\rho=1.3 \mathrm{~kg} / \mathrm{m}^{3}$. The air particles were given an initial translational energy $\mathrm{E}_{0}=$ $253 \mathrm{~kJ} / \mathrm{m}^{3}$ and a ratio of heat capacities $\gamma=1.4$. The initial particle velocities and directions attributed to each particle were randomly selected from the Maxwell-Boltzmann distribution. The HE particles were modeled using the solver's predetermined parameters for C-4 explosive. This ascribes an initial density $\rho=1601 \mathrm{~kg} / \mathrm{m}^{3}$, an initial internal energy $\mathrm{E}_{0}=8.7 \mathrm{GJ} / \mathrm{m}^{3}$, a heat capacity ratio $\gamma=1.4$, a particle initial solid-fill fraction $b=0.35$, and a detonation velocity $\mathrm{D}=8190 \mathrm{~m} / \mathrm{s}$.

Table 4.4. Distribution of air, HE, and soil particles in the simulations for each test shot.

\begin{tabular}{cccc}
\hline Test shot & Air particles & HE particles & Soil particles \\
\hline 1 & 854,468 & 701,310 & 444,222 \\
2 & 617,032 & 514,705 & 868,263 \\
3 & 556,265 & 688,420 & 755,495 \\
4 & 556,838 & 686,445 & 756,717 \\
5 & 468,425 & 585,587 & 945,987 \\
\hline
\end{tabular}


Interactions between the soil particles were treated using a soft particle, penalty contact model [19]. For test Shots 1-3 with glass particles, the solver's predefined wet sand parameters were used in the model. These predefined wet sand parameters were also used in Chapter 3 for validation of the simulation solver for high impact loads. These particle parameters for the wet synthetic (glass particle) sand gave an initial density $\rho=2020 \mathrm{~kg} / \mathrm{m}^{3}$, a soil-soil contact stiffness $k_{0}=4.0 \mathrm{GN} / \mathrm{m}$, a soil-soil contact coefficient of friction $\mu=0.0$, and a soil-soil damping coefficient $\xi=0.005$. The zirconia particles had a dry bulk density of $\rho=2550 \mathrm{~kg} / \mathrm{m}^{3}$ with a $43 \%$ fill fraction. Water was added to fill in the voids, resulting in a measured water-saturated density $\rho=3120$ $\mathrm{kg} / \mathrm{m}^{3}$. To model the zirconia particles within the solver, a series of simulations were conducted in which the stiffness, friction, and damping coefficients were systematically adjusted from the wet glass particle parameters until the impulse applied to the Kolsky bar and the sand front propagation velocity correlated with experimental observations. This resulted in a soil-soil contact stiffness $k_{0}=4.5 \mathrm{GN} / \mathrm{m}$, a soil-soil contact coefficient of friction $\mu=0.05$, and soil-soil damping coefficient $\xi=0.005$. A particle convergence study indicated simulation convergence with two million particles, and so this number of particles was used for all the tests. The division of the two million particles between air, soil, and HE particles was determined by the solver, and since the volume of soil and high explosive was different for each test shot, the particles were distributed differently for each simulated test, Table 4.4.

\subsubsection{FE geometry model}

The charge position dependent geometry of each test was used to construct finite element models as shown in Figure 4.4. The FE models for the test plate, support platform, and Kolsky bar were the same for all the test simulations. However, the suspended explosive test charge, with adjusted inner and outer radius dependent on the charge configuration, was shifted based on the standoff distances, $\mathrm{H}_{\mathrm{p}}$ and $\mathrm{H}_{\mathrm{k}}$, measured from the high-speed cameras, Table 4.2. The experimentally determined dimensions of the Kolsky bar, test platform support frame, and the 2.54 $\mathrm{cm}$ thick stainless steel test plate were used to construct the model. The Kolsky bar was modeled using a bar diameter of $2.54 \mathrm{~cm}$ and total length of $3.81 \mathrm{~m}$. It was constructed in four cylindrical sections, with a $4 \mathrm{~mm}$ length cylinder included at $0.6 \mathrm{~m}$ from the impacted end to represent the strain gauge location. The pressure applied to the bar was calculated by dividing the force-time response of the elements in the $4 \mathrm{~mm}$ length cylinder by the cross-sectional area of the bar. 


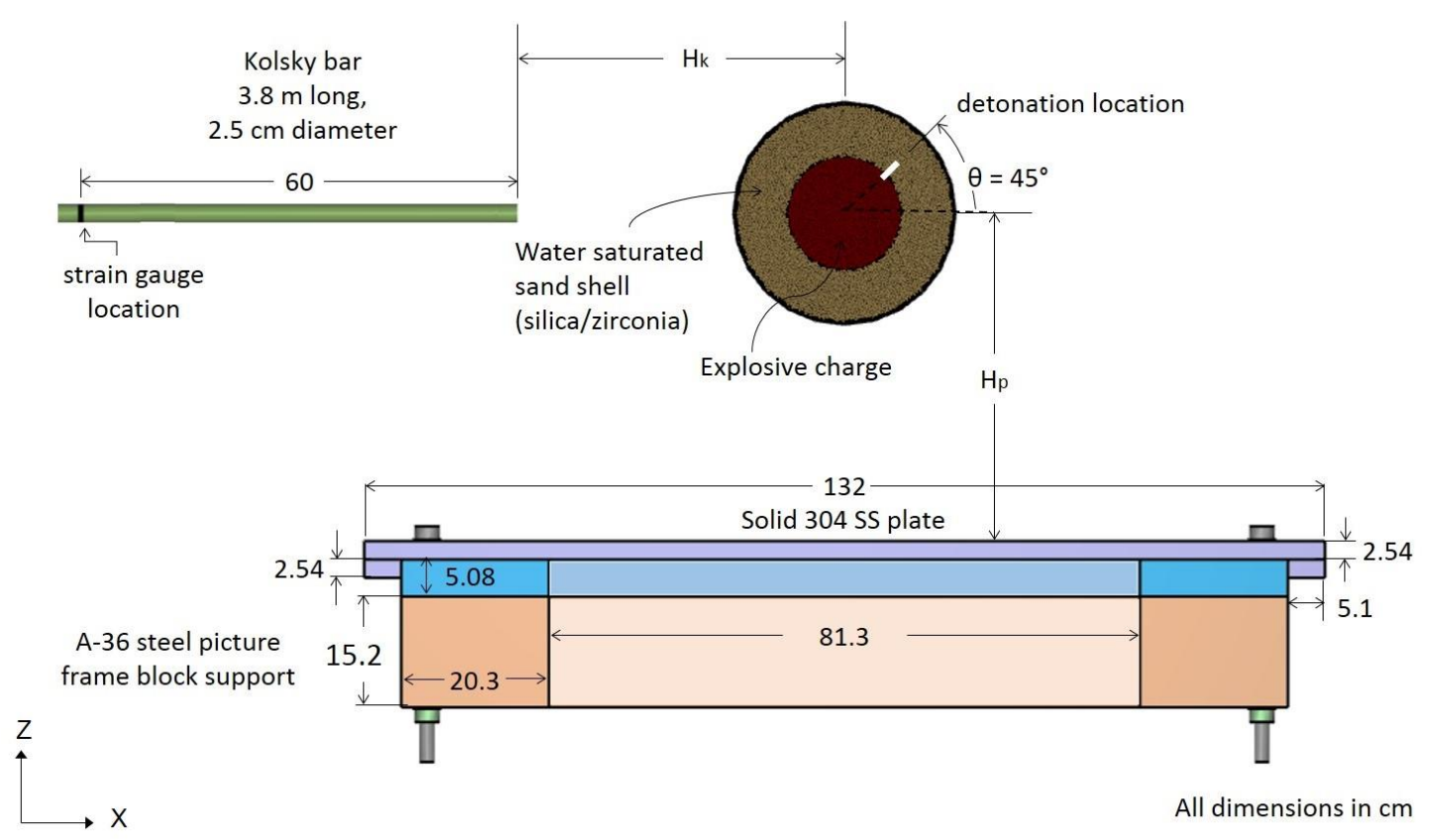

Figure 4.4. Simulation model of test setup with suspended test charge above an edge clamped test plate with a Kolsky bar positioned to measure the applied pressure and impulse loading.

Temporal integration of this pressure-time signal was used to obtain the specific impulse transferred to the Kolsky bar. The Kolsky bar FE model consisted of 39,000 linear hexahedra elements and 43,248 nodes.

The test rigs support frame was modeled as two solid block picture frames with external dimensions of $1.22 \mathrm{~m} \times 1.22 \mathrm{~m}$ with an $81.3 \mathrm{~cm} \times 81.3 \mathrm{~cm}$ center opening. The thickness of the upper block was $5.08 \mathrm{~cm}$ while that of the bottom was $15.2 \mathrm{~cm}$. The four bolts used to secure the plates to the support rig at its four corners were included in the model. The bottom plane of this support frame was constrained in all directions. The stainless steel test plate was given dimensions of $1.32 \mathrm{~m} \mathrm{x} 1.32 \mathrm{~m}$ and a thickness of $2.54 \mathrm{~cm}$. In order to clamp the plate to the support frame, an outer, $5.1 \mathrm{~cm}$ wide and $2.54 \mathrm{~cm}$ thick picture frame was connected to the solid plate using the solver's merge option. This represented the four rectangular bars that had been joined (welded) to the outer edge of the plate. The solid test plate and four attached rectangular bars forming the outer edge of the picture frame, were modeled with a refined mesh (to enhance observation of plate deformation) using 1,492 cubic hexahedra elements with 56,100 nodes. 
The suspended charge was modeled as an inner sphere of outer radius $\mathrm{R}_{1}$ that contained the explosive surrounded by an annulus of granular material with outer radius, $\mathrm{R}_{2}$. The acrylic plastic shell that retained the explosive particles and the outer plastic shell that contained the granular particles were included in the model. To simplify the model, each polymer shell was given a fixed thickness of $3 \mathrm{~mm}$. Since the flange that connected the outer hemispheres did not influence the impulsive load applied to the test plate or Kolsky bar, it was not included in the model. The detonation location of the test charge was set at the outer edge of the explosive sphere, at $\theta=45^{\circ}$ for Shots 1-4 and at $70^{\circ}$ for Shot 5, Figure 4.4. The full FE meshed model (the support structure, test plate, four bolts, Kolsky bar, and acrylic shells) consisted of 43,404 elements (432 linear pentahedra, 1,492 cubic hexahedra, and 41,480 linear hexahedra elements) with 214,202 nodes.

\subsubsection{Material parameters}

A Johnson-Cook model was used to represent the constitutive response of the solid materials in the finite element model. This included the 304 stainless steel test plates, the A-36 support frame, the C-350 grade (age hardened) maraging steel Kolsky bar, and the carbon steel bolts. As described in Chapter 3 (Section 3.3.2), the solver calculates the von Mises flow stress for the Johnson-Cook constitutive model by Eqn. (3.5). The Johnson-Cook parameters for each material are listed in Table 3.1. The test plate was modeled with parameters for annealed 304 stainless steel $[83,84]$. The parameters for A-36 steel were taken from ASTM-A36 [85]. The parameters for the 350 grade maraging steel were those of a similar, VascoMax 300 alloy [86], but with an adjusted yield strength of $2.195 \mathrm{GPa}$ to better represent the C-350 grade steel used here [87]. The Grade 8 steel bolt parameters were assumed to be the same as medium carbon steel AISI 1040 [88]. Since no fracture was observed in the experimental tests, a failure model was not included in the simulations. The plastic spherical shells containing the inner charge and the outer annulus of wet sand were modeled using an elastic constitutive model with parameters for acrylic plastic, $\rho=1180 \mathrm{~kg} / \mathrm{m}^{3}$, Young's modulus $\mathrm{E}=2.80 \mathrm{GPa}$, and Poisson's ratio $v=0.37$. Failure was set at a strain of $10 \%$ at which point the failed elements were eroded. 


\subsection{Results}

\subsubsection{Sand front propagation}

\subsubsection{High speed video observations}

The radial expansions of the particle fronts for the three glass particle tests (Shots 1-3) can be seen in the high-speed video images of Figure 4.5. These expansions were accompanied by an air shock front (not visible in these figures but shown latter) that propagated slightly ahead of the particle fronts. The upper row of images shows the test configuration at the instant of detonator activation $(t=0 \mathrm{~s})$. The white painted edge of the test plate specimen and the end of the Kolsky bar provide a length scale for interpretation of the images. The three vertical image columns show the radial expansion of the glass particle fronts at $\sim 250 \mu$ s and $\sim 650 \mu$ s after detonation for each test charge. A significant local retardation of the sand front by the wide polymer flanges can be seen, but the charge orientation ensured that this had no effect on the impulse loading of the Kolsky bar or test plate. In the image column for Shot 1, Figure 4.5(a) to (c), the rapid radial expansion of the glass particle front resulted in almost simultaneous impact of the granular material with the end of the Kolsky bar and the center of the test plate shortly after $250 \mu$ s, Figure 4.5(b). The lowermost image, Figure 4.5(c), shows the configuration at $650 \mu$ s after detonation when the spherical sand front had made complete contact with the top surface of the test plate. The magnified inset in this image shows the presence of sand fingers, corresponding to spikes of granular material at the leading edge of the sand front with a substantially higher tip velocity compared to the main sand front. Luminescence (white dots) is evident at the tips of a few of these sand fingers.

The effect of increasing the thickness of the sand shell (without changing the explosive mass) can be seen by comparing the images for test Shots 1 and 2. Comparison of Figure 4.5(b) and (e) with Figure 4.5(c) and (f) shows that the radial expansion rate was significantly higher for Shot 1 with a lower (water-saturated) glass particle mass. In contrast, comparison of test Shots 2 and 3 enables the effect of increasing the explosive to be observed. Figure 4.5(h) shows luminescence associated with the impact of glass particles with the test plate and (simultaneously) with the end of the Kolsky bar at $\sim 288 \mu$ s after detonation. Contact of the glass particles began at $\sim 270-280 \mu \mathrm{s}$ after detonation. Comparison of the Shots 2 and 3 images in Figure 4.5 (f) and (i) at about 666 
Shot 1: $(80,152)$; silica
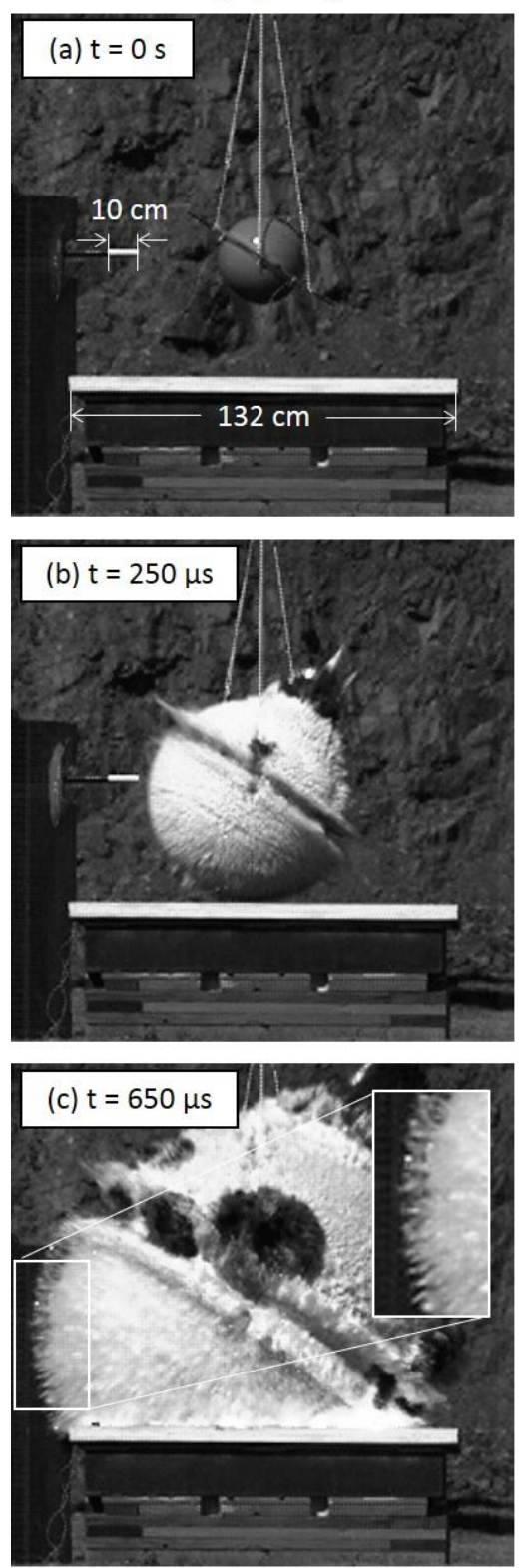

Shot 2: $(80,203)$; silica
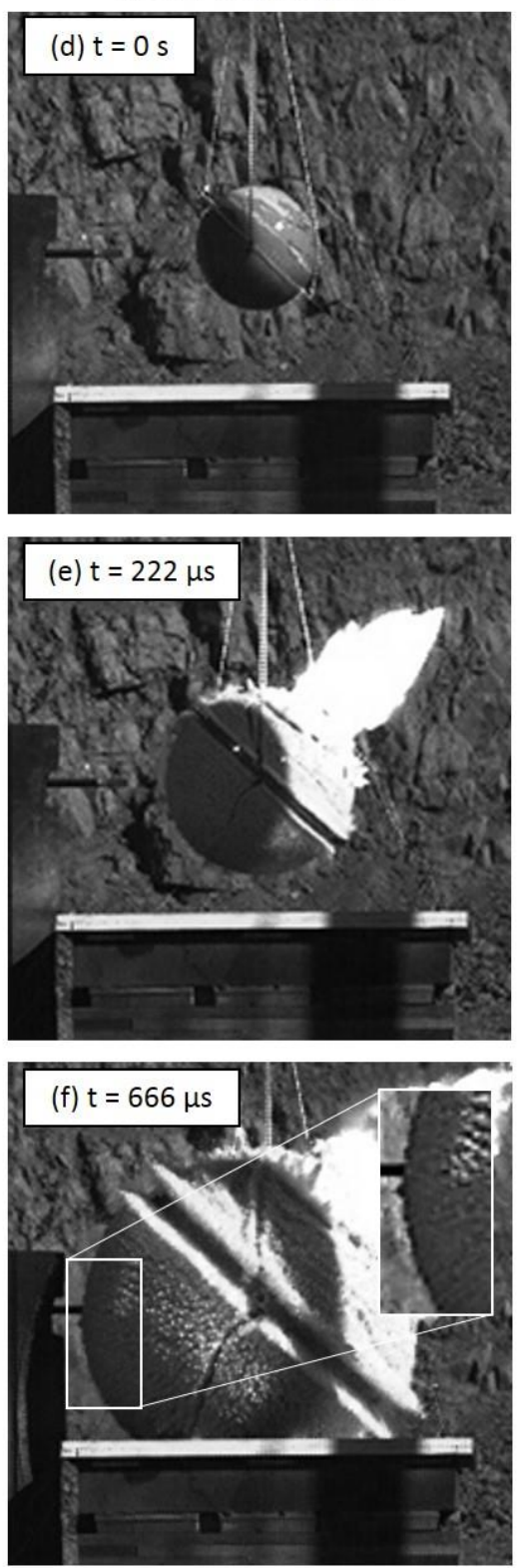

Shot 3: $(90,203)$; silica
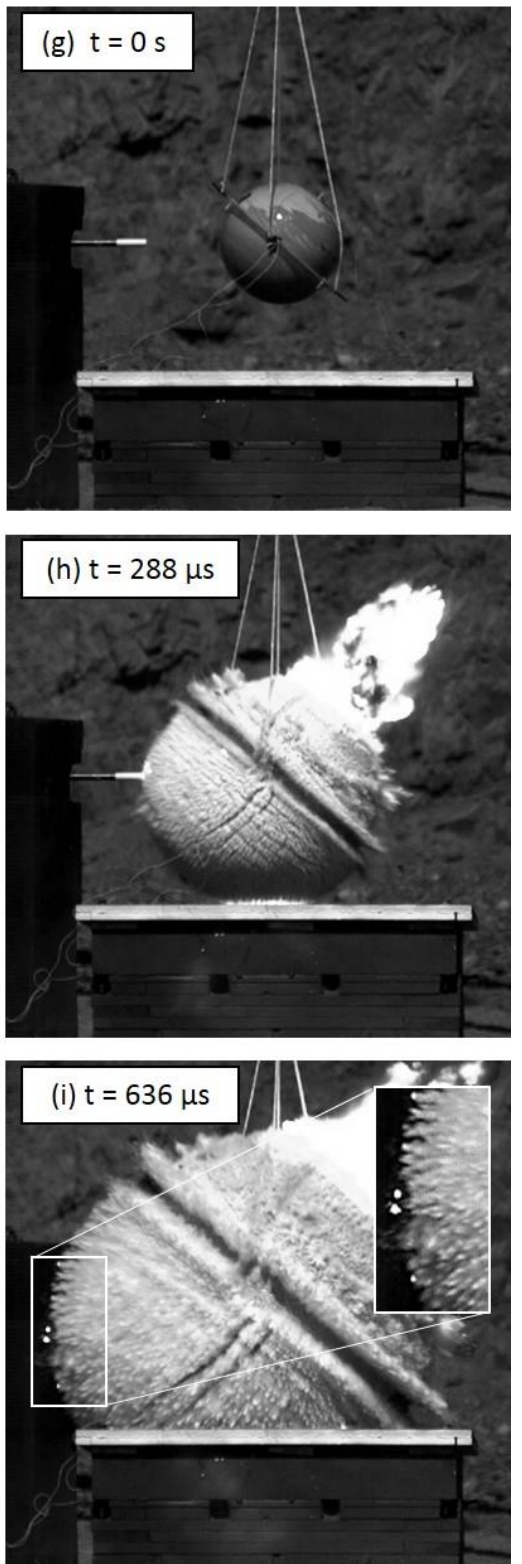

Figure 4.5. High-speed video images of glass microsphere tests. (a) Shot $1(R 1=80 \mathrm{~mm} ; R 2=152 \mathrm{~mm} ; 3$ $\mathrm{kg}$ charge $)(b)$ Shot $2(R 1=80 \mathrm{~mm} ; R 2=203 \mathrm{~mm} ; 3 \mathrm{~kg}$ charge $)$ and (c) Shot $3(R 1=90 \mathrm{~mm} ; R 2=203$ $\mathrm{mm} ; 4.5 \mathrm{~kg}$ charge). The insets in (c), (f) and (i) show "sand fingers" emanating from the main sand fronts.

and $636 \mu$ s after detonation, indicates the sand traveled significantly further for Shot 3, consistent with an increase of the explosive mass resulting in a higher radial glass particle front expansion rate. The sand finger phenomenon can also be clearly seen in the magnified inset of Figure 4.5(i) 
for Shot 3 as well as occasional fingertip luminescence. The fingering instability present in Shot 2 was not as prominent as that seen in the two other higher glass particle expansion rate test shots.

Analogous observations of the radial expansion of the (water-saturated) zirconia particle fronts can be seen in Figure 4.6. The times after detonation for these images were similar to those in Figure 4.5. Note that Shots 3 and 4 used the same mass of explosive and granular material shell thickness $\left(\mathrm{R}_{2}=203 \mathrm{~mm}\right)$, but Shot 4 used a granular shell filled with higher density zirconia particles. Comparison of the third column of Figure 4.5 with the first of Figure 4.6 shows that increasing the granular shell density (and mass) significantly reduced the shell's radial expansion rate. This delayed the arrival of the granular material at the end of the Kolsky bar, and with the top of the test plate. This lower velocity expansion was also accompanied by a much less prominent fingering instability, Figure 4.6(c). The consequence of increasing the zirconia particle shell thickness (and mass) can be seen by comparing the first and second columns of Figure 4.6. The first impact of granular material with the test plate for Shot 5 occurred slightly before $t=641 \mu \mathrm{s}$ after detonation, Figure 4.6(f). This was more than double the time required for the glass particles in test Shot 3 to arrive at the plate, Figure 4.5(h). In that case, first impact with the test plate occurred $\sim 280 \mu$ s after detonation. The fingering instability was almost absent in Shot 5, Figure 4.6(f) which exhibited the slowest particle expansion velocity of the five tests.

The high-speed video images were used to track the granular material main front position (from which the fingering instability emanated) as it radially expanded after detonation. This sand front position data is plotted in Figure 4.7(a) for the glass particle test shots and in Figure 4.8(a) for the zirconia tests. Numerical differentiation of the position data was used to estimate the radial expansion velocity of the fronts. This data is plotted in Figure 4.7(b) for the glass particle tests and in Figure 4.8(b) for the zirconia particle tests. The velocity errors were calculated using the methodology described in Chapter 3 [54]. In all cases, the radial expansion rates rapidly increased to a maximum value typically attained at approximately 100-200 $\mu$ s after detonation. This was followed by an extended period of gradual particle deceleration. The highest radial expansion velocity for the main sand front was approximately $1210 \mathrm{~m} / \mathrm{s}$ for Shot 1, Figure 4.7(b), while the lowest peak velocity of approximately $490 \mathrm{~m} / \mathrm{s}$ was observed for Shot 5, Figure 4.8(b). The peak radial expansion rates and times for the main particle fronts to reach the end of the Kolsky bar are summarized in Table 4.5 for the five tests. 
Shot 4: $(90,203)$; zirconia
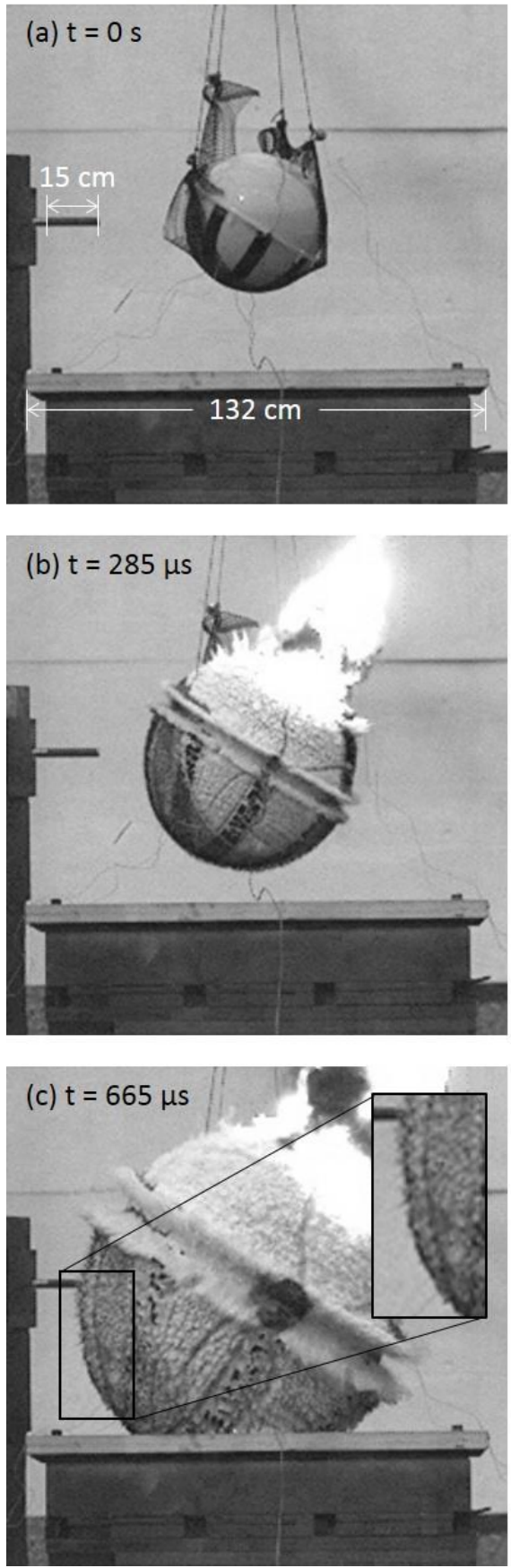

Shot 5: (90, 229); zirconia
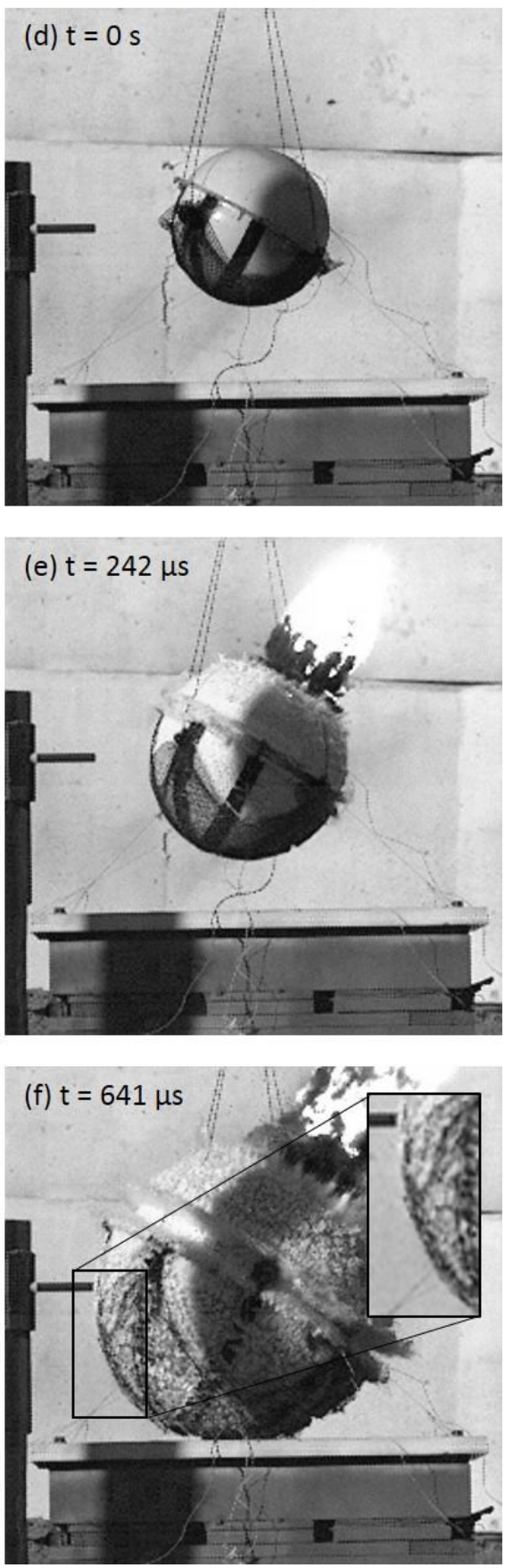

Figure 4.6. High-speed video images from the zirconia particle tests. (a) $R 1=90 \mathrm{~mm} ; R 2=203 \mathrm{~mm} ; 4.5$ $\mathrm{kg}$ charge (b) $R 1=90 \mathrm{~mm} ; R 2=229 \mathrm{~mm} ; 4.5 \mathrm{~kg}$ charge. The insets in $(c)$ and $(f)$ indicate little particle finger formation emanating from the main particle front. 
Table 4.5. The impact times of the main particle front with the Kolsky bar deduced from experimental main particle front position and the peak velocity for each test charge. The calculated air shock pressures and speeds are also listed.

\begin{tabular}{cccccc}
\hline Test Shot & $\begin{array}{c}\text { Impact Time } \\
(\text { Kolsky bar }) \\
(\mu \mathrm{s})\end{array}$ & $\begin{array}{c}\text { Peak velocity } \\
(\text { main front }) \\
(\mathrm{m} / \mathrm{s})\end{array}$ & $\begin{array}{c}\text { Air shock } \\
\text { pressure, } p_{f} \\
(\mathrm{MPa})\end{array}$ & $\begin{array}{c}\text { Air shock } \\
\text { speed, } c_{f} \\
(\mathrm{~m} / \mathrm{s})\end{array}$ & $\begin{array}{c}\text { Simulation } \\
\text { max air speed } \\
(\mathrm{m} / \mathrm{s})\end{array}$ \\
\hline 1 & $275 \pm 20$ & 1210 & 2.3 & 1518 & 1250 \\
2 & $405 \pm 10$ & 780 & 1.1 & 1048 & 770 \\
3 & $300 \pm 19$ & 980 & 1.6 & 1291 & 940 \\
4 & $515 \pm 10$ & 600 & 0.7 & 857 & 745 \\
5 & $580 \pm 8$ & 490 & 0.55 & 756 & 605 \\
\hline
\end{tabular}
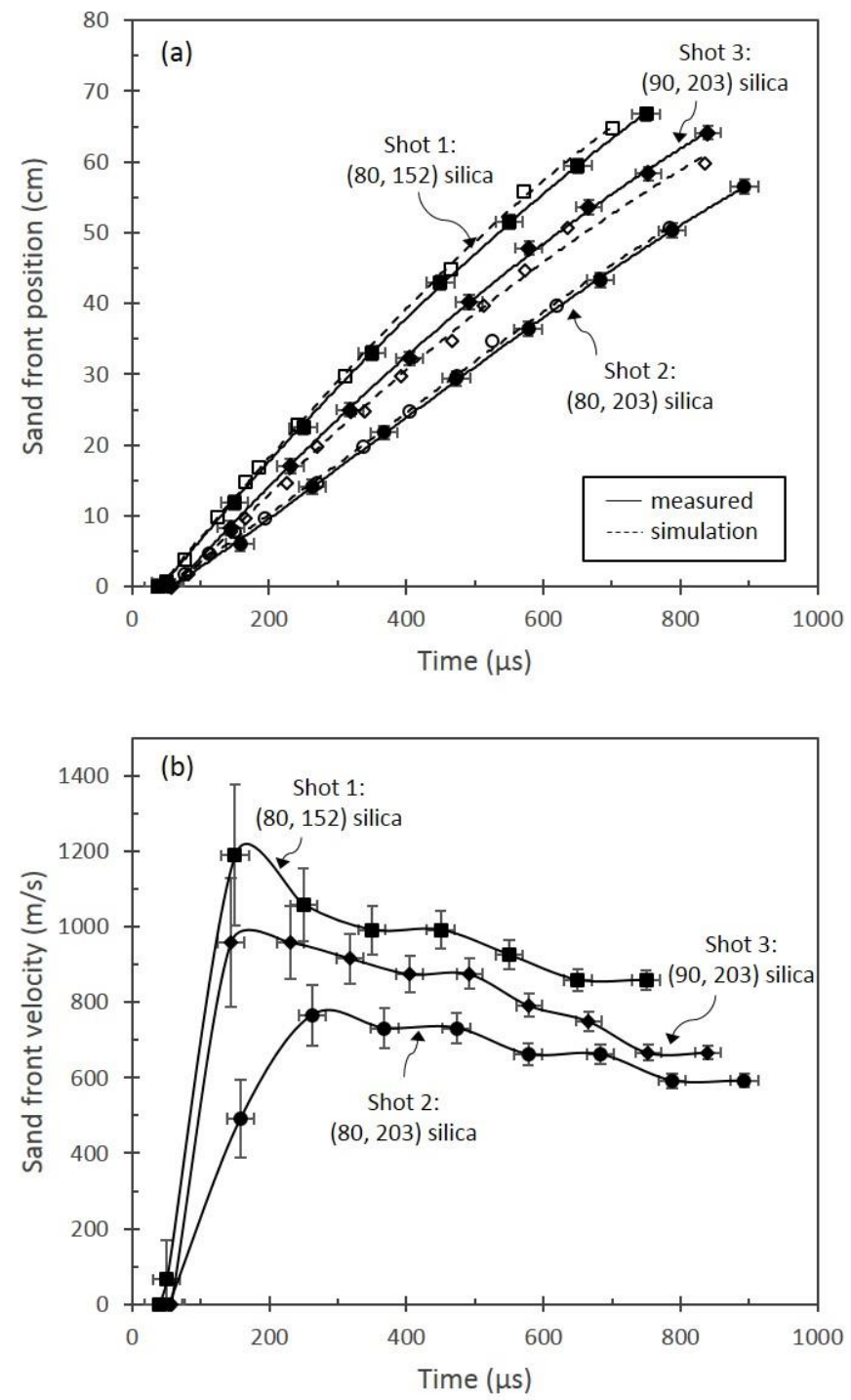

Figure 4.7. (a) Main particle front position and (b) velocity versus time for the glass microsphere test configurations (shots 1-3). In (a) the filled in markers represent the experimental results and the empty markers are from the simulations. 

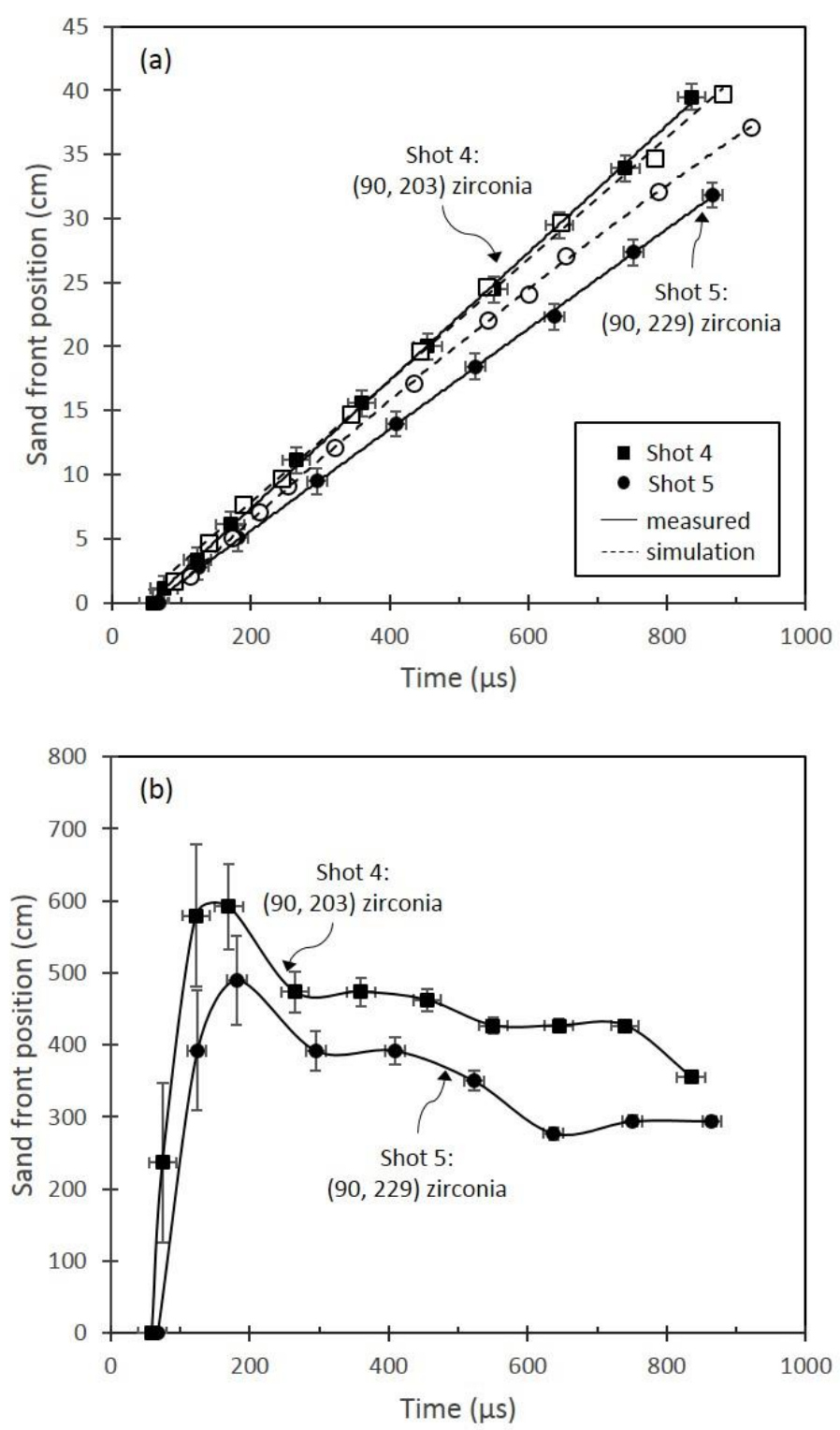

Figure 4.8. (a) Main sand front particle position and (b) velocity versus time for the zirconia test configurations (Shots 4 and 5). In (a) the filled in markers represent the experimental results and the empty markers those from the simulation.

The data in Table 4.1 for Shots 1 and 2 indicate that when the mass of granular material in the annularly filled region was increased while keeping that of the explosive fixed, the maximum particle front velocity decreased from $1210 \mathrm{~m} / \mathrm{s}$ to $780 \mathrm{~m} / \mathrm{s}$. Increasing the explosive mass from 3 $\mathrm{kg}$ (Shot 2) to $4.5 \mathrm{~kg}$ (Shot 3), while keeping the granular material outer radius fixed, resulted in an approximately $200 \mathrm{~m} / \mathrm{s}$ increase in sand front velocity. Switching from glass particles to 
(denser) zirconia particles, while keeping the geometry and explosive mass fixed, significantly reduced the sand front velocity from $980 \mathrm{~m} / \mathrm{s}$ (Shot 3) to $600 \mathrm{~m} / \mathrm{s}$ (Shot 4), and resulted in substantial delay $(\sim 250 \mu \mathrm{s})$ in particle front impact with the Kolsky bar and test plate. Finally, increasing the mass of the zirconia while keeping the explosive mass constant decreased the maximum front speed from $600 \mathrm{~m} / \mathrm{s}$ (Shot 4) to $490 \mathrm{~m} / \mathrm{s}$ (Shot 5) and consequently also increased the time to impact on the Kolsky bar by $90 \mu \mathrm{s}$.

\subsubsection{Particle shell expansion simulations}

The high-speed video observations only enable visualization of particle motions near the exterior surface of the charges during a test event. These observations provided no insight into processes occurring behind the particle fronts, nor quantitative information about the air shocks that developed ahead of the supersonically expanding granular media. Discrete particle simulations of the test shots do not suffer from sand particle or high explosive product obscuration of the event interior. Consequently, they can be used to investigate the transfer of momentum from detonation products to the granular material, the interactions of these particles with background air and solid targets, and for calculation of the impulse transferred during particle impact with the targets.

Activation of detonation on the Northeast surface of the explosive resulted in the propagation of a detonation wave through the explosive core and transmission of a compressive shock through the granular media. Simulations of shock front propagation through the explosive and the watersaturated particles are shown for the five test shots in Figures C1 (Shots 1-3) and C2 (Shots 4 and 5) in Appendix C. The time between activation of detonation and the initiation of particle escape from the charges (by particle spallation at the sand/air interface) depended on charge diameter and varied from 40-67 $\mu$ s, Table 4.3. These times were therefore used to correct the time measured after the trigger event (the breaking of a wire on the exterior of the test charges) so that both the high-speed video and Kolsky bar data used time $t=0 \mathrm{~s}$ to signify the initiation of detonation.

The diametral plane from the simulations of the glass particle tests (Shots 1-3) are shown in Figure 4.9. The simulation times, $\mathrm{t}=0 \mathrm{~s}, 250 \mu \mathrm{s}$, and $650 \mu \mathrm{s}$, correspond to those of the high-speed video images, Figure 4.5. Figure 4.9(a), (d), and (g) show the center cross section of the test plate, support structure, Kolsky bar, and test charge at $\mathrm{t}=0 \mathrm{~s}$ (initiation of detonation). Comparison of Shots 1 and 2 in Figure 4.9 reveals the effect of changing the granular shell thickness (and mass) 

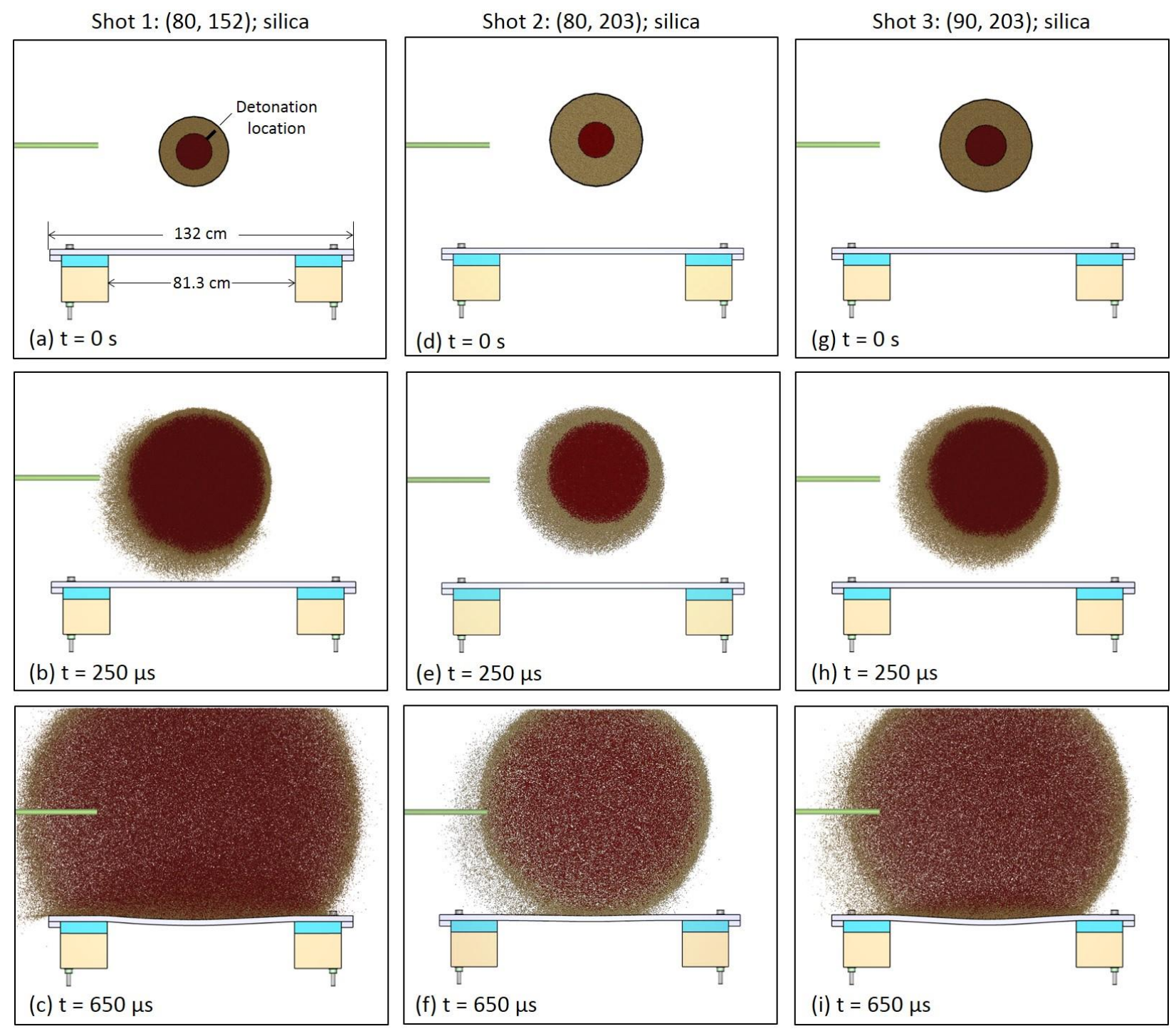

Figure 4.9. Simulation of particle positions for the three glass microsphere tests at times $t=0 \mathrm{~s}, t=250$ $\mu \mathrm{s}$, and $650 \mu \mathrm{s}$. The model is section cut through the center of each test plate.

while comparison of Shots 2 and 3 indicates the effects of changing the radius of the explosive core (or explosive mass). These results show that for Shot 1 particle impact with the Kolsky bar and test plate had begun at about $\mathrm{t}=250 \mu \mathrm{s}$ after detonation. They also show that increasing the thickness of the granular shell (by comparing Shot 1 and Shot 2) slowed the rate of radial particle expansion in the test plate and Kolsky bar directions. This expansion velocity was then increased by increasing the radius/mass of the explosive core (Shot 3). 
The simulations at $\mathrm{t}=650 \mu \mathrm{s}$ after detonation, Figure 4.9(c), (f), and (i) show that for Shot 1 the particles impacting the test plate and the loading of the (darker red) detonation product had progressed further than for the other two shots (Shots 2 and 3), consistent with its higher radial particle velocity. A small rebound of the granular particles occurred where they made perpendicular impact near the test plate center, but this was suppressed by impacts with later arriving particles, resulting in particle accumulation on the test plate. Oblique particle impacts towards the sides of the test plate resulted in the reflection with a horizontal direction component and the formation of a particle layer close to the sample surface that flowed laterally off the edge of the test structure (see lower left of Figure 4.9(c)). Initial deflection of the test plate can also be seen to have occurred by this time.

Figure 4.10 shows analogous results for Shots 4 and 5 that used higher density zirconia particles. Note that the detonator location for Shot 5 was $70^{\circ}$ from horizontal rather than $45^{\circ}$ used in the other tests. Comparison of Figure 4.9(h) and (i) with Figure 4.10(b) and (c) show that increasing the mass of the granular shell by use of higher density particles significantly reduced the radial expansion rate as observed experimentally. Impact of granular particles with the test plate for Shot 5, Figure 4.10(f), occurred slightly before $650 \mu \mathrm{s}$; almost $400 \mu$ s later than that observed for Shot 1, Figure 4.9(b).

The sand front radially expanded outward in a slightly asymmetric manner with the highest particle velocity diametrically opposite the point of detonation (the direction of longest cord length in the explosive), Figures $\mathrm{C} 1$ and $\mathrm{C} 2$. As the sand shell expanded, a velocity and thus particle density gradient developed between the front and back of the expanding granular medium. The leading edge of this expanding particle shell consisted of a low volume fraction of high velocity particles that were spalled from the shell surface during first reflection of the shock at the granular material/air interface. These fast sand particles had a speed consistent with that of the sand fingers seen in the high-speed video images. Comparison of Figure 4.9 and Figure 4.10 indicates that the zirconia test shots had a lower concentration of fast particles at the leading edge of the zirconia particle front consistent with the experimentally observed decrease in sand fingers. The trailing edge of the granular particle shell was composed of a high density of low velocity solid particles that were "pushed" by the expanding detonation product particles. An air shock (not shown in 

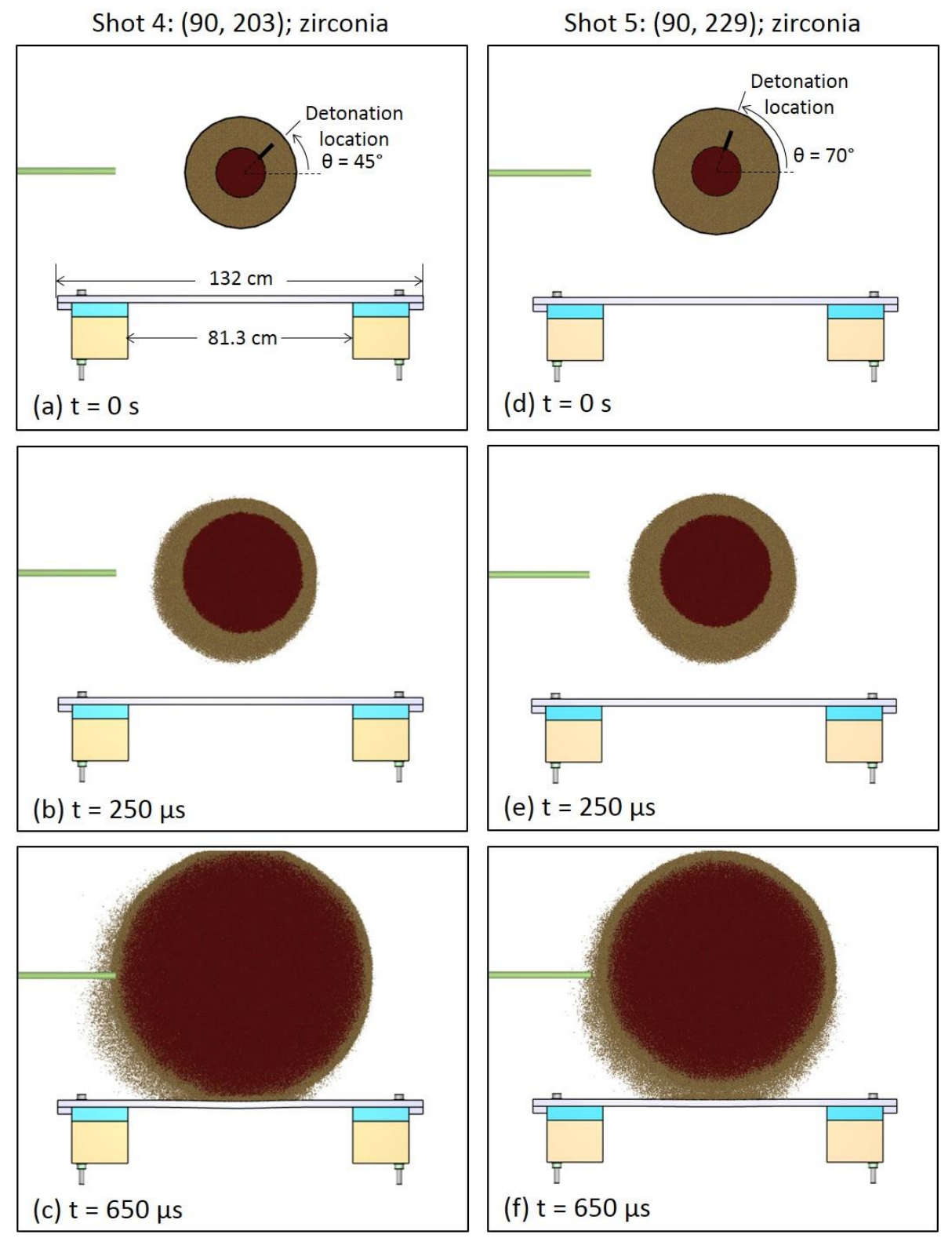

Figure 4.10. Simulation of particle positions for the zirconia particle tests. (a) Shot 4 and (b) Shot 5 at times $t=0 \mathrm{~s}, t=250 \mu \mathrm{s}$, and $650 \mu \mathrm{s}$. The model is section cut along the center of each test plate.

Figure 4.9 and Figure 4.10) formed ahead of the expanding particle front with a maximum speed between $605-1250 \mathrm{~m} / \mathrm{s}$ (Table 4.5) that increased with particle shell (main front) peak velocity.

The region of highest velocity particles was consistent with that occupied by the sand fingers observed experimentally at the leading edge of the sand front. The discrete particle based simulation approach was unable to resolve the evolving surface topology of the instability 
responsible for sand fingering. However, Chapter 3 has applied a result by Kandan et al. [70] to show that the instability originates from the growth of surface perturbations with shapes that suffer the lowest air drag as they penetrate the high air pressure immediately in front of the expanding particles. As the sand front is supersonically pushed outward through the background air with velocity $V_{s}$, an air shock develops ahead of the sand with a shock pressure $p_{f}$ and speed $c_{f}$ [70]. The air shock that developed was observed in the high-speed video for Shot 2 and can be seen in Figure 4.11(a) and (b) as an opaque region propagating outward ahead of the sand front. Figure 4.11(a) shows the air shock wave at $333 \mu$ s just before the shock front impacted the Kolsky bar while Figure 4.11(b) shows it at later time (555 $\mu$ s after detonation). The insets give a magnified view of the air shock and uses a dashed line to show the shock front position. Simulation of Shot 2 at approximately the same times are also shown in Figure 4.11(c) and (d). They show the densified (compressed) shocked region of (blue) air particles that developed ahead of the sand front increased in thickness over time since the air shock velocity exceeded that of the particle front.

The air shock pressures and speeds for each test shot were calculated by the method presented in Kandan et al. [70], and are summarized along with the sand front velocities in Table 4.5. The calculated air shock velocities slightly exceeded those determined from the simulations. It is interesting to note that as the air pressure ahead of the expanding particles decreased (from Shot 1 to Shot 5), the presence of the finger-like instability declined (see the insets of Figure 4.5(c) and Figure 4.6(f)).

The simulated sand front positions are plotted in Figure 4.7(a) and Figure 4.8(a) and found to be in good agreement with the experimentally observed sand front positions. The prolonged period of granular particle deceleration only occurred when background air particles were present in the simulations, consistent with momentum transfer from particles of the granular material to the background air. The peak in granular particle velocity then resulted from the initial transfer of momentum to the solid particles during shock reflection at the particle/air interface of the test charges, followed by solid/air particle collisions which reduced the momentum (and speed) of the solid particles during their propagation towards the target. 

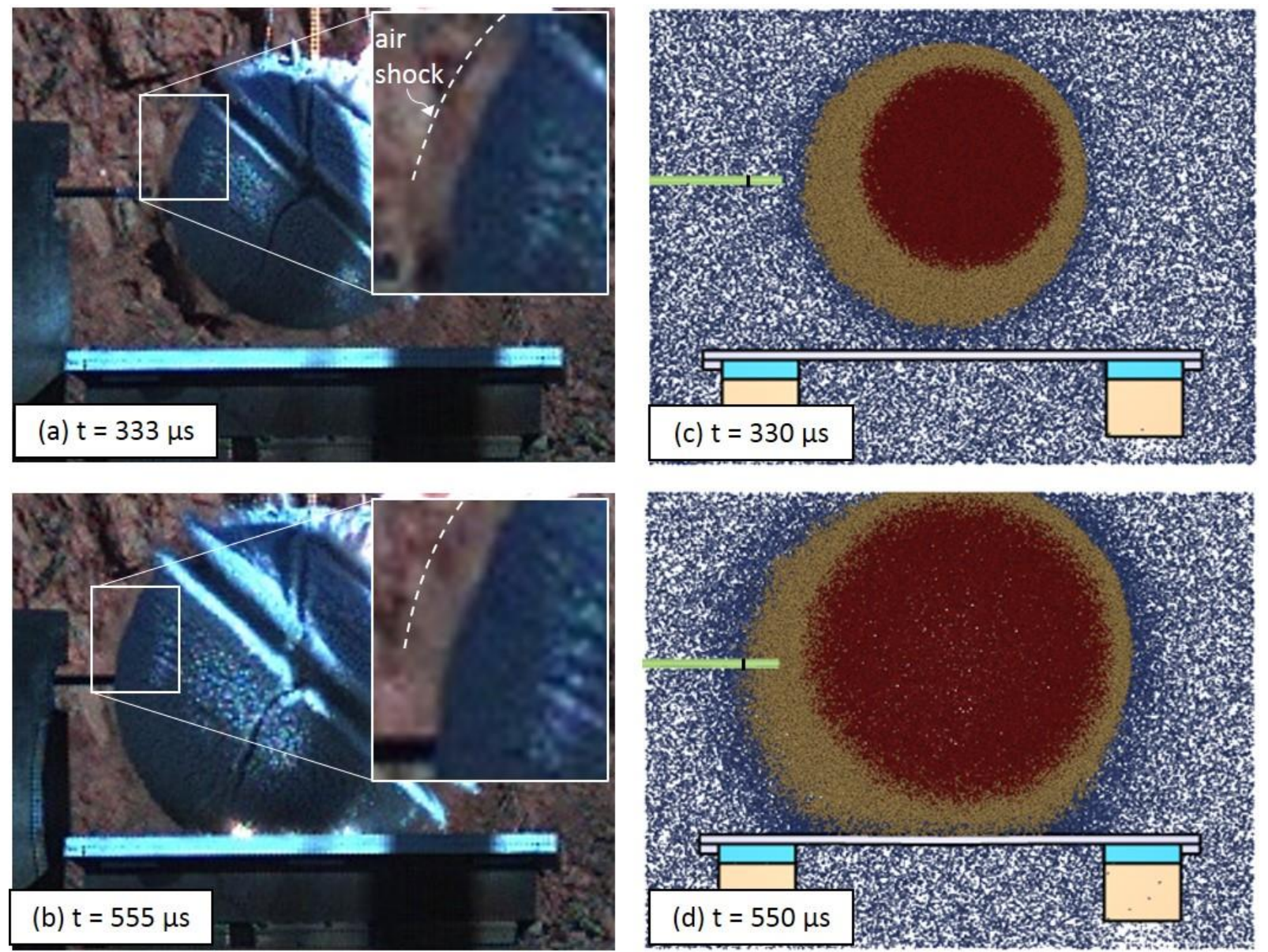

Figure 4.11. High-speed video images showing the air shock that developed in front of the expanding sand shell for Shot $2(a) t=333 \mu$ s and $(b) t=555 \mu$ s after detonation. The insets use a dashed white line to indicate the position of expanding air shock ahead of the particles. Simulation images are shown at approximately equivalent times in (c) at $t=330 \mu \mathrm{s}$ and in $(d)$ at $t=550 \mu \mathrm{s}$ after detonation. The blue dots are air particles, and the region of concentration ahead of the expanding particle front corresponds to the shock. The black line on the Kolsky bar in $(c)$ and $(d)$ indicates the $10 \mathrm{~cm}$ length painted region of the Kolsky bar in the high-speed video image in (a).

\subsubsection{Kolsky bar responses}

The applied pressure (axial stress) determined from the Kolsky bar strain gage measurements are shown in Figure 4.12 for the glass particle tests (Shots 1-3) and in Figure 4.13 for the zirconia particle tests (Shots 4 and 5). Recall that the time for an extensional wave to travel from the impacted end of the Kolsky bar to the strain gauges (where signals were detected) was $125 \mu \mathrm{s}$. Events at the end of the bar observed in the high-speed video images and the simulations therefore correspond to features that appear in the Kolsky bar signals $125 \mu$ s later in time. 

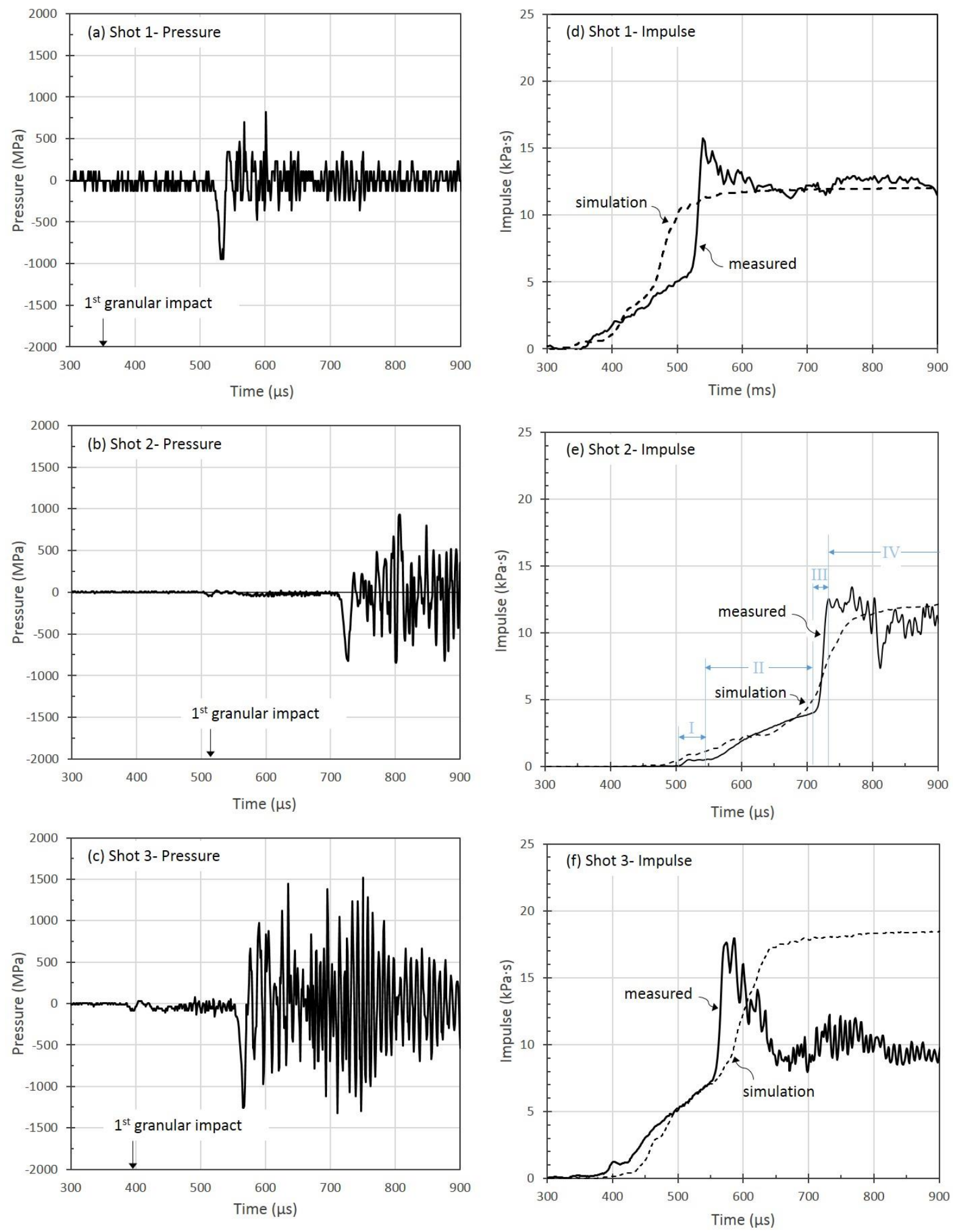

Figure 4.12. Kolsky bar data for the three glass microsphere tests. The waveforms in (a), (b) and (c) show the pressure measured at the strain gage location for Shots 1, 2 and 3. Figures $(d),(e)$ and $(f)$ show the impulse for test Shots 1, 2 and 3. 

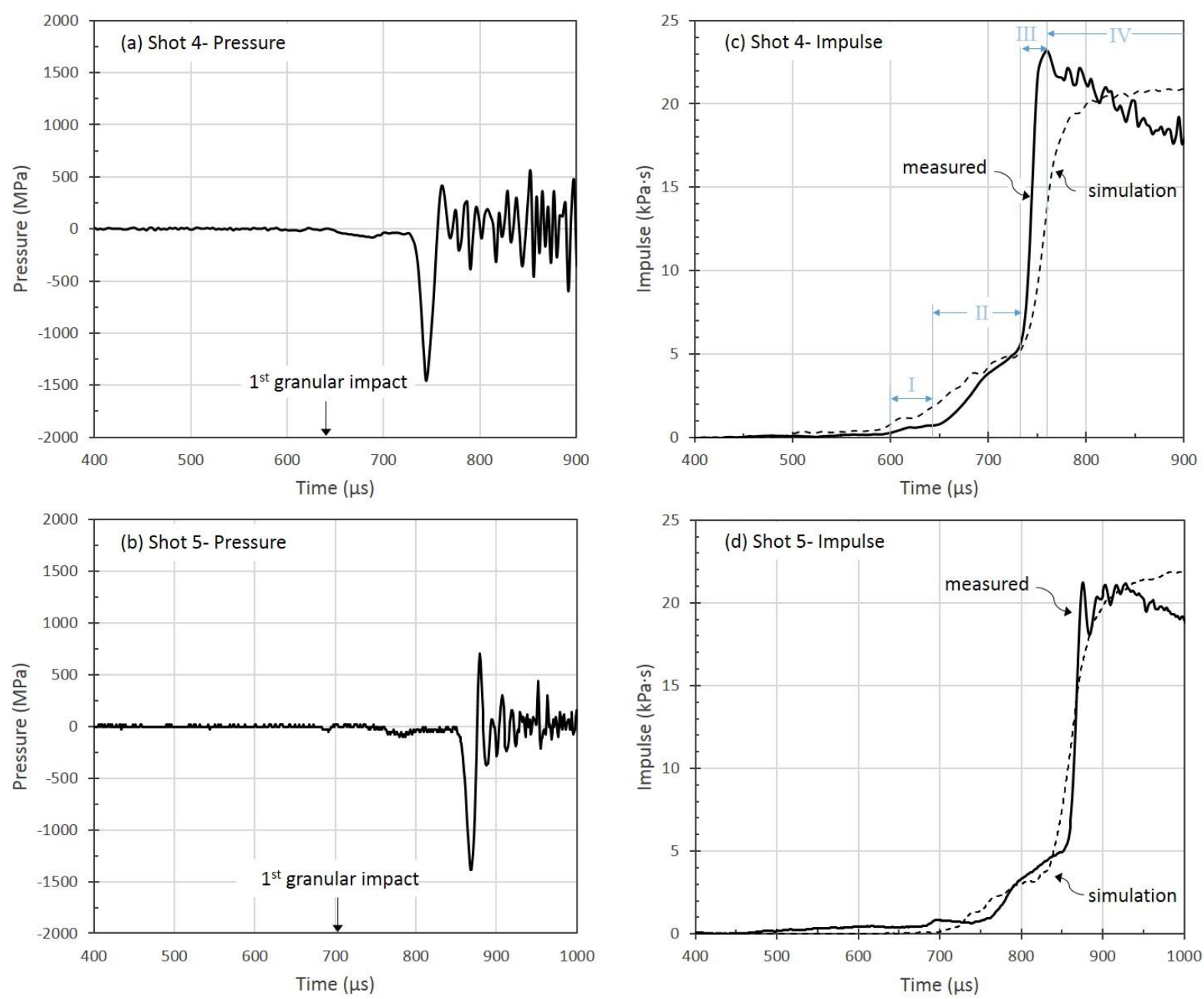

Figure 4.13. Kolsky bar data for the two zirconia tests (Shots 4 and 5). The waveforms in (a) and (b) show the pressure versus time response measured at the stain gage location for Shots 4 and 5. The corresponding impulses are given in $(c)$ and $(d)$.

Examination of the experimentally measured pressure-time responses in Figure 4.12 and Figure 4.13 reveals the presence of an initial, small pressure pulse. This signal was not clearly observable in the noisy pressure data of Shot 1, Figure 4.12(a), but the integrated response shown in Figure 4.12(d) began to rise at about $\sim 350 \mu$ s after detonation consistent with a weak pressure signal in the noisy data. For Shot 2, Figure 4.12(b), this first pulse was observed at $\sim 515 \mu$ s and at $\sim 395 \mu \mathrm{s}$ after detonation in Shot 3, Figure 4.12(c). These three signals correspond to the application of a load at the end of the Kolsky bar at $\sim 225,390$, and $270 \mu$ s after detonation of test Shots 1, 2, and 3 respectively. Recall that Figure 4.5(b) and (h) showed high-speed video images 
from Shots 1 and 3 at 250 and $288 \mu$ s after detonation respectively. The small increases in pressure evident in the pressure versus time waveforms in Figure 4.12 correspond to the arrival of the air shock and fastest sand fingers slightly ahead of the main sand front whose arrival (impact) times are summarized in Table 4.5 .

Figure 4.13 shows the measured Kolsky bar pressure waveforms for the Shots 4 and 5 which used zirconia particles. The first small rise in pressure occurred substantially later for the zirconia test shots, Figure 4.13(a)-(b). For Shot 4, this first rise in pressure occurred at $\sim 620 \mu \mathrm{s}$ corresponding to loading of the end of the Kolsky at about $495 \mu$ s after detonation. The first pressure rise for Shot 5 was observed at $\sim 695 \mu$ s after detonation corresponding to the application of pressure at the end of the Kolsky at about $570 \mu$ s after detonation. Careful analysis of the highspeed video results indicated first granular impact with the end of the Kolsky bar by the main particle front (since there was very little particle fingering) occurred at $515 \pm 10 \mu$ s for Shot 4 and at about $580 \pm 8 \mu$ s for Shot 5 , Table 4.5. When the signal travel time in the Kolsky bar (125 $\mu$ s) is added to these first contact times, Figure 4.13 (c) and (d) show they correspond to the beginning of the rise in impulse.

The initial rise in pressure was followed by a 100-200 $\mu$ s period of weakly oscillating waveform until a sharp, much larger, pressure pulse was observed in all the tests, Figure 4.12 and Figure 4.13. The large pressure pulses were followed by stronger oscillations resulting from the arrival of slower propagating Pochhammer-Chree bar modes [81, 92, 93]. The peak (compressive) pressure transmitted by the glass particle Shot 1 (Figure 4.12(a)) was $950 \mathrm{MPa}$. This was slightly higher than the $825 \mathrm{MPa}$ applied by Shot 2 (Figure 4.12(b)) which used the same mass of explosive but whose water-saturated particle shell mass had been increased from 24 to $\sim 65 \mathrm{~kg}$, Table 4.1 . However, prior to detonation of Shot 1, the center of the charge suffered a $3.37 \mathrm{~cm}$ displacement towards the Kolsky bar, reducing the standoff distance in this direction, Table 4.2, and therefore increasing the speed and density of the granular media that contacted the end of the bar. Simulations where the standoff distance from the center of the test charge to the front of the Kolsky bar, $\mathrm{H}_{\mathrm{k}}=45 \mathrm{~cm}$, revealed that the pressure was increased slightly from Shot 1 to Shot 2 (by about $3 \%)$.

Examination of Figure 4.12(b) and (c) indicates that as the diameter (and mass) of the explosive core was increased while the mass of the granular shell remained fixed, the peak pressure 
for Shot 3 increased from $825 \mathrm{MPa}$ to $1250 \mathrm{MPa}$. The effect of changing the density of the granular particles while maintaining all other charge variables fixed can be seen by comparing the peak pressure of $\sim 1450 \mathrm{MPa}$ for Shot 4, Figure 4.13(a), with that of Shot 3 ( 1250 MPa, Figure 4.13(c)). Examination of Figure 4.13(b) shows that the peak pressure decreased slightly to $\sim 1400 \mathrm{MPa}$ for Shot 5. When the thickness (and therefore mass) of the zirconia particle shell was increased while keeping the explosive mass fixed, the peak pressure was slightly reduced. However, the detonator inclination for Shot 5 was $\theta=70^{\circ}$ (compared to $\theta=45^{\circ}$ for Shots 1-4, Figure 4.4) which slightly reduced the pressure in the Kolsky bar direction. A simulation with the corrected inclination angle for the detonator at $\theta=45^{\circ}$ revealed that the pressure applied to the Kolsky bar was greater (by approximately $4 \%$ ) than when the detonator was inclined at $\theta=70^{\circ}$.

Integration of the pressure signal gave the measured impulse-time response at the Kolsky bar sensor location. This is shown in Figure 4.12(d-f) for the glass particle shots and in Figure 4.13(c) and (d) for the zirconia shots. The five impulse-time responses all exhibited four characteristic regimes. They all showed an initial small jump in impulse at the time when the first small pressure pulse was observed (Region I). Examination of Figure 4.5 for the glass particle tests shows that this occurred as the fastest particles (sand fingers) emitted by the test charges began to make contact with the end of the bar. No fingers were present in the more slowly expanding zirconia particle tests, and in their case, the first impulse was associated with the main particle front contact. This region was followed by a period of slowly rising impulse (Region II) corresponding to the weakly oscillatory period between the initial pressure pulse and the strong pressure peak. The highspeed video and simulation observations indicate the start of Region II coincided with the arrival at the Kolsky bar location, of the main sand front (particles immediately behind the sand fingers), Table 4.5. This region was characterized by a gradual rise in the particle density. Region III corresponded to the sudden jump in impulse associated with the peak in pressure. Since the granular media had engulfed the end the Kolsky bar at the time of these peak pressures, the physical processes responsible for these jumps in pressure could not be deduced from the high-speed video observations. Examination of the simulations, Figure 4.9 and Figure 4.10, shows that this coincided with the arrival of dense, but lower velocity sand that had been pushed to the end of the Kolsky bar by the expanding detonation product gas particles. This was then followed by an oscillatory response (a result of various bar mode elastic arrivals) whose average impulse 
Table 4.6. The experimentally measured and simulated impulse applied to the Kolsky bar and the permanent plate displacement of each test plate. The simulated (maximum) impulse applied to the test plate and maximum transient displacement are also listed.

\begin{tabular}{ccccccc}
\hline $\begin{array}{c}\text { Test } \\
\text { shot }\end{array}$ & $\begin{array}{c}\text { Experimental } \\
\text { Kolsky bar } \\
\text { impulse } \\
(\mathrm{kPa} \cdot \mathrm{s})\end{array}$ & $\begin{array}{c}\text { Simulated } \\
\text { Kolsky bar } \\
\text { impulse } \\
(\mathrm{kPa} \cdot \mathrm{s})\end{array}$ & $\begin{array}{c}\text { Simulated } \\
\text { predicted } \\
\text { plate impulse } \\
(\mathrm{kPa} \cdot \mathrm{s})\end{array}$ & $\begin{array}{c}\text { Experimental } \\
\mathrm{Z}- \\
\text { displacement } \\
\delta(\mathrm{cm})\end{array}$ & $\begin{array}{c}\text { Simulated } \\
\text { Z- } \\
\text { displacement } \\
\delta(\mathrm{cm})\end{array}$ & $\begin{array}{c}\text { Simulated } \\
\text { max Z- } \\
\text { displacement } \\
\delta_{\max }(\mathrm{cm})\end{array}$ \\
\hline 1 & 12.1 & 11.6 & 15.3 & 4.77 & 4.95 & 5.78 \\
2 & 12.5 & 12.2 & 16.5 & 5.02 & 5.68 & 6.44 \\
3 & 17.0 & 17.9 & 22.4 & 7.21 & 7.66 & 8.12 \\
4 & 21.5 & 20.7 & 24.2 & 7.84 & 7.92 & 8.64 \\
5 & 21.3 & 22.3 & 25.4 & 8.16 & 8.39 & 8.88 \\
\hline
\end{tabular}

Table 4.7. The measured and simulated start times (in microseconds) for each region of the sand loading on the Kolsky bar at the signal arrival time at the strain gauge location (corresponding to impacts at the front of the bar $125 \mu$ s earlier).

\begin{tabular}{|c|c|c|c|c|c|c|c|c|}
\hline \multirow[b]{2}{*}{ Test shot } & \multicolumn{2}{|c|}{ Region I } & \multicolumn{2}{|c|}{ Region II } & \multicolumn{2}{|c|}{ Region III } & \multicolumn{2}{|c|}{ Region IV } \\
\hline & $\begin{array}{l}\text { Exp. } \\
(\mu s)\end{array}$ & $\begin{array}{l}\text { Sim. } \\
(\mu \mathrm{s})\end{array}$ & $\begin{array}{l}\text { Exp. } \\
(\mu s)\end{array}$ & $\begin{array}{l}\text { Sim. } \\
(\mu \mathrm{s})\end{array}$ & $\begin{array}{l}\text { Exp. } \\
(\mu s)\end{array}$ & $\begin{array}{c}\text { Sim. } \\
(\mu \mathrm{s})\end{array}$ & $\begin{array}{l}\text { Exp. } \\
(\mu s)\end{array}$ & $\begin{array}{l}\text { Sim. } \\
(\mu \mathrm{s})\end{array}$ \\
\hline 1 & 350 & 340 & 390 & 395 & 520 & 470 & 545 & 525 \\
\hline 2 & 505 & 500 & 540 & 530 & 710 & 700 & 730 & 760 \\
\hline 3 & 385 & 395 & 420 & 435 & 555 & 565 & 570 & 640 \\
\hline 4 & 600 & 590 & 645 & 630 & 735 & 730 & 760 & 800 \\
\hline 5 & 685 & 700 & 745 & 750 & 855 & 830 & 875 & 920 \\
\hline
\end{tabular}

fluctuated around a plateau value (Region IV) which is summarized for each test shot in Table 4.6. This plateau value was reached as the last of the solid (sand) particles and the leading edge detonation products made impact with the bar. The arrival of the remaining detonation products contributed very little additional impulse.

The simulated impulse versus time response at the Kolsky bar strain gauge location is overlaid on the experimental data in Figure 4.12(d)-(f) and Figure 4.13(c) and (d). The four regimes identified in the experimental data were clearly present in the simulated data. Table 4.7 shows the measured and simulated start times for each regime. The simulations were generally in agreement with the measurements. The simulations can therefore be used to investigate the interactions of the later arriving sand particles with the Kolsky bar that cannot be seen in the high-speed videos.

The Kolsky bar data shows that the range of applied impulses varied from $12.1 \mathrm{kPa} \cdot \mathrm{s}$ (Shot 1) to $21.5 \mathrm{kPa} \cdot \mathrm{s}$ (Shot 4). In general, the data from Shots 1 through 4 indicate the impulse transmitted 
to the Kolsky bar, and thus (approximately) to the center of the test plates, increased with thickness of the annular region of particles, with explosive mass, and with the density of the granular medium. The measured plateau impulse for Shot 5 was slightly less than that of Shot 4 . However, when the orientation angle of detonation for Shot 5 was decreased from $\theta=70^{\circ}$ to $45^{\circ}$ (as used for the other tests), the impulse to the Kolsky bar was increased by approximately $2 \mathrm{kPa} \cdot \mathrm{s}$. We therefore conclude that an increase in zirconia particle mass, while keeping the explosive core mass fixed, increased the impulse applied to the Kolsky bar. The simulations indicate that the impulse applied to the Kolsky bar was applied by the three particles types (air, HE, and solid glass or zirconia particles), but the majority ( $90 \%)$ of the impulse loading on the Kolsky bar and the test plate originated from impacts by the solid (sand) particles.

\subsubsection{Panel deformations}

The out of plane (Z-component) displacements of the deformed concave surfaces of the five test plates were measured after testing using a coordinate measuring machine to profile the deformed surface profilometry of the plates in the $\mathrm{X}-\mathrm{X}$ and $\mathrm{Y}-\mathrm{Y}$ directions. Contour plots of the permanent Z-direction displacements of the test plates are shown for the glass particle tests in Figure 4.14 (a)-(c) and for the zirconia test shots in Figure 4.15(a)-(b). The white regions around the border of the contour plots suffered no displacement and surface profile data was therefore not collected for these regions. The simulated displacement contour plots are also shown for comparison in Figure 4.14 (d)-(f) and Figure 4.15(c)-(d). These contour plots show the displacement after $20 \mathrm{~ms}$ when the oscillatory plate movement has decayed to an approximate

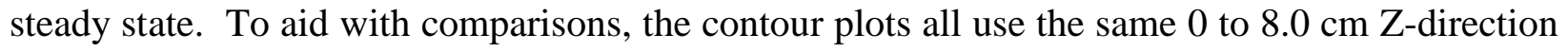
(out of plane) displacement scale. It is evident that the measured and simulated displacement fields are in generally good agreement and that the maximum permanent displacement increased from Shot 1-5 (i.e. with incident impulse). The biggest discrepancy between the measured and simulated displacements was observed for Shots 2 and 3 where the simulations slightly over predict the center displacement. The results also reveal that the permanent displacement extended beyond the 81.3 $\mathrm{cm}$ wide central region between the picture frame supports on which the plates were supported. This can be seen more clearly by plotting the measured permanent plate displacement profile along the $\mathrm{X}$-axis (at the Y-coordinate of maximum $\mathrm{Z}$ displacement) in Figure 4.16(a) for each of the test charges. Figure 4.16(b) compares them with the corresponding simulated displacements. Both 

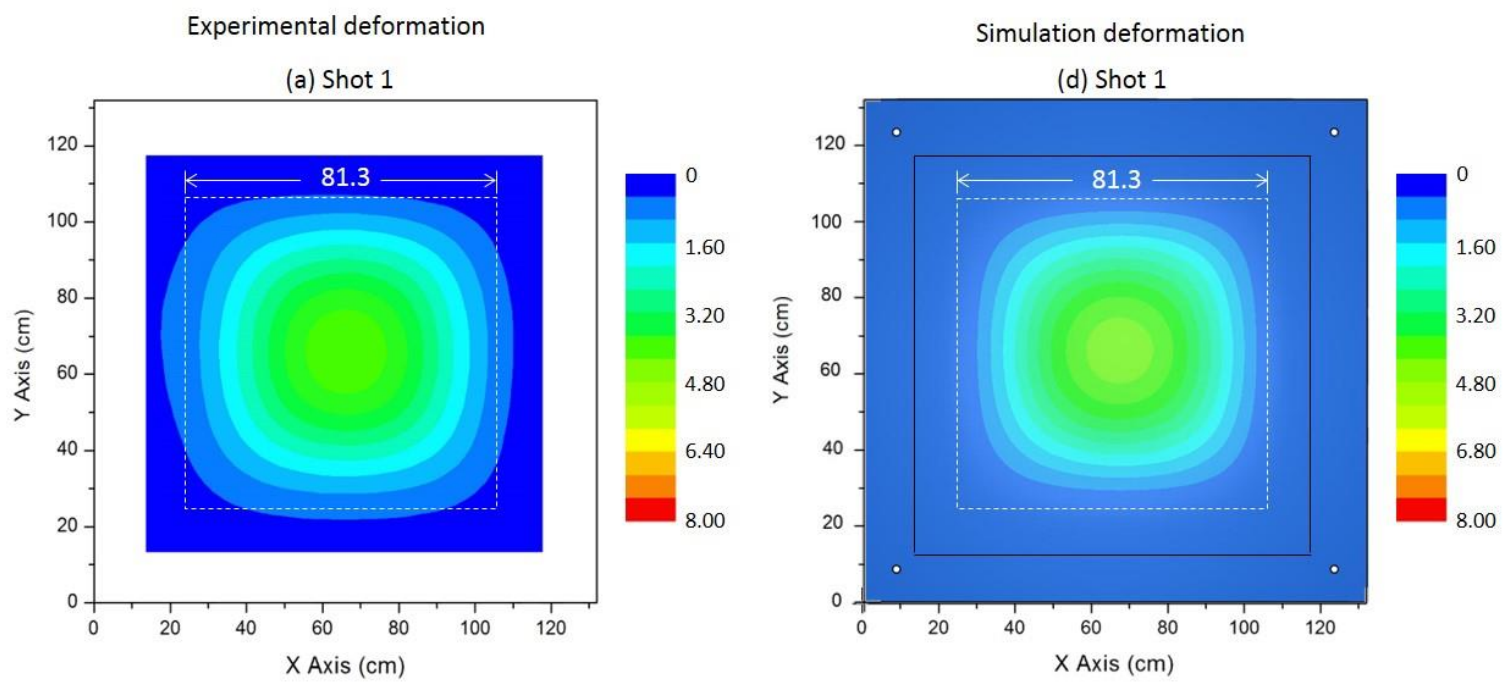

(b) Shot 2
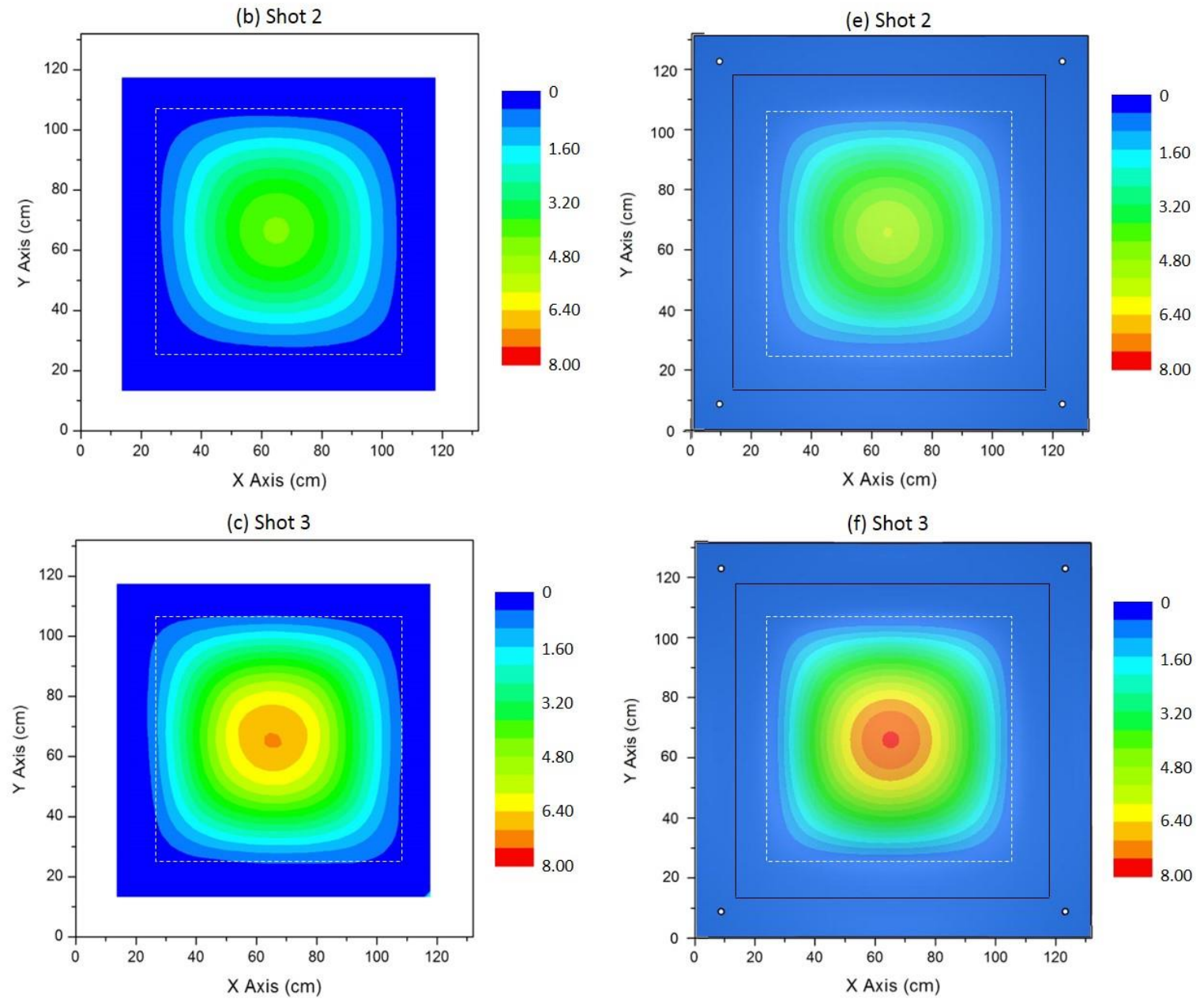

Figure 4.14. Test plate out of plane displacement contour plots for the glass particle tests (Shots 1, 2 and 3). Experimental profilometry results are shown in (a), (b) and (c). The simulated responses at $t=20 \mathrm{~ms}$ after plate oscillations had decayed to an approximate steady state displacement are shown in $(d),(e)$ and (f). The white dashed lines indicate the location of the underlying support structure. Within this region, the panels were not back supported. 
Experimental deformation

(a) Shot 4

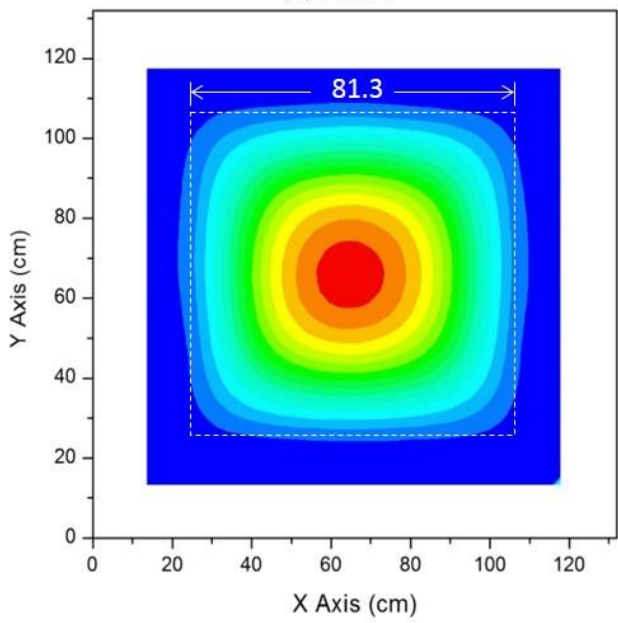

(b) Shot 5

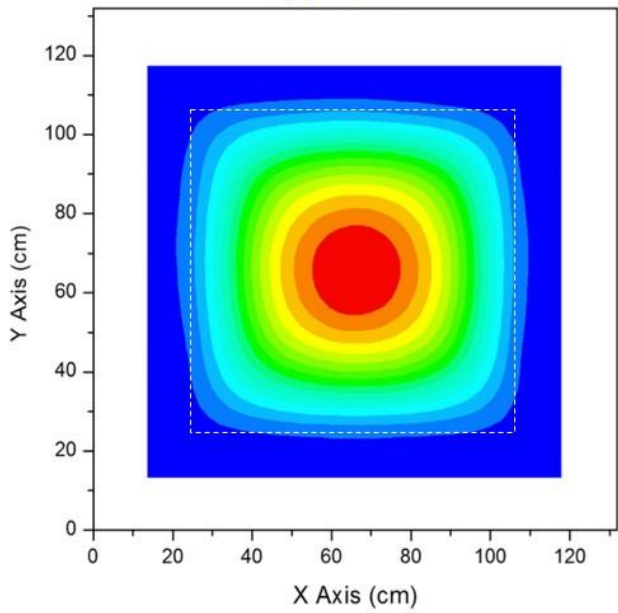

Simulation deformation

(c) Shot 4

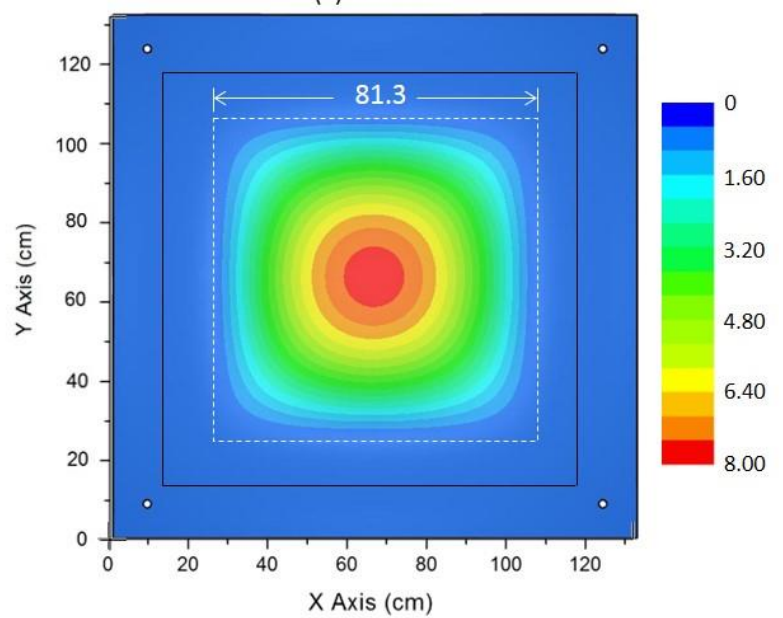

(d) Shot 5

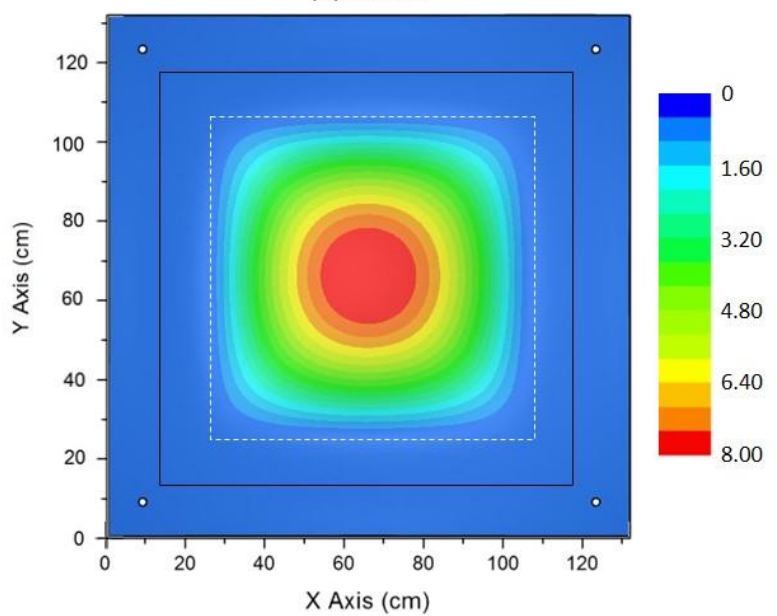

Figure 4.15. Out of plane displacement contour plots for the zirconia tests (Shots 4 and 5). Experimental results are shown in (a) and (b) while the simulated responses after the plate oscillations had decayed to an approximate steady state $(t=20 \mathrm{~ms})$ are shown in $(c)$ and $(d)$. The white dashed lines indicate the outline of the underlying support structure. 

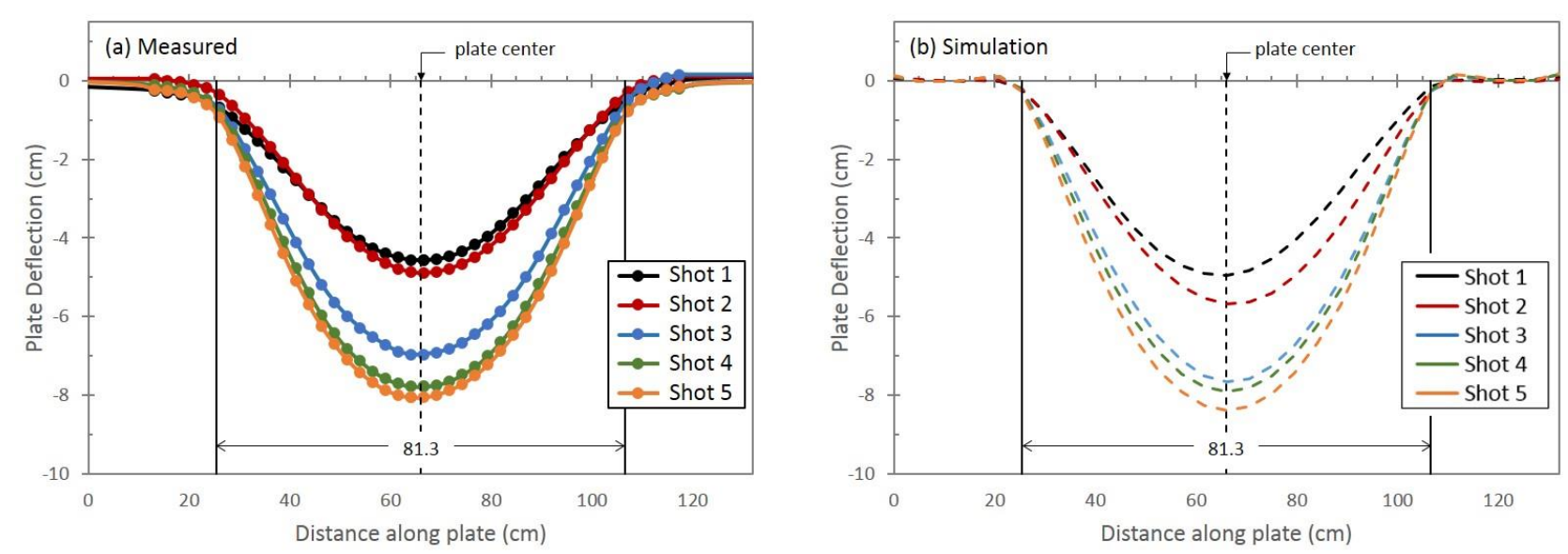

Figure 4.16. Mid-section out of plane deflection profiles for the five test panels. (a) Measured and (b) simulated results.

sets of data confirm that the Z-direction displacement extended outwards beyond the edge of the underlying support structure by a distance that increased with impulse. The maximum permanent displacements of the test plates, $\delta$, are summarized in Table 4.6 for both experimental and simulation results. The maximum permanent displacement increased from Shot 1 to 5 (with increasing impulse) and exceeded the $2.54 \mathrm{~cm}$ plate thickness for all tests.

While the experimental plate displacement results recorded only the permanent deflection after the test event, the simulations allow analysis of the transient test plate behavior including their damped oscillatory displacement which eventually converged to the permanent displacement. This transient Z-displacement behavior is plotted for each test in Figure 4.17 for the first $20 \mathrm{~ms}$ following detonation. The maximum dynamic displacement, $\delta_{\max }$, occurred at approximately $2 \mathrm{~ms}$ after detonation, but exceeded the permanent displacement by less than $1 \mathrm{~cm}$ (Table 4.6). The simulations also enable the specific impulse distribution applied to the test panels to be determined. Figure 4.18 shows the specific impulse distribution on half sections of the test plates for the five tests arranged by increasing impulse (Shot 1 to Shot 5). The peak specific impulses applied to the test plates for each test shot are also summarized in Table 4.6.

In order for the test plates to undergo the permanent Z-direction displacement shown in Figure 4.18 , they must also suffer an in-plane plastic stretching strain. Since the underlying $81.3 \mathrm{~cm}$ span support structure did not inhibit this stretching, the region that contributed to in-plane stretching 


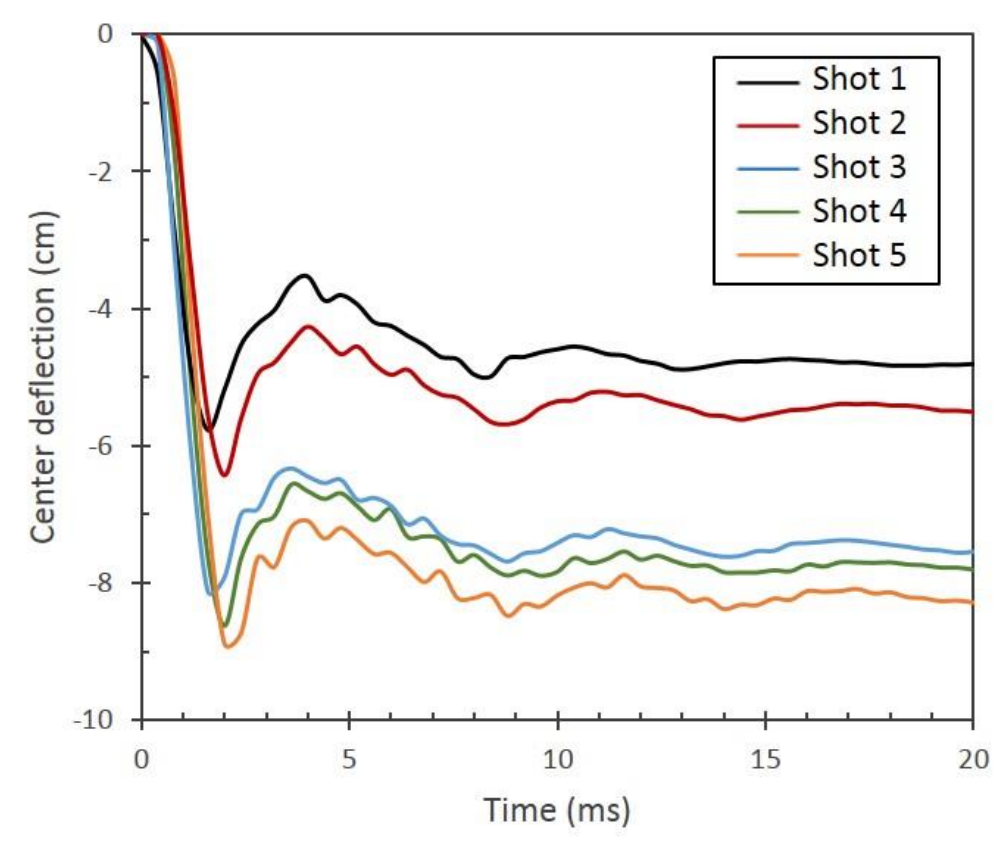

Figure 4.17. Simulated transient out of plane deflection at the center of the test plates impacted by each test charge.

extended to the edge of the test plates where strong in-plane displacement control was imposed. Figure 4.19(a) shows an example of the final total strain in the Y-direction, $\varepsilon_{Y Y}$, on the plate at 20 ms after detonation for test Shot 3. This in-plane strain reached a maximum directly above the inner edge of the underlying picture frame support. Three nodes are indicated in Figure 4.19(a) where the values of the maximum and permanent (after $20 \mathrm{~ms}$ ) displacement magnitude were measured. This data is summarized in Table 4.8 for each test shot along with the final total strain in the Y-direction, $\varepsilon_{Y Y}$, at these node locations. The $\varepsilon_{Y Y}$ strain at these three nodes and their permanent displacements are seen to increase from Shot 1 to 5 . The displacements of the nodes indicate the contribution of the edge material stretching to the final permanent shape (displacement profile) of the test plate. The permanent Y-direction displacement of node 3 is also listed in Table 4.8, which indicates the out of plane displacement near the plate centers was fed by X and Ydirection displacements of plate material from outside the central $81.3 \mathrm{~cm}$ plate span. Figure 4.19(b) shows the effective stress distribution for the same test (Shot 3) after $1 \mathrm{~ms}$ and indicates that the region which exceeded the yield stress of the plate extended through a significant fraction of the width of the back supported (picture frame shaped) periphery of the test plate. 
(a) Shot 1: $(80,152)$; silica

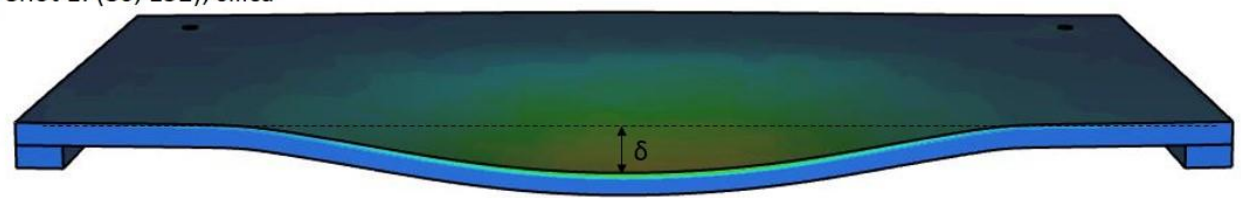

(b) Shot 2: $(80,203)$; silica

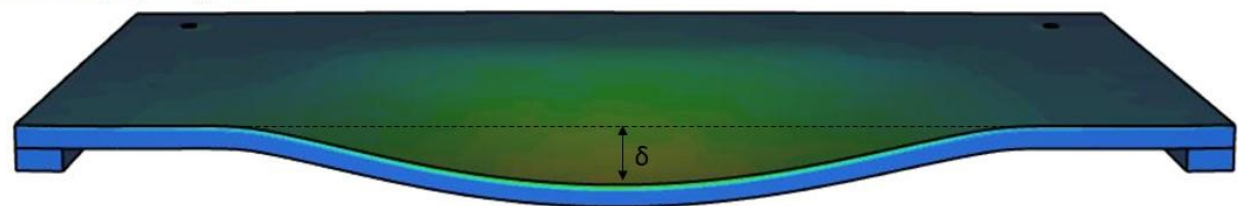

Blast specific impulse (kPa·s)

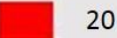

20

16

(c) Shot 3: $(90,203)$; silica

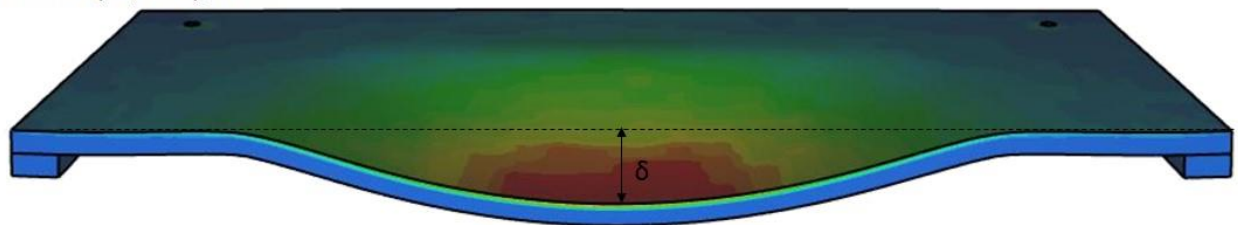

(d) Shot 4: $(90,203)$; zirconia

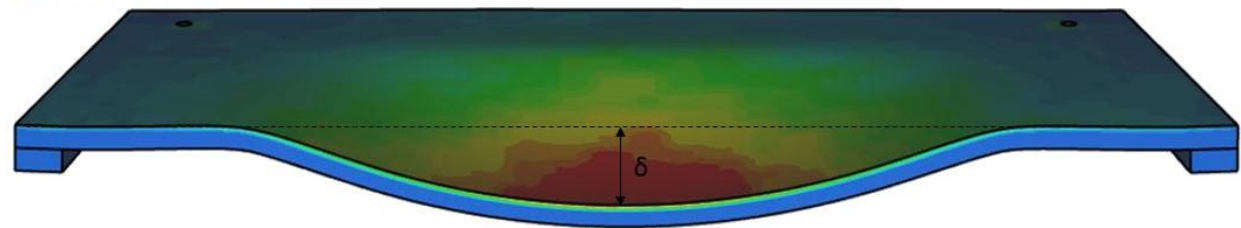

(e) Shot 5: (90, 229); zirconia

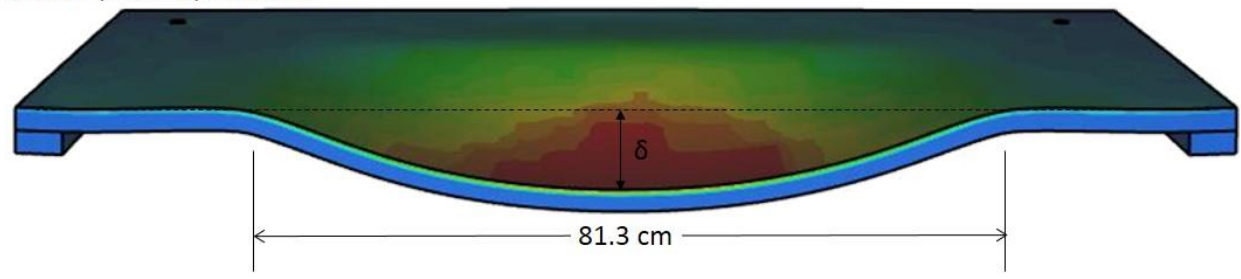

Figure 4.18. The simulated specific impulse distribution applied to the test plates for the five test charge configurations. The maximum deflection $(\delta)$ is also shown for each test and the $81.3 \mathrm{~cm}$ wide region where no back support of the panels was provided. 
(a)

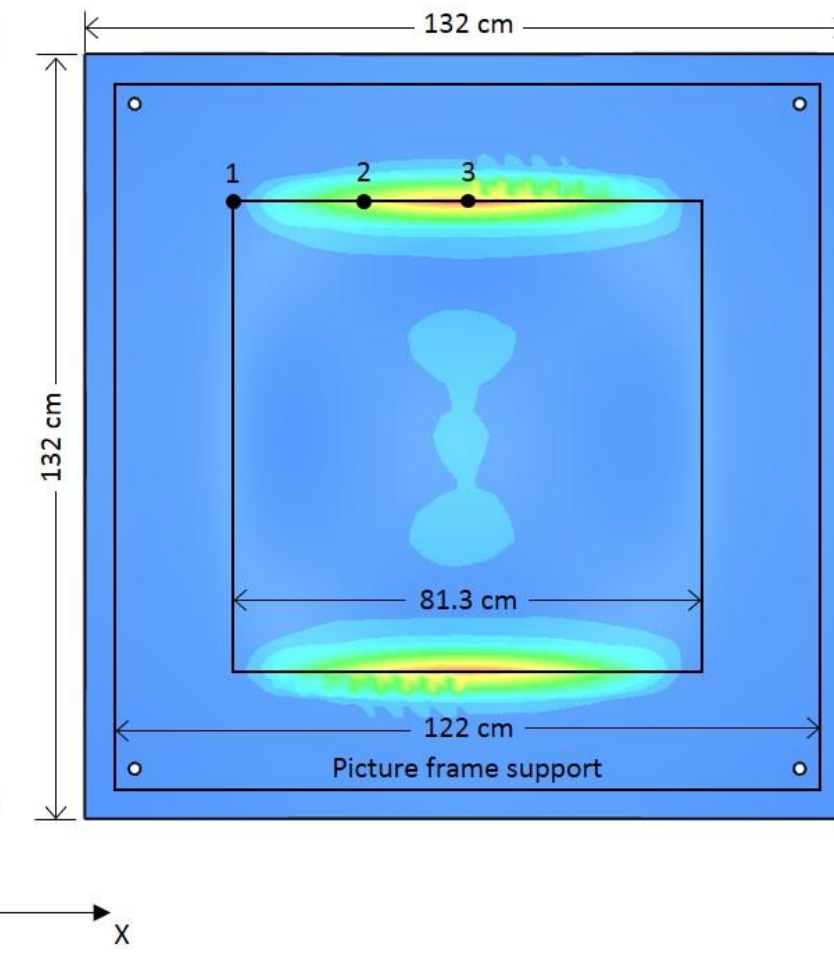

(b)

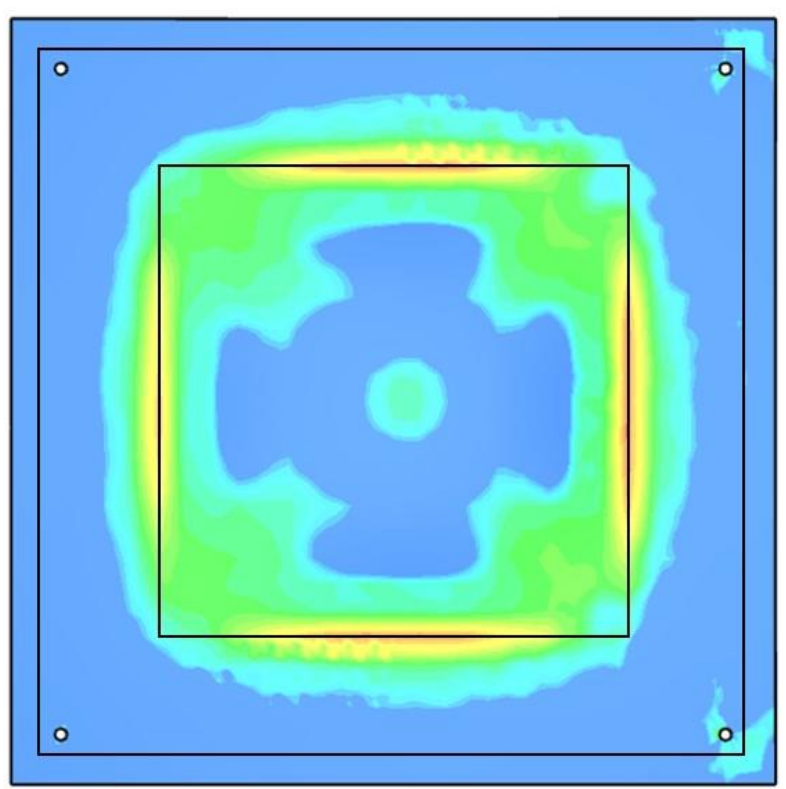

\section{Strain $Y$}

9.000e-02

$8.400 \mathrm{e}-02$

$7.800 \mathrm{e}-02$

$7.200 \mathrm{e}-02$

$6.600 \mathrm{e}-02$

$6.000 \mathrm{e}-02$

$5.400 \mathrm{e}-02$

4.800e- 02

4. $200 \mathrm{e}-02$

$3.600 \mathrm{e}-02$

$3.000 \mathrm{e}-02$

2. $400 \mathrm{e}-02$

$1.800 \mathrm{e}-02$

$1.200 \mathrm{e}-02$

$6.000 \mathrm{e}-03$

$0.000 e+00$

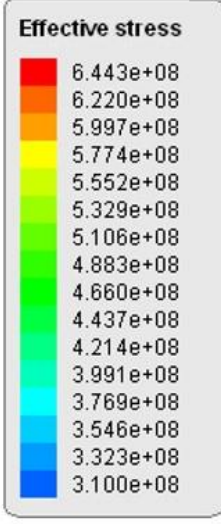

Figure 4.19. (a) The simulated test plate total strain in the $Y$-direction for Shot 3 at $20 \mathrm{~ms}$ after detonation. The samples were edge restrained by the picture frame support (with a span of $122 \mathrm{~cm}$ ). The inner, $81.3 \mathrm{~cm}$ wide center of the panels was not back supported allowing unconstrained out of plane deflection as well as inplane stretching. The outer $20.35 \mathrm{~cm}$ wide picture frame provided rigid back support of the panels but did not restrain in-plane stretching. Three node locations are indicated where the nodal strain and displacement magnitude were recorded for each test shot. (b) The effective stress for Shot 3 at $1 \mathrm{~ms}$ after detonation showing regions of the plate that were stressed beyond the yield strength of the plate material $(310 \mathrm{MPa})$. 
Table 4.8. The maximum out of plane dynamic $\left(\delta_{\max }\right)$ and permanent $(\delta)$ displacements and the $Y$ direction tensile strains at nodes 1,2 , and 3 . The permanent $Y$ displacement $\left(\delta_{Y}\right)$ for node 3 determined at 20 ms after detonation is also listed.

\begin{tabular}{|c|c|c|c|c|c|c|c|c|c|c|}
\hline \multirow[b]{2}{*}{$\begin{array}{l}\text { Test } \\
\text { shot }\end{array}$} & \multicolumn{3}{|c|}{ Node 1} & \multicolumn{3}{|c|}{ Node 2} & \multicolumn{4}{|c|}{ Node 3} \\
\hline & $\begin{array}{c}\delta_{\max } \\
(\mathrm{mm})\end{array}$ & $\begin{array}{c}\delta \\
(\mathrm{mm})\end{array}$ & $\varepsilon_{Y Y}$ & $\begin{array}{l}\delta_{\max } \\
(\mathrm{mm})\end{array}$ & $\begin{array}{c}\delta \\
(\mathrm{mm})\end{array}$ & $\varepsilon_{Y Y}$ & $\begin{array}{l}\delta_{\max } \\
(\mathrm{mm})\end{array}$ & $\begin{array}{c}\delta \\
(\mathrm{mm})\end{array}$ & $\varepsilon_{Y Y}$ & $\begin{array}{c}\delta_{Y} \\
(\mathrm{~mm})\end{array}$ \\
\hline 1 & 6.21 & 0.23 & 0 & 8.62 & 1.19 & 0.03 & 8.91 & 1.92 & 0.04 & 1.87 \\
\hline 2 & 5.57 & 0.32 & 0 & 7.93 & 1.78 & 0.04 & 9.10 & 2.43 & 0.06 & 2.39 \\
\hline 3 & 6.32 & 0.40 & 0 & 9.09 & 3.04 & 0.06 & 10.09 & 4.15 & 0.08 & 3.96 \\
\hline 4 & 7.27 & 0.45 & 0 & 9.66 & 3.23 & 0.07 & 10.21 & 4.48 & 0.09 & 4.30 \\
\hline 5 & 6.94 & 0.56 & 0 & 9.37 & 3.83 & 0.08 & 9.94 & 5.18 & 0.10 & 4.91 \\
\hline
\end{tabular}

To analyze the effect of increasing impulse on the permanent (plastic) plate deflection, the out of plane deflection was measured (after the test) directly beneath the charge center (Table 4.6). This was normalized by both the half span of the unsupported test plate $(2 \mathrm{~L}=81.3 \mathrm{~cm})$ and by that of the edge clamped panel $(2 \mathrm{~L}=122 \mathrm{~cm})$. Both dimensionless displacements are plotted in Figure 4.20 as a function of the normalized impulse, $I_{o} / m_{b} \sqrt{\sigma_{Y} / \rho_{m}}$. The areal mass of the plate, $m_{b}=$ $\rho_{m} h$ (where h is the plate thickness $2.54 \mathrm{~cm}$ ) was $200.6 \mathrm{~kg} / \mathrm{m}^{2}$, with the plate yield strength, $\sigma_{Y}=$ $310 \mathrm{MPa}$, and material density $\rho_{m}=7900 \mathrm{~kg} / \mathrm{m}^{3}$. The peak specific impulses applied to the test plates were determined from the simulations, Table 4.6, rather than the Kolsky bar measured impulse (since there was some variation in the standoff distance between the charge and the Kolsky bar/plate as well as the orientation of the detonator during tests). Additional simulations were also conducted at several lower specific impulses to enable observation of the trend as the impulse decreased to zero. Figure 4.20 shows the permanent out of plane deflection increasing linearly with the incident impulse applied to the plates. It also reveals the need for a critical impulse sufficient to cause permanent (plastic) panel deflection. The use of a back supported picture frameshaped region of the test plates avoided the complications of panel failure in the tests. However, this region was loaded beyond its elastic limit and the resulting inplane plastic displacements permitted a larger deflection of the central, unsupported span. 


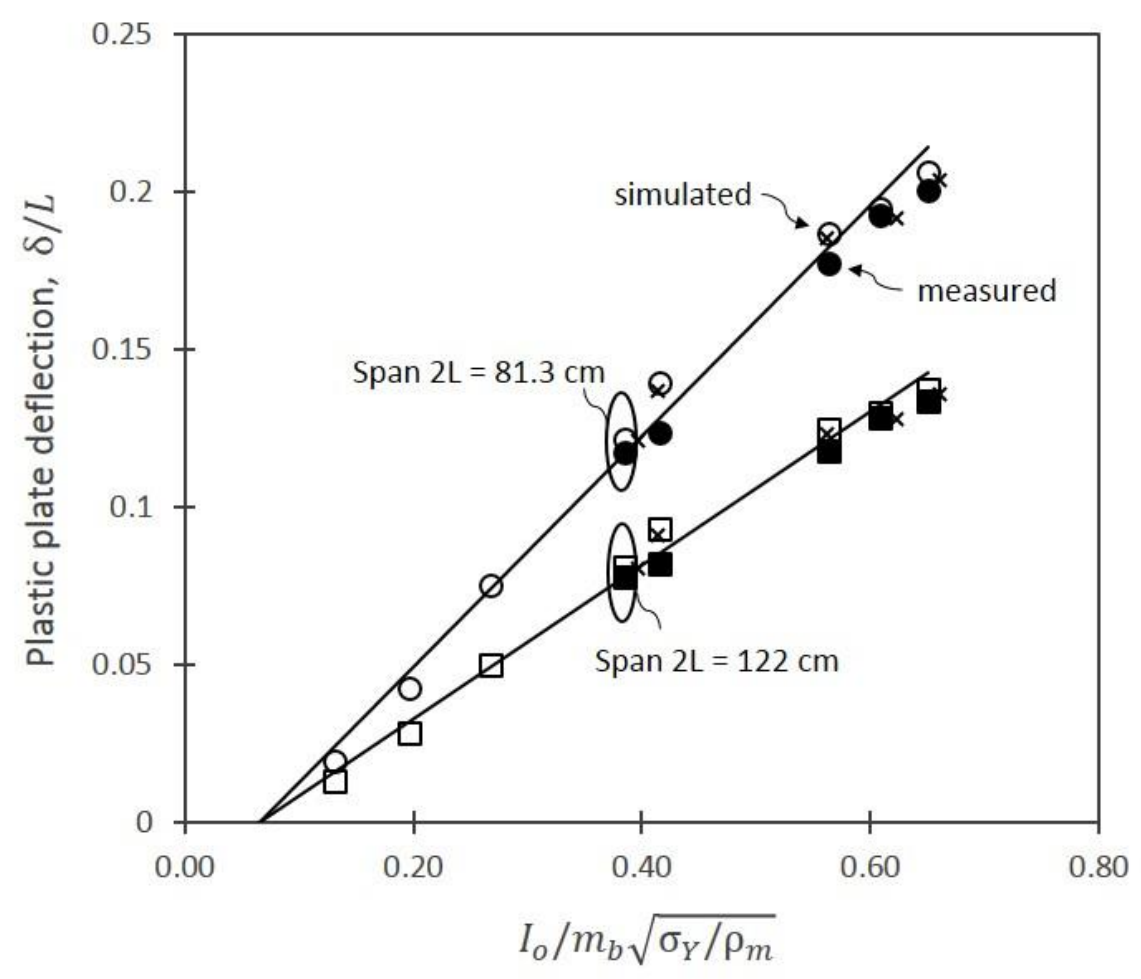

Figure 4.20. The simulated permanent plate deflection scaled by the half span, L versus dimensionless impulse in which $I_{o}$ corresponded to the maximum simulated specific impulse applied near the test plate centers. Results are shown for the $81.3 \mathrm{~cm}$ wide span where no back support existed and the $122 \mathrm{~cm}$ span over which unconstrained inplane panel stretching was allowed. The experimentally measured deflections normalized by the two span lengths are also shown (solid circle and square data). The $x$ data points correspond to simulations in which the background air particles were omitted.

The momentum transfer between the air and granular particles results in a deceleration of the granular particles, Figure 4.7(b) and Figure 4.8(b). To investigate the significance of this momentum transfer mechanism upon the plate's displacement, simulations of each of the test shots were conducted with air particles removed. These displacement results are plotted on Figure 4.20 (the x-data points), and indicate that removal of air particles does not affect either the applied impulse or the plates' permanent displacement. Conservation of momentum dictates that the sum of the impulse of the air and granular particles must be equal to that of the particles in an air free test. Analysis of the simulations confirmed that the reduction in the granular particle momentum (from that observed without air particles) corresponded to the amount transferred to the air shock. Since the resulting panel displacements were also approximately the same, with or without the 
presence of air in the simulations, it appears that the effective momentum transfer efficiency to the plates by the two particle types was similar.

\subsection{Concluding remarks}

The deformation of edge restrained and partially back supported ductile square stainless steel plates has been investigated following impact by high velocity, spherically symmetric granular material shells using a combination of large-scale experiments and numerical simulations implemented in the discrete particle-based IMPETUS Afea code. The study used suspended spherical explosive charges to accelerate 25 to $150 \mathrm{~kg}$ spherical shells of water-saturated glass or higher density zirconia particles to velocities of 500-1200 m/s. The evolution of the granular shell topology following detonation was experimentally characterized by analysis of high-speed video images. This data revealed that the radial velocity of the granular particles reached a maximum within a short distance of the original location of the particles, and then slowly decreased with radial distance due to momentum transfer to the background air. The high-speed video images also revealed the presence of a "particle finger-like" instability at the expanding granular shell-air interface. This was consistent with the analysis in Chapter 3 that applied a result by Kandan et al. [70] to the interaction of supersonic granular particles with a high-pressure air shock just ahead of the radially expanding particle shells. The finger-like instability was most prominent at the leading edge of the fastest expanding granular shells, in agreement with the recent analysis.

The test charges were positioned above the center of $2.54 \mathrm{~cm}$ thick edge clamped $1.32 \mathrm{~m} \mathrm{x}$ $1.32 \mathrm{~m}$ panels made of 304 stainless steel, and their permanent deflection fields were measured after testing. A novel edge restraint approach was utilized to avoid disruption of reflected particle flow over the impacted surface of the sample. Unobstructed out of plane deformation of the plate was permitted within a central $81.3 \mathrm{~cm}$ by $81.3 \mathrm{~cm}$ square opening of the panel. While this gripping approach avoided plate failure near the gripped regions, the $20.3 \mathrm{~cm}$ wide picture frame region between the central opening and the outer edge gripped periphery of the test plates was loaded beyond its elastic limit and contributed an inplane stretching displacement that increased the out of plane deflections.

A Kolsky bar positioned at a location that sampled an impulsive load equivalent to that at the plate center was used to measure both the pressure and specific impulse applied by granular impacts. The expansion of the granular shells, as well as the pressure and impulse transferred to 
the Kolsky bar were all well predicted by the discrete particle-based simulation approach. It also provided insight into the mechanisms of target loading in the region behind the particle front where direct observation with the high-speed cameras was obscured by particles and high explosive reaction product gases. The IMPETUS Afea code was used to predict the impulse distribution applied to the test plates and successfully predicted the test plates out of plane displacement distribution and enabled interpretation of the factors that governed this behavior. The study confirms earlier, simplified model estimates for the approximately linear dependence of the out of plane displacement of edge clamped plates upon incident specific impulse. 


\section{Chapter 5. Impact of granular matter with edge clamped square honeycomb sandwich panels}

This chapter presents the results for a second series of tests almost identical to those presented in Chapter 4. However, here the solid edge clamped 304 stainless steel panels were replaced by square honeycomb core sandwich panels of identical mass per unit area. High-speed video imaging is again used to observe the sand front propagation for each charge configuration while the applied pressure and impulse loading of the explosively accelerated granular media were measured using an instrumented Kolsky bar. Simulations using the IMPETUS Afea Solver are compared to the experimental results. The benefit of the sandwich panel design is investigated by comparing the experimentally measured permanent displacement of the solid plate and sandwich panels when subjected to equivalent impulse loading conditions. 


\subsection{Introduction}

Extensive studies on the benefit of a sandwich panel design have been explored for shock wave impacts resulting from underwater explosions [7-10] and in air [4-6]. The often superior performance of a well-designed sandwich panel compared to an equivalent mass monolithic, solid plate has been attributed to a combination of two effects: (i) the increased bending resistance/strength of the panel, and (ii) the FSI effect which reduces the momentum transferred to some sandwich panel designs (especially during underwater shock loading) [10]. As discussed in Section 2.3.1, Liu et al. [32] used a particle based simulation to investigate the normal incident impact of sand slugs against both monolithic plates and their equivalent mass sandwich designs. This study revealed that low aspect ratio (thick core and small span) sandwich panel designs outperformed their monolithic counterparts, especially when a strong core concept was utilized, Figure 2.2. However, this benefit was mainly a result of the higher bending strength of the sandwich panel, since the small FSI effect did not result in significant variations in transferred impulses.

Chapter 4 described in detail the testing on the five solid monolithic plates, analyzing the sand front expansion rate and the applied impulse of the five charge configurations as well as the resulting test plate deformation. This series of five test shots on solid plates confirmed the previous study of Liu et al. [32] that indicated the out of plane deflection of a normally impacted plate to be a linear function of the applied impulse. The study presented here investigates the loading of an equivalent mass per unit area square honeycomb core sandwich panel (to that of the solid plates) under comparable high intensity impact by the same five charge configurations presented in Chapter 4. The sandwich panel displacement under high impact loading is analyzed and compared to that of the equivalent solid plate. The results reveal a significant benefit of the sandwich panel design compared to its equivalent mass solid plate.

\subsection{Experimental setup}

The experimental tests presented here were also conducted at the outdoor blast testing facility in Edgefield, SC (NEWTEC Services Group Inc.). These square honeycomb sandwich panels were edge clamped on the same test platform as the (equivalent mass) solid plates in Chapter 4. A schematic of the test arrangement used for the test shots is shown in Figure 5.1. This arrangement again consisted of a steel picture frame test platform to support the target panels, a suspended 
spherical wet sand charge, and a steel Kolsky bar with strain gauge instrumentation to measure the applied pressure and impulse. A brief description of the test platform, charge configuration, and Kolsky bar system is described here with greater details provided in Section 4.2.

A comparative test arrangement for the solid, monolithic plates and the square honeycomb sandwich panels are shown, side by side, in Figure 5.2. This schematic shows the concentric spherical charge suspended above the test targets with internal sphere of radius, $\mathrm{R}_{1}$, packed with C-4 explosive, surrounded by an outer sphere of radius $\mathrm{R}_{2}$. The annular shell between the two spheres was filled with either water-saturated silica glass particles or zirconia particles, representing a wet granular medium (wet sand). The impulse applied to a test structure decreases with standoff distance and so a standardized distance was used here. The tests could have been conducted with a constant front face to charge center distance or a fixed distance from charge center to sample back face. Here, the back face of the targets (the previous five solid plates and here the five sandwich panels) was intended to be maintained at a constant standoff distance of $\mathrm{H}_{\mathrm{b}}$ $=47.54 \mathrm{~cm}$ from the center of the test charge. This resulted in the front face of the sandwich panels being $2.54 \mathrm{~cm}$ closer to the center of the test charge than the top of the solid test plates. As a result, the distance from the center of the test charge to the front face of the sandwich panel was $\mathrm{H}_{\mathrm{p}}=$ $42.46 \mathrm{~cm}$ while that to the front face of the solid plate was $\mathrm{H}_{\mathrm{p}}=45 \mathrm{~cm}$.

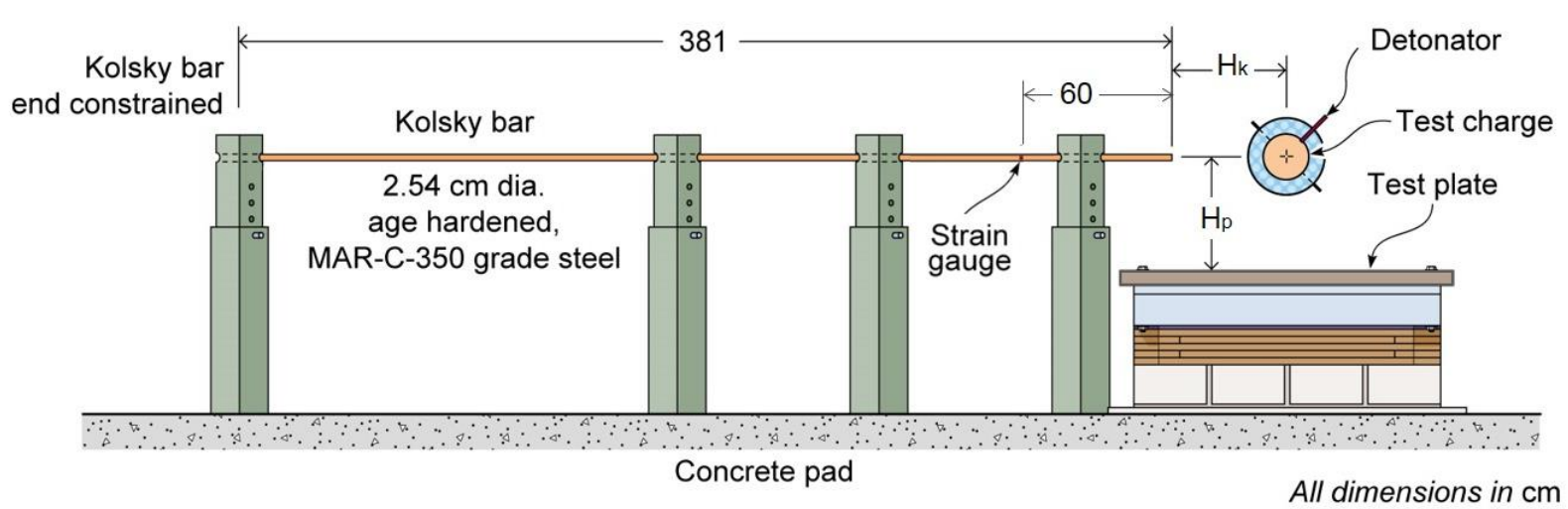

Figure 5.1. Test setup with strain gage instrumented Kolsky bar for impact load measurements. 


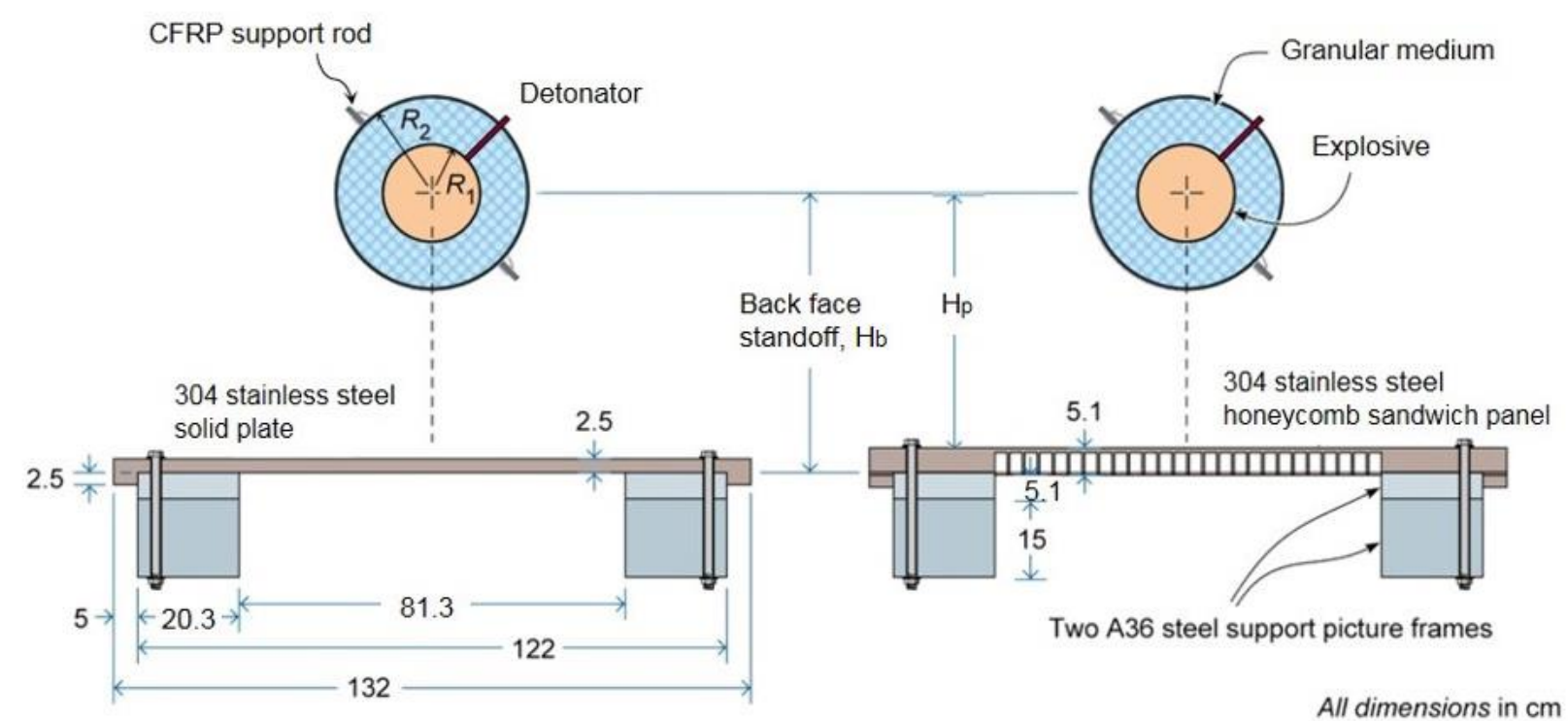

Figure 5.2. Schematic diagram showing the test arrangement for edge clamped panels, solid plate (left) and equivalent mass honeycomb sandwich panel (right). The standoff distance to the back face of both the solid plate and sandwich panel, $H_{b}$, is consistent for all tests.

\subsubsection{Test platform}

The test platform was identical to that used for the five solid plates presented in Chapter 4. Figure 5.1 shows a schematic illustration of the full test setup. Description of the test setup can be found in Section 4.2.1. A view of the honeycomb sandwich panel positioned on the picture frame support base and the suspended spherical test charge is shown in Figure 5.2 on the right. Two high-speed cameras (Vision Research Inc., Phantom V7.3) captured the propagating sand front of each test shot after detonation as they radially expanded towards the Kolsky bar and test panel. The high-speed cameras captured both a wide view of the test event and a closer (magnified) view of the test charge, front edge of the test panel, and the impact end of the Kolsky bar. The front $1.32 \mathrm{~m}$ length of the test panels and the front of the Kolsky bar, a length of $10 \mathrm{~cm}$ for Shots 1 and 2 and $15 \mathrm{~cm}$ for Shots 3-5, were spray painted prior to testing to provide reference lengths.

\subsubsection{Honeycomb panel design and fabrication}

Previous research at the University of Cambridge [32] indicated that sandwich panels with strong, cellular cores outperformed monolithic plates of equivalent weight under impulse loading 
Table 5.1. The design parameters defined for the square honeycomb panels with a relative core density of $\sim 30 \%$.

\begin{tabular}{|c|c|c|c|c|c|c|c|}
\hline $\begin{array}{l}\text { Cell } \\
\text { size }\end{array}$ & $\begin{array}{c}\text { Web } \\
\text { thickness }\end{array}$ & $\begin{array}{l}\text { Unit cell } \\
\text { size }\end{array}$ & $\begin{array}{l}\text { Core } \\
\text { height }\end{array}$ & $\begin{array}{l}\text { Front } \\
\text { face }\end{array}$ & $\begin{array}{l}\text { Back } \\
\text { face }\end{array}$ & $\begin{array}{c}\text { Panel } \\
\text { thickness }\end{array}$ & $\begin{array}{l}\text { Corner } \\
\text { radius }\end{array}$ \\
\hline $\mathrm{D}(\mathrm{mm})$ & $\mathrm{t}(\mathrm{mm})$ & 1 (mm) & $\mathrm{H}_{\mathrm{c}}(\mathrm{mm})$ & $\mathrm{t}_{\mathrm{f}}(\mathrm{mm})$ & $\mathrm{t}_{\mathrm{b}}(\mathrm{mm})$ & $\mathrm{t}_{\mathrm{p}}(\mathrm{mm})$ & $\mathrm{r}(\mathrm{mm})$ \\
\hline 44.45 & 6.35 & 50.80 & 36.50 & 7.90 & 6.40 & 50.8 & 9.5 \\
\hline
\end{tabular}
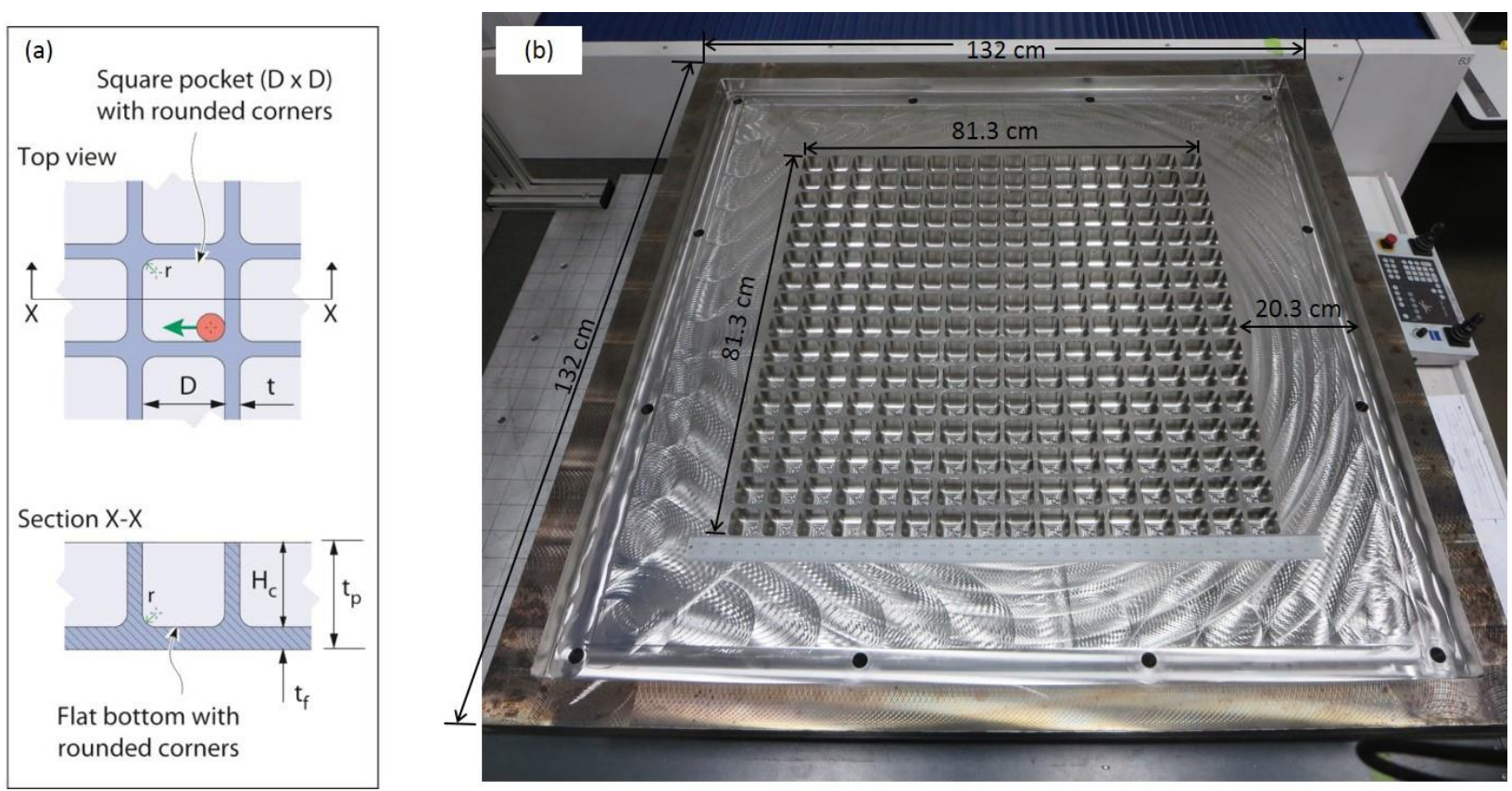

Figure 5.3. Machined, square honeycomb core sandwich panel. The pockets are machined such that the impact side face sheet is integrated to the core structure. (a) Top and section view of the cells. (b) Machined front face sheet/core integrated panel before back face sheet was welded on.

scenarios. Square honeycomb core sandwich panels are ideal candidates since they have large thru thickness load bearing capabilities and are able to resist the in-plane stretching associated with panel stretching (as it suffers out of plane bending) $[29,96]$. A core relative density, $\bar{\rho}=\rho_{c} / \rho_{m}$, of $30 \%$ was selected for design of the cellular core investigated here. As a result, approximately a third of the solid plate mass was assigned to the core and two thirds distributed between the front and back face sheets. Thus, the honeycomb core density was $\rho_{c}=\bar{\rho} \rho_{m}=2370 \mathrm{~kg} / \mathrm{m}^{2}$ for a 304 stainless steel sandwich panel with material density $\rho_{m}=7900 \mathrm{~kg} / \mathrm{m}^{2}$. Figure 5.3(a) shows two views (top and section) of the square honeycomb core pattern, with the design parameters listed in Table 5.1. 
The sandwich panels were fabricated from $1.32 \mathrm{~m}$ x $1.32 \mathrm{~m} \mathrm{x} 7.6 \mathrm{~cm}$ thick plates that were first stress relieved at Rex Heat Treat (Warrendale, PA) at $538^{\circ} \mathrm{C}$ for eight hours and then machined at KVK Precision Technologies (Shenandoah, VA). This was the same stress relief process used for the fabrication of the five solid test plates. A $1.22 \mathrm{~m}$ x $1.22 \mathrm{~m}$ and $2.54 \mathrm{~cm}$ deep center pocket was milled out of the plate to form a $5.08 \mathrm{~cm}$ wide picture frame edge. This allowed the panels to tightly fit over the test platform support base and form the edge clamped condition. Within the center $81.3 \mathrm{~cm} \times 81.3 \mathrm{~cm}$ square span of the panel, a matrix of $256,44.45 \mathrm{~mm} \times 44.45$ $\mathrm{mm}$, square cell pockets were milled in a $16 \times 16$ square array. These cells were spaced $50.8 \mathrm{~mm}$ apart with a depth of $36.5 \mathrm{~mm}$ (the core height, $\mathrm{H}_{\mathrm{c}}=36.5 \mathrm{~mm}$ ). The corners and bottoms of the cell pockets were rounded off with a $9.5 \mathrm{~mm}$ radius, ball mill cutter as shown in Figure 5.3(a), to provide smooth corners and to minimize areas of stress concentrations. In comparison to a regular, square honeycomb core where the openings completely penetrate the plate thickness, the modified design here incorporated an integrated face sheet on one side. The integrated face sheet had thickness $t_{\mathrm{f}}=7.9 \mathrm{~mm}$ and was used as the front face sheet of the sandwich panel. This was chosen such that there was an integral face sheet/core structure on the impact side of the test panels, which did not require any form of joining or welding at the face sheet/core intersections. This machined honeycomb panel (integral face sheet/core structure) had a total height of $44.4 \mathrm{~mm}\left(\mathrm{H}_{\mathrm{c}}+\mathrm{t}_{\mathrm{f}}\right)$, and is shown in Figure 5.3(b).

The back face sheet with thickness $t_{b}=6.4 \mathrm{~mm}$ was then attached by electron beam welding at Sciaky, Inc. (Chicago, IL) to form the complete sandwich panel structure with a total thickness (panel height) of $t_{p}=50.8 \mathrm{~mm}$. Electron beam weld parameters were developed for the through thickness welding of T-joint configurations where the $6.4 \mathrm{~mm}$ thick, back face sheet intersected with the $6.35 \mathrm{~mm}$ thick, honeycomb webs. A linear welding pattern both in the X- and Yorthogonal directions was adopted for welding at each of the honeycomb web and back face sheet intersections. Since the honeycomb cell matrix pattern was only defined in the center square 81.3 $\mathrm{cm} \times 81.3 \mathrm{~cm}$ unsupported area, a series of external perimeter welds were also done to cover the area between the inner honeycomb cell matrix pattern and the outer picture frame edges. A schematic illustration of the electron beam procedure and the weld pattern is shown in Figure 5.4. The electron beam welding was performed with a $50 \mathrm{kV}$ beam voltage and $100 \mathrm{~mA}$ beam current. The weld width was $0.51-0.64 \mathrm{~mm}$ with a penetration depth of $10.16 \mathrm{~mm}$. A view of the weld through the back face sheet into the web of the sandwich cell is shown in Figure 5.5. Since the 


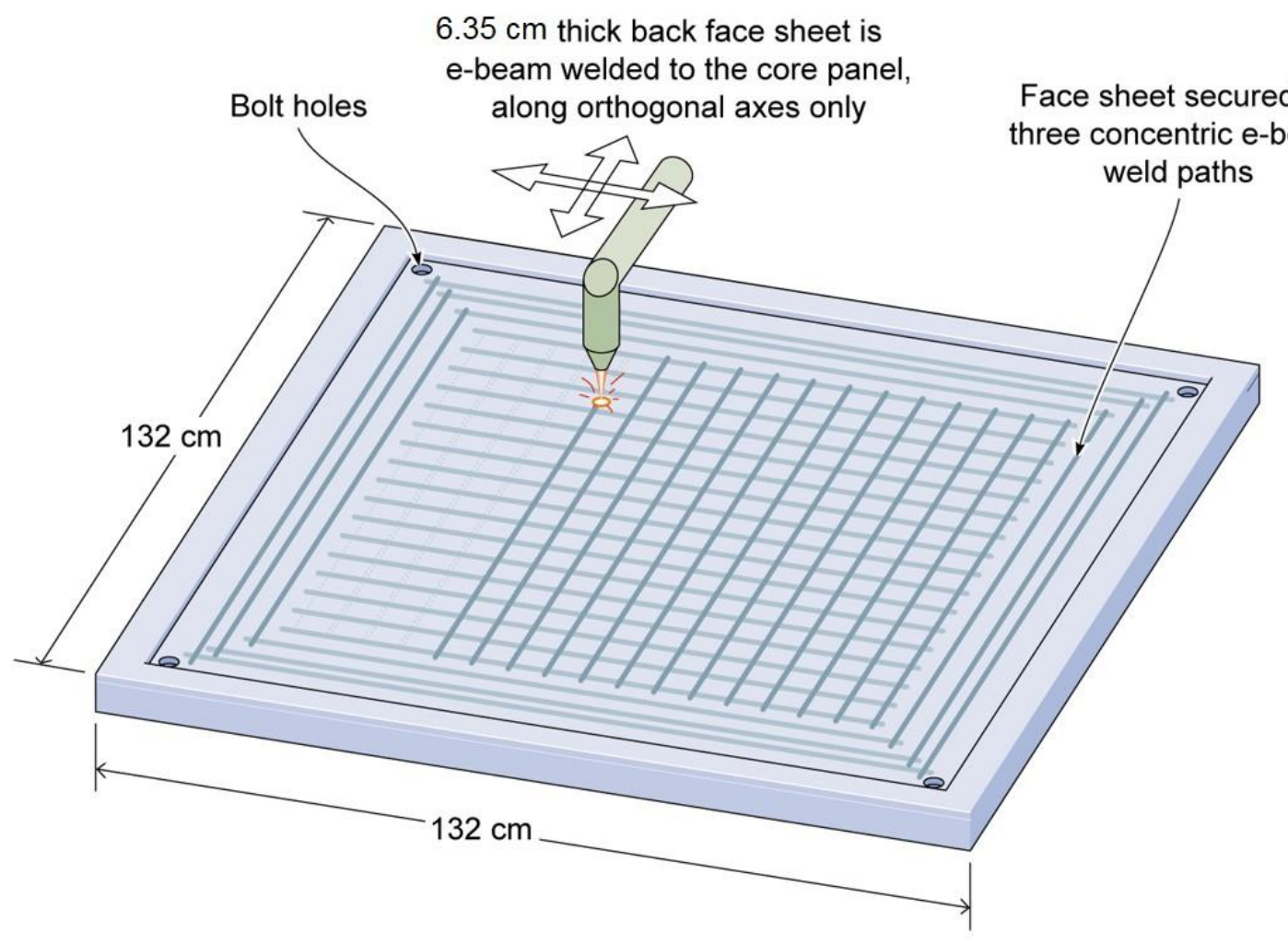

Figure 5.4. Electron beam welding of linear welds attaching the back face sheet to the square honeycomb core in alignment with the honeycomb web walls.

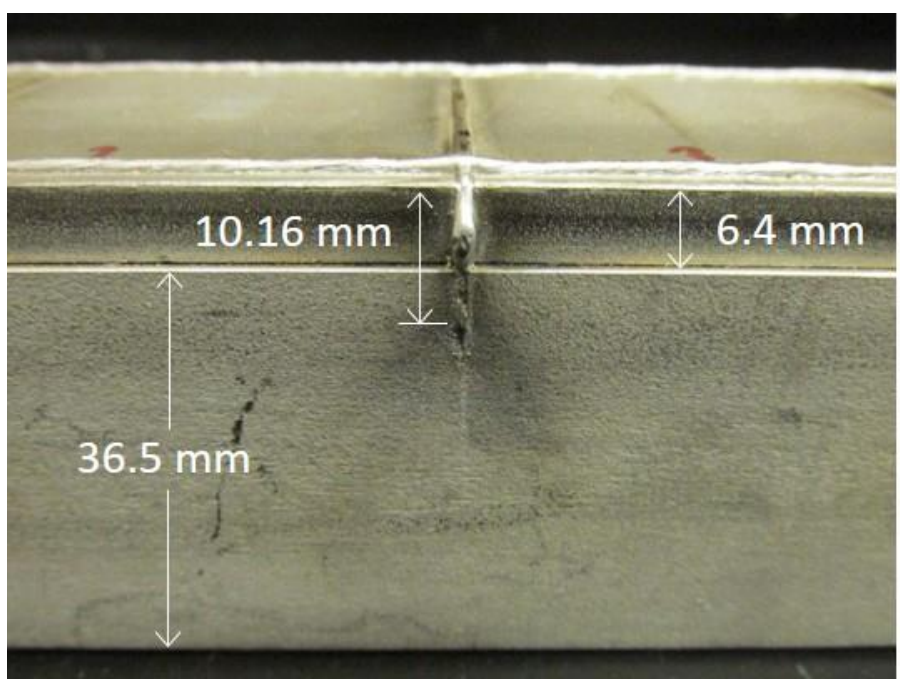

Figure 5.5. Electron beam weld bonding the back face and the integral sandwich panel core structure. 
back face sheet was $6.40 \mathrm{~mm}$ thick, the weld penetrated $3.76 \mathrm{~mm}$ into the honeycomb core web. The narrow weld width parameter was chosen to minimize thermal distortion of the panels. However, the $\sim 0.635 \mathrm{~mm}$ weld was small compared to the $6.35 \mathrm{~mm}$ web thickness, covering only about $10 \%$ of the web cross sectional area. These welds were not sufficiently robust to withstand the high intensity loading conditions for this test series.

\subsubsection{Test charges}

The five test charge configurations were identical to those described in Chapter 4 for the loading on the solid test plates. Briefly, the suspended spherical test charge consisted of an inner acrylic sphere of radius, $\mathrm{R}_{1}$, filled with explosive ( 3 or $4.5 \mathrm{~kg}$ of $\mathrm{C}-4$ ) and an outer sphere of radius $\mathrm{R}_{2}$, defined to the outer surface of the acrylic spheres. The annular region between the two shells was filled with a granular medium of either silica glass particles or zirconia particles and then fully saturated with water to fill the void space between the particles. Shots 1-3 were filled with silica glass particles which Shots 4 and 5 were filled with higher density (heavier) zirconia particles. The measured values of the granular medium (sand), water, and saturated granular medium (wet sand) mass for the test charge configurations are summarized in Table 5.2. These measured mass values were very similar to those from the previous five test shots on the solid plates (Table 4.1). A carbon fiber reinforced polymer (CFRP) suspension rod, inserted through the center of the test charges during the charge configuration, was used to assist in the suspension and orientation of the test shots above the panels. The heavier mass test shots (Shots 3-5) required the additional use of a mesh net to suspend the charges above the test panels. The test shots were oriented such that the detonation location was $\theta=45^{\circ}$ from the test panel normal, Figure 5.6. Test Shot 5 was difficult to rotate due to its significant mass, and consequently the rotation of the charge was restricted to $\theta$ $=49^{\circ}$. After setup, an instantaneous detonator (manufactured by Dyno Nodel, Inc. in Salt Lake City, Utah; model SP/SM (12-0)) was inserted into the explosive charge just prior to detonation. 
Table 5.2. Charge configurations for the five sandwich panel test shots.

\begin{tabular}{ccccccccc}
\hline $\begin{array}{c}\text { Test } \\
\text { shot }\end{array}$ & $\begin{array}{c}\text { Inner } \\
\text { radius } \\
\mathrm{R}_{1}(\mathrm{~mm})\end{array}$ & $\begin{array}{c}\text { Explosive } \\
\text { mass } \\
(\mathrm{kg})\end{array}$ & $\begin{array}{c}\text { Outer } \\
\text { radius } \\
\mathrm{R}_{2}(\mathrm{~mm})\end{array}$ & $\begin{array}{c}\text { Annular } \\
\text { shell width } \\
(\mathrm{mm})\end{array}$ & $\begin{array}{c}\text { Particle } \\
\text { type }\end{array}$ & $\begin{array}{c}\text { Particle } \\
\text { mass } \\
(\mathrm{kg})\end{array}$ & $\begin{array}{c}\text { Water } \\
\text { mass } \\
(\mathrm{kg})\end{array}$ & $\begin{array}{c}\text { Annular } \\
\text { shell mass } \\
(\mathrm{kg})\end{array}$ \\
\hline 1 & 80 & 3.0 & 152 & 72 & Silica & 19.30 & 4.36 & 23.66 \\
2 & 80 & 3.0 & 203 & 123 & Silica & 51.92 & 12.58 & 64.50 \\
3 & 90 & 4.5 & 203 & 113 & Silica & 50.24 & 11.88 & 62.12 \\
4 & 90 & 4.5 & 203 & 133 & $\mathrm{ZrO}_{2}$ & 86.33 & 14.75 & 101.08 \\
5 & 90 & 4.5 & 229 & 139 & $\mathrm{ZrO}_{2}$ & 126.88 & 22.05 & 148.93 \\
\hline
\end{tabular}

Table 5.3. The standoff distance from the center of the test charge to the front face of the sandwich panel and to the impact end of the Kolsky bar. The delay time for the Kolsky bar signal initiation and the impact time of the main sand front on the Kolsky bar are also listed.

\begin{tabular}{ccccc}
\hline Test shot & $\begin{array}{c}\text { Standoff to } \\
\text { test plate } \\
\mathrm{H}_{\mathrm{p}}(\mathrm{cm})\end{array}$ & $\begin{array}{c}\text { Standoff to } \\
\text { Kolsky bar } \\
\mathrm{H}_{\mathrm{k}}(\mathrm{cm})\end{array}$ & $\begin{array}{c}\text { Total } \\
\text { delay time } \\
(\mu \mathrm{s})\end{array}$ & $\begin{array}{c}\text { Impact Time } \\
(\text { Kolsky bar }) \\
(\mu \mathrm{s})\end{array}$ \\
\hline 1 & 43.18 & 48.50 & 40 & 350 \\
2 & 38.47 & 45.35 & 54 & 445 \\
3 & 41.90 & 45.61 & 53 & 325 \\
4 & 44.68 & 44.03 & 58 & 470 \\
5 & 43.01 & 45.80 & 67 & 640 \\
\hline
\end{tabular}

The test charges were suspended $\mathrm{H}_{\mathrm{p}}=42.46 \mathrm{~cm}$ above the top face of the sandwich panels. Since a distance of $\mathrm{H}_{\mathrm{b}}=47.54 \mathrm{~cm}$ was maintained from the charge center to the back face sheet of both the solid plates and the sandwich panels (Figure 5.2). Recall, this placed the thicker sandwich panel $2.54 \mathrm{~cm}$ closer to the test charge than the top of the solid plate. Prior to detonation, sufficient time elapsed after setup such that the test charges experienced small shifts in their initial standoff locations. The high-speed videos were used to measure the exact standoff distances to the top of the test panels, $\mathrm{H}_{\mathrm{p}}$, and the front impact end of the Kolsky bar, $\mathrm{H}_{\mathrm{k}}$, just prior to detonation. These are listed in Table 5.3.

\subsubsection{Kolsky bar}

A strain gage instrumented $3.81 \mathrm{~m}$ long, $2.54 \mathrm{~cm}$ diameter, age hardened, maraging steel C350 grade, Kolsky bar was used to measure the axial stress (pressure) and impulse-time response of the loading for the different test charge configurations. The long Kolsky bar was positioned $42.46 \mathrm{~cm}$ above the front face sheet of the sandwich panel using four adjustable height pedestal supports, to align the center of the Kolsky bar with the explosive charge center. The standoff 
distances from the charge center to the front of the Kolsky bar, $\mathrm{H}_{\mathrm{k}}$, measured by the high-speed videos prior to detonation are recorded in Table 5.3. Ideally, the applied pressure and impulse measured by the strain gauges mounted on the Kolsky bar represent the impact experienced by the test panels.

Two T-rosette type strain gauges (Vishay Precision group, CEA-06-125UT-350) were mounted diametrically opposite each other and wired in a full Wheatstone bridge circuit $0.6 \mathrm{~m}$ from the front (impact) end of the Kolsky bar. The signal recorded by the strain gauges was used to calculate the axial stress (pressure) and applied impulse to the front of the bar by the granular media impacts. A detailed description of the strain gauge instrumentation and measurement mode is found in Chapter 4. The strain gauge signal recording was again initiated by the break of a trigger wire that was attached to the outer shell of the test charge configuration. Therefore, there was a time delay from the moment of detonation before the initiation of the signal recording. For consistency, the detonation activation is defined as $\mathrm{t}=0 \mathrm{~s}$, and the Kolsky bar data from each test shot was shifted by the time it took for the compressive sand shock to reach the outer shell. The total delay times for the shock to reach the outer shell of each test shot (Shots 1-5) are summarized in Table 5.3, varying from 40-67 $\mu$ s. This delay time was a combination of the time for the detonation shock to reach the HE/soil interface, and the time for the resultant compressive shock front to propagate through the annular region of the wet granular medium (detailed for each charge configuration in Appendix C).

Recall, the elastic wave speed in a C-350 steel bar is $4800 \mathrm{~m} / \mathrm{s}$, and thus the time for a signal initiated by impacts on the front of the Kolsky bar to reach the strain gauge located $0.6 \mathrm{~m}$ from the impacted end of the bar was $125 \mu \mathrm{s}$. The $3.81 \mathrm{~m}$ length of the bar ensured that the signal measurement of interest was not complicated by distal reflections that arrived $1.34 \mathrm{~ms}$ after the initial impact signal reaches the strain gauge.

\subsection{Simulation methodology}

The five honeycomb sandwich panel tests were simulated using IMPETUS Afea Solver [50], a discrete particle based solver that models air, soil, and high explosive (HE) particles, and employs a corpuscular method to model the particle interactions. Based on a Lagrangian formulation, the solver fully couples the discrete particle method with finite element (FE) models and allows for interactions between the particles and structures to be observed. A more detailed description of 
Table 5.4. Distribution of air, HE, and soil particles in the simulations for each test shot.

\begin{tabular}{cccc}
\hline Test shot & Air particles & HE particles & Soil particles \\
\hline 1 & 849,692 & 707,078 & 443,230 \\
2 & 617,878 & 508,193 & 873,929 \\
3 & 556,265 & 688,240 & 755,495 \\
4 & 557,828 & 684,505 & 757,667 \\
5 & 468,104 & 586,956 & 944,940 \\
\hline
\end{tabular}

the particle model and implementation used for the analysis of high impact loading on solid test plates is found previously in Chapters 3 and 4.

As previously determined, 2 million particles were again confirmed to be sufficient to analyze the particle/FE interactions. The distribution of these particles is summarized for each simulated test shot in Table 5.4. Due to the volume change of the soil and high explosive (HE) for each test shot, the particle division between air, soil, and HE defined by the solver is slightly different for each simulation.

\subsubsection{FE geometry model}

The model geometry for the square honeycomb sandwich panel defined in the solver is shown in Figure 5.6. The geometry were modeled according to experimental dimensions with a consistent Kolsky bar, test rig support frame, and a $5.08 \mathrm{~cm}$ thick honeycomb sandwich panel. The suspended charge was the only inconsistency between the simulations. As in Chapter 4 with the same charge configurations, the charge consisted of an inner spherical charge and an outer annulus of wet sand (glass or zirconia particles). An acrylic plastic shell with radius, $\mathrm{R}_{1}$, was modeled to constrain the inner HE particles and a second outer acrylic shell of radius, $\mathrm{R}_{2}$, was modeled around the annular region of saturated sand. Both shells were modeled with a $3 \mathrm{~mm}$ thickness. The spherical charge was suspended in space with specified standoff distances in Table 5.3 from the charge center to the front of the Kolsky bar, $\mathrm{H}_{\mathrm{k}}$, and the top of the sandwich panel, $\mathrm{H}_{\mathrm{p}}$, measured by the high-speed videos. The detonation location shown in Figure 5.6 was defined as $\theta=45^{\circ}$ from the test panel normal (X-axis) at the outer surface of the HE charge for Shots $1-4$ and $\theta=49^{\circ}$ for test Shot 5. A detailed description of the FE model for the Kolsky bar and test platform can be found in Section 4.3.2. 


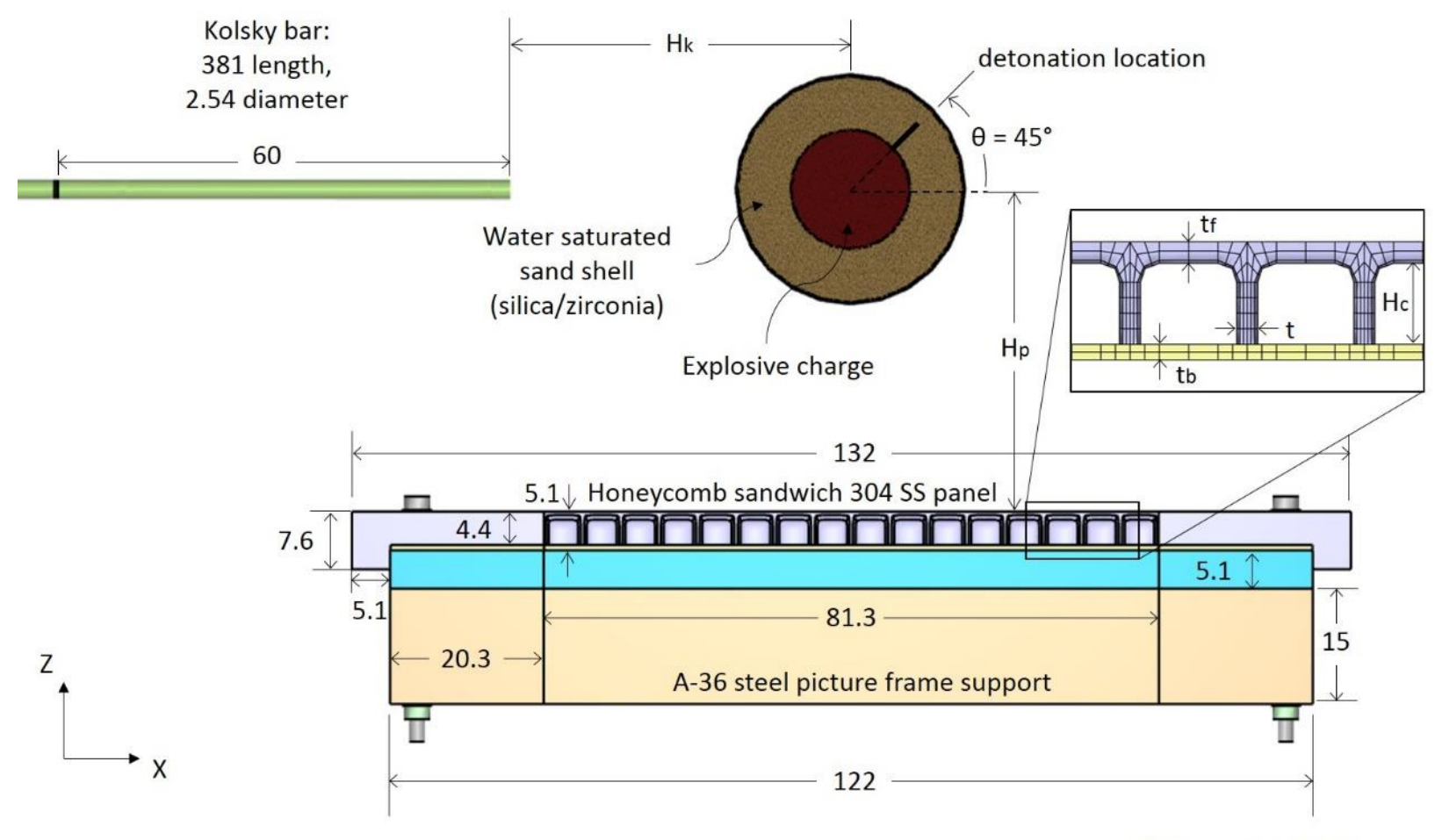

All dimensions in $\mathrm{cm}$

Figure 5.6. Model geometry used for simulations for solid plate and honeycomb sandwich panel showing standoff distance of charge to the back face of the panels.

The sandwich panel model consisted of two separate parts, the integral front face/core structure and the back face sheet. The sandwich panel integral core consisted of an array of $16 \mathrm{x}$ 16 honeycomb cells within the $81.3 \mathrm{~cm}$ x $81.3 \mathrm{~cm}$ unsupported center of the panel and a front face sheet with a $7.9 \mathrm{~mm}$ thickness. Each cell was $50.8 \mathrm{~mm}$ x $50.8 \mathrm{~mm}$ with a core height of $36.5 \mathrm{~mm}$. The model contained a $6.4 \mathrm{~mm}$ thick back face sheet that was initially connected to the honeycomb core panel using the merger option within the solver. A merge failure force of $2.5 \cdot 10^{5} \mathrm{~N}$ was defined in each simulation to represent the weld failure and debonding of the back face sheet from the sandwich panel core that occurred in the experiments during impact. This force failure criteria was determined as $F=\sigma / A$ assuming the area of one line of the weld $(81.3 \mathrm{~cm} \times 0.1 \mathrm{~cm})$ with the material yield stress $310 \mathrm{MPa}$ for stainless steel. The honeycomb panel was modeled with a refined mesh in the $81.3 \mathrm{~cm} \times 81.3 \mathrm{~cm}$ center region of the honeycomb core to observe panel deformation. The $40.6 \mathrm{~cm} \times 40.6 \mathrm{~cm}$ center meshed region of the panel was refined even further to observe any core compression of the cells at the panel center. The sandwich panel FE model had a total of 
188,816 linear hexahedra elements and 253,290 nodes. The full FE model (Kolsky bar, test support base, bolts, acrylic spherical shells, and sandwich panel) consisted of 230,728 elements (432 linear pentahedra and 230,296 linear hexahedra elements) with 1,352,484 nodes.

Johnson-Cook parameters were used to define the material properties for the 304 stainless steel honeycomb panels $[83,84]$, the A-36 test support frame [85], the C-350 grade age hardened, maraging steel Kolsky bar [86, 87], and the carbon steel bolts [88]. The material constants and Johnson-Cook parameters are listed in Table 3.1. There was no fracture of the honeycomb panels or cells observed after testing. Therefore, a damage failure model was not included in the simulations (since the back face debonding was modeled with a merge failure criteria).

\subsection{Results}

\subsubsection{Sand front propagation}

Observations from the high-speed videos for the five honeycomb sandwich panel test shots confirm previous observations in Section 4.4.1 for the same series of five test shots on the equivalent mass solid plates. Examination of the sand shell expansion reveals the effect of increasing the granular shell mass, explosive driver mass, and granular particle density. For the five test shots presented here, the sand shell expansion captured by the high-speed videos after detonation is shown in Figure 5.7 for test Shots 1-3 (glass particles) and Figure 5.8 for test Shots 4 and 5 (zirconia particles). The images are captured approximately 250 and $650 \mu$ s after detonation. For consistency, $\mathrm{t}=0 \mathrm{~s}$ is defined as the moment of detonation for all tests. The first and second columns of Figure 5.7 show a decrease in the radial expansion rate of the sand front from Shot 1 to Shot 2, resulting from an increase in mass of the glass particles in the annular region around the explosive charge. Examination of the second and third columns in Figure 5.7 reveals that the expansion rate increases when the explosive charge mass is increased from 3 to $4.5 \mathrm{~kg}$ of C-4 (Shot 2 to 3) while the granular mass remained approximately the same. A more distinguishable sand fingering effect, shown in the insets of Figure 5.7(c), (f), and (i), develops at the leading edge of the faster expanding sand fronts of Shots 1 and 3 (i.e. higher velocity particles). These sand fingers develop as a result of instabilities between the sand/air interfaces, recently discussed by Kandan et al. [70]. 

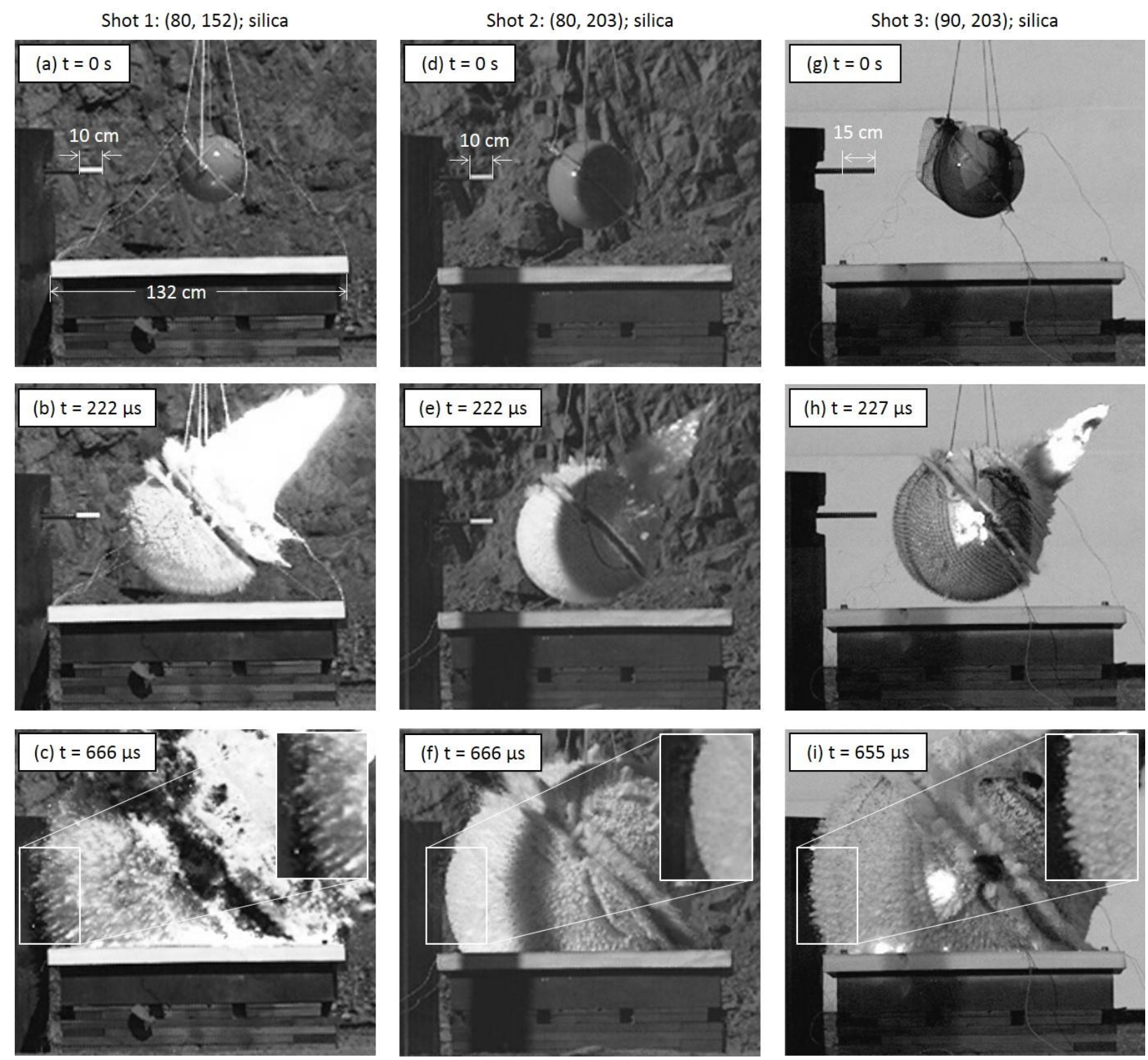

Figure 5.7. Silica glass microsphere sand shots with honeycomb sandwich panel target showing approximately equivalent time snapshots for Shot $1(R 1=80 \mathrm{~mm} ; R 2=152 \mathrm{~mm} ; 3 \mathrm{~kg}$ charge $)$ Shot $2(R 1$ $=80 \mathrm{~mm} ; R 2=203 \mathrm{~mm} ; 3 \mathrm{~kg}$ charge $)$ and Shot $3(R 1=90 \mathrm{~mm} ; R 2=203 \mathrm{~mm} ; 4.5 \mathrm{~kg}$ charge $)$.

Replacing the glass particles in Shot 3 (Figure 5.7 (g)-(i)) with denser (heavier mass) zirconia particles in Shot 4 (Figure 5.8(a)-(c)), while the test charge (4.5 kg C-4) and outer shell radius $\left(\mathrm{R}_{2}\right.$ $=203 \mathrm{~mm}$ ) remain the same, results in a slower expansion velocity of the sand front. Increases to the zirconia particle mass by increasing the outer shell radius to $\mathrm{R}_{2}=229 \mathrm{~mm}$ resulted in a further reduction of the particle expansion velocity. The magnified insets of Figure 5.8(c) and (f) reveal the slower sand front velocity of the zirconia particle test shots results in less observable sand 

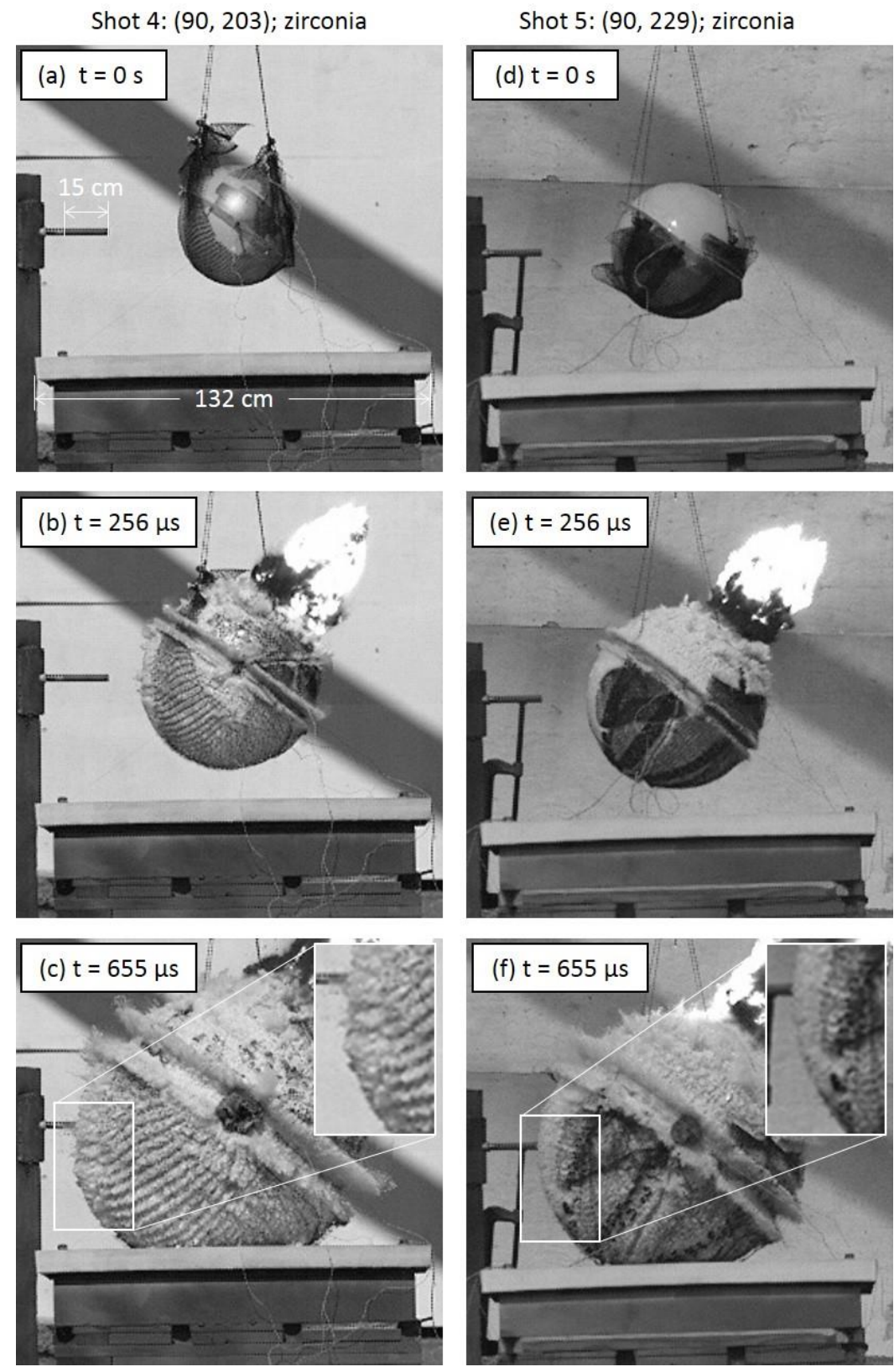

Figure 5.8. Zirconia sand shots with honeycomb sandwich panel target showing approximately equivalent time snapshots for Shot $4(R 1=90 \mathrm{~mm} ; R 2=203 \mathrm{~mm} ; 4.5 \mathrm{~kg}$ charge $)$ and Shot $5(R 1=90 \mathrm{~mm} ; R 2=229$ $\mathrm{mm} ; 4.5 \mathrm{~kg}$ (harge).

fingers at the forefront of the shell expansion compared to the higher velocity sand fronts of the three glass particle test shots, Figure 5.7(c), (f), and (i). The impact time of the main sand front (directly behind the sand fingers) on the Kolsky bar for each test shot is summarized in Table 5.3. The flange around the spherical test charge impedes the sand front expansion, but the orientation 
of the test charge is such that this had no effect on the loading of the Kolsky bars or sandwich panels.

While the observations from the high-speed videos are limited to viewing the outer sand front expansion and the developing sand fingers, the simulations allow for investigation of the sand particles propagation within the inner shell region and the development of the particle density and velocity gradients as the shell radially expands. The corresponding simulated images at $\mathrm{t}=0,250$, and $650 \mu \mathrm{s}$ as well as $2 \mathrm{~ms}$ are shown as cross sections through the center of the FE model in Figure 5.9 for the glass particle tests (Shots 1-3) and Figure 5.10 for the zirconia particle tests (Shots 4 and 5). The first three rows of images in Figure 5.9 correspond to the high-speed video images in Figure 5.7 for Shots 1-3. Consistent with the experimental observations discussed above, the particle expansion velocity is seen to decrease from Shot 1 to 2 with increased granular mass (outer shell diameter) and then increase from Shot 2 to 3 when the explosive charge (inner shell diameter) is increased. These changes in the particle expansion velocity are clearly seen in the third row of images in Figure 5.9 at $650 \mu \mathrm{s}$ after detonation at which the time the sandwich panel has begun to deform. The first three rows of Figure 5.10 correspond to the images in Figure 5.8. The slower expansion of the zirconia particle test shots observed by the high-speed videos is also seen in the simulated test for Shots 4 and 5 in Figure 5.10. Panel deformation is not yet initiated after $650 \mu \mathrm{s}$ with granular particle impacts on the test panel occurring just shortly before this time. The last row of images in Figure 5.9 and Figure 5.10 shows the panel deformation after $2 \mathrm{~ms}$ at which time the granular (sand) and HE particles have dispersed.

The simulations reveal that as the wet sand shell expands particle velocity and density gradients develop within the expanding annular sand region observed in the first three rows of Figure 5.9 and Figure 5.10 as the sand spreads radially outward. A densified region of air (not shown) develops in front of the expanding sand particles. This air shock impacts the Kolsky bar just prior to the first particle impacts. A region of fast, low density particles are seen at the outer edge of the expanding sand front in Figure 5.9 (at the air/sand interface) which correlates to the sand figures in Figure 5.7. These fast particles are followed by a main sand front region of increased density which is trailed by a region of dense, low velocity particles that are pushed by the HE particles. This ring of dense particles is clearly seen in the third row of images in Figure 5.9 at $\mathrm{t}=650 \mu \mathrm{s}$. At this time, impact of the sand particles with the honeycomb sandwich panel is 

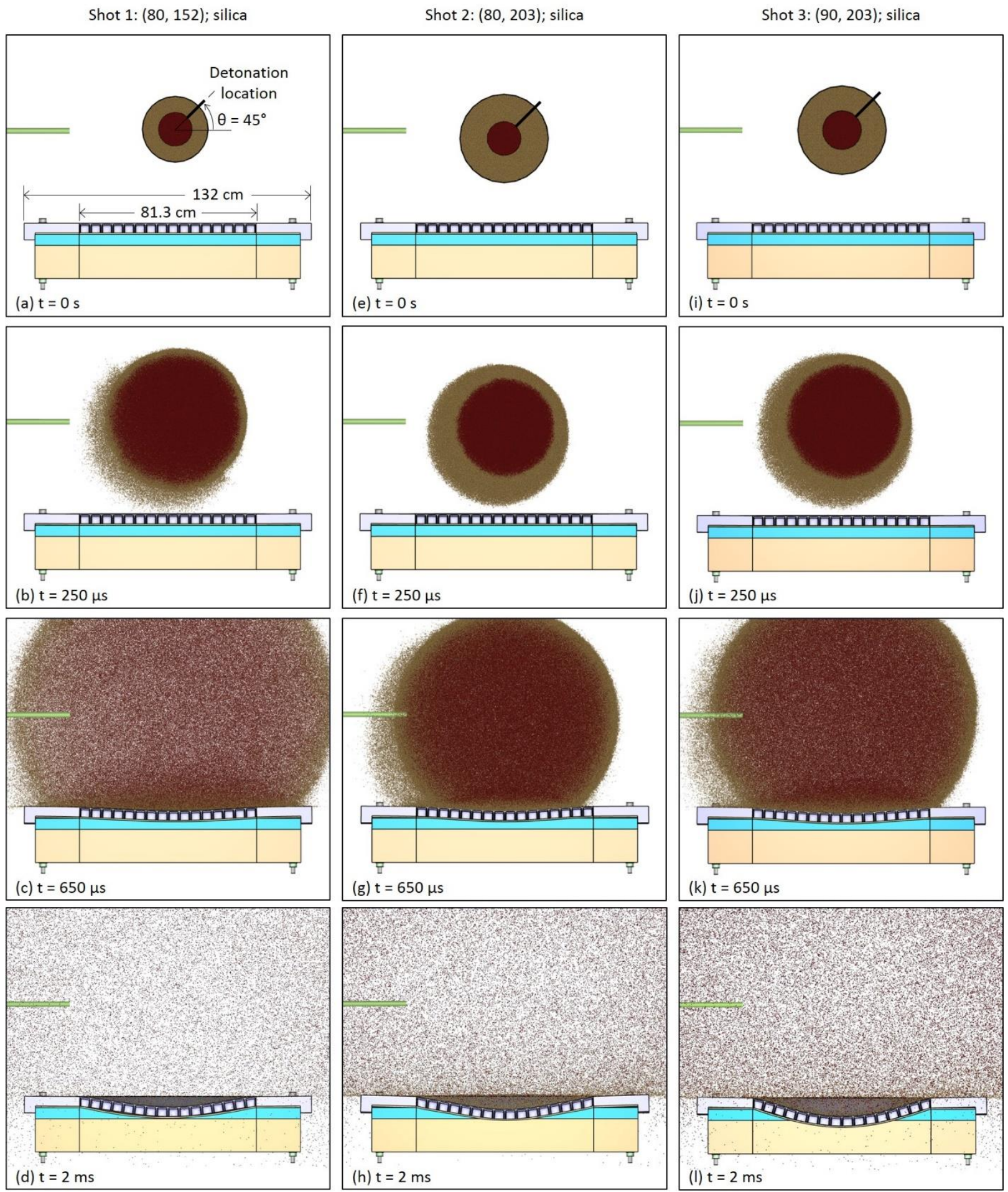

Figure 5.9. Simulated sand front propagation of the three silica test shots (Shots 1-3) for $t=0 \mathrm{~s}$ the moment of detonation, $t=250 \mu \mathrm{s}, t=650 \mu \mathrm{s}$, and $t=2 \mathrm{~ms}$. The sand particles are tan and the inner red particles are the $H E$. 
Shot 4: $(90,203)$; zirconia
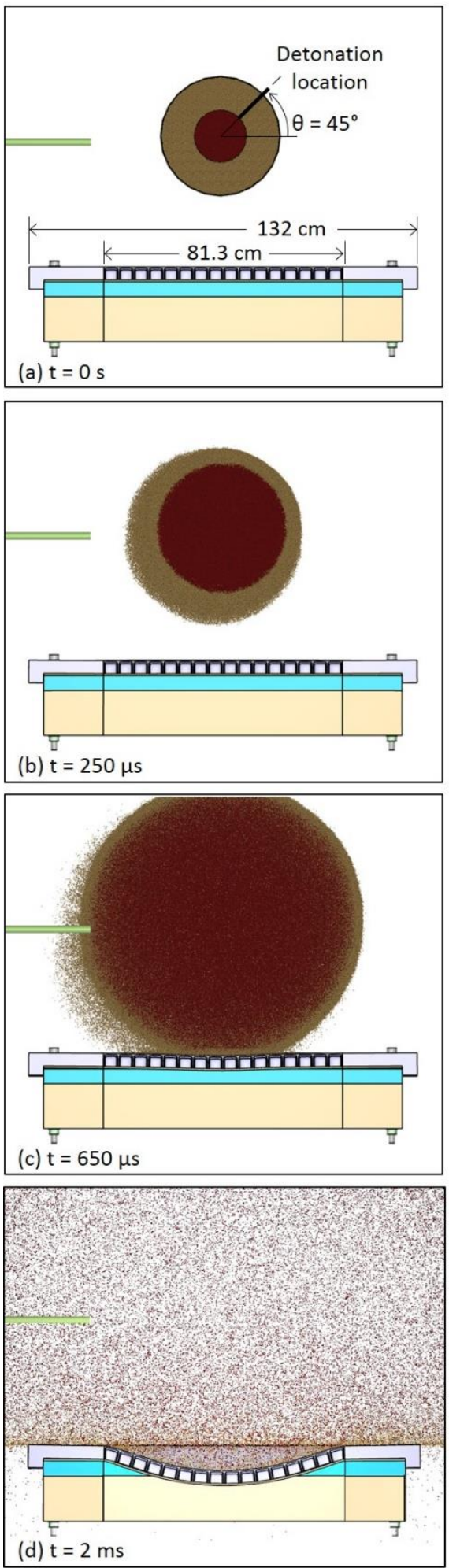

Shot 5: $(90,229)$; zirconia
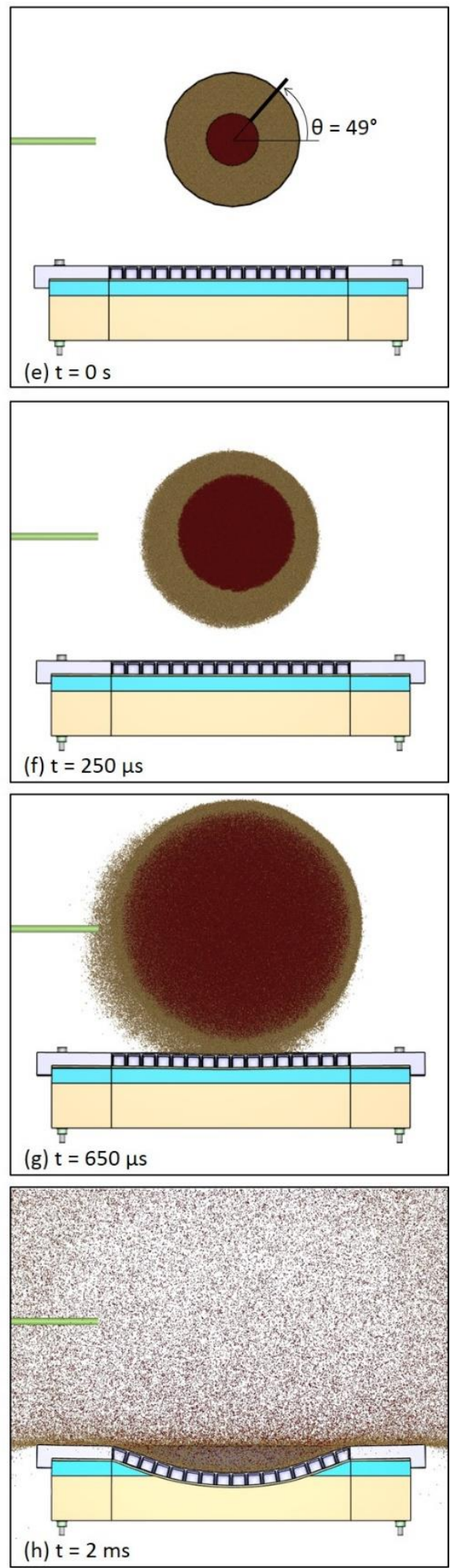

Figure 5.10. Simulated sand front propagation of the two zirconia test shots (Shots 4 and 5) for $t=0 \mathrm{~s}$ the moment of detonation, $t=250 \mu \mathrm{s}, t=650 \mu \mathrm{s}$, and $t=2 \mathrm{~ms}$. The sand particles are tan and the inner HE particles are red. 
also observed and panel deformation has already begun. The particles accumulate on the front face of the panels and are pushed laterally out along the panel surface by later arriving particles seen in Figure 5.9(c). The three regions of various particle density and velocity are also observed for the zirconia test shots Figure 5.10, with less particle dispersion after $650 \mu \mathrm{s}$, consistent with a less prominent sand fingering effect seen in Figure 5.8(c) and (f).

\subsubsection{Kolsky bar responses}

The sand front impact and loading response on the Kolsky bar for each test shot was measured by the strain gauges located $0.6 \mathrm{~m}$ from the impact end of the bar. The experimentally measured pressure-time waveforms and integrated impulse-time response for the test shots are shown in Appendix D. The observations from the strain gauge signal response is consistent with that from Chapter 4 (with the almost identical five charge configurations). The impulse-time response reveals four distinct regions of loading that correspond to variations in the pressure waveforms. As the spherical sand shell expanded radially, velocity and density gradients developed within the shell. The simulations in Section 5.4.1 revealed, a region of high velocity, low density particles at the leading edge of the sand front that was consistent with the sand fingers observed in the highspeed videos. These fast, low density particles are followed by the main sand front with an increased density trailed by a region of low velocity, high density sand particles pushed by the detonation products. Impacts by these different regions of varying particle velocities and densities correspond to the four distinct regions of loading identified in the Kolsky bar signal. The impulse reaches a plateau value as the latter arriving granular particles and HE products impact the Kolsky bar. The measured and simulated plateau impulse values are recorded in Table 5.5. In general, the simulations are seen to be in good agreement with the experimental results. The simulations show that the majority of the impulse applied to the Kolsky bar result from the granular media impacts with only a small contribution from the initial air shock and late arriving HE particles. 
Table 5.5. Experimental permanent Z-displacement for the sandwich panels from each test shot for both the integral corelfront face sheet structure and the back face, as well as the permanent and maximum simulation Z-displacement of the sandwich panels.

\begin{tabular}{ccccccc}
\hline $\begin{array}{c}\text { Test } \\
\text { shot }\end{array}$ & $\begin{array}{c}\text { Experimental } \\
\text { Kolsky bar } \\
\text { impulse } \\
(\mathrm{kPa} \cdot \mathrm{s})\end{array}$ & $\begin{array}{c}\text { Simulated } \\
\text { Kolsky bar } \\
\text { impulse } \\
(\mathrm{kPa} \cdot \mathrm{s})\end{array}$ & $\begin{array}{c}\text { Simulated } \\
\text { plate } \\
\text { impulse } \\
(\mathrm{kPa} \cdot \mathrm{s})\end{array}$ & $\begin{array}{c}\text { Experimental } \\
\text { plate } \\
\text { displacement } \\
\delta(\mathrm{cm})\end{array}$ & $\begin{array}{c}\text { Simulated } \\
\text { plate } \\
\text { displacement } \\
\delta(\mathrm{cm})\end{array}$ & $\begin{array}{c}\text { Experimental } \\
\text { back face } \\
\text { displacement } \\
\delta(\mathrm{cm})\end{array}$ \\
\hline 1 & 8.7 & 8.9 & 17.6 & 3.32 & 3.65 & 3.37 \\
2 & NA & 14.6 & 26.1 & 5.50 & 6.33 & 5.20 \\
3 & 19.5 & 19.4 & 28.3 & 6.35 & 7.23 & 5.90 \\
4 & 21.7 & 21.1 & 31.7 & 7.21 & 7.28 & 6.93 \\
5 & 23.1 & 22.5 & 33.7 & 8.17 & 8.24 & 7.49 \\
\hline
\end{tabular}

\subsubsection{Panel deflection}

An in-depth displacement mapping on the concave side of the deformed panels was performed at KVK Precision Technologies (Shenandoah, Virginia) for each of the square honeycomb sandwich panels. A coordinate measuring machine (CMM) measured the surface profilometry of the top face of the sandwich panels in the $\mathrm{X}-\mathrm{X}$ and $\mathrm{Y}-\mathrm{Y}$ directions. Contour plots of the permanent Z-direction displacement are shown for the sandwich panels in Figure 5.11 for the glass particle test shots and Figure 5.12 for the zirconia particle test shots. The simulated panel contour plots after $20 \mathrm{~ms}$ are also shown for comparison. At this time, the oscillatory response of the test panels has sufficiently dampened to a final permanent plastic deformation. The $81.3 \mathrm{~cm} \mathrm{x} 81.3 \mathrm{~cm}$ center region of the honeycomb cell pattern is indicated for all the images along with the $40.6 \mathrm{~cm} \times 40.6$ $\mathrm{cm}$ region of further refined mesh on the simulated images. For consistency, the same 0 to $8.0 \mathrm{~cm}$ Z-direction deformation scale is used for all plots. The permanent plastic $\mathrm{Z}$ displacement of the measured and simulated panels is summarized in Table 5.5. The panel deflections increased from Shot 1 to Shot 5, consistent with an increasing impulse applied to the test panels. During testing, the electron beam welds securing the back face sheet to the honeycomb sandwich panel core failed and the back face sheet debonded from the core. The final permanent deflection of the back face sheet was also measured using the CMM and is recorded in Table 5.5. These deflections were less than the permanent displacement measured for the top face sheet of the sandwich panels, suggesting core compression. However, the final core height was easily measured after testing (since the back face sheet was no longer attached), and these measurements revealed that there was only a slight compression of the core during the impulsive loading (detailed below). 
Experimental displacement

(a) Shot 1

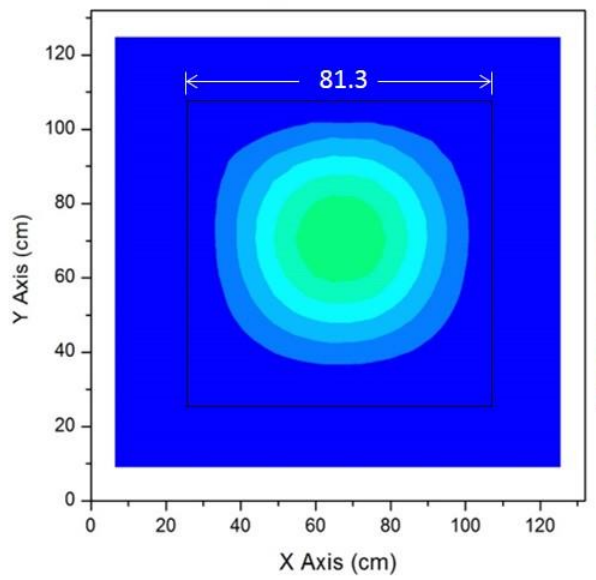

(b) Shot 2

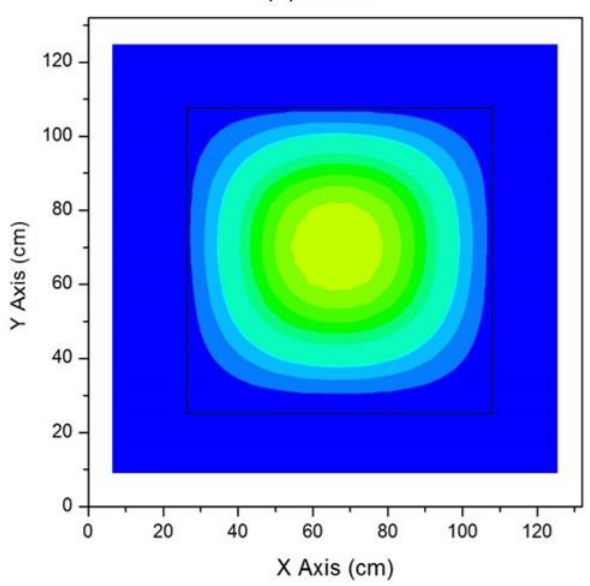

(c) Shot 3

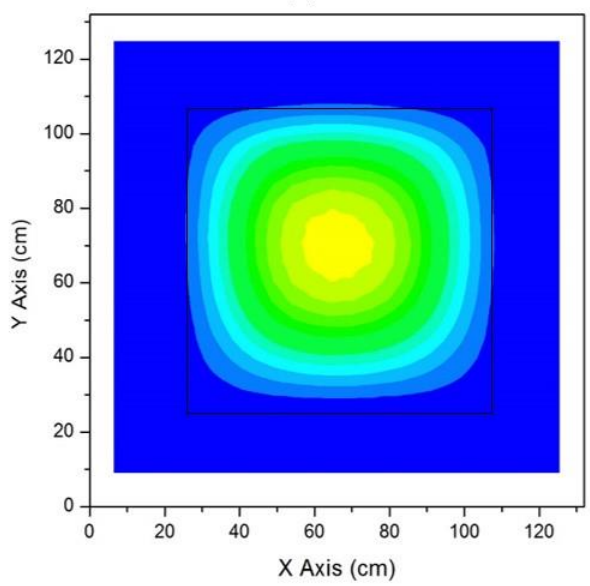

Simulated displacement

(d) Shot 1

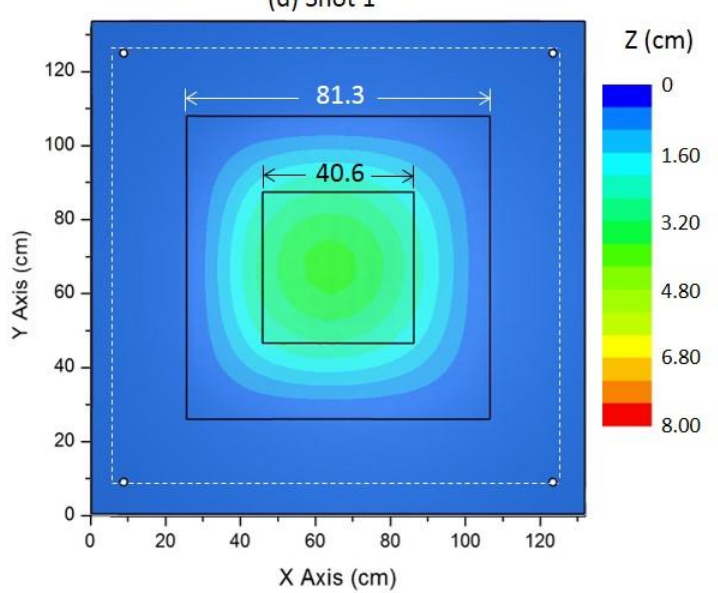

(e) Shot 2

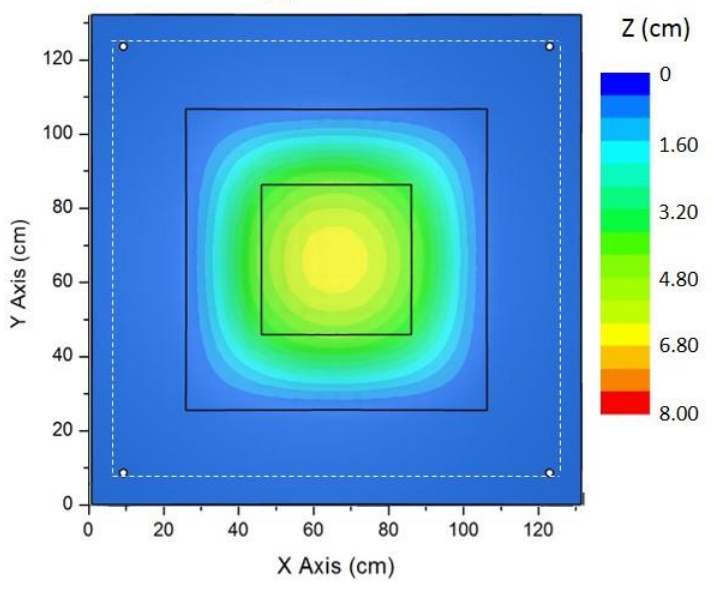

(f) Shot 3

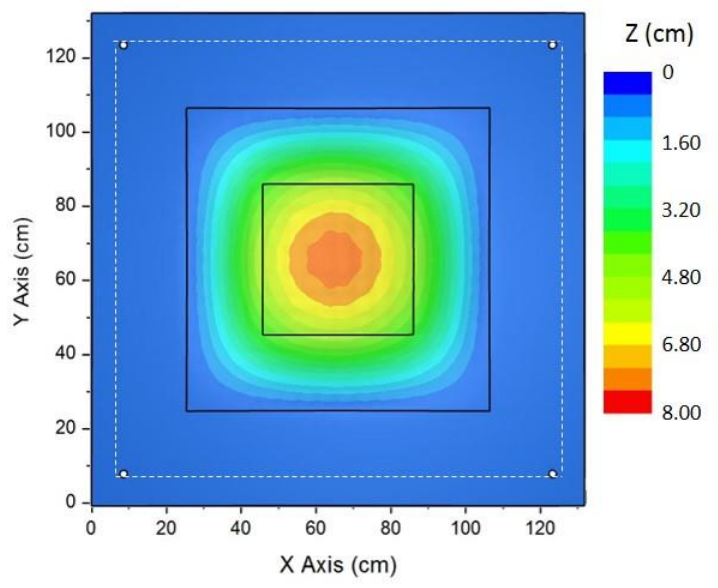

Figure 5.11. Test panel permanent plastic displacement contour plot in the $Z$ direction for the silica test shots (Shots 1-3). Experimental profilometry results are shown in (a), (b) and (c). The simulated responses at $t=20 \mathrm{~ms}$ are shown in $(d),(e)$, and $(f)$. The larger black box indicates the underlying support base and region of honeycomb cells. The inner black box indicates the refined mesh region in the FE simulated model. 
Experimental displacement

(a) Shot 4

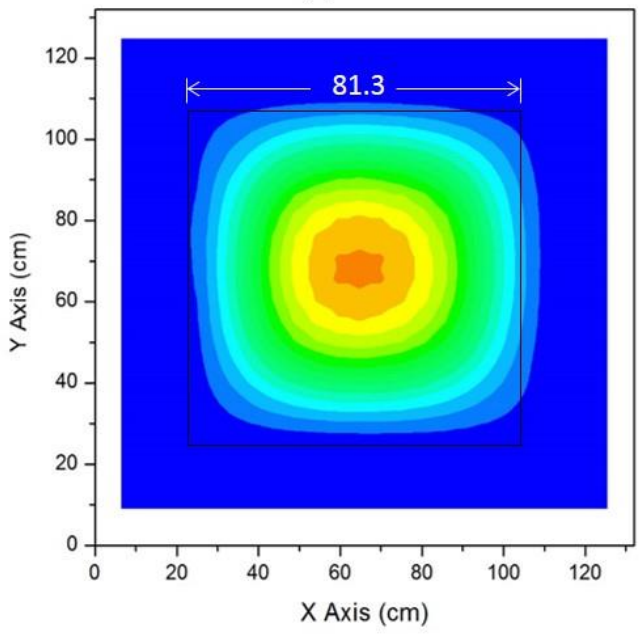

(b) Shot 5

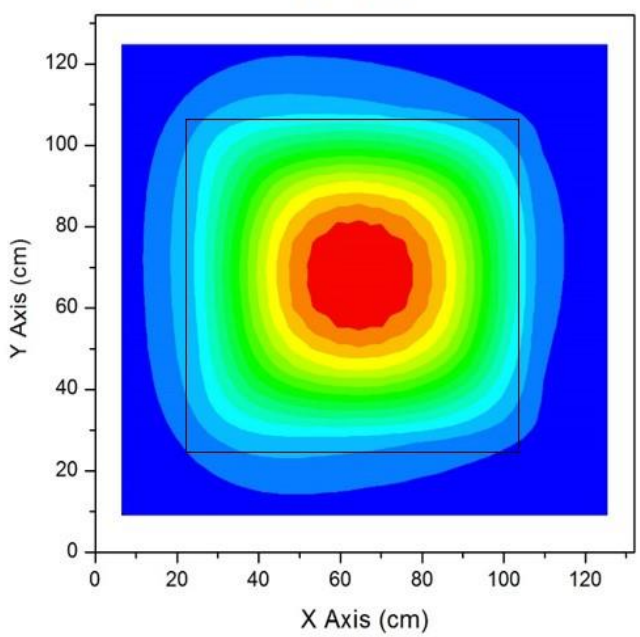

Simulated displacement

(c) Shot 4

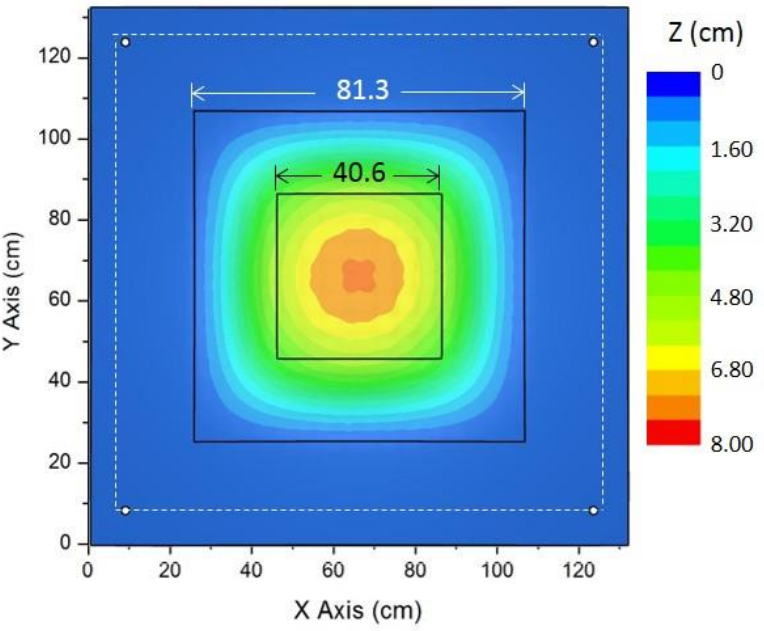

(d) Shot 5

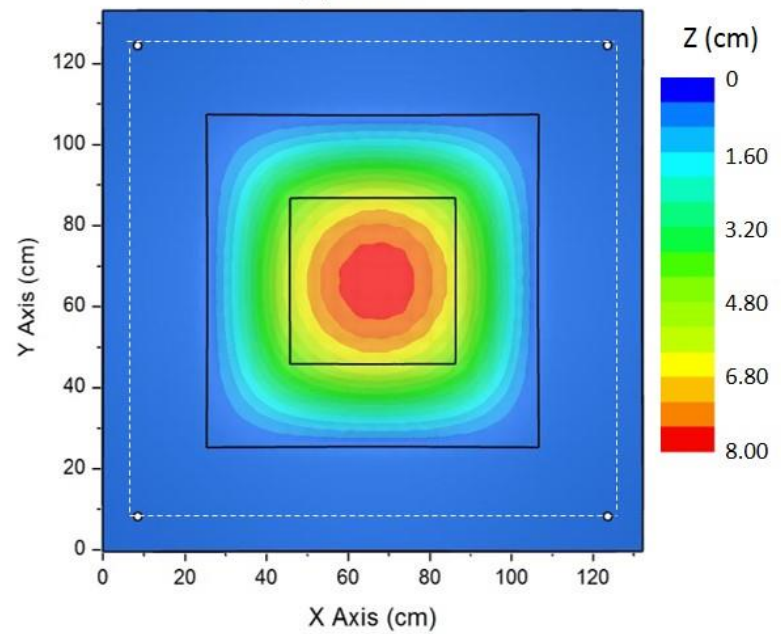

Figure 5.12. Test panel permanent plastic displacement contour plot in the $Z$ direction for the zirconia test shots (Shots 4 and 5). Experimental profilometry results are shown in (a) and (b). The simulated responses at $t=20 \mathrm{~ms}$ are shown in $(c)$ and $(d)$. The larger black box indicates the underlying support base and region of honeycomb cells. The inner black box indicates the refined mesh region of the FE model. 
(a) Shot 1: $(80,152)$

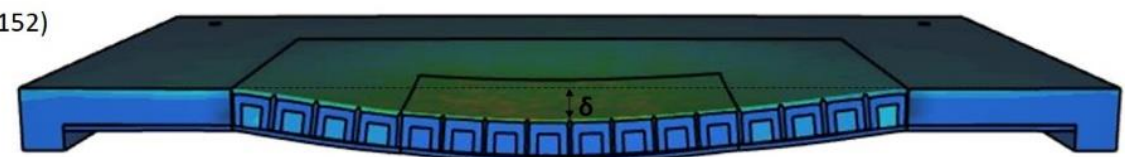

(b) Shot 2: $(80,203)$

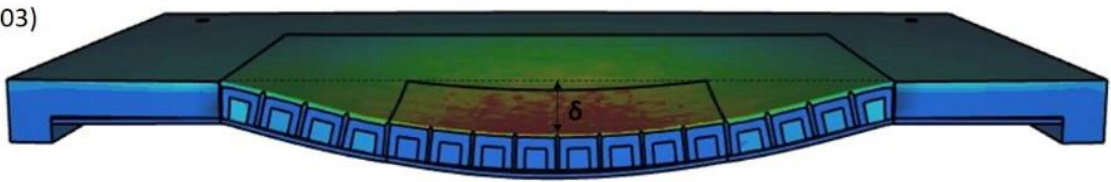

(c) Shot 3: $(90,203)$

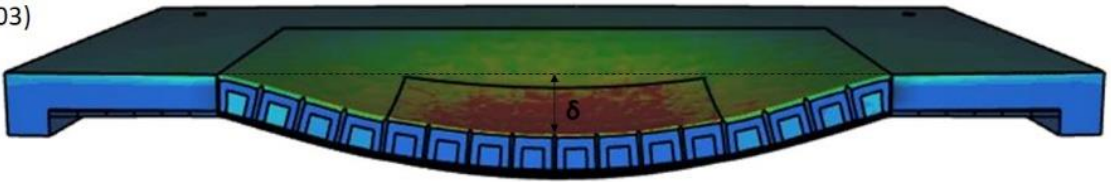

(d) Shot 4: $(90,203)$ zirconia
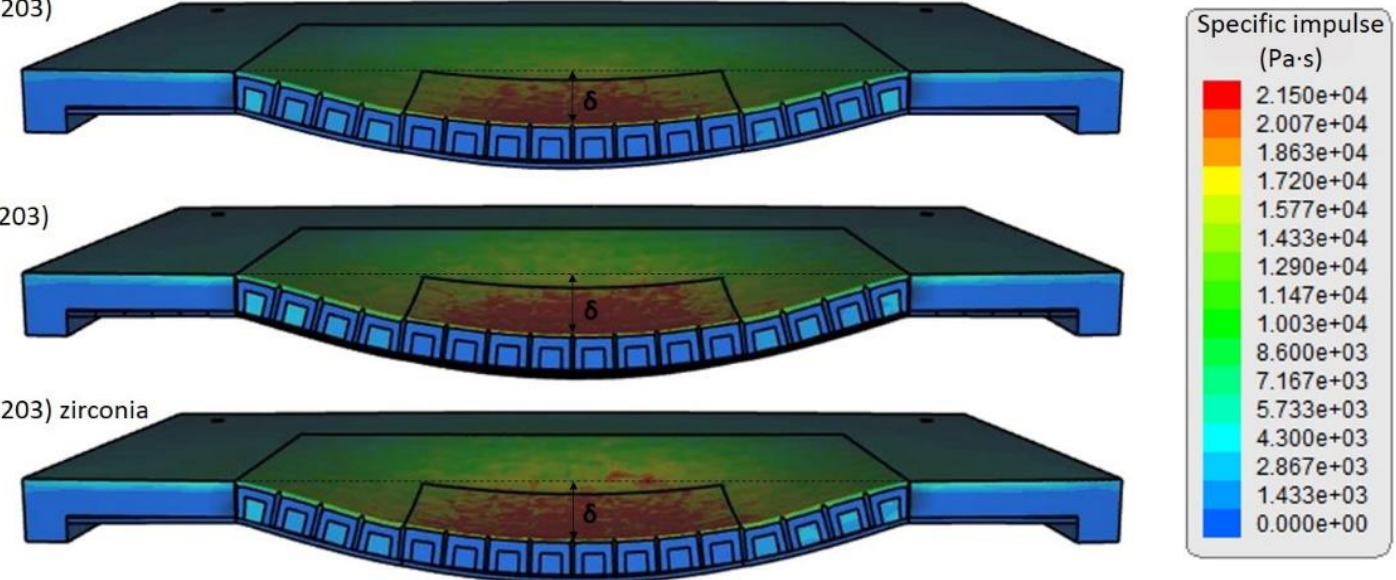

(e) Shot 5: $(90,229)$ zirconia

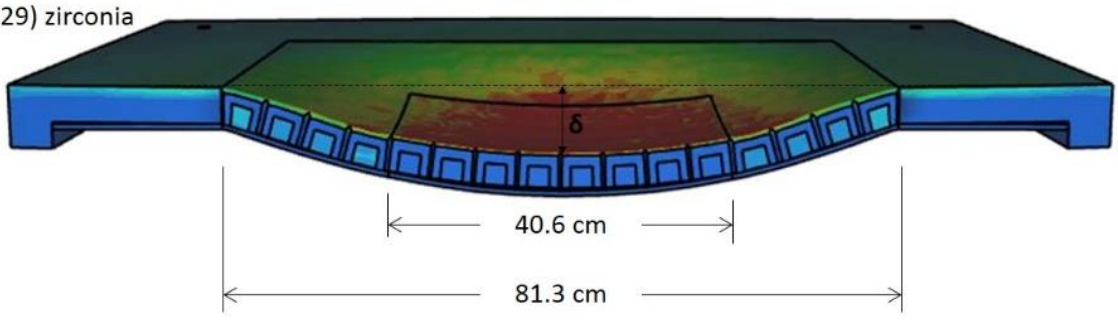

Figure 5.13. The cross section of the sandwich panels shows the specific impulse distribution applied to the surface by the five test shots. The $81.3 \mathrm{~cm}$ wide region of the honeycomb cells is indicated along with the refined mesh center region of $40.6 \mathrm{~cm}$. The permanent deflection $(\delta)$ is also shown for each test.

While the Kolsky bar offers the only measure of estimating the pressure and impulse applied to the test panels during the event, the shifts in the test charge location and orientation prior to detonation complicates the accuracy to which this represents the loading experienced by the panels. The simulations allow for measurement of the maximum specific impulse applied to the top face of the panels. These peak impulse values are summarized in Table 5.5 along with the permanent panel deflections. Figure 5.13 shows the blast impulse loading on cross sections for the sandwich panels for the five test shots. These cross sections show the increasing peak impulse applied to the panels consistent with increased impulse for each test shot from Shot 1-5. The debonded back face sheet is also shown no longer attached (merged) to the sandwich panel core. The impulse transferred to the front fact (impact surface) of the sandwich panels was greater than 
that transferred to the top surface of the solid plates. The front face (impact surface) of the sandwich panel was $2.54 \mathrm{~cm}$ closer to the center of the test charge than the top of the solid plates.

The final total strain in the $\mathrm{X}$ and $\mathrm{Y}$ directions, $\varepsilon_{X X}$ and $\varepsilon_{Y Y}$, on the sandwich panel after 20 $\mathrm{ms}$ for test Shot 3 is shown in Figure 5.14. The greatest strain is seen above the inner edge of the steel picture frame support within the center area containing the cell matrix of the honeycomb sandwich panel. The majority of this strain is within the unsupported $81.3 \mathrm{~cm}$ span of the honeycomb panel with only a small amount of in-plane plastic pull-in strain along the outer edge. Three nodes are indicated in Figure 5.14 (corresponding to those on the solid plate in Figure 4.19(a)). The final total strain in the Y-direction, $\varepsilon_{Y Y}$, at these node locations is summarized in Table 5.6 along with their maximum and permanent (after $20 \mathrm{~ms}$ ) displacement magnitude. Similar to the observations for the solid plates, the strain increases with increasing impulse loading from Shot 1 to Shot 5. However, the region contributing to the in-plane stretching from the back supported edge of the plate was much less for the sandwich panels (Figure 5.14) than the solid plates (Figure 4.19(a)). The maximum displacement magnitude, $\delta_{\max }$, measured for node 3 (after impact by Shot 3) on the honeycomb sandwich panel (Table 5.6) was approximately 40\% less than that measured for the solid plate (Table 4.8). This indicates there was less edge material stretching contributing to the final permanent displacement of the sandwich panels than was previously observed for the solid plates.

The permanent plastic deflection of the panels (Table 5.5) is normalized by their half span L $(2 \mathrm{~L}=81.3 \mathrm{~cm})$. The normalized impulse, $I_{o} / \mathrm{m}_{\mathrm{b}} \sqrt{\sigma_{\mathrm{Y}} / \rho_{\mathrm{m}}}$, is a function of the maximum impulse applied to the panel, $I_{O}$ (Table 5.5), the areal mass of the sandwich panel, the panel material yield strength, $\sigma_{\mathrm{Y}}=310 \mathrm{MPa}$, and material density $\rho_{\mathrm{m}}=7900 \mathrm{~kg} / \mathrm{m}^{3}$. The areal mass of the sandwich panel, $\mathrm{m}_{\mathrm{b}}=\rho_{\mathrm{m}} t_{f}+\rho_{\mathrm{m}} t_{b}+\rho_{c} H_{c}$, is $199.5 \mathrm{~kg} / \mathrm{m}^{2}$ and depends on the thickness and material density of the two face sheets as well as the core height and density. Figure 5.15 shows the dimensionless plot of deflection as a function of the impulse. For comparison, the results from Section 4.4.3 for the five solid plates are also shown (normalized by the $81.3 \mathrm{~cm}$ center span). Recall, the front (impact) face of the honeycomb sandwich panels was located $2.54 \mathrm{~cm}$ closer to the center of the test charges than the top of the solid test plates. This resulted in the sandwich panels experiencing a larger impulsive loading compared to the solid test plates for the same five charge configurations. There were no reductions observed in the transferred impulse to the 


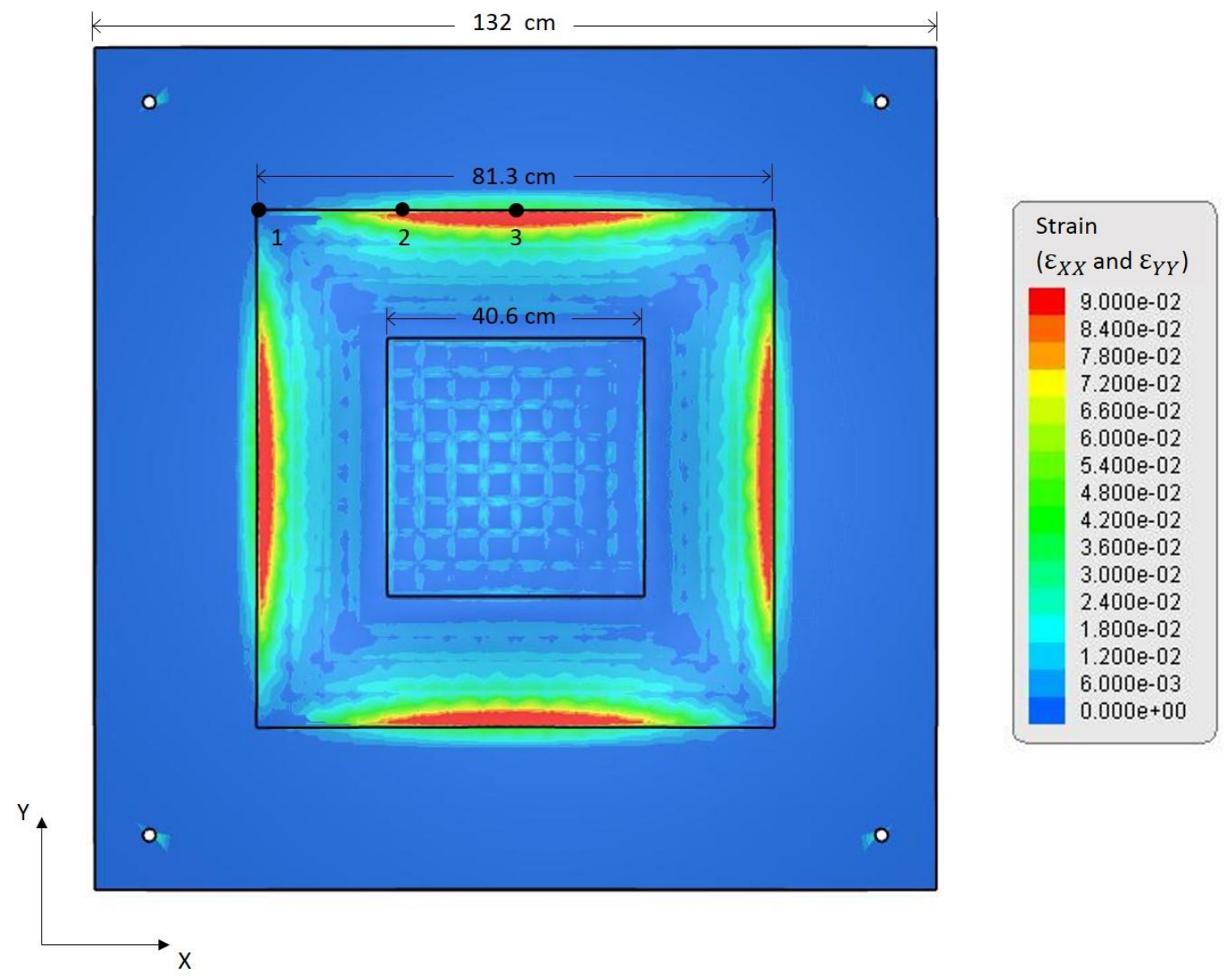

Figure 5.14. Strain in the $X$ and $Y$ directions $\left(\varepsilon_{X X}\right.$ and $\left.\varepsilon_{Y Y}\right)$ on the top face of the sandwich panel for Shot 3 at $t=20 \mathrm{~ms}$.

Table 5.6. The maximum out of plane dynamic $\left(\delta_{\max }\right)$ and permanent $(\delta)$ displacements along with the $Y$ direction tensile strains at nodes 1,2 , and 3 . The permanent $Y$ displacement $\left(\delta_{Y}\right)$ for node 3 determined at $20 \mathrm{~ms}$ after detonation is also listed.

\begin{tabular}{|c|c|c|c|c|c|c|c|c|c|c|}
\hline \multirow[b]{2}{*}{$\begin{array}{l}\text { Test } \\
\text { shot }\end{array}$} & \multicolumn{3}{|c|}{ Node 1} & \multicolumn{3}{|c|}{ Node 2} & \multicolumn{4}{|c|}{ Node 3} \\
\hline & $\begin{array}{l}\delta_{\max } \\
(\mathrm{mm})\end{array}$ & $\begin{array}{c}\delta \\
(\mathrm{mm})\end{array}$ & $\varepsilon_{Y Y}$ & $\begin{array}{c}\delta_{\max } \\
(\mathrm{mm})\end{array}$ & $\begin{array}{c}\delta \\
(\mathrm{mm})\end{array}$ & $\varepsilon_{Y Y}$ & $\begin{array}{l}\delta_{\max } \\
(\mathrm{mm})\end{array}$ & $\begin{array}{c}\delta \\
(\mathrm{mm})\end{array}$ & $\varepsilon_{Y Y}$ & $\begin{array}{c}\delta_{Y} \\
(\mathrm{~mm})\end{array}$ \\
\hline 1 & 3.39 & 0.34 & 0 & 4.39 & 0.53 & 0.01 & 5.03 & 0.75 & 0.02 & 0.44 \\
\hline 2 & 4.45 & 0.54 & 0 & 5.49 & 1.00 & 0.02 & 5.81 & 1.36 & 0.04 & 1.33 \\
\hline 3 & 4.56 & 0.60 & 0 & 5.99 & 1.08 & 0.03 & 6.50 & 1.53 & 0.05 & 1.50 \\
\hline 4 & 5.11 & 0.89 & 0 & 5.98 & 1.22 & 0.03 & 6.41 & 1.65 & 0.05 & 1.62 \\
\hline 5 & 5.22 & 0.83 & 0 & 6.13 & 1.40 & 0.04 & 6.57 & 2.06 & 0.06 & 2.06 \\
\hline
\end{tabular}




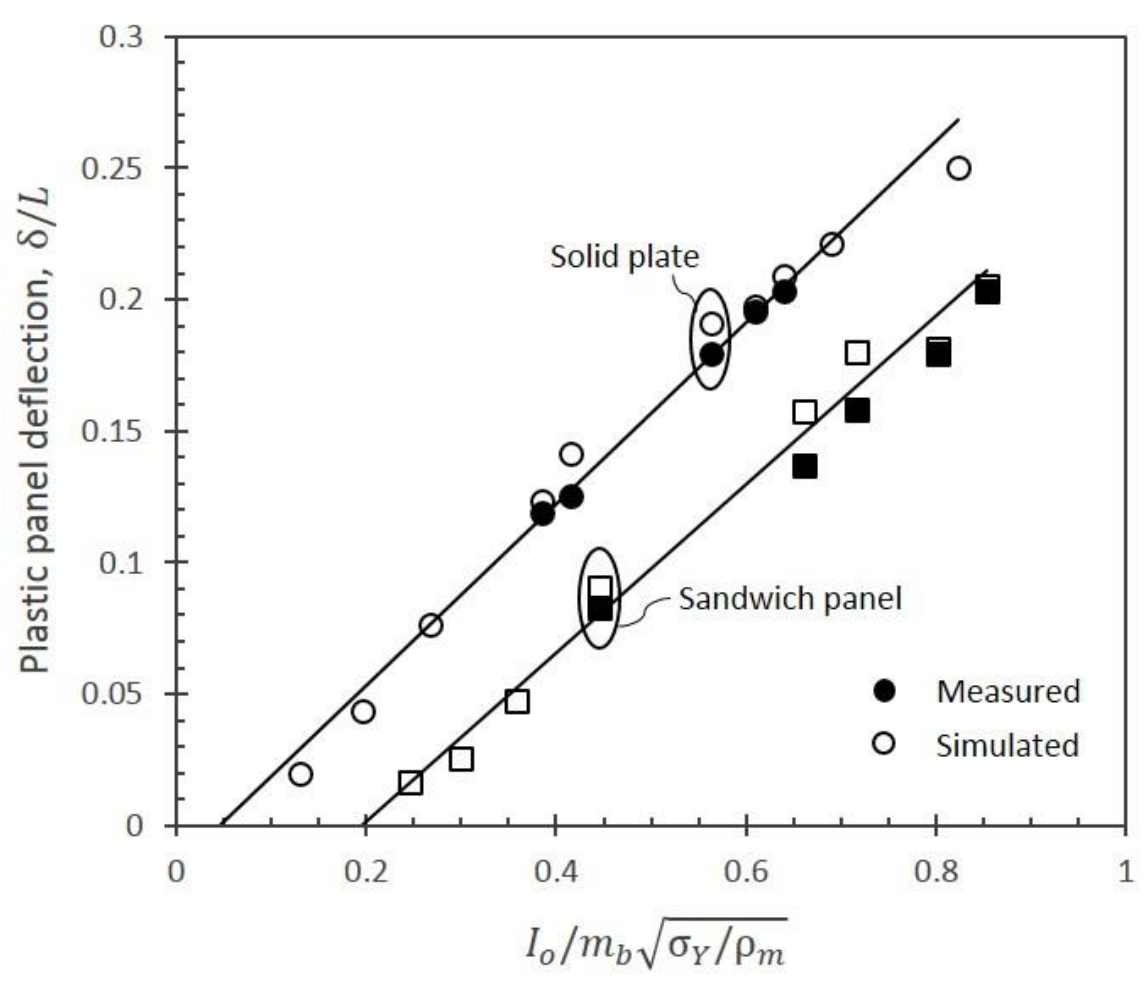

Figure 5.15. Normalized plot of center permanent deflection versus the impulse comparing the solid plates and the honeycomb sandwich panels (measured solid circle and square and simulated open circle and square). The deflection is normalized by the panel span $2 L=81.3 \mathrm{~cm}$.

sandwich panel resulting from an FSI effect. Simulations were performed with larger impulse loading conditions on the solid plates to compare their response to the sandwich panels at equivalent impulsive loading conditions. Several smaller impulse loading conditions were also simulated in order to observe the deformation as the impulse decreased to zero. Comparing the solid plate and sandwich panel permanent normalized deflections as a function of the applied impulse, the honeycomb sandwich panel design outperformed the solid plate for each of the impulse loading conditions presented here.

The core compression, $\Delta \mathrm{H}_{\mathrm{c}}$, is the difference between the initial core height of $\mathrm{H}_{\mathrm{c}}=36.5 \mathrm{~mm}$ and the final core height in the location of maximum out of plane deflection measured after testing (accessible since the back face sheets debonded from the integral core) and in the simulations at $t$ $=20 \mathrm{~ms}$ after detonation. The measured and simulated core compression summarized in Table 5.7 increased from Shot 1 to 5 as the impulse applied to the test plates increased. The core compression strain $\varepsilon_{\mathrm{c}}=\Delta \mathrm{H}_{\mathrm{c}} / \mathrm{H}_{\mathrm{c}}$ for each shot is also listed in Table 5.7. The measured core compression varied 
Table 5.7. The measured and simulated (after $20 \mathrm{~ms}$ ) core compression (change in core height) and the compression strain for each test shot.

\begin{tabular}{ccccc}
\hline $\begin{array}{c}\text { Test } \\
\text { shot }\end{array}$ & $\begin{array}{c}\text { Measured } \\
\text { core compression } \\
\Delta \mathrm{H}_{\mathrm{c}}(\mathrm{mm})\end{array}$ & $\begin{array}{c}\text { Simulated } \\
\text { core compression } \\
\Delta \mathrm{H}_{\mathrm{c}}(\mathrm{mm})\end{array}$ & $\begin{array}{c}\text { Measured Core } \\
\text { compression strain } \\
\varepsilon_{\mathrm{c}}=\Delta \mathrm{H}_{\mathrm{c}} / \mathrm{H}_{\mathrm{c}}(\%)\end{array}$ & $\begin{array}{c}\text { Simulated Core } \\
\text { compression strain } \\
\varepsilon_{\mathrm{c}}=\Delta \mathrm{H}_{\mathrm{c}} / \mathrm{H}_{\mathrm{c}}(\%)\end{array}$ \\
\hline 1 & $0.40 \pm 0.79$ & 0.65 & 1.12 & 1.78 \\
2 & $0.79 \pm 0.79$ & 0.95 & 2.27 & 2.60 \\
3 & $1.19 \pm 0.79$ & 1.09 & 3.45 & 2.99 \\
4 & $1.59 \pm 0.79$ & 1.26 & 4.65 & 3.45 \\
5 & $1.98 \pm 0.79$ & 1.38 & 5.88 & 3.78 \\
\hline
\end{tabular}

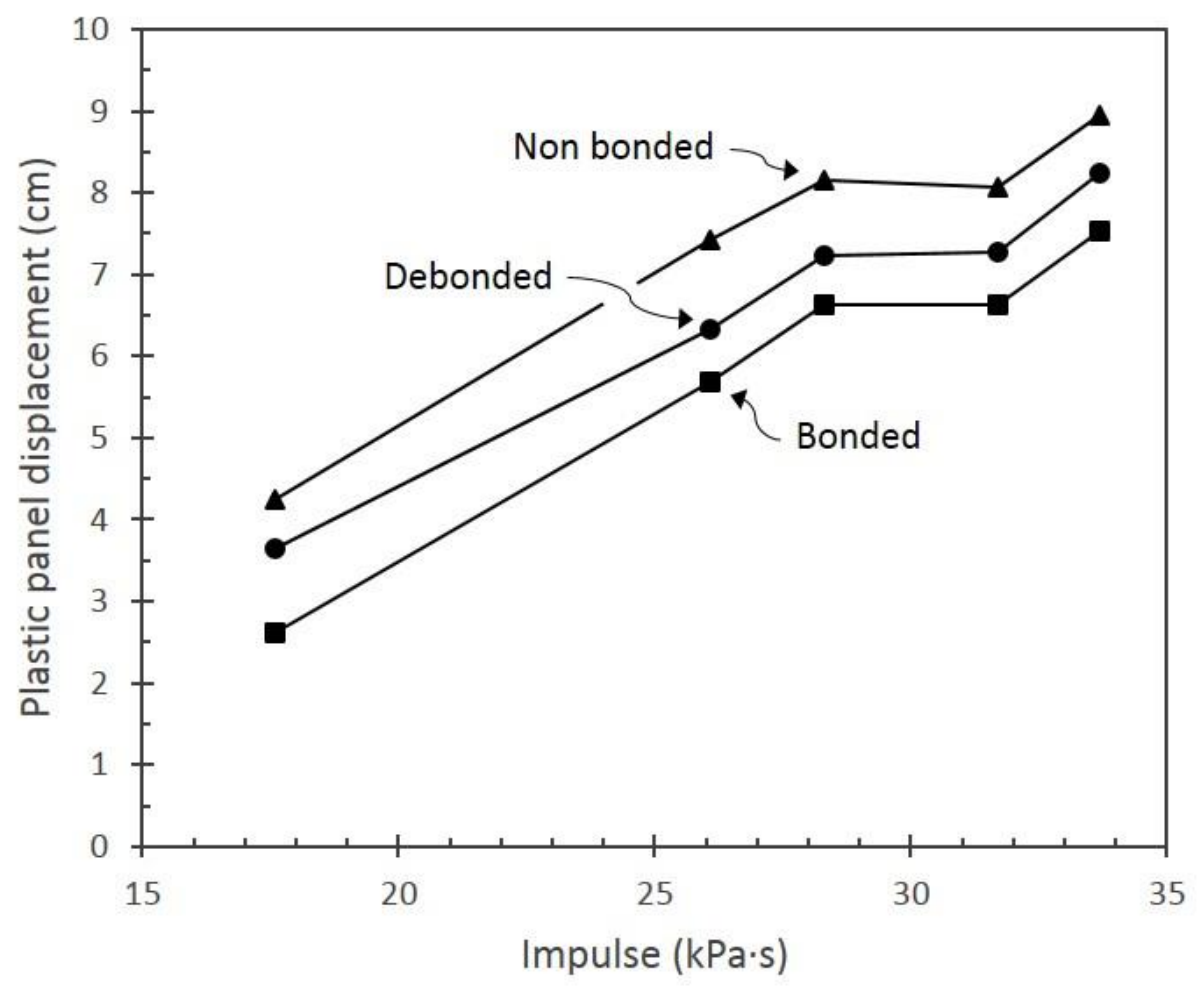

Figure 5.16. Simulated panel displacement for different cases of back face sheet bonding to the sandwich panel. Case 1: A merge failure allowed debonding of the back face sheet during impact. Case 2: A merged sandwich panel where the back face sheet remained bonded ("welded") to the sandwich panel for all high intensity tested impacts. Case 3: The back face sheet was never connected (non bonded) to the sandwich panel. 
from 0.40 to $1.98 \pm 0.79 \mathrm{~mm}$ from Shot 1 to 5 . For each test shot, there was minimal core crushing observed after testing; the measured core compression was less than $2 \mathrm{~mm}$ with less than $6 \%$ core compression strain for all test shots. The simulated core compression varied from 0.65 to 1.38 $\mathrm{mm}$ with less than $4 \%$ core compression strain. For each test shot, the simulated values were within the margin of error of the measured core compression values. This minimal core compression indicates the high strength of the square honeycomb sandwich panel core and that the benefit of the panel design results from the increased panel stretching resistance.

The electron beam welding attaching the back face sheet to the sandwich panel core failed during testing. Recall that to predict this response, the simulations were modeled with a merge failure criteria. During the simulations (modeled with the merge failure), the back face sheet debonded from the integral honeycomb sandwich panel core early in the loading process. To examine the effect of the back face sheet debonding on the permanent deformation, two other cases of simulations were modeled. A second case (Case 2: bonded) was modeled in which the back face remained merged (welded) to the sandwich panel core for the duration of the testing and no debonding occurred. A third case (Case 3: non-bonded) was modeled such that the back face sheet was never merged (welded) to the sandwich panel. The results for the permanent plastic deflection for these three cases where the back face sheet was either dedonded, bonded, or non-bonded to the sandwich panel core during high intensity granular impacts is shown in Figure 5.16. For each test shot, the bonded sandwich panel (in which no weld failure occurred) outperformed the panel when a merge failure was included in the model to allow the back face sheet to debond during loading (as observed in the experimental testing). However, simulations with a non-bonded back face sheet revealed that the debonded case resulted in less permanent deflection than if the back face was never bonded to the sandwich panel.

\subsection{Conclusions}

This study analyzed the five shots tested on the square honeycomb sandwich panel with comparisons drawn to the previous chapter for almost identical charge configurations tested on equivalent mass solid plates. The radial expansion for each test charge was examined and observations corresponded to those made in Chapter 4. The pressure and impulse applied to the Kolsky bar again revealed four regions of granular media impacts on the front of the bar, 
corresponding to different regions of particle velocity and density gradients that develop within the expanding sand shell.

A comparison of the deformation of solid plates and equivalent honeycomb sandwich panels impacted by the same test shots indicated a substantial benefit of the sandwich panel design for reduction of panel deflection under equivalent loading conditions. Minimal core compression was observed for the sandwich panels due to the high strength of the core. Even though the rear face sheet of the sandwich panel debonded early in the loading process, most of the stiffness of the design was retained, and the benefit of this sandwich panel design resulted from its resistance to bending rather than changes to the transferred impulse. 


\section{Chapter 6. Momentum transfer during the impact of granular matter with inclined sliding surfaces}

This chapter is based upon a recently published paper in the Journal of the Mechanics and Physics of Solids ${ }^{3}$. It explores ways in which the impulse applied by the impact of granular matter with a structure can be reduced by first changing its inclination and then adding a sliding structure to reduce frictional interactions between granular media and the structure. The study presented here experimentally compares the impulse transferred to three target types as a function of the distance between the sand surface and the target (to vary the average sand velocity, its applied pressure, and the duration of the impact process). Two of the targets are used to assess the effect of surface inclination on the transferred impulse. Then, a novel target design is presented in which a sacrificial sliding plate attached to an inclined surface is allowed to freely move along the inclined lubricated surface during granular media impact. A vertical impulse measurement test apparatus is used to measure the applied pressure and impulse transferred to the targets. Highspeed video imaging is used to observe the sand/target interaction and motion of the sliding plates

after ejection from the targets. Discrete particle-based simulations implemented in the IMPETUS Afea simulation code [50] are used to analyze the experiments and clarify the impulse mitigation mechanism.

\footnotetext{
${ }^{3}$ A. Kyner, V. Deshpande, H. Wadley, Momentum transfer during the impact of granular matter with inclined sliding surfaces, Journal of the Mechanics and Physics of Solids, (2017).
} 


\subsection{Introduction}

As discussed in Chapter 2, numerous concepts have been proposed for mitigating the effects of soil impact with the underside of vehicles $[15,17,36,37,58]$. These included increasing the standoff distance by raising the height of a vehicle above the surface. This reduces the applied impulse as the sand density decreases as it expands both axially and laterally, and as air drag decelerates the soil particles before they reach the structure $[13,54,78,97]$. The use of a V-shaped hull has also been shown to reduce the fraction of momentum that is transferred to an impacted surface $[12,71-75]$. Combinations of these approaches, when combined with the use of damage resistant materials and structures are widely used to reduce the damage caused by shallow buried landmines [76].

Recent laboratory studies of Uth et al. [78] and Goel et al. [98] have sought to gain a deeper understanding of granular matter/structure interactions with inclined surfaces. These studies (discussed in Section 2.3.2) enabled determination of the transmitted impulse, $I_{T}$, to rigidly clamped flat surfaces inclined at either $\alpha=90^{\circ}$ (perpendicular) or $45^{\circ}$ to the slug propagation direction, Figure 2.3(a). The results confirmed that the impulse transferred to an inclined target was significantly reduced from a normally oriented target. However, the transferred momentum was shown to depended not only upon the orientation of the impacted surface, but also depended upon the frictional interaction between the granular particles and this surface. Smooth/hard surfaces with the lowest friction coefficient acquired less momentum at a fixed inclination than rough surfaces. However, the momentum transferred in all cases (even for the smooth/hard surfaces) exceeded that predicted to be transferred to a frictionless target of the same inclination angle. This extra transferred momentum resulted from frictional particle interactions with the impact surface which reduced their velocity and transferred more of their momentum to the inclined structure. The study described here explores if it is possible to avoid this frictional interaction and thereby reduce the fraction of momentum transferred.

The idea to be explored here is the use of a sacrificial thin plate attached to an inclined surface by a low friction lubricant (ideally a shear thinning fluid). When granular matter from a planar buried explosive event makes impact with the inclined sacrificial plate, it is able to slide over the lubricated surface of a target to which it is attached. The hypothesis is that when the sliding plate's exterior surface is impacted by soil, it is accelerated, and acquires a momentum with resolved 
components perpendicular and parallel to the plate surface. The latter induce plate sliding across a very low friction coefficient interface, thereby reducing the momentum transferred to the underlying structure. Figure 6.1(b) shows a V-shaped structure with two inclined surfaces equipped with lubricated sliding plates. Impact of sand with these plates transfers momentum, enabling the sliding of the plate along the surface of the underlying target with momentum $P_{\text {plate }}$. In the limiting frictionless interface scenario, the final momentum of the granular matter and sliding plate combination (parallel to the inclined surface) approaches $I_{O} \cos \alpha$ while the impulse transmitted to the sample (perpendicular to its inclined surface) is $I_{T}=I_{O} \sin \alpha$. Since the vertical component of the reaction momentum of the target is $I_{T}^{Z}=I_{T} \sin \alpha$, the vertical impulse component is $I_{T}^{Z}=I_{O} \sin ^{2} \alpha$ which is approximately $I_{O} / 2$ for $\alpha=45^{\circ}$.

The study uses a recently developed vertical impulse measurement facility [10, 22] to measure the impulse transferred by an explosively accelerated, $5 \mathrm{~cm}$ thick, wet sand layer whose distance of travel to the target can be systematically varied. The impulse transferred by stagnating wet sand against a flat-bottomed structure oriented normal to the sand impact direction is measured first. This is then compared to that for a $\mathrm{V}$-shaped sample whose surfaces are inclined at $53^{\circ}$ to the incident sand direction, Figure 6.1(a). For uniform sand impact, symmetry dictates that the Ycomponent of the reaction momentum, $I_{T}^{y}$, will be zero. Momentum conservation further dictates that the $\mathrm{Z}$ (vertical) component of momentum, $I_{T}^{Z}$, arising from the impact of granular matter with an incident impulse $I_{O}$, can be no less than $I_{O} \sin ^{2} \alpha$ (the resolved frictionless limit) where $\alpha$ is the angle of inclination. In the absence of significant rebound of the sand, the effective friction force, $f_{t}$, at the sample-granular matter interface will dictate how close one can approach the lower limit. 
(a)
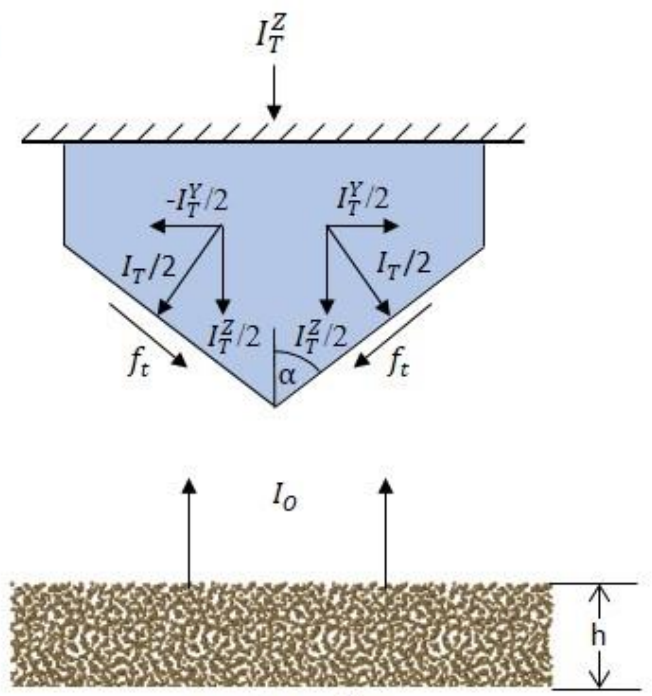

granular matter mass $m$, velocity $v_{0}$ (b)

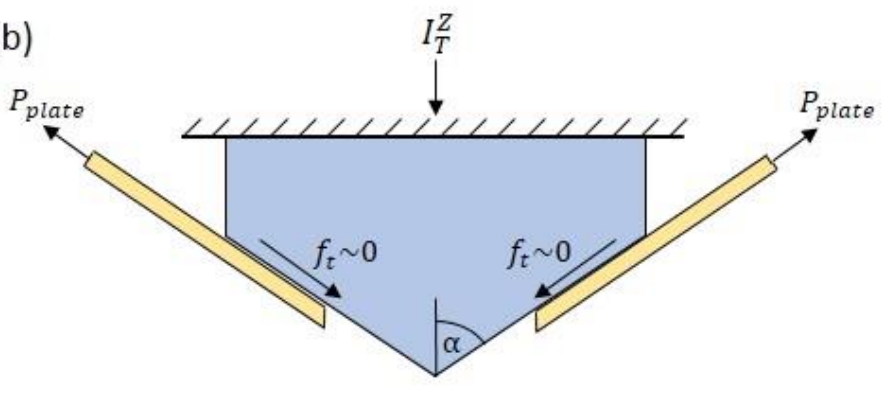

Figure 6.1. Reaction momenta following impact of a granular matter slab of thickness $h$ with (a) a V-block target and (b) a $V$-shaped target with attached slider plates.

\subsection{Experimental methodology}

A series of experimental tests were again performed at the outdoor explosives test site operated by NEWTEC Services Group, Inc. in Edgefield, South Carolina. The tests used a vertical impulse test apparatus recently developed and analyzed by Holloman et al. [13, 14]. The tests measured the pressure and impulse transferred to solid aluminum target designs impacted by explosively accelerated wet sand at three standoff distances (in an analogous manner to that used to investigate

underwater fluid structure interactions) $[8,9,99]$. The target designs enabled the effect of a sacrificial plate sliding on the momentum transferred to the inclined surfaces to be compared to that resulting from a change of surface inclination during loading by explosively accelerated wet sand.

\subsubsection{Sample designs and fabrication}

Three aluminum targets were developed for the tests, Figure 6.2. A flat bottom (baseline) 6061-T6 aluminum target, Figure 6.2(a), that had an area of $203 \mathrm{~mm}$ x $203 \mathrm{~mm}$ and thickness of $89 \mathrm{~mm}$, was used to measure impulse transfer for $\alpha=90^{\circ}$ sand impacts. The target was milled to shape and then welded to a $305 \mathrm{~mm}$ x $305 \mathrm{~mm}$ x $6.35 \mathrm{~mm}$ attachment plate along the four contact 
edges with the target, and by a series of $12.7 \mathrm{~mm}$ diameter, through thickness, plug welds. Eight holes were also drilled along the outer perimeter of the attachment plate to accommodate $12.7 \mathrm{~mm}$ diameter bolts used for attaching the target to the bottom of the vertical impulse test rig.

The second target had the same $203 \mathrm{~mm}$ x $203 \mathrm{~mm}$ square footprint as the baseline target, but had a V-shaped bottom with two slanted faces inclined at $37^{\circ}$ to the horizontal plane, such that the angle between the sand impact direction and the inclined surface was $\alpha=53^{\circ}$, Figure 6.2(b). The geometry of the target was adjusted to ensure that the target mass was identical to the baseline target. As a result, the distance between the apex of the V-bottom and the underside of the attachment was $127 \mathrm{~mm}$ compared to $89 \mathrm{~mm}$ for the flat-bottomed target.

The third target maintained the same $\mathrm{V}$-shaped bottom as the second with an inclined surface angle, $\alpha=53^{\circ}$, and with the same $203 \mathrm{~mm} \times 203 \mathrm{~mm}$ square footprint. However, its height was reduced to accommodate the addition of a pair of $6.3 \mathrm{~mm}$ thick, $0.468 \mathrm{~kg}$, sliding 6061-T6 aluminum plates, Figure 6.2(c). One edge of each of the slider plates was beveled to ensure good apex contact when the pair of slider plates were assembled on the target. Since the slider plates were aluminum, the target with these plates attached was identical in volume and external shape to the V-shaped target, Figure 6.2(b). The mass of the three target designs was therefore the same.

The sliding plates were held in position using a set of clamps, Figure 6.3. Six holes were drilled into each inclined side of the target, and four clamps were bolted onto the target, forming edge rails to ensure the sliding plates remained in place prior to the sand loading. A Molykote M77 solid lubricant paste from Dow Corning was applied to both interfaces between the sliding plates and the rigid V-shaped target and to the interfaces of the plates with the clamps to minimize friction forces between surfaces when the sliding plate was ejected. This silicone oil based paste has been successfully used between metal/metal contact surfaces and has a base oil viscosity of $125 \mathrm{~mm}^{2} / \mathrm{s}$ at $25^{\circ} \mathrm{C}$ [100]. The impact surface of the baseline and the V-block target (without slider plates) as well as the impact surface of the sliding plates were grit blasted using a coarse grit with a mesh size of 12 to 40 . The same grit, spray pressure $(690 \mathrm{kPa})$ and surface modification procedure was used for the impact faces of the three target types so their average surface roughness $(5.9 \mu \mathrm{m})$ was the same. 
(a) Flat target

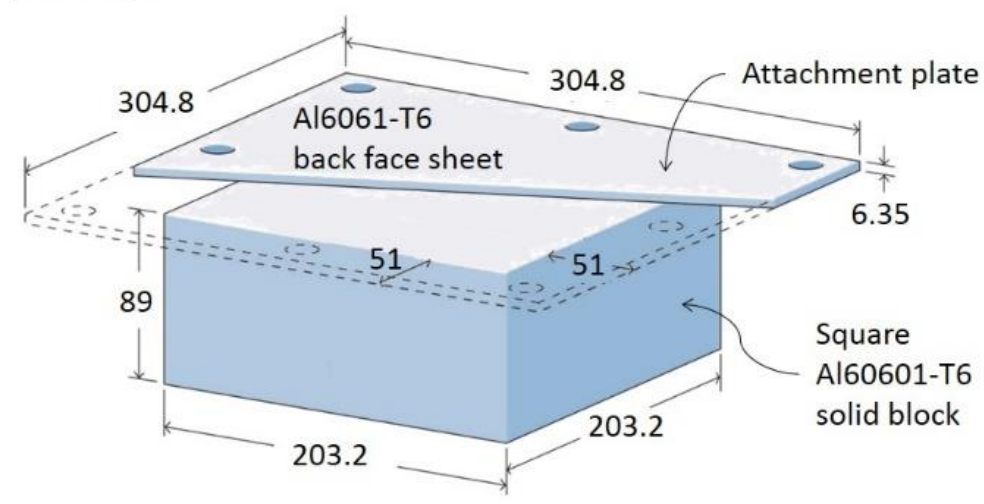

(b) V shaped target

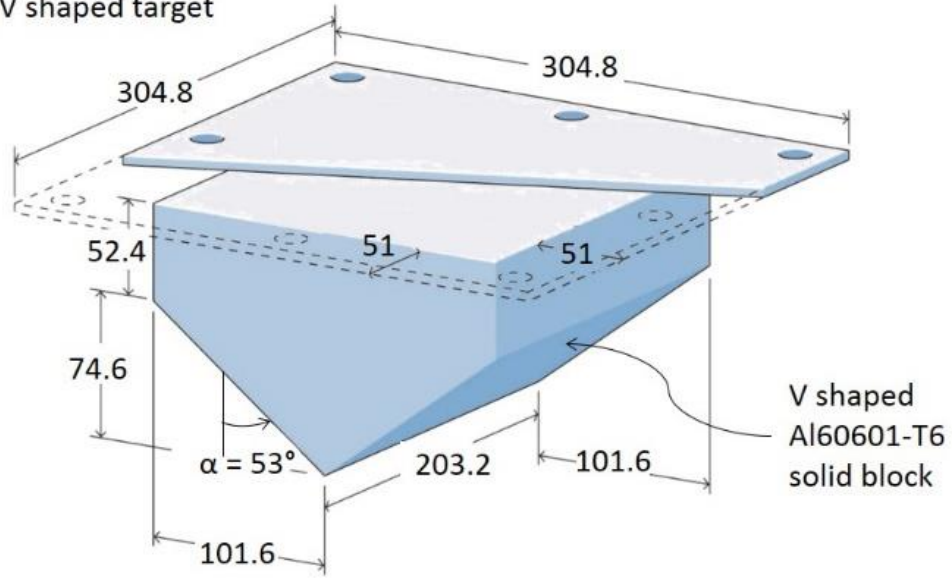

(c) V shaped target with slider plates

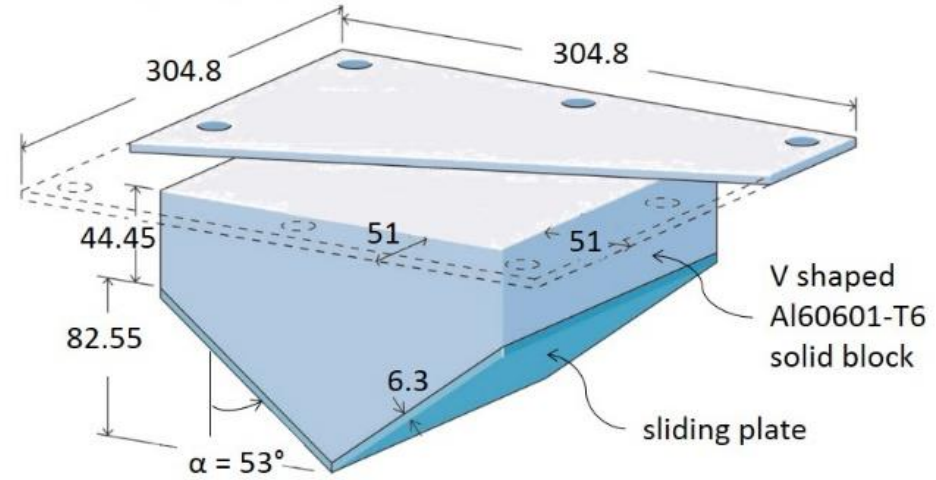

All dimensions in $\mathrm{mm}$

Figure 6.2. The three Al 6061-T6 targets used for the impulse transfer study: (a) The flat bottom target, (b) the $V$-shaped target with surfaces inclined at $53^{\circ}$ to the incident sand and (c) the V-shaped target with a pair of sliding plates attached. 


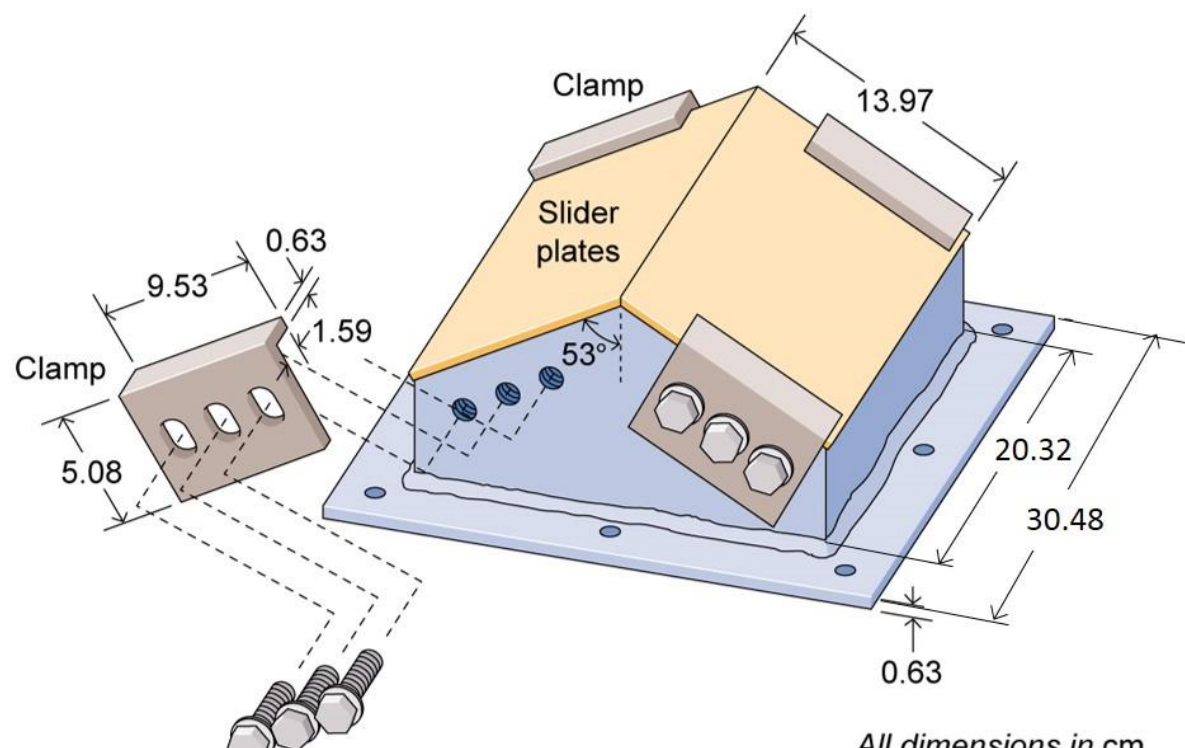

Figure 6.3. The sliding plate target geometry showing the use of four edge clamps that act as guides for the sliding plates during sand impact.

\subsubsection{Test apparatus and procedures}

The vertical impulse test rig used for the impulsive loading of the targets is shown in Figure 6.4. This rig consisted of an "A" shaped structural steel frame mounted on a steel base plate that supported a set of four, $5 \mathrm{~cm}$ diameter, $2 \mathrm{~m}$ long, Kolsky bars, Figure 6.4(a). The 6061-T6 aluminum Kolsky bars were spaced $10 \mathrm{~cm}$ apart in a square pattern and were attached to the rear corners of a test sample, Figure 6.4. The test rig enables an impulse measurement to be made in one of two ways. One approach allows free sliding of the four bars in a vertical pendulum manner, and the impulse is inferred from the jump height of the pendulum and its mass which can be varied by adding cylindrical plates to the top of the bar system, Figure 6.4(b). The second approach, utilized here, used a fixed configuration that restrains the pendulum's vertical movement following an impact [13]. In this arrangement, impulse was computed by integrating the axial compressive stress (pressure) transmitted by the target to the Kolsky bars using strain gauges attached to each bar. To facilitate this arrangement, the top plate of the Kolsky bar assembly shown in Figure 6.4(b) was securely bolted to the "A-frame" to restrain the pendulum movement when targets attached to the bottom end of the bars were impulsively loaded. 
(a)

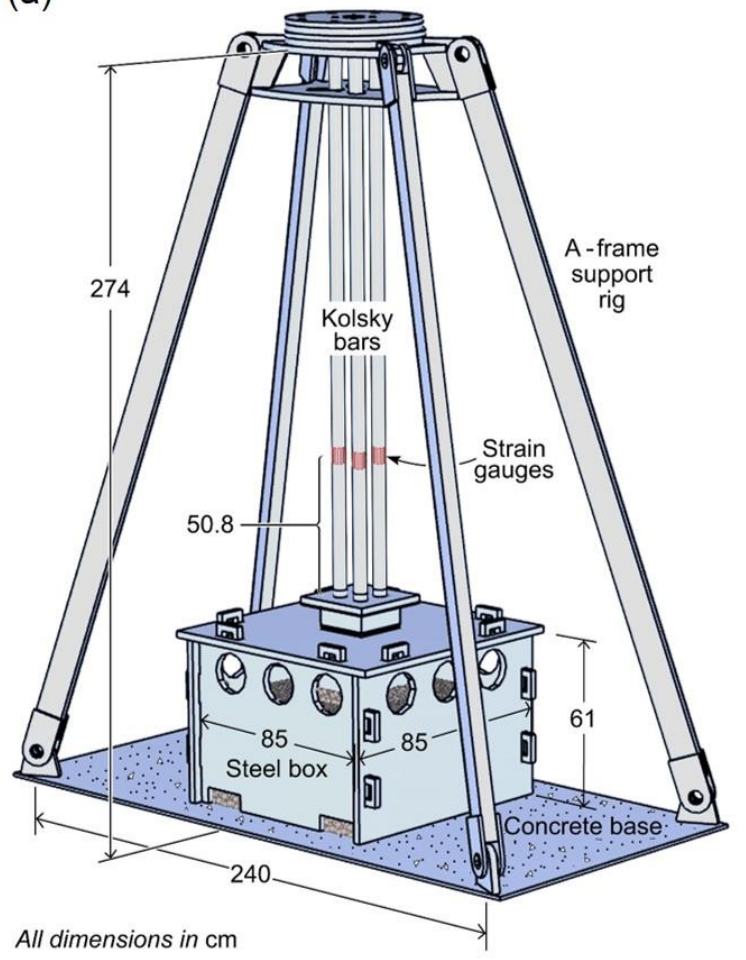

(b)

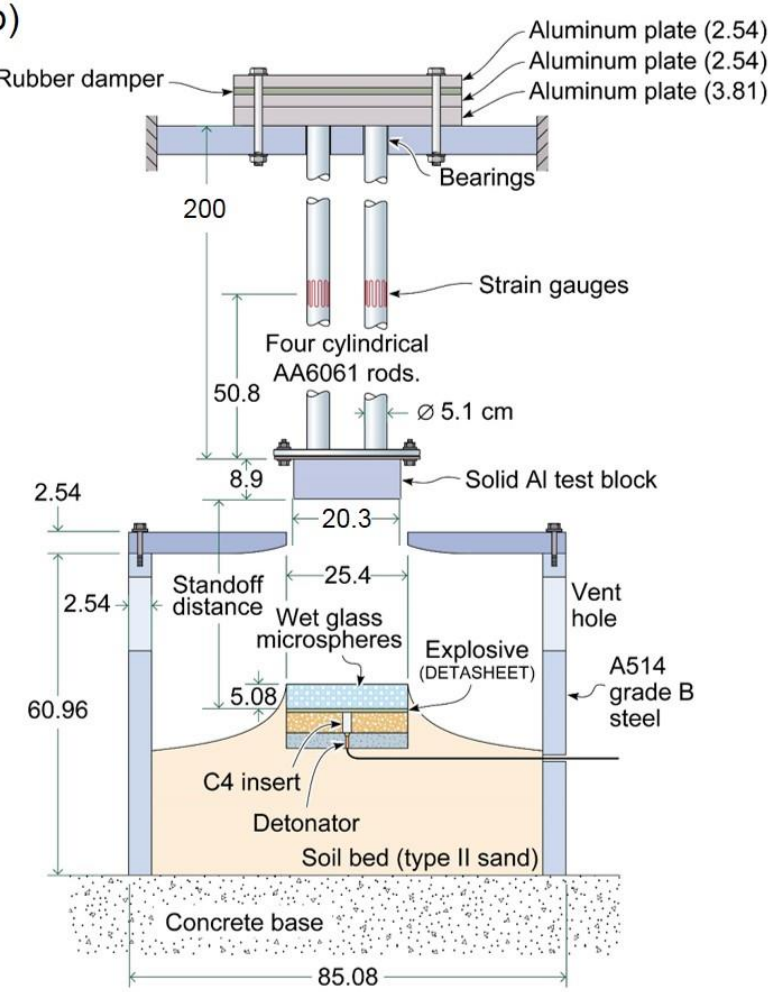

Figure 6.4. (a) Schematic illustration of the vertical impulse test rig with a flat-bottomed solid aluminum target attached. (b) An expanded view of the interior of the steel box, the test charge design, the sample location, and the strain gauge location on the Kolsky bars.

Variations in the standoff distance (defined from the underside of the flat bottom target to the top surface of a test charge) was achieved by altering the height of the explosive test charge located in a sandbox below the target. To standardize the tests, the bottom surface of the attachment plate was held fixed for the three targets at each of the three standoff distances. As a result, the apex of the two V-shaped targets was $(127 \mathrm{~mm}-89 \mathrm{~mm}) 38 \mathrm{~mm}$ closer to the test charge, Figure 6.2. An $86 \mathrm{~cm} \times 86 \mathrm{~cm} \times 61 \mathrm{~cm} \mathrm{A514} \mathrm{grade} \mathrm{B} \mathrm{steel} \mathrm{sandbox} \mathrm{contained} \mathrm{the} \mathrm{sand} \mathrm{and} \mathrm{explosive} \mathrm{charge,}$ Figure 6.4(b). To reduce ejecta impact with the support structure, a $2.54 \mathrm{~cm}$ thick A514 grade steel lid was secured to the top of the steel box during experiments. It contained a $25.4 \mathrm{~cm} \times 25.4$ $\mathrm{cm}$ square opening through which explosively accelerated sand freely propagated to strike the targets positioned above. The tests were conducted by partially filling the sand box with dry, Type II sand to a depth that would ultimately set the standoff distance for the test. A test charge was 
then placed on top of this Type II sand and covered with $5.08 \mathrm{~cm}$ of water-saturated synthetic sand identical to that used by Holloman et al. [13].

The test charge, Figure 6.5, consisted of a $3 \mathrm{~mm}$ thick planar explosive together with a high explosive booster and detonator, all assembled with the aid of a polymer foam plate. The planar energetic material consisted of a $25.4 \mathrm{~cm}$ x $25.4 \mathrm{~cm}, 300 \mathrm{~g}$ Detasheet C which consists of $63 \%$ pentaerythritol tetranitrate (PETN), 8\% nitrocellulose, and 29\% acetryl tributyl citrate (ATBC). The Detasheet was positioned on top of a $25.4 \mathrm{~cm}$ x $25.4 \mathrm{~cm}$ x $3.81 \mathrm{~cm}$ thick, low-density polyurethane foam pad. To introduce a more uniform shockwave in the Detasheet, an inverted, truncated, conical hole was drilled in the center of the foam pad and packed with $25 \mathrm{~g}$ of C-4 to boost the detonation. Detonation was initiated using a model SP/SM(12-0) instantaneous electric detonator (manufactured by Dyno Nobel Inc., Salt Lake City, Utah) held in position with a $1.9 \mathrm{~cm}$ thick sheet of polystyrene foam. The Detasheet charge assembly was placed on the Type II sand whose height was adjusted to result in a standoff distance of 19, 24, or $29 \mathrm{~cm}$, Figure 6.4(b). A $5.08 \mathrm{~cm}$ thick, $25.4 \mathrm{~cm}$ x $25.4 \mathrm{~cm}$ wide layer of synthetic (Type I) sand, consisting of 150-200 $\mu \mathrm{m}$ diameter soda-lime glass microspheres (grade GL-0201, Mo-Sci Corporation), was placed on the top surface of the Detasheet. This was the same fused silica glass particles used previously for the spherical test charges in Chapters 3, 4, and 5. This synthetic sand layer had a mass of $4.4 \mathrm{~kg}$ and was initially saturated with an additional $1.2 \mathrm{~kg}$ of water. However, in the 10-30 minute time period between setting up and conducting the test, some of the water drained out of the charge assembly. Subsequent measurements indicated that the water content dropped to about $80 \%$ of the saturated limit during this time. The total mass of the wet sand at detonation was therefore taken to be $5.36 \mathrm{~kg}$.

A Vision Research Inc., Phantom V7.3 high-speed camera was used to record the nine test events ( 3 standoff distances for 3 targets). For the sliding plate test, a second camera with a much wider field of view was used to attempt visualization of the sliding plate trajectories as they were ejected into free flight from the rig. 


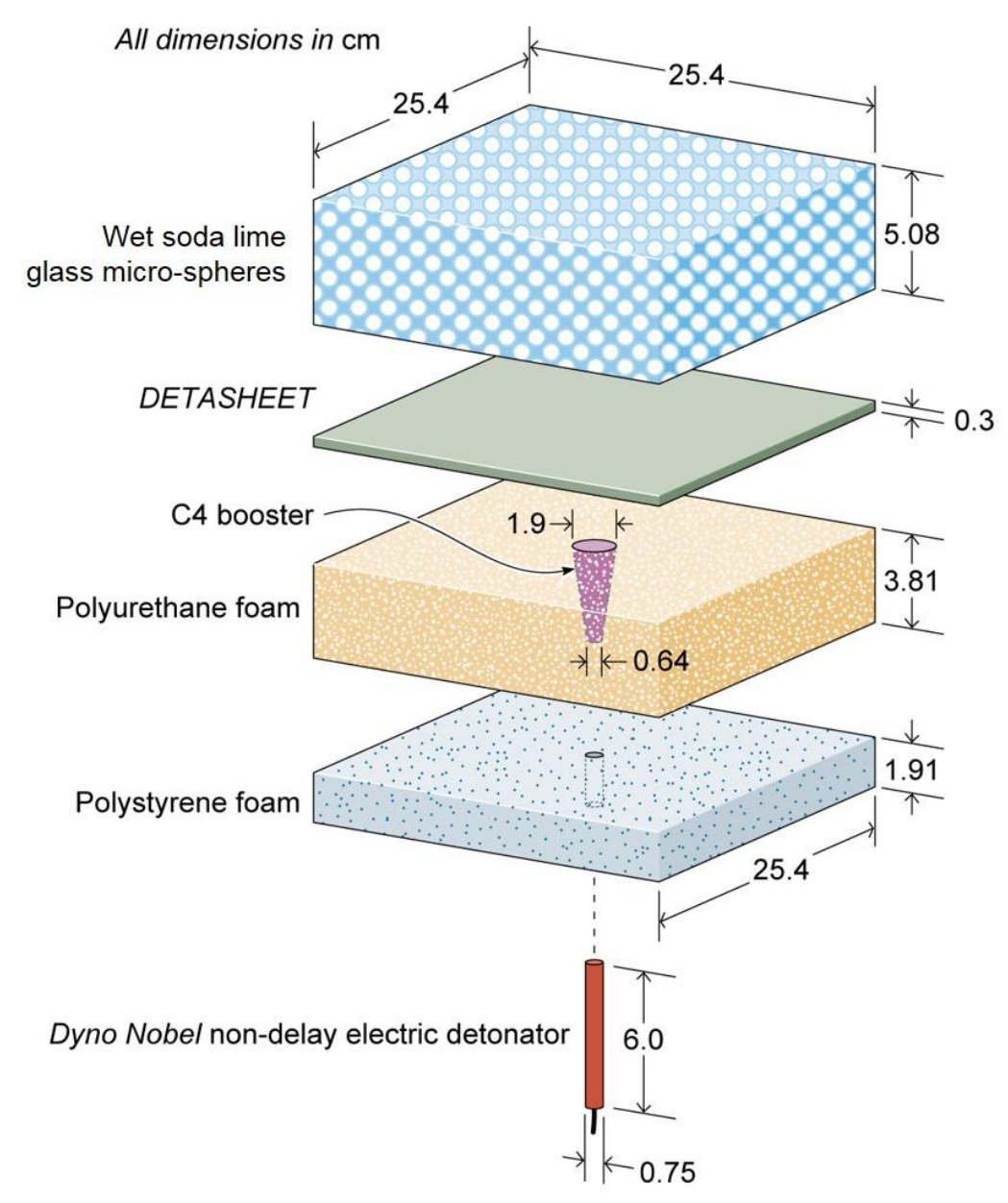

Figure 6.5. Schematic illustration of the test charge.

\subsubsection{Kolsky bar instrumentation}

Four aluminum Kolsky bars were used to measure the transmitted pressure and deduce the vertical impulse transferred to the targets. A pair of Vishay Micro-Measurements axial-transverse T-rosette strain gages (model CEA-13-125UT-350) were mounted on each Kolsky bar using Vishay AE-10 epoxy adhesive, at a distance of $50.8 \mathrm{~cm}$ from the bottom end of the bars, and diametrically opposite to each other [13, 14]. A layer of protective coating (Vishay Gage Kote \#5) was applied to protect the gauges and prevent detachment during the experiments. The pair of strain gauges on each bar were connected in a full Wheatstone bridge circuit, and the output voltage on each bar was recorded on separate channels of an analogue to digital converter. The recording of the signal was triggered by the electrical pulse used for initiation of detonation. For protection 
from the blast and the ejected sliding plates, the instrumentation was placed in a steel box behind a concrete barrier to the side of the vertical rig.

The pressure transmitted to the Kolsky bars was determined from the axial strain of the bars using the method described by Holloman et al. [13] and in the previous chapters. The relationship between the output voltage and input voltage, $V_{o} / V_{i}$, of the strain gauge forming the full Wheatstone bridge circuit is defined in Eqn. (3.1). For the aluminum Kolsky bars used here, the gage factor is again GF $=2.15, \varepsilon$ is the axial strain in the Kolsky bar, $v=0.33$ is the bar's Poisson's ratio, and $\mathrm{V}_{\mathrm{i}}=10 \mathrm{~V}$ is the input excitation voltage. Hooke's law was used to relate the axial strain to the pressure (axial stress) using the measured Young's modulus for the aluminum bars, $\mathrm{E}=70$ $\mathrm{GPa}$. The force transmitted by the rear of the target was found by multiplying the pressure in each bar by its cross sectional area $\left(20 \mathrm{~cm}^{2}\right)$. The summed forces for the four bars was then divided by the sample's area $\left(0.041 \mathrm{~m}^{2}\right)$ to determine the pressure transmitted by the test sample. The pressure-time response was digitally recorded for $7 \mathrm{~ms}$ and subsequently integrated to determine the impulse-time relationship for each target and standoff distance.

Since the bars were $2 \mathrm{~m}$ in length and the strain gauges were positioned $50.8 \mathrm{~cm}$ from the impacted end of the bars, elastic wave reflections from the ends of the bars eventually arrived at the sensors and greatly increased the complexity of signal interpretation. Since the speed of the longitudinal wave in Al6061-T6 is $5350 \mathrm{~m} / \mathrm{s}$, the first distal reflection arrived at the strain gauges $558 \mu \mathrm{s}$ after the first direct signal arrival was sensed. A reflection from the impacted end of the bar is expected to arrive at the strain gauges $190 \mu$ s later, and would be followed by a second distal reflection $748 \mu$ s after the first. Since the bars had a relatively large diameter (to avoid inelastic responses), many Pochhammer-Chree modes [81, 92, 93] are expected to contribute to the observed response, and the impulse, determined by integration of the bar pressure, gradually degraded over time.

\subsection{Numerical discrete particle simulations}

The experimental results were once again interpreted with the assistance of particle-based simulations performed using the IMPETUS Afea solver following similar procedures described in detail by Holloman et al. [13] and Chapter 3. Recent studies investigating the ballistic penetration of granular media [101-103] have revealed that there is a degree of inter-particle sensitivity within 
the IMPETUS Afea solver that effects the validity of the results. The inter-particle friction was found to effect the transferred impulse, and the friction coefficient value should be adjusted to best match the experimental loading response. Here a slight adjustment to the inter particle friction coefficient from that used by Holloman et al. [13] was necessary for a best match with the measured signal response. After validation, the simulations were used to determine the impulse that was eventually transmitted to the test structures. They were also used to investigate the activation of sliding plate motion and its effect upon pressure and impulse transfer to the targets.

\subsubsection{Discrete particle methodology}

Recall, the solver models the transfer of linear momentum through an array of discrete particles whose properties and inter particle contact laws are representative of air, high explosive (HE), and sand [13, 19, 20,54]. The approach does not address the angular momentum of the particles (it implicitly assumes they have an infinite moment of inertia). Each simulation particle represents many actual particles, and their interactions are governed by spherically symmetric inter particle contact laws. The inter particle contact laws and momentum transfer during the elastic collisions of air and HE particles are consistent with Maxwell's kinetic theory of gases [19, 20]. Air particles are modeled as an ideal gas and given initial velocities and randomized propagation directions consistent with the Maxwell-Boltzmann distribution. The predefined air particle parameters used by the solver are consistent with an air density of $1.3 \mathrm{~kg} / \mathrm{m}^{3}$, a pressure of 100 $\mathrm{kPa}$, an initial internal energy of $253 \mathrm{~kJ} / \mathrm{m}^{3}$, and assume a ratio of specific heats $\gamma=1.4$.

The HE particle parameters for Detasheet $\mathrm{C}$ have not been directly determined; however, this explosive material consists of $63 \%$ PETN for which particle contact law parameter values are available in the IMPETUS Afea Solver. Since the solver allows the definition of only one HE particle type in a simulation, the PETN and nitrocellulose in the $3 \mathrm{~mm}$ thick Detasheet and the C4 booster charge were modeled as PETN. The high explosive model was implemented by filling a $254 \mathrm{~mm}$ x $254 \mathrm{~mm}$ x $2.4 \mathrm{~mm}$ volume with PETN particle properties to represent the PETN and the nitrocellulose, in the $300 \mathrm{~g}$ Detasheet. The ATBC plasticizer was not included in this model, resulting in a reduced HE model sheet thickness of $2.4 \mathrm{~mm}$. To simplify the model, the mass of the $25 \mathrm{~g} \mathrm{C}-4$ booster was represented by a PETN cylinder $0.6 \mathrm{~cm}$ in height and diameter. This produced a total mass of PETN HE particles of $0.273 \mathrm{~g}$. The solvers predefined PETN parameters correspond to a density $\rho=1765 \mathrm{~kg} / \mathrm{m}^{3}$ and initial internal energy $E_{0}=10.2 \mathrm{GJ} / \mathrm{m}^{3}$. The model 
also specifies the ratio of heat capacities $\gamma=1.4$, the initial particle solid-fill fraction $b=0.35$, and the detonation velocity $\mathrm{D}=8350 \mathrm{~m} / \mathrm{s}$. During the simulations, detonation was initiated at the center of the bottom surface of the PETN cylinder representing the C-4 booster at $\mathrm{t}=0 \mu \mathrm{s}$, whereupon initially stationary detonation particles were released at a time $t=R / D$ where $R$ was the particle distance from the detonation location and D the detonation velocity.

The normal and tangential soil particle motions following inter particle contact were modeled with a penalty based contact model defined in Section 1.5. The contact model used in the solver for both dry and water-saturated soil was determined by Borvik et al. [19] using the same 150-200 $\mu \mathrm{m}$ diameter, soda-lime glass microspheres used here and in the previous chapters with spherical test charges. Based on a $60 \%$ sand particle fill fraction, the initial density of the dry sand was 1620 $\mathrm{kg} / \mathrm{m}^{3}$. Filling the remaining volume with water leads to fully water-saturated sand with a density of $2020 \mathrm{~kg} / \mathrm{m}^{3}$. The water is not explicitly modeled in the solver. Instead, the soil parameters were adjusted by Borvik et al. [19] until the simulation matched the experimental results with watersaturated sand. These calibrated parameter values are available as the default parameters for both dry and water-saturated soil in the solver, and are listed in Table 6.1. The solver also allows user specified parameter values to be utilized. Holloman et al. [13] previously determined soil parameters for a water saturation of $80 \%$ corresponding to a wet sand density of $\rho=1940 \mathrm{~kg} / \mathrm{m}^{3}$, and these are also listed in Table 6.1. The Type I and II sand were modeled using the wet sand parameters in Table 6.1 with an adjusted contact friction coefficient of 0.08 which was found to give a slightly better match between the simulations and experimental test results.

Table 6.1. Soil parameters for predefined options in IMPETUS [19] and calibrated wet sand by Holloman et al. [13].

\begin{tabular}{|c|c|c|c|c|}
\hline & $\begin{array}{l}\text { Density } \\
\rho\left(\mathrm{kg} / \mathrm{m}^{3}\right)\end{array}$ & $\begin{array}{c}\text { Contact stiffness } \\
\mathrm{k}_{0}(\mathrm{GN} / \mathrm{m})\end{array}$ & $\begin{array}{c}\text { Contact friction } \\
\text { coefficient } \\
\mu \\
\end{array}$ & $\begin{array}{c}\text { Damping } \\
\text { coefficient } \\
\xi \\
\end{array}$ \\
\hline Dry & 1620 & 0.4 & 0.1 & 0.0 \\
\hline Saturated & 2020 & 4.0 & 0.0 & 0.005 \\
\hline Wet & 1940 & 0.76 & 0.0 & 0.005 \\
\hline
\end{tabular}


To model the friction created on the impact surface of the grit blasted experimental targets, a soil-structure contact coefficient of friction was defined in the model with a value $\mu_{s}=0.5$. The friction coefficient of the lubricated interface between the slider plates and the target surface was modeled using $\mu_{\mathrm{p}}=0.05$. A convergence study was performed to identify the optimum number of particles, and showed no further improvement in the simulations beyond 2,000,000 particles. The IMPETUS Afea Solver distributed these particles as approximately 70,000 air particles, 10,000 HE particles and 1,920,000 soil particles with exact values differing slightly for each simulation setup (different target and standoff distances). The velocity and density of the soil particles that were explosively accelerated by the detonation event were characterized at $0.5 \mu$ s time intervals using four sets of twenty, equidistant virtual spherical sensors ("monitors") each of $5.08 \mathrm{~mm}$ radius and located at 2, 7, 12, and $17 \mathrm{~cm}$ below the bottom surface of the baseline target.

\subsubsection{Finite element model}

The full vertical impulse test rig geometry was modeled as a set of finite element (FE) parts, Figure 6.6(a). The model included the "A-frame" supported vertical pendulum rig and the four, strain gage instrumented Kolsky bars. Figure 6.6(b) shows a view of the model setup within the sandbox and the attached V-shaped target with aluminum sliding plates. The other two targets are also shown in Figure 6.6(b). The finite element (FE) geometry was modeled based on the measured dimensions from the test rig schematic, Figure 6.4, and the test targets, Figure 6.2. The four, $5.08 \mathrm{~cm}$ diameter Kolsky bars were modeled using the IMPETUS Afea Solver finite element package as multiple cylindrical parts that were merged to form the full $2 \mathrm{~m}$ length Kolsky bars. A $2.54 \mathrm{~cm}$ long cylinder region was used at the strain gauge location to determine the axial force between these cylindrical parts and the adjoining parts of the bars. The force on each of the bars was summed and divided by the surface area of the baseline target $\left(0.041 \mathrm{~m}^{2}\right)$ to determine the pressure transferred to the target. The impulse was calculated as the time integral of the pressuretime waveform. The Kolsky bars were restrained at the top of the rig, and a damping parameter was introduced to better model the oscillatory pressure-time response. The support structure "A frame" and concrete base of the model were defined as rigid structures that remained fixed in space. The sandbox was modeled with dimensions that matched the experimental specifications, including a $25.4 \mathrm{~cm}$ x $25.4 \mathrm{~cm}$ opening in the lid for the explosively accelerated sand to escape. 
(a)

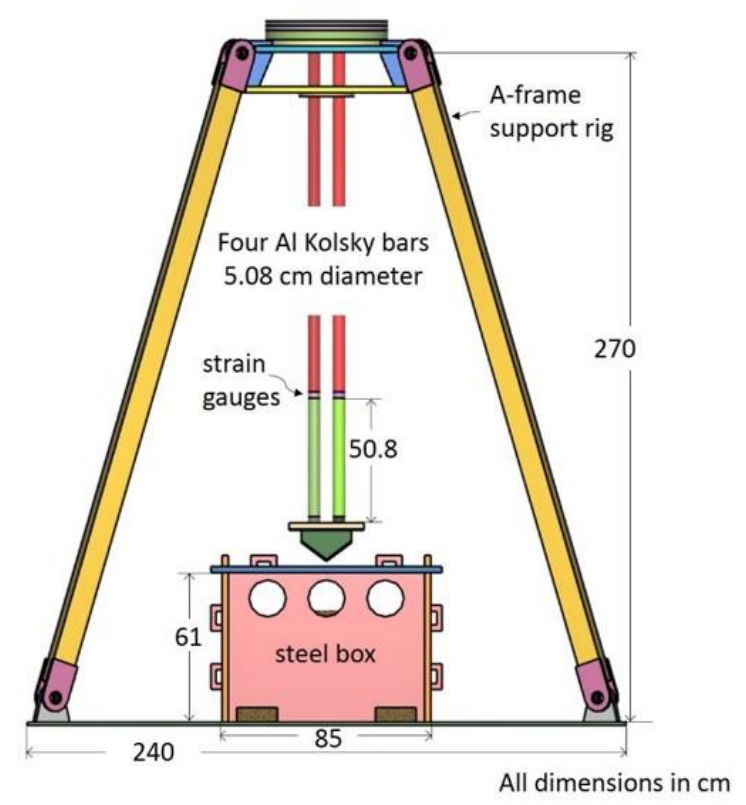

(b)

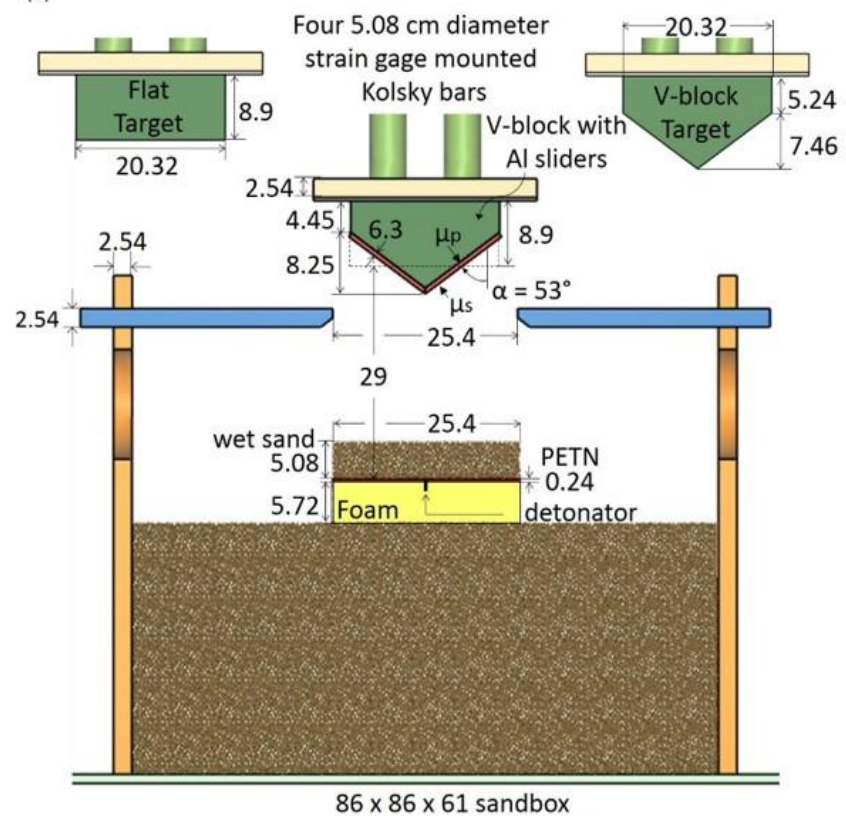

Figure 6.6. (a) The FE model of the vertical impulse test rig. Each color-coded part used a different model and material combination. (b) The simulation geometry within the sandbox. The V-block target with sliding plates is shown for a standoff distance of $29 \mathrm{~cm}$. The model geometries of the flat block and V-block targets are also shown.

After a mesh sensitivity study, the converged FE model of the vertical impulse test rig was meshed with 31,832 elements and 50,221 nodes. Each of the targets was modeled with a finer mesh and was attached to the attachment plate part directly below the Kolsky bars. The baseline flat bottom block was meshed with 3,600 elements and 4,410 nodes. The V-block target was modeled with 480 linear pentahedra elements, 3,560 linear hexahedra elements, and 4,599 nodes. The V-block target with the aluminum slider plates was modeled with 360 linear pentahedra elements, 3,240 linear hexahedra elements and 4,294 nodes with slider plates each made up of 520 linear hexahedra elements and 882 nodes.

\subsubsection{Material parameters}

The material properties for the aluminum AA6061-T6 and A514 steel alloys were defined using the Johnson-Cook constitutive model that defines the von Mises flow stress $\sigma_{\mathrm{y}}$ (Eqn. (3.5)). The aluminum parts were modeled using the parameters for AA6061-T6 aluminum obtained by Wadley et al. [37]. The sandbox was modeled as A514 grade B steel using Johnson-Cook 
Table 6.2. Johnson-Cook material constants for AA6061-T6 aluminum and A514-grade B steel.

\begin{tabular}{|c|c|c|c|c|c|c|c|c|c|c|c|}
\hline \multirow[t]{2}{*}{ Material } & \multicolumn{3}{|c|}{$\begin{array}{l}\text { Density and } \\
\text { elastic constant }\end{array}$} & \multicolumn{3}{|c|}{$\begin{array}{l}\text { Yield stress and } \\
\text { strain hardening }\end{array}$} & \multicolumn{2}{|c|}{$\begin{array}{l}\text { Strain rate } \\
\text { hardening }\end{array}$} & \multicolumn{3}{|c|}{$\begin{array}{c}\text { Temperature } \\
\text { softening and } \\
\text { adiabatic heating }\end{array}$} \\
\hline & $\begin{array}{c}\rho \\
\left(\mathrm{kg} / \mathrm{m}^{3}\right)\end{array}$ & $\begin{array}{c}\mathrm{E} \\
(\mathrm{GPa})\end{array}$ & $v$ & $\begin{array}{c}\mathrm{A} \\
(\mathrm{MPa})\end{array}$ & $\begin{array}{c}\mathrm{B} \\
(\mathrm{MPa})\end{array}$ & $\mathrm{n}$ & $\mathrm{C}$ & $\begin{array}{c}\dot{\varepsilon}_{0} \\
\left(\mathrm{~s}^{-1}\right)\end{array}$ & $\begin{array}{l}T_{0} \\
(\mathrm{~K})\end{array}$ & $\begin{array}{l}\mathrm{T}_{\mathrm{m}} \\
(\mathrm{K})\end{array}$ & $\mathrm{m}$ \\
\hline AA6061 & 2700 & 70 & 0.3 & 270 & 98 & 0.22 & 0.001 & $5 \cdot 10^{-4}$ & 293 & 893 & 1 \\
\hline A514 & 7850 & 210 & 0.3 & 796 & 510 & 0.26 & 0.014 & $1 \cdot 10^{-2}$ & 293 & 1793 & 1 \\
\hline
\end{tabular}

parameters from Johnson et al. [104]. These Johnson-Cook parameters and other material constants for both materials are summarized in Table 6.2.

The foam layers below the Detasheet were included in the simulation using a model built into the solver for an isotropic crushable foam. The foam was modeled with density $\rho=33 \mathrm{~kg} / \mathrm{m}^{3}$ and Young's modulus $\mathrm{E}=0.9 \mathrm{MPa}$. A criteria for geometric strain failure was introduced to the foam by defining a failure strain, $\varepsilon_{\text {fail }}=0.1$, at which point the foam lost all strength and was eroded from the simulation.

\subsection{Results and analysis}

\subsubsection{Flat bottom target}

Figure 6.7(a) shows a high-speed video image of the initial interaction of explosively accelerated sand (at $0.47 \mathrm{~ms}$ after detonation) with the flat bottom target at a standoff distance of $29 \mathrm{~cm}$. The interaction between the high-speed sand front and shock compressed air ahead of the sand caused the development of a sand front instability [54, 70]. This instability results in the formation of sand "fingers" whose tip velocity was up to $18 \%$ higher than that of the main sand front. The motion of the sand "fingertips" and the main (continuous) sand front were tracked using the high-speed video images, and the sand position vs time is plotted in Figure 6.7(b). The main sand front position data was numerically differentiated to obtain the main sand front velocity as a function of time, Figure 6.7(c). Standard error propagation techniques described in reference [91] were used to estimate the error bars. The data in Figure 6.7(c) shows that the main sand front was accelerated to a maximum velocity of about $500 \mathrm{~m} / \mathrm{s}$ during the first $250 \mu$ s following detonation before undergoing rapid deceleration (by momentum transfer to air particles) prior to reaching the target at a $29 \mathrm{~cm}$ standoff. The estimated main sand front impact times with the flat-bottomed 
target are shown for each of the standoff distances in Figure 6.7(c), and each incident main sand front speed is listed in Table 6.3.

A simulation of the test is shown in Figure 6.8 and allows the important physical processes involved in (initially) planar sand front propagation towards the target to be investigated. The figure shows the sand particles' positions and color codes their vertical (Z-direction) velocity at selected times after detonation. The sandbox lid and the test fixture hardware have been hidden in these images. Detonation of the HE layer resulted in the launching of a compressive shock front into the $5 \mathrm{~cm}$ thick layer of wet sand. This front can be easily seen in the sand particle velocity data at $30 \mu \mathrm{s}$ after detonation, Figure 6.8(a). The wet sand shock speed was estimated to be 1690 $\mathrm{m} / \mathrm{s}$ which is consistent with previous estimates by Holloman et al. [13] for the same sand. Similar to the spherical charges, upon reaching the air/sand interface, the compressive shock was reflected back into the sand as a release wave. To conserve momentum, this resulted in outward spallation of the sand particles from an interface that propagated back through the sand layer, Figure 6.8(b). The first particles released in this manner had a velocity in excess of $500 \mathrm{~m} / \mathrm{s}$ while those launched later were significantly slower, Figure 6.8(c). As a result, the initially $5 \mathrm{~cm}$ thick slab of sand was stretched in length as it advanced perpendicular to the original plane of the sand surface, and therefore in a manner consistent with recent sand slug experiments [49, 105]. Some lateral expansion of the sand also occurred. Later in the process, Figure 6.8(d-f), expanding high pressure detonated HE particles (not shown) "pushed" the slower (lower) end of the sand slab towards the target.

The simulation image of sand particle positions at $0.45 \mathrm{~ms}$ after detonation, Figure 6.8(f), corresponds to the high-speed video image shown in Figure 6.7(a). The impact of the fastest sand particles (red) with the bottom of the target is consistent with the experimental observation of sand fingertip impacts in Figure 6.7(a). However, the topology of the fingers that are initiated by an interfacial instability [70] is not resolved in the simulation. Alternative analysis methods capable of analyzing the aerodynamic forces upon these highly deformable granular structures (travelling at a velocity approaching the Mach 2 range into shock compressed air) are necessary for this. 

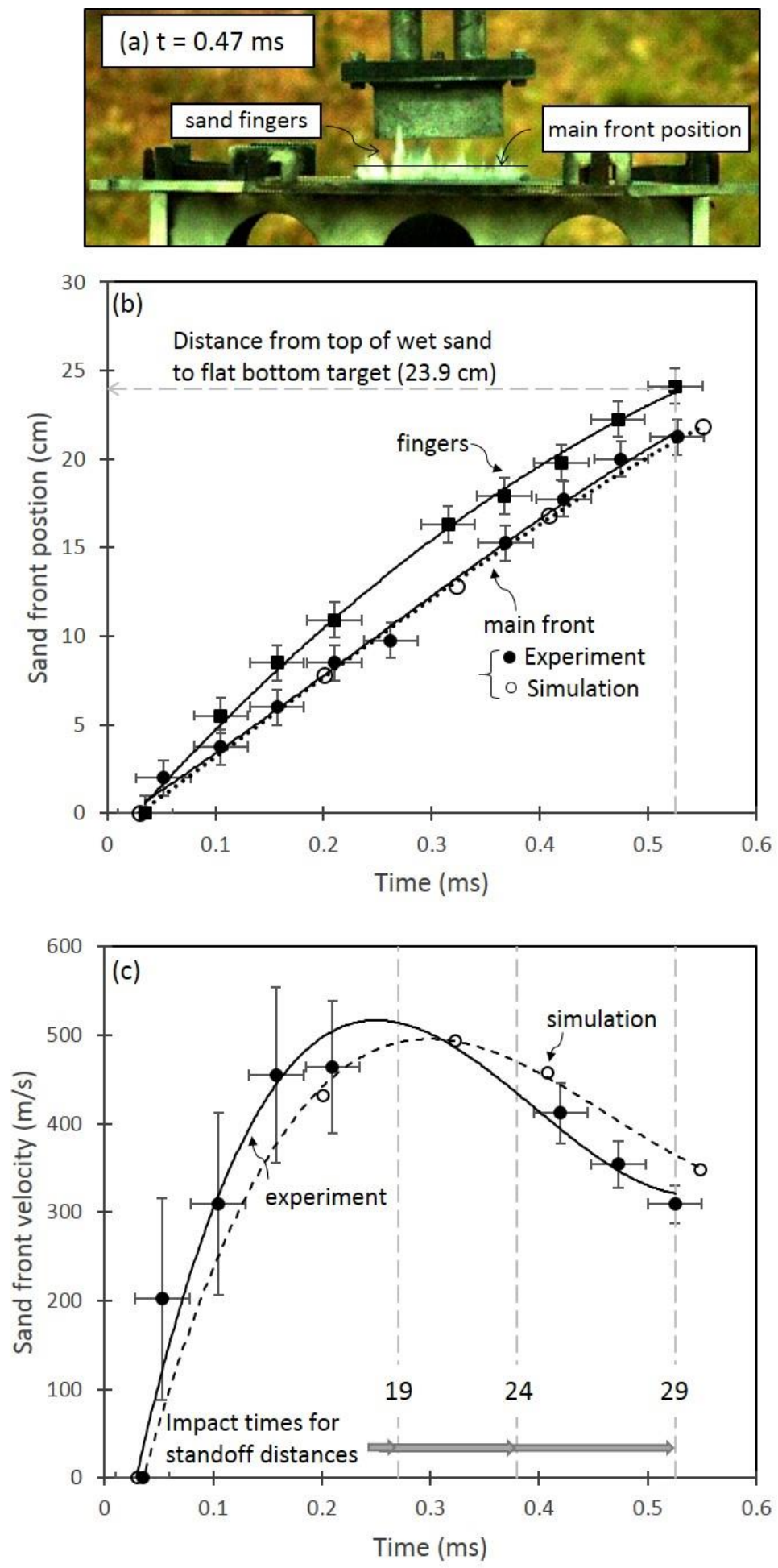

Figure 6.7. (a) A photograph of the sand front at $0.47 \mathrm{~ms}$ after detonation showing the main front position and the presence of sand fingers for a test with the flat bottom sample at a standoff distance of $29 \mathrm{~cm}$. (b) The measured and simulated main sand front position versus time and measured fingers position versus time. (c) The measured and simulated main sand front velocity versus time with the impact times for the flat bottom sample for each of the standoffs. 
(a) $\mathrm{t}=0.03 \mathrm{~ms}$

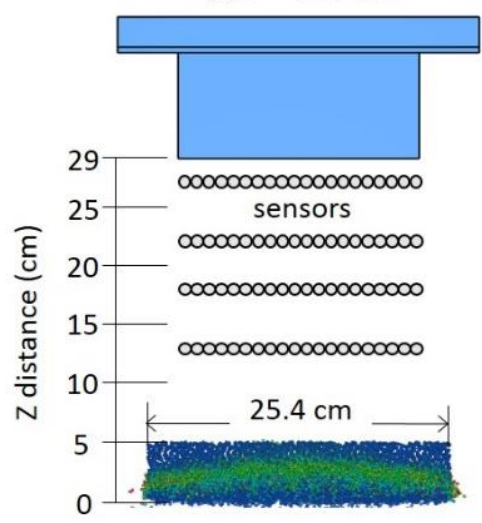

(d) $\mathrm{t}=0.27 \mathrm{~ms}$

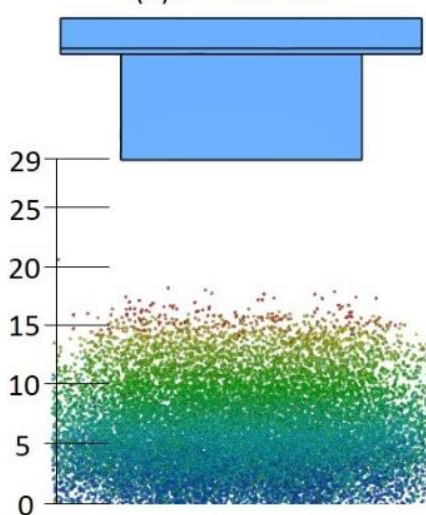

(g) $\mathrm{t}=0.60 \mathrm{~ms}$

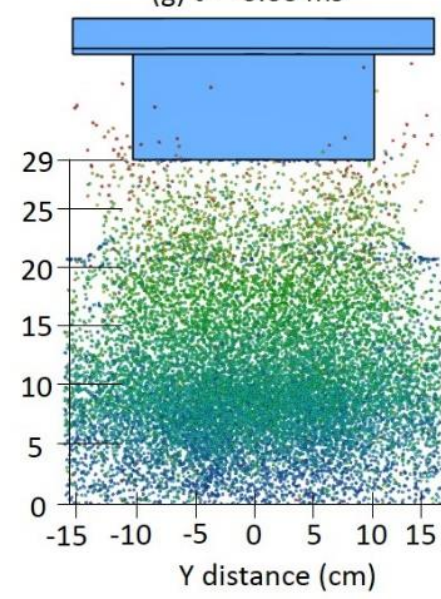

(b) t $=0.06 \mathrm{~ms}$

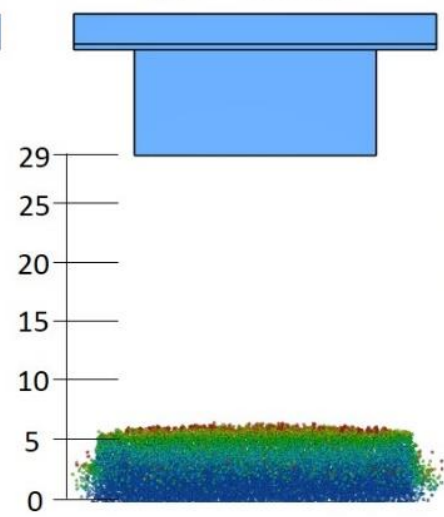

(e) $\mathrm{t}=0.36 \mathrm{~ms}$

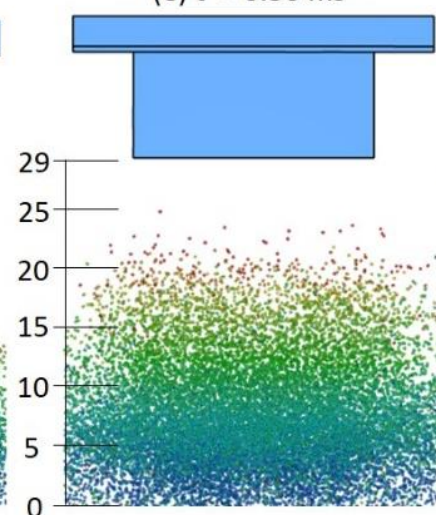

(h) $\mathrm{t}=0.75 \mathrm{~ms}$

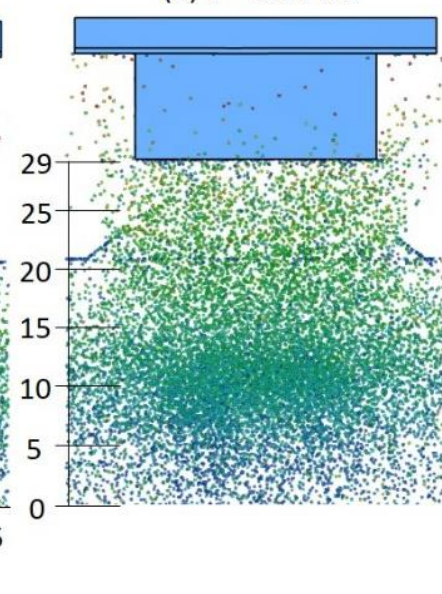

(c) $\mathrm{t}=0.15 \mathrm{~ms}$

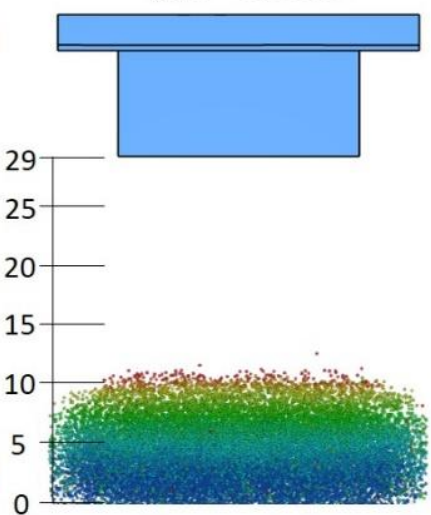

(f) $\mathrm{t}=0.45 \mathrm{~ms}$

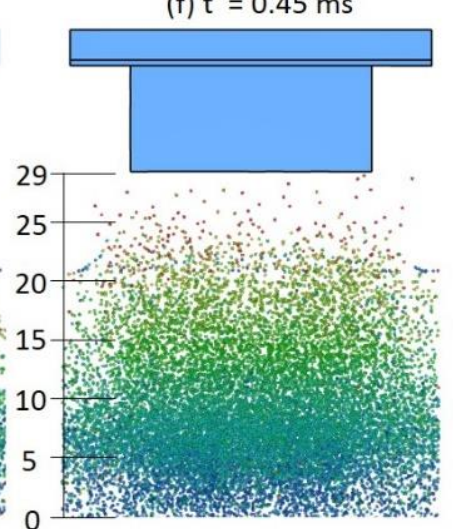

(i) $\mathrm{t}=0.90 \mathrm{~ms}$

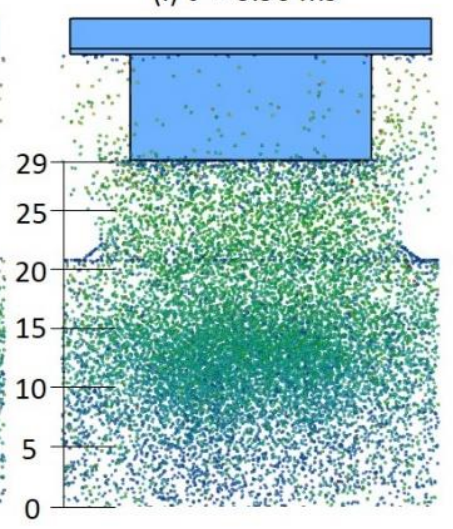

Z Velocity $(\mathrm{m} / \mathrm{s})$

500

440

380

320

260

200

Figure 6.8. Simulation of sand particle progression towards the baseline test target for a standoff distance of $29 \mathrm{~cm}$. The particle velocity is color coded. The position of the sensors used to monitor the particle position and velocity are indicated in $(a)$. 
The position of the main sand front at various time steps was measured using the four rows of sensors shown in Figure 6.8(a). The main sand front position data is shown as a function of time in Figure 6.7(b), and the corresponding velocity versus time response is shown in Figure 6.7(c). These simulation results can be seen to be in good agreement with the experimental observations. The main sand front was strongly accelerated by the detonation, but then began to decelerate due to momentum transferring collisions with background air particles. This resulted in the attainment of a maximum main sand front velocity of $\sim 500 \mathrm{~m} / \mathrm{s}$ at about $0.2-0.3 \mathrm{~ms}$ after detonation as the sand front passed through the $19 \mathrm{~cm}$ standoff distance location. As the standoff distance was increased, the sand that reached the target surface had suffered more air drag, resulting in a main sand front velocity of about $350 \mathrm{~m} / \mathrm{s}$ by the time it reached the sample at the $29 \mathrm{~cm}$ standoff distance, Figure 6.7(c). This, combined with greater stretching (and lateral expansion) at later times, resulted in a reduction of sand density as the standoff distance increased.

The sand particles that impacted the flat surface suffered a weak reflection and began to rebound in the $-Z$ direction. However, they then suffered impact with later arriving particles which resulted in accumulation of particles against the target surface, consistent with calculations of sand slug impacts on normally oriented rigid targets $[32,48]$. Further particle impacts eventually caused particles to flow radially outwards, towards and past the edges of the target surface, Figure 6.8(i). Because of the lateral stretching of the sand, a small fraction (5-10\%) of the sand particles did not impact the target and is seen to instead impact the underside of the attachment plate, Figure 6.8(h) and (i). This is likely to increase the impulse transferred to the instrumented Kolsky bars above that applied by the target and is corrected for in Section 6.5 .

The pressure-time signals experimentally determined from the Kolsky bar strain gauge data are plotted for each standoff distance for the flat target and compared with simulations in Figure 6.9(a), (c), and (e). Since signal reflections from the ends of the bars complicate the data and the damping of the Kolsky bars was not well understood, data is only plotted for the first $2.15 \mathrm{~ms}$ of loading corresponding to a time shortly after the arrival of the $2^{\text {nd }}$ distal reflection at the Kolsky bars sensors for the $29 \mathrm{~cm}$ standoff distance test. The first distal reflection arrived $558 \mu \mathrm{s}$ after the initial (direct) signal arrival while the second reflection was delayed by an additional $748 \mu$ s. These times are indicated for each standoff distance on Figure 6.9. 

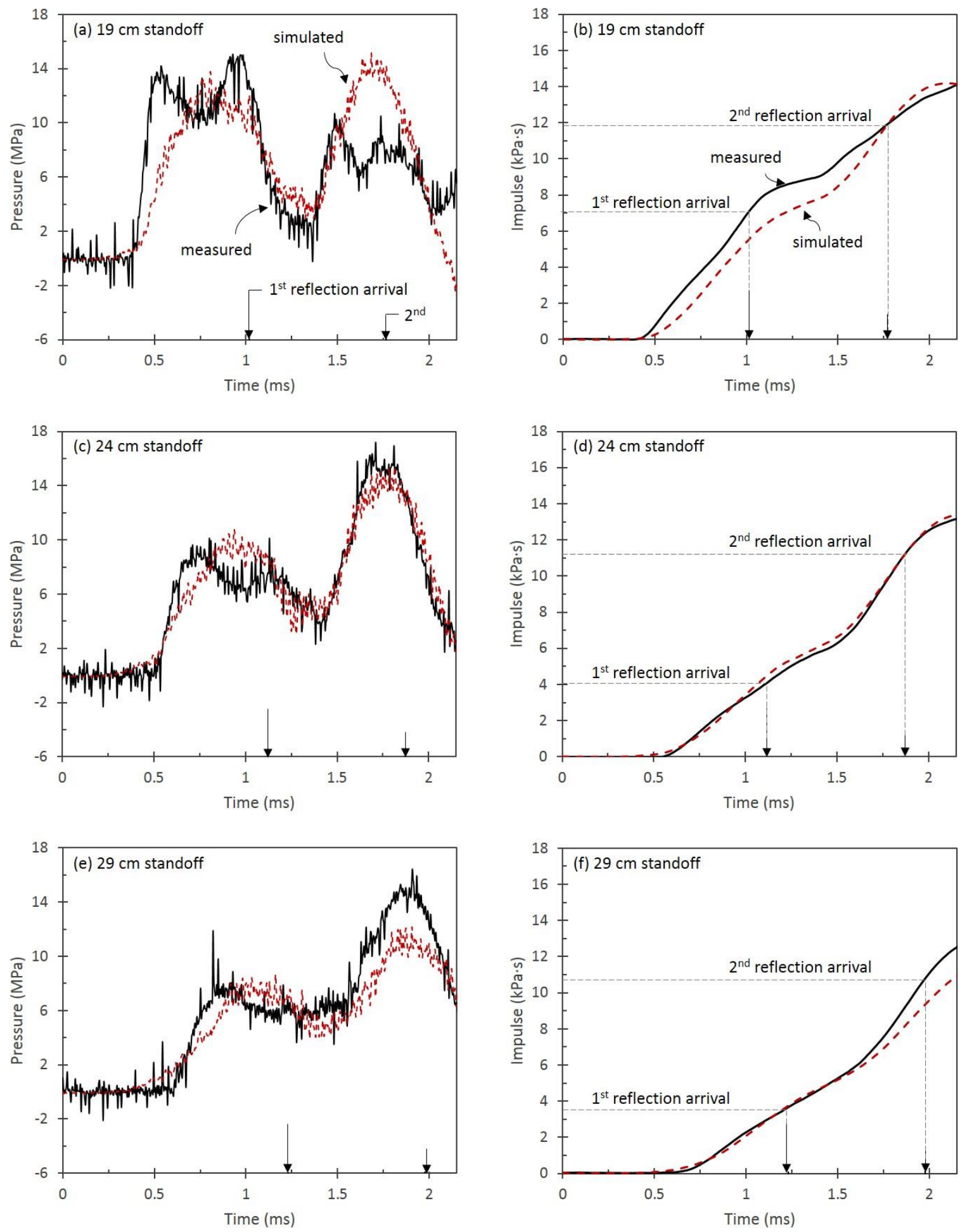

Figure 6.9. Comparison of the strain gauge measured (black line) and simulated (red dashed line) pressure-time waveforms for the flat-bottomed target at standoff distances of (a) $19 \mathrm{~cm},(\mathrm{c}) 24 \mathrm{~cm}$ and (e) $29 \mathrm{~cm}$. The impulse-time waveforms are also shown for each standoff distance and the arrival times of the $1^{\text {st }}$ and $2^{\text {nd }}$ distal reflection at the strain gauges are indicated on $(b),(d)$, and $(f)$. The time $t=0 \mathrm{~s}$ corresponds to initiation of detonation of the Detasheet. 
At each standoff distance, the pressure exhibits a sharp rise to an initial peak pressure (reported in Table 6.3) followed by a secondary peak of equal or greater value. The simulated responses are in generally good agreement with the measurements. Increasing the standoff distance delayed the rise in pressure due to the delay in impact time that resulted from the increased distance and declining velocity of sand that impacted the sample. The initial peak pressure was also influenced by standoff distance falling from $13.0 \mathrm{MPa}$ at $19 \mathrm{~cm}$ to $7.1 \mathrm{MPa}$ at the $29 \mathrm{~cm}$ standoff distance.

The transmitted impulse-time plots (obtained by integrating the pressure-time plots) are shown in Figure 6.9(b), (d) and (f) for the three standoff distances. The impulse plots show an initial rise in slope with a slope change between the first and second reflection arrival times. The initial impulse rate, $\dot{I}$, was determined from the initial slope of the impulse-time response (during the first $200 \mu$ s following the first arriving signal) and is summarized in Table 6.3. The initial impulse rates while slightly lower than the initial peak pressures, usually declined with standoff distance. The exception was the sliding response at the longest standoff distance where the sliding plate had not begun to slide during the measurement window. The arrival times of the first and second distal reflection at the strain gauge location are shown for each impulse plot in Figure 6.9(b), (d), and (f). The measured impulse at each of these times, indicated by dotted lines in Figure 6.9, is summarized for each standoff distance in Table 6.4. The simulated data is also shown on Figure 6.9 and agrees well with the experiments. The impulse accumulated up to the arrival of the first distal reflection decreased rapidly with standoff distance due to a combination of the drop in sand particle velocity, Figure 6.7(c), and axial and lateral stretching of the sand column [13]. These impulse values are shown as a function of standoff distance in Figure 6.10.

Table 6.3. Incident main sand front speed at the time of impact with the flat bottom target and experimentally measured initial pressure peaks and impulse rates for the three targets for each standoff distance.

\begin{tabular}{|c|c|c|c|c|c|c|c|}
\hline \multirow{3}{*}{$\begin{array}{c}\text { Standoff } \\
\text { distance } \\
(\mathrm{cm})\end{array}$} & \multirow{3}{*}{$\begin{array}{c}\text { Sand } \\
\text { speed } \\
(\mathrm{m} / \mathrm{s})\end{array}$} & \multicolumn{2}{|c|}{ Flat block } & \multicolumn{2}{|c|}{ V-block } & \multicolumn{2}{|c|}{$\begin{array}{c}\text { V-block } \\
\text { with Al slider plates }\end{array}$} \\
\hline & & $\begin{array}{c}\text { Initial } \\
\text { peak pressure }\end{array}$ & $\frac{\mathrm{dI}}{\mathrm{dt}}=\dot{\mathrm{I}}$ & $\begin{array}{c}\text { Initial } \\
\text { peak pressure }\end{array}$ & $\frac{\mathrm{dI}}{\mathrm{dt}}=\dot{\mathrm{I}}$ & $\begin{array}{c}\text { Initial } \\
\text { peak pressure }\end{array}$ & $\frac{\mathrm{dI}}{\mathrm{dt}}=\dot{\mathrm{I}}$ \\
\hline & & (MPa) & $(\mathrm{MPa})$ & $(\mathrm{MPa})$ & $(\mathrm{MPa})$ & (MPa) & (MPa) \\
\hline 19 & 510 & 13.0 & 11.6 & 8.4 & 8.0 & 8.1 & 7.8 \\
\hline 24 & 440 & 8.7 & 7.6 & 5.8 & 5.0 & 3.4 & 3.3 \\
\hline 29 & 310 & 7.1 & 6.4 & 4.7 & 4.2 & 4.1 & 3.9 \\
\hline
\end{tabular}



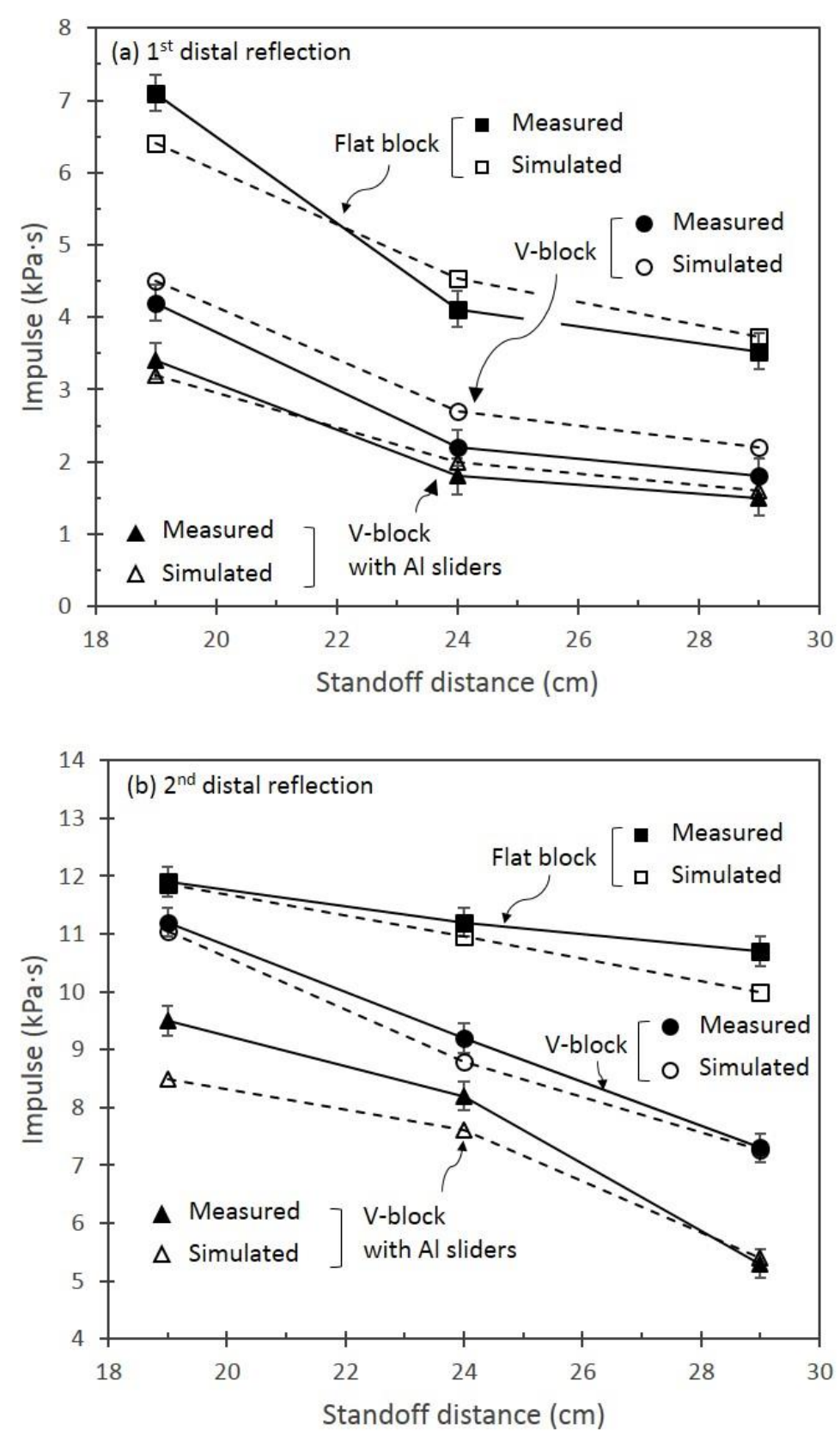

Figure 6.10. (a) Impulse at the first distal reflection (up to $558 \mu$ s after the signal first arrives at the strain gauge) for each of the three standoff distances for the flat (baseline) target, the solid V-block target, and the V-block target with the aluminum slider plates. (b) Impulse at the second reflection (748 $\mu$ s after the first reflection) for each of the targets. 
Table 6.4. Experimentally measured transmitted impulses at the arrival time of the first and second distal reflections for the three targets.

\begin{tabular}{|c|c|c|c|c|c|c|}
\hline \multirow[b]{3}{*}{$\begin{array}{l}\text { Standoff } \\
\text { distance } \\
(\mathrm{cm})\end{array}$} & \multicolumn{2}{|c|}{ Flat block } & \multicolumn{2}{|c|}{ V-block } & \multicolumn{2}{|c|}{$\begin{array}{c}\text { V-block } \\
\text { with Al slider plates }\end{array}$} \\
\hline & \multicolumn{2}{|c|}{ Transferred impulse } & \multicolumn{2}{|c|}{ Transferred impulse } & \multicolumn{2}{|c|}{ Transferred impulse } \\
\hline & $\begin{array}{c}1^{\text {st }} \text { distal } \\
\text { reflection } \\
(\mathrm{kPa} \cdot \mathrm{s})\end{array}$ & $\begin{array}{c}2^{\text {nd }} \text { distal } \\
\text { reflection } \\
(\mathrm{kPa} \cdot \mathrm{s})\end{array}$ & $\begin{array}{c}1^{\text {st }} \text { distal } \\
\text { reflection } \\
(\mathrm{kPa} \cdot \mathrm{s})\end{array}$ & $\begin{array}{l}2^{\text {nd }} \text { distal } \\
\text { reflection } \\
(\mathrm{kPa} \cdot \mathrm{s})\end{array}$ & $\begin{array}{l}1^{\text {st }} \text { distal } \\
\text { reflection } \\
(\mathrm{kPa} \cdot \mathrm{s})\end{array}$ & $\begin{array}{l}2^{\text {nd }} \text { distal } \\
\text { reflection } \\
(\mathrm{kPa} \cdot \mathrm{s})\end{array}$ \\
\hline 19 & 7.1 & 11.9 & 4.2 & 11.2 & 3.4 & 9.5 \\
\hline 24 & 4.1 & 11.2 & 2.2 & 9.2 & 1.8 & 8.2 \\
\hline 29 & 3.5 & 10.7 & 1.8 & 7.3 & 1.5 & 5.3 \\
\hline
\end{tabular}

\subsubsection{V-shaped target}

High-speed video images of sand impact with a V-block target at a standoff distance of $29 \mathrm{~cm}$ are shown in Figure 6.11. Careful examination of the images in Figure 6.11(d)-(e) shows reflection of the sand off the edge of the target (indicated by white lines) parallel to the inclined surface of the V-block. However, important details are obscured by the sand that traveled between the target and camera.

Results from a simulation of the test are shown in Figure 6.12 and can be used to understand the test results. The fastest sand began to first impact the target at about $0.45 \mathrm{~ms}$ after detonation (not shown). The simulation in Figure 6.12(a) shows sand particle locations at about the same time as the experimental image in Figure 6.11(a). It shows that the fastest sand had contacted the target in a manner consistent with the experimental image. The subsequent simulations show that sand particles were forward reflected from the target's inclined faces and traveled towards the outer edges of the target. Many of these reflected particles suffered collisions with later arriving incoming particles causing their redirected trajectory to remain close and parallel to the inclined target surface. Some of these particles can be seen leaving the target in Figure 6.12(d). As time progressed, the concentration of the near surface reflected particles increased, and a thin line of particles flowing away from the target edge can be clearly seen in Figure 6.12(e) and (f) consistent with experimental observations, Figure 6.11(d) and (e). The accumulation of particles is clearly seen in Figure 6.12(g) and (h) with the densest region of the propagating sand impacting the target between 2.0 to $2.8 \mathrm{~ms}$. It remained close to the inclined edge as it flowed parallel to the surface and then beyond the $\mathrm{V}$ target edge. Once again, a small fraction of the sand particles made impact 

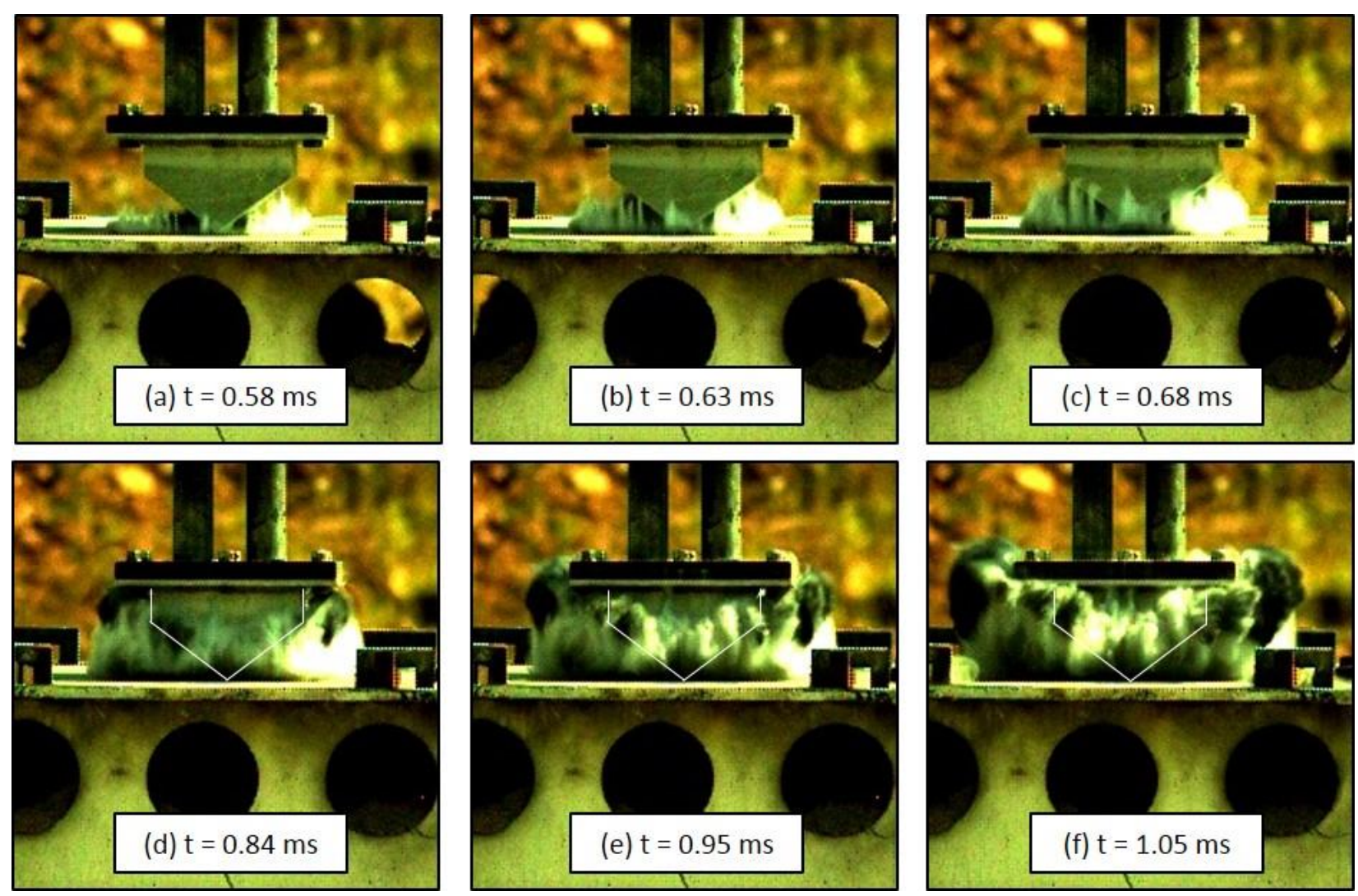

Figure 6.11. High-speed video image sequence of sand impact with the V-shaped target at a standoff distance of $29 \mathrm{~cm}$. The target geometry is indicated by white lines in $(d)-(f)$.

with the underside of the attachment plate rather than the test target. Their impulse would have been added to that of the samples in both the experimental and simulated transmitted impulse data measured by the Kolsky bars.

Figure 6.13 shows the measured and simulated pressure-time waveforms and the integrated impulse-time plots for the V-shaped target. The simulated and measured signal arrival times and waveforms are in good agreement. Like the experimental results, the simulated signal shows an initial pressure peak followed by a second pressure peak of equal or greater value. When these results are compared to those for the flat bottom sample, Figure 6.9, it is evident that the rise time of the V-block target pressure was substantially longer. This arose because of the variation in sand propagation distance (and therefore impact time) with lateral position for the V-block target. The V-block target pressure waveforms again exhibited two peaks with the peak positions corresponding to the arrival of sequential Kolsky bar distal reflections. The maximum pressure 

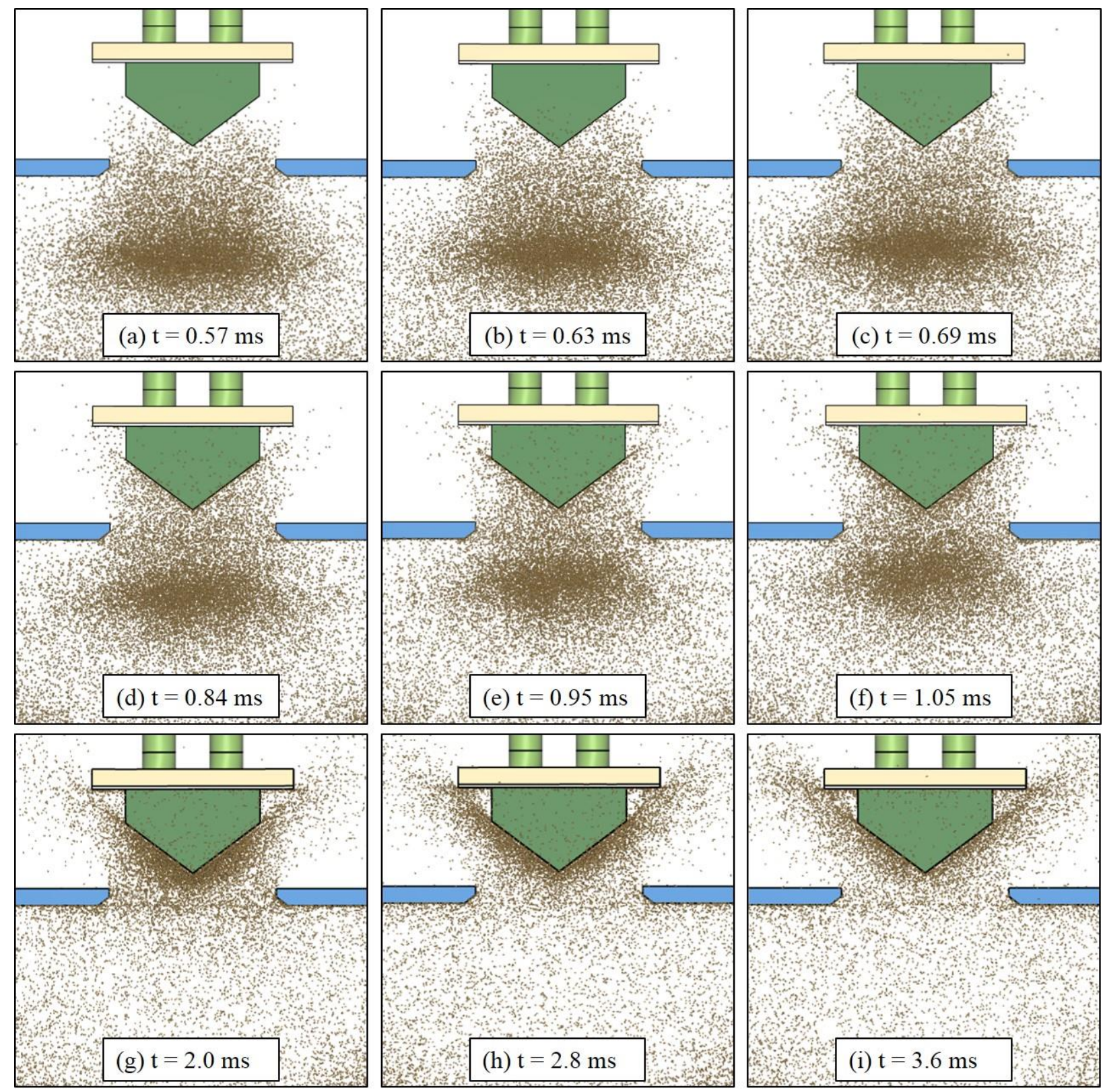

Figure 6.12. Simulation of sand impact with the V-shaped target for a standoff distance of $29 \mathrm{~cm}$. 

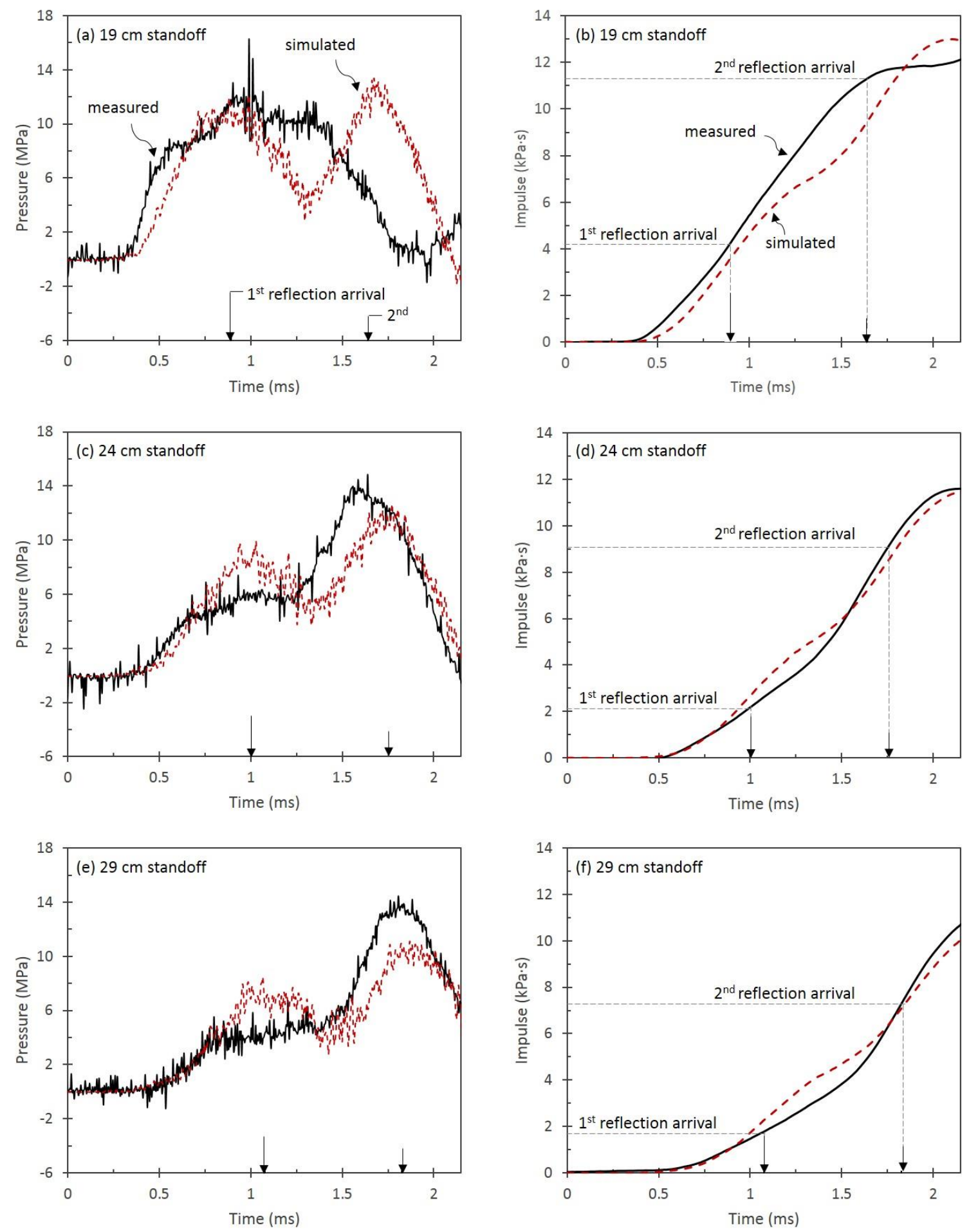

Figure 6.13. Comparison of the strain gauge measured (black line) and simulated (red dashed line) pressure-time waveforms for the V-shaped target at standoff distances of (a) $19 \mathrm{~cm},(\mathrm{c}) 24 \mathrm{~cm}$ and (e) 29 $\mathrm{cm}$. Figures $(b),(d)$, and $(f)$ show the impulse-time waveforms for each standoff distance together with the arrival times of the $1^{\text {st }}$ and $2^{\text {nd }}$ distal reflections at the strain gauges. Time $t=0 \mathrm{~s}$ again corresponds to the instant of detonation. 
attained during the first peak is summarized in Table 6.3. It decreased from 8.4 MPa at a standoff distance of $19 \mathrm{~cm}$ to $4.7 \mathrm{MPa}$ at the $29 \mathrm{~cm}$ standoff distance consistent with a reduction in particle velocity and decrease in density with increasing standoff distance. Since the apex of the V-block target was $3.8 \mathrm{~cm}$ closer to the sand surface than the flat bottom geometry sample, Figure 6.2 , the apex of the V-shaped targets at a nominal standoff distance of $19 \mathrm{~cm}$ was only $\sim 15 \mathrm{~cm}$ from the Datasheet surface. At this standoff, examination of Figure 6.8(c) and (d) shows that the sand first arrived at the apex location at approximately $0.2 \mathrm{~ms}$ after detonation and with a velocity near the peak in Figure 6.7(c). The initial signal arrival time at the strain gauge location is slightly earlier for the V-block targets compared to the arrival times of the flat bottom target, consistent with this observation. The measured impulse transmitted by the V-block target at the first and second distal reflection arrival times is summarized in Table 6.4, and plotted against standoff distance in Figure 6.10. Like the flat bottomed sample, the impulses fell sharply with standoff distance.

\subsubsection{V-shaped target with sliding plates}

Figure 6.14 shows high-speed video images of the impact of the sand with the V-block target with attached aluminum slider plates (using the same time sequence as Figure 6.11) for a standoff distance of $29 \mathrm{~cm}$. The sand fingers had already begun to make contact with the target in the image collected at $0.58 \mathrm{~ms}$ after detonation, Figure 6.14(a). While redirection of the sand upon impact is evident in Figure 6.14(d)-(f), details are again obscured by intervening sand.

A simulation of the test is shown in Figure 6.15. Initial impact of sand fingers with the sample apex (not shown) occurred at approximately $0.45 \mathrm{~ms}$. The result shown in Figure 6.15(a) at 0.57 $\mathrm{ms}$ after detonation is consistent with the experimental observation, Figure 6.14(a), of fastest sand contact with the sample at this time. During the following $0.27 \mathrm{~ms}$ of loading, Figure 6.15(a)-(d), the slider plates acquired sufficient impulse to begin sliding at approximately $0.84 \mathrm{~ms}$ after detonation, Figure 6.15(d). Figure 6.15(d)-(f) show that the sand impact with the slider caused continued acceleration of the sliders resulting in exposure of the V-block apex to impact by late arriving sand. Some sand particles again missed the V-block and made impact with the underside of the attachment plate. However, as the slider plates began to propagate outwards, they intersected this sand and began to shield the underside of two areas of the attachment plate from further impact (and impulse), Figure 6.15(g)-(i). Departure of the plates from the rigid V-block surfaces is seen in Figure 6.15(i) to have occurred at approximately $3.6 \mathrm{~ms}$ after detonation. The 

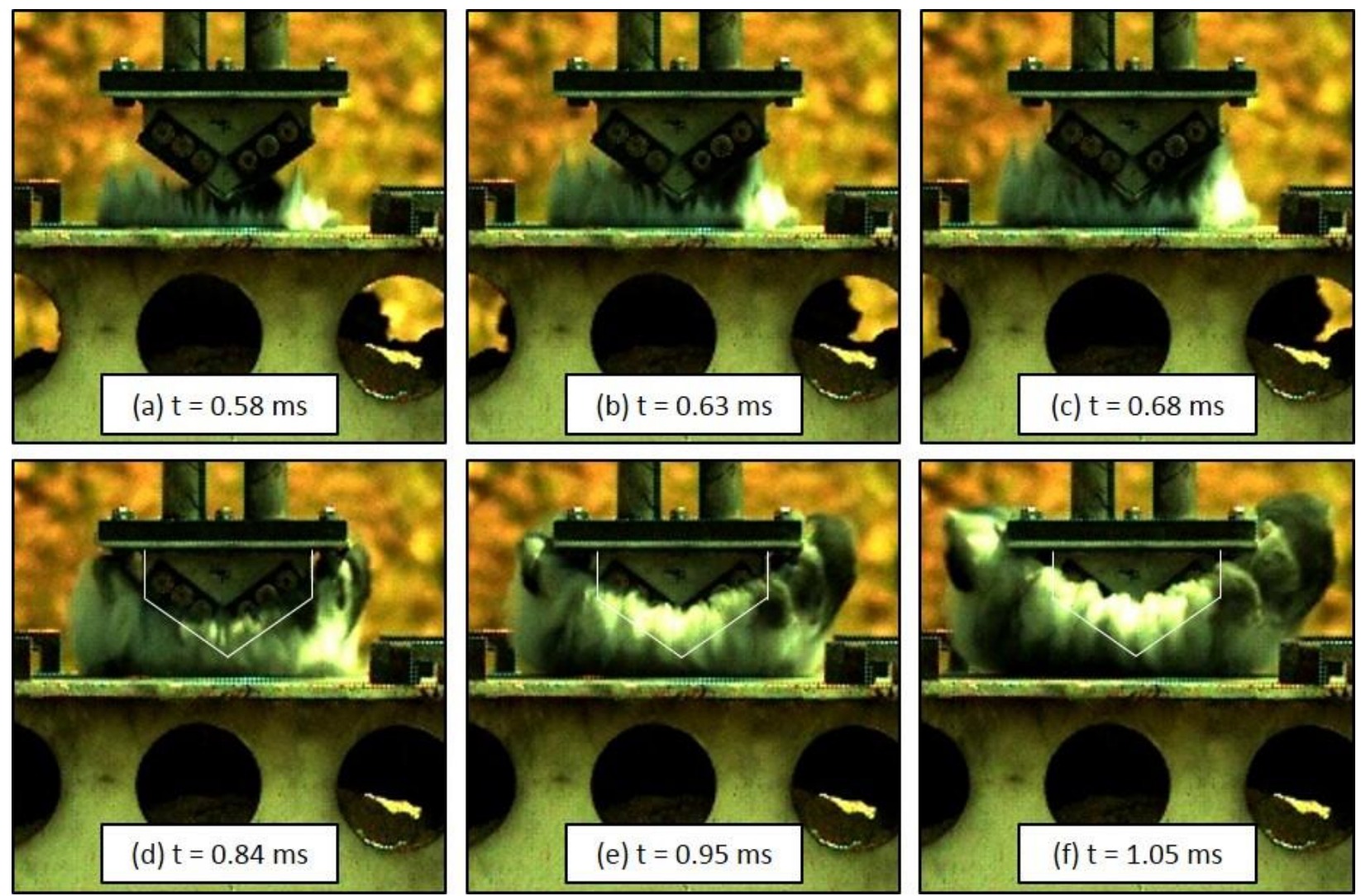

Figure 6.14. High-speed video image sequence for the V-shaped target with aluminum slider plates impacted by sand for a $29 \mathrm{~cm}$ standoff distance. The target geometry is indicated by white lines in (d)-(f).

ballistic trajectory of one of the sliding plates for this $29 \mathrm{~cm}$ standoff distance test was successfully tracked using the wider field of view camera. Images from this camera have been overlaid at approximately $100 \mathrm{~ms}$ time intervals in Figure 6.16 to show the sliding plate's parabolic trajectory (and plate rotation). The sliding plate was subsequently recovered $12.5 \mathrm{~m}$ to the right of the vertical rig apparatus at a location orthogonally offset by $1.8 \mathrm{~m}$ towards the camera. 

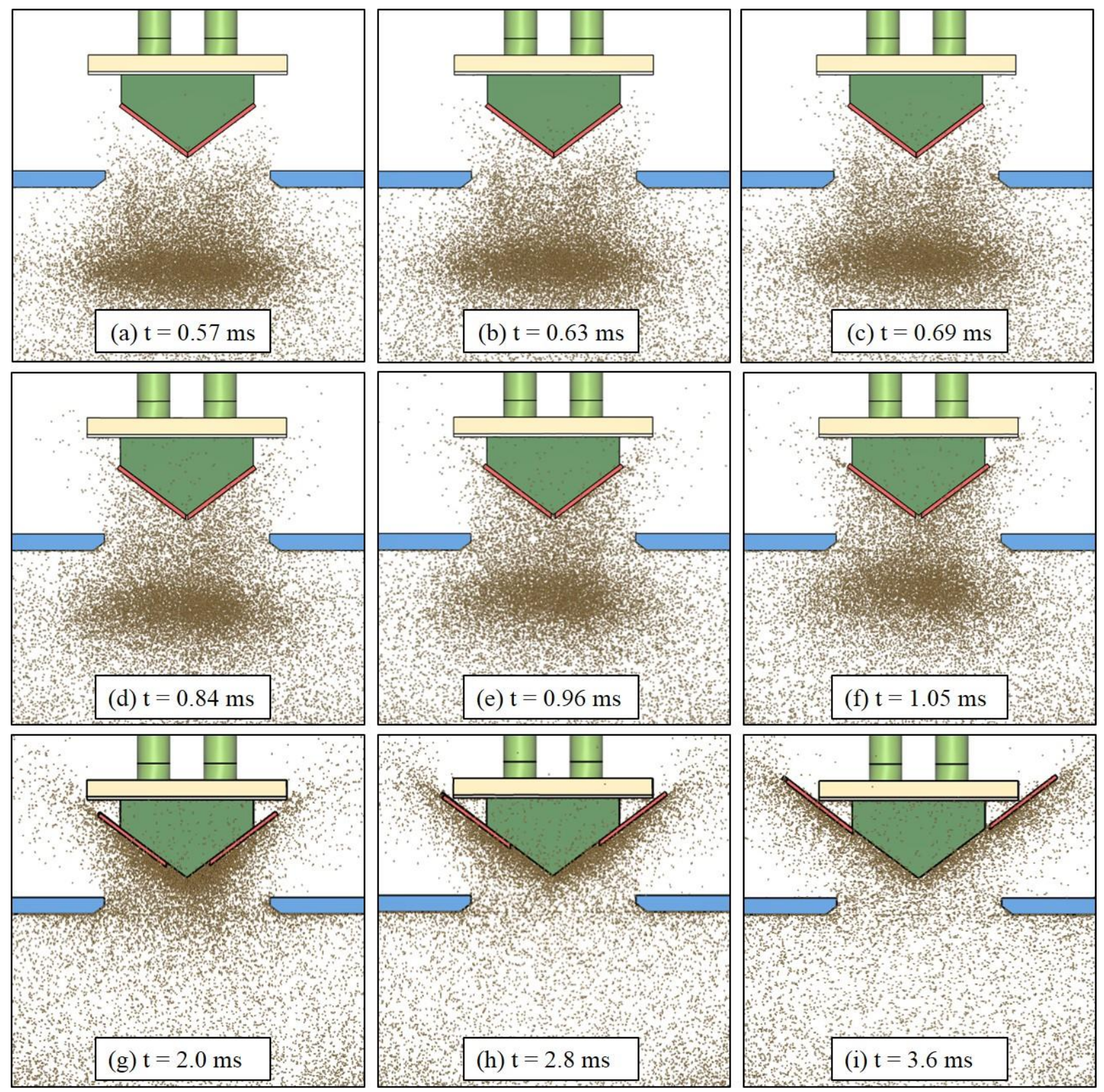

Figure 6.15. Simulation time sequence of sand impact with the $V$-shaped target with attached slider plates for $29 \mathrm{~cm}$ standoff distance. 


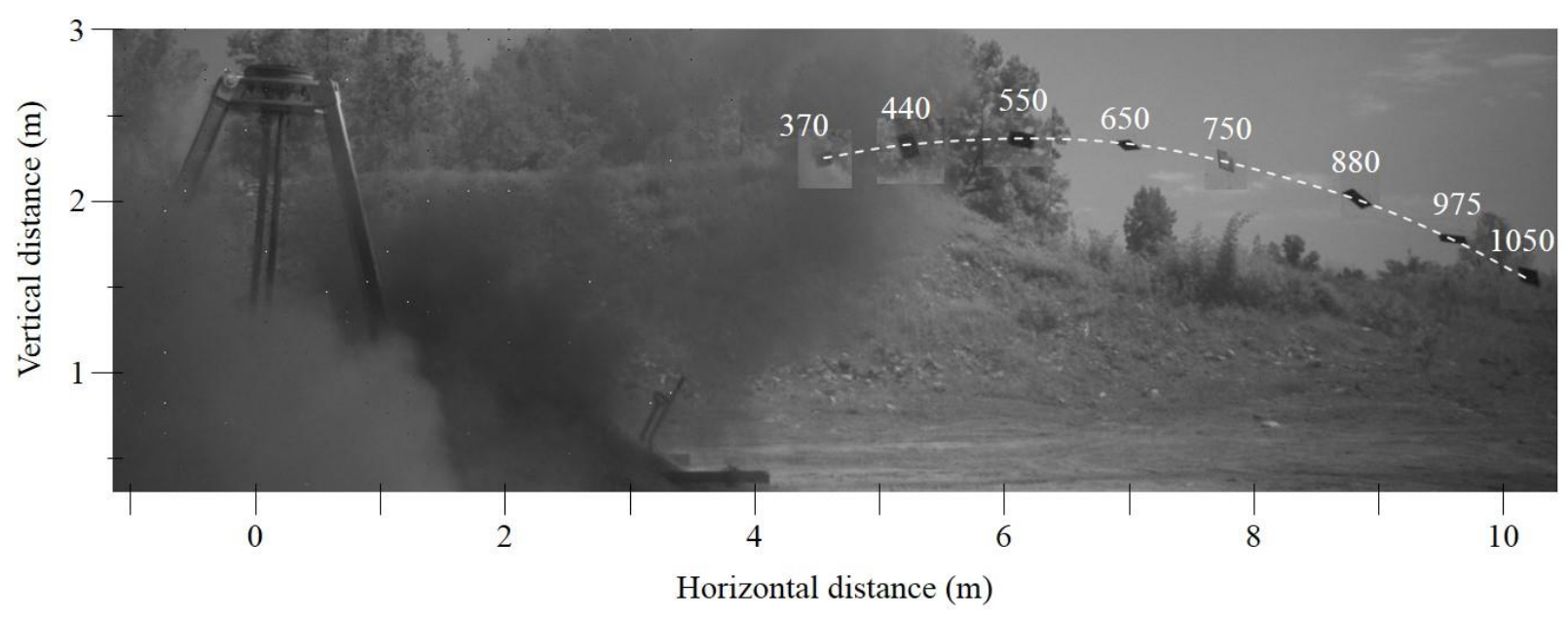

Figure 6.16. Multiple exposure image of a sliding plate target test at a $29 \mathrm{~cm}$ standoff distance. The sliding plate (left) location at $t=370 \mathrm{~ms}$ after detonation is shown and its trajectory over time is indicated by the dotted line. The time for each slider plate position is shown (in milliseconds) at approximately $100 \mathrm{~ms}$ intervals.

High-speed video observations of the interaction of sand at a $19 \mathrm{~cm}$ standoff distance are shown in Figure 6.17. Figure 6.17(a) is the initial setup at the instant of detonation. It shows the support rig, the steel sandbox, the Kolsky bar system (including its strain gauges), the trigger wire for detonation initiation, the V-target with aluminum sliding plates, and the concrete barrier protecting the instrumentation. Figure 6.17(b) shows the sand position at $0.3 \mathrm{~ms}$ after detonation. The inset shows that contact of sand with the apex of the target had already occurred, consistent with the prediction of Figure 6.8 that the first sand would arrive at approximately $0.2 \mathrm{~ms}$ after detonation. Subsequent interactions between the sand and target shortly thereafter are hidden by reflected sand and (black) detonation products. Nevertheless, Figure 6.17(c) collected at $1.2 \mathrm{~ms}$ after detonation shows that substantial sand reflection from the $\mathrm{V}$-shape of the target had occurred. The sand then completely obstructed the view of the target until about $35 \mathrm{~ms}$ after detonation. In Figure 6.17(e) and (f), the sand had expanded to a sufficiently low density that the sliding plates could be seen after their ejection from the target. Their initial position is identified, and dotted lines are provided to indicate their probable trajectory to their first observed positions. The sliding plates were eventually recovered from a location that was $39 \mathrm{~m}$ from the original position. 

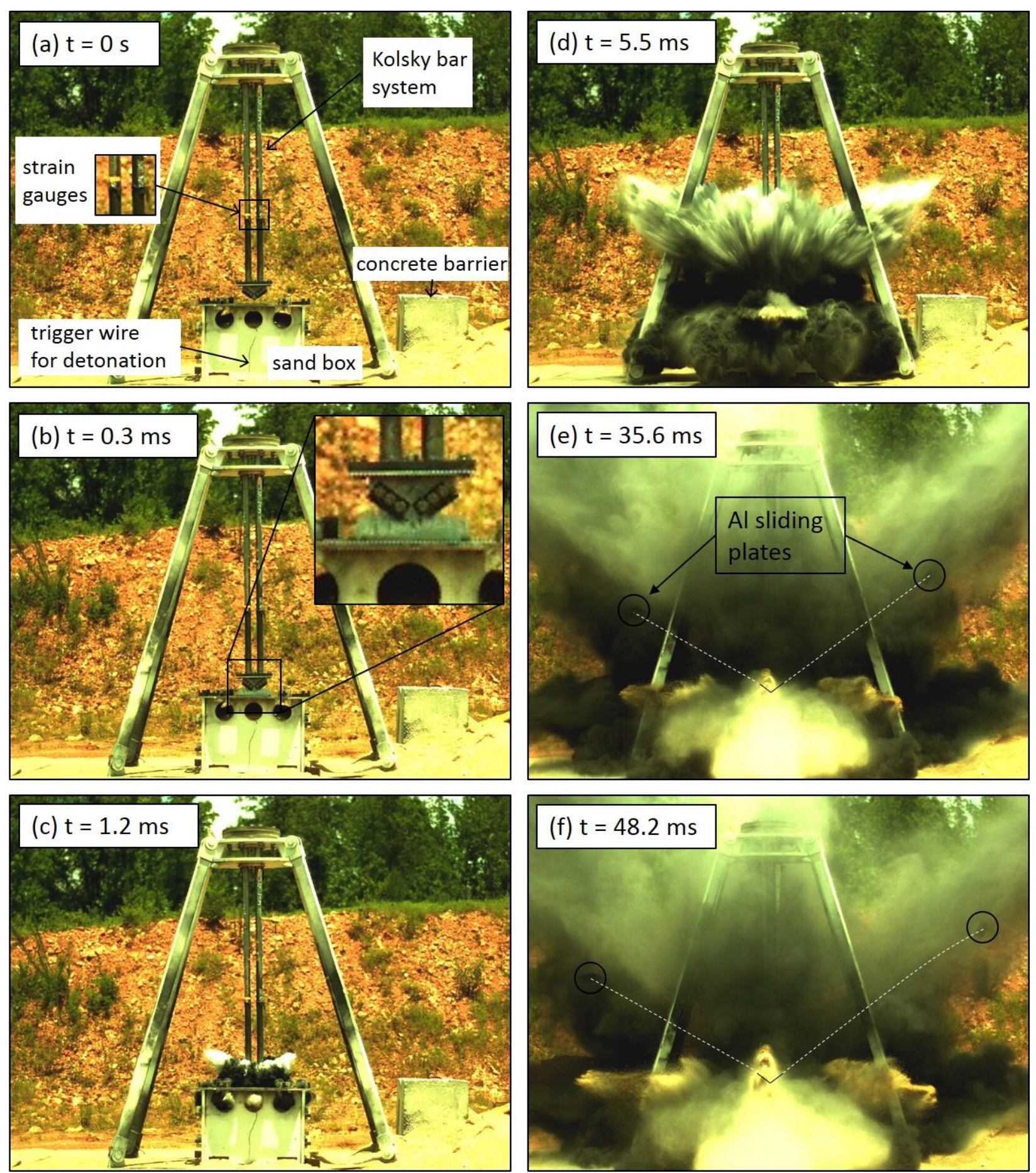

Figure 6.17. High-speed video images of V-block target with aluminum slider plates at $19 \mathrm{~cm}$ standoff distance. (a) The test rig at $t=0 \mathrm{~s}$ (the moment of detonation), (b) $t=0.3 \mathrm{~ms},(c) t=1.2 \mathrm{~ms},(d) t=5.5 \mathrm{~ms}$, (e) $t=35.6 \mathrm{~ms}$, and $(f) t=48.2 \mathrm{~ms}$. The two aluminum slider plates are visible as they were ejected from the target in $(e)$ and $(f)$. The white dotted lines show the approximate trajectory. 


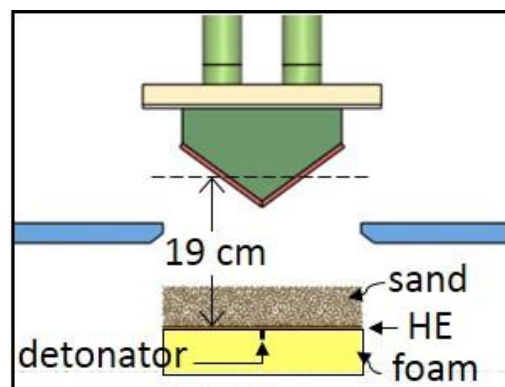

(a) $0.0 \mathrm{~ms}$
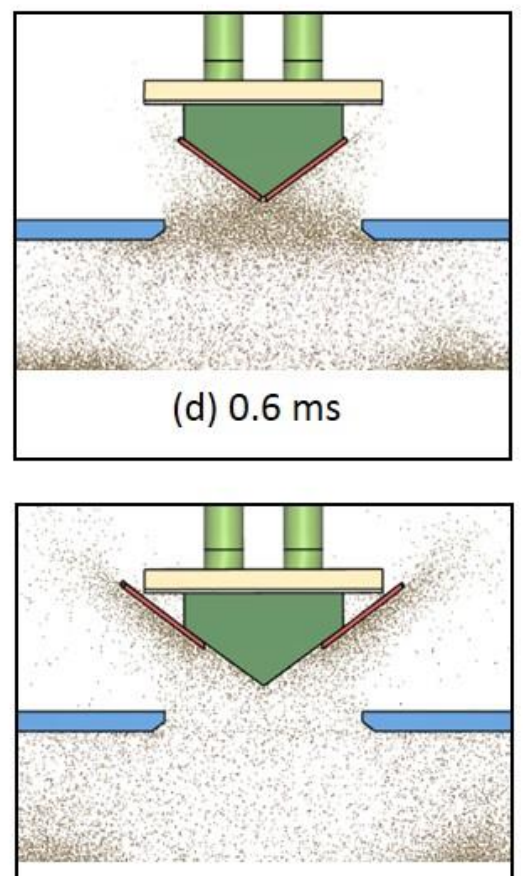

(g) $2.1 \mathrm{~ms}$
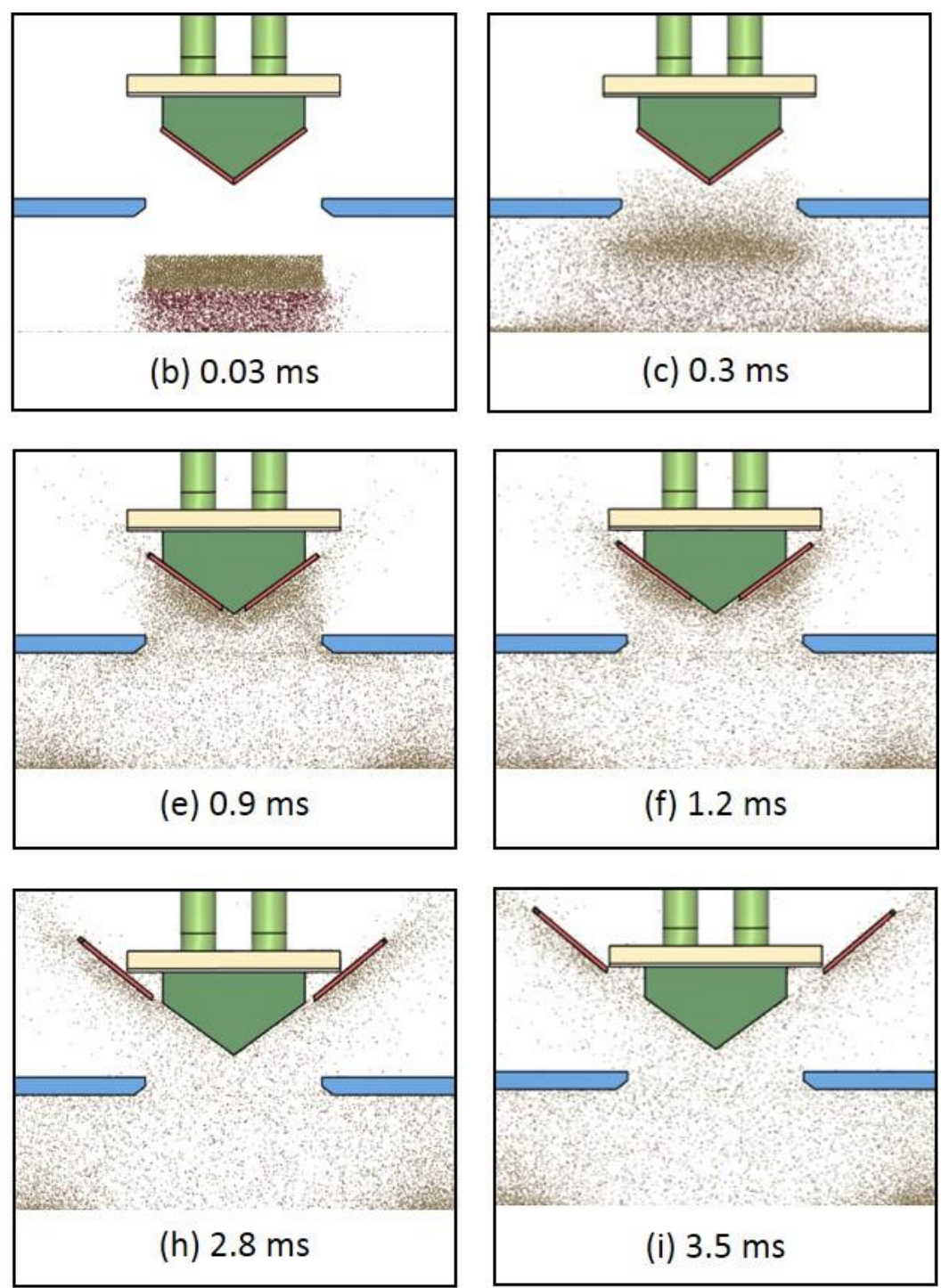

Figure 6.18. Sand particle propagation simulations for a V-block target with sliding plates showing the sliding plate movement after impact. The standoff distance was $19 \mathrm{~cm}$. The dark red particles represent high explosive particles and the brown particles the wet sand.

The simulation of the $19 \mathrm{~cm}$ standoff distance test can be used to infer the target's response sequence. Figure 6.18 shows particle progression, impact with the target, and slider plate ejection for the $19 \mathrm{~cm}$ standoff distance test. In Figure 6.18(b), the detonation has transmitted a compressive shock through the wet sand. This is subsequently reflected at the air/sand interface resulting in spallation of fast sand from the interface. Figure 6.18(c) shows that the accelerated sand had begun to impact the target slightly before $0.3 \mathrm{~ms}$ after detonation, consistent with experimental observations, Figure 6.17(b). Plate sliding began shortly before Figure 6.18(d) at 
Table 6.5. Simulation predicted slider plate escape time for a $V$ shaped target, together with the plates $Y$ and $Z$ component velocities and plate speed at the time of escape. The $P_{Z}$ momentum and $I_{Z}$ impulse component of the each plate is also given.

\begin{tabular}{ccccccc}
$\begin{array}{c}\text { Standoff } \\
(\mathrm{cm})\end{array}$ & $\begin{array}{c}\text { Escape time } \\
(\mathrm{ms})\end{array}$ & $\begin{array}{c}\mathrm{V}_{\mathrm{Y}} \\
(\mathrm{m} / \mathrm{s})\end{array}$ & $\begin{array}{c}\mathrm{V}_{\mathrm{Z}} \\
(\mathrm{m} / \mathrm{s})\end{array}$ & $\begin{array}{c}\text { Plate speed } \\
\mathrm{V}(\mathrm{m} / \mathrm{s})\end{array}$ & $\begin{array}{c}\text { Z-plate } \\
\text { momentum } \\
\mathrm{P}_{\mathrm{Z}}(\mathrm{N} \cdot \mathrm{s})\end{array}$ & $\begin{array}{c}\text { Z-plate } \\
\text { impulse } \\
\mathrm{I}_{\mathrm{Z}}(\mathrm{kPa} \cdot \mathrm{s})\end{array}$ \\
\hline 19 & 2.60 & 53 & 43 & 69 & 20 & 0.97 \\
24 & 2.97 & 51 & 41 & 66 & 19 & 0.93 \\
29 & 3.45 & 43 & 35 & 57 & 16 & 0.79 \\
\hline
\end{tabular}

approximately $0.6 \mathrm{~ms}$ after detonation. At this standoff distance, the sliding plates escaped the target between Figure 6.18(g) and (h) at approximately $2.6 \mathrm{~ms}$ after detonation. Once the slider plate is impacted and accelerates outward, the V-target apex and underlying inclined surfaces become exposed to late arriving sand impacts.

The simulations indicated that the time taken for the slider plates to slide off the target surface increased with standoff distance from approximately 2.60 to $3.45 \mathrm{~ms}$. The plate escape times at each of the standoff distances were determined from the simulations and are summarized in Table 6.5 , together with their horizontal $\left(\mathrm{V}_{\mathrm{Y}}\right)$ and vertical $\left(\mathrm{V}_{\mathrm{Z}}\right)$ component velocities, their departure speed, and their Z-component momentum and impulse at the time of escape. As the standoff distance increased, the sliding plate velocity and momentum decreased consistent with the reduction in sand velocity (Figure 6.7(c)) and density resulting from collisions of sand particles with air and the axial and lateral stretching of the sand plume. It is interesting to observe the accumulation of the sand particles near the sliding plates in Figure 6.18(d) to (i), indicating cessation of motion in the reference frame of the sliding plates and the end of the momentum transfer to the plates. This phenomenon was also observed for $29 \mathrm{~cm}$ standoff distance in Figure $6.15(\mathrm{~g})$ and $(\mathrm{h})$.

The measured and simulated pressure-time waveforms for the V-block targets with the aluminum slider plates are shown for each standoff distance in Figure 6.19. The pressure waveforms had a similar initial slope and peak pressure to the V-block target, Table 6.3. The transmitted impulse at the first and second distal arrival times are also listed in Table 6.4, and their dependence upon standoff distance shown on Figure 6.10. While the maximum pressures of the first peaks were similar to those of the tests conducted on V-block samples without a sliding plate, 

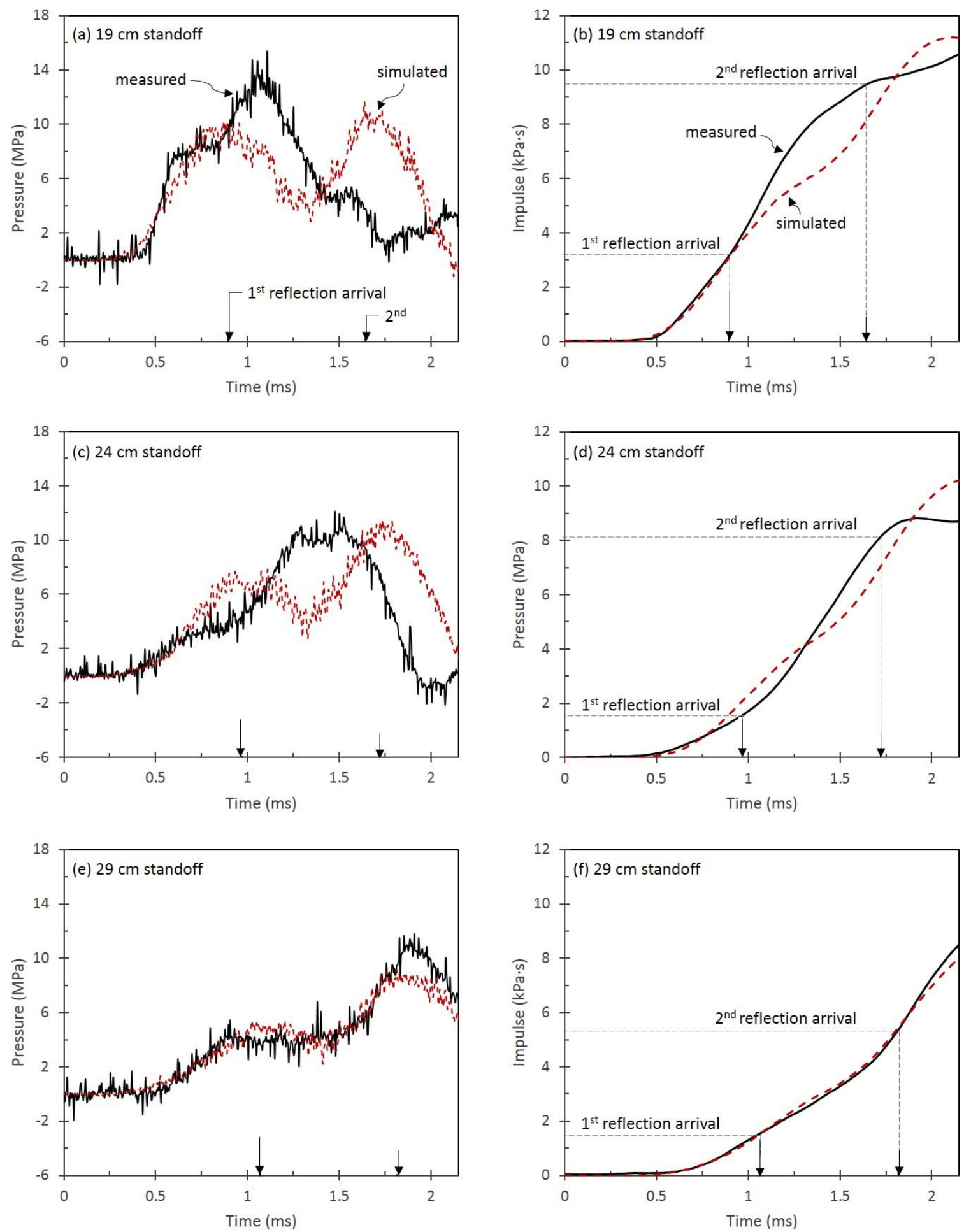

Figure 6.19. Comparison of the strain gauge measured (black line) and simulated (red dashed line) pressure-time waveforms for the $V$-shaped target with aluminum slider plates at standoff distances of $(a)$ $19 \mathrm{~cm},(\mathrm{c}) 24 \mathrm{~cm}$ and (e) $29 \mathrm{~cm}$. The impulse-time waveforms for each standoff distance are shown in (b), $(d)$, and $(f)$ together with the arrival times of the $1^{\text {st }}$ and $2^{\text {nd }}$ distal reflections. 
the impulses later in the loading process were substantially reduced as the inertia of the sliding plates was overcome.

\subsection{Discussion}

The experimental study has confirmed that significant reductions in the pressure applied by impact of granular matter with a target can be achieved by reducing the inclination of impact and increasing the standoff distance, Table 6.3. Integration of the measured pressure up to the arrival of the first distal reflection, Table 6.4 and Figure 6.10(a), also showed that the impulse transmitted to the targets decreased with reduction of incidence angle and increase in standoff distance. For example, at a standoff distance of $19 \mathrm{~cm}$, the impulse fell from $7.1 \mathrm{kPa} \cdot \mathrm{s}$ for the flat bottom target to $4.2 \mathrm{kPa} \cdot \mathrm{s}$ for the $\mathrm{V}$-block target. The combination of increasing the standoff distance from 19 to $29 \mathrm{~cm}$ and reducing $\alpha$ from $90^{\circ}$ to $53^{\circ}$ decreased the impulse from $7.1 \mathrm{kPa} \cdot \mathrm{s}$ to only $1.8 \mathrm{kPa} \cdot \mathrm{s}$. The discrete particle simulations gave similar peak pressures and impulses to those measured, Figure 6.9 and Figure 6.13, supporting their use for interpretation of the tests.

The spatial distribution of the impulse transferred to the samples is shown in Figure 6.20 for each standoff distance. Note that some impulse was also transmitted to the underside of the attachment plates supporting each target, and its contribution to the transmitted impulse will be estimated below. The impulse applied to the flat-bottomed target was uniformly distributed across the surface of the impact face of the target, and declined as the standoff distance was increased. The impulse transmitted to the V-block target was much more concentrated near the apex of the target. Recall from Figure 6.6(b) that the distance between the apex of the V-block target and the explosive sheet was $3.8 \mathrm{~cm}$ less than that to the surface of the flat-bottomed sample. Even though this local standoff distance was smaller, the impulse applied to the inclined surfaces of the V-block was less than to the flat-bottomed target because the reflected sand retained a significant fraction of its incident momentum in the $\mathrm{Z}$ direction.

The mechanisms responsible for the reductions of pressure and impulse with standoff distance for $\alpha=90^{\circ}$ targets have been analyzed by Holloman et al. [13] and shown to result from axial and lateral spreading of the granular medium, and reductions in its velocity due to momentum transferring collisions with air particles. This interpretation is consistent with the simulations shown in Figure 6.8. The surface inclination effect observed here in both the experimental and 


\section{Specific impulse (kPa) $\mathrm{s}) \mathrm{t}=2.0 \mathrm{~ms}$}

$\begin{array}{llllll}0 & 2 & 4 & 6 & 8 & 10\end{array}$

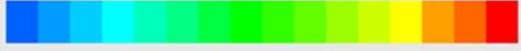
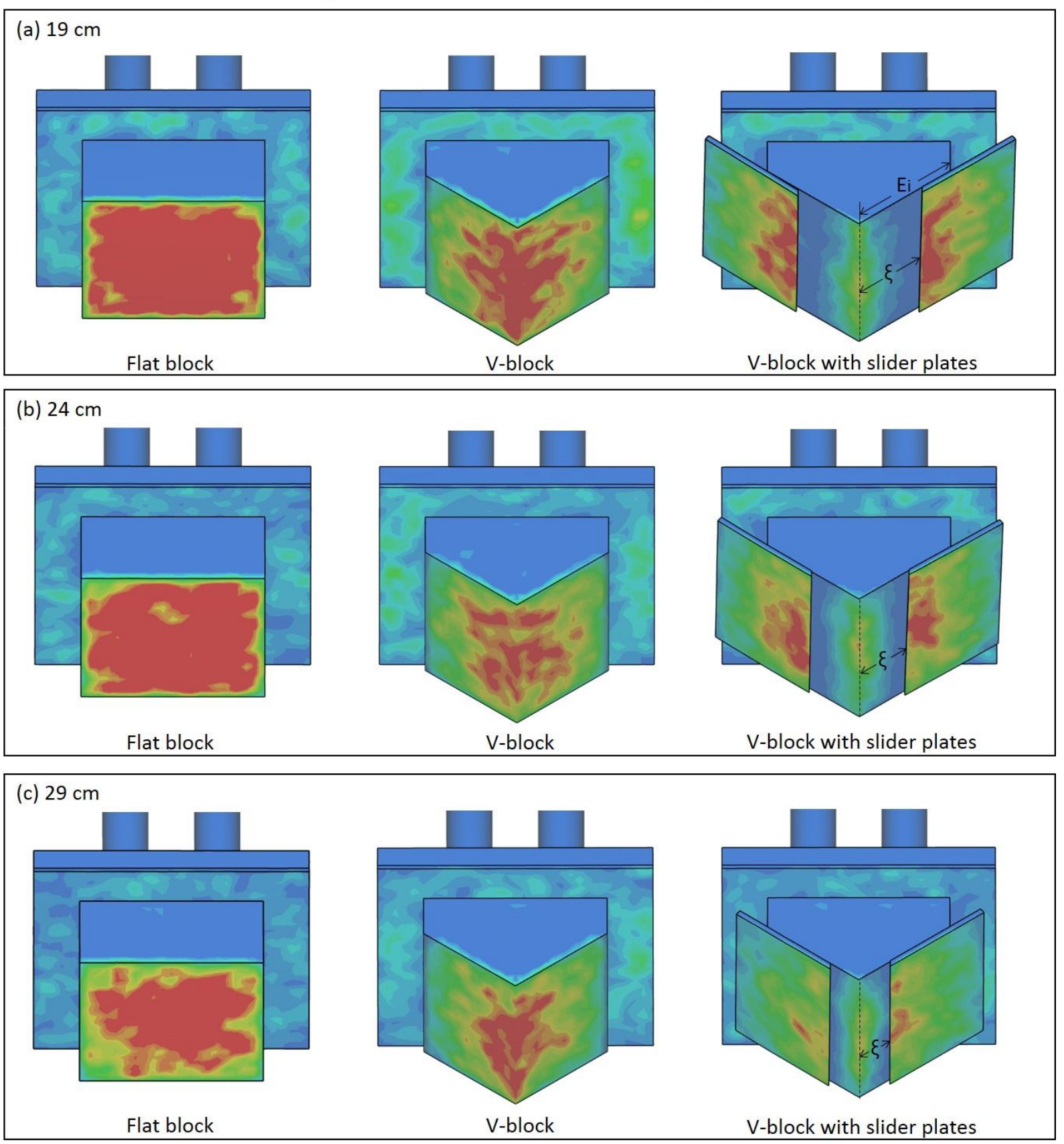

Figure 6.20. Impulse distributions on the bottom of the flat block, V-block target, and the V-block with slider plates accumulated during the first $2 \mathrm{~ms}$ after detonation for each standoff distance. $\xi$ is the distance the sliding plates have traveled along the inclined underlying surface. The simulations used a coefficient of friction $\mu_{s}=0.5$ between the sand and roughened target surface, and a friction value of $\mu_{p}=0.05$ for the lubricated interface between the slider and target. 
simulation results also support the observations of Goel et al. [98]. By avoiding the arrest or rebound reflection of a granular flow against the target, a reduction of $\alpha$ from $90^{\circ}$ to $53^{\circ}$ reduced the impulse by a factor of 0.4-0.5 at the first distal reflection, Figure 6.10(a).

The experimental and simulation results, Figure 6.10, indicated that a further reduction in the applied pressure and the $I_{T}^{Z}$ component of transferred momentum was achieved by allowing the lubricated sliding of a pair of $6.3 \mathrm{~mm}$ thick plates attached to the $\mathrm{V}$-shaped target. Since the measured and simulated pressures and impulses are in very good agreement, the simulations can be used to analyze the mechanisms of interaction of the targets with the granular medium. The effect of the sliding plates upon the impulse distribution on the V-shaped structure is also shown in Figure 6.20. The majority of the impulse was transmitted to the apex end of the sliding plates with the underlying V-block directly receiving only a small fraction of the incident impulse at all standoff distances. The time dependent impulse transmitted to the V-block and sliding plate Vtargets has been calculated for the first $4 \mathrm{~ms}$ of loading and is shown in Figure 6.21(a) for the three standoff distances. The difference in transmitted impulse between the two target types was initially small, but then began to grow after a delay time that increased with standoff distance. This delay in impulse reduction is consistent with the time taken for the initiation of plate sliding.

The distance the sliding plate moved can be scaled by the length of the inclined edge of the V-block target, $E_{i}=12.7 \mathrm{~cm}$, and the normalized displacement is plotted versus time in Figure 6.21(b). It shows that the distance the slider plates had moved, $\xi$, decreased with increasing standoff distance (i.e. with decrease in incident impulse). The escape distance is shown by a dotted grey line along with the points of intersection with the plate distance at each standoff, and vertical dotted lines indicate the slider plate escape times (summarized in Table 6.5). This analysis rationalizes the experimental results shown in Figure 6.10 where the impulse accumulated up to the time of arrival of the first distal reflection $(558 \mu$ s after the direct signal reached the strain gauges), showed a smaller effect of the sliding plates, but their beneficial effect then increased over time as seen in Figure 6.10(b) (748 $\mu$ s later).

The $I_{T}^{Z}$ component of impulse transferred to each target design after 4 ms of loading can be calculated from the simulated strain gauge pressure and is plotted as a function of standoff distance in Figure 6.22(a). This (total) impulse is the sum of the impulse applied to the test targets and that applied to the underside of the attachment plate, Figure 6.20. This attachment plate impulse varied 

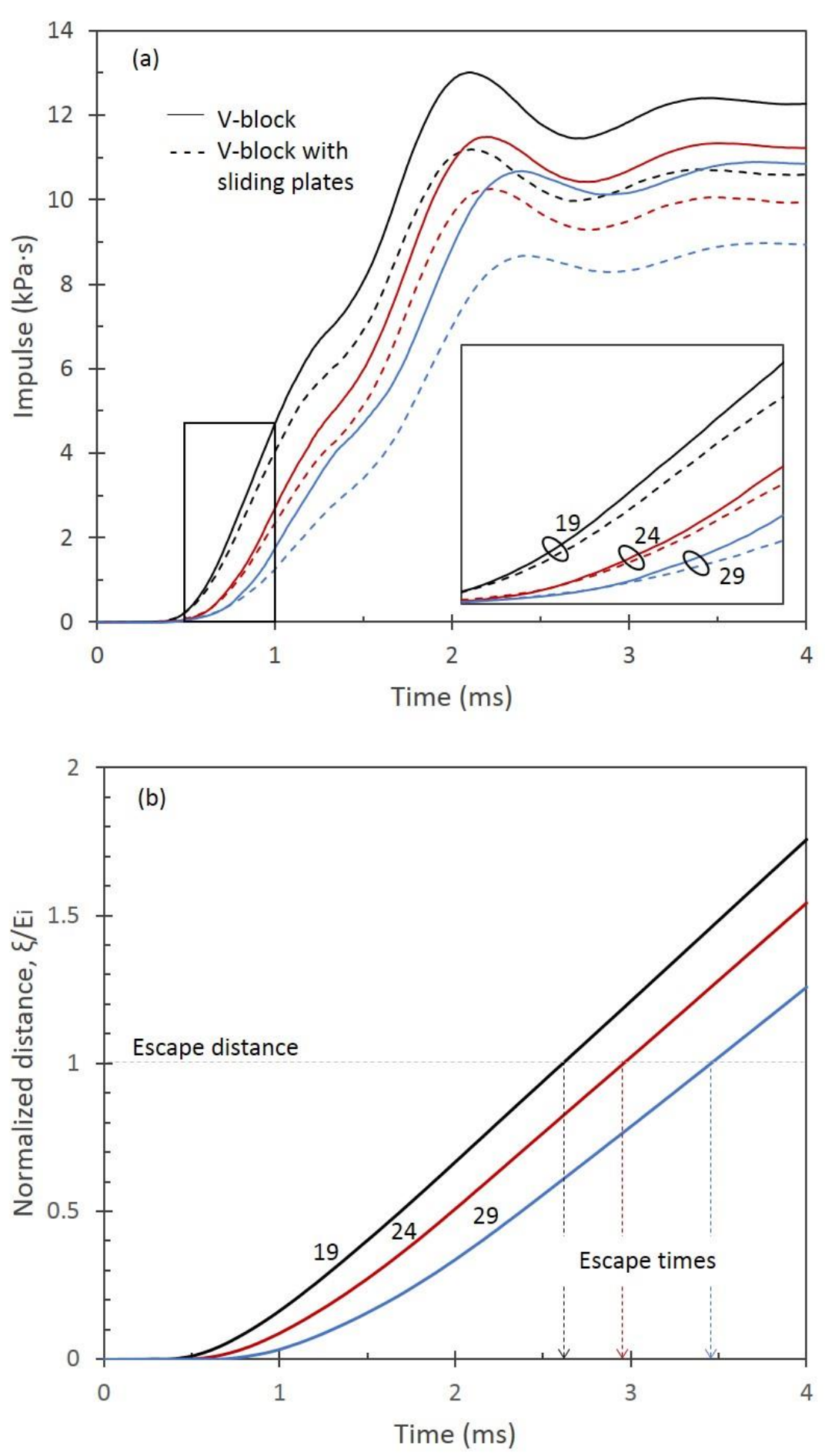

Figure 6.21. (a) Comparison of simulated impulse transfer to the V-block target (solid lines) and the $V$ block target with aluminum sliding plates (dashed lines) for the three standoff distances. (b) The normalized plate sliding distance versus time during sand impact for the three standoff distances. The distance and time of sliding for plate escape from the V-block are also shown for each standoff distance. 

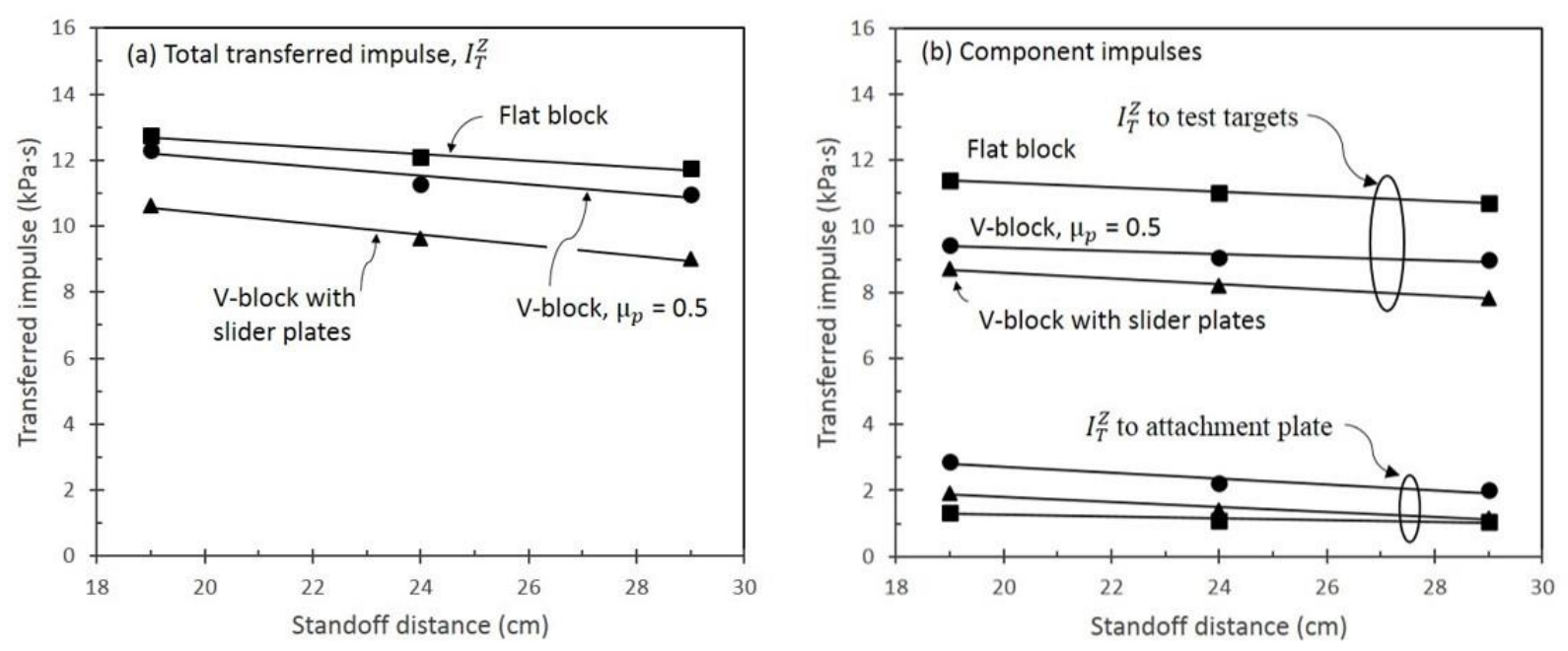

Figure 6.22. (a) The total simulated transferred impulse in the Z-direction after 4 ms calculated at the strain gauge location. (b) The impulse contributions transferred to the test targets and to the periphery of their attachment plate.

from 1 to $3 \mathrm{kPa} \cdot \mathrm{s}$, and resulted from the lateral expansion of the sand front beyond the width of the test targets, Figure 6.8, Figure 6.12, and Figure 6.15. It is plotted on Figure 6.22(b) for the three targets at each standoff distance. The impulse transferred to only the target surfaces is summarized in Table 6.6 and plotted on Figure 6.22(b). It shows that the reduction of impulse by the use of the sliding plates was approximately half of the reduction achieved by the change of incidence angle.

To quantify the effects of the target geometry and use of a sliding plate for the buried charge test geometry studied here, the ratio of the transmitted to incident impulse for each target design is also shown in Table 6.6. It can be seen that the ratio of the transmitted to incident impulse for the flat-bottomed target was approximately 1.02 while that transferred to the V-block was 0.85 and the target with sliding plates was 0.76 . The Z-component impulse of each slider plate at its time of escape from the targets was calculated by dividing the product of the plate mass and $\mathrm{V}_{\mathrm{Z}}$ velocity component (Table 6.5$)$ by the area normal to the plate $(203.2 \mathrm{~mm}$ x $101.6 \mathrm{~mm}$ ). The results indicate that approximately $8 \%$ of the incident impulse was transferred to each slider plate as it escaped the V-block base target. The inclined V-block target $\left(\alpha=53^{\circ}\right)$ reduced the impulse 
Table 6.6. Simulated impulse and normalized impulse applied to the three target designs for each standoff distance.

\begin{tabular}{|c|c|c|c|c|c|c|c|}
\hline \multirow[b]{2}{*}{$\begin{array}{l}\text { Standoff } \\
(\mathrm{cm})\end{array}$} & \multirow[b]{2}{*}{$\begin{array}{c}I_{O} \\
(\mathrm{kPa} \cdot \mathrm{s})\end{array}$} & \multicolumn{2}{|c|}{ Flat bottom } & \multicolumn{2}{|c|}{$\begin{array}{c}\text { V-block } \\
\left(\mu_{\mathrm{s}}=0.5\right)\end{array}$} & \multicolumn{2}{|c|}{$\begin{array}{c}\text { V-block } \\
\text { with slider plates }\end{array}$} \\
\hline & & $\begin{array}{c}I_{T}^{Z} \\
(\mathrm{kPa} \cdot \mathrm{s})\end{array}$ & $I_{T}^{Z} / I_{O}$ & $\begin{array}{c}I_{T}^{Z} \\
(\mathrm{kPa} \cdot \mathrm{s})\end{array}$ & $I_{T}^{Z} / I_{O}$ & $\begin{array}{c}I_{T}^{Z} \\
(\mathrm{kPa} \cdot \mathrm{s})\end{array}$ & $I_{T}^{Z} / I_{O}$ \\
\hline 19 & 11.10 & 11.40 & 1.03 & 9.48 & 0.85 & 8.71 & 0.78 \\
\hline 24 & 10.93 & 11.00 & 1.01 & 9.06 & 0.83 & 8.21 & 0.75 \\
\hline 29 & 10.44 & 10.71 & 1.03 & 8.97 & 0.86 & 7.83 & 0.75 \\
\hline
\end{tabular}

transferred to the normally oriented target $\left(\alpha=90^{\circ}\right)$ by about $17 \%$. An additional impulse reduction of approximately $11 \%$ was achieved by the use of sliding plates.

To compare the results in Table 6.6 with those of Goel et al. [98], the impulse ratios $I_{T}^{Z} / I_{O}$ are plotted versus standoff distance in Figure 6.23 (the incident main sand front speed for each standoff is summarized in Table 6.3). The reduction of impulse resulting from a decrease of the inclination angle from $90^{\circ}$ to $53^{\circ}$ can be clearly seen. The simulations again indicate that this "inclination effect" is dependent upon the friction coefficient between the sand particles and target surface. Inclined target impacts with a friction coefficient of $\mu_{s}=0.5$ result in an impulse ratio reduction from 1.02 to 0.85 whereas frictionless impact (with a friction coefficient $\mu_{s}=0.0$ ) results in an impulse reduction from 1.02 to 0.72 . This change of impulse is shown as the particle friction effect in Figure 6.23, and confirms the significant effect of surface friction reported by Goel et al. [98].

Examination of Figure 6.23 shows that the impulse ratios for tests with the sliding plates (solid triangles) resulted in a transferred impulse ratio of 0.76 which was higher than the predicted value of 0.72 for the frictionless impact of the V-block target (the open circles). Examination of Figure 6.20 shows that some of the impulse transferred to the sliding plate targets impacted the apex region beneath the plate (after it had slid past this region). These test samples therefore experienced two phases of loading. During early Phase I loading, the fastest sand impacted the sliding plates attached to the V-block target, transferring some of its incident momentum to the plates and thereby initiating low friction plate sliding across the surface of the underlying V-block target. The exposed surface of the underlying V-block surface was then impacted by later arriving (lower 


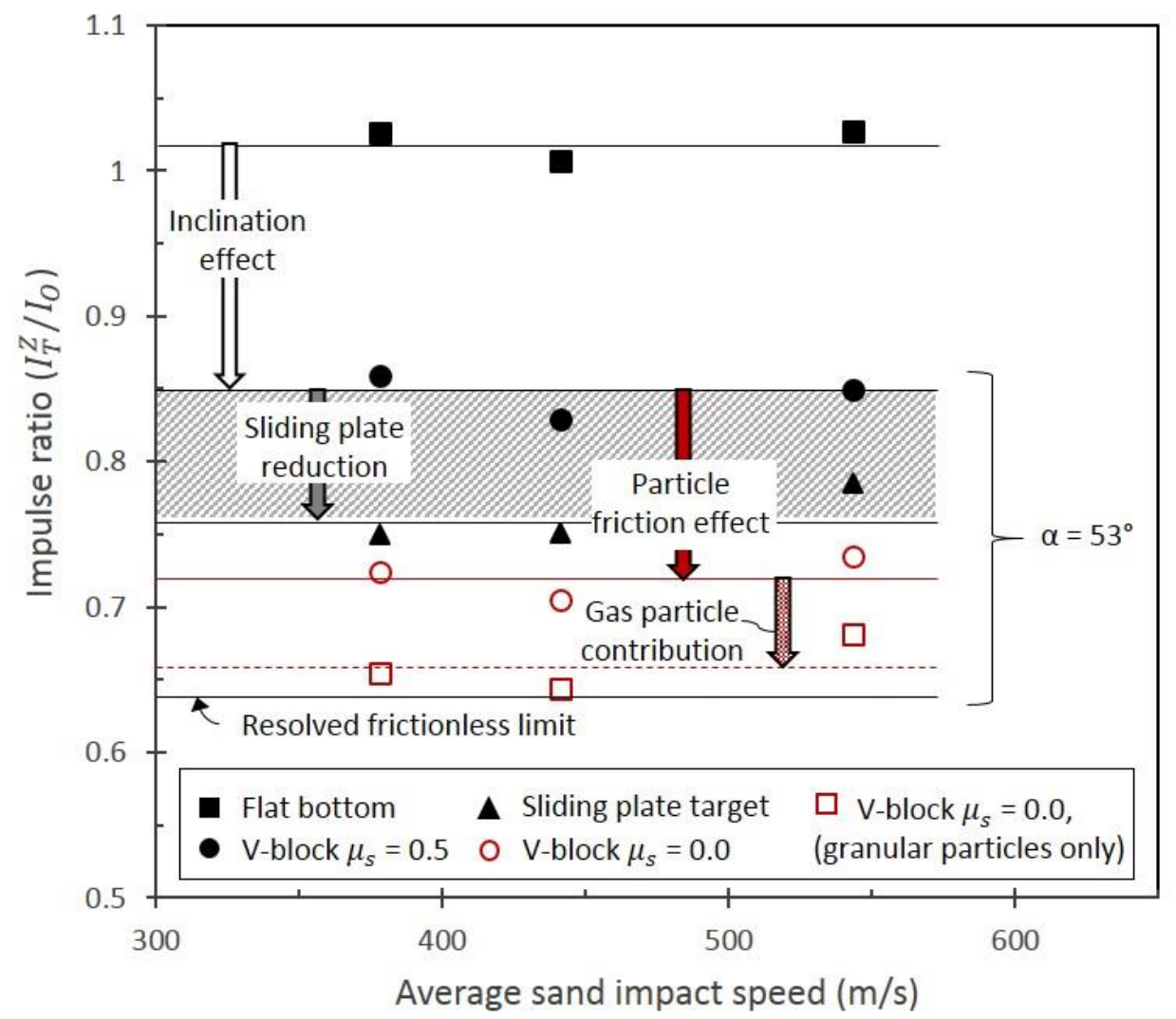

Figure 6.23. The simulated transferred impulse ratio in the Z-direction versus average sand particle velocity. The impulse of the (flat-bottomed) target with $\alpha=90^{\circ}$ dropped from 1.02 to 0.85 when $\alpha=53^{\circ}$ and a particle-target friction coefficient $\mu_{s}=0.5$ was used. The effect of the frictional interaction between sand particles and the inclined plate can be seen by comparing the $\mu_{s}=0.5$ and 0.0 results. This resulted in a decrease in impulse ratio from 0.85 to 0.72 . The use of a sliding plate reduced the impulse ratio from 0.85 to 0.76 . A fraction of the impulse was transferred to the targets by air and detonation product particles with no frictional interaction. When their contribution was subtracted from the results, the impulse transferred by frictionless sand particles approached the $\alpha=53^{\circ}$ resolved frictionless limit.

velocity) particles during Phase II loading. The magnitude of the Phase II impulse depends upon the speed with which the sliding plate exposes the underlying target surface, and by the impulse carried by late arriving particles. It is therefore a complex function of the target design and placement, and by the buried charge configuration. For the target design and test configuration explored here, the Phase II impulse transferred to the underlying V-block target varied from 1.8 $\mathrm{kPa} \cdot \mathrm{s}$ at a $19 \mathrm{~cm}$ standoff distance to $2.13 \mathrm{kPa} \cdot \mathrm{s}$ at a standoff distance of $29 \mathrm{~cm}$. This delayed impulse was the primary reason why the sliding plate tests did not reach the frictionless sand impulse ratio (the open circle data in Figure 6.23) of $\sim 0.72$. 
Table 6.7. Impulse transferred to the V-block target by each particle type (sand, air, and HE). The sand particles were modeled with a friction coefficient $\mu_{s}=0.0$.

\begin{tabular}{ccccc}
\hline \multirow{2}{*}{$\begin{array}{c}\text { Standoff } \\
(\mathrm{cm})\end{array}$} & \begin{tabular}{c} 
Total \\
\cline { 2 - 5 }
\end{tabular} & $\begin{array}{c}\text { V-block }\left(\mu_{s}=0.0 ; \alpha=53^{\circ}\right) \\
(\mathrm{kPa} \cdot \mathrm{s})\end{array}$ & $\begin{array}{c}\text { Air } \\
(\mathrm{kPa} \cdot \mathrm{s})\end{array}$ & $\begin{array}{c}\text { HE } \\
(\mathrm{kPa} \cdot \mathrm{s})\end{array}$ \\
\hline 19 & 8.16 & 7.56 & 0.55 & 0.04 \\
24 & 7.77 & 7.10 & 0.60 & 0.07 \\
29 & 7.56 & 7.00 & 0.65 & 0.10 \\
\hline
\end{tabular}

It is also interesting to note that even if a means were found to avoid impulse transfer to the structure exposed behind a sliding plate, the reduction of impulse ratio to the frictionless sand $\left(\mu_{\mathrm{s}}\right.$ $=0.0$ ) result of $\sim 0.72$ still substantially exceeds the resolved momentum on a plate with an inclination angle $\alpha=53^{\circ}$. For the case considered here, momentum conservation gives $I_{T}^{Z} / I_{O}=$ 0.64. The simulations reveal that this discrepancy arises because some of the impulse from the buried tests was transferred by gaseous (air or high explosive) particles, which have a very weak frictional interaction with a solid target. This component of the impulse is therefore unaffected by the sliding plate mitigation concept. Table 6.7 shows the contributions to the impulse transferred to the V-block target for the three particle types (sand, air, and HE). The impulse applied by the air and HE particles was approximately $8.5 \%$ of the total impulse. When the impulse contribution of gaseous particles is removed from the simulations, the (open square) results in Figure 6.23 are very close to the resolved frictionless limit of 0.64 .

\subsection{Concluding Remarks}

A combined experimental and discrete particle based simulation approach has been used to investigate impulse transfer from granular matter with an incident main front velocity of 350-500 $\mathrm{m} / \mathrm{s}$ to rigidly supported targets. While the discrete particle method used by the solver ignores both particle rotation and fracture (to reduce the computational cost of the simulations), the good agreement with experimental results indicates that this modeling approach, when combined with particle contact parameter calibration results in a surprisingly useful means for interpreting granular loading by explosively accelerated particles.

This study investigated both the effect of target inclination angle using designs with $\alpha=90^{\circ}$ (normally oriented) to $\alpha=53^{\circ}$ and a sliding plate concept that reduced the effects of friction during loading by impulsively accelerated sand particles. The inclination angle investigation confirmed 
the reduction in impulse transferred to an inclined surface was dependent upon frictional interactions between the particles and the target surface as well as the angle of inclination. A novel target design that implemented lubricated (low friction) sliding plates on the inclined target surface was successfully tested. Simulations of the tests revealed that these plates acquired a velocity between $55-70 \mathrm{~m} / \mathrm{s}$ from momentum transfer by the incident granular impact, and led to a substantial reduction of transferred impulse. In scenarios where most of the impulse is transferred by soil, and the rate of uncovering of the underlying target surface is negligible, the lubricated sliding plate design results in impulse reductions comparable to those achievable by changes of inclination angle. 


\section{Chapter 7. Parametric study of impulse mitigation of granular impacts to inclined sliding surfaces}

The aim of this chapter is to examine the effect of the sliding plate design on the impulse transferred to the test target through a series of parametric studies. A pair of steel slider plates replaced the aluminum plates previously used in the same test arrangement described in Chapter 6. This allowed experimental investigation of the effect of sliding plate mass on the impulse transferred to the test target. Good agreement is observed between the experimental results for the steel sliding plates and the simulated results. This motivated a simulation study on variables that influence the sliding plate effectiveness. This chapter uses simulations to investigate the roles of the sliding plate mass, the explosive charge mass, and the number of sliding plates that are stacked against the inclined surfaces of the rigid V-shaped target. These simulations reveal the limits to which the sliding plate design can mitigate the transferred impulse. 


\subsection{Introduction}

The previous chapter, Chapter 6, looked at the effect of impulse transferred to three different equivalent mass target geometry designs; a flat bottom (baseline) target, an inclined V-block target, and a V-shaped target with attached sliding plates. The study confirmed that an inclined target oriented with angle $\alpha=53^{\circ}$ to the impacting granular media reduced the transferred impulse compared to a normally oriented $\left(\alpha=90^{\circ}\right)$ rigid flat bottom target. It also showed that the use of a lubricated sliding plate attached to the inclined V-shaped target led to substantial further reductions of the transferred impulse.

Here, a series of experimental tests with steel slider plates (replacing the lighter aluminum plates) is reported together with discrete particle simulations. The discrete particle simulations were then used to systematically study the effect of the sliding plate mass (density of the plate material), the explosive charge mass, and the number of attached (stacked) slider plates to analyze the transferred impulse to the target and the impulse reduction compared to a V-shaped target without sliding plates.

\subsection{Test configuration}

The same test arrangement presented in Chapter 6, using a vertical rig impulse test apparatus, was used for the series of tests presented here. The tests were also performed at NEWTEC Services Group, Inc., an outdoor blast testing site in Edgefield, SC. A detailed description of the test arrangement can be found in Section 6.2.2. This test arrangement allowed for the measurement of the applied pressure and impulse loading to a target from explosively accelerated granular media impacts. The test target was the same as in Section 6.2.1 for the aluminum reduced height Vshaped base target, with inclined surfaces at $\alpha=53^{\circ}$ angle from incoming, incident granular media, that allowed for the attachment of sliding plates. To examine the effect of slider plate mass, a pair of $6.3 \mathrm{~mm}$ thick, $1.326 \mathrm{~kg}$, ASTM-A514 steel slider plates replaced the lighter aluminum plates used in Chapter 6. The volume of the plates was equivalent to the aluminum plates with surface area $20.32 \mathrm{~cm}$ x $13.97 \mathrm{~cm}$. Prior to testing, the steel slider plates were grit blasted, similar to the aluminum plates, with a coarse grit mesh size (12 to 40) and a spray pressure of $690 \mathrm{kPa}$ to increase the surface roughness of the plates. Clamps (guide rails) were attached to the sides of these inclined surfaces to secure the sliding plates onto the target prior to granular impacts. The surfaces between the steel sliding plates and the inclined target surface and the clamps were lubricated with 
a Molykote M-77 solid lubricant paste from Dow Corning to minimize the frictional forces between the metal contact surfaces as the slider plates acquired momentum and moved along the underlying V-block inclined surface.

The test charge was once again contained in a steel "sandbox". This consisted of a $25.4 \mathrm{~cm} \mathrm{x}$ $25.4 \mathrm{~cm}, 300 \mathrm{~g}$ Detasheet charge placed below a $5.08 \mathrm{~cm}$ thick layer of water-saturated $(80 \%)$ glass silica microspheres. The Detasheet was detonated by a $25 \mathrm{~g} \mathrm{C}-4$ explosive booster located at the bottom center of the Detasheet. The test target was secured to an attachment plate at the base of four, $5 \mathrm{~cm}$ diameter, $2 \mathrm{~m}$ long, aluminum Kolsky bars. These four bars were instrumented with strain gauges for the measurement of the applied pressure and transferred impulse by granular impact (instrumentation detailed in Section 6.2.3). The target was tested at the same three standoff distances as defined for the previous targets (Chapter 6) which were 19, 24, and $29 \mathrm{~cm}$.

\subsection{Simulation approach}

Simulations were performed using the IMPETUS Afea Solver [50], previously used to analyze the impulsive load applied to the three equivalent mass aluminum targets and for the spherical charges. The modeling approach (including HE and soil parameters) and FE design are described in Section 6.3.

Briefly, for all the simulations presented here, the explosive charge is defined as again PETN high explosive particles with parameters predefined in the solver. The wet sand particles were modeled with a density of $\rho=1940 \mathrm{~kg} / \mathrm{m}^{3}$, previously determined by Holloman et al. [13] for an $80 \%$ water saturation. The frictional contact between the sand particles and the surface of the slider plates was defined as $\mu_{\mathrm{s}}=0.5$ to model the friction created on the surface of the slider plates caused by grit blasting. The lubricated (low friction) contact surfaces between the sliding plates and the underlying surface of the V-block were modeled with a friction coefficient of $\mu_{\mathrm{p}}=0.05$. Particle convergence was once again reached at a total of 2 million particles that were divided by the solver into the air, HE, and sand particles. The exact number of particles depended on the model setup (i.e. standoff distance) averaging around 72,000 air, 9,000 HE, and 1,919,000 sand particles.

The FE model is based on the experimental dimensions and includes the "A-frame" vertical test apparatus, the four Kolsky bars with a small cylindrical part representing the strain gauge 
Table 7.1. Material properties and Johnson Cook parameters for aluminum [37], titanium [106], steel [104], and tungsten alloy $(0.07 \mathrm{Ni}, 0.03 \mathrm{Fe})$ [107] used for the sliding plates.

\begin{tabular}{lcccccccccc}
\hline Material & \multicolumn{2}{c}{$\begin{array}{c}\text { Elastic } \\
\text { constant }\end{array}$} & \multicolumn{3}{c}{$\begin{array}{c}\text { Yield stress and } \\
\text { strain hardening }\end{array}$} & \multicolumn{2}{c}{$\begin{array}{c}\text { Strain rate } \\
\text { harding }\end{array}$} & \multicolumn{3}{c}{$\begin{array}{c}\text { Temperature softening and } \\
\text { adiabatic heating }\end{array}$} \\
\hline & $\mathrm{E}$ & $v$ & $\mathrm{~A}$ & $\begin{array}{c}\mathrm{B} \\
\mathrm{E}\end{array}$ & $\mathrm{n}$ & $\mathrm{C}$ & $\dot{\varepsilon}_{0}$ & $\mathrm{~T}_{0}$ & $\mathrm{~T}_{\mathrm{m}}$ & $\mathrm{m}$ \\
& $(\mathrm{GPa})$ & & $(\mathrm{MPa})$ & $(\mathrm{MPa})$ & & & $\left(\mathrm{s}^{-1}\right)$ & $(\mathrm{K})$ & $(\mathrm{K})$ & \\
AA6061 & 70 & 0.3 & 270 & 98 & 0.22 & 0.001 & $5 \cdot \mathrm{e}^{-4}$ & 293 & 893 & 1.0 \\
Ti-6A1-4V & 110 & 0.31 & 998 & 653 & 0.45 & 0.0198 & 1.0 & 298 & 1941 & 0.7 \\
A514 steel & 210 & 0.3 & 796 & 510 & 0.26 & 0.014 & $5 \cdot \mathrm{e}^{-2}$ & 293 & 1793 & 1.0 \\
W alloy & 343 & 0.28 & 1506 & 177 & 0.12 & 0.016 & 1.0 & 300 & 1723 & 1.0 \\
\hline
\end{tabular}

located $50.8 \mathrm{~cm}$ from the impact end of the bars, an attachment plate, the steel "sandbox", and the test target (detailed in Section 6.3.2). The materials are modeled using a Johnson-Cook (J-C) constitutive model implemented in the solver with the von Mises flow stress defined by Eqn. (3.5). The aluminum V-block target and Kolsky bars were modeled with J-C parameters for AA6061 defined by Wadley et al. [37] with a density of $\rho=2700 \mathrm{~kg} / \mathrm{m}^{3}$. The "sandbox" and steel slider plates were modeled as A514 grade B steel, $\rho=7850 \mathrm{~kg} / \mathrm{m}^{3}$, with JC parameters obtained from Johnson et al. [104]. Their material properties and JC parameters are listed in Table 7.1.

\subsection{Steel sliding plate results}

\subsubsection{Sand particle impacts}

High-speed video images of the sand particles impacting the V-shaped target with attached steel sliding plates at $29 \mathrm{~cm}$ standoff distance is shown in Figure 7.1. For consistency, $\mathrm{t}=0 \mathrm{~s}$ is defined as the moment of detonation. As previously observed in Section 6.4.3 (with aluminum sliding plates), the first particle impacts by the sand fingers occur slightly before $t=0.58 \mathrm{~ms}$, Figure 7.1(a). The redirection of the sand particles by the V-shaped target is seen in Figure 7.1(d)(f). However, details of the particle interactions are obscured by the sand traveling between the target and the high-speed camera.

Simulations allow for the observation of the sand particle interactions with the target and the initiation of the slider plate movement. Time snapshots of the simulation at $29 \mathrm{~cm}$ standoff distance is shown in Figure 7.2. Images shown (after detonation $t=0 \mathrm{~s}$ ) in Figure 7.2(a)-(f) correspond to the high-speed video images in Figure 7.1. Initial particle impacts occur at approximately $0.48 \mathrm{~ms}$ (not shown). The redirection of the sand particles is seen in Figure 7.2(d)(i). Approximately $1.05 \mathrm{~ms}$ after detonation in Figure 7.2(f), the sliding plates have acquired 

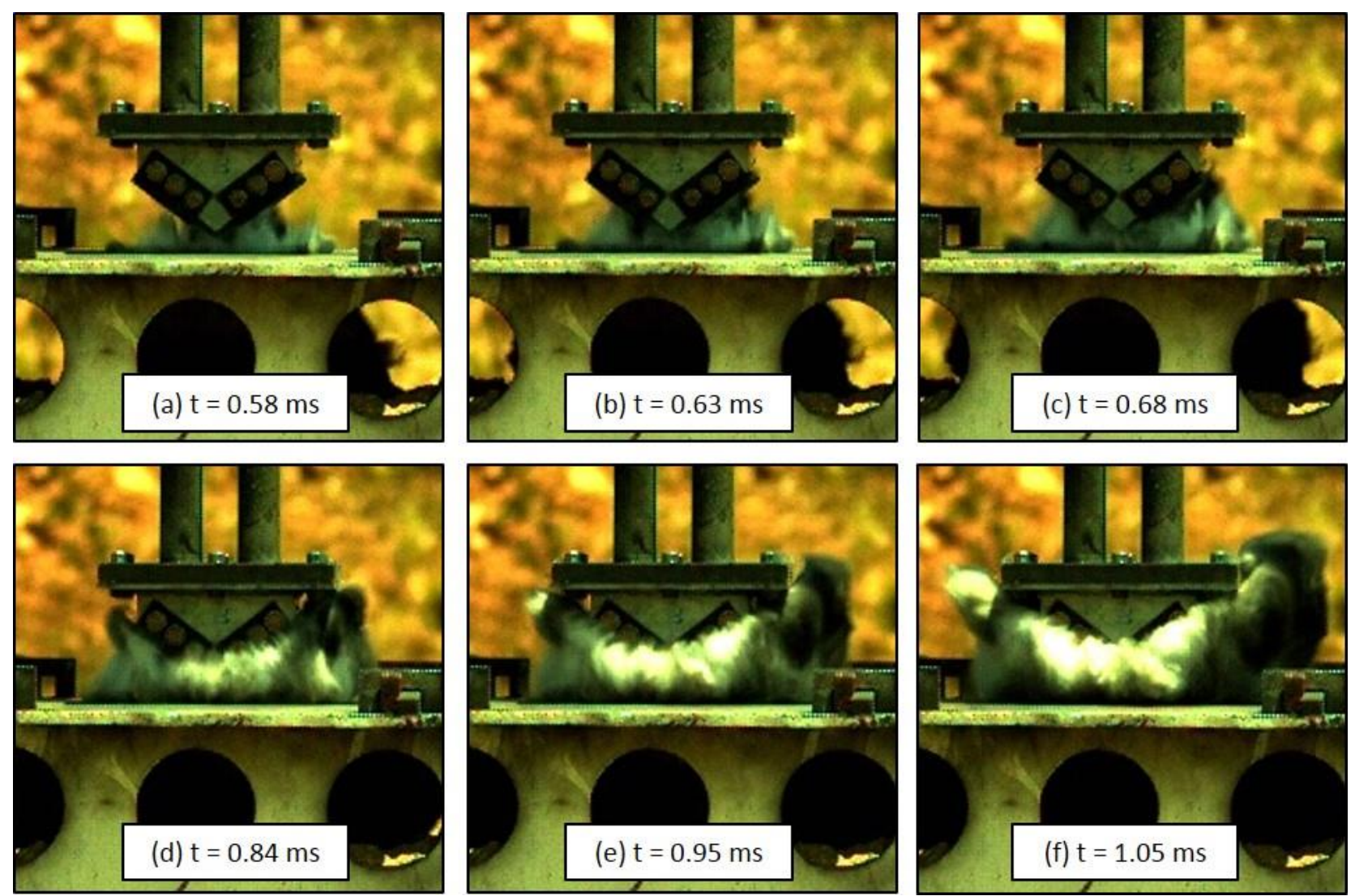

Figure 7.1 High-speed video images of particle impacts on V-shaped target with attached steel plates at $29 \mathrm{~cm}$ standoff distance. These times represent the time after detonation ( $t=0$ is moment of detonation).

enough momentum to begin their movement. The accumulation of the sand particles on the target during impact by the densest region of particles is seen in Figure 7.2(g). At this time after $2 \mathrm{~ms}$, the sliding plates have not advanced far along the inclined surface. The sliding plates escape the surface of the rigid base target just after $6 \mathrm{~ms}$ (Figure 7.2(i)).

The specific impulse applied normal to the inclined surfaces of the $\mathrm{V}$-shaped targets $2 \mathrm{~ms}$ after detonation is shown in Figure 7.3. The first column shows the impulse loading on the V-block target with no sliding plates, clearly showing the decrease in applied impulse as the standoff distance increases from Figure 7.3(a)-(c). The images for the impulsive impacts on the sliding plate targets are shown in the second (aluminum plates) and third (steel plates) columns. 

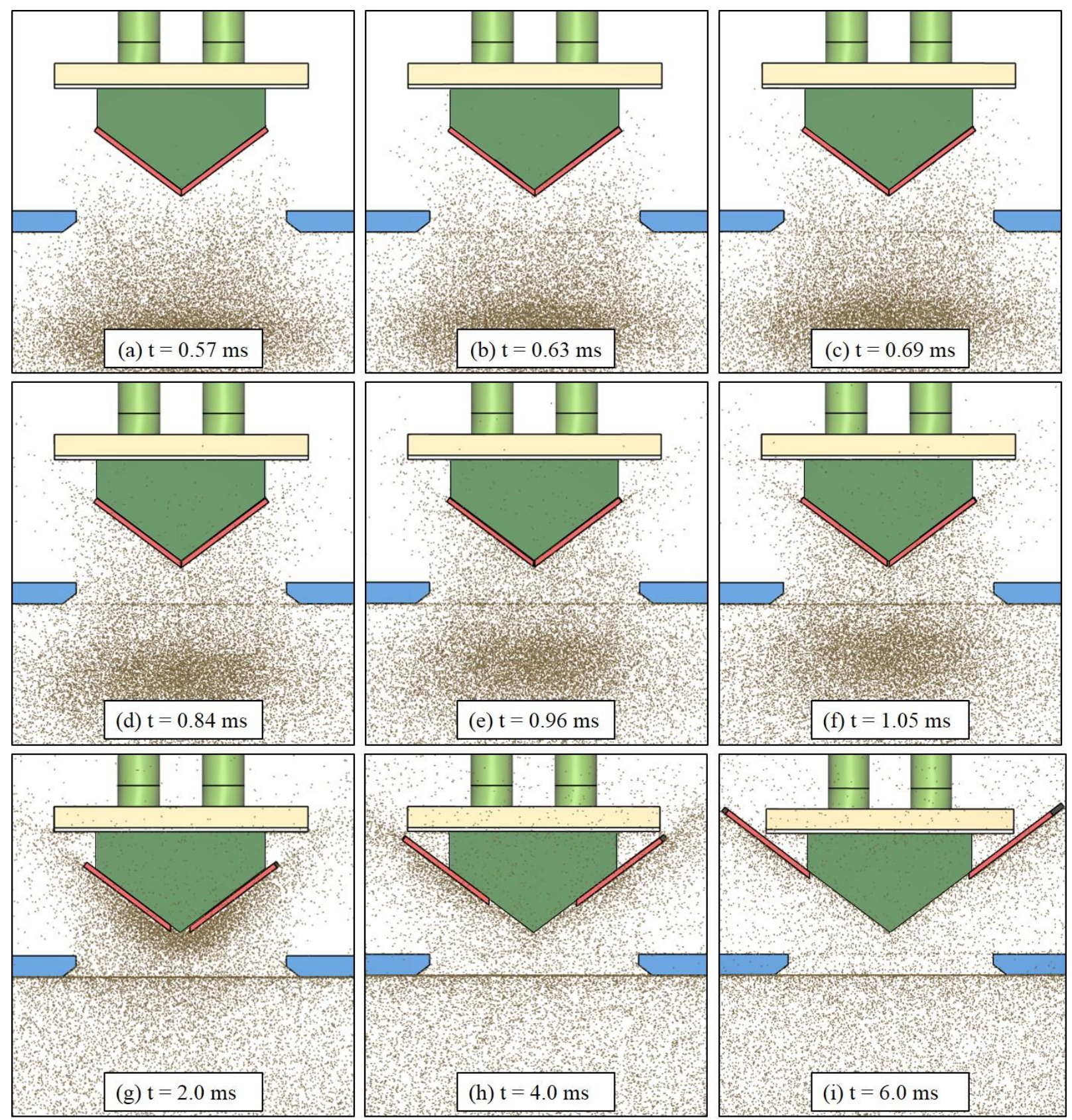

Figure 7.2. Simulation images of sand particle impacts on the V-target with attached sliding plates, transferring momentum to the plate movement for $29 \mathrm{~cm}$ standoff distance.

As identified in the previous chapter, there are two phases of impulse loading on these sliding plate targets observed in Figure 7.3. In Phase I, the initial high velocity particles (sand fingers) 
and main sand front impact the sliding plates transferring momentum to the plates and initiating their movement. In Phase II, the late arriving sand particles impact the underlying inclined surface of the V-block target that has become exposed as the sliding plates move away from the $\mathrm{V}$-apex. There is significantly less loading on the apex and underlying inclined surfaces during Phase II compared to the impacts on the sliding plates during Phase I. The mass of the steel slider plates was approximately 2.9 times greater than that of the aluminum plates. The heavier steel plates acquire less velocity than the aluminum plates. As such for each standoff distance in Figure 7.3, the steel plates have moved a much shorter distance, $\xi$, along the inclined underlying rigid surface after $2 \mathrm{~ms}$. These slower moving plates protect the underlying target longer from late arriving particle impacts, thus increasing the time of Phase I loading (particle impacts and momentum transfer to the slider plates). This resulted in less impulse applied to the apex of the V-target during Phase II loading (third column in Figure 7.3), compared to the faster moving aluminum plates that expose the apex and underlying surface earlier (second column in Figure 7.3).

The distance that the sliding plates have moved at time $\mathrm{t}=1,2$, and $3 \mathrm{~ms}$ after detonation is shown for the $19 \mathrm{~cm}$ standoff distance in Figure 7.4(a)-(c) for the aluminum and (d)-(f) for the steel sliding plates. This further emphasizes the slow movement of the heavier steel plates, resulting in increased protection and less impulse applied to the underlying V-block surface. After $3 \mathrm{~ms}$, Figure 7.4(c), the aluminum plates have already escaped the rigid inclined target while the steel plates are seen to only have traveled about halfway along the inclined rigid surface. The steel plate's escape time, speed, and Z-direction momentum are summarized for each standoff distance in Table 7.2, along with those for the aluminum plates. The $\mathrm{Z}$ plate impulse, also listed, was calculated by $I=m v / A$ where A is the cross sectional area normal to the plates, $203.2 \mathrm{~mm} \times 101.6$ $\mathrm{mm}$. The heavier steel sliding plates have a much lower plate speed (approximately 45-50\% slower) and longer escape time ( 2.3 to $2.7 \mathrm{~ms}$ longer) at each standoff distance compared to the lighter aluminum plates. 


\section{Specific impulse $(\mathrm{kPa} \cdot \mathrm{s}): \mathrm{t}=2.0 \mathrm{~ms}$}

$\begin{array}{llllll}0 & 2 & 4 & 6 & 8 & 10\end{array}$

(a) $19 \mathrm{~cm}$
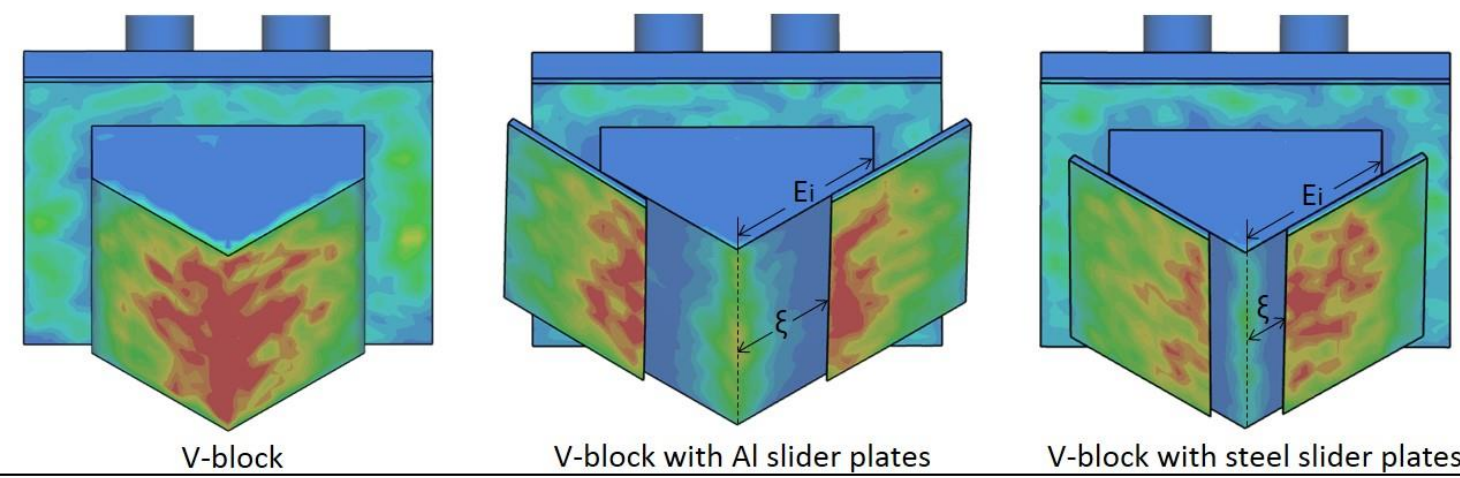

V-block with Al slider plates

V-block with steel slider plates

(b) $24 \mathrm{~cm}$
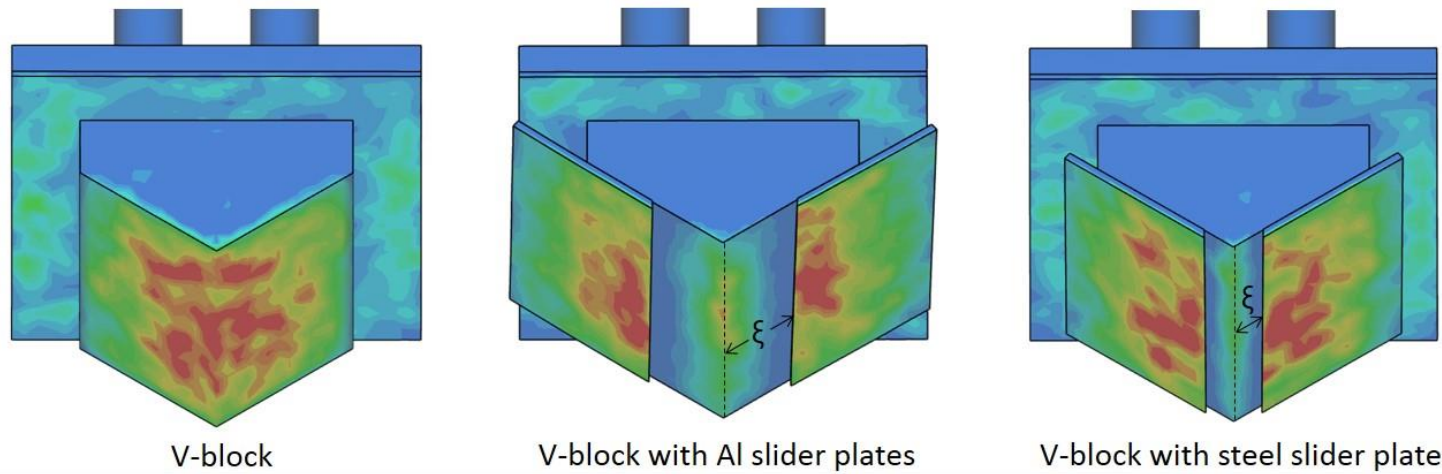

V-block with Al slider plates

V-block with steel slider plates

(c) $29 \mathrm{~cm}$

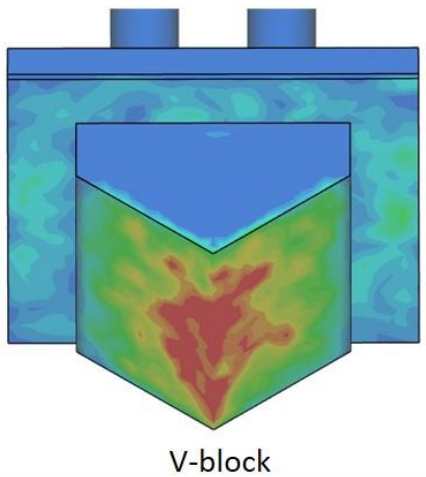

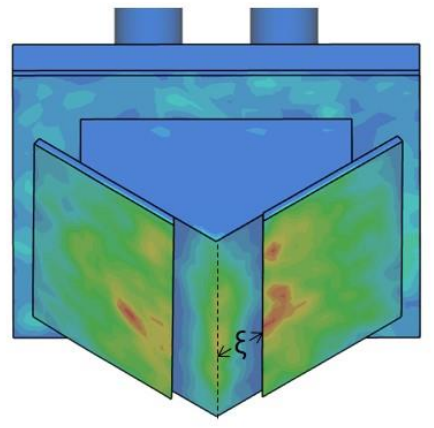

V-block with Al slider plates

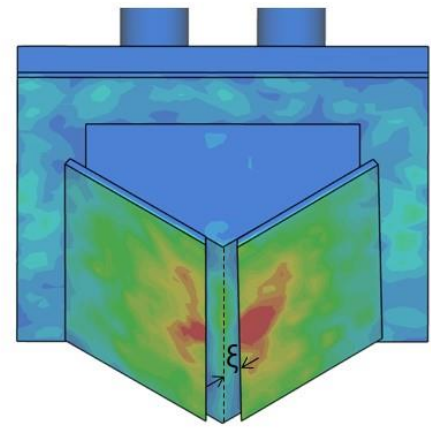

V-block with steel slider plates

Figure 7.3. Impulse distributions on the impact surfaces of the V-block target, and the V-block targets with aluminum and steel slider plates $2 \mathrm{~ms}$ after detonation for each standoff distance. $\xi$ is the distance the slider plate has traveled along the inclined surface. 
Table 7.2. The simulated velocity and momentum at escape times for both the aluminum and steel sliding plates for the three standoff distances.

\begin{tabular}{cccccccccc}
\hline \multirow{2}{*}{$\begin{array}{c}\text { Standoff } \\
\text { distance } \\
(\mathrm{cm})\end{array}$} & $\begin{array}{c}\text { Plate } \\
\text { speed } \\
(\mathrm{m} / \mathrm{s})\end{array}$ & $\begin{array}{c}\text { Aluminum slider plates } \\
\text { momentum } \\
(\mathrm{N} \cdot \mathrm{s})\end{array}$ & $\begin{array}{c}\text { Escape } \\
\text { time } \\
(\mathrm{ms})\end{array}$ & $\begin{array}{c}\text { Z-plate } \\
\text { impulse } \\
(\mathrm{kPa} \cdot \mathrm{s})\end{array}$ & & $\begin{array}{c}\text { Plate } \\
\text { speed } \\
(\mathrm{m} / \mathrm{s})\end{array}$ & $\begin{array}{c}\text { Z-plate } \\
\text { momentum } \\
(\mathrm{N} \cdot \mathrm{s})\end{array}$ & $\begin{array}{c}\text { Escape } \\
\text { time } \\
(\mathrm{ms})\end{array}$ & $\begin{array}{c}\text { Z-plate } \\
\text { impulse } \\
(\mathrm{kPa} \cdot \mathrm{s})\end{array}$ \\
\hline 19 & 69 & 20 & 2.60 & 0.97 & & 32 & 26 & 4.92 & 1.25 \\
24 & 66 & 19 & 2.97 & 0.93 & & 30 & 25 & 5.37 & 1.20 \\
29 & 57 & 16 & 3.45 & 0.79 & & 29 & 23 & 6.12 & 1.13 \\
\hline
\end{tabular}
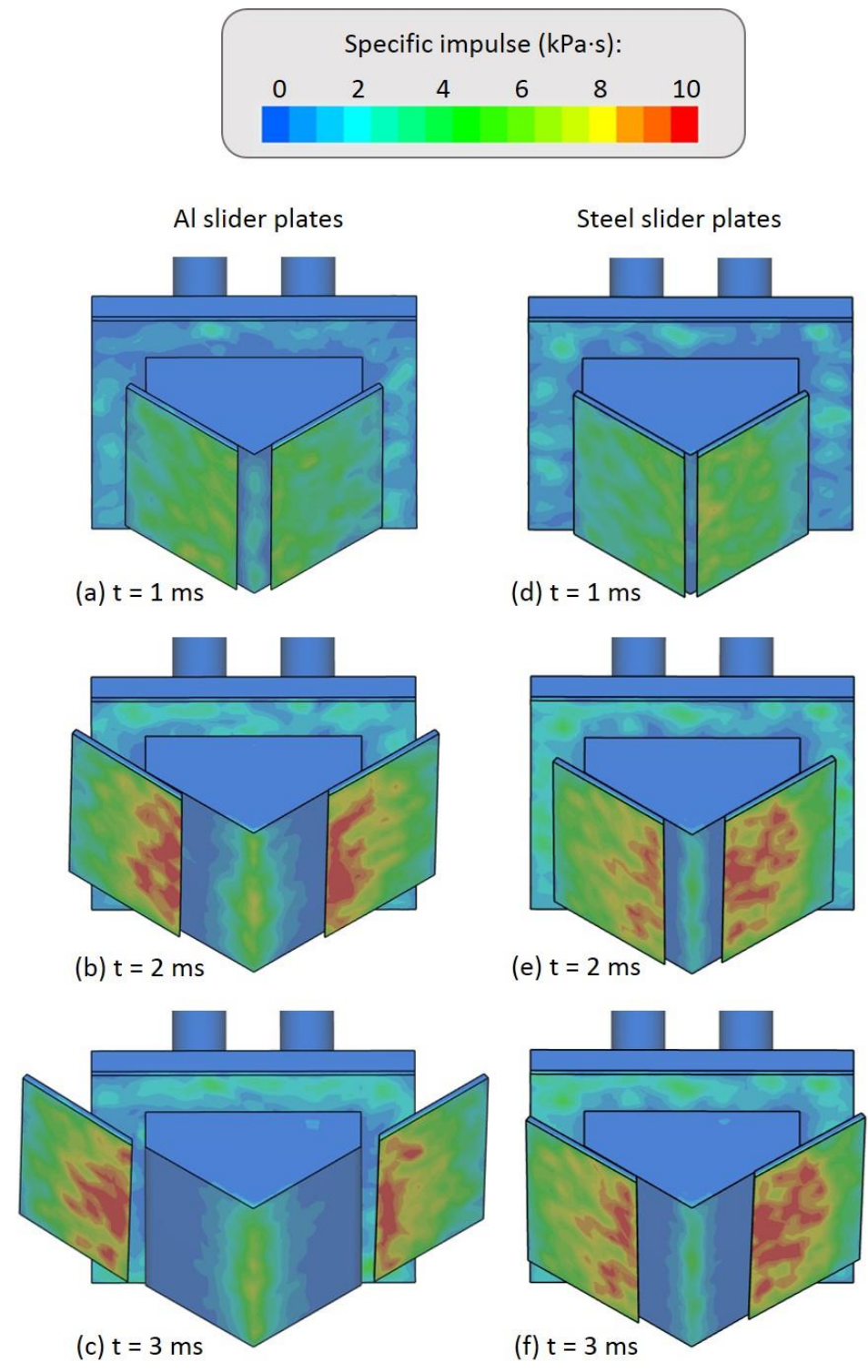

Figure 7.4. Impulse distributions on the impact surfaces of the V-block targets with (a)-(c) aluminum and (d)-(f) steel slider plates at $19 \mathrm{~cm}$ standoff distance, showing snapshots of the slider plate movement 1, 2, and $3 \mathrm{~ms}$ after detonation. 


\subsubsection{Kolsky bar response}

The pressure and impulse measured by the $2 \mathrm{~m}$ long Kolsky bars at each standoff distance (19, 24 , and $29 \mathrm{~cm}$ ) are shown in Figure 7.5 for the steel sliding plate target. Due to the distal reflection complications described in Chapter 6, the measured pressure and impulse-time response is only recorded here for the first $2.15 \mathrm{~ms}$ after detonation (shortly after the arrival of the second distal reflection). The first distal reflection arrived at the strain gauges, positioned $50.8 \mathrm{~cm}$ from the impact end of the bars, $558 \mu$ s after the initial recorded signal. This was followed by a second distal reflection $748 \mu$ s after the first. The arrival times of these distal reflections are indicated on the plots in Figure 7.5. The initial pressure peak decreases as the standoff distance increases, Figure 7.5(a)-(c), consistent with previous observations of the decrease in particle velocity and density as a sand column expands both axially and laterally [13]. The integrated impulse-time response with the arrival times of the first and second distal reflections indicated for each standoff distance is shown in Figure 7.5(d)-(f). The impulse measured at the distal reflection arrival times is summarized in Table 7.3. The results for the target with aluminum plates is also listed for comparison. The impulse measured experimentally for the three targets from Chapter 6 (Figure 6.10) are shown here in Figure 7.6 for each standoff distance with the additional results for the steel sliding plate target. At the arrival time of the first distal reflection, the steel results are less than those for the aluminum slider plates at a standoff distance of 19 and $29 \mathrm{~cm}$ (though not at 24 $\mathrm{cm})$. This is also seen at the second distal reflection with a slight reduction also at the $24 \mathrm{~cm}$ standoff distance. While this impulse difference is less than $1 \mathrm{kPa} \cdot \mathrm{s}$, the results indicated that the heavier mass steel plates may be used to reduce the transferred impulse though further examination is necessary.

The simulated pressure and impulse-time responses are shown alongside the measured responses in Figure 7.5. The simulated response is seen to be in good agreement with the experimentally measured data. The accuracy of the simulated signal allows for the simulations to be used for further analysis and calculation of the total impulse transferred to the targets by the granular media impacts. They also allow for more insight into the effect of the slider plate mass on impulse reduction and examination of the two phases of loading on the sliding plate targets (discussed earlier in Section 7.4.1). 

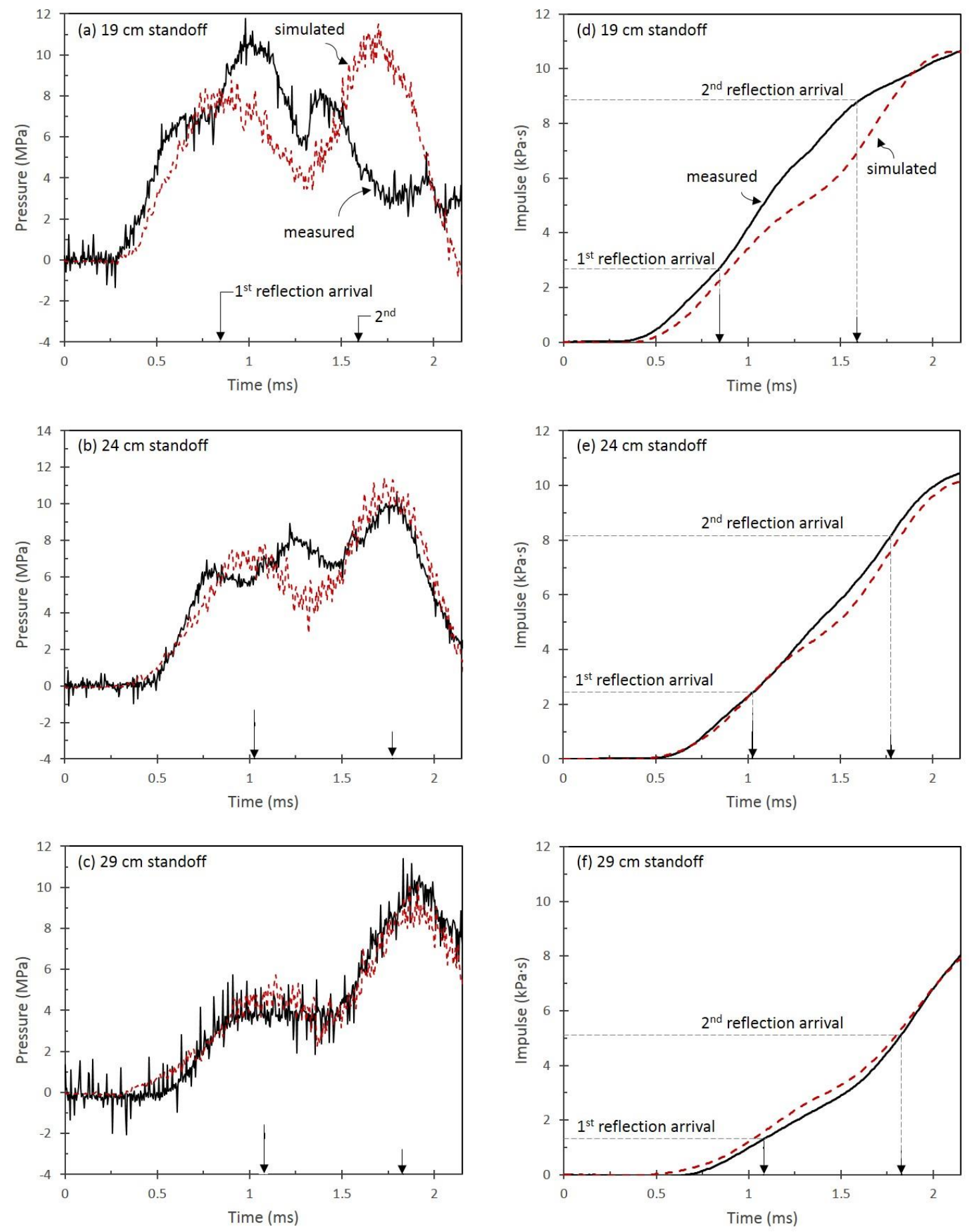

Figure 7.5. Comparison of the strain gauge measured (black line) and simulated (red dashed line) pressure-time waveforms for the V-block target with attached steel slider plates at standoff distances of (a) $19 \mathrm{~cm}$, (b) $24 \mathrm{~cm}$ and (c) $29 \mathrm{~cm}$. The impulse-time waveforms are also shown for each standoff distance and the arrival times of the $1^{\text {st }}$ and $2^{\text {nd }}$ distal reflection at the strain gauges are indicated on $(d),(e)$, and $(f)$. The time $t=0$ s corresponds to initiation of detonation of the Detasheet. 
Table 7.3. Impulse measured at the arrival times of the $1^{\text {st }}$ and $2^{\text {nd }}$ distal reflections for each standoff distance for the $V$-shaped targets with aluminum and steel slider plates attached.

\begin{tabular}{cccccc}
\hline \multirow{2}{*}{$\begin{array}{c}\text { Standoff } \\
\text { distance } \\
(\mathrm{cm})\end{array}$} & $\begin{array}{c}\text { Impulse } 1^{\text {st }} \\
\text { distal reflection } \\
(\mathrm{kPa} \cdot \mathrm{s})\end{array}$ & $\begin{array}{c}\text { Impulse } 2^{\text {nd }} \text { distal } \\
\text { reflection } \\
(\mathrm{kPa} \cdot \mathrm{s})\end{array}$ & & $\begin{array}{c}\text { Impulse } 1^{\text {st }} \\
\text { distal reflection } \\
(\mathrm{kPa} \cdot \mathrm{s})\end{array}$ & $\begin{array}{c}\text { Impulse } 2^{\text {nd }} \text { distal } \\
\text { reflection } \\
(\mathrm{kPa} \cdot \mathrm{s})\end{array}$ \\
\hline 19 & 3.4 & & & & \\
24 & 1.8 & 5.5 & & 2.7 & 8.8 \\
29 & 1.5 & 5.3 & & 1.3 & 8.1 \\
\hline
\end{tabular}
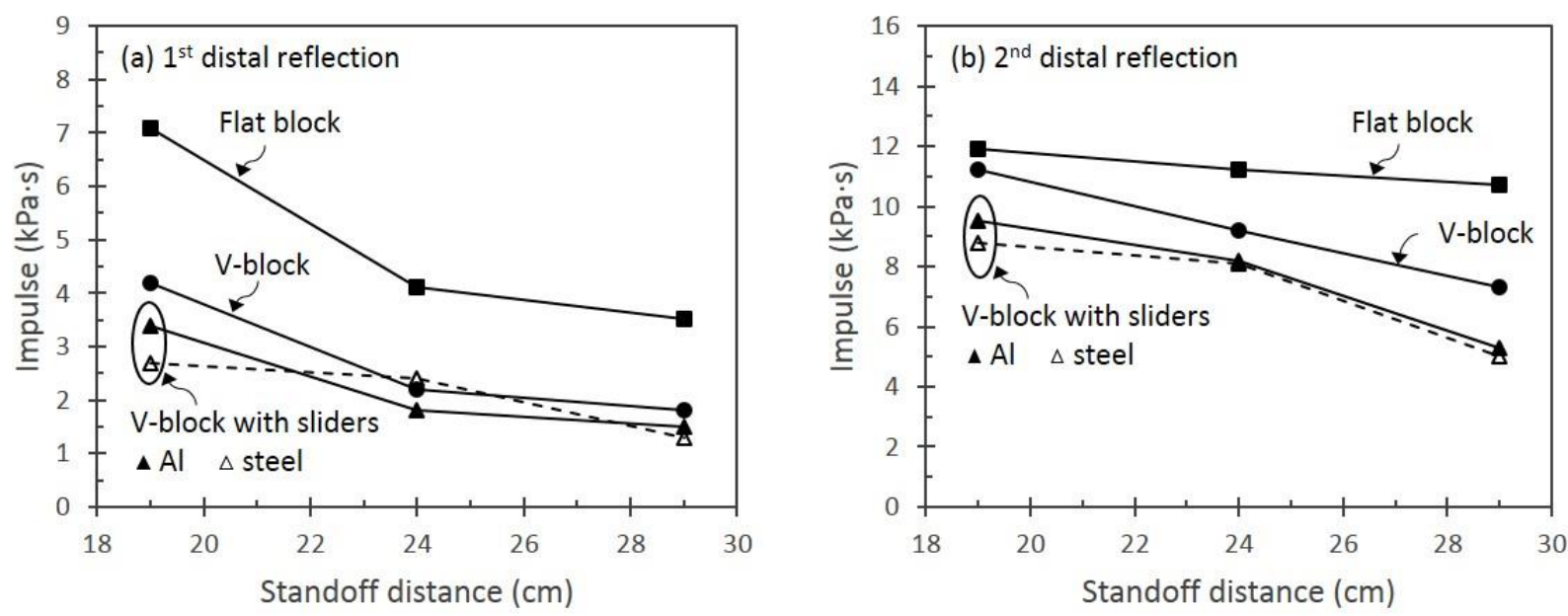

Figure 7.6. Impulse experimentally measured by the Kolsky bars at the arrival times of the (a) $1^{\text {st }}$ and (b) $2^{\text {nd }}$ distal reflections at each standoff distance for each of the targets; flat bottomed, V-block, and V-block with slider plates (Al and steel).

The simulations allow calculation of the total impulse transferred to the test targets in the Zdirection after $4 \mathrm{~ms}$. The results from Chapter 6 are again plotted here in Figure 7.7(a) for the transferred impulse, $I_{T}^{Z}$, for each standoff distance alongside the simulated transferred impulse to the target with steel sliding plates. The impulse ratio, $I_{T}^{Z} / I_{o}$, is the transferred Z-direction impulse normalized by the incident impulse of the incoming sand, shown for each standoff distance in Figure 7.7(b) for each of the target designs. The results from Chapter 6 showed that the impulse ratio for the flat block was $\sim 1.02$. The transferred impulse was approximately equal to the incident impulse indicating weak reflection of the particles from the target surface. The impulse ratio for the V-block target was $\sim 0.85$. This revealed an impulse reduction when the inclination angle of 

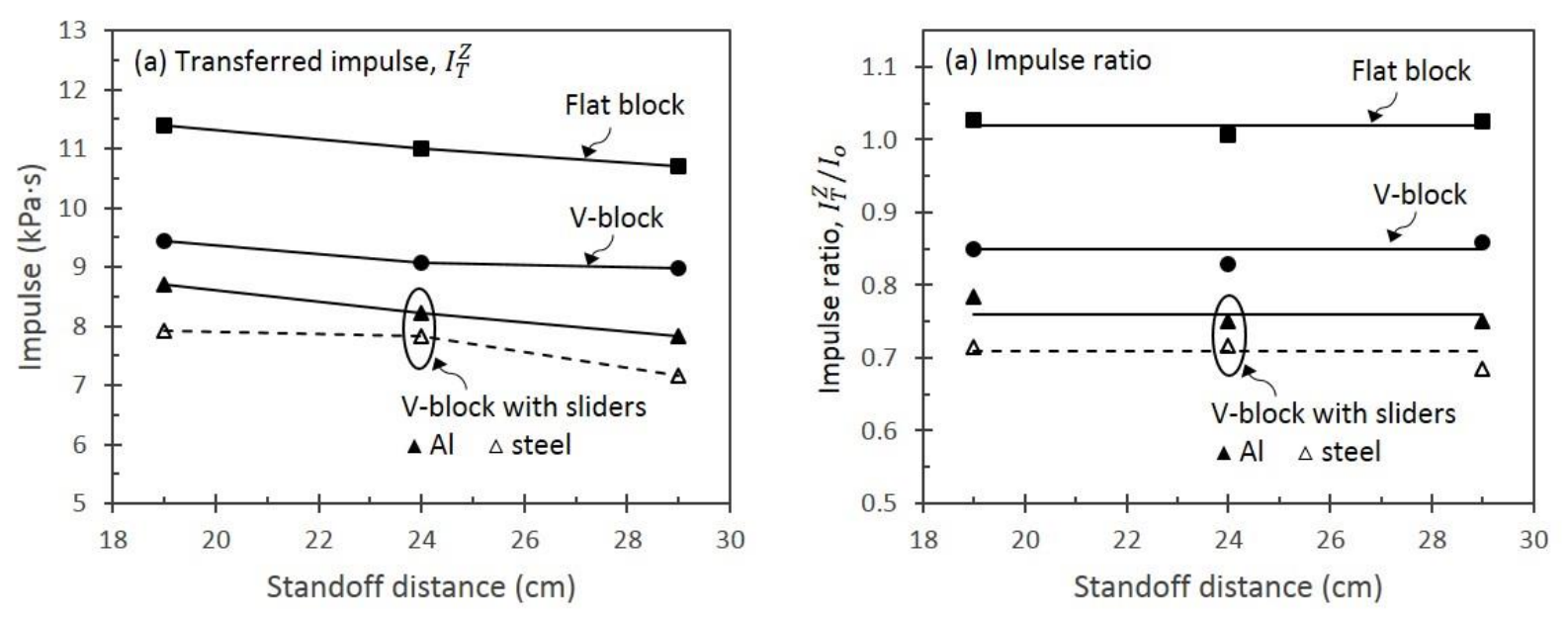

Figure 7.7. (a) Transferred impulse in the vertical Z-direction to all targets measured in the simulation after $4 \mathrm{~ms}$. (b) Calculated impulse ratio for each target.

particle impact was changed from $\alpha=90^{\circ}$ (flat block) to $\alpha=53^{\circ}$ (V-block). The addition of aluminum sliding plates to the inclined surface resulted in a further impulse reduction to $\sim 0.76$. Figure 7.7 shows that the steel sliding plates can reduce the transferred impulse even further. The impulse ratio for the inclined target with steel plates was $\sim 0.71$. This was approximately $7 \%$ less than the results for the aluminum sliding plates. These indicates that a heavier mass sliding plate can further reduce the impulse transferred to a test target.

In Chapter 6, the effect of friction between the incident particles and impacted structure was investigated. The impulse ratio for the frictionless V-block target $\left(\mu_{\mathrm{s}}=0.0\right)$ was 0.72 . The aluminum sliding plate result (0.76) exceeded this frictionless result. However, the impulse ratio for the steel sliding plate target $(0.71)$ was approximately that transferred to the frictionless target. Examination of the simulations revealed that this was a consequence of impacts by late arriving sand particles during Phase II loading. These late arriving particles impacted the apex of the target and the exposed inclined surfaces after the sliding plates had moved from their initial positions, transferring impulse directly to the base target. The increased mass of the steel sliding plate resulted in an increased Phase I loading and decreased Phase II loading. The slower steel plates protected the rigid inclined surface longer, resulting in less impulse transferred to the exposed Vapex and the underlying inclined surfaces by late arriving particles during the Phase II loading than observed for the aluminum sliding plate targets (Figure 7.4). 


\subsection{Parametric study}

Motivated by the reduction in impulse resulting from changing the slider plate mass (from aluminum to steel) presented above, a series of simulations were performed to analyze the effects of several different variables on the sliding plate effectiveness for impulse mitigation. Three variables were selected for the parametric study; the slider plate mass $m_{p}$, the explosive charge mass $\mathrm{m}_{\mathrm{c}}$, and the number of attached slider plates, $n$. All simulations presented here have been performed using IMPETUS Afea Solver with the same FE model for the vertical rig impulse test apparatus and test setup discussed above and previously in Chapter 6. The rigid V-block, reduced height, aluminum target that allowed for the attachment of sliding plates was used for all simulations presented here.

\subsubsection{Sliding plate mass}

The mass of the sliding plates was varied from $330 \mathrm{~g}$ to $5.8 \mathrm{~kg}$ by adjusting the density of the pair of $6.35 \mathrm{~mm}$ thick plates. The volume of the plates was fixed with surface area $20.32 \mathrm{~cm} \mathrm{~s}$ $13.97 \mathrm{~cm}$ and only the material properties of the plates was adjusted. Simulations were performed at three standoff distances $(14,19$, and $29 \mathrm{~cm})$ to investigate a range of incident impulses impacting the target. The incident sand particle velocity, along with the incident impulse for each standoff distance, are summarized in Table 7.4. The sand velocity reached a peak value of $500 \mathrm{~m} / \mathrm{s}$ around $19 \mathrm{~cm}$ standoff distance before it began to decay due to momentum transfer to surrounding air particles. At the $14 \mathrm{~cm}$ standoff distance, the velocity was $475 \mathrm{~m} / \mathrm{s}$, as the particles were still being accelerated by expanding detonation products. This velocity at $14 \mathrm{~cm}$ was slightly less than that for the $19 \mathrm{~cm}$ standoff distance. However, the incident impulse was greater at the shorter standoff distance (Table 7.4) due to the higher density of the impacting incident particles, since less sand stretching in the axial and lateral directions had occurred at this time. The Z-direction impulses transferred to the flat (baseline) target and V-block target (without sliding plates) are listed in Table 7.4, indicating the results from Chapter 6 where an impulse reduction was achieved by surface inclination.

Four materials of various densities were chosen to investigate the effect of the sliding plate mass, $m_{p}$, on the impulse transferred to the target: (i) aluminum $\rho=2700 \mathrm{~kg} / \mathrm{m}^{3}$, (ii) titanium $\rho=$ $4430 \mathrm{~kg} / \mathrm{m}^{3}$, (iii) steel $\rho=7850 \mathrm{~kg} / \mathrm{m}^{3}$, and (iv) tungsten $\rho=17900 \mathrm{~kg} / \mathrm{m}^{3}$. The material properties 
Table 7.4. Incident velocity and impulse of incoming sand particles and the Z-direction impulse transferred to the rigid flat block and $V$-block target (without sliding plates).

\begin{tabular}{ccccc}
\hline $\begin{array}{c}\text { Standoff } \\
\text { distance } \\
(\mathrm{cm})\end{array}$ & $\begin{array}{c}\text { Incident } \\
\text { velocity } \\
(\mathrm{m} / \mathrm{s})\end{array}$ & $\begin{array}{c}\text { Incident } \\
\text { Impulse } \\
(\mathrm{kPa} \cdot \mathrm{s})\end{array}$ & $\begin{array}{c}\text { Flat block } \\
\text { Z-direction impulse } \\
(\mathrm{kPa} \cdot \mathrm{s})\end{array}$ & $\begin{array}{c}\text { V-block } \\
\text { Z-direction impulse } \\
(\mathrm{kPa} \cdot \mathrm{s})\end{array}$ \\
\hline 14 & 475 & 11.60 & 11.75 & 9.60 \\
19 & 500 & 11.10 & 11.40 & 9.43 \\
29 & 350 & 10.44 & 10.71 & 8.97 \\
\hline
\end{tabular}

Table 7.5. The material density and mass of the sliding plates, along with their mass normalized by the 5 $\mathrm{cm}$ thick wet sand mass, $m_{s}=5.36 \mathrm{~kg}$. The Z-direction impulse transferred to the targets for each different material slider plate is also listed for each standoff distance.

\begin{tabular}{ccccccc}
\hline Material & Density & $\begin{array}{c}\text { Sliding plates } \\
\text { mass }\end{array}$ & $\begin{array}{c}\text { Normalized } \\
\text { mass }\end{array}$ & \multicolumn{2}{c}{ Transferred Z- direction impulse } \\
& $\begin{array}{c}\rho \\
\left(\mathrm{kg} / \mathrm{m}^{3}\right)\end{array}$ & $\begin{array}{c}\mathrm{m}_{\mathrm{p}} \\
(\mathrm{kg})\end{array}$ & $\mathrm{m}_{\mathrm{p}} / \mathrm{m}_{\mathrm{s}}$ & $\begin{array}{c}14 \mathrm{~cm} \\
(\mathrm{kPa} \cdot \mathrm{s})\end{array}$ & $\begin{array}{c}19 \mathrm{~cm} \\
(\mathrm{kPa} \cdot \mathrm{s})\end{array}$ & $\begin{array}{c}29 \mathrm{~cm} \\
(\mathrm{kPa} \cdot \mathrm{s})\end{array}$ \\
\hline-- & 1000 & 0.33 & 0.06 & 8.69 & 8.45 & 7.90 \\
AA6061 & 2700 & 0.89 & 0.17 & 8.35 & 8.11 & 7.71 \\
Ti-6Al-4V & 4430 & 1.5 & 0.27 & 8.10 & 7.85 & 7.36 \\
A514 steel & 7850 & 2.6 & 0.49 & 7.91 & 7.62 & 7.15 \\
W alloy & 17900 & 5.8 & 1.09 & 7.67 & 7.64 & 7.14 \\
\hline
\end{tabular}

and $\mathrm{J}-\mathrm{C}$ parameters used for modeling these four materials are listed in Table 7.1. To investigate very light plates, an additional study was performed with slider plates modeled with a density of $\rho$ $=1000 \mathrm{~kg} / \mathrm{m}^{3}$. The material properties and JC parameters other than density were defined to those of aluminum for the purpose of investigating a low density (light weight) sliding plate and their influence on the transferred impulse. The density and corresponding mass of each material for the different plates used in the model are listed in Table 7.5. The mass of the pair of sliding plates, $m_{p}$, ranged from $330 \mathrm{~g}$ for a light plate with density $\rho=1000 \mathrm{~kg} / \mathrm{m}^{3}$ to $5.8 \mathrm{~kg}$ for the heavy tungsten (W) alloy $(0.07 \mathrm{Ni}, 0.03 \mathrm{Fe})$ plates with density $\rho=17,900 \mathrm{~kg} / \mathrm{m}^{3}$.

As the standoff distance increased from 14 to $29 \mathrm{~cm}$, the incident impulse of the wet sand decreased, Table 7.4. This decreased incident impulse resulted in less momentum transferred to the sliding plates at greater standoff distances, completely consistent with previous observations in Section 6.4. The plate velocity, $v=p / m_{p}$, depends upon the incident momentum of the sand, $p$, and the mass of the plates, $m_{p}$. The escape velocity, $v_{p}$, of each slider plate of varying mass (i.e. 

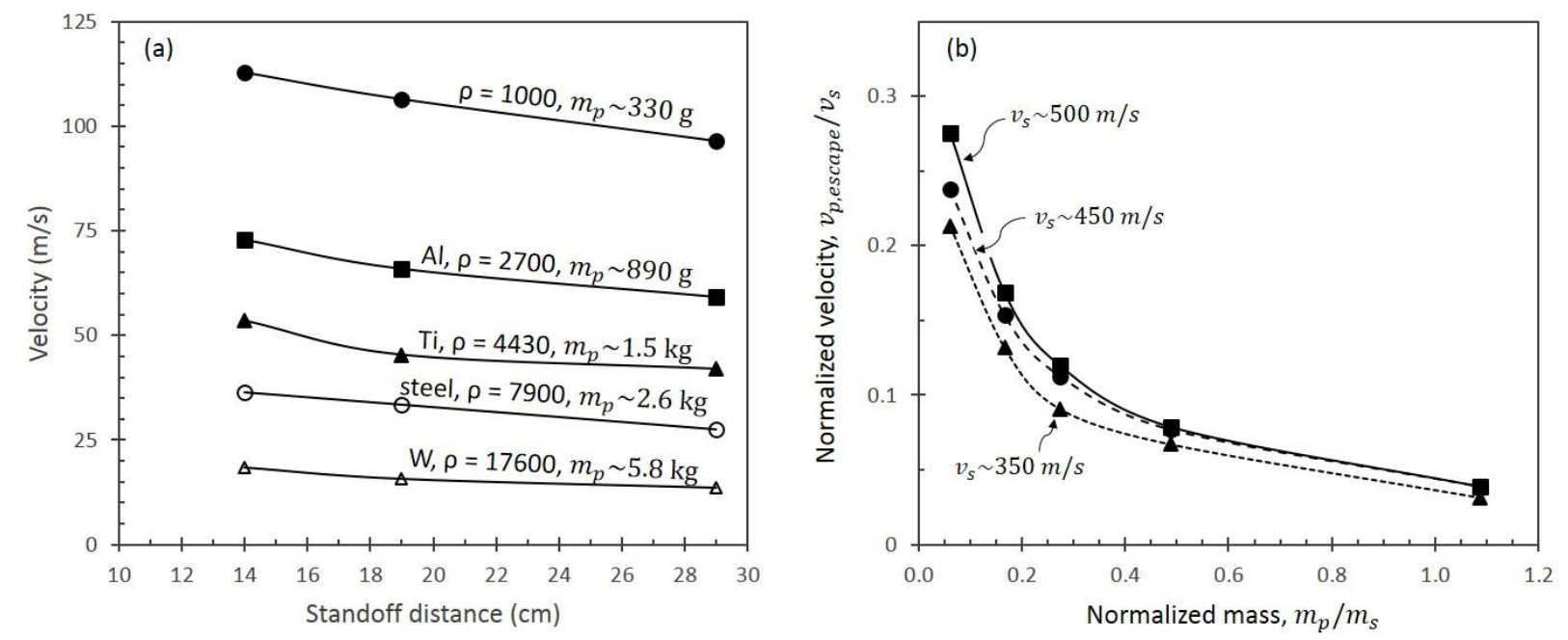

Figure 7.8. The sliding plate escape velocity for the five different sliding plate masses. (a) The escape velocity at the three standoff distances. (b) The plates' normalized escape velocity (normalized by the incident sand velocity) versus the normalized mass of the slider plates, $m_{p} / m_{s}\left(m_{s}=5.36 \mathrm{~kg}\right.$ is the initial mass of the sand).

material density) is shown in Figure 7.8(a) for each standoff distance. The escape velocity of the sliding plates decreased with increasing standoff distance consistent with the experimental results presented in Table 7.2. Figure 7.8(a) also shows that the escape velocity of the sliding plates decreased with the increasing mass (density) of the plates. At a $14 \mathrm{~cm}$ standoff distance, the slider plate escape velocity varied from $\sim 110 \mathrm{~m} / \mathrm{s}$ for the lightest plate to $\sim 20 \mathrm{~m} / \mathrm{s}$ for the heaviest plate. The sliding plate escape velocity was then normalized by the average velocity of the main front of the incident sand, $v_{s}$ (Table 7.4). The normalized plate mass, $\mathrm{m}_{\mathrm{p}} / \mathrm{m}_{\mathrm{s}}$, of each slider plate material is summarized in Table 7.5, where $\mathrm{m}_{\mathrm{s}}=5.36 \mathrm{~kg}$ is the mass of the initial $5.08 \mathrm{~cm}$ thick wet sand volume. Examination of Figure 7.8(b) shows that the normalized velocity exponentially decays as the plate mass increases. It is also noted that only the tungsten plate mass exceeds the mass of the sand $\left(\mathrm{m}_{\mathrm{p}} / \mathrm{m}_{\mathrm{s}}>1\right)$.

The simulated (Z-direction) impulse transferred to the aluminum V-block rigid base target was measured for the three standoff distances after $4 \mathrm{~ms}$ when a plateau value was reached. These are shown in Figure 7.9(a) for each of the targets with the five varying sliding plate masses and summarized in Table 7.5. The transferred impulse decreased with increasing standoff distance consistent with previous observations. The sliding plates have been shown to reduce the impulse 

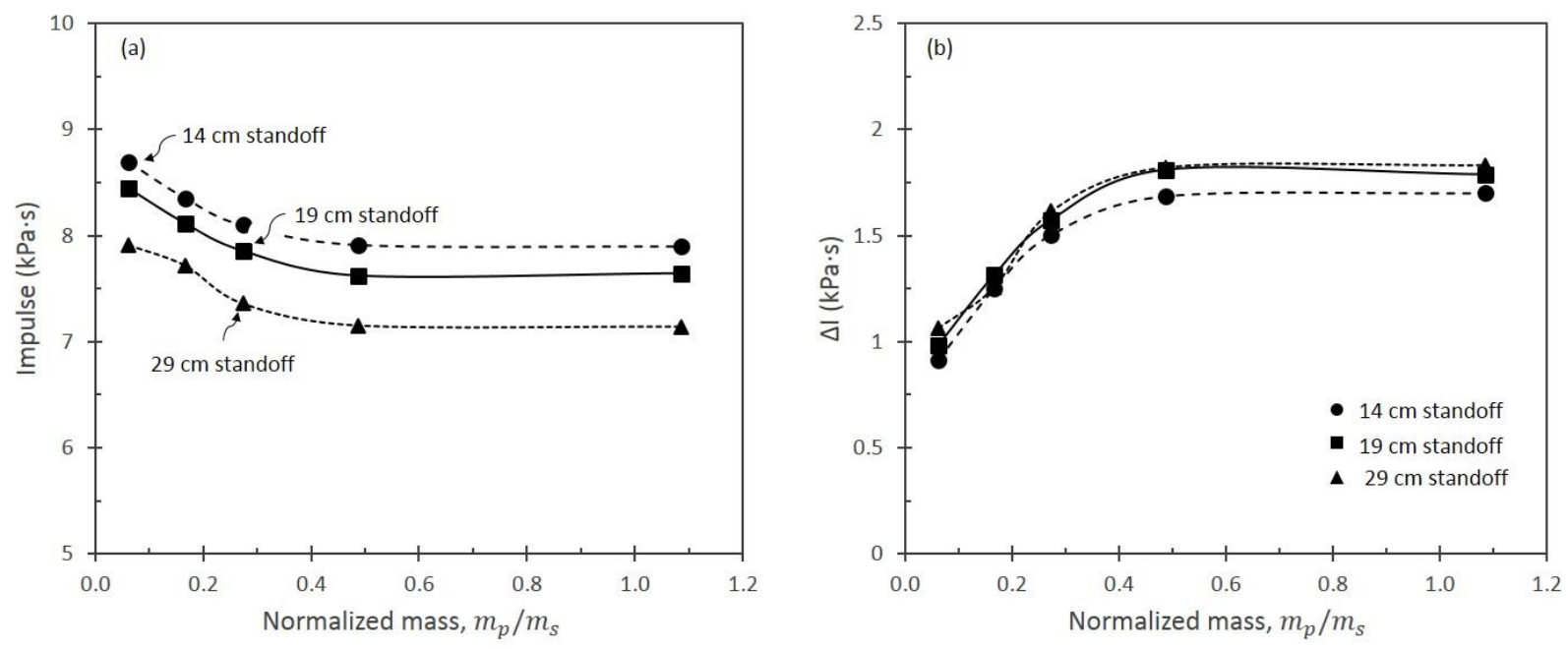

Figure 7.9. Impulse transferred to the $V$-shaped target with varying slider plate mass. (a) The transferred impulse versus the normalized slider plate mass, $m_{p} / m_{s}$, where the initial mass of the sand is $m_{s}=5.6 \mathrm{~kg}$. (b) Change in impulse, $\Delta I$, between the V-block target without sliding plates and the targets with different mass sliding plates as a function of the normalized plate mass.

compared to a rigid V-block target without plates (Table 7.4). Varying the plate mass from $\mathrm{m}_{\mathrm{p}}=$ $330 \mathrm{~g}$ to $2.6 \mathrm{~kg}$ (steel plates) revealed that as the mass increased the impulse transferred to the target decreased. The light plates $\left(\rho=1000 \mathrm{~kg} / \mathrm{m}^{3}\right)$ reduced the impulse from that achieved by surface inclination by $9.5 \%, 10.4 \%$ and $11.9 \%$ for the 14,19 , and $29 \mathrm{~cm}$ standoff distances respectively, while the heavier steel plates $\left(\rho=7850 \mathrm{~kg} / \mathrm{m}^{3}\right)$ reduced the impulse by $17.6 \%, 19.2 \%$, and $20.3 \%$ for the same standoff distances. Figure 7.9(b) shows the change in transferred impulse, $\Delta I=I_{\text {Vblock }}-I_{\text {sliders }}$, between the $\mathrm{V}$-block target without and with sliding plates as a function of the normalized mass, $m_{p} / m_{s}$ (Table 7.5).

For the incident impulses considered here, the impulse decreased with the increasing density from the lightest sliding plates, $\rho=1000 \mathrm{~kg} / \mathrm{m}^{3}$, to the steel sliding plates, $\rho=7850 \mathrm{~kg} / \mathrm{m}^{3}$ beyond which point increasing the density appeared to have no further effect on impulse reduction. The heaviest tungsten alloy $\left(\mathrm{m}_{\mathrm{p}}=5.8 \mathrm{~kg}\right)$ plate resulted in no further reduction beyond that achieved with the steel plate. The reduction in impulse achieved by increasing the plate mass is limited here by the mass of the steel plates. The plates must then be sufficiently heavy to protect the inclined surface during Phase II loading, but also sufficiently light enough to acquire enough momentum to sliding along the surface. 


\subsubsection{Charge mass}

The mass of the explosive charge, $m_{c}$, was varied from $100 \mathrm{~g}$ to $1.2 \mathrm{~kg}$ for the three target geometries (flat bottom, V-block, and V-block with attached sliding plates). For this study, both aluminum and steel slider plates were used in order to examine the effect of the plate mass alongside the variations in the charge mass. The standoff distance remained fixed at $19 \mathrm{~cm}$ from the top of the explosive charge to the base (impact side) of the flat bottom target. The $25.4 \mathrm{~cm} \mathrm{x}$ $25.4 \mathrm{~cm}$ by $5.08 \mathrm{~cm}$ thick volume of wet sand located above the explosive charge also remained fixed for each simulation. As mentioned in Section 7.3, the explosive charge was defined in the solver as PETN consistent with previous studies with a surface area of $25.4 \mathrm{~cm} \mathrm{x} 25.4 \mathrm{~cm}$. Here, the thickness of the HE particles was varied to adjust the PETN charge mass, $\mathrm{m}_{\mathrm{c}}$.

Figure 7.10(a) shows the results for the impulse transferred to the test targets as the charge mass was increased from $100 \mathrm{~g}$ to $1.2 \mathrm{~kg}$. The impulse applied to the targets is seen to gradually increase as the explosive charge mass increases. Further, as the charge increased, the impulse reduction, resulting from surface inclination, increased as well. There was also consistently an additional reduction in the transferred impulse by the addition of sliding plates. The difference in the transferred impulse, $\Delta \mathrm{I}$, between the aluminum V-block target and the V-block target with attached sliding plates is shown in Figure 7.10(b) for both the aluminum (filled triangles) and steel (empty triangles) slider plates. As the charge mass was increased, the impulse difference, $\Delta \mathrm{I}$, for both the aluminum and steel slider plates increased. The impulse reduction for the steel plates was consistently greater than the aluminum sliding plates for each charge mass studied here, consistent with observations in Section 7.4.

Table 7.6. Impulse transferred to each target when the charge mass was varied from $100 \mathrm{~g}$ to $1.2 \mathrm{~kg}$.

Impulse ( $\mathrm{kPa} \cdot \mathrm{s})$

\begin{tabular}{ccccc}
\cline { 2 - 5 } $\begin{array}{c}\text { Charge mass } \\
(\mathrm{g})\end{array}$ & Flat block & V-block & $\begin{array}{c}\text { V-block with } \\
\text { Al sliding plates }\end{array}$ & $\begin{array}{c}\text { V-block with } \\
\text { steel sliding plates }\end{array}$ \\
\hline 100 & 5.08 & 4.79 & 4.56 & 4.31 \\
200 & 9.15 & 8.48 & 7.87 & 7.39 \\
300 & 11.04 & 10.19 & 9.27 & 8.88 \\
500 & 16.42 & 14.19 & 13.04 & 12.69 \\
750 & 20.17 & 17.70 & 16.06 & 15.62 \\
1000 & 26.64 & 22.13 & 20.30 & 19.93 \\
1200 & 28.90 & 24.70 & 22.20 & 21.34 \\
\hline
\end{tabular}



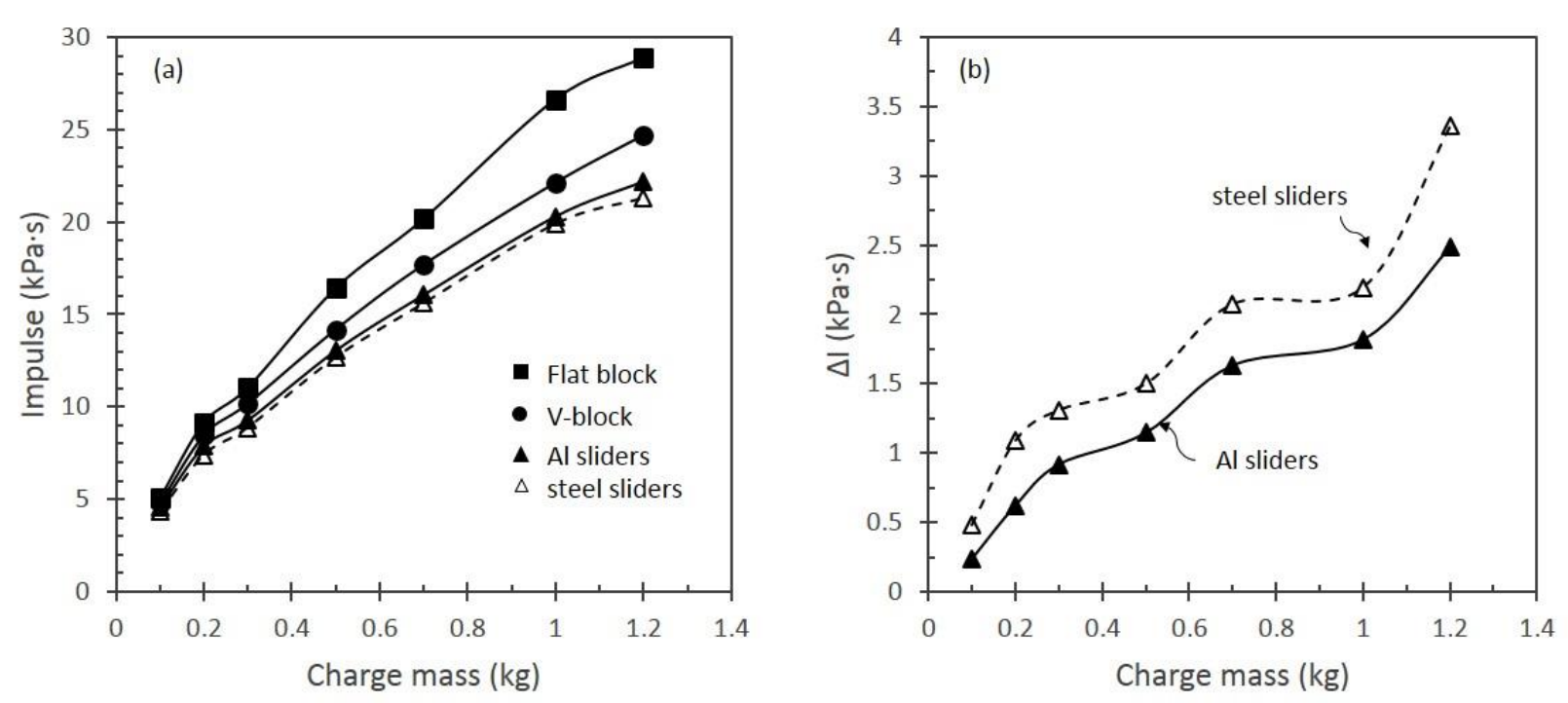

Figure 7.10. Impulse transferred to test targets as the charge mass increased at a fixed $19 \mathrm{~cm}$ standoff distance. (a) Transmitted impulse to the flat block, $V$-block, and V-block target with aluminum and steel slider plates. (b) Difference in transferred impulse between the V-block target and V-block target with slider plates $\left(\Delta I=I_{V b l o c k}-I_{\text {sliders }}\right)$ for the aluminum (filled triangles) and steel (empty triangles) sliding plates.

\subsubsection{Number of sliding plates}

The parametric study of the slider plate mass revealed that the heaver, slower moving slider plates reduced the impulse below that of the lighter sliding plates (within a certain limit for the incident impulses studied). This result correlated to the two phases of loading on the sliding plate targets. Initial, high velocity particles impacted the sliding plates during Phase I, accelerating the sliding plates along the inclined surface. Late arriving particles impacted the exposed apex and underlying target surface in Phase II loading. The heavier plates protected (shielded) the underlying surface longer than the lighter plates which acquired a much higher velocity upon particle incident impact and escaped much earlier. This resulted in an increased Phase I loading for the heavier plates and decreased the impulse applied to the exposed surface during Phase II loading after the sliding plates had moved away from their initial position. The intent here was to see if it was possible to progressively release stacked plates so the target base surface remained covered by a plate for a greater fraction of the loading time. The hypothesis is that the initial sand impacts will transfer momentum to an outer sliding plate and then later arriving sand impacts will 
(a) $\mathrm{n}=1$

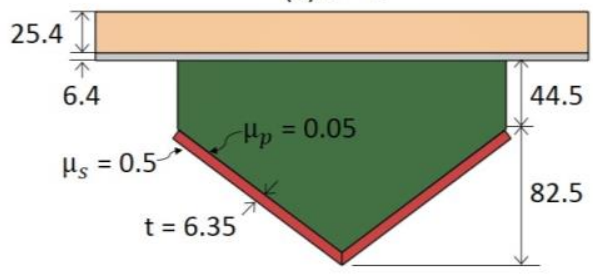

(b) $n=2$

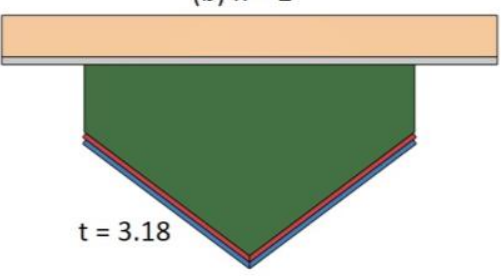

(c) $n=3$

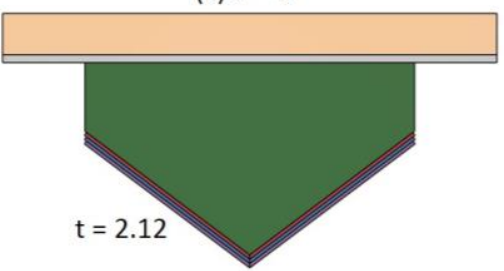

All dimensions in $\mathrm{mm}$

Figure 7.11. The targets with varied number of sliding plates, (a) one, $6.35 \mathrm{~mm}$ thick plate, (b) two, 3.18 mm thick plates, (c) three, 2.12 mm thick plates.

transfer momentum to a second (or third) plate, reducing the impulse applied to the V-apex and the underlying exposed inclined surface. For simplicity, the mass of the PETN charge remained fixed at $300 \mathrm{~g}$ below a $5.08 \mathrm{~cm}$ thick wet sand volume.

For this study, the number of sliding plates, n, was varied from 1-3 shown in Figure 7.11. Simulations at 19 and $29 \mathrm{~cm}$ standoff distances were performed with these stacked sliding plates. The overall thickness of the stacked slider plates remained constant, with either two $(n=2), 3.18$ $\mathrm{mm}$ thick plates or three $(\mathrm{n}=3), 2.12 \mathrm{~mm}$ thick plates modeled to replace the original $6.35 \mathrm{~mm}$ thick slider plate. The surface area of all the plates remained constant, $20.32 \mathrm{~cm} \mathrm{x} 13.97 \mathrm{~cm}$. A friction coefficient of $\mu_{\mathrm{p}}=0.05$ was modeled between the surfaces of the stacked sliding plate to represent a lubricated (low friction) metal/metal contact surface.

The impulse transferred to the targets is summarized in Table 7.7 for $n=0,1,2$, and 3 sliding plates (both aluminum and steel) at 19 and $29 \mathrm{~cm}$ standoff distances. Figure 7.12(a) and (b) shows the impulse transferred to the targets with the varying number of stacked sliding plates. Consistent with standoff distance effects the impulses transferred to the targets at $29 \mathrm{~cm}$ standoff (Figure 7.12(b)) were less than those transferred at $19 \mathrm{~cm}$ standoff (Figure 7.12(a)). The difference, $\Delta \mathrm{I}$, between the V-block target and the V-block with $\mathrm{n}$ aluminum or steel plates is shown in Figure 7.12(c) and (d). For the steel sliding plates, the impulse is not seen to reduce any further below $\mathrm{n}$ $=1$ slinging plate when increasing to $\mathrm{n}=2$ or 3 stacked plates. However, increasing the number of lighter aluminum plates from $n=1$ to 3 reduced the transferred impulse slightly compared to $n$ $=1$ plate. This was observed as a $2 \%$ reduction at $19 \mathrm{~cm}$ standoff distance and $6 \%$. While 
Table 7.7. Transferred impulse to the targets at 19 and $29 \mathrm{~cm}$ standoff distance with varying number of stacked aluminum or steel sliding plates, $n=0,1,2$, or 3 .

\begin{tabular}{|c|c|c|c|c|}
\hline \multirow{2}{*}{$\begin{array}{l}\text { Number of } \\
\text { plate, } n\end{array}$} & \multicolumn{2}{|c|}{$\begin{array}{c}19 \mathrm{~cm} \\
\text { Impulse }(\mathrm{kPa} \cdot \mathrm{s})\end{array}$} & \multicolumn{2}{|c|}{$\begin{array}{c}29 \mathrm{~cm} \\
\text { Impulse }(\mathrm{kPa} \cdot \mathrm{s})\end{array}$} \\
\hline & Al plates & Steel plates & Al plates & Steel plates \\
\hline 0 & 10.19 & -- & 9.64 & -- \\
\hline 1 & 9.27 & 8.88 & 9.20 & 8.62 \\
\hline 2 & 9.26 & 8.87 & 9.32 & 8.74 \\
\hline 3 & 9.09 & 8.96 & 8.64 & 8.69 \\
\hline
\end{tabular}
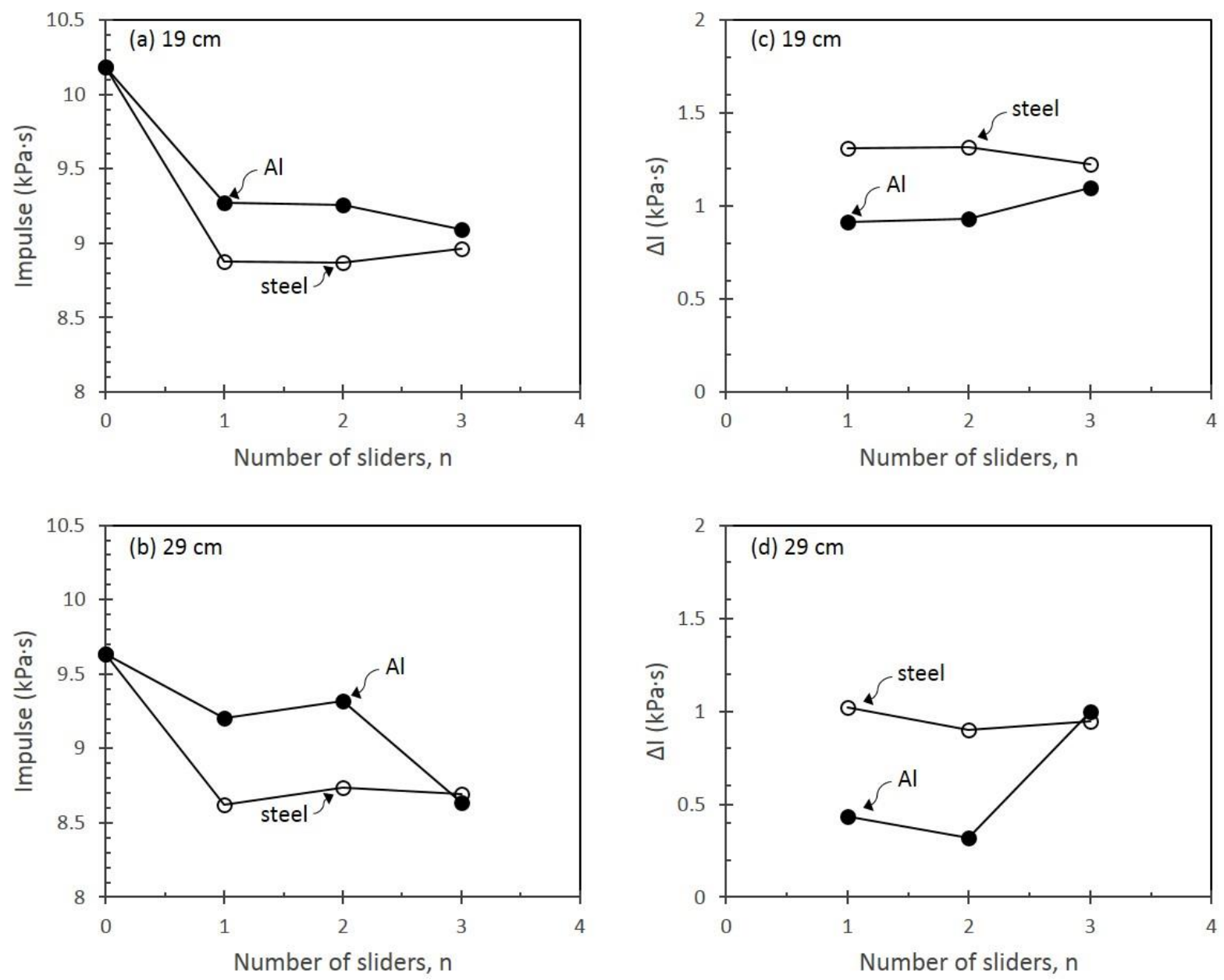

Figure 7.12. Effect of changing the number of sliding plates attached to the V-block target. Impulse transferred to the $V$-block target without slider plates (0 sliders) compared to the impulse transferred to the target with 1-3 attached slider plates (Al and steel) at standoff distance (a) $19 \mathrm{~cm}$ and (b) $29 \mathrm{~cm}$. The difference in transferred impulse between the V-block target without slider plates and the target with attached plates (both Al and steel) at standoff distances (c) $19 \mathrm{~cm}$ and (d) $29 \mathrm{~cm}$. 
Table 7.8. Impulse transferred to the sliding plate targets at a standoff distance of $19 \mathrm{~cm}$ with two plates and three plates with varying material properties for the series of stacked plates.

\begin{tabular}{ccccc}
\hline \multicolumn{2}{c}{ Two slider plates } & & \multicolumn{2}{c}{ Three slider plates } \\
\cline { 1 - 2 } \cline { 5 - 5 } Material & Impulse $(\mathrm{kPa} \cdot \mathrm{s})$ & & Material & Impulse $(\mathrm{kPa} \cdot \mathrm{s})$ \\
Al-Al & 9.26 & & Al-Al-Al & 9.09 \\
steel-steel & 8.87 & & steel-steel-steel & 8.96 \\
Al-steel & 8.84 & & Al-Al-steel & 8.87 \\
Ti-steel & 8.82 & & Al-Ti-steel & 8.92 \\
\hline
\end{tabular}

The impulse transferred to the target with $\mathrm{n}=3$ stacked aluminum sliding plates was $9.09 \mathrm{kPa} \cdot \mathrm{s}$ at $19 \mathrm{~cm}$ standoff distance reduced from $9.27 \mathrm{kPa} \cdot \mathrm{s}$ for a single aluminum plate. This was still greater than the impulse transferred to the target with $\mathrm{n}=1$ steel plate $(8.88 \mathrm{kPa} \cdot \mathrm{s})$. At $29 \mathrm{~cm}$, the impulse transferred to the target with $n=3$ aluminum plates was $8.64 \mathrm{kPa} \cdot \mathrm{s}$ which was approximately that transferred to a target with $\mathrm{n}=1$ steel plate $(8.62 \mathrm{kPa} \cdot \mathrm{s})$. The results show that three stacked aluminum sliding plates can reduce the impulse transferred to a target compared to a single thick aluminum sliding plate to the limit reached by the use of a heavier mass steel plate. This indicates that increasing the number of stacked plates has approximately the same effect as increasing the sliding plates mass.

Sliding plates of different masses (densities) were then stacked to analyze the resultant impulse loading at $19 \mathrm{~cm}$ standoff distance. The results are shown in Table 7.8 comparing $\mathrm{n}=2$ and $\mathrm{n}=3$ sliding plates, using a combination of aluminum, titanium, and steel plates. For the combinations listed in Table 7.8, the first material listed indicates the slider plate impacted first (outer plate), and the last material listed indicates the slider plate lying directly against the inclined V-block surface. The combinations of Al-steel and Ti-steel sliding plates resulted in $8.84 \mathrm{kPa} \cdot \mathrm{s}$ and $8.82 \mathrm{kPa} \cdot \mathrm{s}$ impulse transferred to the target respectively. This was marginally less (less than $1 \%$ ) than the loading applied to the target with $n=2$ steel-steel sliding plate combination $(8.87$ $\mathrm{kPa} \cdot \mathrm{s})$. This was an impulse reduction of $\sim 4-5 \%$ from that applied to the $\mathrm{n}=2, \mathrm{Al}-\mathrm{Al}$ target. The result for the $\mathrm{n}=3$ stacked sliding plate target with the attached Al-Al-steel sliding plate combination reduced the transferred impulse from that transferred to $\mathrm{n}=3$ stacked aluminum sliding plates by $\sim 2.4 \%$. This transferred impulse $8.87 \mathrm{kPa} \cdot \mathrm{s}$ (Al-Al-steel target) was once again approximately that observed for the single steel plate, $8.88 \mathrm{kPa} \cdot \mathrm{s}$. Increasing the number of plates from $\mathrm{n}=1$ to $\mathrm{n}=3$ did not significantly reduce the transferred impulse below that achieved by a 
single heavy (steel) plate $(8.88 \mathrm{kPa} \cdot \mathrm{s})$. However, combinations of $\mathrm{n}>1$ stacked plates reduced the impulse transferred to a target below that transferred to a target with $n=1$ aluminum plate $(9.27$ $\mathrm{kPa} \cdot \mathrm{s})$.

\subsection{Conclusions}

Experimental testing on a vertical impulse test rig allowed measurement of the pressure and impulse applied to several different target designs. Chapter 6 indicated the benefit of aluminum sliding plates attached to a V-shaped target to further reduce the transmitted impulse below that achieved by simply inclining the impact surface at an angle $\alpha$ to incident granular media impacts. A further series of tests were done with steel sliding plates attached to the same V-shaped aluminum base target tested at various standoff distances (i.e. incident impulse). Experimental results indicated that the heavier steel sliding plates could reduce the impulse below that achieved with the aluminum plates. A validated simulation model was shown to have good agreement with the measured response. Two phases of impulse loading were again identified for granular impacts on a sliding plate target. During Phase I the initial high velocity particles impacted the sliding plates, transferring momentum to the plates to begin their motion. As the sliding plates moved away from their initial location, the $\mathrm{V}$-apex of the rigid base target and the underlying surface became exposed to later arriving sand particle impacts during Phase II loading. Results indicated that by increasing the sliding plate mass the time of Phase I loading could be increased; thus reducing the impulse transferred to the exposed apex and underlying inclined surface.

These simulation were further used to systematically vary the sliding plate mass to investigate the effect on the transferred impulse. Increasing the plate mass led to a decrease in transferred impulse within a certain limit for the incident impulses studied. Increasing the mass of the sliding plates beyond that of the density of steel did not reveal any further reduction in transferred impulse. The mass of the explosive charge, accelerating the $5.08 \mathrm{~cm}$ thick layer of wet sand, was also systematically varied. Results indicated the benefit of the sliding plate exists for all explosive charge masses investigated here. As the explosive mass increased, the difference between the impulse transferred to a $\mathrm{V}$-shaped target and a target with sliding plates also increased, suggesting the scalability of this design for higher charges. Lastly a study was modeled to investigate the effect of the number of stacked sliding plates by changing the number of plates from $\mathrm{n}=1,2$, and 3. This progressively released the stacked plates from the inclined surface in order to cover the 
underlying surface by a sliding plate for a greater fraction of the loading. The stacked sliding plates revealed that the impulse could be approximately reduced to the limit achieved by a single steel sliding plate. However, no further significant benefit beyond the limit achieved by the single steel plate was observed in the simulations. Increasing the number of stacked plates had approximately the same effect as increasing the mass (density) of the plate. 


\section{Chapter 8. Discussion}

The goals of this dissertation were to investigate, both experimentally and with validated discrete particle simulations, granular media impacts with deformable structures, and explore novel concepts to mitigate damage to these structures. The discrete particle method for high velocity impact loads has been shown to give pressure and impulse waveforms that are in good agreement with the experimental results. The simulation approach also predicts the ensuing structural deformation well. Sufficient confidence in the simulations was obtained, and they were then used to validate the use of a sandwich panel concept to reduce the deflections of edge clamped plates under very high intensity granular particle loading. Additional studies then explored the use of inclined target surfaces in conjunction with sliding plates to reduce the momentum transferred by particle impacts. 


\subsection{Explosively accelerated granular media propagation}

Explosively accelerated granular media traveling at supersonic velocities has been investigated. The development of velocity and density gradients within the expanding plume of the granular particles is shown to effect the loading applied to nearby structures. These gradients develop as a result of the longitudinal and radial stretching of the particle volume. An experimental test setup was designed in which a large (several kg mass) spherical test charge, consisting of an inner sphere of high explosive material (composition C-4) surrounded by an annular region of water-saturated particles, was suspended above the center of an edge clamped test plate. This spherically suspended test charge experimental setup extended that recently devised by Dharmasena et al. [17] by explosively accelerating granular media to velocities in excess of 1200 $\mathrm{m} / \mathrm{s}$. The test charge was suspended such that it was located equidistant from the top of the test plate and to the front of a Kolsky bar (although shifts in this positioning occurred prior to detonation). This bar was instrumented with strain gauges to measure the axial stress (pressure) and impulse applied by the granular media impacts. The test plate was edge clamped to a picture frame support base where out of plane deflection occurred in the unsupported region of the plate. The clamping method was designed to permit in-plane stretching of the test plates while avoiding shear-off near the gripped region. The test platform was raised to allow for the visualization of test events and measurements of the granular front speeds by high-speed video imaging.

An initial model test validated the discrete particle based simulation method of the IMPETUS Afea code for very high velocity granular particle impacts. The solver employs the discrete particle based method by modeling the particle/particle interactions between air, high explosive, and soil particles. This is coupled to a finite element (FE) model to determine contact forces between the particles and FE structures (defined here by the Johnson-Cook (J-C) constitutive model) and the resulting structural response. The model test consisted of a charge of $3 \mathrm{~kg}$ of high explosive (C4) surrounded by $23.8 \mathrm{~kg}$ of water-saturated fused silica glass particles (synthetic wet sand) that was detonated above a $1.32 \mathrm{~m}$ x $1.32 \mathrm{~m}, 2.54 \mathrm{~cm}$ thick, edge clamped, high ductility 304 stainless steel test plate. The plate was gripped in a manner that avoided local impulse amplification resulting from the upward reflection of particles that was previously observed in studies with square plates where the edge grips extended above the top surface of the test plate [17]. The detonation event accelerated the main sand front, tracked by high-speed imaging, to a supersonic velocity of $\sim 1200 \mathrm{~m} / \mathrm{s}$. The measured peak impulse applied to the instrumented Kolsky bar by 
these granular impacted reached a final value of $\sim 9.5 \pm 1.5 \mathrm{kPa} \cdot \mathrm{s}$. The simulated response showed good agreement reaching a final value of $9.2 \mathrm{kPa} \cdot \mathrm{s}$. The Kolsky bar served as the only means for measuring the impulse applied to the test plates in the experimental test event. However, the simulations allowed for the measurement of the impulse applied directly to the test plate. The maximum simulated impulse applied to the center of the test plate was $12.8 \mathrm{kPa} \cdot \mathrm{s}$, slightly higher than that applied to the Kolsky bar (a result of the $\sim 5 \mathrm{~cm}$ closer standoff distance). The impulse then rapidly decayed with radial distance outward from this maximum peak. This decay resulted from the longer standoff distance (before particle impact) and the oblique angle of incidence which reduces the fraction of particle momentum transferred to the plate surface. The maximum plate deflection was measured as $3.56 \mathrm{~cm}$, and the simulated plate deflection profile revealed excellent agreement with the experimentally measured final permanent deflection after testing, Figure 3.20. For this model test, the plate was secured to an I-beam picture frame support base. The simulations revealed a region of elastic rotation and localized plastic strain on the I-beam support frame (shown in Figure B3) where failure occurred during a subsequent test (not discussed in this dissertation). This indicated that for further testing at these high impact loading conditions a sturdier support base was required, and the I-beam frame was replaced by a solid picture frame plate for the succeeding tests.

The discrete particle based simulations were in very good agreement with the measured (expanding) sand front position and velocity, its impulse-time response, and plate deformation for the initial model test. The now validated simulation method was then used to design a series of five test charge configurations with systematically increasing impulsive loading conditions. This was achieved by varying the mass of the granular media and the explosive. The granular media mass was adjusted by either increasing the annular region of saturated particles (number of particles) or increasing the density of the particles (switching from silica glass to higher density zirconia particles). The explosive mass was varied from 3 to $4.5 \mathrm{~kg}$ of C-4 (limited by restrictions for the test range). These five charge configurations with total mass varying from $25-150 \mathrm{~kg}$ were then experimentally tested using the same test setup as the model test but with a reinforced support picture frame base onto which the test plates were secured.

The radial expansion of each of the spherically suspended test charge configurations was observed by high-speed imaging techniques. The leading edge of the sand front expansion for 
each charge was examined in Figure 4.5 (silica glass test shots) and Figure 4.6 (zirconia test shots). Sand fingering was observed at the leading edge of the sand front, where localized regions of the front traveled faster than the main front, consistent with a jetting phenomenon $[53,59,70]$. Recent studies $[62,70]$ have shown this fingering effect to be consistent with the development of a Richtmyer-Meshkov type instability at the interface between the air and granular media. A denser main sand front was seen directly behind the sand fingers and was tracked to determine the position and velocity of the expanding main particle front shown in Figure 4.7(b) (silica glass test shots) and Figure 4.8(b) (zirconia test shots). The sand front velocity was observed to vary between 500$1200 \mathrm{~m} / \mathrm{s}$ depending on the ratio of the masses of the granular particles and the high explosive. When the granular particle mass was increased while the explosive mass remained fixed, the expansion velocity of the sand front was observed to decrease. However, the sand front velocity increased when the explosive mass was increased for a fixed mass of granular particles. The use of higher density zirconia particles (to increase the particle mass) resulted in much lower expansion velocities. Examination of the leading edge of the expanding sand front for the five charge configurations in Figure 4.5 and Figure 4.6 revealed that the sand fingering phenomena was dependent on the sand front velocity. A greater fingering effect (instability) was observed at the leading edge of the charge configurations with higher particle velocities. The development of these sand fingers progressively decreased as the radial speed of the particle expansion decreased from $1200 \mathrm{~m} / \mathrm{s}$ to $500 \mathrm{~m} / \mathrm{s}$.

While high-speed imaging was limited to observations of the outer expanding shell, discrete particle simulations allowed for the examination of the granular media acceleration by the inner explosive charge and further analysis of the particle velocity and density gradients that developed within the expanding shell. The detonation shock wave was observed propagating radially outward from the point of detonation, initiating a compressive shock in the granular media upon reaching the interface between the high explosive/granular media. This compressive shock propagated through the annular sand shell reaching the interface between the granular media and acrylic outer shell, where the shock was reflected backwards towards the detonation location. To conserve momentum during this reflection, spallation of sand particles from the outer edge of the particle shell occurred. Accelerated by the momentum transfer from the expanding HE particles, the sand particles reached a peak in velocity at a short distance ahead of the original outer surface of the particle shells approximately $100-200 \mu$ s after detonation (depending on the test charge 
granular media/explosive mass ratio). The competing drag resulting from particle interactions and momentum transfer with background air particles then resulted in a decay in velocity after this peak velocity was achieved.

As the sand shell radially expanded (Figure 3.12), accelerated by the high explosive products, a significant variation in particle density developed, Figure 3.15 (also observed for the five charge configurations in Chapter 4). The particles at the leading edge of the sphere acquired the highest momentum and were accelerated ahead of the main sand front. Trailing particles, released from the shell as the (decaying strength) sand shock reflection reached the inner particle shell location, had a lower velocity even though they were located directly in front of the detonation products. The density of these slower velocity particles was much higher than that at the leading edge of the sand particle plume. The lower sand particle density at the outer edge of the sand shell resulted from a combination of the radial stretching of the sand (due to the velocity dispersion) as well as the growth in surface area of the spherically expanding front. This high velocity, low density region of sand particles corresponded to the sand fingering instability that was observed in the high-speed videos at the leading edge of the sand front. However, the topology of these fingering instabilities was not resolved in the simulations.

High-speed video and particle based simulations revealed the development of an air shock ahead of the expanding shell of granular media in Figure 4.11. This was observed as a densified (compressed) region of air forming ahead of the leading edge of the sand front. The air shock pressures and velocities were dependent on the sand front velocity and summarized in Table 4.5. The simulations modeled with and without air particles allowed for a detailed investigation of the effect of the air on the sand front expansion. In simulations modeled without air particles, the sand front was accelerated to a maximum velocity and then continued to propagate radially outward at this (constant) velocity, with no deceleration. Simulations performed with air particles, however, revealed a decay in velocity after this peak (maximum) velocity was achieved, which was consistent with the high-speed video observations. This revealed that the deceleration of the sand front velocity observed in the experiments was a result of the momentum transfer between the particle (air/sand) interactions. Air particles are thus necessary to accurately model the radial expansion (position) and velocity of the sand particles. 


\subsection{High impact loading and structural response of solid plates}

Ideally the test charge was positioned equidistant from the impacted front end of the instrumented Kolsky bar (used to measure the applied pressure and impulse by granular media impacts) and the top center of the test plate. The Kolsky bar strain gauge data then represents a measurement of the impulsive loading applied to the test targets. However, the test charge experienced shifts in positioning prior to detonation and was thus not equidistant from the test plate and impact end of the bar. The Kolsky bar was still used as an approximate measure of the loading on the test plate and as a means to validate the simulated response. Particle impacts at the front of the Kolsky bar were observed later in the signal depending on the elastic wave speed of the bar and the location of the strain gauges from the impacted end.

The Kolsky bar signals for each of the spherical charge configurations revealed distinct characteristics in the pressure waveform that corresponded to four regions of loading observed in both the experimental and simulation impulse-time responses, Figure 4.12 and Figure 4.13. These four regions of impulse loading were characterized by the density and velocity of the incident particles. The explosively accelerated sand shell developed particle density and velocity gradients as the sand stretched and expanded. Examination of the high-speed videos (Figure 4.5 and Figure 4.6) and simulations (Figure 4.9 and Figure 4.10) revealed that impacts by these sand particle gradients corresponded to these four distinct regions of loading. While high-speed video imaging showed the impact times of the leading edge of the sand front (sand fingers) and main sand front, the simulations allowed for a further examination of the sand particle impacts.

Each pressure waveform (Figure 4.12 and Figure 4.13) exhibited a small pressure pulse at the arrival time of the initial particle impacts. This was followed by weakly oscillating waveforms before a large peak pressure rise was observed for each charge configuration. This was then followed by much stronger oscillations resulting from the Pochhammer-Chree modes [81, 92, 93]. Integration of the pressure signal resulted in the impulse-time response where four distinct regions of granular particle loading were observed for each spherically suspended test charge. An initial rise (bump) in impulse was characteristic of Region I. The arrival time of this region corresponded to the small pressure pulse observed in the pressure waveform. Observations from the high-speed videos and simulations revealed that Region I correlated to the initial sand particle impacts. For the highest velocity sand fronts (the silica glass particles), this corresponded to the initial impact 
by the sand fingers. However, for the slower expanding sand fronts (the zirconia particle test charges), the sand fingering effect at the leading edge of the sand front was less prominent and initial impact was by the main sand front. Following this initial bump in impulse, Region II was characterized by a slowly rising impulse region lasting between 100-200 $\mu$ s that corresponded to the period of weak oscillations observed in the pressure signal. This region coincided with particle impacts of the main sand front which was characterized by a gradual rise in the particle density and decreasing particle velocity. After this gradual rise, a sharp jump in impulse was observed in Region III, corresponding to the large, peak pressure arrival time. Simulations revealed that this sharp jump in impulse corresponded to impacts on the Kolsky bar by the low velocity, high density region of trailing sand particles. This dense region of particles was directly followed by the high explosive detonation products. Impacts by the HE particles and any remaining sand particles contributed very little additional impulse, and the impulse reached a plateau in Region IV for the remainder of the observed signal (before the distal reflection arrival).

The measured and simulated Kolsky bar responses were in good agreement each displaying the four distinct regions of impulse loading that correlated to the granular particle impacts of various density and velocity gradients. The simulations showed that the majority ( 90\%) of the impulse applied to the Kolsky bar was a result of the granular (sand) particle impacts. Only small contributions were observed from impacts by the initial air shock and the late arriving high explosive (detonation product) particles as shown in Figure 3.18 for the different particle contributions (air, HE, and sand) to the total impulse. Simulations performed without air particles revealed that the total impulse applied to the Kolsky bar was approximately the same as when air particles were present in the simulation. In simulations without air, there was no momentum transfer between the air/sand particles, and as such, the sand particles applied a larger contribution to the total impulse applied to the Kolsky bar. In simulations with air, the momentum transfer during particle interactions resulted in a deceleration of the sand particles, which resulted in less impulse applied by the granular impacts (than in the simulations without air). However, the total applied impulse remained relatively unchanged in both cases with the additional impulse applied by the air shock in simulations with air.

The five test charge configurations were impacted against $1.32 \mathrm{~m}$ x $1.32 \mathrm{~m}, 2.54 \mathrm{~cm}$ thick edge clamped, 304 stainless steel solid test plates. The structural response of the test targets could 
not be observed in the high-speed images, but the permanent plastic deflection was measured after testing and summarized in Table 4.6. The final deflection increased from $4.77 \mathrm{~cm}$ (Shot 1) to 8.16 $\mathrm{cm}$ (Shot 5) consistent with an increased impulse loading. The particle based simulation code allowed for the transient nature of the panels to be simulated until oscillatory decay occurred and a permanent steady state deformation was reached (after $\sim 20 \mathrm{~ms}$ ). The dimensionless final permanent deflection (normalized by the half span, $\delta / \mathrm{L}$ ) and dimensionless impulse (normalized by the areal mass and material properties of the plate, $I_{o} / m_{b} \sqrt{\sigma_{Y} / \rho_{m}}$ ) were plotted in Figure 4.20 to analyze the plate's structural response when increasing the applied incident impulse. Since the test charges shifted slightly prior to detonation (some shots more significantly than others), varying the standoff distance from the test charge and Kolsky bar, the impulse response measured by the Kolsky bar did not accurately measure the impulse applied to the test plates in certain cases. The simulations allowed for the measurement of the impulse applied directly to the test plates by the granular impacts which varied from 15.3 to $25.4 \mathrm{kPa} \cdot \mathrm{s}$ (Table 4.6). Results indicated a linear relationship between the permanent plastic plate deformation and the applied impulse. In-plane stretching, shown for Shot 3 in Figure 4.19, was observed in the back supported region of the solid test plates. This revealed that edge material was pulled inward and contributed to the final out of plane plate displacement. The normalized permanent deflection was thus plotted for two definitions of the plate span, the unsupported region of the plate $(2 \mathrm{~L}=81.3 \mathrm{~cm})$ and the length of the plate defined by the supported picture frame base $(2 \mathrm{~L}=122 \mathrm{~cm})$.

Simulations modeled without air particles revealed that the presence of the air particles in the model did not affect the plate's permanent displacement. This was consistent with the results for the impulse loading on the Kolsky bar observed without air particles. The conservation of momentum dictates that the sum of the impulse of the air and sand particles must be equal to the particles in the absence of air. Thus, the structural response of the test plates remained relatively unchanged whether or not air particles were modeled in the simulations.

\subsection{Structural response of sandwich panels}

Recent sand slug investigations revealed that a sandwich panel design with a strong core and increased bending strength suffers smaller deflections than equivalent (same mass per unit area and material properties), solid (monolithic) test plates [32]. A strong square honeycomb core was identified as an ideal strong-core candidate. To limit core crushing, a $\sim 30 \%$ relative core density 
was selected for the sandwich panels. The square honeycomb sandwich panels were fabricated from $1.32 \mathrm{~m} \times 1.32 \mathrm{~m}$ plates to have an equivalent mass as the solid plates. An array of $16 \times 16$ square honeycomb cells were milled within the center $81.3 \mathrm{~cm}$ x $81.3 \mathrm{~cm}$ square span of the plates. The panel consisted of an integral front face sheet/core structure, which was chosen such that the impact side of the panel did not require welding between the front face sheet and the core. The back face sheet was attached by electron beam welding along the intersection between the face sheet and the cell walls. The total thickness of the sandwich panel was then $5.08 \mathrm{~cm}$. For testing, the standoff distance from the spherical test charge center to the back face sheet was fixed at $\mathrm{H}_{\mathrm{b}}=$ $47.54 \mathrm{~cm}$ for both the solid plates and sandwich panels. This required that the sandwich panel front face sheet be placed $2.54 \mathrm{~cm}$ closer to the test charge than the top of the solid test plates.

The impulse applied to the sandwich panels varied from $17.6 \mathrm{kPa} \cdot \mathrm{s}$ (Shot 1) to $33.7 \mathrm{kPa} \cdot \mathrm{s}$ (Shot 5). This was greater than that applied to the solid test plates since the impact (front face) surface of the sandwich panels was $2.54 \mathrm{~cm}$ closer to the test charges. The maximum permanent plastic displacement of the sandwich panels increased from 3.32 to $8.17 \mathrm{~cm}$ (Shot 1 to Shot 5), as the applied impulse increased (Table 5.5). During the loading process, the welding attaching the back face sheet to the core failed, and the back face debonded. This was observed early in the loading process in the simulations where the back face sheet was modeled with a merge failure criteria. The stiffness of the square honeycomb sandwich panel design was retained and minimal core compression was observed for each test shot after testing for the strong (high strength), cellular core. The measured core compression was only $0.40 \pm 0.79 \mathrm{~mm}$ for Shot 1 , approximately $1 \%$ core compression strain. While the core compression increased slightly for each test shot with increasing applied impulse (Table 5.7), the maximum core compression measured for Shot 5 was $1.98 \pm 0.79 \mathrm{~mm}$, less than $6 \%$ core compression strain.

The dimensionless final permanent deflection was calculated for the square honeycomb sandwich panels (normalized by their half span, $\delta / \mathrm{L}$ ). The span of the panel was defined by the unsupported center region, $2 \mathrm{~L}=81.3 \mathrm{~cm}$. This was plotted in Figure 5.15 as a function of the dimensionless impulse, again calculated using the peak impulse applied to the panels normalized by the panel's areal mass and material properties. The results for the solid plates are also plotted in Figure 5.15 for analysis of the sandwich panel design benefit. For the impulse loading conditions considered here, the square honeycomb sandwich panel outperformed its equivalent 
mass solid plate counterpart. The sandwich panels also exhibited less in-plane stretching of the back supported edge material than was observed in the solid plates (Figure 5.14). There was no measured reduction in the transferred impulse to the sandwich panel (the impulse was greater than that applied to the solid plates), indicating there was no benefit of the FSI effect as observed for impacts in water. Here, the benefit of the square honeycomb sandwich panel resulted from its increased bending/stretching resistance due to the strong core of the sandwich panel design.

Simulations were used to further investigate the debonding of the back face sheet and its effect on the permanent deflection of the panels. Three cases were investigated in which (i) the back face remained bonded during the entirety of the granular impulsive loading, (ii) the back face sheet debonded during impacts, and (iii) the back face sheet was never attached to the sandwich panel core. The debonding of the back face sheet resulted in an increased permanent deflection of the sandwich panel by 6-10 mm, Figure 5.16 (compared to if the back face had remained welded for the entire loading process). However, if the back face sheet had never been attached to the core, the permanent deflection of the panel would have been even 6-11 mm greater than observed for the debonded case.

\subsection{Impulse mitigation}

The momentum per unit area transferred to a test structure by the impact of granular media depends upon that of the incident material and the efficiency which this momentum is transferred to a test structure. In scenarios where the media is brought to arrest by stagnation against a surface that is oriented perpendicularly to the impact direction, the full momentum is transferred. If the granular particles bounced back in the direction of origin, then additional momentum (equal to that of the reflected material) would also be transferred. If a test structure were inclined to the incident granular flow, some of the flows momentum in the incident direction is retained by the particles and so the target receives less momentum than that of the incident particles. This inclination effect therefore provides a significant opportunity to mitigate the impulse transferred by a buried explosion to a structure.

To investigate this opportunity, a vertical impulse test apparatus equipped with four strain gauge, instrumented Kolsky bars that had previously been developed by Holloman et al. [13, 14], was utilized to measure the pressure and impulse time response of explosively accelerated granular media impacts against three different target designs. The baseline target was a flat bottomed 
rectangular aluminum block. One of its faces was oriented normal to the incident granular particle direction $\left(\alpha=90^{\circ}\right)$ to measure the impulse transferred when sand was stagnated against its surface. The second target had a pair of V shaped impact surfaces each inclined at $\alpha=53^{\circ}$ to the sand impact direction. This target allowed the effect of partial momentum transfer to be examined. The third sample was also a $\mathrm{V}$ shape with inclined surfaces $\alpha=53^{\circ}$. However, aluminum or steel sliding plates were attached to its surface so the effect of reducing the effective friction coefficient of the granular particle/target interaction could be assessed. Using the same buried explosive test configuration, experiments were conducted at three standoff distances of 19, 24, and $29 \mathrm{~cm}$.

High-speed video imaging was used to view the sand front propagation and initial sand particle impacts. In some instances, a wider camera angle was able to capture the sliding plates' parabolic trajectory after departure from the rigid V shaped target. Simulations of the test events were used to observe sand particle interactions with each of the test targets, the initiation of the sliding plate movement, and their escape from the rigid base target. A sand fingering instability (similar to that observed in the spherical test charges) was seen at the leading edge of the sand front, with localized regions of particles moving faster than the bulk of the sand. This instability was again not resolved in the simulations. However, similar to the previously reported results for the spherical sand shell charges, the simulations revealed particle density and velocity gradients with a region of low density, high velocity sand particles at the leading edge of the sand front. The main sand front (directly behind the sand fingers) was tracked experimentally with the high-speed cameras and in the simulations by sensors. Good agreement was observed between the experiments and simulations. The main sand front velocity, Figure 6.7(c), was observed to vary from $350-500 \mathrm{~m} / \mathrm{s}$, depending on the standoff distance, with the sand fingers accelerating up to $18 \%$ faster than the main front. As the sand propagated away from the detonation location, it expanded both axially (stretching in the direction of propagation normal to the sand surface) and laterally. This resulted in a density gradient within the expanding sand volume. Further, the sand front experienced a deceleration after a peak velocity was reached, resulting from momentum transfer with surrounding air particles.

The simulated pressure and impulse responses, up to the arrival of the second distal reflection, were also shown to be in good agreement for each target design with the experimentally measured Kolsky bar signal. This was shown in Figure 6.9, Figure 6.13, and Figure 6.19 for the baseline, flat block target, the inclined V-block target, and the V-block target with attached aluminum 
sliding plates respectively. The simulations were then used to investigate the impulse transferred to the test targets after $4 \mathrm{~ms}$, when a plateau value was reached. This allowed for the investigation of the impulse ratio $\left(I_{T}^{Z} / I_{o}\right)$ for each of the target designs to determine impulse reductions resulting from surface inclination and the addition of the attached pair of sliding plates. The reduction of the impulse ratio by these effects is shown in Figure 6.23.

The discrete particle simulations allowed for observations of the particle interactions with the test targets that was obscured from view by particles that traveled between the target and camera. The sand particles were seen to impact the flat target with a weak reflection in Figure 6.8. Impacts by later arriving particles resulted in an accumulation against the target surface that eventually caused the particles to flow laterally outwards past the edges of the target surface. These observations were consistent with sand slug impacts on a normally oriented target $[32,48]$. The impulse ratio $\left(I_{T}^{Z} / I_{o}\right)$ was calculated for each standoff distance (i.e. varying incident impulse, $\left.I_{o}\right)$, where $I_{T}^{Z}$ is the impulse transferred in the vertical $\mathrm{Z}$ direction. For the baseline target, oriented normally to the incoming particle impacts $\left(\alpha=90^{\circ}\right)$, the impulse ratio was approximately 1.02 , indicating the transferred impulse was approximately equivalent to the incident impulse of the incoming sand. This was consistent with the weak particle reflection upon impact and a weak FSI (fluid structure interaction) effect by granular particles. The impulse was observed to be uniformly distributed to the flat impact target surface in Figure 6.20 with the impulse decreasing as the standoff distance increased. This was consistent with the decelerating particle velocity due to air drag as well as the decreased density resulting from stretching of the sand column.

Observation from the high-speed videos of the V-shaped target in Figure 6.14 caused significant redirection of the sand particles. Upon impact on the inclined surfaces, oriented $\alpha=$ $53^{\circ}$ from incident particle impacts, the sand particles were forward reflected and traveled towards the outer edges of the target. The simulation sequence in Figure 6.15 showed that later arriving particles resulted in an accumulation of particles on the inclined surface. These particles flowed parallel to the target surface and then beyond the edge of the target. For experimental comparison with particle impacts on a rough (grit blasted) surface, the discrete particle simulations were modeled with a soil/structure friction coefficient of $\mu_{\mathrm{s}}=0.5$. The transferred impulse ratio for the V-block target inclined $\alpha=53^{\circ}$ was $\sim 0.85$. This resulted in an approximately $17 \%$ reduction from the impulse transferred to the baseline target (1.02), revealing the effect of reducing the surface 
inclination from $\alpha=90^{\circ}$ to $53^{\circ}$ seen in Figure 6.23. The study confirmed previous results in which an inclined surface, at an angle $\alpha$ from the incident granular particle impacts, reduces the impulse transferred to the target. The majority of the impulse applied to the V-block target was concentrated at the apex of the target (Figure 6.20), again decreasing with standoff distance. The impulse applied to the inclined V-block target was consistently less than that applied to the flat baseline target.

The impulse reduction due to surface inclination was previously shown to depend on the coefficient of friction between the impacted inclined surface and the granular particles. Impacts against smooth (low friction) surfaces resulted in greater impulse reductions compared to rough (high friction) surfaces. Further simulations were performed with a soil/structural interaction friction coefficient $\mu_{\mathrm{s}}=0.0$ to investigate this friction effect on the impulse transferred to the target. The expected (calculated) vertical impulse transferred in the frictionless limit (i.e. the absence of friction) is $I_{T}^{Z}=I_{o} \sin ^{2} \alpha$. For the inclination angle studied here, $\alpha=53^{\circ}$, the calculated frictionless limit is then $I_{T}^{Z} / I_{o}=0.64$. However, the simulated transferred impulse ratio for the frictionless target $\left(\mu_{\mathrm{s}}=0.0\right)$ was 0.72 which exceeded this calculated value. The impulse contributions by the different particle type (air, HE, and sand) impacts summarized in Table 6.7 revealed that the majority of the impulse transferred to the targets resulted from sand particle impacts. However, a small portion, $\sim 8.5 \%$, of the impulse was applied by the initial air shock and the late arriving detonation products. The discrepancy of the impulse transferred in the resolved frictionless limit was determined to be a result of this additional impulse transferred by the gaseous (air and high explosive) particles.

These impulse mitigation results for impacts on low friction surfaces suggests that hard polished coatings applied to an inclined surface may be used to significantly reduce the impulse transferred to a target. However, soil particles that impact with high velocity can result in surface roughening which may limit the benefit of a coating to create a smooth impact surface. This dissertation introduced an alternate concept with a new target design in which sliding plates (attached to an inclined surface) are accelerated by granular particle impacts. These sliding plates acquire momentum from these incident impacts and when sliding along a very low friction coefficient interface, reduces the momentum transferred to the rigid base target. For experimental testing a lubricant paste was applied to the metal contact surfaces between the inclined V-target 
surface and the sliding plates to create a low friction sliding surface. For the simulations, contact between the sliding plates and base target was modeled with a low friction coefficient of $\mu_{\mathrm{p}}=0.05$.

Particle impacts on the sliding plate test target revealed two phases of granular media loading. During Phase I, incident sand particles impacted the sliding plates transferring momentum to the plates and initiating their movement. The majority of the applied impulse occurs during Phase I. Similar to the impacts on the V-block target, the particles are redirected upon impact with the inclined surfaces, accumulating and flowing parallel to the inclined target surface (Figure 6.18). As the sliding plates gain momentum and move away from their initial position, the apex of the V-shaped target and the underlying inclined surface become exposed to impacts by later arriving sand particles during Phase II loading, evident in Figure 6.20. The plates were accelerated to velocities ranging between $55-70 \mathrm{~m} / \mathrm{s}$, resulting in the plates escaping the rigid $\mathrm{V}$ shaped base target 2.6-3.5 ms after detonation.

The transferred impulse ratio for the targets with the attached pair of aluminum sliding plates was $\sim 0.76$, which was an additional $11 \%$ impulse reduction from that transferred to the inclined V-block target (without sliding plates), Figure 6.23. This result was slightly above that transferred to the frictionless inclined target $\left(\mu_{\mathrm{s}}=0.0\right)$. Simulations revealed this was a consequence of the Phase II loading on the V-apex and underlying inclined surface that become exposed as the sliding plates were accelerated away from their initial position.

In further experimental tests, a pair of steel sliding plates replaced the aluminum plates to investigate the effect of the plate mass on the transferred impulse. For these tests, the rigid aluminum V shaped base target remained constant. The Kolsky bar signal responses in Figure 7.5 for the simulated and experimental pressure and impulse were again shown to be in good agreement. Simulations shown in Figure 7.4 revealed that the steel sliding plates took longer to accelerate and escape the rigid base target than the lighter aluminum plates. This increased the Phase I loading time where the sliding plates were impacted by the initial incident granular particles and reduced the time for Phase II loading where the V-apex and exposed underlying inclined surfaces were impacted by later arriving particles. The heavier steel plates protected the underlying inclined surfaces longer than the lighter aluminum plates. The transferred impulse ratio to the targets with steel plates was 0.71 which is reduced by $\sim 6.5 \%$ from the aluminum slider target (0.76) and is approximately that transferred to the frictionless target (0.72). This motivated a 
parametric study that investigated the influence of several variables on the sliding plate effectiveness using discrete particle simulations. These included the slider plate mass, the explosive charge mass, and the number of attached (stacked) slider plates.

The material properties (density) of the sliding plates were adjusted to investigate the plate mass varying from $330 \mathrm{~g}$ to $5.8 \mathrm{~kg}$. The heavier plates took longer to escape the underlying target and thus protected the surfaces longer. This resulted in a decreased period of Phase II loading on the apex and exposed inclined surfaces of the target by late arriving particles. The mass of the sliding plates was shown to influence the transferred impulse in Figure 7.9. The transferred impulse decreased as the plate mass was increased up to a certain limit. For the range of incident impulses studied here, 10.4-11.6 kPa·s (standoff distances 14, 19 and $29 \mathrm{~cm}$ ), it was shown that the greatest impulse reduction was achieved with steel sliding plates, $m_{p}=2.6 \mathrm{~kg}$. No further reduction was observed with heavier, tungsten sliding plates, where the ratio between the plate mass and sand mass was greater than 1 .

The explosive charge mass (below a fixed volume of wet sand) was varied from $100 \mathrm{~g}$ to 1.2 $\mathrm{kg}$. The impulse transferred to the target without sliding plates as well as with aluminum and steel plates was determined by the simulations. As the charge mass increased, the transferred impulse increased. For the charges investigated, the sliding plate target continuously resulted in an impulse reduction beyond that of surface inclination. The change in impulse reduction between the Vblock target and the target with sliding plates increased as the explosive charge mass increased (Figure 7.10). The continued impulse reduction at higher charge masses suggests that the sliding plate design can be used as an impulse mitigation technique for a larger scale test. The steel sliding plates continuously resulted in a greater impulse reduction than the aluminum plates, consistent with the result from the sliding plate mass study.

To reduce the impulse applied during Phase II loading by late arriving particles, simulations were performed in which multiple sliding plates were stacked on top of each other. The multiple stacked plates were progressively released as they were impacted by the incident particles. Stacking the plates ensured that the base surface was covered for a greater fraction of the loading time and reduced the impulse transferred to the exposed inclined surfaces. Results in Figure 7.12 indicated that three aluminum sliding plates reduced the impulse transferred to the target compared to a single aluminum plate. However, the results were not much improved beyond that transferred 
to the target with a single steel sliding plate. Using different combinations of various sliding plate density, the impulse was marginally reduced (by less than 1\%) below that transferred to a target with a single steel plate. Increasing the number of stacked sliding plates could result in approximately the same impulse reduction achieved by increasing the sliding plate mass. 


\section{Chapter 9. Conclusions}

The objective of this dissertation was to use a combination of experiments and numerical simulations to investigate the processes by which a buried explosion transfers momentum and applies stresses to nearby structures, and to use this insight to explore mitigation concepts. Model granular materials have been used in this study to avoid the complexities of real burial materials. The test geometries have also been simplified to reduce the effects of unknown or poorly characterized variables of the test arrangements. The work presented here began by providing insight into the radial expansion of spherical explosive charges with an inner explosive core surrounded by an outer annular region of water-saturated granular media (fused silica particles or zirconia particles). Upon detonation, these charges accelerated $25-150 \mathrm{~kg}$ of the granular media towards large (meter-scale) square stainless steel test targets. An initial target design investigated the structural response of solid (monolithic) plates. A second set of identical mass/unit area (and material) targets were configured as square honeycomb core sandwich panels to investigate their potential benefit for reducing panel deflections during impulsive loading. The study has also investigated the use of inclined targets which do not fully arrest impacting particles and therefore do not acquire all the momentum (impulse) of an incoming granular flow. For these tests, a planar explosive charge was submersed beneath a thin layer of wet granular particles (the same fused silica used in the spherical charges). The study then investigated the use of a sacrificial sliding plate on inclined surfaces to reduce frictional effects during granular particle impacts and has shown that substantial additional reductions of transferred momentum can be achieved.

The detailed conclusions from this dissertation are organized into four subsections: (i) Sand particle expansion following explosive acceleration, (ii) air particle effects upon granular propagation, (ii) impulse transfer during high intensity impacts, and (iv) impulse mitigation concepts with sliding plates. This chapter ends with a brief discussion of several aspects of this work that were beyond the scope of this dissertation but may be appropriate for future work. 


\subsection{Sand particle expansion}

- Experimental high-speed video recordings of model explosive events have allowed the observation of the leading edge of both spherical and nominally planar sand fronts. This allowed the main sand front position and velocity for each of the charge configurations to be tracked. However, these experimental observations were limited to the leading edge of the expanding sand fronts. The granular particles and later emerging detonation products obscured visual observation of the structural interactions. Discrete particle simulations were performed in the IMPETUS Afea Solver which allows for interparticle interactions and particle/structural interactions (with an FE model). These simulations were used to further analyze the sand particle expansion after detonation. The main sand front position and velocity were also tracked in the simulations, and results from the high-speed videos and simulations were in good agreement.

- The discrete particle simulations allowed for the detonation event to be modeled and the behavior of the shock front propagation to be inferred. The detonation event resulted in a shock front propagating through the high explosive (HE) particles outward from the detonation location. Upon reaching the interface between the HE/granular media, the detonation front of $\mathrm{HE}$ particles compressed the granular media outward. When this compressive shock front reached the interface between the granular media and air (acrylic shell), it was reflected, resulting in spallation along this interfacial region.

- The radially expanding sand front in the high-speed videos quickly accelerated to a peak velocity, a result of the momentum transfer from the explosive detonation event. This was then followed by a longer period of deceleration of the sand front velocity.

- A series of five spherical charge configurations with varying granular particle and explosive mass were investigated to systematically increase the applied impulsive loading. The main sand front peak velocity of these charges varied from 500-1200 m/s, depending on the mass of the granular particles and the explosive. Three of the charges were filled 
with water-saturated glass silica microsphere particles. Increasing the mass of these particles with a fixed explosive charge resulted in a reduced sand front expansion velocity. For a fixed mass of the granular particles, increasing the mass of the high explosive charge then resulted in a higher expansion velocity. To further increase the granular mass of the test charges, the silica glass particles were replaced with denser (heavier) zirconia particles, which resulted in a significantly slower particle expansion velocity.

- In the high-speed videos, localized regions of the sand front (i.e. sand fingers) were observed moving substantially faster than the bulk of the sand with velocities that were up to $50 \%$ faster than the main sand front (for the fastest expanding spherical charge).

- The discrete particle simulations allowed for further examination of the sand particle expansion. Simulations revealed that the radial distance of the particle propagation was not uniform within the sand shell as velocity and density gradients developed within the radially expanding granular shell. Sand spalled from the outer edge of the shell formed a region of high velocity, low density particles at the leading edge of the simulated sand front consistent with the sand fingers observed experimentally. A main sand front was observed directly behind these high velocity particles with increased particle density. At the trailing edge of the expanding shell, a region of low velocity, high density sand particles accumulated directly in front of the high explosive products.

\subsection{Air particle effects}

- Localized regions of the sand front were observed in the high-speed videos as sand fingers (jetting phenomena) that advanced (in some cases) well ahead of the bulk of the sand particles. Surface instabilities have been previously determined at the interface between the high explosive products/granular media as well as at the interface between the granular media/air. The latter was responsible for the sand fingering effect observed in the highspeed videos. This was consistent with a Richtmyer-Meshkov type instability developing along these interfacial regions $[62,70]$. 
- Observations from the high-speed videos revealed that the charge configurations with higher sand front velocities (1000-1200 m/s) had a more defined sand fingering effect at the leading edge of the expanding shell. The sand fingering instability was much less prominent for the denser (heavier mass), zirconia particle test charges, with much slower expansion velocities $(500-600 \mathrm{~m} / \mathrm{s})$.

- The discrete particle simulations revealed that the long period of deceleration of the sand front velocity observed in the high-speed videos was a consequence of the interactions with the air particles. In simulations performed without air particles, no deceleration of the granular particles was observed. Instead, the sand was accelerated to a peak velocity, which it then maintained. However, a deceleration of the granular particles, after the peak velocity was attained, occurred when air particles were present in the simulations. This velocity decay was consistent with the momentum transfer between the granular media and the surrounding air particles.

- An air shock developed in front of the leading edge of the sand particles, observed in the high-speed videos and in the simulations. The simulations and numerical calculations indicated that both the pressure and speed of this shock were dependent on the expanding sand front velocity of the granular media. Higher shock velocities correlated to the higher sand front velocities. The air shock impacted the Kolsky bar just prior to the first granular particle impacts with a small initial impulse. Conservation of momentum dictates that the sum of the impulse of the air and granular particles must be the same as in the absence of air. Simulations confirmed that the reduction in the granular particle momentum resulting from the presence of air particles corresponded to the amount transferred to the air shock during particle interactions.

- Since the impulse was equivalent for simulations with and without air particles due to momentum conservation, the momentum transferred to the test targets was roughly 
equivalent in either case. This resulted in approximately similar panel displacements in both instances whether air particles were present or not in the simulations.

\subsection{High intensity impacts}

- An instrumented Kolsky bar measured the applied pressure and impulse resulting from the granular media impacts from the explosively accelerated charge configurations. These measurements approximately represented that experienced by the test targets and was used for validation with the simulated responses. The experimental and simulated Kolsky bar responses were shown to be in good agreement.

- The discrete particle simulations revealed that the total impulse transferred to the Kolsky bar and test targets was the sum of the impulse transferred by the three different particle contributions: air, sand, and high explosive particles. The majority of the impulse $\sim 90 \%$ was due to the sand particle impacts. The air shock impacted the Kolsky bar just prior to the first sand particles, contributing a small amount of impulse to the initial signal. The high explosive particles trailed behind the radially expanding sand shell and made impacts much later in time with also only small contributions to the total impulse.

- For each of the spherical charge configurations, the pressure and impulse time responses obtained from the Kolsky bar data (both measured and simulated) revealed four distinct regions of granular media (sand) impact loading. Region I was characterized by an initial bump in impulse that correlated to a small pressure pulse. Detailed examination of the high-speed videos and simulations revealed that this corresponded to the impacts of the sand fingers at the leading edge of the sand front, i.e. the region of high velocity, low density sand particles. For the zirconia particle test shots (where barely any sand fingering instability was observed), this initial impact resulted from the main sand front impact. This initial bump in impulse was followed by a region of slowly rising impulse, Region II. The start of Region II corresponded to the impact time of the main sand front directly behind the sand fingers. This was then followed by Region III characterized by a sharp jump in 
impulse, correlating to the arrival time of a peak pressure signal. Examination of the radially expanding sand shell in the simulations revealed that this jump in impulse (Region III) corresponded to the arrival time of the trailing, low velocity, high density, particles that accumulated directly in front of the high explosive particles. The signal reached a plateau in Region IV, during which time later arriving particles and the high explosive particles (detonation products) impacted the bar with no further contributions to the applied impulse.

- The five spherical charge configurations were impacted against solid test plates. These charges were designed to systematically increase the impulsive loading on the test plates. The permanent plastic displacements of the test targets were measured after the event. The simulated final plate displacements were in good agreement with the measured. The normalized deflection of the solid test plates was observed to increase linearly with increasing impulse. Discrete particle simulations allowed for investigation of the particle/structural interactions and the transient structural response upon impact.

- In-plane stretching of the edge material outside of the unsupported center span of the solid test plates was observed. This edge material contributed to the final plastic out of plane deformation of the test plates.

- A square honeycomb, sandwich panel, designed with $\sim 30 \%$ relative core density, outperformed its equivalent mass (and material) solid plate counterpart for the impulse loading conditions considered here. The sandwich panel design had a strong core and marginal core compression was measured after testing. The impulse transferred to the sandwich panel was greater than that transferred to the solid plates (due to the $2.54 \mathrm{~cm}$ closer standoff distance of the top face sheet of the sandwich panel). The benefit of the sandwich panel design was thus a result of its increased stretching and bending strength.

- The back face sheet of the honeycomb panels was insufficiently welded to the integral core of the honeycomb panels and became debonded (welding failed) when subjected to large impulsive loading conditions. Simulations revealed that this debonding occurred early in 
the loading process. Simulations were also performed in which the back face sheet remained bonded (welded) for the entirety of the loading process. The back face sheet debonding resulted in greater panel deformation than if the back face sheet had remained welded for the duration of the loading. However, the results were less than those for the simulated case where the back face sheet was never bonded (welded) to the core before testing.

\subsection{Impulse mitigation}

- Surface inclination was first investigated as an impulse mitigation technique, and then the use of a sliding plate (attached to the inclined surface) was explored as a potential means to further reduce the momentum transfer during granular particle impact. Three aluminum equivalent mass target designs were experimentally tested; flat bottom (baseline) target, Vblock (inclined) target, and a V-block target with attached sliding plates. Each target was impacted by an explosively accelerated, $5.08 \mathrm{~cm}$ thick layer of granular media at three standoff distances $(19,24$, and $29 \mathrm{~cm})$. Instrumented Kolsky bars were used to measure the pressure and impulse applied to the test targets. The data was only presented for the first $2.15 \mathrm{~ms}$ of loading since the arrival of the distal reflections complicated the signal later in time. The strain gauge measurements showed that the inclination of the target impact surface (V-block target) resulted in a longer rise time in the pressure-time response and less applied pressure than that of the normally oriented target. The impulse measured at the first distal reflection arrival time for the V-block target was observed to be reduced by a factor of 0.4-0.5 from the normally oriented flat bottom target.

- The discrete particle simulations were in good agreement with the measured pressure and impulse time responses up to the arrival of the second distal reflection. The simulations were used to investigate the particle interaction with the three targets at each standoff distance as well as determine the total vertical impulse transferred to the targets. 
- Inclining the surface of the target to $\alpha=53^{\circ}$ reduced the transferred impulse by $\sim 17 \%$ compared to a normally oriented target $\left(\alpha=90^{\circ}\right)$. Examination of the particle/structural interactions in the simulations revealed that inclining the target surface allowed the incident particles to flow parallel and along the inclined surface of the $\mathrm{V}$-shaped target rather than stagnate against the impact surface.

- Previous research has indicated a strong frictional effect of the impulse transferred to an inclined target. Simulations modeling the V-block target were performed with friction coefficients of 0.5 and 0.0 (between the granular particles and impacted structure). The results confirmed a frictional effect; $\sim 15 \%$ less impulse was transferred to the frictionless target. However, the simulated frictionless result exceeded the resolved frictionless limit predicted by calculations for a surface with an inclination angle of $\alpha=53^{\circ}$. Examinations of the simulations revealed that this was a result of impulse transferred by the gaseous particles (air and high explosive particles) that suffered a weak frictional interaction with the target.

- An additional impulse reduction of $\sim 11 \%$ was achieved beyond that of the surface inclination by attaching aluminum sliding plates to the inclined surface. This resulted in an approximately $28 \%$ reduction from the normally oriented (flat bottom) target. However, the impulse was greater than that transferred to the frictionless V-shaped target. Examination of discrete particle simulations revealed this discrepancy resulted from late particle impacts transferring momentum to the underlying exposed $\mathrm{V}$ apex and inclined surfaces after the sliding plates had moved from their initial position.

- Two phases of impact loading were observed for particle interactions with the sliding plate targets. In Phase I, the initial high velocity sand particles impacted the surface of the sliding plates, transferring momentum to the plates and resulting in their subsequent movement along the inclined surface. As the sliding plates gained momentum and moved along the 
inclined surface, they exposed the apex of the $\mathrm{V}$ shaped target and the underlying surface. Later arriving sand particles impacted these exposed surfaces during Phase II of loading.

- The use of heavier steel plates rather than aluminum sliding plates resulted in an increased Phase I loading on the sliding plates and thus less impulse transferred to the underlying exposed V-apex and inclined surface (less Phase II loading). This resulted in a further impulse reduction below that achieved by the use of aluminum sliding plates. The impulse reduction achieve with steel sliding plates was approximately that transferred to the frictionless inclined target (without sliding plates). This motivated a parametric study using the simulation solver to investigate the effect of sliding plate mass, explosive charge mass, and number of stacked sliding plates on the impulse mitigation achieved by the use of sliding plates.

- Increasing the mass of the sliding plates resulted in reduced impulse. Heavier plates required more momentum from particle impacts to begin their movement and resulted in an increase period of Phase I loading. Consequently, this decreased the duration of the Phase II loading, resulting in less impulse transferred to the exposed inclined surfaces by later arriving particle impacts. For the incident impulses investigated here, steel sliding plates with a $2.6 \mathrm{~kg}$ mass resulted in the greatest impulse reduction. Increasing the plate mass further resulted in no further impulse reduction.

- The impulse transferred to the targets increases as the explosive charge mass was increased up to $1.2 \mathrm{~kg}$ (limited by the aluminum target design). An impulse reduction resulting from surface inclination was observed for the range of charges investigated, and the impulse transferred to the sliding plate targets continued to be less than that transferred to a V-block target (without sliding plates) as the explosive charge mass was increased. The difference between the V-block target and the target with sliding plates increased as the charge mass was increased, indicating the continued benefit of the sliding plate design when the target is subjected to greater impulsive loads. 
- The number of sliding plates attached to the target was varied by stacking either two or three plates on the inclined surface to replace the single plate. The target with three aluminum plates reduced the impulse compared to one aluminum plate but no further impulse was achieved below that for the limiting case using a single steel sliding plate. Stacking sliding plates could have approximately the same effect as increasing the plate mass by protecting the underlying surface longer.

\subsection{Future work}

This dissertation examined high intensity granular impacts on several different structural designs and their response in order to reduce structural deformation and transferred impulse. Reducing structural deformation was accomplished by replacing a solid plate with an equivalent mass and material square honeycomb sandwich panel design with a strong core. The benefit of the square honeycomb sandwich panels over their monolithic plate counterparts at the same impulsive loading conditions was confirmed. The panel design for this dissertation was constructed with a very strong core and thus suffered minimal core compression. Reducing the transferred impulse was accomplished by the addition of lubricated sliding plates attached to the surface of an inclined (V-shape) target. Once impacted by the explosively accelerated granular media, these sliding plates acquired a momentum and began sliding along the underlying surface. The transfer of momentum to the sliding plates reduced the total impulse transferred to the underlying target surface.

It was beyond the scope of this study to investigate an optimized square honeycomb sandwich panel design. The design presented here is based on a very strong core topology and results indicate minimal core crushing, attributing the sandwich panel benefit to the increased stretching resistance of the core. Presumably a core that also allows for some degree of core compression would further reduce the impulse transferred to the back face sheet and result in less back face deflection. An optimization study of the honeycomb sandwich panel design would help to understand the panel's maximum benefit when subjected to high intensity loads. Further, it would be essential to understand the welding required to avoid the back face sheet debonding during loading. Investigation of the weld thickness required to retain the sandwich panel construction would be necessary for future testing. 
The sliding plate technique is a new structural design. As such, there are a variety of parametric studies that can be investigated to determine the effectiveness of these sliding plates. This dissertation only examined three variables, but variations of the target size should be explored. This would aid in understanding the effects of scaling this target design to larger targets as well as much larger charges which is important for determining future applications.

Further, future efforts for combining these two techniques into a V-shaped target design would be favorable in which an optimized sandwich panel design is fabricated as a V-shaped target and sliding plates are then attached to the inclined surface. This would exploit the benefit of the increased structural bending resistance of the sandwich panel design with the reduction of impulse resulting from the momentum transfer to sliding plates. 


\section{Appendix A. Effect of air particles in simulation}

Simulations were run in the IMPUTUS Afea Solver with and without air particles to understand the effects of the air particles in the simulation, and how these particles effect the sand particle propagation. Figure A1 shows snapshots of a contour plot of the radial sand speed for a simulation with air particles present (left) and without air particles (right) at equivalent times. Figure A1(a) shows the fastest moving (red) sand particles in both simulations traveled in the south-west direction (direction of detonation). However, there was a higher fraction of fast sand particles in the airless simulation. As the sand expanded over time, Figure A1(b)-(d), the radial sand speed of the simulations conducted with air particles decreased (less red particles) while in the airless simulation the sand speed appears to have been less affected. To quantify these differences, Figure A2 shows the maximum radial sand speed for the fastest sand particles in both simulations. The two plots show that after almost instantaneously acquiring a maximum speed at around $\sim 40 \mu$ s after detonation, the particle speed in the airless simulation remained constant while that with air reached a maximum velocity at between 80-150 $\mu$ s after detonations and was followed by a prolonged deceleration, consistent with momentum transfer to the air. 
Air particles
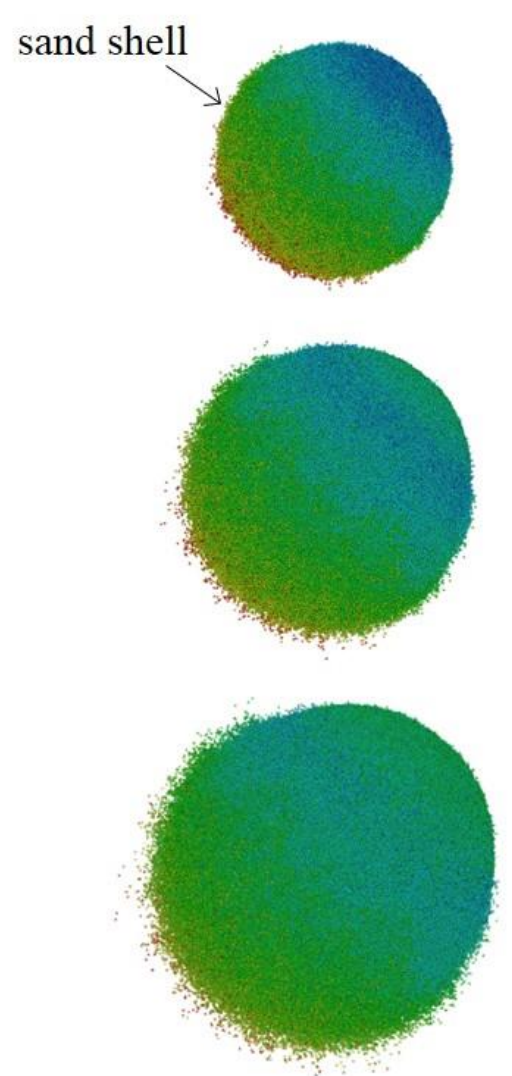

(c) $\mathrm{t}=200 \mu \mathrm{s}$

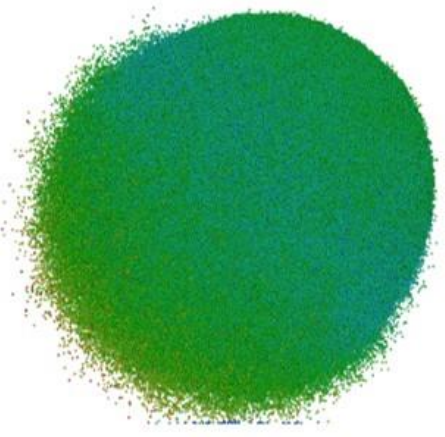

(a) $\mathrm{t}=100 \mu \mathrm{s}$

(b) $\mathrm{t}=150 \mu \mathrm{s}$

(d) $\mathrm{t}=250 \mu \mathrm{s}$
No air particles
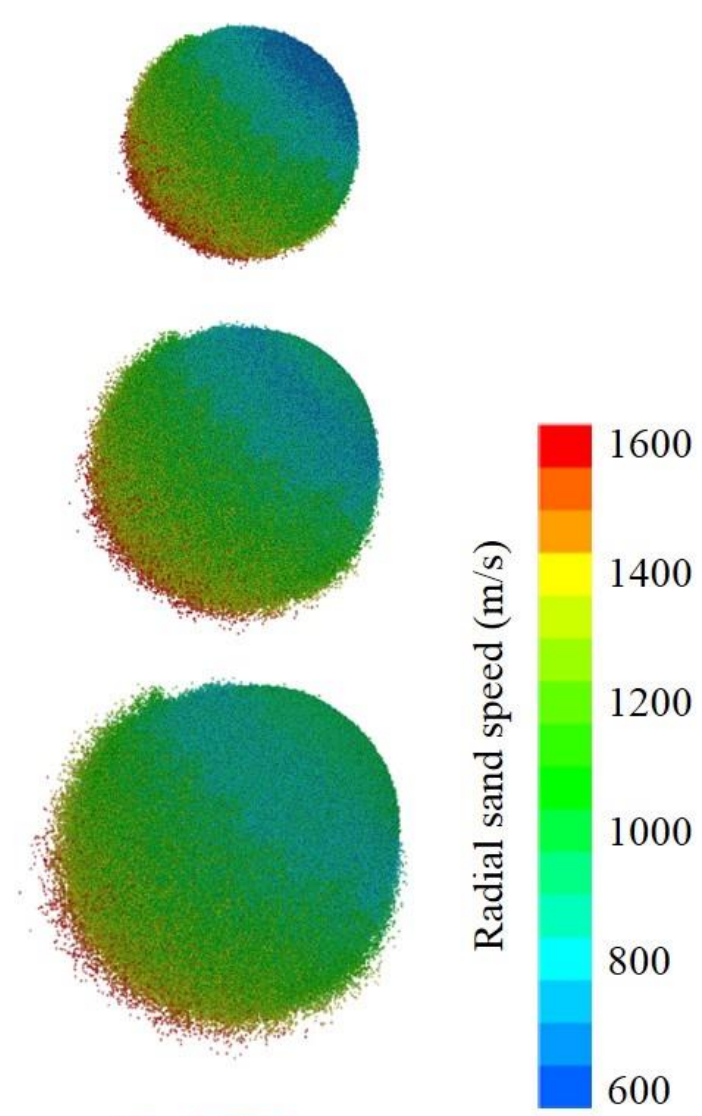

$$
\frac{\text { scale }}{304 \mathrm{~mm}}
$$

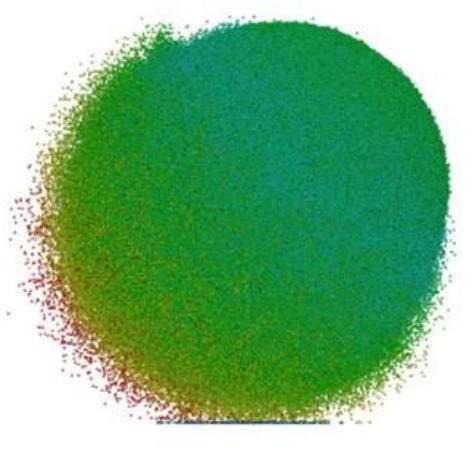

Figure A1. Simulation snapshots indicating the radial sand particle speeds for simulations with and without air particles present in the simulation. The presence of air particles significantly reduced the speed of the leading edge sand particles. 


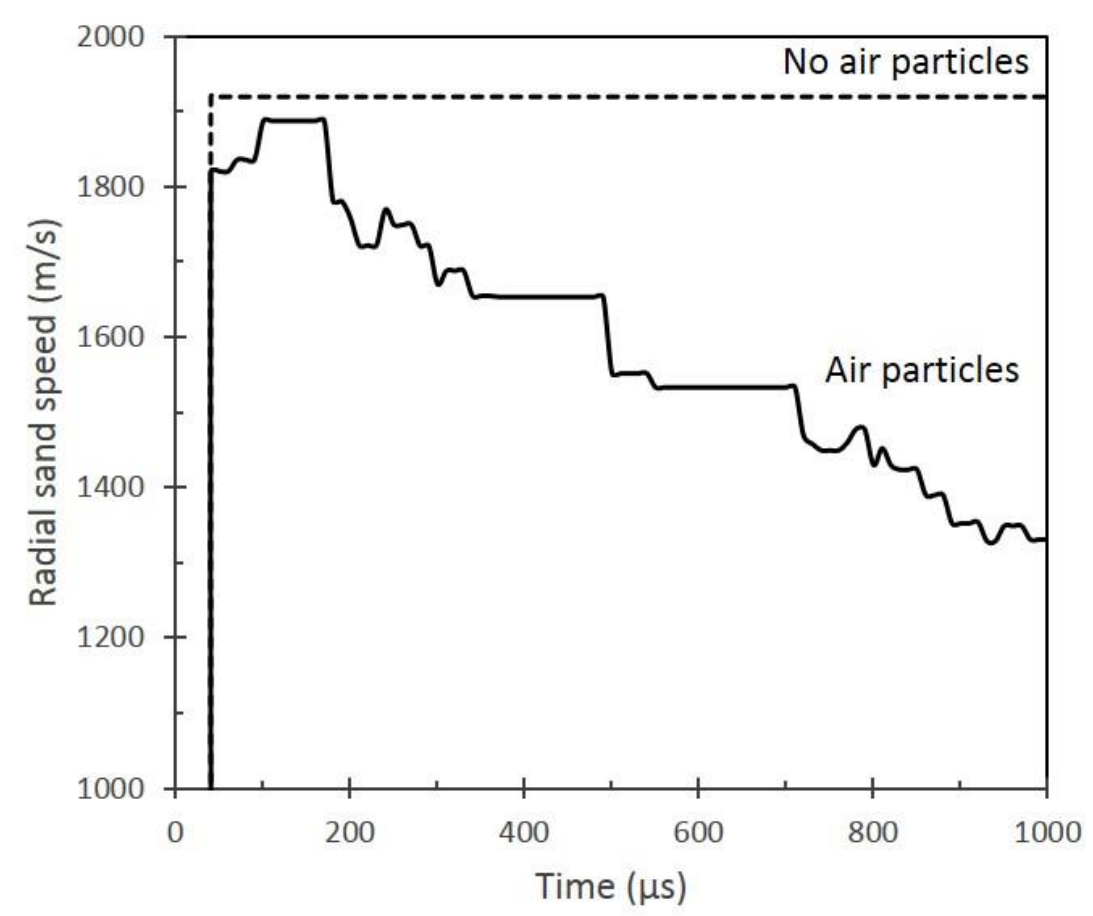

Figure A2. The maximum radial speed of sand particles as a function of time for simulations performed with and without air particles. 


\section{Appendix B. Rotation of I-beam support frame}

Simulations of the test have revealed that shortly after sand impact with the test plate had been completed, the I-beam and support frame of the test facility was inelastically bent and suffered substantial rotation. B1(a) shows the edge of the test plate connected to the A-36 grade steel support plate and I-beam flange of the test facility structure. Figure B1(b) shows the deformed geometry at $2.2 \mathrm{~ms}$ after detonation. This corresponded to the time required for the support plate to reach its maximum rotation (of about 5 degrees) from the initial edge orientation. The maximum effective stress on the I-beam support frame predicted by the simulation was $535 \mathrm{MPa}$.

B2(a) shows the time dependent displacement of three nodes located on the test plate, the support plate, and the I-beam picture frame, B1(b). The maximum displacement of node 22570 on the I-beam picture frame was $7.3 \mathrm{~mm}$ with permanent deformation of $3.8 \mathrm{~mm}$ and the permanent deformation of the support plate (node 20100) was $6.1 \mathrm{~mm}$. B2(b) shows the corresponding angle of rotation at these nodes as the support frame rotated and bent after impact. This rotation of the
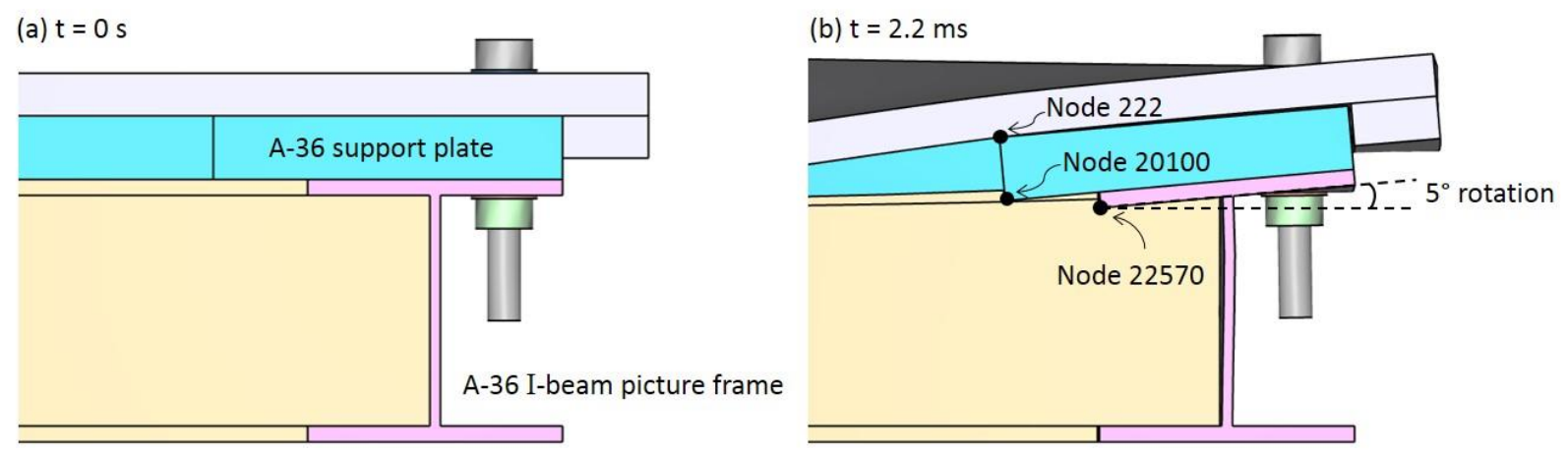

Figure B1. Simulated bending and rotation of the I-beam picture frame and support plate. (a) Shows the geometry prior to sand impact. (b) Shows the deformed structure at the time of maximum I-beam rotation $(t=2.2 \mathrm{~ms})$. 

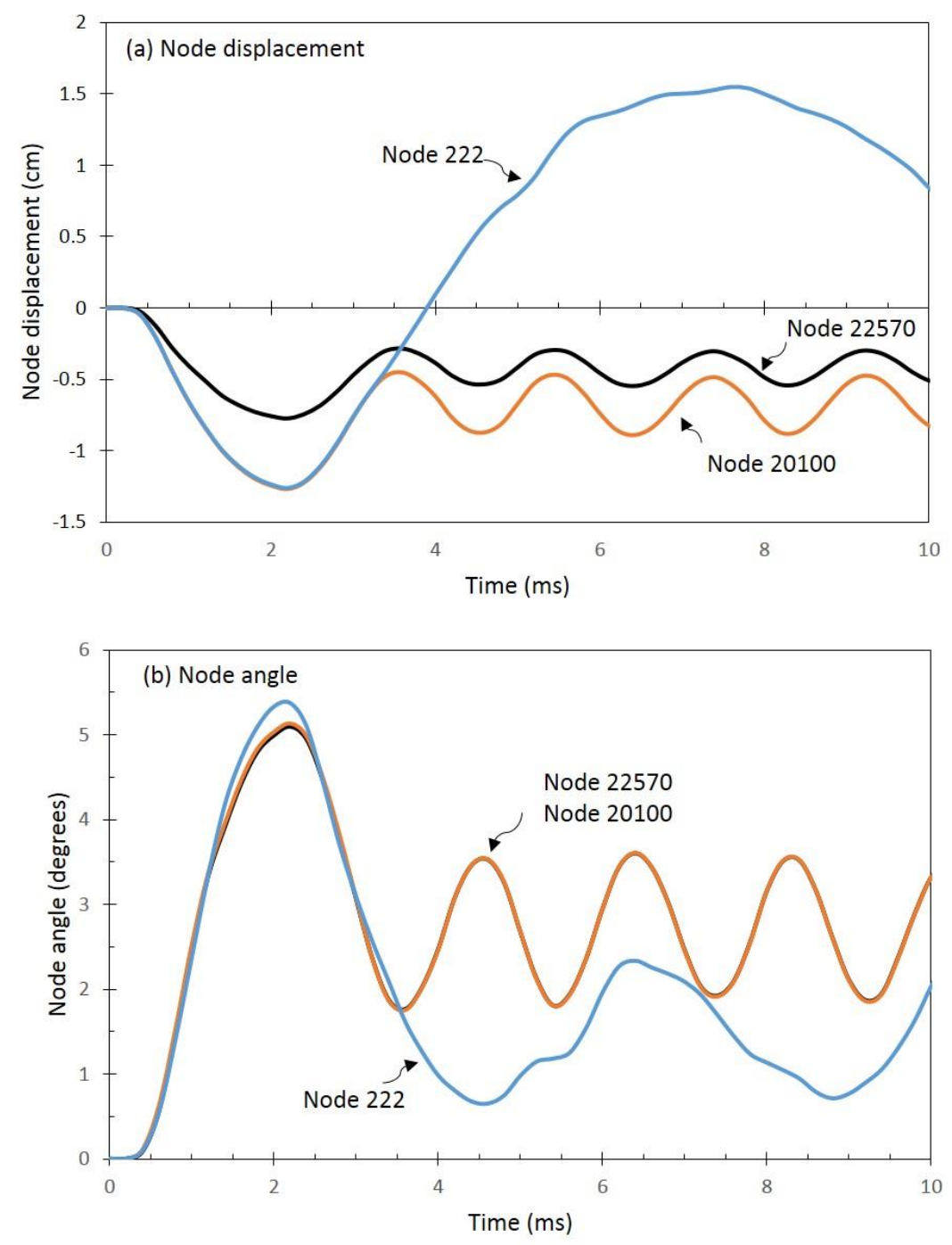

Figure B2. (a) Nodal displacements for node 22570 located on the rotating I-beam picture frame, node 20100 located on the support plate, and node 222 located on the 304 SS plate and (b) corresponding plot of the angle of rotation at the three nodes.

I-beam support frame resulted in development of a slightly more compliant edge restraint during the period of test plate oscillation, Figure 3.21.

In subsequent tests using a higher impulse loading, the I-beam flange near the corner of the picture frame support (parallel to the edge of the frame) ruptured, Figure B3(b). Figure B3(a) shows the effective plastic strain on the meshed I-beam for the loading condition used here at 5 $\mathrm{ms}$ after detonation. This area of maximum strain coincided with that of rupture and was consistent with the location of the point of rotation of the I-beam, Figure B3(b). 
(a) $\mathrm{t}=5 \mathrm{~ms}$
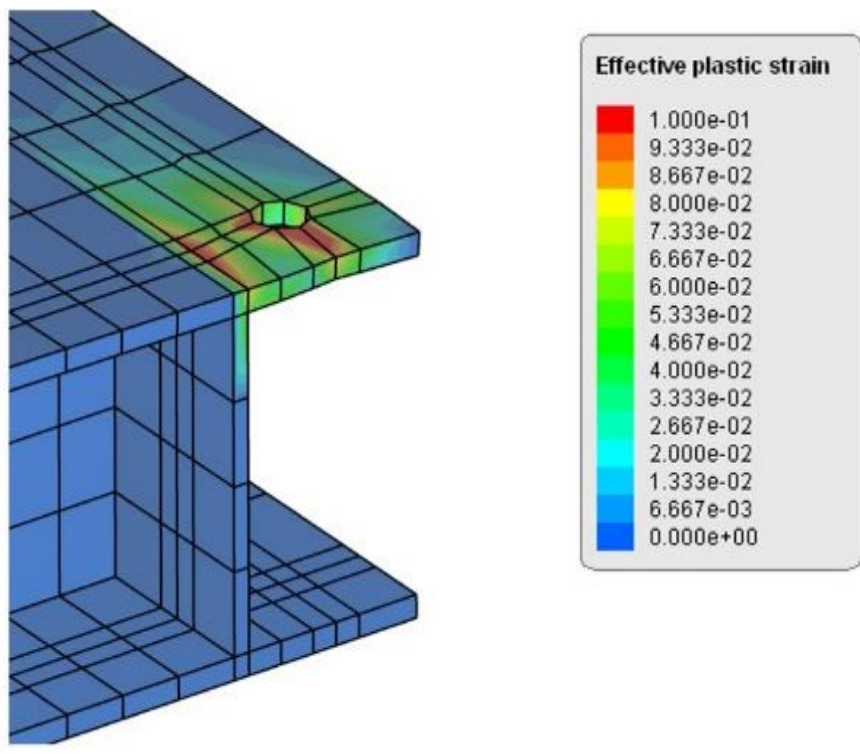

(b)
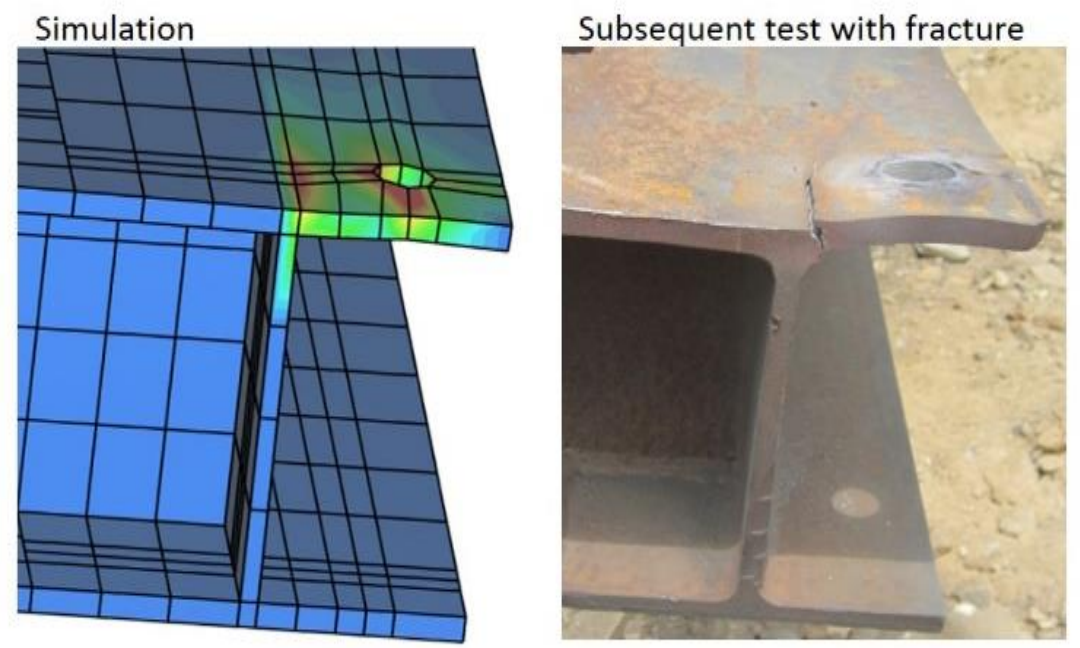

Figure B3. (a) Meshed corner of the I-beam frame with effective plastic strain contours showing the region of maximum strain in the flange near the I-beam web-flange transition. (b) Comparison of the effective plastic strain simulation with I-beam deformation and fracture location. 


\section{Appendix C. Shock front propagation of five test shots}

Upon detonation, a shock front propagates through the HE particles until reaching the interface between the HE and sand particles. A compressive shock is then launched into the wet sand and the shock propagates outward until reaching the outer surface of the test charge, thus breaking the acrylic shell. These shock propagations are shown for each test charge in Figure $\mathrm{C} 1$ (Shots 1-3) and Figure C2 (Shots 4 and 5). The acrylic shell is not shown in these images. The white dotted lines indicate the location of the detonation shock in the HE particles, and the black dotted lines mark the location of the compressive shock in the wet sand region. The changes in the inner and outer shell diameters can be seen for the different charge configurations. These changes influence the shock propagation time and thus the time for the outer acrylic shell to break. The propagation times were consistent with a C-4 detonation velocity of $8190 \mathrm{~m} / \mathrm{s}$ defined in the solver, $3600 \mathrm{~m} / \mathrm{s}$ shock speed in the wet synthetic (glass particle) sand and $3100 \mathrm{~m} / \mathrm{s}$ shock speed in a wet zirconia particle aggregate consistent with reported velocities for shock propagation in water-saturated granular media [89, 90]. These simulation times correlated with the times calculated in Table 4.3 where a handbook detonation velocity of $8040 \mathrm{~m} / \mathrm{s}$ [80] was used to estimate the detonation wave propagation time. 
Shot 1: $(80,152)$
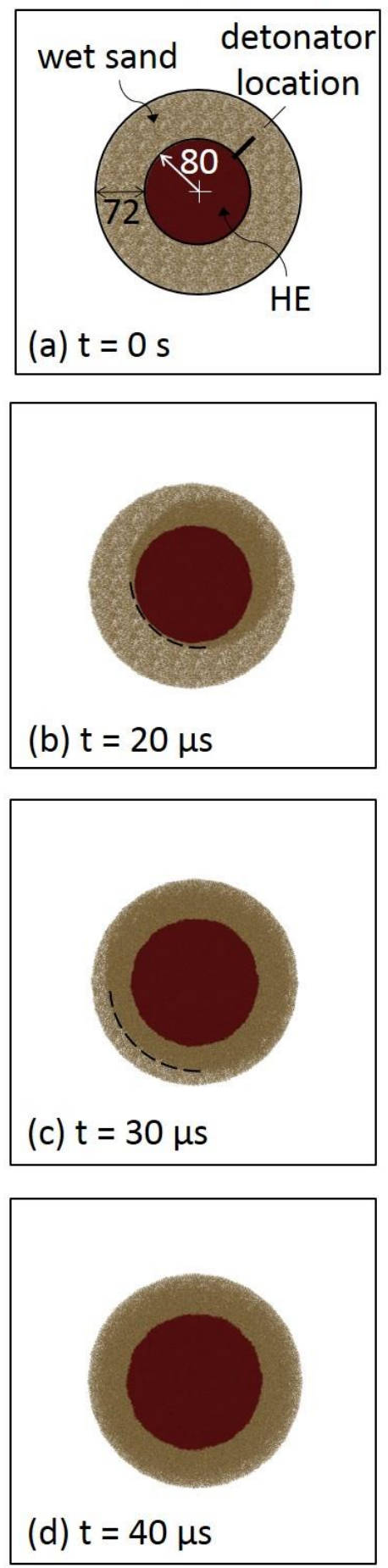

Shot 2: $(80,203)$
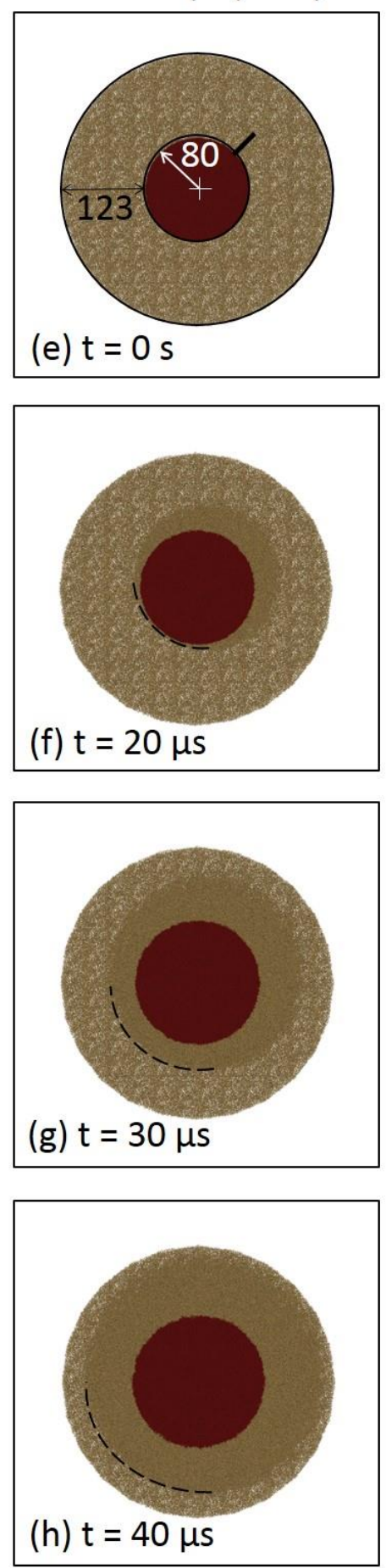

Shot 3: $(80,203)$
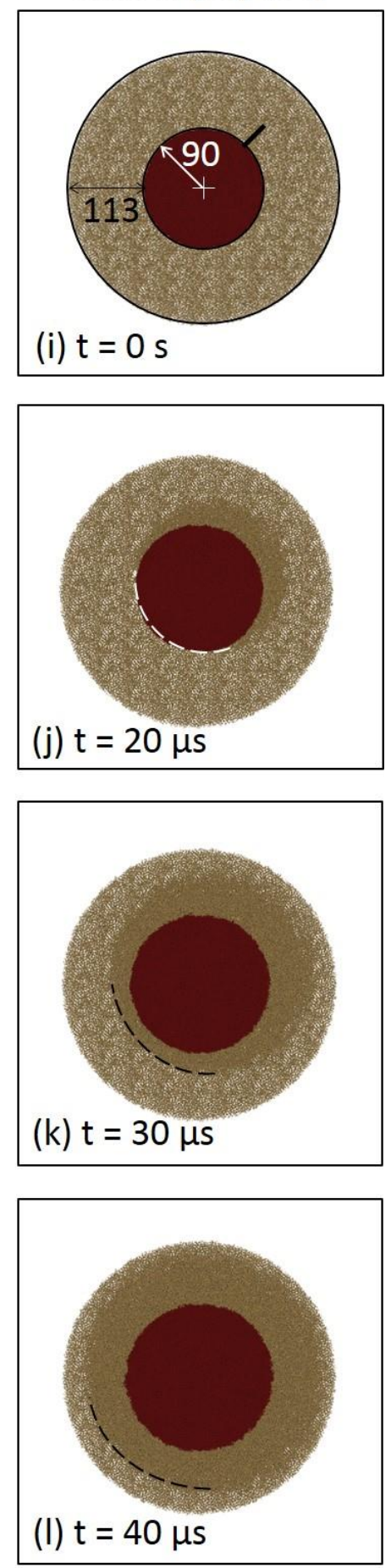

Figure C1. Simulated results showing shock front propagation through the three glass particle charges (Shots 1-3). The dashed white line in (j) shows the approximate location of the detonation front in the explosive while the dashed black lines show the position of the wet sand shock fronts. 
Shot 4: $(90,203)$

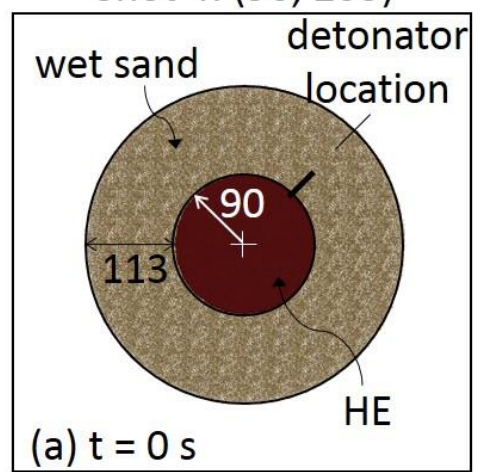

\section{(d)}
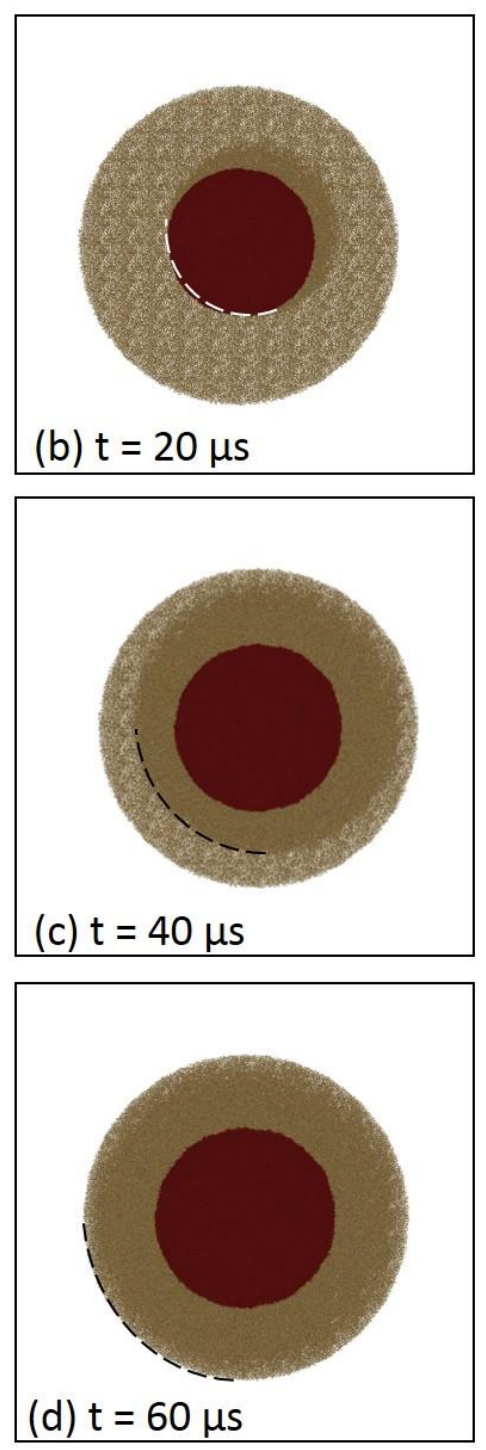

Shot 5: $(90,229)$
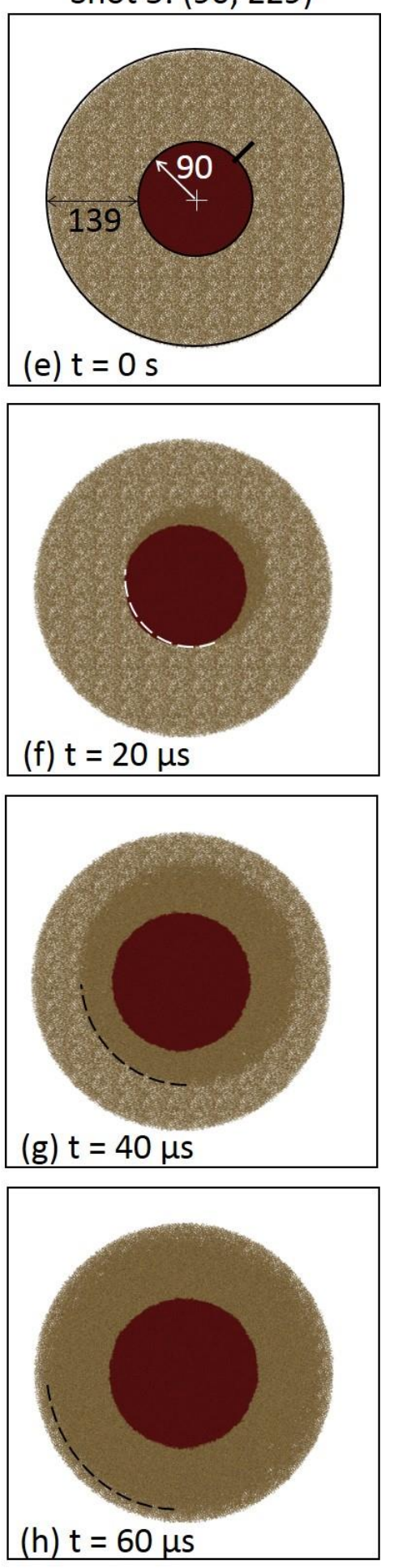

Figure C2. Simulated results showing shock front propagation through the two water-saturated zirconia charges (Shots 4 and 5). The dashed white line shows the approximate location of the detonation front in the explosive while the dashed black line shows the shock front locations in the granular media at various times after detonation. 


\section{Appendix D. Kolsky bar measurements for the sandwich panel tests}

The instrumented Kolsky bar was used to measure the applied pressure and impulse by granular media impacts to represent the loading experienced by the square honeycomb sandwich panels. The five charge configurations were almost identical to those used and then characterized for the testing on the solid plates (Chapter 4). The measured pressure-time waveforms and impulse-time response are shown in Figure D.1 for the glass particle test shots (Shots 1 and 3) and Figure D.2 for the zirconia test shots (Shots 4 and 5). The strain gauge signal corresponds to particle impacts that occurred $125 \mu$ s earlier on the front of the bar. Kolsky bar data was not recorded for test Shot 2 in this test series. Examination of each of the pressure-time signals revealed several distinct characteristics which correspond to four distinct regions of granular particle loading observed in the impulse-time response. Initial impacts resulting in an initial small pressure pulse, followed by small waveform oscillations to a much larger pressure peak resulting from impact by the densest particle region. These observations are consistent with those previously made for the same five charge configurations (Shots 1-5) that were tested on the solid plates. The four regions of impulse loading are indicated in Figure D.1(b) and Figure D.2(b) and are briefly discussed here with more details in Chapter 4.

The initial small pressure pulse correlates to an initial bump in impulse (Region I) that corresponds to initial impacts by the fastest, low density sand particles (the sand fingers observed in the high-speed videos). Since there is not a prominent sand fingering effect at the leading edge of the sand front for the zirconia test shots, the main sand front impact corresponds to the initial Region I impact. This is followed by a region of slowly increasing impulse (Region II). The start of Region II correlates to the impacts of the main sand front recorded in Table 5.3 for the glass particle test Shots 1-3. This period of gradually increasing impulse of Region II is followed by a sharp jump in impulse (Region III) at the arrival time of the pressure peak. Simulations reveal that this jump in impulse is a result of impacts by the low velocity, high density trailing granular particles. Impacts by the detonation products directly follow but contribute very little additional impulse as the impulse reaches a plateau value in Region IV. 

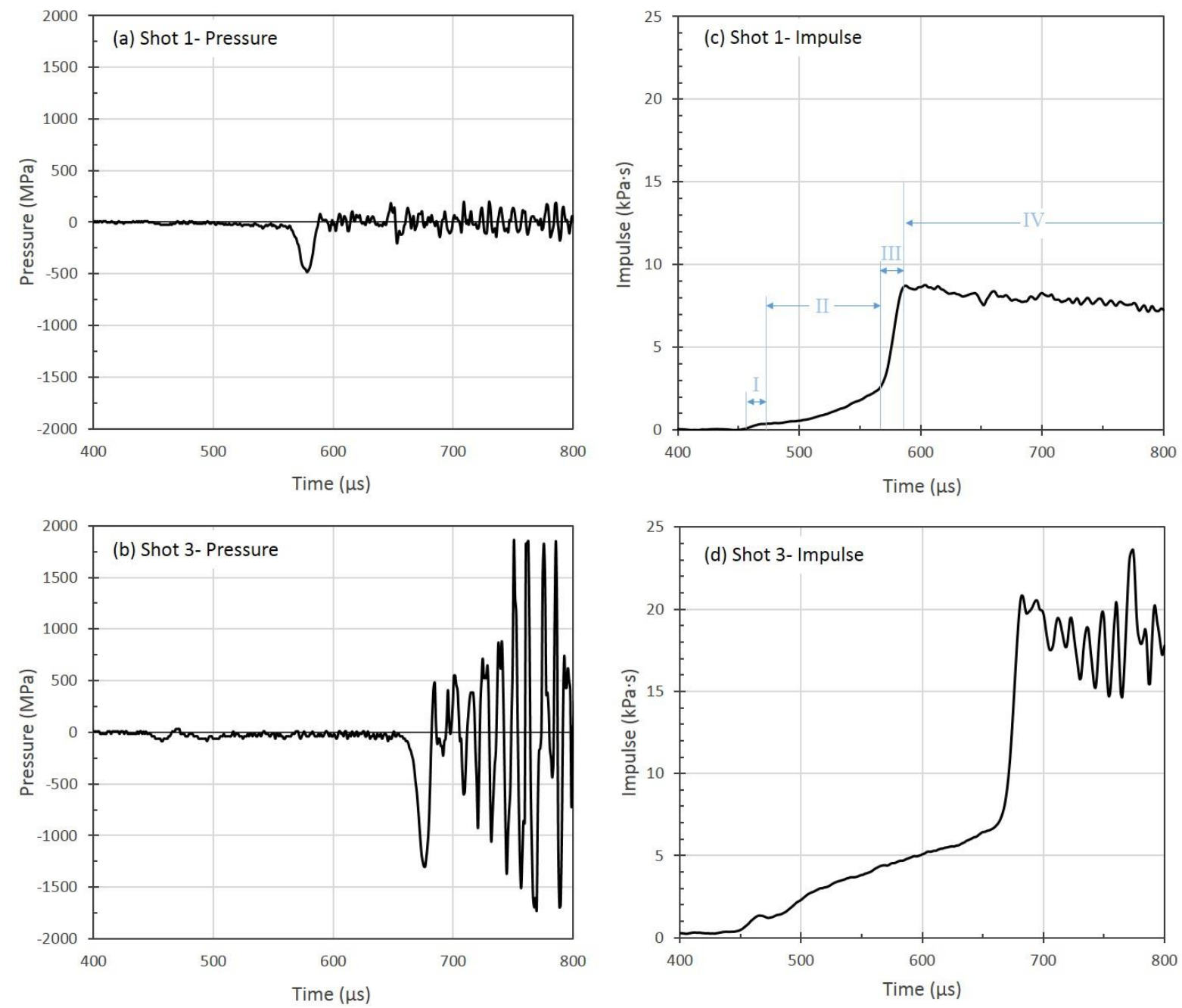

Figure D.1. Kolsky bar data for the glass microsphere particle tests. The waveforms in (a) and (b) show the pressure measured at the strain gage location for Shots 1 and 3. Figures $(c)$ and $(d)$ show the impulse for test Shots 1 and 3. 

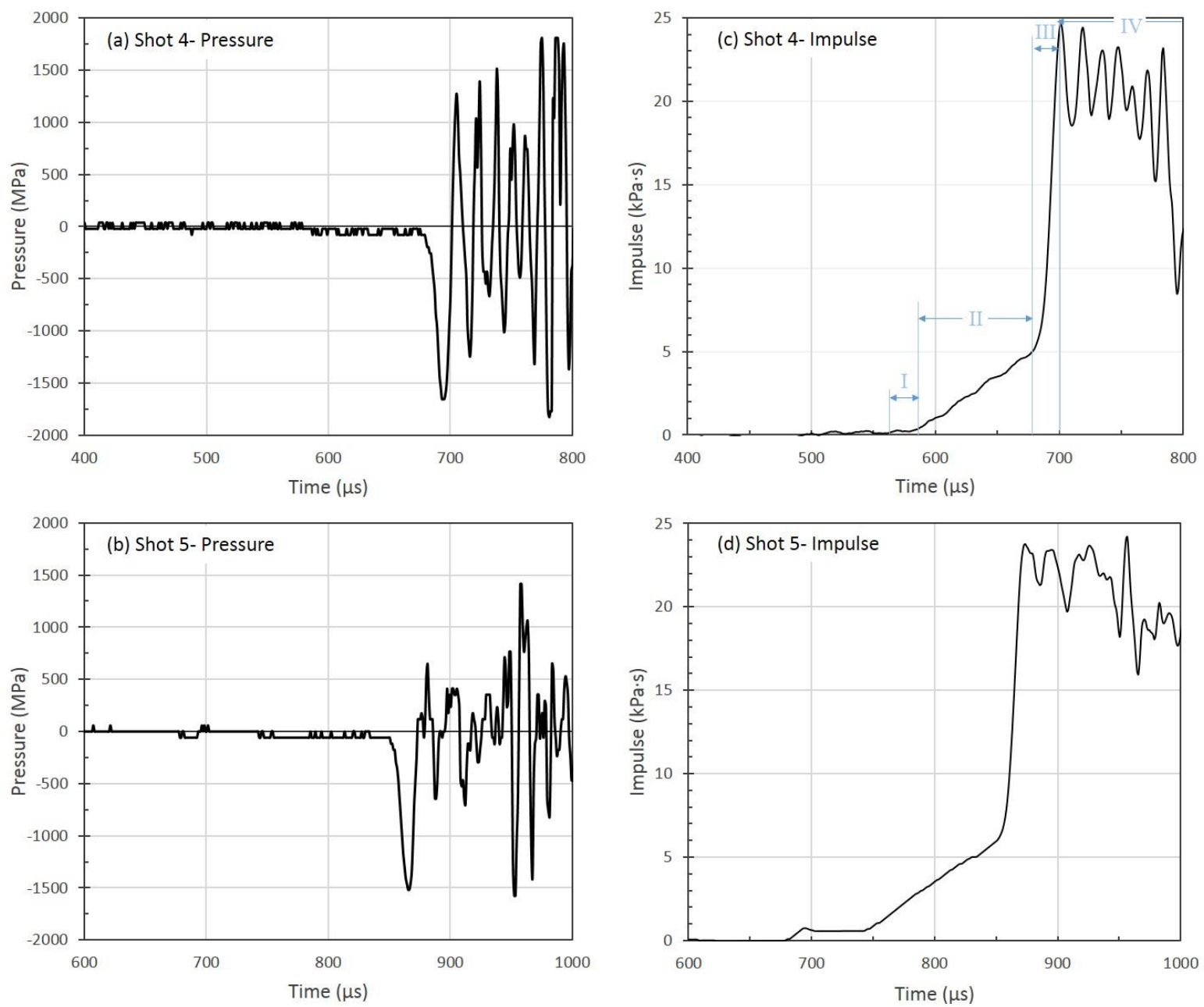

Figure D.2. Kolsky bar data for the zirconia particle tests. The waveforms in (a) and (b) show the pressure measured at the strain gage location for Shots 4 and 5. Figures (c) and (d) show the impulse for test Shots 4 and 5. 


\section{References}

[1] A. Ramasamy, A.-M. Hill, A. Hepper, A.M. Bull, J. Clasper, Blast mines: physics, injury mechanisms and vehicle protection, Journal of the Royal Army Medical Corps, 155 (2009) 258-264.

[2] D.M. Bergeron, R. Walker, C. Coffey, Detonation of 100-gram Anti-personnel Mine Surrogate Charges in Sand, a Test Case for Computer Code Validation, National Defence, Defence Research Establishment Suffield, 1998.

[3] V. Deshpande, R. McMeeking, H. Wadley, A. Evans, Constitutive model for predicting dynamic interactions between soil ejecta and structural panels, Journal of the Mechanics and Physics of Solids, 57 (2009) 1139-1164.

[4] K.P. Dharmasena, H.N. Wadley, K. Williams, Z. Xue, J.W. Hutchinson, Response of metallic pyramidal lattice core sandwich panels to high intensity impulsive loading in air, International journal of impact engineering, 38 (2011) 275-289.

[5] A. Vaziri, J.W. Hutchinson, Metal sandwich plates subject to intense air shocks, International Journal of Solids and Structures, 44 (2007) 2021-2035.

[6] Z. Xue, J.W. Hutchinson, Preliminary assessment of sandwich plates subject to blast loads, International Journal of Mechanical Sciences, 45 (2003) 687-705.

[7] N. Fleck, V. Deshpande, The resistance of clamped sandwich beams to shock loading, Journal of Applied Mechanics, 71 (2004) 386-401.

[8] Z. Wei, K. Dharmasena, H. Wadley, A. Evans, Analysis and interpretation of a test for characterizing the response of sandwich panels to water blast, International journal of impact engineering, 34 (2007) 16021618.

[9] K. Dharmasena, D. Queheillalt, H. Wadley, P. Dudt, Y. Chen, D. Knight, A. Evans, V. Deshpande, Dynamic compression of metallic sandwich structures during planar impulsive loading in water, European Journal of Mechanics-A/Solids, 29 (2010) 56-67.

[10] J.W. Hutchinson, Z. Xue, Metal sandwich plates optimized for pressure impulses, International Journal of Mechanical Sciences, 47 (2005) 545-569.

[11] W. Fourney, U. Leiste, R. Bonenberger, D. Goodings, Mechanism of loading on plates due to explosive detonation, Fragblast, 9 (2005) 205-217.

[12] D. Fox, X. Huang, D. Jung, W. Fourney, U. Leiste, J. Lee, The response of small scale rigid targets to shallow buried explosive detonations, International journal of impact engineering, 38 (2011) 882-891.

[13] R.L. Holloman, V. Deshpande, H.N.G. Wadley, Impulse transfer during sand impact with a solid block, International journal of impact engineering, 76 (2015) 98-117.

[14] R.L. Holloman, V. Deshpande, H.N.G. Wadley, Impulse transfer during sand impact with a cellular structure, International journal of impact engineering, 82 (2015) 36-58.

[15] E.G. Pickering, S.C.K. Yuen, G. Nurick, P. Haw, The response of quadrangular plates to buried charges, International journal of impact engineering, 49 (2012) 103-114. 
[16] A. Neuberger, S. Peles, D. Rittel, Scaling the response of circular plates subjected to large and closerange spherical explosions. Part II: buried charges, International journal of impact engineering, 34 (2007) 874-882.

[17] K.P. Dharmasena, H.N. Wadley, T. Liu, V.S. Deshpande, The dynamic response of edge clamped plates loaded by spherically expanding sand shells, International journal of impact engineering, 62 (2013) 182195.

[18] T. Børvik, A. Hanssen, M. Langseth, L. Olovsson, Response of structures to planar blast loads-A finite element engineering approach, Computers \& Structures, 87 (2009) 507-520.

[19] T. Børvik, L. Olovsson, A. Hanssen, K. Dharmasena, H. Hansson, H. Wadley, A discrete particle approach to simulate the combined effect of blast and sand impact loading of steel plates, Journal of the Mechanics and Physics of Solids, 59 (2011) 940-958.

[20] L. Olovsson, A.G. Hanssen, T. Børvik, M. Langseth, A particle-based approach to close-range blast loading, European Journal of Mechanics - A/Solids, 29 (2010) 1-6.

[21] T. Ngo, P. Mendis, A. Gupta, J. Ramsay, Blast loading and blast effects on structures-an overview, Electronic Journal of Structural Engineering, 7 (2007) 76-91.

[22] I. Cullis, Blast waves and how they interact with structures, Journal of the Royal Army Medical Corps, 147 (2001) 16-26.

[23] B.M. Dobratz, Properties of chemical explosives and explosive simulants, in, comp. and ed.; California Univ., Livermore (USA). Lawrence Livermore Lab., 1972.

[24] J.M. Dewey, The shape of the blast wave: studies of the Friedlander equation, in: Proceeding of the 21st International Symposium on Military Aspects of Blast and Shock (MABS), Israel, 2010, pp. 1-9.

[25] M.D. Goel, V.A. Matsagar, A.K. Gupta, S. Marburg, An abridged review of blast wave parameters, Defence Science Journal, 62 (2012) 300-306.

[26] G. Taylor, The pressure and impulse of submarine explosion waves on plates, The scientific papers of GI Taylor, 3 (1963) 287-303.

[27] N. Kambouchev, L. Noels, R. Radovitzky, Nonlinear compressibility effects in fluid-structure interaction and their implications on the air-blast loading of structures, Journal of Applied Physics, 100 (2006) 063519.

[28] J.W. Hutchinson, Energy and momentum transfer in air shocks, Journal of Applied Mechanics, 76 (2009) 051307.

[29] Z. Xue, J.W. Hutchinson, A comparative study of impulse-resistant metal sandwich plates, International journal of impact engineering, 30 (2004) 1283-1305.

[30] H. Wadley, K. Dharmasena, Y. Chen, P. Dudt, D. Knight, R. Charette, K. Kiddy, Compressive response of multilayered pyramidal lattices during underwater shock loading, International journal of impact engineering, 35 (2008) 1102-1114.

[31] Z. Wei, V. Deshpande, A. Evans, K. Dharmasena, D. Queheillalt, H. Wadley, Y. Murty, R. Elzey, P. Dudt, Y. Chen, The resistance of metallic plates to localized impulse, Journal of the Mechanics and Physics of Solids, 56 (2008) 2074-2091.

[32] T. Liu, N. Fleck, H. Wadley, V. Deshpande, The impact of sand slugs against beams and plates: Coupled discrete particle/finite element simulations, Journal of the Mechanics and Physics of Solids, 61 (2013) 1798-1821. 
[33] J. Akhavan, The chemistry of explosives, Royal Society of Chemistry, 2004.

[34] M. Grujicic, B. Pandurangan, R. Qiao, B. Cheeseman, W. Roy, R. Skaggs, R. Gupta, Parameterization of the porous-material model for sand with different levels of water saturation, Soil Dynamics and Earthquake Engineering, 28 (2008) 20-35.

[35] S.L. Hlady, Effect of soil parameters on landmine blast, in: 18th International symposium on the Military Aspects of Blast and Shock (Bad Reichenhall, Germany, 2004.

[36] J. Rimoli, B. Talamini, J. Wetzel, K. Dharmasena, R. Radovitzky, H. Wadley, Wet-sand impulse loading of metallic plates and corrugated core sandwich panels, International journal of impact engineering, 38 (2011) 837-848.

[37] H.N. Wadley, T. Børvik, L. Olovsson, J.J. Wetzel, K.P. Dharmasena, O.S. Hopperstad, V. Deshpande, J.W. Hutchinson, Deformation and fracture of impulsively loaded sandwich panels, Journal of the Mechanics and Physics of Solids, 61 (2013) 674-699.

[38] G.I. Kerley, Numerical modeling of buried mine explosions, in, DTIC Document, 2001.

[39] J. Wang, Simulation of landmine explosion using LS-DYNA3D software: benchmark work of simulation of explosion in soil and air, in, DTIC Document, 2001.

[40] J.D. Baum, E. Mestreau, H. Luo, R. Löhner, D. Pelessone, M.E. Giltrud, J.K. Gran, Modeling of nearfield blast wave evolution, in: Collection of Technical Papers-44th AIAA Aerospace Sciences Meeting, 2006, pp. 2310-2320.

[41] Z. Wang, Y. Lu, H. Hao, K. Chong, A full coupled numerical analysis approach for buried structures subjected to subsurface blast, Computers \& Structures, 83 (2005) 339-356.

[42] A.D. Gupta, Modeling and Analysis of a 3-D Asymmetric Mine-Soil-Hull Floor Interaction Problems with Mine Buried in Dry and Wet Sand, in, DTIC Document, 2001.

[43] LSTC, LS-DYNA Keyword User's Manual, Version 971. Livermore Software Technology Corporation, (2007).

[44] Z. Wang, H. Hao, Y. Lu, A three-phase soil model for simulating stress wave propagation due to blast loading, International Journal for Numerical and Analytical Methods in Geomechanics, 28 (2004) 33-56.

[45] L. Laine, A. Sandvik, Derivation of mechanical properties for sand, in: Proceedings of the 4th AsiaPacific Conference on Shock and impact loads on structures, CI-Premier PTE LTD, Singapore, ANSYS Inc., 2001, pp. 368.

[46] D.C. Drucker, W. Prager, Soil mechanics and plastic analysis or limit design, Quarterly of applied mathematics, 10 (1952) 157-165.

[47] Z. Wang, Y. Lu, Numerical analysis on dynamic deformation mechanism of soils under blast loading, Soil Dynamics and Earthquake Engineering, 23 (2003) 705-714.

[48] S. Pingle, N. Fleck, H. Wadley, V. Deshpande, Discrete element calculations of the impact of a sand column against rigid structures, International journal of impact engineering, 45 (2012) 74-89.

[49] S. Park, T. Uth, N. Fleck, H. Wadley, V. Deshpande, Sand column impact onto a Kolsky pressure bar, International journal of impact engineering, 62 (2013) 229-242.

[50] A.G. Hanssen, L. Olovsson, Welcome to IMPETUS AFEA, in, IMPETUS AFEA, Flekkefjord, Norway. 
[51] J. Attwood, S. Khaderi, K. Karthikeyan, N. Fleck, H. Wadley, V. Deshpande, The out-of-plane compressive response of Dyneema ${ }^{\circledR}$ composites, Journal of the Mechanics and Physics of Solids, 70 (2014) 200-226.

[52] D. Frost, C. Ornthanalai, Z. Zarei, V. Tanguay, F. Zhang, Particle momentum effects from the detonation of heterogeneous explosives, Journal of Applied Physics, 101 (2007) 113529.

[53] F. Zhang, D. Frost, P. Thibault, S. Murray, Explosive dispersal of solid particles, Shock Waves, 10 (2001) 431-443.

[54] A. Kyner, K. Dharmasena, K. Williams, V.S. Deshpande, H.N.G. Wadley, High intensity impulsive loading by explosively accelerated granular matter, International Journal of Impact Engineering, (2017).

[55] G. Nurick, G. Shave, The deformation and tearing of thin square plates subjected to impulsive loadsan experimental study, International journal of impact engineering, 18 (1996) 99-116.

[56] M. Olson, G. Nurick, J. Fagnan, Deformation and rupture of blast loaded square plates-predictions and experiments, International journal of impact engineering, 13 (1993) 279-291.

[57] M. Grujicic, B. Pandurangan, B. Cheeseman, The effect of degree of saturation of sand on detonation phenomena associated with shallow-buried and ground-laid mines, Shock and Vibration, 13 (2006) 41-61.

[58] N. Jacob, G. Nurick, G. Langdon, The effect of stand-off distance on the failure of fully clamped circular mild steel plates subjected to blast loads, Engineering Structures, 29 (2007) 2723-2736.

[59] D.L. Frost, Y. Grégoire, O. Petel, S. Goroshin, F. Zhang, Particle jet formation during explosive dispersal of solid particles, Physics of Fluids (1994-present), 24 (2012) 091109.

[60] T. Xu, F.-S. Lien, H. Ji, F. Zhang, Formation of particle jetting in a cylindrical shock tube, Shock Waves, 23 (2013) 619-634.

[61] A. Milne, C. Parrish, I. Worland, Dynamic fragmentation of blast mitigants, Shock Waves, 20 (2010) 41-51.

[62] L.C. Taylor, W.L. Fourney, D.P. Lathrop, Surface Instabilities from Buried Explosives, in, DTIC Document, 2009.

[63] M. Brouillette, The richtmyer-meshkov instability, Annual Review of Fluid Mechanics, 34 (2002) 445468.

[64] C.E. Needham, Instabilities, in: Blast Waves, Springer, 2010, pp. 127-137.

[65] R.L. Holmes, G. Dimonte, B. Fryxell, M.L. Gittings, J.W. Grove, M. Schneider, D.H. Sharp, A.L. Velikovich, R.P. Weaver, Q. Zhang, Richtmyer-Meshkov instability growth: experiment, simulation and theory, Journal of Fluid Mechanics, 389 (1999) 55-79.

[66] J.W. Grove, R. Holmes, D.H. Sharp, Y. Yang, Q. Zhang, Quantitative theory of Richtmyer-Meshkov instability, Physical review letters, 71 (1993) 3473.

[67] V. Rodriguez, R. Saurel, G. Jourdan, L. Houas, Solid-particle jet formation under shock-wave acceleration, Physical Review E, 88 (2013) 063011.

[68] V. Rodriguez, R. Saurel, G. Jourdan, L. Houas, External front instabilities induced by a shocked particle ring, Physical Review E, 90 (2014) 043013.

[69] V. Rodriguez, R. Saurel, G. Jourdan, L. Houas, Impulsive dispersion of a granular layer by a weak blast wave, Shock Waves, (2016) 1-12. 
[70] K. Kandan, S. Khaderi, H.N.G. Wadley, V.S. Deshpande, Instabilities in shock loaded granular media, Journal of the Mechanics and Physics of Solids, Submitted (2017).

[71] S. Chung Kim Yuen, G.S. Langdon, G.N. Nurick, E.G. Pickering, V.H. Balden, Response of V-shape plates to localised blast load: Experiments and numerical simulation, International journal of impact engineering, 46 (2012) 97-109.

[72] C.E. Anderson, T. Behner, C.E. Weiss, Mine blast loading experiments, International journal of impact engineering, 38 (2011) 697-706.

[73] W. Fourney, H. Leiste, A. Hauck, D. Jung, Distribution of specific impulse on vehicles subjected to IED's, International Journal for Blasting \& Fragmentation, 4 (2010) 117-134.

[74] R.R. Sahu, P.K. Gupta, Blast diffusion by different shapes of vehicle hull, International Journal of Automotive Engineering and Technologies, 2 (2013) 130-139.

[75] M.-A. Saeimi-Sadigh, A. Paykani, A. Afkar, D. Aminollah, Design and energy absorption enhancement of vehicle hull under high dynamic loads, Journal of Central South University, 21 (2014) 1307-1312.

[76] C. Lester, Protection of Light Skinned Vehicles Against Landmines-A Review, in, DTIC Document, 1997.

[77] M. Glauert, The wall jet, Journal of Fluid Mechanics, 1 (1956) 625-643.

[78] T. Uth, H.N.G. Wadley, V.S. Deshpande, The effect of inclination and stand-off on the dynamic response of beams impacted by slugs of a granular material, International Journal of Solids and Structures, 56-57 (2015) 154-174.

[79] A. Goel, T. Uth, H. Wadley, V. Deshpande, Effect of surface properties on momentum transfer to targets impacted by high-velocity sand slugs, International journal of impact engineering, (2017).

[80] M. Explosives, Department of the Army Technical Manual, in, TM 9-1300-214, September, 1984.

[81] J.A. Simmons, E. Drescher-Krasicka, H. Wadley, Leaky axisymmetric modes in infinite clad rods. I, The Journal of the Acoustical Society of America, 92 (1992) 1061-1090.

[82] J.C. Maxwell, Illustrations of the dynamical theory of gases., The London, Edinburgh, and Dublin Philosophical Magazine and Journal of Science, 19 (1860) 19-32.

[83] J. Dean, C. Dunleavy, P. Brown, T. Clyne, Energy absorption during projectile perforation of thin steel plates and the kinetic energy of ejected fragments, International journal of impact engineering, 36 (2009) $1250-1258$.

[84] L. Mori, S. Lee, Z. Xue, A. Vaziri, D. Queheillalt, K. Dharmasena, H. Wadley, J. Hutchinson, H. Espinosa, Deformation and fracture modes of sandwich structures subjected to underwater impulsive loads, Journal of mechanics of materials and structures, 2 (2007) 1981-2006.

[85] E.L. Guzas, C.J. Earls, Simulating blast effects on steel beam-column members: Methods, Computers \& Structures, 89 (2011) 2133-2148.

[86] J.D. Cinnamon, A.N. Palazotto, Z. Kennan, Material characterization and development of a constitutive relationship for hypervelocity impact of 1080 Steel and VascoMax 300, International journal of impact engineering, 33 (2006) 180-189.

[87] U.K. Viswanathan, R. Kishore, M.K. Asundi, Effect of thermal cycling on the mechanical properties of 350-grade maraging steel, MMTA, 27 (1996) 757-761. 
[88] G. Cohen, P. Gilles, S. Segonds, M. Mousseigne, P. Lagarrigue, Thermal and mechanical modeling during dry turning operations, The International Journal of Advanced Manufacturing Technology, 58 (2012) 133-140.

[89] M. Prasad, M. Zimmer, P. Berge, B. Bonner, Laboratory measurements of velocity and attenuation in sediments, University of California, Lawrence Livermore National Laboratory, Report, 205155 (2004).

[90] D. Chapman, K. Tsembelis, W. Proud, The behavior of water saturated sand under shock-loading, in: Proceedings of the 2006 SEM Annual Conference and Exposition on Experimental and applied mechanics, 2006, pp. 834-840.

[91] J.R. Taylor, Error Analysis: The study of uncertainties in physical measurements, Sausalito: University Science Book, (1997).

[92] R. Davies, A critical study of the Hopkinson pressure bar, Philosophical Transactions of the Royal Society of London A: Mathematical, Physical and Engineering Sciences, 240 (1948) 375-457.

[93] A.D. Puckett, M. Peterson, Individual longitudinal Pochhammer-Chree modes in observed experimental signals, Acoustics Research Letters Online, 6 (2005) 268-273.

[94] G. Nurick, M. Gelman, N. Marshall, Tearing of blast loaded plates with clamped boundary conditions, International journal of impact engineering, 18 (1996) 803-827.

[95] S. Mendes, H. Opat, Tearing and shear failures in explosively loaded clamped beams, Exp. Mech, 13 (1973) 480-486.

[96] D. Radford, G. McShane, V. Deshpande, N. Fleck, Dynamic compressive response of stainless-steel square honeycombs, Journal of Applied Mechanics, 74 (2007) 658-667.

[97] A. Goel, T. Uth, T. Liu, H. Wadley, V. Deshpande, Coupled discrete/continuum simulations of the impact of granular slugs with clamped beams: Stand-off effects, Mechanics of Materials, (2017).

[98] A. Goel, T. Uth, H.N.G. Wadley, V.S. Deshpande, Effect of surface properties on momentum transfer to targets impacted by high velocity sand slugs, Submitted for publiction., (2016).

[99] K. Dharmasena, D. Queheillalt, H. Wadley, Y. Chen, P. Dudt, D. Knight, Z. Wei, A. Evans, Dynamic response of a multilayer prismatic structure to impulsive loads incident from water, International journal of impact engineering, 36 (2009) 632-643.

[100] Molykote M-77 Solid Lubricant Paste, in: Form No. 71-0186E-01, Dow Corning, 2014.

[101] T. Børvik, S. Dey, L. Olovsson, Penetration of granular materials by small-arms bullets, International journal of impact engineering, 75 (2015) 123-139.

[102] J.K. Holmen, L. Olovsson, T. Børvik, Discrete modeling of low-velocity penetration in sand, Computers and Geotechnics, 86 (2017) 21-32.

[103] J.K. Holmen, T. Børvik, O.S. Hopperstad, Experiments and simulations of empty and sand-filled aluminum alloy panels subjected to ballistic impact, Engineering Structures, 130 (2017) 216-228.

[104] G.R. Johnson, W.H. Cook, A constitutive model and data for metals subjected to large strains, high strain rates and high temperatures, in: Proceedings of the 7th International Symposium on Ballistics, The Hague, The Netherlands, 1983, pp. 541-547.

[105] T. Uth, V. Deshpande, Response of clamped sandwich beams subjected to high-velocity impact by sand slugs, International journal of impact engineering, 69 (2014) 165-181. 
[106] S. Seo, O. Min, H. Yang, Constitutive equation for Ti-6Al-4V at high temperatures measured using the SHPB technique, International journal of impact engineering, 31 (2005) 735-754.

[107] L. Westerling, P. Lundberg, B. Lundberg, Tungsten long-rod penetration into confined cylinders of boron carbide at and above ordnance velocities, International journal of impact engineering, 25 (2001) 703-714. 